2. To: (Receiving Organization)

Distribution

5. Proj-/Prog./Dept./Div.:

Tank 241-B-108/Waste

Management/PPC/AS

8. Originator Remarks:

This document is being released into the supporting document system for retrievability purposes.

11. Receiver Remarks:

For release.

\section{From: (originating Organization) Production Planning and Control}

6. Design Authority/ Design Agent/Cog. Engr.:

Jennifer L. Nuzum
4. Related EDT No.:

N/A

7. Purchase order No.:

$N / A$

9. Equip./Component No.: $N / A$

10. Systen/Bldg./Facility: 241-B-108

12. Major Assm. Dwg. No.: N/A

13. Permit/Permit Application No.: N/A

14. Required Response Date: $03 / 04 / 97$

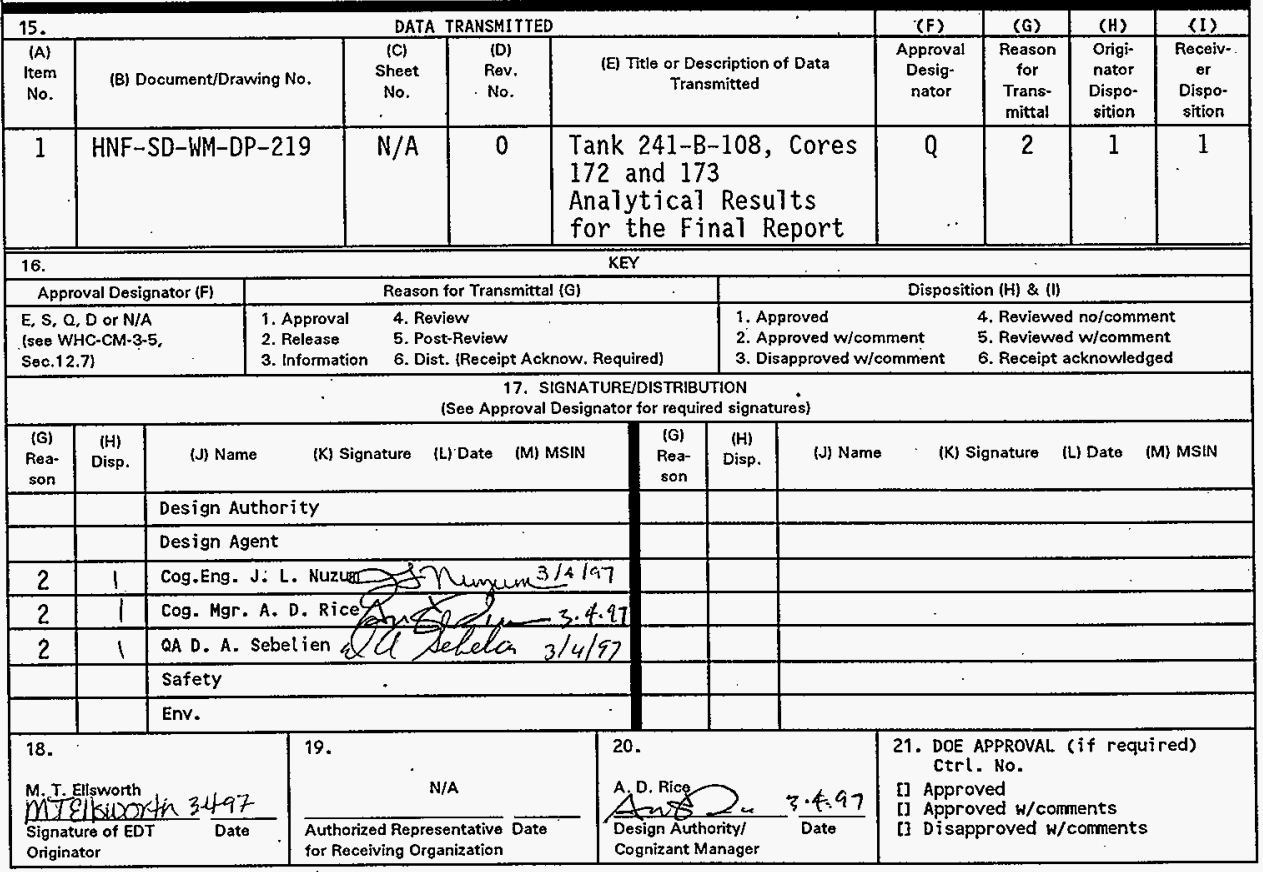

BD-7400-172-2(05/96) GEF097 


\title{
Tank 241-B-108, Cores 172 and 173 Analytical Results for the Final Report
}

\author{
Jennifer L. Nuzum \\ Rust Federal Services of Hanford, Inc., Richland, WA 99352 \\ U.S. Department of Energy Contract DE-AC06-96RL13200 \\ EDT/ECN: EDT-620388 UC: 2070 \\ Org Code: 7576B Charge Code: MD378 \\ B\&R Code: EW 3120074 Total Pages: 747 \\ Key Words: Tank 241-B-108, 241-B-108, B-108, B Farm, Core 172, Core \\ 173, Analytical Results, Final Report \\ Abstract: $N / A$
}

TRADEMARK DISCLAIMER. Reference herein to any specific comercial product, process, or service by trade name, trademark, manufacturer, or otherwise, does not necessarily constitute or imply its endorsement, recommendation, or favoring by the United States Government or any agency thereof or its contractors or subcontractors.

Printed in the United States of America. To obtain copies of this document, contact: Document Control Services, P.0. Box 950, Mailstop H6-08, Richland WA 99352, Phone (509) 372-2420; Fax (509) 376-4989.

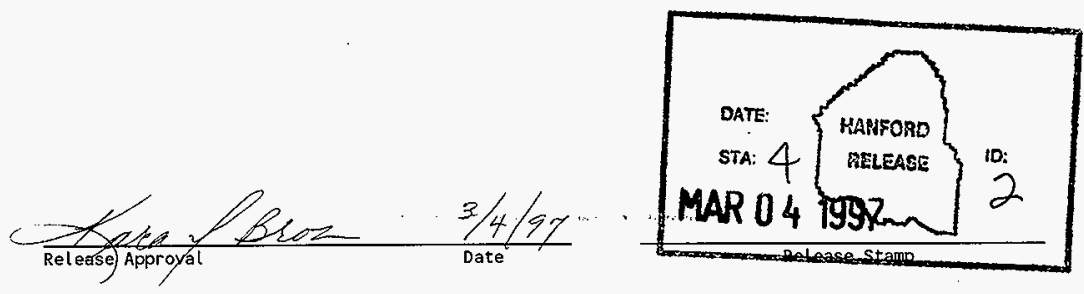

Approved for Public Release 
HNF-SD-WM-DP-219, REV. 0

ANALYTICAL SERVICES

TANK 241-B-108, CORES 172 AND 173 ANALYTICAL RESULTS FOR THE FINAL REPORT

\author{
Project Coordinator: JENNIFER L. NUZUM \\ Prepared for the U.S. Department of Energy \\ office of Environmental Restoration \\ and Waste Management \\ by \\ 222-S Laboratory \\ Rust Federal Services of Hanford Inc. \\ P. 0. Box 700 \\ Richland, Washington
}


HNF-SD-WM-DP-219, REV. 0

THIS PAGE INTENTIONALLY LEFT BLANK 
HNF-SD-WM-DP-219, REV. 0

TABLE OF CONTENTS

Narrative ........................ 1

B-108 Sample Breakdown (Attachment 1) ............ 13

Data Verification and Deliverable Summary Report (Attachment 2) . . . . 21

Sample Data Summary . . . . . . . . . . . . . 120

Chain of Custody Forms . . . . . . . . . . . . . . 142

Photographs .................... . . 149

Core Composite Worksheets . . . . . . . . . . . . . . 156

Extrusion Analysis ........................ 160

Sample Preparations . . . . . . . . . . . . . . . 167

Fusion Digestion Worklist \# $14104(5468,5477,5478,5487) \ldots 169$

Fusion Digestion Workl ist \# $14172(5510,5511,5512) \ldots . . . .171$

Fusion Digestion Workl ist \# 16005 (000001, 000002) . . . . . 171.1

Water Digestion Workl ist \# $14105(5469,5479,5470,5481) \ldots . . .172$

Water Digestion Work ist \# $14106(5480,5488,5482,5489) \ldots . . .176$

Water Digestion Work 1 ist \# $14173(5513,5516,5514,5519) \ldots . . .180$

Water Digestion Workl ist \# 16245 (000015, 000016, 000017, 000019) 180.1

B-108 Bulk Density Worksheets . . . . . . . . . . . . . . . 182

Inorganic Anlaysis . . . . . . . . . . . . . . . 189

Differential Scanning Calorimetry (DSC)

DSC Work 1 ist \# $14390(5466,5473) \ldots \ldots \ldots 1 . \ldots \ldots 1$

DSC Workl ist \# $14391(5474,5485) \ldots \ldots . \ldots . . . . . . .197$

DSC Workl ist \# $14656(5463) \ldots \ldots . \ldots . . \ldots 203$

DSC Workl jst \# $14715(5523) \ldots \ldots . . . . . . . .208$

DSC Work1 ist \# $14718(5505,5506,5507) \ldots 213$

DSC Workl ist \# $14742(5505,5506,5507,5473,5474,5485,5463,5523$,

$5466) \ldots \ldots . \ldots 221$

Thermogravimetric Analys is (TGA)

TGA Work 1 ist \# $14377(5463) \ldots \ldots$. . . . . . . . . . 224

TGA Work 1 ist \# $14383(5466,5473) \ldots \ldots . . \ldots . . . . .228$

TGA Work 1 ist \# $14384(5474,5485) \ldots . . . . . . . . . .234$

TGA Work ist \# 14707 (5523) . . . . . . . . . . . 240

TGA Workl ist \# $14711(5505,5506,5507) \ldots \ldots . \ldots 245$

Specific Gravity Analysis (SpG)

SpG Workl ist \# $14392(5463) \ldots \ldots . \ldots . . \ldots 253$ 
HNF-SD-WM-DP-219, REV. 0

TABLE OF CONTENTS (Continued)

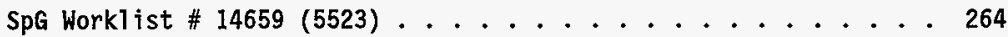

Ion Chromatographic Analys is (IC)

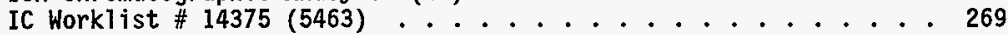

IC Worklist \# $14415(5469,5479) \ldots \ldots . . . . . . .278$

IC Workl ist \# $14416(5480,5488) \ldots \ldots . . . . . . . . .291$

IC Workl ist \# $14654(5523) \ldots \ldots . . . . . . . . .304$

IC Work list \# $14853(5514,5513,5515) \ldots . . . . . . . . .313$

IC Workl ist \#16305 $(000015,000017) \ldots \ldots . \ldots . . \ldots 327$

Inductively Coupled Plasma Spectroscopy (ICP)

ICP Worklist \# $14793(5463,5523) \ldots . . . . . . . . .339$

ICP Workl ist \# $14797(5468,5477,5478,5487) \ldots \ldots 345$

ICP Workl ist \# $14798(5510 ; 5511,5512) \ldots . . . . . . . . .354$

ICP Workl ist \#14799 (5489) . . . . . . . . . . . . 361

ICP Workl ist \# 15994 (5519) . . . . . . . . . . . . . . . . . 367

ICP Workl ist \# 15995 (5481) . . . . . . . . . . . 385

ICP Workl ist \# $16230(000001,000002) \ldots . . \ldots 403$

ICP Worklist \# $16262(000016) \ldots . . . . . . . . .411$

Total Organic Carbon / Total Inorganic Carbon (TICTOC)

TICTOC Work1 ist \# $14372(5485) \ldots . . . . . . . .4417$

Uranium Analysis (U)

U Worklist \# $14449(5487) \ldots . . . . . . . . .4437$

U Worklist \# 16021 (5477) . . . . . . . . . . . . . . . 446

U Worklist \# $16022(5511) \ldots . . . . . . . . . . .4455$

U Worklist \# $16257(000002 \ldots . . . . . . . . .4464$

Radiochemical Analysis ............... 472.1

Alpha/Beta Analys is (AB)

$A B$ Workl ist \# $14725(5487) \ldots . . . . . . . . . .443$

$A B$ Work 1 ist \# $16409(000002) \ldots . . . . . . . . .487$

Total Alpha Analysis (AT)

AT Workl jst \# $14351(5463) \ldots \ldots$. . . . . . . . 500

AT Worklist \# $14361(5477,5478)$. . . . . . . . . . 506 506

AT Workl ist \# $14608(5523) \ldots \ldots . . . . . . . . . .5515$

AT Workl ist \# $14788(5511,5512) \ldots . . . . . . . . . .522$

AT Worklist \# 14949 (5511) . . . . . . . . . . . . . 531

AT Workl ist \# $16349(000001)$. . . . . . . . . . 538

Total Beta Analysis (TB)

TB Work1 ist \#16016 (5511) . . . . . . . . . . . 545

TB Workl ist \# 16015 (5477) $\ldots \ldots \ldots \ldots 52$

Gamma Energy Analysis (GEA)

GEA Workl ist \#15416 (5487) . . . . . . . . . . . 559 
HNF-SD-WM-DP-219, REV. 0

TABLE OF CONTENTS (Continued)

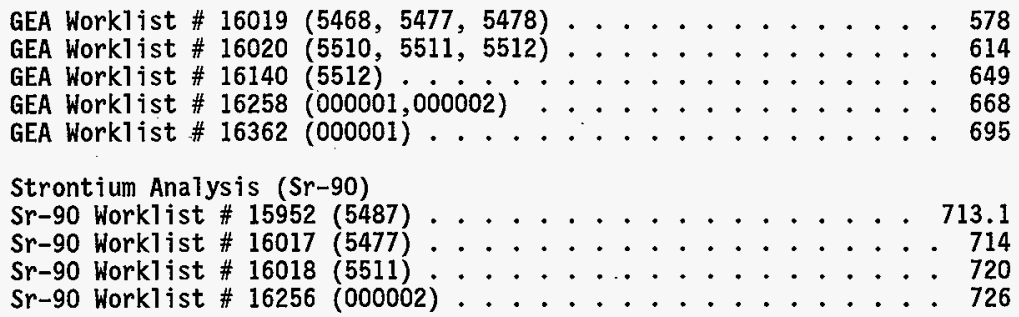

This Document consists of pages 1 through 731. Pages $i i, 2,14,22,121,142$, $150,157,161,168,183,190,472.2,485$ and 486 were intentional1y left blank.

\section{TRADEMARKS:}

Perkin-Elmer is a Registered Trademark of Research and Manufacturing Company, Inc. Mettler is a Registered Trademark of Mettler Electronics. 
HNF-SD-WM-DP-219, REV . 0

NARRATIVE 
HNF-SD-WM-DP-219, REV. 0

222-S ANALYTICAL SERVICES

\section{TANK 241-B-108, CORES 172 AND 173, ANALYTICAL RESULTS FOR THE FINAL REPORT}

This document is the final laboratory report for Tank 241-B-108. Push mode core segments were removed from Risers 3 and 6 between September 4, 1996, and September 6, 1996. The field blank was prepared September 4, 1996. Segments were received and extruded at 222-S Analytical Laboratory. Analyses were performed in accordance with Tank 241-B-108 Push Mode Core Sampling and Analysis Plan (TSAP) (Schreiber, 1996), Tank Safety Screening Data Quality Objective (SSDQO) (Dukelow, et al., 1995), and Historical Model Evaluation Data Requirements (HistoricalDQO) (Simpson, et al., 1995).

None of the subsamples exceeded notification limits as described in TSAP. Statistical evaluation of results by calculating the $95 \%$ upper confidence limit is not performed by $222-\mathrm{S}$ Laboratory and is not considered in this report.

\section{Appearance and Sample Handling}

Samples were removed from Tank 241-B-108 between September 4, 1996, and September 5, 1996. These samples were delievered to 222-S Laboratory on October 11, 1996. TSAP states that core samples shall be transported to the laboratory within three calendar days from the time each segment is taken from the tank. The laboratory does not control the shipment of segments; this issue is beyond the scope of this report.

Attachment 1 is a cross reference to relate the tank farm identification numbers to 222-S Laboratory sample numbers. The subsamples generated in the laboratory for analysis are identified in these diagrams with their sources shown.

A core composite was prepared for Core 173 and underwent analysis as directed by TSAP. Limited sample recovery from Core 172 , segment 2 precluded the preparation of a core composite. Lower Half Segment 1 of Core 172 and Lower Half Segment 1 of Core 173 were identified for secondary analyses as required by TSAP and HistoricalDQO.

\section{Core 172}

Two push mode core segments were removed from Tank 241-B-108 Riser 6 on September 4, 1996. Both segments were received by 222-S Laboratory on October 11, 1996. Table 1 summarizes the extrusion information. 


\section{HNF-SD-WM-DP-219, REV. 0}

\section{Core 173}

Two push mode core segments were removed from Tank 241-B-108 Riser 3 between September 5, 1996 and September 6, 1996. Both segments were received by 222-S Laboratory on October 11, 1996. Table 2 summarizes the extrusion information.

\section{Field Blank}

A field blank was provided to 222-S Laboratory with Core 172 . This sample was treated as a drainable liquid in accordance with TSAP.

\section{Hydrostatic Head Fluid}

There is no indication of the use of hydrostatic head fluid (HHF) in procuring these samples; a HHF blank was not provided to 222-S Laboratory. 


\begin{tabular}{|c|c|c|c|c|c|c|c|c|}
\hline 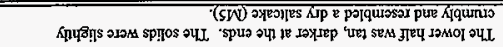 & 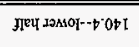 & $0^{\circ} 0$ & 0.5 & $96 / 9 \mathrm{I} / 0 \mathrm{~T}$ & $96 / 1 \mathrm{l} / 0 \mathrm{I}$ & $96 / 9 / 6$ & $z$ & $\operatorname{LOS}-96$ \\
\hline 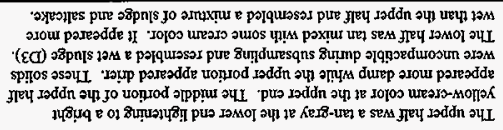 & 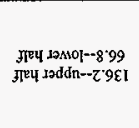 & 0.0 & 06 & $96 / 9 \mathrm{I} / 0 \mathrm{I}$ & $96 / \mathrm{II} / 0 \mathrm{I}$ & $96 / 5 / 6$ & I & $905-96$ \\
\hline 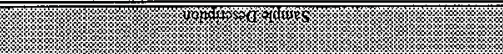 & (1) & (6) & 19.7. & 13\% & 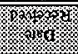 & 8 & 8 & 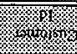 \\
\hline
\end{tabular}

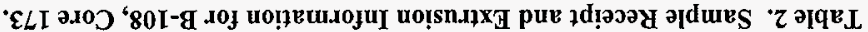

\begin{tabular}{|c|c|c|c|c|c|c|c|c|}
\hline 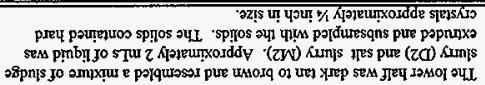 & गष्प $39 M O 1--662$ & 00 & $5+1$ & $96 / 12 / 01$ & $96 / 11 / 01$ & $96 / t / 6$ & $z$ & $505-96$ \\
\hline 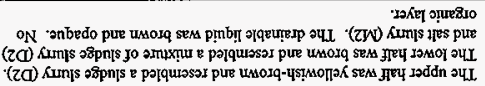 & 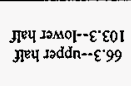 & 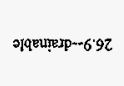 & $S \mathcal{E l}$ & $96 / 9 \mathrm{~L} / 0 \mathrm{I}$ & $96 / 1 \mathrm{I} / 0 \mathrm{I}$ & $96 / t / 6$ & I & $505-96$ \\
\hline 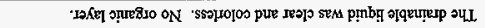 & 00 & 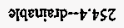 & 00 & $96 / 9 \mathrm{I} / 0 \mathrm{I}$ & $96 / 11 / 01$ & $96 / t / 6$ & 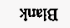 & त्रथए। $\mathrm{O}^{2} \mathrm{H}$ \\
\hline flow & (2) & 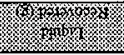 & (3) & 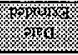 & 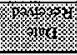 & \%o & 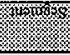 & 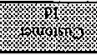 \\
\hline
\end{tabular}

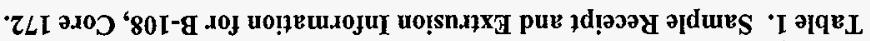

0 *ATY '6IZ-dU-WM-US-HNH 


\section{HNF-SD-WM-DP-219, REV. 0}

\section{Results Summary}

The Data Summary Table (Table 3) included in this report compiles analytical results in compliance with all applicable DQOs.

Liquid subsamples that were prepared for analysis by an acid adjustment of the direct subsample are indicated by a "D" in the A\# column in Table 3. Solid subsamples that were prepared for analysis by performing a fusion digest are indicated by an " $\mathrm{F}$ " in the A\# column in Table 3 . Solid subsamples that were prepared for analysis by performing a water digest are indicated by a "W" or an "I" in the A\# column of Table 3.

Due to poor precision and accuracy in original analysis of both Lower Half Segment 2 of Core 173 and the core composite of Core 173, fusion and water digests were performed for a second time. Precision and accuracy improved with the repreparation of Core 173 Composite. Analyses with the repreparation of Lower Half Segment 2 of Core 173 did not show improvement and suggest sample heterogeneity. Results from both preparations are included in Table 3 .

\section{Inorganic Analyses}

\section{DSC - Differential Scanning Calorimetry}

There are no exceptions to the quality control (QC) parameters stated in TSAP for these samples.

\section{TGA- Thermogravimetric Analysis}

There are no exceptions to the QC parameters stated in TSAP for these samples.

\section{Sp.G.-Specific Gravity/Bulk Density}

There are no exceptions to the QC parameters stated in TSAP for these samples.

\section{IC - Ion Chromatography}

Only required analyte results $(\mathrm{Br}, \mathrm{PO} 4, \mathrm{SO} 4, \mathrm{NO} 3$, and $\mathrm{F})$ are considered in this report. Other opportunistic analyte results are included in Table 3. These analytes do not have customer defined QC parameters and are not discussed. Lower Half Segment 2 of Core 172 (S96T005515) exhibited high relative percent difference (RPD) between sample and duplicate results and elevated spike recoveries. Rerun analyses did not improve precision or accuracy and suggest sample heterogeneity. Continuing reruns were not requested. 


\section{HNF-SD-WM-DP-219, REV. 0}

RPD between sample and duplicate for the Lower Half Segment 2 of Core 173 (S97T000017) for NO3 and SO4 were $32.0 \%$ and $44.4 \%$, respectively. This sample was a repreparation of the water digest. Consistently high RPDs between the old and new water digest suggest the sample was heterogenous and further rerun analyses were not requested.

\section{ICP - Inductively Coupled Plasma Spectroscopy}

Only required analyte results $(\mathrm{Li}, \mathrm{Na}$, and $\mathrm{Al})$ are considered in this report. Other "opportunistic" analyte results are included in Table 3. These analytes do not have customer defined QC parameters and are not discussed.

RPDs between sample and duplicate for the Lower Half Segment 2 of Core 173 (S97T000001) and the core composite of Core 173 (S97T000002) for Al was $63.4 \%$ and $22.2 \%$, respectively. These samples were repreparations of the fusion. Consistently high RPDs between the old and new fusions suggest the samples are heterogenous. Further rerun analyses were not requested.

The Na spike recovery reported in Table 3 for Drainable Liquid Segment 1 of Core 172 (S96T005523) is invalid because the spike concentration (1 ppm) is too low in comparison to the sample concentration (less than $25 \%$ ). A second spike with an analyte level of $10 \mathrm{ppm}$ was performed, and the resulting spike recovery was $99.8 \%$. This calculation can be found in the raw data.

Preparation blanks showed Na results above the detection level. The levels of these analytes in the preparation blank are inconsequential when compared to the result for the sample. This contamination does not impact sample data quality.

\section{TOC - Total Organic Carbon}

There are no exceptions to the QC parameters stated in TSAP for theses samples.

\section{TIC - Total Inorganic Carbon}

There are no exceptions to the $\mathrm{QC}$ parameters stated in TSAP for these subsamples.

\section{U - Total Uranium}

The preparation blank results for uranium were above the detection level. This level is inconsequential when compared to sample results. RPD between sample and duplicate for the core composite of Core 173 (S97T000002) was $25.0 \%$. This sample was a repreparation of the fusion. Consistently high RPDs between the old and new fusion suggest the sample was heterogenous and further rerun analyses were not requested. 


\section{HNF-SD-WM-DP-219, REV. 0}

\section{Radiochemical Analyses}

Attachment 2 contains the Data Verification and Deliverable (DVD) summary report for radionuclide analyses. This report summarizes results from radiochemical analyses and provides data qualifiers and total propagated uncertainty (TPU) values for results. The TPU values are based on the uncertainties inherent in each step of the analysis process. They may be used as an additional reference to determine "reasonable" RPD values which may be used to accept valid data that do not meet the TSAP acceptance criteria. A report guide is provided with the report to assist in understanding this summary report.

\section{AT - Total Alpha}

One preparation blank showed AT activity above the detection level. The activity was inconsequential when compared to the results for the samples. This contamination does not impact sample data quality.

Two subsamples showed RPDs greater than 20\%. Elevated RPD (38.1\%) for S96T005477 (Lower Half Segment 1 of Core 173) was attributed to low alpha activity and a rerun was not requested. S97T000001 (Lower Half Segment 2 of Core 173) was a repreparation of the fusion. Consistently high RPDs between the old and new fusion suggest the sample was heterogenous and further renun analyses were not requested.

AT analysis of two subsamples resulted in low spike recoveries. Rerun results were similar, suggesting the cause to be matrix interference. Continuing reruns were not requested.

\section{TB - Total Beta}

The preparation blanks showed TB activity above the detection level. The activity in these preparation blanks is inconsequential when compared to the results for the samples. This contamination does not impact sample data quality.

RPD between sample and duplicate for the core composite of Core 173 (S97T000002) was 21.6. Rerun analyses suggest poor precision is due to sample heterogeneity. Further reruns were not requested. 


\section{HNF-SD-WM-DP-219, REV. 0}

\section{GEA - Gamma Energy Analysis}

Only required analyte results $\left({ }^{137} \mathrm{Cs}\right)$ are considered in this report. Other "opportunistic" analyte results are included in Table 3 . These analytes do not have customer defined QC parameters and are not discussed.

RPD. between sample and duplicate exceeded $20 \%$ for two subsamples. Lower Half Segment 2 of Core 172 (S96T005512) and Lower Half Segment 2 of Core 173 (S97T000001) had RPDs of $22.7 \%$ and $39.8 \%$, respectively. Consistently high RPDs between original and rerun data suggest these samples are heterogenous and further rerun analyses were not requested.

\section{${ }^{90} \mathrm{Sr}$ - Strontium-90}

The preparation blanks showed ${ }^{90} \mathrm{Sr}$ activity above the detection level. The activity in these preparation blanks is inconsequential when compared to the results for the samples. This contamination does not impact sample data quality. 


\section{HNF-SD-WM-DP-219, REV, 0}

\section{Procedures}

Table 4 lists the analytical procedures used for performing the sample analyses. Abbreviations for analyses are defined in the table notes.

Table 4. Analytical Procedures

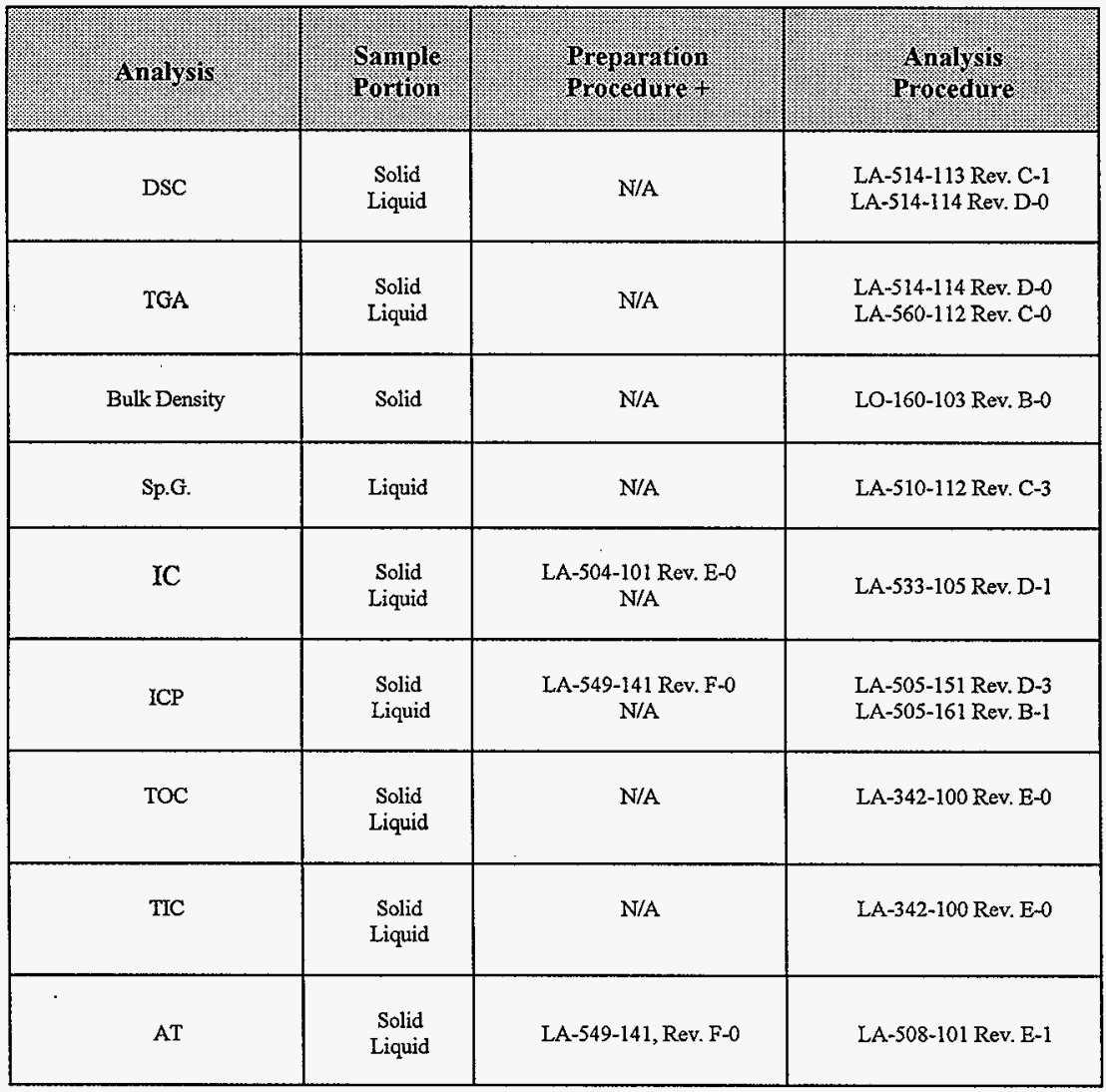


HNF-SD-WM-DP-219, REV. 0

\begin{tabular}{|c|c|c|c|}
\hline TB & Solid & LA-549-141 Rev. F-0 & LA-508-101 Rev. E-1 \\
\hline GEA & Solid & LA-549-141 Rev. F-0 & LA-548-121 Rev. E-0 \\
\hline${ }^{\text {s0 Sr }}$ & Solid & LA-549-141 Rev. F-0 & LA-220-101 Rev. D-1 \\
\hline U & Solid & LA-549-141 Rev. F-0 & LA-925-009 Rev. A-1 \\
\hline
\end{tabular}

Abbreviations:
N/A = not applicable (these are direct samples)
DSC = differential scanning calorimetry
TGA = thermogravimetric analysis
Sp.G. = specific gravity
IC $=$ ion chromatography
ICP = inductively coupled plasma
TOC = total organic carbon
TIC = total inorganic carbon
AT = total alpha activity
$\mathrm{TB} \quad=$ total beta
GEA = gamma energy analysis
$\mathrm{U}=$ total uranium 


\section{HNF-SD-WM-DP-219, REV. 0}

\section{References}

Dukelow, G. T., J. W. Hunt, H. Babad, and J. E. Meacham, 1995, Tank Safety Screening Data Quality Objective, WHC-SD-WM-SP-004, Rev. 2, Westinghouse Hanford Company, Richland, WA 99352.

Schreiber, R.D., 1996, Tank 241-B-108 Push Mode Core Sampling and Analysis Plan, WHC-SD-WM-TSAP-075, Rev. 1-A, Westinghouse Hanford Company, Richland, WA 99352.

Meznarich, H. K., et. al, 1995, Quality Assurance Plan for the 222-S Laboratories, WHC-SDCP-QAPP-016, Rev. 0, Westinghouse Hanford Company, Richland, WA 99352.

Simpson, B. C., and D. J. McCain, 1996, Historical Model Evaluation Data Requirements, WHC-SD-WM-DQO-018, Rev. 1, Westinghouse Hanford Company, Richland, WA 99352.

Turner, D. A., H. Babad, L. L. Buckley, and J. E. Meacham, 1995, Data Quality Objective to Support Resolution of the Organic Complexant Safety Issue, WHC-SD-WM-DQO-006, Rev. 2, Westinghouse Hanford Company, Richland, WA 99352. 
HNF-SD-WM-DP-219, REV . 0

\section{ATTACHMENT 1 \\ B-108 SAMPLE BREAKDOWN}


HNF-SD-WM-DP-219, REV. 0

\section{THIS PAGE INTENTIONALLY LEFT BLANK}


B-108

Core: 172

Field Blank (Blank $\mathrm{H} 2 \mathrm{O}$ )

Attachment 1

S96T005393
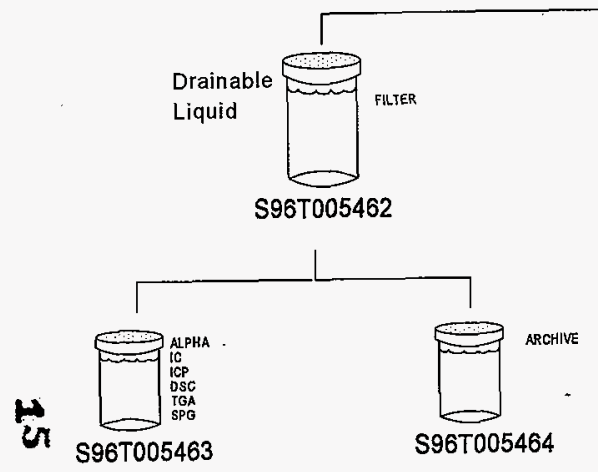

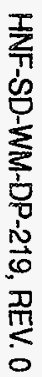


B-108

Core:172

Seg: 1 (96-504)

Attachment 1

S96T005394
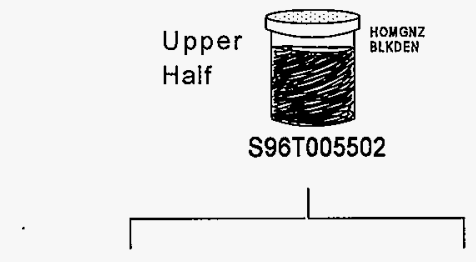

H.

S96T005505
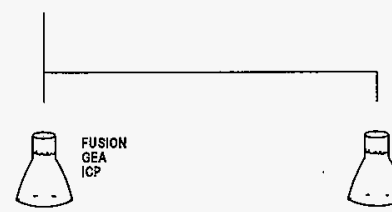

S96T005510

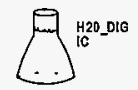

S96T005513

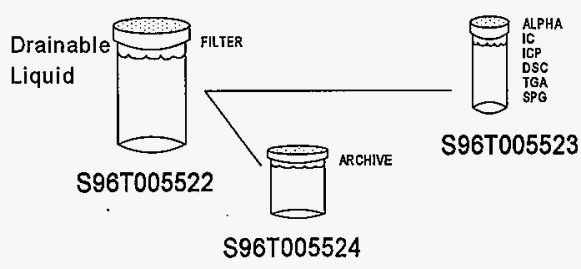

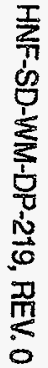

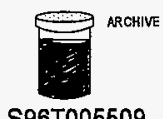

S96T005509

S96T005506

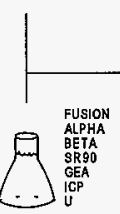

S96T005511

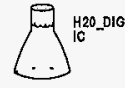

S96T005514

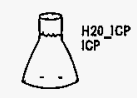

S96T005519 
B-108

Core:172

Seg: 2 (96-505)

S96T005395

Attachment 1
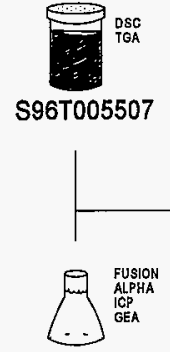

S96T005512

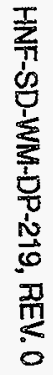

S96T005515 


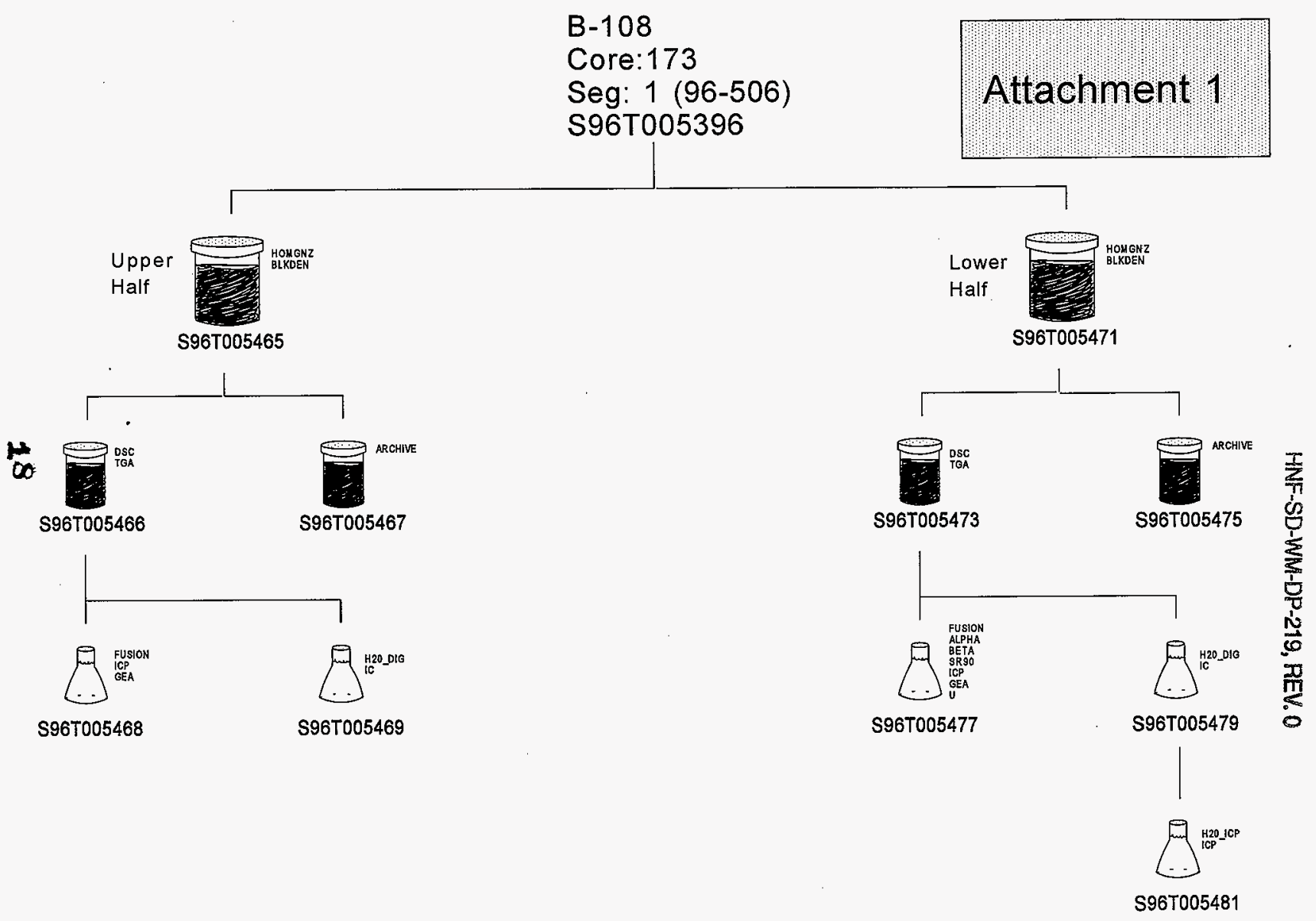


B-108

Core:173

Seg: 2 (96-507)

S96T005397

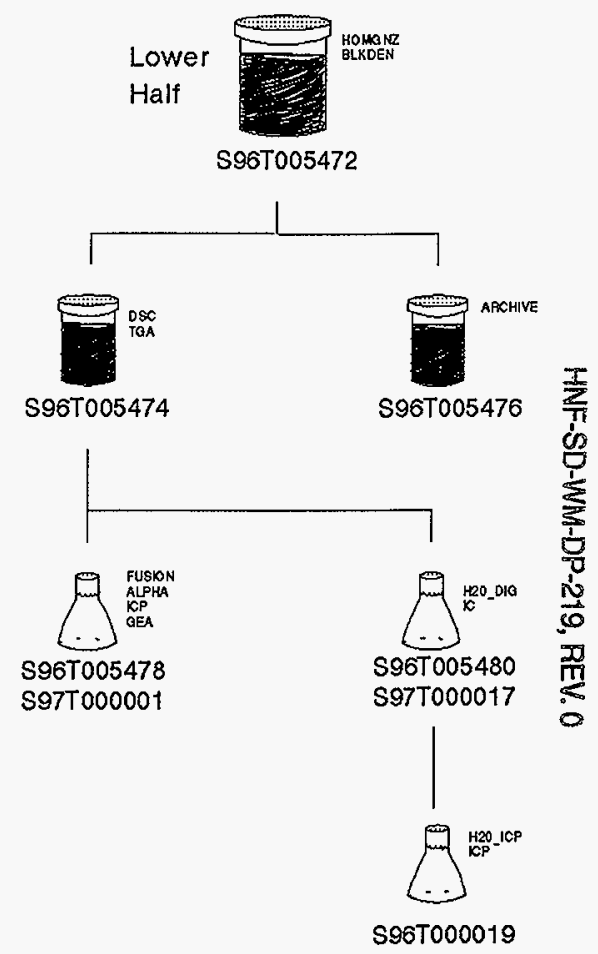




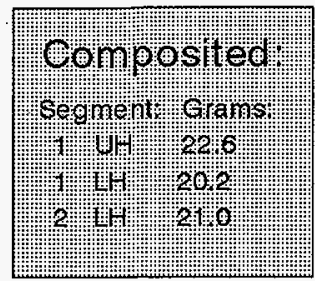

\section{B-108}

Core:173

Composite

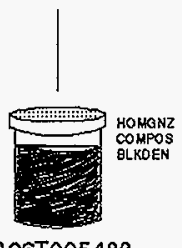

S96T005483

$\approx$

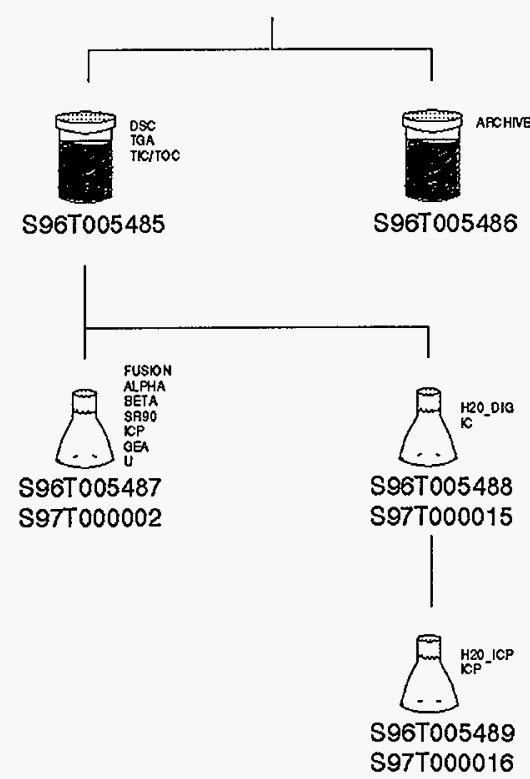

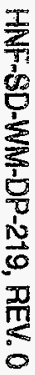

3967005489 
HNF-SD-WM-DP-219, REV. O

ATTACHMENT 2

DATA VERIFICATION AND DELIVERABLE SUMMARY REPORT 
HNF-SD-HM-DP-219, REV. 0

THIS PAGE INTENT IONALLY LEFT BLANK 
HNF-SD-WM-DP-219, REV. 0

$222-S$ I A B O R A T O R Y

TANK 241-B-108, CORE 172

SDG 96001379

contact I. L. Fritts
Client TWRS

Tank 241-B-108

S U M M A R Y D A T A S E C T I O N

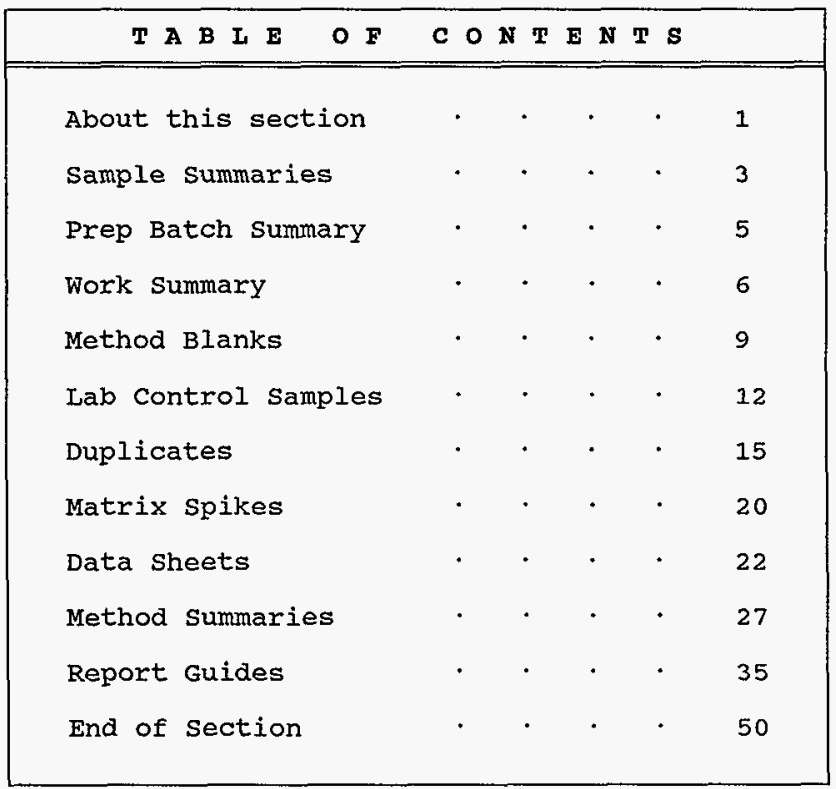

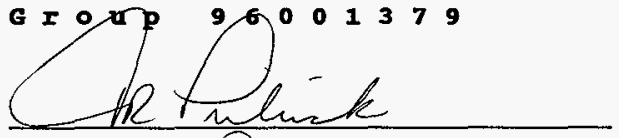

Reviewed by

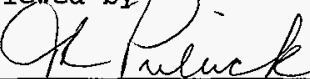

Approved by
Lab id 222-S

Protocol SST

Version 1.0

Form DVD-TOC

version 3.08

Report date $\underline{02 / 24 / 97}$ 
HNF-SD-WM-DP-219, REV. 0

$222-S$ I A B OR A TOR Y

TANK 241-B-108, CORE 172

SDG 96001379

Contact L. L. Fritts

R E P O R T G DI D E

Client TWRS

Tank $241-\mathrm{B}-108$

\section{A BOUT THE DATA SUMMARY SECTION}

The Data Summary Section of a Data Package has all data, in several useful orders, necessary for first level, routine review of the data package for a Sample Delivery Group (SDG). This section follows the Data Package Narrative, which has an overview of the data package and a discussion of special problems. It is followed by the Raw Data section, which has full details.

The Data Summary Section has several groups of reports:

\section{SAMPLE SUMMARIES}

The Sample and QC Summary Reports show all samples, including QC samples, reported in one SDG. These reports cross-reference client and lab sample identifiers.

\section{PREPARATION BATCH SUMMARY}

The Preparation Batch Summary Report shows all preparation batches (lab groupings reflecting how work was organized) relevant to the reported SDG with information necessary to check the completeness and consistency of the SDG.

WORK SUMMARY

The Work Summary Report shows all samples and work done on them relevant to the reported SDG.

METHOD BLANKS

The Method Blank Reports, one for each Method Blank relevant to the SDG, show all results and primary supporting information for the blanks.

LAB CONTROL SAMPLES

The Lab control sample Reports, one for each Lab Control sample relevant to the SDG, show all results, recoverieg and primary supporting information for these QC samples.

DUPLICATES

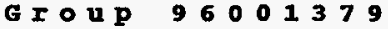

REPORT GUIDES

Page 1

SUMMARY DATA SECTION

Page 1
Iab id $222-5$

protocol SST

Version 1.0

Form DVD-RG

Version 3.08

Report date $02 / 24 / 97$ 
$222-S$ I A B O R A T OR Y TANK 241-B-108, CORE 172

SDG 96001379

\section{A B O T THE DATA SUMMARY BECTION}

The Duplicate Reports, one for each Duplicate and Original sample pair relevant to the SDG, show all results, differences and primary supporting information for these $\mathrm{QC}$ samples.

MATRIX SPIKES

The Matrix Spike Reports, one for each Spiked and Original sample pair relevant to the SDG, show all results, recoveries and primary supporting information for these QC samples.

DATA SHEETS

The Data Sheet Reports, one for each client sample in the SDG, show all results and primary supporting information for these samples.

METHOD SUMMARIES

The Method Summary Reports, one for each test used in the SDG, show all results, $\mathrm{QC}$ and method performance data for one analyte on one or two pages. (A test is a short code for the method used to do certain work to the client's specification.)

\section{REPORT GUIDES}

The Report Guides, one for each of the above groups of reports, have documentation on how to read the associated reports.

\section{G r up 96001379}

\section{REPORT GUIDES}

Page 2

SUMMARY DATA SECTION

Page 2
Lab id 222-s

Protocol SST

Version 1.0

Form DVD-RG

Version 3.08

Report date $02 / 24 / 97$ 
HNF-SD-WMA-DP-219, REV.O

222 -S LABORATORY

TANK 241-B-108, CORE 172

SAMPLE SUMMARY
Client TWRS

Tank 241-B-108

\begin{tabular}{|c|c|c|c|c|c|c|}
\hline CLIENT SAMPLE ID & LOCATION & & MATRIX & LEVEL & PRIORITY COLLECTED & RECEIVED \\
\hline B108 C172 FB Direct 1116 & R: 6 s: Field Blank $\mathrm{C}:$ ? & & LIQUID & & s967005463 & $10 / 17 / 9606: 11$ \\
\hline 8108 C172 FB Direct J116 & R: 6 S: Field Blank C: 1 & & LIQUID & & s96T0054630 & \\
\hline B108 C172S1 DL Direct V\# & $\mathrm{R}: 6 \mathrm{~S}: 1 \mathrm{C}: 172$ & & LIQUID & & \$96T005523 & $10 / 21 / 96 \quad 12: 27$ \\
\hline B108 C172S1 DL Direct V\# & $R: 6 \mathrm{~S}: 1 \mathrm{C}: 172$ & & L. IQUID & & S96T005523D & \\
\hline B108 C172S1 DL Direct V\# & $R: 6 \mathrm{~S}: 1 \mathrm{C}: 172$ & & LIQUID & & \$96T005523s & \\
\hline B108 C172s1 LH Fusion & $R: 6 \mathrm{~s}: 1 \mathrm{C}: 172$ & FUSION & SOLID & & s96roos511 & $10 / 21 / 96 \quad 12: 08$ \\
\hline B108 C172S1 LH Fusion-DU & $R: 6 \mathrm{~S}: 1 \mathrm{C}: 172$ & FUSION & SOL. ID & & S96T005511D & \\
\hline 8108 c172s1 LH Fusion-SP & $R: 6$ s: 1 C: 172 & FUSION & SOLID & & S96T005511S & \\
\hline B108 C172st UH Fusion & $R: 6 \mathrm{~S}: 1 \mathrm{C}: 172$ & FUSION & SOLID & & S96T005510 & $10 / 21 / 96 \quad 12: 06$ \\
\hline B108 C172s1 UH Fusion-DU & R: 6 S: 1 C: 172 & FUSION & SOLID & & S96T005510D & \\
\hline B108 c172s2 LH Fusion & $R: 6$ s: 2 C: 172 & FUSION & SOLID & & S96T005512 & $10 / 21 / 9612: 08$ \\
\hline B108 C172S2 LH Fusion-DU & $R: 6$ S: 2 C: 172 & FUSION & SOLID & & S96T005512D & \\
\hline DI Blank & & & LIQUID & & B14351-2 & \\
\hline DI B lank & & & LIQUID & & B14608-2 & \\
\hline Method B!ank & & & SOLID & & B14788-2 & \\
\hline Method Blank & & & SOLID & & B14949-2 & \\
\hline Method Blank & & & SOLID & & $B 16016-2$ & \\
\hline Method Blank & & & SOL.ID & & B16018-2 & \\
\hline Method Blank & & & SOLID & & B16020-2 & \\
\hline Method B lank & & & SOLID & & B16140-2 & \\
\hline Lab Control Sample & & & LIQUID & & $\mathbf{s} 14351-1$ & \\
\hline Lab Control Sample & & & LIQUID & & $\$ 14608-1$ & \\
\hline Lab Control Sample & & & SOLID & & s14788-1 & \\
\hline Lab Control Sample & & & SOLID & & $\$ 14949-1$ & \\
\hline Lab Control Sample & & & SOLID & & $s 16016-1$ & \\
\hline Lab Control Sample & & & SOLID & & $s 16018-1$ & \\
\hline Lab Control Sample & & & SOL.ID & & $\mathbf{s} 16020-1$ & \\
\hline Lab Control Sample & & & SOL:D & & $s 16140-1$ & \\
\hline
\end{tabular}

\section{Group 96001379}

Lab id 222-S

Protocol SST

Version 1.0

Form DVD-CS

Version 3.08

Report date $02 / 24 / 97$ 
HNF-SD-WMM-DP-219, REV. 0

$222-S$ LABORATORY

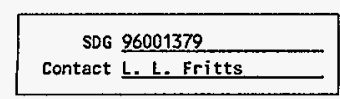

TANK 241-B-108, CORE 172

\section{QC SUMMARY}

Client IWRS

Tank 241-B-108

\begin{tabular}{|c|c|c|c|c|c|c|c|c|c|c|c|}
\hline & & & & & & & & DAYS F & ROH/TO & & \\
\hline QC ВАТСH & $\begin{array}{l}\text { CHAIN OF } \\
\text { CUSTOOY }\end{array}$ & CL.IENT SAMPLE ID & & MATRIX & $\frac{x}{\text { MoIsT }}$ & $\begin{array}{l}\text { SAMPLE } \\
\text { AMOUNT }\end{array}$ & $\begin{array}{l}\text { BASIS } \\
\text { AMOUNT }\end{array}$ & $\begin{array}{l}\text { COLL } \\
\text { RCVD }\end{array}$ & $\begin{array}{l}\text { RCVD } \\
\text { RPTD }\end{array}$ & $\begin{array}{c}\text { LAB } \\
\text { SAMPLE ID }\end{array}$ & $\begin{array}{l}\text { DEPARTMENT } \\
\text { SAMPLE ID }\end{array}$ \\
\hline $96001379-F$ & $\mathrm{n} / \mathrm{a}$ & B108 C172s1 LH Fusion & FUSION & SOL.iD & & & & & 126 & s96ro05511 & \\
\hline & & B108 C172S1 UH Fusion & FUSION & SOLID & & & & & 126 & $\$ 96 T 005510$ & \\
\hline & & B108 C172s2 LH Fusion & FUSION & SOLID & & & & & 126 & s96T005512 & \\
\hline & & B108 c172s1 LH Fusion-DU & FUSION & SOLID & & & & & 126 & s96r0055110 & \\
\hline & & B108 C172S1 LH Fusion-SP & FUSION & SOLID & & & & & 126 & s96r005511s & \\
\hline & & B108 C172S1 UH Fusion-DU & FUSION & SOLID & & & & & 126 & S967005510D & \\
\hline & & B108 C172S2 LH Fusion-DU & FUSION & SOLID & & & & & 126 & S96T005512D & \\
\hline $96001379-1$ & & B108 C172 FB Direct J116 & & LIQUID & & & & & 130 & S96T005463 & \\
\hline & & B108 C172S1 DL Direct V\# & & LIQUID & & & & & 126 & S96T005523 & \\
\hline & & 8108 C172 F8 Direct $J 116$ & & LIQUID & & & & & 130 & S96T0054630 & \\
\hline & & B108 C172s1 DL Direct V\# & & LIQUID & & & & & 126 & S96T0055230 & \\
\hline & & B108 C172S1 DL Direct V\# & & LIQUID & & & & & 126 & \$96T005523S & \\
\hline LIQUID & & DI Blank & & LIQUID & & & & & & B14351-2 & \\
\hline & & DI Blank & & LIQUID & & & & & & B14608-2 & \\
\hline & & Lab Control Sample & & LIQUID & & & & & & s14351-1 & \\
\hline & & Lab Control Sample & & LIQUID & & & & & & $514608-1$ & \\
\hline SOLID & & Method Blank & & SOL ID & & & & & & B14788-2 & \\
\hline & & Method Blank & & SOL ID & & & & & & B14949-2 & \\
\hline & & Method Blank & & SOL ID & & & & & & B16016-2 & \\
\hline & & Method Blank & & SOLID & & & & & & B16018-2 & \\
\hline & & Method Blank & & SOL ID & & & & & & B16020-2 & \\
\hline & & Method Blank & & SOLID & & & & & & B16140-2 & \\
\hline & & Lab Control Sample & & SOLID & & & & & & s14788-1 & \\
\hline & & Lab Control Sample & & SOL. ID & & & & & & \$14949-1 & \\
\hline & & Lab Control Sample & & SOLID & & & & & & $516016-1$ & \\
\hline & & Lab Control Sample & & SOLID & & & & & & s16018-1 & \\
\hline & & Lab Control Sample & & SOLID & & & & & & $\$ 16020-1$ & \\
\hline & & Lab Control Sample & & SOLID & & & & & & $\$ 16140-1$ & \\
\hline
\end{tabular}

\section{Group 96001379}

QC SUMMARY

Page 1

SUMMARY DATA SECTION

Page 4
Lab id 222-S

Protocol SST

Version 1.0

Form DVD-QS

Version 3.08

Report date $02 / 24 / 97$ 


\section{HNF-SD-WHA-DP-219, REV. O}

222-S LABORATORY

TANK 241-B-108, CORE 172

SDG 96001379

Contact L. L. Fritts

PREP BATCH SUMMARY
Client TWRS

Tank 241-B-108

\begin{tabular}{|c|c|c|c|c|c|c|c|c|c|c|c|c|}
\hline & \multirow[b]{2}{*}{ MATRIX } & \multirow[b]{2}{*}{ METHOD } & \multicolumn{2}{|c|}{ PREPARATION ERROR } & \multirow[b]{2}{*}{ CLIENT } & \multirow[b]{2}{*}{ MORE } & \multicolumn{2}{|c|}{ PLANCHETS } & \multicolumn{3}{|c|}{$\longrightarrow$} & \multirow{2}{*}{$\begin{array}{l}\text { OUALI - } \\
\text { FIERS }\end{array}$} \\
\hline & & & BATCH & $2 \sigma \%$ & & & RE & BLANK & LCS & DUP/ORIG & MS/ORIG & \\
\hline \multicolumn{13}{|c|}{ Gas Proportional Counting } \\
\hline $\mathbf{S R}$ & SOLiD & Strontium- $89 / 90$ & 96012651 & 15.0 & 1 & & & 1 & 1 & $1 / 1$ & & \\
\hline \multicolumn{13}{|c|}{ Gas Proportional Counting } \\
\hline \multirow[t]{4}{*}{ AT } & LIQUID. & Alpha Analysis & 96010892 & 15.0 & 1 & & & 1 & 1 & $1 / 1$ & & \\
\hline & & & 96011188 & 15.0 & 1 & & & 1 & 1 & $1 / 1$ & $1 / 1$ & \\
\hline & SOLID & Alpha Analysis & 96011382 & 15.0 & 1 & & & 1 & 1 & $1 / 1$ & & \\
\hline & & & 96011553 & 15.0 & 1 & & & 1 & 1 & $1 / 1$ & $1 / 1$ & \\
\hline TB & SOLID & Beta Analysis & 96012647 & 15.0 & 1 & & & 1 & 1 & $1 / 1$ & $1 / 1$ & \\
\hline \multicolumn{13}{|c|}{ Gamma Energy Analysis } \\
\hline \multirow[t]{2}{*}{ GEA } & SOLID & Gamma Spectroscopy & 96012649 & 15.0 & 2 & & & 1 & 1 & $3 / 2$ & & \\
\hline & & & 96012785 & 15.0 & 1 & & & 1 & 1 & $0 / 1$ & & \\
\hline
\end{tabular}

Dupticates and Matrix Spikes are those with original (Client) sample in this Sample Delivery Group. Blank and LCS planchets are those in the same preparation batch as some Client, Duplicate or Spike sample.

\section{Group 96001379}

PREP BATCH SUMUARY

Page 1

SUMWARY DATA SECTION

Page 5
Lab id $222-s$

Protocol SST

Version 1.0

Form DVD-PBS

Version 3.08 Report date $02 / 24 / 97$ 
HNF-SD-WMA-DP-219, REV. 0

$222-S$ IABORATORY

TANK 241-B-108, CORE 172

WORK SUMMARY
Client TWRS

Tank 241-B-108
CLIENT SAMPLE ID

LOCATION

CustooY

B108 C172 FB Direct $\mathrm{j116}$

R: 6 S: Field Blank C: 1 LIQUID
LAB SAMPLE ID

COLLECTED

RECEIVED
SUF-

FIX ARALYZED REVIEUED BY METHOO

$\begin{array}{lllll}\text { S96T005463 } 14351-4 & \text { AT } & 10 / 29 / 96 & \text { SLF Alpha Analysis }\end{array}$

$10 / 17 / 96$

B108 C172 FB Direct $\mathrm{J} 116$

R: 6 S: Field Blank C: 1 LIQULD

59670054630

$14351-5$

AT

$10 / 29 / 96$

$10 / 17 / 96$

B108 C172S1 DL Direct $V \#$
R: 6 S: 1 C: 172

R: 6 S: 1 C: 172

$\begin{array}{llll}\$ 96 T 005523 & 14608.4 & \text { AT } & 11 / 06 / 96\end{array}$

$10 / 21 / 96$

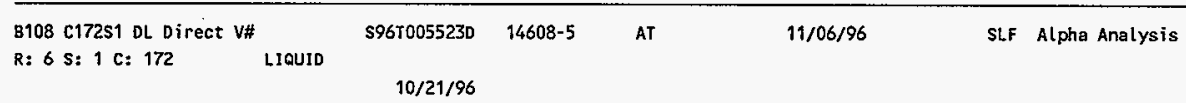

\begin{tabular}{|c|c|c|c|c|c|c|c|c|}
\hline $\begin{array}{l}\text { B108 C172S } 1 \text { DL Direct V\# } \\
\text { R: } 6 \text { S: } 1 \text { C: } 172\end{array}$ & LIQUID & $\begin{array}{r}\text { s96T005523s } \\
10 / 21 / 96\end{array}$ & $14608+6$ & AT & & $11 / 06 / 96$ & SLF & Alpha Analys is \\
\hline 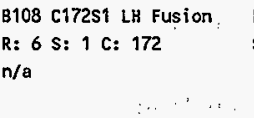 & $\begin{array}{l}\text { FUSION } \\
\text { SOLID }\end{array}$ & $\begin{array}{r}\text { s96ro05511 } \\
10 / 21 / 96\end{array}$ & $\begin{array}{l}14949-4 \\
16020-5 \\
16018-4 \\
16016-4\end{array}$ & $\begin{array}{l}\text { AT } \\
\text { GEA } \\
\text { SR } \\
\text { TB }\end{array}$ & 01 & $\begin{array}{l}11 / 20 / 96 \\
01 / 09 / 97 \\
01 / 08 / 97 \\
01 / 09 / 97\end{array}$ & $\begin{array}{l}\text { SLF } \\
\text { PPB } \\
\text { SAC } \\
\text { SLF }\end{array}$ & $\begin{array}{l}\text { Alpha Analysis } \\
\text { Gamma Spectroscopy } \\
\text { Strontium- } 89 / 90 \\
\text { Beta Analysis }\end{array}$ \\
\hline $\begin{array}{l}\text { B108 C172S1 LH Fusion-DU } \\
\text { R: } 6 \text { S: } 1 \text { C: } 172\end{array}$ & $\begin{array}{l}\text { FUSION } \\
\text { SOLID }\end{array}$ & $\begin{array}{r}\$ 9670055110 \\
10 / 21 / 96\end{array}$ & $\begin{array}{l}14949-5 \\
16020-6 \\
16018-5 \\
16016-5\end{array}$ & $\begin{array}{l}\text { AT } \\
\text { GEA } \\
\text { SR } \\
\text { TB }\end{array}$ & 01 & $\begin{array}{l}11 / 20 / 96 \\
01 / 09 / 97 \\
01 / 08 / 97 \\
01 / 09 / 97\end{array}$ & $\begin{array}{l}\text { SLF } \\
\text { PPB } \\
\text { SAC } \\
\text { SLF }\end{array}$ & $\begin{array}{l}\text { Alpha Analysis } \\
\text { Gamma Spectroscopy } \\
\text { Strontium-89/90 } \\
\text { Beta Analysis }\end{array}$ \\
\hline $\begin{array}{l}\text { B108 C172S1 LH Fusion-SP } \\
\text { R: } 6 \text { s: } 1 \text { C: } 172\end{array}$ & $\begin{array}{l}\text { FUSION } \\
\text { SOLIO }\end{array}$ & $\begin{array}{r}S 96 \mathrm{~T} 005511 \mathrm{~S} \\
10 / 21 / 96\end{array}$ & $\begin{array}{l}14949-6 \\
16016-6\end{array}$ & $\begin{array}{l}\text { AT } \\
\text { TB }\end{array}$ & 01 & $\begin{array}{l}11 / 20 / 96 \\
01 / 09 / 97\end{array}$ & $\begin{array}{l}\text { SLF } \\
\text { SLF }\end{array}$ & $\begin{array}{l}\text { Alpha Analysis } \\
\text { Beta Analys is }\end{array}$ \\
\hline $\begin{array}{l}\text { B108 C172s1 UH Fusion } \\
\text { R: } 6 \text { S: } 1 \mathrm{C}: 172 \\
\mathrm{n} / \mathrm{a}\end{array}$ & $\begin{array}{l}\text { FUSION } \\
\text { SOLID }\end{array}$ & $\begin{array}{l}\$ 961005510 \\
10 / 21 / 96\end{array}$ & $16020-3$ & GEA & & $01 / 09 / 97$ & PPB & Gamma Spectroscopy \\
\hline
\end{tabular}

\section{Group 96001379}

WORK SUMMARY

$$
\text { Page } 1
$$

SUMHARY DATA SECTION

Page 6
Lab id 222-s Protocol \$ST Version 1.0 form DVD-CWS Version 3.08 Report date 02/24/97 
HNF-SD-WM-DP-219, REV.O

\section{2-S LABORATORY}

TANK 241-B-108, CORE 172

SDG 96001379

Contact L. L. Fritts
WORK SUMMARY, cont.
Client TWRS

Jank 241-8-108
CLIENT SAMPLE ID LOCATION CUSTOOY

8108 c172s1 UH Fusion-DU FUSION R: 6 s: 1 C: 172 SOLID
LAB SAMPLE ID

MATRIX Priority
COLLECTED RECEIVED
S96T005510D

$16020-4$

$10 / 21 / 96$
B108 C172S2 LH Fusion

R: 6 S: 2 C: 172

n/a
FUSION

SOLID
S967005512

$10 / 21 / 96$
14788-7 AT

16140-3 GEA

$11 / 11 / 96$

$01 \quad 01 / 14 / 97$

$11 / 11 / 96$

$01 / 10 / 97$

14788-8 AT

16020-8 GEA

SUF-

FIX ANALYZED REVIEHEO BY METHOO

$01 / 09 / 97$

PPB Gamma Spectroscopy

\section{B108 C172S2 LH Fusion-DU FUSION}

R: 6 S: 2 C: 172

\$96T0055120

$10 / 21 / 96$ $\begin{array}{llll}\text { B14351-2 } & 14351-2 & \text { AT } & \end{array}$

LIQUID

DI Blank

LIOUID

Method Blank

SOLID

B14788-2 $14788-2 \quad$ AT

B14949-2 14949-2 AT

SOL:D

Method Blank

B16016-2

16016-2

TB

SOLID

Method Blank

B16018-2

SOLID

Method Blank

B16020-2

SOLID

Method Blank

B16140-2 16140-2

GEA

SOLED

Lab Control Sample

\$14351-1 14351-1 AT

\$14351-1 14351-1 AT

LIQUTD
SLF Alpha Analysis

PPB Gamma Spectroscopy

SLF Alpha Analysis

PPB Ganma Spectroscopy

SLF Alpha Analysis

SLF Alpha Analysis

SLF Alpha Analysis

SLF Alpha Analysis

$11 / 20 / 96$

$01 / 09 / 97$

SLF Beta Analysis

01/08/97 SAC strontium-89/90

$01 / 09 / 97$

PPB Garma Spectroscopy

PPB Gamma Spectroscopy

\section{Group 96001379}

WORK SUMAMARY

Page 2

SUMMARY DATA SECTION

Page 7

Lab id 222-s

Protocol SST

version 1.0

Form DVD-CWS

Version 3.08

Report date $02 / 24 / 97$ 
HNF-SD-WM-DP-219, REV. 0

\section{$222-S$ LABORATORY}

TANK 241-B-108, CORE 172

SDG 96001379

Contact L.L. Fritts
FORK SUMMARY, cont.
Client TURS

Tank 241-B-108

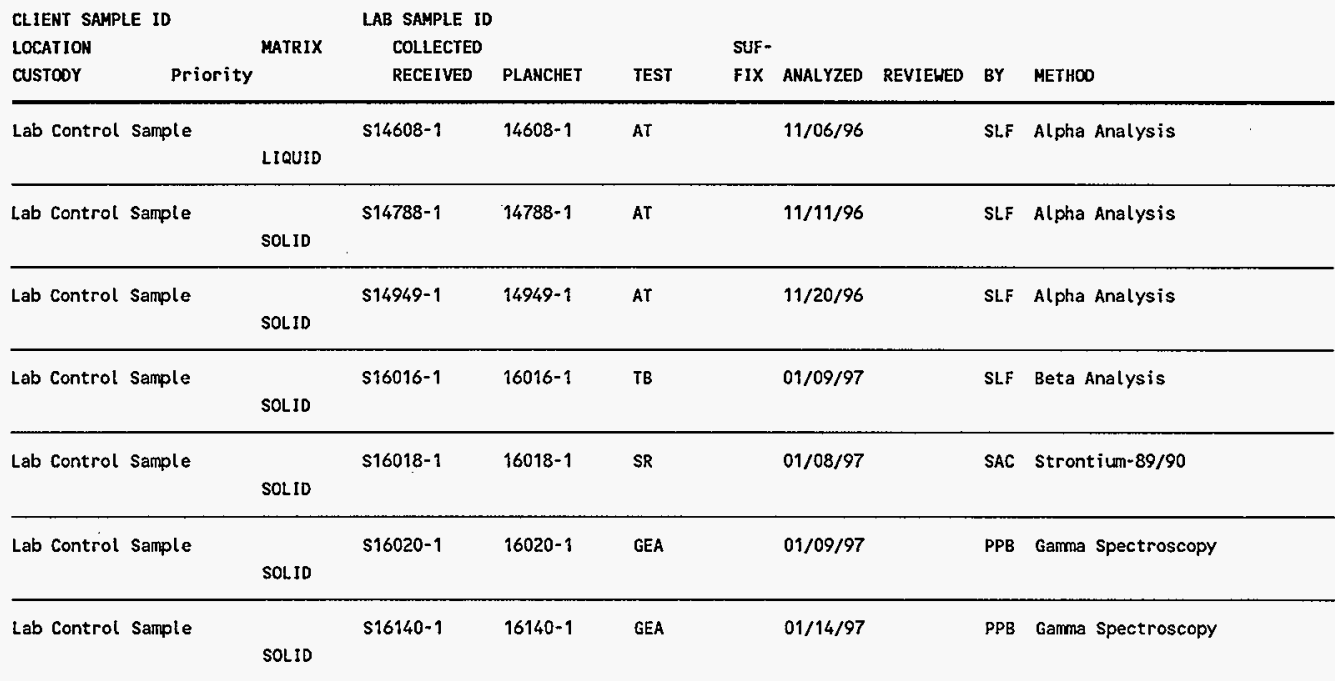

\begin{tabular}{|c|c|c|c|c|c|c|c|c|c|c|c|c|}
\hline TEST & Priority & COUNTS & $O F$ & $\underset{\text { REFERENCE }}{\text { TESTS }}$ & $\begin{array}{r}\text { SAMPIE } \\
\text { CLIENT }\end{array}$ & $\begin{array}{l}\text { TY } \mathbf{Y} \mathbf{E} \\
\text { MORE }\end{array}$ & RE & BLANK & LCS & DUP & SPIKE் & TOTAL \\
\hline AT & & Alpha Anatysis & & 222-S Lab Analytical & Al Procedure 2 & & & 2 & 2 & 2 & 1 & 9 \\
\hline AT & & Alpha Analysis & & 222-S Lab Analytical & Lrocedure 2 & & & 2 & 2 & 2 & 1 & 9 \\
\hline GEA & & Garma Spectroscopy & & 222-S Lab Analytical & I Procedure 3 & & & 2 & 2 & 3 & & 10 \\
\hline SR & & Strontiun- $89 / 90$ & & 222-S Lab Analytical & Lrocedure 1 & & & 1 & 1 & 1 & & 4 \\
\hline TB & & Beta Analysis. & & 222-S Lab Analytical & Procedure 1 & & & 1 & 1 & 1 & 1 & 5 \\
\hline TOTALS & & & & & 9 & & & 8 & 8 & 9 & 3 & 37 \\
\hline
\end{tabular}

\section{Group 96001379}

WORK SUMALARY

Page 3

SUMMARY DATA SECTIDN

Page 8
Lab id 222-s

Protocol SST

Version 1.0

Form DVD-CWS

Version 3.08

Report date $02 / 24 / 97$ 
HNF-SD-WM-DP-219, REV. O

222 -S LABORATORY

TANK 241-B-108, CORE 172

SDG 96001379

Contact L. L. Fritts

BLANKS

Client TWRS

Tank 241-B-108

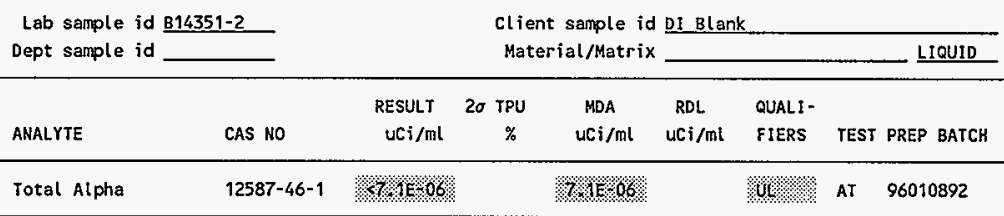

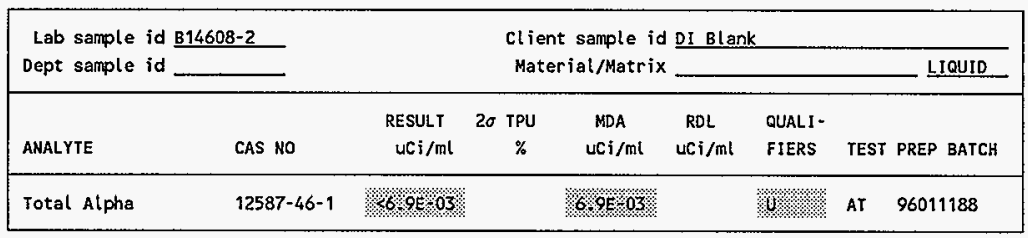

\begin{tabular}{|c|c|c|c|c|c|c|c|c|}
\hline \multicolumn{2}{|c|}{$\begin{array}{l}\text { Lab sample id B14788-2 } \\
\text { Dept sample id }\end{array}$} & \multicolumn{3}{|c|}{$\begin{array}{l}\text { client sample id } \\
\text { Material/Matrix }\end{array}$} & \multicolumn{2}{|c|}{ Method BLank } & \multicolumn{2}{|r|}{ SOL ID } \\
\hline ANALYTE & CAS NO & $\begin{array}{r}\text { RESULT } \\
\mathrm{uCi} / \mathrm{g}\end{array}$ & $\begin{array}{c}2 \sigma \mathrm{TPU} \\
\%\end{array}$ & $\begin{array}{l}\text { MDA } \\
\mathrm{UCi} / \mathrm{g}\end{array}$ & $\begin{array}{c}\mathrm{RDL} \\
\mathrm{UCi} / \mathrm{g}\end{array}$ & $\begin{array}{l}\text { QUAL I - } \\
\text { FIERS }\end{array}$ & TEST & PREP BATCH \\
\hline Total Alpha & $12587-46-1$ & 448103 & & $48=03$ & & $0 \%$ 楼 & AT & 96011382 \\
\hline
\end{tabular}

\begin{tabular}{|c|c|c|c|c|c|c|c|c|}
\hline \multirow{2}{*}{\multicolumn{2}{|c|}{$\begin{array}{l}\text { Lab sample id B14949-2 } \\
\text { Dept sample id }\end{array}$}} & \multirow{2}{*}{\multicolumn{3}{|c|}{$\begin{array}{l}\text { client sample id } \\
\text { Material/Matrix }\end{array}$}} & \multirow{2}{*}{\multicolumn{2}{|c|}{ Method Blank }} & \multirow{2}{*}{\multicolumn{2}{|c|}{ SOLID }} \\
\hline & & & & & & & & \\
\hline ANALYTE & CAS NO & $\begin{array}{r}\text { RESULT } \\
\text { UCi } / g\end{array}$ & $\begin{array}{c}2 \sigma \mathrm{TPU} \\
\%\end{array}$ & $\begin{array}{l}\text { MDA } \\
\mathrm{uCj} / \mathrm{g}\end{array}$ & $\begin{array}{l}\mathrm{RDL} \\
\mathrm{uCi} / \mathrm{g}\end{array}$ & $\begin{array}{l}\text { QUAL1- } \\
\text { FIERS }\end{array}$ & TEST & PREP BATCH \\
\hline Total Atpha & $12587-46-1$ & 442503 & & - 2 2 030 & & $0 \mathrm{O} / \mathrm{k}$ & AT & 96011553 \\
\hline
\end{tabular}

Group 96001379

BLANKS
Page 1
SUMMARY DATA SECTION

Page 9
Lab id 222-S

Protocol SST

Version 1.0

Form DVD-BLANK

Version 3.08

Report date $\underline{02 / 24 / 97}$ 
HNF-SD-WM-DP-219, REV.O

$222-S$ LABORATORY

TANK 241-B-108, CORE 172

BLANKS

Client TWRS

Tank 241-B-108

\begin{tabular}{|c|c|c|c|c|c|c|c|c|}
\hline \multicolumn{2}{|c|}{$\begin{array}{l}\text { Lab sample id B16016-2 } \\
\text { Dept sample id }\end{array}$} & \multicolumn{3}{|c|}{$\begin{array}{l}\text { client sample id } \\
\text { Material/Matrix }\end{array}$} & \multicolumn{2}{|c|}{ Method Blank } & \multicolumn{2}{|r|}{ SOLID } \\
\hline ANALYTE & CAS NO & $\begin{array}{l}\text { RESULT } \\
\text { uCi/g }\end{array}$ & $\begin{array}{c}2 \sigma \mathrm{TPU} \\
\%\end{array}$ & $\begin{array}{l}\text { MDA } \\
\mathrm{uCi} / \mathrm{g}\end{array}$ & $\begin{array}{l}\text { RDL } \\
\mathrm{uCi} / \mathrm{g}\end{array}$ & $\begin{array}{l}\text { QUALI - } \\
\text { FIERS }\end{array}$ & TEST & PREP BATCH \\
\hline Total Beta & $12587-47-2$ & 4. 802 & & Kot & & \% & TB & 96012647 \\
\hline
\end{tabular}

\begin{tabular}{|c|c|c|c|c|c|c|c|c|}
\hline \multicolumn{2}{|c|}{$\begin{array}{l}\text { Lab sample id B16018-2 } \\
\text { Dept sample id }\end{array}$} & \multicolumn{5}{|c|}{$\begin{array}{l}\text { Client sample id Method Blank } \\
\text { Material/Matrix }\end{array}$} & & SOLID \\
\hline ANALYTE & CAS NO & $\begin{array}{r}\text { RESULT } \\
\text { UCi/g }\end{array}$ & $\begin{array}{c}2 \sigma \mathrm{TPU} \\
\%\end{array}$ & $\begin{array}{l}\text { MDA } \\
\mathrm{UCi} / \mathrm{g}\end{array}$ & $\begin{array}{c}\text { ROL } \\
\mathrm{UC}: / \mathrm{g}\end{array}$ & $\begin{array}{l}\text { QUALI - } \\
\text { FIERS }\end{array}$ & TEST & PREP BATCH \\
\hline Strontium $\$ 0$ & $10098-97-2$ & 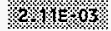 & 120 & 36403 & & 湆 & SR & 96012651 \\
\hline
\end{tabular}

\begin{tabular}{|c|c|c|c|c|c|c|c|c|}
\hline \multicolumn{2}{|c|}{$\begin{array}{l}\text { Lab sample id B16020-2 } \\
\text { Dept sample id }\end{array}$} & \multicolumn{5}{|c|}{$\begin{array}{l}\text { Client sample id Method Blank } \\
\text { Material/Matrix }\end{array}$} & \multicolumn{2}{|r|}{ SOLID } \\
\hline ANALYTE & CAS NO & $\begin{array}{l}\text { RESULT } \\
\mathrm{UCi} / \mathrm{g}\end{array}$ & $\begin{array}{c}2 \sigma \text { TPU } \\
\%\end{array}$ & $\begin{array}{c}\text { MDA } \\
\mathrm{uCi} / \mathrm{g}\end{array}$ & $\begin{array}{c}\text { RDL } \\
\text { uCi } / g\end{array}$ & $\begin{array}{l}\text { QUAL I - } \\
\text { FIERS }\end{array}$ & TEST & PREP BATCH \\
\hline GEA Analytes & & & & & & & & \\
\hline Cobalt 60 & $10198-40-0$ & $1 \times 4802$ & & $1 / 41007$ & & \% & GEA & 96012649 \\
\hline Cẹsium 137 & $10045-97-3$ & $2 \%=02$ & & $2 \times 1802$ & & \% & GEA & 96012649 \\
\hline Europium 154 & $15585-10-1$ & 34402 & & 3. 6102 & & U. & GEA & 96012649 \\
\hline Europium 155 & $14391-16-3$ & 3.11 .02 & & 3. 1802 & & 济 & GEA & 96012649 \\
\hline Americium 241 & $14596-10-2$ & 470000 & & \% de 02 & & & GEA & 96012649 \\
\hline
\end{tabular}

Group 96001379

8LANKS

Page 2

SUMAARY DATA SECTION

Page 10
Lab id 222-S

Protocol SST

Version 1.0

Form DVD-BLANK

Version 3.08

Report date $02 / 24 / 97$ 
HNF-SD-WM-DP-219, REV. 0

$222-5$ LABORATORY

TANK 241-8-108, CORE 172

SDG 96001379

Contact L. L. Fritts

BLANKS

Client TWRS

Tank 241-B-108

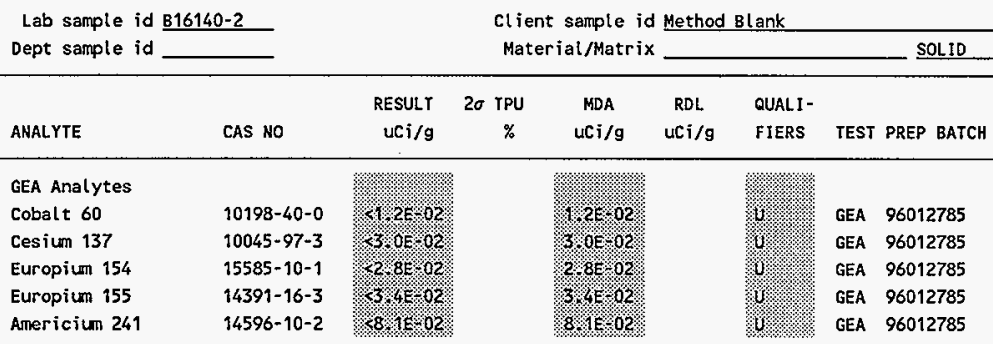

Group 96001379

BLANKS

Page 3

SUMMARY DATA SECTION

Page 11
Lab id 222-S

Protocol SST

Version 1.0

Form DVD-BLANK

Version 3.08

Report date $02 / 24 / 97$ 
222 -S LABORATORY

TANK 241-B-108, CORE 172

SOG 96001379

Contact L. L. Fritts

\section{LAB CONTROL SAMPLES}

Client IWRS

Tank 241-B-108
Lab sample id $\underline{\text { s14351-1 }}$

Dept sample id

\begin{tabular}{|c|c|c|c|c|c|c|}
\hline ANALYTE & $\begin{array}{l}\text { RESULT } \\
\mathrm{uCi} / \mathrm{ml}\end{array}$ & $\begin{array}{c}2 \sigma \mathrm{TPU} \\
\%\end{array}$ & $\begin{array}{c}\text { MDA } \\
\mathrm{uCj} / \mathrm{ml}\end{array}$ & $\begin{array}{c}\text { ROL } \\
\mathrm{UCi} / \mathrm{ml}\end{array}$ & $\begin{array}{l}\text { QUALI - } \\
\text { FIERS }\end{array}$ & IEST \\
\hline Totat Alpha & 1482504 & 15 & $1 \times 10_{0}$ & & \%र/א & AT \\
\hline
\end{tabular}

Client sample id Lab Control Sample Material/Matrix LIQUID

ADDED $2 \sigma$ ERR REC $3 \sigma$ LMTS PROTOCOL

UCi $/ \mathrm{mL} \%$ (TOTAL) LIMITS PREP BATCH

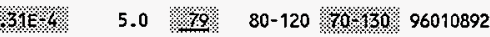

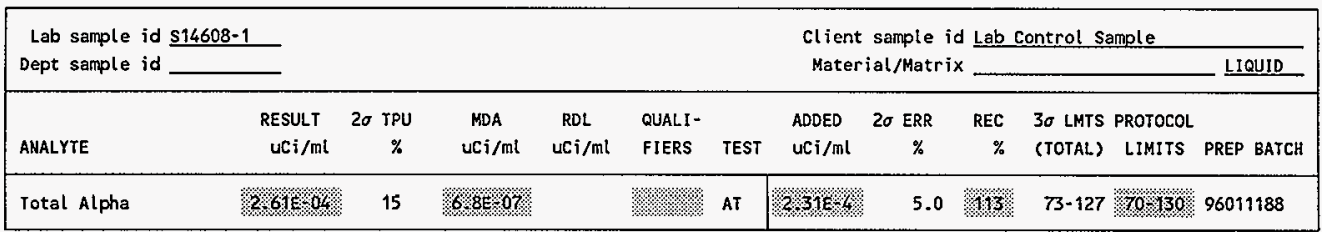

Lab sample id \$14788-1

Dept sample id

\begin{tabular}{|c|c|c|c|c|c|c|c|c|c|c|c|c|}
\hline ANALYTE & $\begin{array}{r}\text { RESULT } \\
\mathrm{uCi} / \mathrm{g}\end{array}$ & $\begin{array}{c}2 \sigma \mathrm{TPU} \\
\%\end{array}$ & $\begin{array}{c}\text { MDA } \\
\mathrm{UCi} / \mathrm{g}\end{array}$ & $\begin{array}{c}\text { RDL } \\
\mathrm{UCi} / \mathrm{g}\end{array}$ & $\begin{array}{l}\text { QUALI - } \\
\text { FIERS }\end{array}$ & TEST & $\begin{array}{l}\text { ADDED } \\
U C j / g\end{array}$ & $\begin{array}{c}2 \sigma \text { ERR } \\
\%\end{array}$ & $\begin{array}{r}\text { REC } \\
\%\end{array}$ & $\begin{array}{l}3 \sigma \text { LMTS } \\
\text { (TOTAL) }\end{array}$ & $\begin{array}{l}\text { PROTOCOL } \\
\text { LIMITS }\end{array}$ & PREP BATCH \\
\hline Total Alpha & $1420 \mathrm{n} 0$ & 15 & $480 \% 4$ & & & AT & Xor & 5.0 & $98 \%$ & $76-124$ & $70 \times 130$ & 96011382 \\
\hline
\end{tabular}

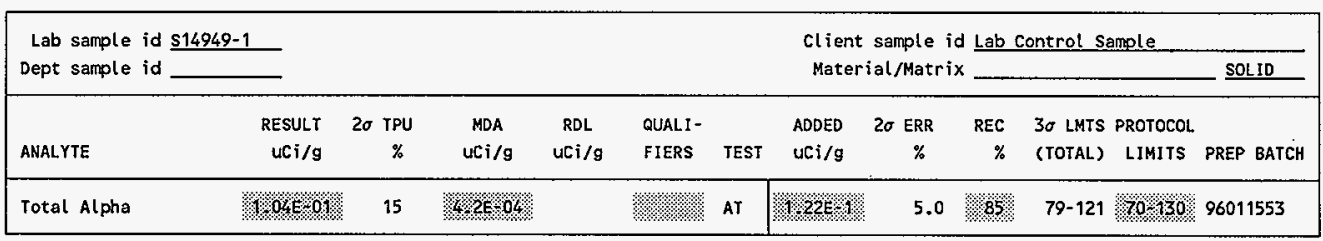

\section{Group 96001379}

LAB CONTROL SAMPLES

Page $?$

SUMMARY DATA SECTIOH

Page 12
Client sample id Lab Control Sample

Material/Matrix SOL.1D

lient sample id Lab control Sample Material/Matrix

9.0 .130 73-127 *30-130\% 96011188 
HNF-SD-WM-DP-219, REV. 0

222 -S IABORATORY

TANK 241-B-108, CORE 172

LAB CONTROL SAMPLES
Client TWRS

Tank $241-B-108$

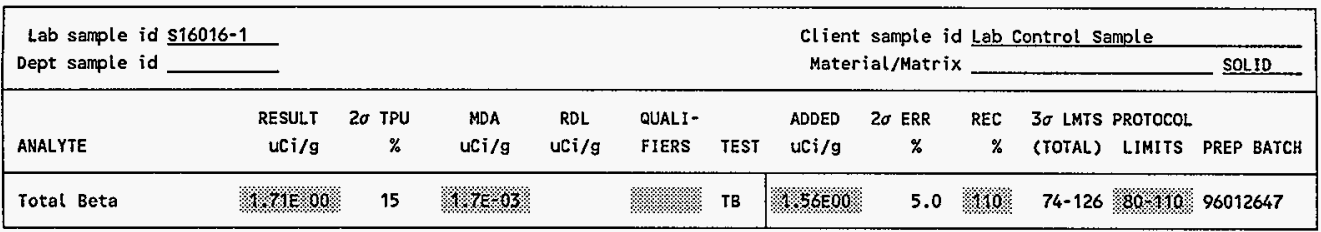

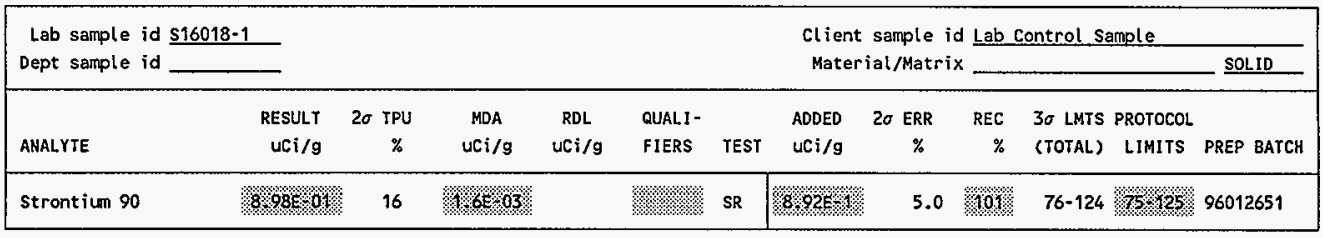

\section{Group 96001379}

LAB CONTROL SAMPLES

Page 2

SURMARY DATA SECTION

Page 13
Client sample id Lab control Sample

Material/Matrix SOLLD

Lab sample id
Dept sample id

RESULT $2 \sigma$ TPU MDA RDL QUALI-

UCi/g UCi/g

FIERS

ADDEO

20 ERR

REC 3o LHTS PROTOCOL

UCi/g $\%$

TEST UCi/g

$\% \quad \% \quad$ (TOTAL) LIMITS PREP BATCH

GEA Analytes

Cobalt 60

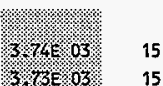

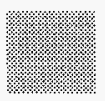

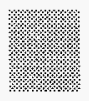

GEA

379003

GEA \% 26003

5.0

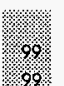

$76-124$
$76-124$

96012649

96012649
Lab id 222-S

Protocol SST

Version 1.0

Form DVD-LCS

Version 3.08

Report date $02 / 24 / 97$ 
HNF-SD-WM-DP-219, REV.O

SDG 96001379

Contact L. L. Fritts
222-S IABORATORY

TANK 241-B-108, CORE 172

LAB CONTROL SAMPLES
Client TWRS

Tank 241-B-108

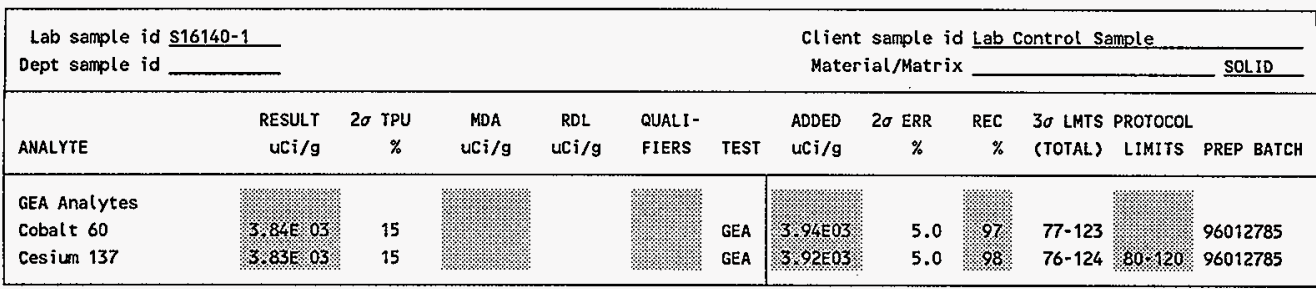

\section{Group 96001379}

LAB CONTROL SAMPLES

Page 3

SUMMARY DATA SECTION

Page 14
Lab id 222-s

Protocol SST

Version 1.0

Form DVD-LCS

Version 3.08

Report date $02 / 24 / 97$ 


\section{2 -S LABORATORY}

TAMK 241-B-108, CORE 172

DUPIICATE

SDG $\underline{96001379}$

Contact L. L. Fritts DUPLICATE

Lab sample id $\$ 96 \mathrm{T0054630}$ Dept sample id
Lab sample id \$96T005463

Dept sample id

Received $10 / 17 / 96$
Client JWRS

Tank 241-8-108

Client sample id B108 C172 FB Direct J116

Location/Matrix R: 6 S: Field Blank C: 1 LIQUID Collected

Chain of custody id

\begin{tabular}{|c|c|c|c|c|c|c|c|c|c|c|c|c|c|}
\hline ANALYTE & $\begin{array}{r}\text { DUPLICATE } \\
\text { UCi/ml }\end{array}$ & $\begin{array}{c}2 \sigma \text { TPU } \\
\%\end{array}$ & $\begin{array}{c}\text { MDA } \\
\text { uCi/ml }\end{array}$ & $\begin{array}{c}\text { RDL } \\
\mathrm{uCi} / \mathrm{mL}\end{array}$ & $\begin{array}{l}\text { QUALI- } \\
\text { FIERS }\end{array}$ & TEST & $\begin{array}{r}\text { ORIGINAL } \\
\text { UCi/ml }\end{array}$ & $\begin{array}{c}2 \sigma \text { TPU } \\
x\end{array}$ & $\begin{array}{c}\text { MDA } \\
\text { uci/ml }\end{array}$ & $\begin{array}{l}\text { QUALI - } \\
\text { FIERS }\end{array}$ & $\begin{array}{r}\text { RPD } \\
\%\end{array}$ & $\begin{array}{r}30 \\
\text { TOT }\end{array}$ & $\begin{array}{r}\text { PROT } \\
\text { LIMIT }\end{array}$ \\
\hline Total Alpha & K $/ \mathrm{k} 06$ & & $1 \% 106$ & & Uut & AT & $471 \mathrm{E} 06$ & & \% 106 & UL & $\%$ & & \%) \\
\hline
\end{tabular}

Loc: Riser: 6 Seg: Field 8lank Core: 172
Samp: B108 C172 FB Direct $\mathbf{J 1 1 6 0 0}$

Loc: Riser: 6 Seg: Field Blank Core: 172

\section{Group 96001379}

DUPLICATES

Page 1

SUMAMAR DATA SECTION

Page 15
Lab id 222-s

Protocol. SST

Version 1.0

Form DVD-DUP

Version 3.08 Report date $02 / 24 / 97$ 
HNF-SD-WM-DP-219, REV. 0

222-S LABORATORY

TANK 241-B-108, CORE 172

DUPLICATE

SDG 26001379

Contact L. L. Fritts DUPLICATE

Lab sample id \$96T0055100

Dept sample id
ORIGINAL

Lab sample id \$96T005510 Dept sample id Received 10/21/96
Client TWRS

Tank 241-B-108

Client sample id B108 C172S1 UH Fusion Location/Matrix R: 6 S: 1 C: 172 FUSION Collected

Chain of custody id $n / a$

\begin{tabular}{|c|c|c|c|c|c|c|c|c|c|c|c|c|c|}
\hline ANALYTE & $\begin{array}{l}\text { DUPLICATE } \\
\text { UCi/g }\end{array}$ & $\begin{array}{c}2 \sigma \text { TPU } \\
\%\end{array}$ & $\begin{array}{c}\text { MDA } \\
\mathrm{uCi} j / \mathrm{g}\end{array}$ & $\begin{array}{c}\text { RDL } \\
\mathrm{uCi} / \mathrm{g}\end{array}$ & $\begin{array}{l}\text { QUALI- } \\
\text { FIERS }\end{array}$ & TEST & $\begin{array}{l}\text { ORIGINAL } \\
\text { UCi/g }\end{array}$ & $\begin{array}{c}2 \sigma \text { TPU } \\
x\end{array}$ & $\begin{array}{c}\text { MDA } \\
\mathrm{uCi} / \mathrm{g}\end{array}$ & $\begin{array}{l}\text { QUALI - } \\
\text { FIERS }\end{array}$ & $\begin{array}{r}\text { RPD } \\
\%\end{array}$ & $\begin{array}{r}3 \sigma \\
\text { TOT }\end{array}$ & $\begin{array}{r}\text { PROT } \\
\text { LIKIT }\end{array}$ \\
\hline GEA Analytes & & & & & & & & & & & & & \\
\hline Cobalt 60 & Y2102 & & $1 \times 2 \mathrm{E} 02$ & & \% & GEA & $\times 1 \times 202$ & & $12 \times 02$ & $\mathrm{U}$ & & & \\
\hline Cesium 137 & \% $540 \%$ & 15 & $0 \% 0 \mathrm{~g} 00$ & & & GEA & $508=02$ & 15 & 0.0000 & & 199 & 45 & 20. \\
\hline Europium 154 & 3 8502 & & $3 \times 80 \%$ & & \% & GEA & $3 \times 8002$ & & 3.80102 & $\mathrm{u}$ & \& & & \\
\hline Europiun 155 & 142401 & & $1 / 2=01$ & & $\%$ & GEA & $1 / 124 \times 01$ & & $1 \times 20 \% 0 \%$ & u & \% & & \\
\hline Americium 241 & $3 \times 1001$ & & 31500 & & U. & GEA & $\times 3 / 101$ & & $3 \times 1201$ & $u$ & \% & & \\
\hline
\end{tabular}

\section{Group 96001379}

Lab id 222-s

DUPLICATES

Page 2

SUMLARY DATA SECTION

Page 16

Protocol SST

Version 1.0

Form DVD-DUP

Version 3.08

Report date $\underline{02 / 24 / 97}$ 
HNF-SD-WM-DP-219, REV.O

$222-S$ LABORATORY

TANK 241-B-108, CORE 172

\section{DUPLICATE}

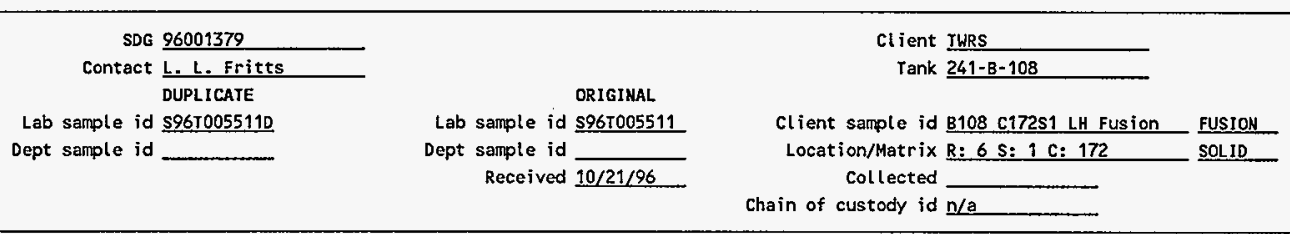

\begin{tabular}{|c|c|c|c|c|c|c|c|c|c|c|c|c|c|}
\hline ANALYTE & $\begin{array}{l}\text { DUPLICATE } \\
\text { uCi/g }\end{array}$ & $\begin{array}{c}2 \sigma \text { TPU } \\
\%\end{array}$ & $\begin{array}{c}\text { MDA } \\
\text { uCi/g }\end{array}$ & $\begin{array}{c}\text { RDL } \\
\text { uCi } / g\end{array}$ & $\begin{array}{l}\text { QUALI- } \\
\text { FIERS }\end{array}$ & TEST & $\begin{array}{l}\text { ORIGINAL } \\
\qquad \mathrm{uCj} / \mathrm{g}\end{array}$ & $\begin{array}{c}2 \sigma \text { TPU } \\
\%\end{array}$ & $\begin{array}{c}\text { MDA } \\
\mathrm{uCi} / \mathrm{g}\end{array}$ & $\begin{array}{l}\text { QUALI - } \\
\text { FIERS }\end{array}$ & $\begin{array}{r}\text { RPD } \\
x\end{array}$ & $\begin{array}{r}3 \sigma \\
\text { Tot }\end{array}$ & $\begin{array}{l}\text { PROT } \\
\text { LIMIT }\end{array}$ \\
\hline Total Alpha & 804003 & 89 & $45=203$ & & $\%$ & AT & 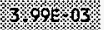 & 93 & 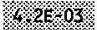 & $\mathrm{U}$ & & & \\
\hline Total Beta & 85000 & 15 & $1 \times 6802$ & & & TB & 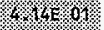 & 15 & 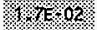 & & 4 & 32 & 20. \\
\hline Strontium 90 & $5888=03$ & 15 & $288=03$ & & & $S R$ & $425=01$ & 15 & 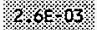 & & 9 & 33 & 20 \\
\hline GEA Analytes & & & & & & & & & & & & & \\
\hline Cobalt 60 & 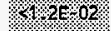 & & 12602 & & 4 & GEA & YKE KOZ & & 10202 & U & & & \\
\hline Cesium 137 & 300504 & 15 & 000000 & & & GEA & $1 \% 635 \% 2$ & 14 & $0 \times 0500 \%$ & & 199 & 45 & 20. \\
\hline Europium 154 & 3. & & 80.02 & & 16 & GEA & 3. & & $501=02$. & U & & & \\
\hline Europium 155 & 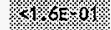 & & $1 \% 60 \%$ & & 6 & GEA & $4 \% 601$ & & $16=01$ & U & & & \\
\hline Americium 241 & $4 \times 1 E 01 \%$ & & $411=201$ & & $4 \%$ & GEA & 241607 & . & (1E0\% & U & & & \\
\hline
\end{tabular}

\section{Group 96001379}

DUPLICATES

Page 3

SUMALARY DATA SECTION

Page 17
Lab id 222-S

Protocol SST

Version 1.0

Form DVD-DUP

Version 3.08

Report date $02 / 24 / 97$ 


\section{HNF-SD-WM-DP-219, REV. 0}

\section{2 -S LABORATORY}

TANK 241-B-108, CORE 172

DUPLICATE

SDG 96001379

Contact L. L. Fritts

DUPLICAIE

Lab sample id $\mathbf{5 9 6 \mathrm { T } 0 0 5 5 1 2 0}$

Dept sample id
ORIGINAL

Lab sample id \$967005512

Dept sample id

Received 10/21/96
Client TWRS

Tank 24i-B-108

Client sample id B108 C172\$2 LH Fusion FUSION

Location/Matrix R: 6 s: 2 C: 172 SOLID Collected

Chain of custody id $n / a$

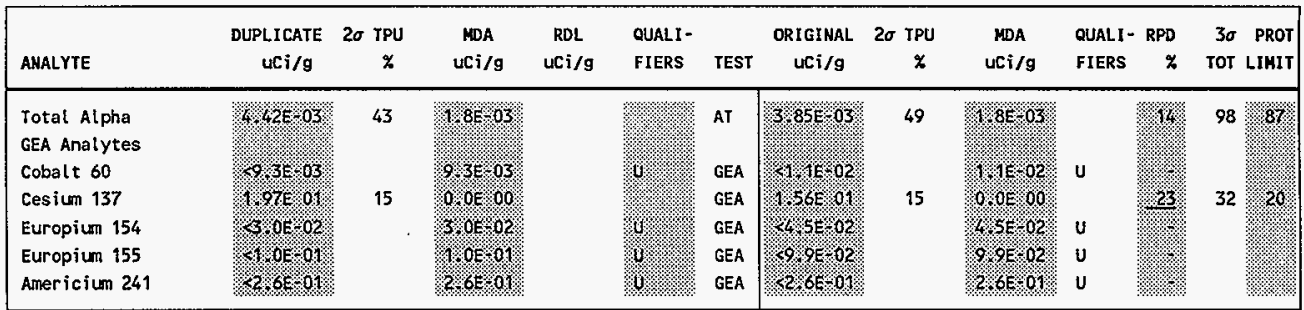

\section{Group 96001379}

Lab id 222-S

Protocol SST

Version 1.0

Form DVD-DUP

Version 3.08

Report date $\underline{02 / 24 / 97}$ 
$222-S$ LABORATORY

TANK 241-B-108, CORE 172

DUPLICATE

SDG 96001379

Contact L. L. Fritts DUPLICATE

Lab sample id \$96T0055230

Dept sample id
ORIGINAL.

Lab sample id \$967005523 Dept sample id

Received 10/21/96
Client TWRS

Tank 241-B-108

Client sample id B108 C172S1 DL Direct V\# Location/Matrix R: $6 \mathrm{~S}: 1 \mathrm{C}: 172$ Collected

Chain of custody id

\begin{tabular}{|c|c|c|c|c|c|c|c|c|c|c|c|c|}
\hline AHALYTE & $\begin{array}{r}\text { DUPLICATE } \\
\text { uCi/ml }\end{array}$ & $\begin{array}{c}2 \sigma \text { TPU } \\
\%\end{array}$ & $\begin{array}{c}\text { MDA } \\
\text { uCi /ml }\end{array}$ & $\begin{array}{c}\text { RDL } \\
\text { uCi /ml }\end{array}$ & $\begin{array}{l}\text { QUALI- } \\
\text { FIERS }\end{array}$ & TEST & $\begin{array}{r}\text { ORIGINAL } \\
\text { uCi/mi }\end{array}$ & $\begin{array}{c}2 \sigma \mathrm{TPU} \\
\times\end{array}$ & $\begin{array}{c}\text { MDA } \\
\text { uCi/ml }\end{array}$ & $\begin{array}{l}\text { QUALI - } \\
\text { FIERS }\end{array}$ & $\begin{array}{r}\text { RPD } \\
\mathbf{x}\end{array}$ & $\begin{array}{l}3 \sigma \text { PROT } \\
\text { TOT LIMIT }\end{array}$ \\
\hline Total Alpha & $\% 6,9103$ & & 69803 & & 誉 & AT & 66.9903 & & $6 \% 03$ & U & \% & 稵 \\
\hline
\end{tabular}

Lab id 222-s

Protocol SST Version 1.0 Form DVD-DUP Version 3.08 Report date $02 / 24 / 97$ 
HNF-SD-WM-DP-219, REV. 0

222-S LABORATORY

TANK 241-B-108, CORE 172

MATRIX SPIKE

SDG 96001379

Contact 1. L. Fritts MATRIX SPIKE

Lab sample id s96T005511s

Dept sample id
ORIGINAL

Lab sample id \$96r005511

Dept sample id

Received $10 / 21 / 96$
Client TWRS

Tank 241-8-108

Client sample id B108 C172s1 LH Fusion Location/Matrix R: 6 s: 1 C: 172 Collected

Chain of custody id $\underline{n / a}$

\begin{tabular}{|c|c|c|c|c|c|c|c|c|c|c|c|c|}
\hline ANALYTE & $\begin{array}{l}\text { SPIKE } \\
\mathrm{UCi} / \mathrm{g}\end{array}$ & $\begin{array}{c}2 \sigma \text { TPU } \\
\%\end{array}$ & $\begin{array}{c}\text { MDA } \\
\mathbf{u C i} / \mathbf{g}\end{array}$ & $\begin{array}{c}\text { RDL. } \\
\text { uCî/g }\end{array}$ & $\begin{array}{l}\text { QUALI- } \\
\text { FIERS }\end{array}$ & TEST & $\begin{array}{l}\text { ADDED } \\
\text { uCi/g }\end{array}$ & $\begin{array}{c}2 \sigma \text { ERR } \\
\%\end{array}$ & $\begin{array}{l}\text { ORIGINAL } \\
\text { uCi/g }\end{array}$ & $\begin{array}{c}2 \sigma \mathrm{TPU} \\
\%\end{array}$ & $\begin{array}{r}\text { REC } 3 \sigma \text { LMTS } \\
\times \text { (TOTAL) }\end{array}$ & $\begin{array}{l}\text { PROTOCOL } \\
\text { LIMITS }\end{array}$ \\
\hline $\begin{array}{l}\text { Total Alpha } \\
\text { Total Beta }\end{array}$ & 16.8\% & & & & & $\begin{array}{l}A T \\
T B\end{array}$ & MoE0/ & $\begin{array}{l}5.0 \\
5.0\end{array}$ & 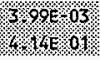 & $\begin{array}{l}93 \\
15\end{array}$ & $\begin{array}{l:l}45 & 87-113 \\
65 & 64-136\end{array}$ & $35 \times 125$ \\
\hline
\end{tabular}

\section{Group 96001379}

MATRIX SPIKES

Page 1

SURAARY DATA SECTION

Page 20
Lab id 222-S

Protocol SST

Version 1.0

Form OVD-MS

Version 3.08

Report date $02 / 24 / 97$ 


\section{2 -S LABORATORY}

TANK 241-8-108, CORE 172

MATRIX SPIRE

SDG 96001379

Contact L. L. Fritts MATRIX SPIKE

Lab sample id s967005523s

Dept sample id

\section{ORIGINAL}

Lab sample id \$96T005523

Dept sample id Received $10 / 21 / 96$
Client TWRS

Tank 241-B-108

$C l$ ient sample id B108 C172S1 DL Direct V\#

Location/Matrix R: 6 S: 1 C: 172 LIQUID Collected Chain of custody id

\begin{tabular}{|c|c|c|c|c|c|c|c|c|c|c|c|c|}
\hline ANALYTE & $\begin{array}{l}\text { SPIKE } \\
\mathrm{uCi} / \mathrm{ml}\end{array}$ & $\begin{array}{c}2 \sigma \text { TPU } \\
x\end{array}$ & $\begin{array}{c}\text { MDA } \\
\text { uCi/ml }\end{array}$ & $\begin{array}{c}\mathrm{RDL} \\
\mathrm{uCi} / \mathrm{ml}\end{array}$ & $\begin{array}{l}\text { QUALI- } \\
\text { FIERS }\end{array}$ & TEST & $\begin{array}{l}\text { ADDED } \\
\mathrm{uCj} / \mathrm{ml}\end{array}$ & $\begin{array}{c}2 \sigma \text { ERR } \\
x\end{array}$ & $\begin{array}{r}\text { ORIGINAL } \\
\text { UCi/ml }\end{array}$ & $\begin{array}{c}2 \sigma \text { TPU } \\
\%\end{array}$ & $\begin{array}{r}\text { REC } 3 \sigma \text { LHTS } \\
\times \text { (TOTAL) }\end{array}$ & $\begin{array}{l}\text { PROTOCOL } \\
\text { LIMITS }\end{array}$ \\
\hline Total Alpha & $6535 \%$ & & 1/. & & & AT & $3595 \%$ & 5.0 & & & 98 . $77-123$ & 754125 \\
\hline
\end{tabular}

Group 96001379

MATRIX SPIKES

Page 2

SUMMARY DATA SECTION

Page 21
Lab id 222-s Protocol SST Version 1.0 Form DVD-MS

Version 3.08 Report date $02 / 24 / 97$ 
$222-S$ I A B O R A T OR Y TANK 241-B-108, CORE 172

D A T A S H E T
SDG 96001379

Contact L. L. Fritts

Lab sample id s96T005463

Dept sample id

Received $10 / 17 / 96$
Client TWRS

Tank 241-B-108

Client sample id B108 C172 FB Direct J116

Location/Matrix R: 6 S: Field Blank C: 1 LIQUID Collected

Chain of custody id

\begin{tabular}{|c|c|c|c|c|c|c|c|}
\hline ANALYTE & CAS NO & $\begin{array}{l}\text { RESULT } \\
\text { uCi } i / m l\end{array}$ & $\begin{array}{c}2 \sigma \\
\text { TPU } \\
8\end{array}$ & $\begin{array}{c}\mathrm{MDA} \\
\mathrm{uCi} / \mathrm{ml}\end{array}$ & $\begin{array}{c}\mathrm{RDL} \\
\mathrm{uCi} / \mathrm{ml}\end{array}$ & $\begin{array}{l}\text { QUALI- } \\
\text { FIERS }\end{array}$ & TEST \\
\hline Total Alpha & $12587-46-1$ & $\% 11 \mathrm{E}-0 \delta$ & & \% $1 \mathrm{~F}-06$ & & $61 \%$. & $\mathrm{AT}$ \\
\hline
\end{tabular}

Samp: B108 C172 FB Direct $J 11600$

Loc: Riser: 6 Seg: Field Blank Core: 172

G r U p 96001379

DATA SHEETS

Page 1

SUMMARY DATA SECTION

Page 22
Lab id 222-S

Protocol SST

Version 1.0 Form DVD-DS

Version 3.08 Report date $02 / 24 / 97$ 
$222-S$ IA BORATORY TANK 241-B-108, CORE 172

D A T A $S$ H E T
SDG 96001379

Contact I. L. Fritts

Lab sample id $596 \mathrm{T005523}$

Dept sample id Received $10 / 21 / 96$
Client TWRS

Tank 241-B-108

Client sample id B108 C172S1 DL Direct V\# Location/Matrix R: 6 S: 1 C: 172 collected

chain of custody id

\begin{tabular}{|c|c|c|c|c|c|c|c|}
\hline ANALYTE & CAS NO & $\begin{array}{l}\text { RESULT } \\
\text { uci/ml }\end{array}$ & $\begin{array}{c}2 \sigma \text { TPU } \\
8\end{array}$ & $\begin{array}{c}\mathrm{MDA} \\
\mathrm{uCi} / \mathrm{ml}\end{array}$ & $\begin{array}{c}\mathrm{RDI} \\
\mathrm{uCi} / \mathrm{ml}\end{array}$ & $\begin{array}{l}\text { QUALI- } \\
\text { FIERS }\end{array}$ & TEST \\
\hline Total Alpha & $12587-46-1$ & $46 \% \mathrm{n}^{\circ} 0 \mathrm{~s}$ & & $1609 \mathrm{p}-03$ & & U & AT \\
\hline
\end{tabular}

Samp: B108 C172S1 DI Direct V\#11647

G r o p p $9 \begin{array}{lllllllll} & 0 & 0 & 1 & 3 & 7 & 9\end{array}$

DATA SHEETS

Page 2

SUMMARY DATA SECTION

Page 23
Iab id $222-s$

protocol SST

version 1.0 Form DVD-DS

Version 3.08 Report date $\underline{02 / 24 / 97}$ 
HNF-SD-WM-DP-219, REV.O

$222-5$ I A B OR A T OR Y

TANK 241-B-108, CORE 172

D A T A H E T

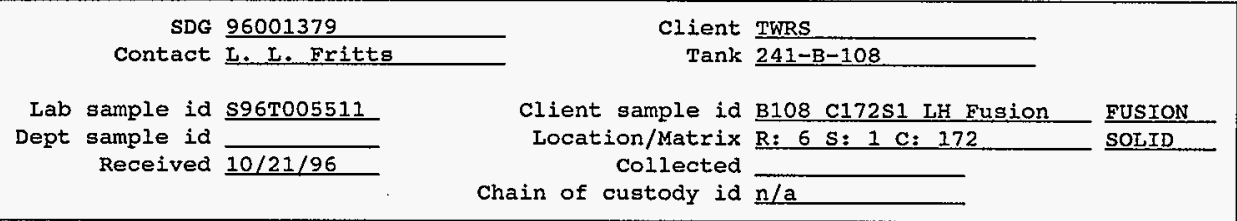

\begin{tabular}{|c|c|c|c|c|c|c|c|}
\hline ANALYTE & CAS No & $\begin{array}{r}\text { RESULT } \\
\mathrm{uci} / \mathrm{g}\end{array}$ & $\begin{array}{c}2 \sigma \text { TPU } \\
8\end{array}$ & $\begin{array}{c}\text { MDA } \\
u C_{i} / g\end{array}$ & $\begin{array}{c}\text { RDL } \\
u C i / g\end{array}$ & $\begin{array}{l}\text { QUALI- } \\
\text { FIERS }\end{array}$ & TEST \\
\hline Total Alpha & $12587-46-1$ & 3. $9900 \%$ & 93 & $1 \% 20003$ & & 6 & AT \\
\hline Total Beta & $12587-47-2$ & $4014 \mathrm{~F} .67$ & 15 & $1 \% 7 \mathrm{~s} .02$ & & & TB \\
\hline strontium 90 & $10098-97-2$ & $4 \% 5 B=0 \%$ & 15 & $206 \% 3$ & & & SR \\
\hline GEA Analytes & & & & & & & \\
\hline Cobalt 60 & $10198-40-0$ & $142 \mathrm{r}, 02$ & & $1 \% 23=02$ & & 0 & GEA \\
\hline Cesium 137 & $10045-97-3$ & $16 \% 3402$ & 14 & $000 \mathrm{H}, 00$ & & & GEA \\
\hline Europium 154 & $15585-10-1$ & $Y 36,9=02$ & & $3.9 \mathrm{E}-02$ & & 4 & GEA \\
\hline Europium 155 & $14391-16-3$ & 1.6. & & $1 \% \mathrm{E}-\mathrm{og}$ & & 4 & GEA \\
\hline Americium 241 & $14596-10-2$ & $44+\mathrm{s}=01$ & & $4 \times 1 \mathrm{~s} .0 \%$ & & 16 & GEA \\
\hline
\end{tabular}

Group 96001379

DATA SHEETS

Page 3

SUMMARY DATA SECTION

Page 24
Lab id 222-s

Protocol SST

Version 1.0 Form DVD-DS

Version 3.08 Report date $\underline{02 / 24 / 97}$ 
HNF-SD-WH-DP-219, REV. 0

$222-5$ L A B O R A T O R Y TANK 241-B-108, CORE 172

D A A S HE T

SDG 96001379

Contact L. L. Fritts

Lab sample id S96T005510

Dept sample id

Received $10 / 21 / 96$
Client TWRS

Tank 241-B-108

Client sample id B108 C172S1 UH Fusion

Location/Matrix R: 6 S: 1 C: 172 collected

Chain of custody id $\mathrm{n} / \mathrm{a}$
FUSION

SOLID

\begin{tabular}{|c|c|c|c|c|c|c|c|}
\hline ANALYTE & cas No & $\begin{array}{r}\text { RESULT } \\
\text { uCi/g }\end{array}$ & $\begin{array}{c}20 \mathrm{TPU} \\
\frac{8}{6}\end{array}$ & $\begin{array}{c}\mathrm{MDA} \\
\mathrm{uC} \dot{\mathrm{I}} / \mathrm{g}\end{array}$ & $\begin{array}{c}\text { RDL } \\
\text { uCi/g }\end{array}$ & $\begin{array}{l}\text { QUALI- } \\
\text { FIERS }\end{array}$ & TEST \\
\hline $\begin{array}{l}\text { GEA Analytes } \\
\text { Cobalt } 60\end{array}$ & $10198-40-0$ & 1.2. & & $102 \mathrm{~F}=02$. & & & GEA \\
\hline Cesium 137 & $10045-97-3$ & $5.08 \mathrm{~F}=0.2$ & 15 & 0.00 E .00. & & & GEA \\
\hline Europium 154 & $15585-10-1$ & $<3,8 \mathrm{E}=02$ & & $3.8 \mathrm{E}-02$ & & W. & GEA \\
\hline Europium 155 & $14391-16-3$ & $4.9 .2 \mathrm{~F}-01$ & & $1.20 \% 04$ & & 0 & GEA \\
\hline Americium 241 & $14596-10-2$ & 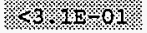 & & $2.1 \mathrm{E}=0 \mathrm{O}$ & & & GEA \\
\hline
\end{tabular}

G I U P 96001379

DATA SHEETS

Page 4

SUMMARY DATA SECTION

Page 25
Lab id 222-s

Protocol SST

version 1.0

Form DVD-DS

Version 3.08

Report date $02 / 24 / 97$ 
HNF-SD-WM-DP-219, REV. 0

\section{$222-S$ I A BORATOR Y} TANK 241-B-108, CORE 172

\section{A A SHE T}

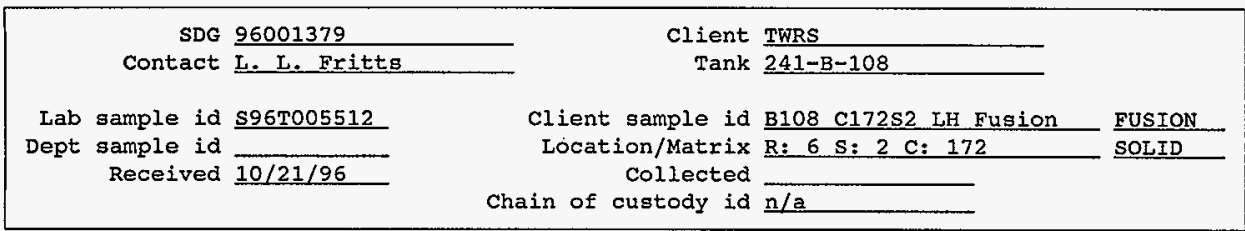

\begin{tabular}{|c|c|c|c|c|c|c|c|}
\hline ANALYTE & CAS NO & $\begin{array}{r}\text { RESULT } \\
\mathrm{uCi} / \mathrm{g}\end{array}$ & $\begin{array}{c}20 \mathrm{TPU} \\
\frac{8}{8}\end{array}$ & $\begin{array}{l}\text { MDA } \\
u C i / g\end{array}$ & $\begin{array}{c}\text { RDL } \\
\mathrm{uCi} / \mathrm{g}\end{array}$ & $\begin{array}{l}\text { QUALI- } \\
\text { FIERS }\end{array}$ & TEST \\
\hline $\begin{array}{l}\text { Total Alpha } \\
\text { GEA Analytes }\end{array}$ & $12587-46-1$ & \% 85 E 08 & 49 & $1 / 8 \mathrm{~B}-03$ & & & $\mathrm{AT}$ \\
\hline Cobalt 60 & $10198-40-0$ & $1+15=02$ & & $1 \times 1=02$ & & (1) & GEA \\
\hline Cesium 137 & $10045-97-3$ & $1.56 \mathrm{H} .04$ & 15 & $0.00 .00 \%$ & & & GEA \\
\hline Europium 154 & 15585-10-1 & \%4 $5 \mathrm{~B}=102$ & & $4 \cdot 5 \mathrm{P} \cdot 02$. & & 18 & GEA \\
\hline Europium 155 & $14391-16-3$ & $29.9 P-02$ - & & $9.9 \mathrm{~B}=02$ & & 13 & GEA \\
\hline Americium 241 & $14596-10-2$ & $12.6 \mathrm{E}=011$ & & $2.6 \mathrm{E}-01$ \% & & 80 & GEA \\
\hline
\end{tabular}

G r u p 96001379

DATA SHEETS

Page 5

SUMMARY DATA SECTION

Page 26
Lab id 222-S

Protocol SST

Version 1.0

Form DVD-DS

Version 3.08

Report date $\underline{02 / 24 / 97}$ 


\title{
HNF-SD-WM-DP-219, REV. 0
}

\section{2 -S LABORATORY}

TANK 241-B-108, CORE 172

Test $\underline{\mathrm{SR}}$ Matrix SOLIO

SDG 96001379

Contact L. L. Fritts

\section{METHOD SUMMARY \\ STRONT IUM-89/90 \\ GAS PROPORTIONAL COUNTIKG}

Client TWRS

Tank 241-B-108

\section{RESUITS \\ CLIENT SAMPLE IO \\ LAB RAW SUF- \\ SAMPLE ID TEST FIX PLAHChET Strontium 90 \\ Preparation batch 96012651 \\ B108 C172s1 LH Fusion FU 596T005511 \\ B108 C172S1 LH Fusion-DU \$96T0055110 \\ Method Blank \\ B16018-2 \\ Lab control Sample \\ \$16018-1

$16018-4$
$16018-5$
$16018-2$
$16018-1$

Hominal values and limits fron method

RDLs (uCi/g)

\section{METHOD PERFORMANCE}

CLIENT SAMPLE ID

LAB RAH SUF- MDA

ALIQ PREP DILU- YIELD EFF COUNT FWHM DRIFI DAYS

ANAL-

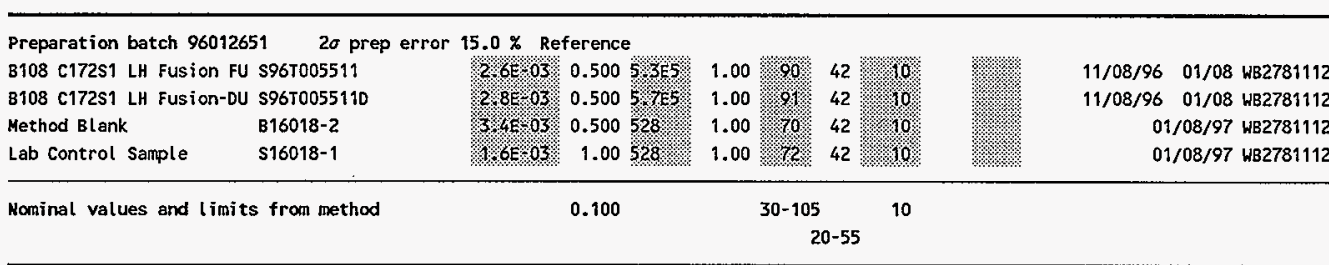

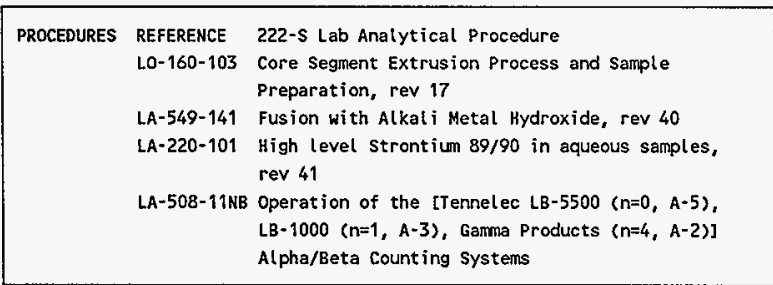

\begin{tabular}{|lr|}
\hline AVERAGES \pm 2 SD & MOA $\frac{2.6 E-03}{81} \pm \frac{1.5 E-03}{23}$ \\
FOR 4 SAMPLLS & YIELL \\
& EFFICIENCY
\end{tabular}

\section{Group 96001379}

\author{
METHOO SUMMARIES \\ Page 1 \\ SUMMARY DATA SECTION
}

Page 27

Lab id 222-s

Protocol SST

Version 1.0

Form DVD-CMS

Version 3.08

Report date $02 / 24 / 97$ 
HNF-SD-WM-DP-219, REV. 0

$222-S$ LABORATORY

TANK 241-B-108, CORE 172

Test AT Matrix LIQUID

SDG 26001379

Contact L. L. Fritts
METHOD SUMMARY

ALPHA ANALYSIS

GAS PROPORTIONAL COUNTING
$C l$ jent IWRS

Tank $\underline{241-B-108}$
RESUITS
CLIENT SAMPLE IO
LAB RAU SUF-
1: Total
2: sum, Alpha
RESULT RATIO (\%)
SAMPLE ID TEST FIX PLANCHET
Alpha
Emitters
$2 \div 1 \quad 2 \sigma$

Preparation batch 96010892

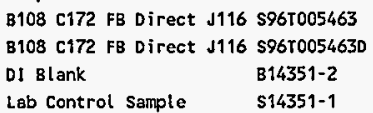

$14351-4$

14351-5

14351-2

14351-1
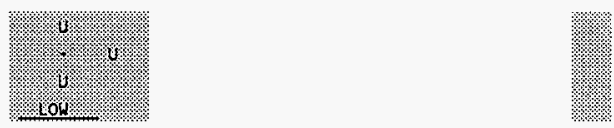

Preparation batch 96011188

B108 C172S1 DL Direct V\# S96r005523

B108 C172S1 DL Direct V\# \$96T005523D

B108 C172s1 DL Direct V\# S96T005523S

DI Blank

B14608-2

Lab Control Sample

s14608-1

$14608-4$
$14608-5$
$14608-6$
$14608-2$
$14608-1$

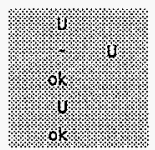

Nominal values and limits from method

RDLs (uci/ml)

80

Average

\section{METHOD PERFORMANCE \\ LAB RAH SUF- MDA ALIQ PREP DILU- RESID EFF COUNT FHHM DRIFT DAYS \\ ANAL-}

CLIENT SAMPLE ID

SAMPLE ID TEST FIX UCi/mL

mL FAC TION mS

\% min keV KeV held PREPAREd

YZED DETECTOR

Preparation batch $96010892 \quad 2 \sigma$ prep error $15.0 \%$ Reference B108 C972 FB Direct J116 \$96T005463

B108 C172 FB Direct 1116 \$96T005463D

DI Blank

B $14351-2$

rote 6 \%

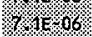

1\% 1006

$0.100 \%$ \%o

0.100 林

\% $1 \mathrm{~F} \mathrm{oz}$

0.100

$1.00 \% 1 \% 0$

1.00
1.00
1.00
1.00

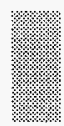

$10 / 29 / 96$ WB27807

$10 / 29 / 96$ WB27807

$10 / 29 / 96$ W827807

$10 / 29 / 96$ WB27807

Preparation batch 9601118820 prep error $15.0 \%$ Reference

B108 C172S1 DL. Direct V\# S96T005523

B108 C172S1 DL Direct V\# \$96T005523D

B108 C172S1 DL. Direct V\# S96T005523S

DI Blank

B14608-2

Lab Control Sample

s14608-1

\begin{tabular}{|c|c|c|c|}
\hline $601 \% 03$ & 1.00 & 100 & 10200 \\
\hline 6.1003 & 1.00 & 100 & 10200 \\
\hline & 1.00 & $1 \times 00$ & 1.00 \\
\hline $1690 \times 03$ & 1.00 & & 10200 \\
\hline 608507 & 1.00 & 100 & 1.00 \\
\hline
\end{tabular}

21
21
21
21
21

$11 / 06 / 96$

WB27809

$11 / 06 / 96$ WB27809

$11 / 06 / 96$ WB27809

$11 / 06 / 96$ WB27809

$11 / 06 / 96$ WB27809

Hominal values and limits from method

0.100

30

$20-55$

\section{Group 96001379}

METHOD SUMMARIES

Page 2

SUMMARY DATA SECTION

Page 28
Lab id 222-S

Protocol SST

Version 1.0

Form DVD-CMS

Version 3.08 Report date $02 / 24 / 97$ 
HNF-SD-WM-DP-219, REV.O

\section{2-S LABORATORY}

TANK 241-B-108, CORE 172

Test Ar Matrix

SDG 96001379

Contact L. L. Fritts

\section{METHOD SUMMARY, cont. ALPHA ANALYSIS \\ GAS PROPORTIONAL CONNING}

Client IWRS

Tank 241-8-108
PROCEDURES REFERENCE 222-S Lab Analytical Procedure

L0-160-103 Core Segment Extrusion Process and Sample Preparation, rev 17

LA-508-101A Alpha in liquid samples, rev 42

LA-508-11NA Operation of the [Tennelec LB-5500 ( $n=0, A-5)$, LB-1000 ( $n=1, A-3)$, Gamma Products $(n=4, A-2)]$

Alpha/Beta Counting Systems
AVERAGES \pm 2 SD

FOR 9 SAMPLES
MDA $2.6 \mathrm{E}-03 \pm 7.1 \mathrm{E}-03$

EFFICIENCY $22 \pm \underline{2.1}$

\section{Group 96001379}

METHCD SUMMARIES

Page 3

SUMALARY DATA SECTION

Page 29
Lab id 2?2-s Protocol SST

Version 1.0

Form DVD-CMS

Version 3.08

Report date $02 / 24 / 97$ 


\section{2 - S IABORATORY}

TANK 241-B-108, CORE 172

Iest AT Matrix SOLID SOG 96001379

Contact L. L. Fritts

\section{METHOD SUMMARY}

ALPHA ANALYSIS

GAS PROPORTIONAL COUNTING
Client TWRS

Tank 241-B-108

\section{RESULTS \\ CLIENT SAMPLE ID \\ LAB RAL SUF- \\ 1: Total 2: Sum, Alpha \\ RESULT RATIO (\%) \\ SAMPLE ID TEST FIX PLANCHET Alpha Emitters \\ $2 \div 1 \quad 2 \sigma$}

Preparation batch 96011382

B108 C172S2 LH Fusion FU S96T005512

B108 C172S2 LH Fusion-DU \$96T005512D

Method Blank

814788-2

Lab Control Sample

\$14788-1

14788-7

$14788-8$

14788-2

14788-1

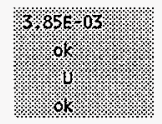

$\begin{array}{lll}01 & 14949-4 & \\ 01 & 14949-5 & \\ 01 & 14949-6 & \text { \% } \\ & 14949-2 & \text { \% } \\ & 14949-1 & \end{array}$

RDLS (uCi/g)
Preparation batch 96011553

B108 C172S1 LH Fusion Fu S96T005511

B108 C172s1 LH Fusion-DU S96T0055110

B108 C172S1 LH Fusion-SP S96T005511s

Method Blank

B $94949-2$

Lab Control Sample

s14949-1
80

Average

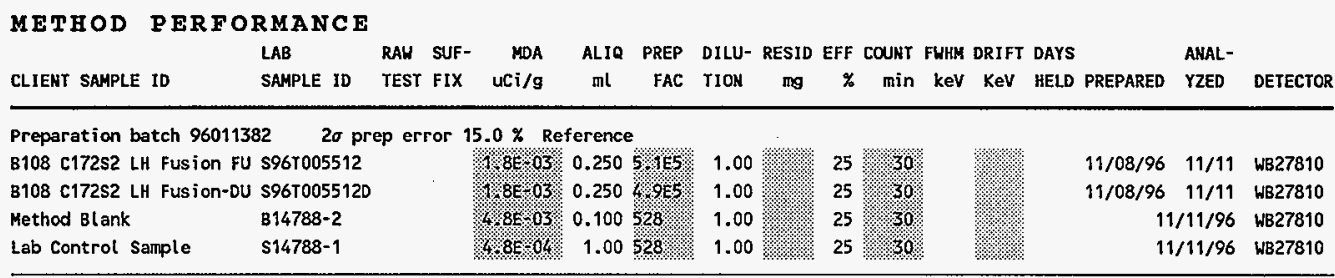

\section{Preparation batch $96011553 \quad 2 \sigma$ prep error $15.0 \%$ Reference}

B108 C172S1 LH Fusion FU S96T005511

B108 C172\$1 Lh Fusion-DU \$96r0055110

B108 C172s1 LH Fusion-SP S96r005511S

Method Blank

B14949-2

Lab Control Sample

s14949-1

1.00
1.00
1.00
1.00
1.00

27
27
27
27
27
$370 \% 0$
$30 \%$

$11 / 08 / 96 \quad 11 / 20 \quad$ WB27806

$11 / 08 / 96 \quad 11 / 20$ WB27806

$11 / 08 / 96 \quad 11 / 20 \quad$ WB27806

$11 / 20 / 96$ WB27806

$11 / 20 / 96$ WB27806

\section{Group 96001379}

\section{METHOD SUIMMARIES}

Page 4

SIMMARY DATA SECTION

Page 30
Lab id 222-s Protocol SST Version 1.0 Form DVD-CMS

Version 3.08 Report date $02 / 24 / 97$ 
HNF-SD-WM-DP-219, REV. 0

22 2-S LABORATORY

TANK 241-B-108, CORE 172

Test AT Matrix

SDG 96001379

Contact L. L. Fritts

\section{METHOD SUMMARY, cOnt. ALPHA ANALYSIS}

GAS PROPORTIONAL COUNTING
Client TWRS

rank 241-B-108
PROCEDURES REFERENCE 222-S Lab Analytical Procedure

10-160-103 Core Segment Extrusion Process and Sample Preparation, rev 17

LA-549-141 Fusion with Alkali Metal Hydroxide, rev 40

LA-508-101A Alpha in liquid samples, rev 42

L.A-508-11WA Dperation of the [Tennelec $1 B-5500$ ( $n=0, A-5)$, L.B-1000 ( $n=1, A-3)$, Gamma Products $(n=4, A-2)]$

Alpha/Beta Counting Systens
MDA 2.8E-03 $\pm \underline{3.7 \mathrm{E}-03}$

FOR 9 SAMPLES EFFICIENCY $26 \div$

\section{Group 96001379}

METHOD SUMMARIES

Page 5

SUMAARY DATA SECTION

Page 31
Lab id 222-S

Protocol SST

Version $\mathbf{1 . 0}$

Form DVD-CMS

Version 3.08

Report date $02 / 24 / 97$ 
HNF"-SD-WM-DP-219, REV. O

\section{$222-S$ LABORATORY}

TANK 241-B-108, CORE 172

Test $\mathrm{IB}$. Matrix SOL ID

SDG 96001379

Contact L.L. Fritts
METHOD SUMMARY

BETA ANALYSIS

GAS PROPORTIONAL COUNTING
$C t$ ient rWRS

Tank 241-B-108

\section{RESULTS}

\section{CLIENT SAMPLE ID}

LAB RAU SUF-

SAMPLE ID TEST FIX PLANCHET
1: Total 2: Sum, Beta Beta Emitters
RESULT RATIO (\%)

$2 \div 1 \quad 20$
Preparation batch 96012647

8108 c172s1 L\& Fusion FU S96T005511

B108 C172s1 LH Fusion-DU S96T005511D

B108 C172S1 LH Fusion-SP S96T005511S

Method Blank

Lab Control Sample

B16016-2

\$16016-1

$16016-4 \quad \%$ 相
$16016-5$
$16016-6$
$16016-2$
$16016-1$

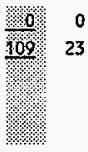

80

Nominal values and limits from method

RDLS (uCi/g)

Average 54

\section{METHOD PERFORMANCE}

\begin{tabular}{|c|c|c|c|c|c|c|c|c|c|c|c|c|c|c|c|}
\hline CLIENT SAMPLE ID & $\begin{array}{l}\text { LAB } \\
\text { SAMPLE ID }\end{array}$ & $\begin{array}{l}\text { RAW } \\
\text { TEST }\end{array}$ & $\begin{array}{l}\text { SUF- } \\
\text { FIX }\end{array}$ & $\begin{array}{r}\text { MDA } \\
\text { uCi/g }\end{array}$ & $\begin{array}{c}\text { ALIQ } \\
\mathrm{mL}\end{array}$ & $\begin{array}{r}\text { PREP } \\
\text { FAC }\end{array}$ & $\begin{array}{l}\text { DItU- } \\
\text { TION }\end{array}$ & $\begin{array}{c}\text { RESID } \\
\mathrm{mg}\end{array}$ & $\begin{array}{l}\text { EFF COUNT } \\
\% \min \end{array}$ & $\begin{array}{r}\text { FWHM } \\
\text { keV }\end{array}$ & $\begin{array}{l}\text { DRIFT } \\
\text { KeV }\end{array}$ & $\begin{array}{l}\text { DAYS } \\
\text { HELD }\end{array}$ & PREPARED & $\begin{array}{l}\text { ANAL } \\
\text { YZED }\end{array}$ & DETECTOR \\
\hline
\end{tabular}

Preparation batch 9601264720 prep error $15.0 \%$ Reference

B108 C172S1 LH Fusion FU S96T005511

B108 C172S1 LH Fusion-DU S96T005511D

8108 C172S1 L.H Fusion-SP S96T005511S

Method Blank

B16016-2

Lab Control Sample

\$16016-1

\begin{tabular}{|c|c|}
\hline $1 / 5=02$ & 0.1005 .315 \\
\hline 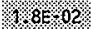 & $0.1005 \%$ \\
\hline & 0.100528 \\
\hline 1870.02 & 0.100528 \\
\hline Yros & 1.00528 \\
\hline
\end{tabular}

0.100

1.00
1.00
1.00
1.00
1.00

$11 / 08 / 96 \quad 01 / 09$ WB26872

$11 / 08 / 96 \quad 01 / 09 \quad$ WB26872

$11 / 08 / 96 \quad 01 / 09$ WB26872

$01 / 09 / 97$ WB26872

$01 / 09 / 97$ WB26872

Hominal values and Limits from method

\subsection{0}
30
$20-55$

AVERAGES \pm 2 SD

MDA $1.3 \mathrm{E}-02 \pm \underline{1.6 \mathrm{E}-02}$

FOR 5 SAMPLES EFFICIENCY $42 \pm 0$

\section{Group 96001379}

\section{METHOO SUMMARIES}

Page 6

SUNMARY DATA SECTION

Page 32
Lab id 222-s

Protocol SST

Version 1.0

Form DVD-CMS

Version 3.08 Report date $02 / 24 / 97$ 


\section{HNF-SD-WM-DP-219, REV. 0}

\section{$222-8$ IABORATORY}

TANK 241-B-108, CORE 172

Test GEA Matrix SOLID

SDG 96001379

Contact L.L. Fritts
METHOD SUMMARY

GAMHA SPECTROSCOPY

GAMMA ENERGY ANALYSIS
Client TWRS

Tank 241-B-108

\section{RESULTS}

CLIENT SAMPLE ID

LAB RAH SUF-

SAMPLE ID TEST FIX PLANChET Cobalt 60 Cesiun 137 Europium 154 Europium 155

Americium

241

Preparation batch 96012649

B108 C172S1 LH Fusion FU \$96T005511

B108 C172\$1 LH Fusion-DU S96T0055110

8108 C17251 UH Fusion FU S967005510

B108 C172S1 UH Fusion-DU 596T005510D

B108 C172S2 LH Fusion-DU S96T005512D

Method Blank

B16020-2

Lab Control Sample
16020-5

16020-6

16020-3

16020-4

16020-8

$16020-2$

$16020-1$
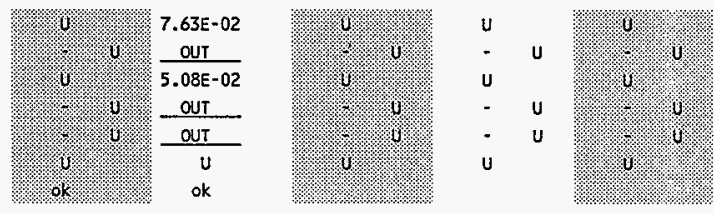

Preparation batch 96012785

B108 c172S2 LH Fusion FU S96T005512

Method Blank

B16140-2

Lab Control Sample

\$16140-1
$01 \quad 16140-3$
16140-2
$16140-1$
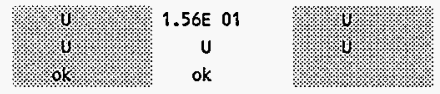

U

Nominal values and limits from method

RDLS (UCi/g)

RAW SUF- MAX MDA ALIQ PREP DILU- YIELD EFF COUNT FUHM DRIFT DAYS

LAB
uCi/g mL FAC IION

ANAL-

CLIENT SAMPLE ID

$2 \sigma$ prep error $15.0 \%$ Reference

Preparation batch $96012649 \quad 2 \sigma$ pr
8108 C172s1 LH Fusion FU S96T005511
B108 C172S1 LH Fusion-DU $\$ 96 T 0055110$
B108 C172S1 UB Fusion FU S96r005510
B108 C172S1 UH Fusion-DU S96T005510D
B108 C172S2 LH Fusion-DU S96T005512D
Method Blank
Lab Control Sample

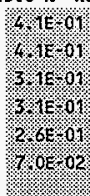

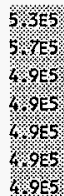

1.00

1.00

1.00

1.00

1.00

1.00

1.00
* $* \min \mathrm{keV}$ KeV held prepared

YZED
Preparation batch $960127852 \sigma$ prep error $15.0 \%$ Reference B108 Cj72S2 LH Fusion FU \$96T005512 Method Blank B16140-2

Lab Control Sample s16140-1

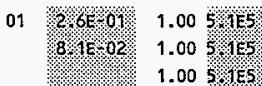

0.100
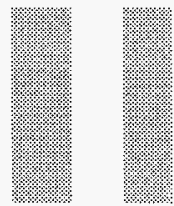

$11 / 08 / 96$

01/09 GEA03

11/08/96 01/09 GEA03

$11 / 08 / 96$ 01/09 GEA03

$11 / 08 / 96 \quad 01 / 09$ GEA03

$11 / 08 / 96 \quad 01 / 10$ GEA03

01/09/97 GEA03

01/09/97 GEA03

Nominal values and limits from method

1.00
1.00
1.00

.

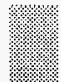

$11 / 08 / 96 \quad 01 / 14$ GEA03 01/14/97 GEA03 01/14/97 GEA03

\section{Group 96001379}

METHOD SUMMARIES

Page 7

SUMOLARY DATA SECTION

Page 33
Lab id 222-5

Protocol SST

Version $\mathbf{1 . 0}$

Form DVD-CMS

Version 3.08 Report date $02 / 24 / 97$ 
HNF-SD-WM-DP-219, REV.O

\section{2 -S LABORATORY}

Test GEA Matrix SDG 96001379

Contact L.L. Fritts
TANK 241-B-108, CORE 172

METHOD SUMMARY, cOnt. GAMMA SPECTROSCOPY

GAMMA ENERGY ANALYSIS
Client ThRS

Tank $\underline{241-B-108}$
PROCEOURES REFERENCE 222-s Lab Analytical Procedure

L0-160-103 Core Segment Extrusion Process and Sample Preparation, rev 17

LA-549-141 Fusion with Alkali Metal Hydroxide, rev 40

LA-548-121 Preparation of Sample Mounts for Gamms Energy Analysis, rev 41

LA-508-162 Ganma Energy Analysis - the Genie System, rev 11
AVERAGES \pm 2 SD

FOR 8 SAMPLES
MDA 2.6E-01 $\pm \underline{2.6 E-01}$ YIELD
Group 96001379

METHOD SUMMARIES

Page 8

SUMMARY DATA SECTION

Page 34
Lab id 222-S

Protocol SST

Version 1.0

Form DVD-CMS

Version 3.08

Report date $02 / 24 / 97$ 
222 - S I A B OR A T O R X TANK 241-B-108, CORE 172

SDG 96001379

Contact I. L. Fritts
R E POR T G U I D E
Client TWRS

Tank $241-B-108$

\section{S A M P L E S O M A R Y}

The Sample and QC Summary Reports show all samples, including QC samples, reported in one Sample Delivery Group (SDG).

The Sample Summary Report fully identifies client samples and gives the corresponding lab sample identification. The gC summary Report shows at the sample level how the lab organized the samples into batches and generated $\mathrm{QC}$ samples. The Preparation Batch and Method Summary Reports show this at the analysis level.

The following notes apply to these reports:

*

*

*

w

LAB SAMPLE ID is the lab's primary identification for a sample.

DEPARTMENT SAMPLE ID is an alternate lab id, for example one assigned by a radiochemistry department in a lab.

CLIENT SAMPLE ID is the client's primary identification for a sample. It includes any sample preparation done by the client that is necessary to identify the sample.

QC BATCH is a lab assigned code that groups samples to be processed and gCed together. These samples should have similar matrices.

QC BATCH is not necessarily the same as SDG, which reflects samples received and reported together.

All Lab Control Samples, Method Blanks, Duplicates and Matrix spikes are shown that $\mathrm{QC}$ any of the samples. Due to possible reanalyses, not all results for all these QC samples may be relevant to the SDG. The Lab Control Sample, Method Blank, Duplicate, Matrix spike and Method summary Reports detail these relationships.

G I O

REPORT GUIDES

Page 1

SUMMARY DATA SECTION

Page 35
Lab id $222-\mathrm{S}$

Protocol SST

Version 1.0

Form DVD-RG

Version 3.08

Report date 02/24/97 
$222-S$ L A B O R A T OR Y

TANR 241-B-108, CORE 172

SDG 96001379

Contact L. L. Fritts
RE POR T G U I DE
Client TWRS

Tank 241-B-108

\section{P R E A R A ION BATCH SUMMAR Y}

The Preparation Batch Summary Report shows all preparation batches in one sample Delivery Group (SDG) with information necessary to check the completeness and consistency of the SDG.

The following notes apply to this report:

* The preparation batches are shown in the same order as the Method Summary Reports are printed.

* Only analyses of planchets relevant to the SDG are included.

* Each preparation batch should have at least one Method Blank and LCs in it to validate client sample results.

* The QUALIFIERS shown are all qualifiers other than U, J, B, I and $\mathrm{H}$ that occur on any analysis in the preparation batch. The Method Summary Report has these qualifiers on a per sample basis.

These qualifiers should be reviewed as follows:

$x$ Some data has been manually entered or modified. Transcription errors are possible.

P One or more results are 'preliminary'. The data is not ready for final reporting.

2 There were two or more results for one analyte on one planchet imported at one time. The results in DVD may not be the same as on the raw data sheets.

Other lab defined qualifiers may occur. In general, these should be addressed in the SDG narrative.

\section{G I O u p 99600011379}

\section{REPORT GUIDES}

Page 2

SUMMARY DATA SECTION

Page 36
Lab id 222-S

protocol SST

Version 1.0

Form DVD-RG

Version 3.08 
$222-5$ I A B O R A T OR Y TANR 241-B-108, CORE 172

SDG 96001379

Contact L. L. Fritts
R E POR T G UI D E
Client TWRS

Tank 241-B-108

\section{W O R R S U M M A R Y}

The Work Summary Report shows all samples, including Qc samples, and all relevant analyses in one Sample Delivery Group (SDG). This report is often useful as supporting documentation for an invoice.

The following notes apply to this report:

*

TEST is a code for the method used to measure associated analytes. Results and related information for each analyte are on the Data Sheet Report. In special cases, a test code used in the summary data section is not the same as in associated raw data. In this case, both codes are shown on the Work summary.

* SUFFIX is the lab'g code to distinguish multiple analyses (recounts, reworks, reanalyses) of a fraction of the sample. The suffix indicates which result is being reported. An empty suffix normally identifies the first attempt to analyze the sample.

* The LAB SAMPLE ID, TEST and SUFFIX uniquely identify all supporting data for a result. The Method summary Report for each TEST has method performance data, such as yield, for each lab sample id and suffix and procedures used in the method.

* PLANCHET is an alternate lab identifier for work done for one test. It, combined with the TEST and sUFFIX, may be the begt link to raw data.

* For QC samples, only analyses that directly QC some regular sample are shown. The Lab Control Sample, Method Blank, Duplicate, Matrix Spike and Method Summary Reports detail these relationships.

*

The SAS (Special Analytical Services) Number is a client or lab assigned code that reflects special processing for samples, such as rapid turn around. Counts of tests done are lists by SAS number since it is likely to affect prices.

G r u p 96001379

REPORT GUIDES

Page 3

SUMMARY DATA SECTION

Page 37
Lab id 222-s

Protocol SST

Version 1.0

Form DVD-RG

Version 3.08

Report date $02 / 24 / 97$ 
222 - S I A B O R A T OR Y

SDG 96001379

Contact I. L. Frittg
TANK 241-B-108, CORE 172

R E P O R T G U I D E
Client TWRS

Tank 241-B-108

\section{DA TA S H E T}

The Data Sheet Report shows all results and primary supporting information for one client sample or Method Blank. This report corresponds to both the CLP Inorganics and organics Data sheet.

The following notes apply to this report:

* TEST is a code for the method used to measure an analyte. If the TEST is empty, no data is available; the analyte was not analyzed for.

* The IAB SAMPLE ID and TEST uniquely identify work within the Summary Data Section of a Data Package. The Work Summary and Method Summary Reports further identify raw data that underlies this work.

The Method Summary Report for each TEST has method performance data, such as yield, for each Lab Sample ID and a list of procedures used in the method.

* ERRors can be labeled total or count. total implies a preparation (non-counting method) error has been added, as square root of sum of squares, to the counting error denoted by COUNT. The preparation errors, which may vary by preparation batch, are shown on the Method summary Report.

* A RESULT can be 'N.R.' (Not Reported). This means the lab did this work but chooses not to report it now, possibly because it was reported at another time.

* When reporting a Method Blank, a RESUlT can be 'N.A.' (Not Applicable). This means there is no reported client sample work in the same preparation batch as the Blank's result. This is likely to occur when the Method Blank is associated with reanalyses of selected work for a few samples in the SDG.

The following qualifiers are defined by the DVD system:

U The RESULT is less than the MDA (Minimum Detectable Activity). If the MDA is blank, the BRROR is used as the limit.

REPORT GUIDES

Page 4

SUMMARY DATA SECTION

Page 38
Lab id 222-s

Protocol SST

Version 1.0

Form DVD-RG

Version 3.08

Report date $02 / 24 / 97$ 
$222-S$ I A B O R A T O R Y

TANK 241-B-108, CORE 172

SDG 96001379

Contact L. L. Fritts
GUIDE, cont.

\section{A T A S H E E T}

Client TWRS

Tank 241-B-108

J The RESULT is less than the RDL (Required Detection Limit) and no $U$ qualifier is assigned.

B A Method Blank associated with this sample had a result without a $U$ flag and, after correcting for possibly different aliquots, that result is greater than or equal to the MDA for this sample.

Normally, $B$ is not assigned if $U$ is. When method blank subtraction is shown on this report, B flags are assigned based on the unsubtracted values while $U^{\prime} B$ are assigned based on the subtracted ones. Both flags can be assigned in this case.

For each sample result, all Method Blank results in the same preparation batch are compared. The Method Summary Report documents this and other $Q \mathrm{C}$ relationships.

I Some Lab Control Sample that QC's this sample had a low recovery. The lab can disable assignment of this qualifier.

H Similar to ' $L$ ' except the recovery was high.

p The RESULT is 'preliminary' .

$X$ Some data necessary to compute the RESULT, ERROR or MDA was manually entered or modified.

2 There were two or more results available for this analyte. The reported result may not be the same as in the raw data.

Other qualifiers are lab defined. Definitions should be in the SDG narrative.

The following values are underlined to indicate possible problems:

* An MDA is underlined if it is bigger than its RDL.

* An ERROR is underlined if the 1.645 sigma counting error is bigger than both the MDA and the RESULT, implying that the MDA

GI0 4 P 96001379

REPORT GUIDES Page 5

SUMMARY DATA SECTION

Page 39
Lab id 222-S

Protocol SST

Version 1.0

Form DVD-RG

version 3.08

Report date $02 / 24 / 97$ 
HNF-SD-WM-DP-219, REV. 0

$222-8$ I A B O R A T O Y

SDG 96001379

Contact L. L. Fritts
TANK 241-B-108, CORE 172

GUIDE, cont.
Client IWRS

Tank 241-B-108

D A T A S H E T

may not be a good estimate of the 'real' minimum detectable activity.

* A negative REsULT is underlined if it is less than the negative of its 2 sigma counting ERROR.

* When reporting a Method Blank, a RESULT is underlined if greater than its MDA. If the MDA is blank, the 2 sigma counting error is used in the comparison.

Gx० u 996001379

REPORT GUIDES

Page 6

SUMMARY DATA SECTION

Page 40
Lab id 222-s

Protocol SST

Version 1.0 Form DVD-RG

version 3.08

Report date $\underline{02 / 24 / 97}$ 
$222-S$ I A B O R T OR Y

TANK 241-B-108, CORE 172

SDG 96001379

Contact L. L. Fritts
RE POR T G U I D E

Client TWRS

Tank 241-B-108

\section{A B CONTROL SAM P L E}

The Lab Control Sample Report shows all results, recoveries and primary supporting information for one Lab Control Sample.

The following notes apply to this report:

* All fields in common with the Data Sheet Report have similar usage. Refer to its Report Guide for details.

* An amount ADDED is the lab's value for the actual amount spiked into this sample with its ERROR an estimate of the error of this amount.

An amount added is underlined if its ratio to the corresponding RDL is outside protocol specified limits.

* REC (Recovery) is RESULT divided by ADDED expressed as a percent.

* The first, computed limits for the recovery reflect:

1. The error of RESULT, including that introduced by rounding the result prior to printing.

If the limits are labeled (TOTAL), they include preparation error in the result. If labeled (COUNT), they do not.

2. The error of ADDED.

3. A lab specified, per analyte bias. The bias changes the center of the computed limits.

* The second limits are protocol defined upper and lower QC limits for the recovery.

* The recovery is underlined if it is outside either of these ranges.

* Laboratory control limits are defined in procedure LQ-543-101.

\section{G I U U 96001379}

\section{REPORT GUIDES}

Page 7

\section{SUMMARY DATA SECTION}

Page 41
Lab id 222-s

Protocol SST

Version 1.0 Form DVD-RG

version 3.08 Report date $02 / 24 / 97$ 
HNF-SD-WM-DP-219, REV. 0

$222-S$ I A B O R A T O R Y

TANK 241-B-108, CORE 172

G U I D E, cont.

Client TWRS

Tank 241-B-108

\section{A B CONTROI SAM PLE}

DVD reported limits are based on total propagated uncertainty, a part of which is the laboratory control limits.

G I O L 960001379

REPORT GUIDES

Page 8

SUMMARY DATA SECTION

Page 42
Iab id 222-s

Protocol SST

Version 1.0 Form DVD-RG

Version 3.08

Report date $02 / 24 / 97$ 
$222-8$ I A B ORA T OR Y

TANK 241-B-108, CORE 172

SDG 96001379

Contact L. L. Fritts

RE PORT G UIDE

Client TWRS

Tank 241-B-108

\section{U I I C A E}

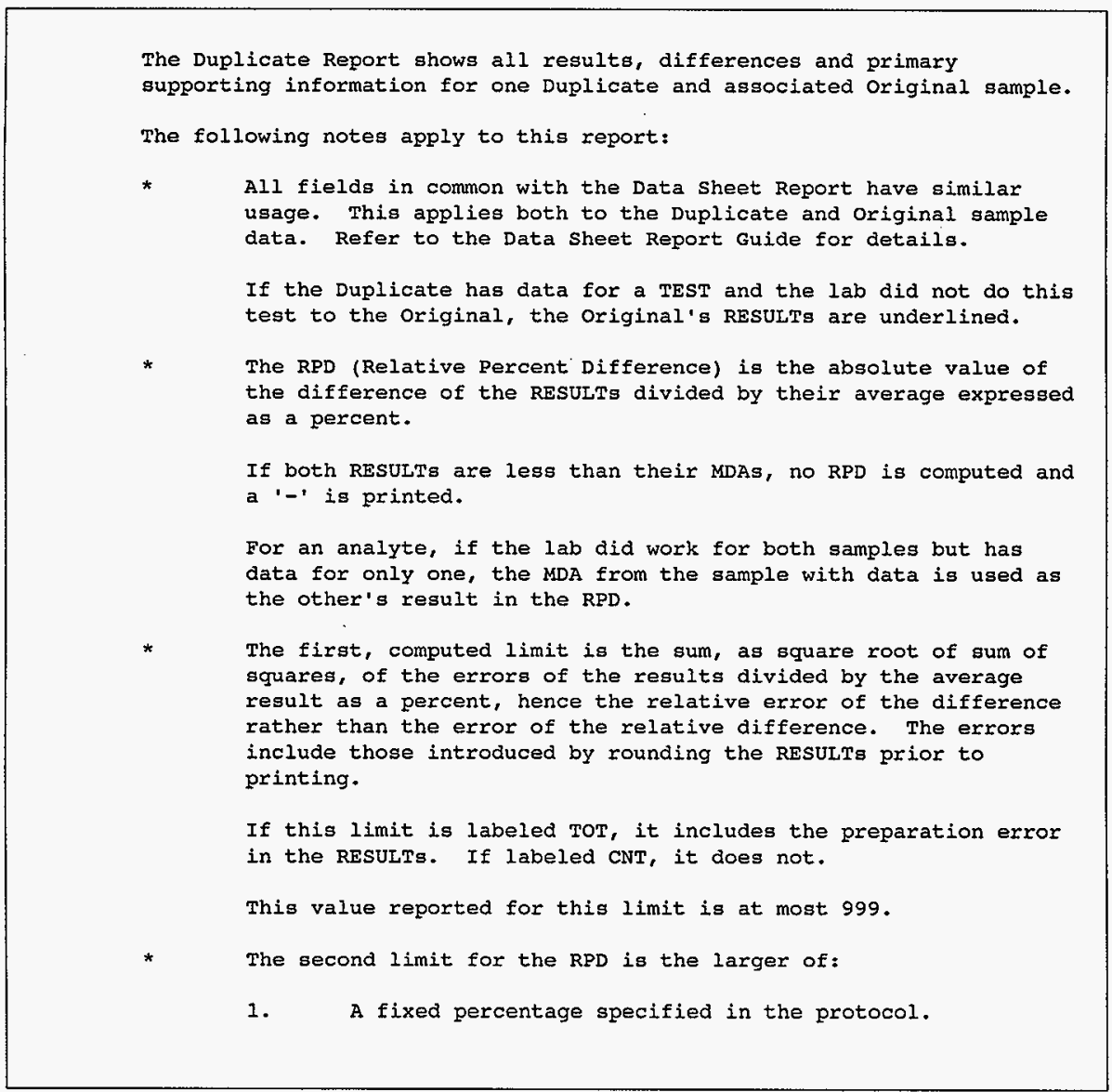

\section{G r U p 96001379}

REPORT GUIDES

page 9

SUMMARY DATA SECTION

Page 43
Lab id 222-s

protocol SST

Version 1.0

Form DVD-RG

Version 3.08

Report date $02 / 24 / 97$ 
HNF-SD-WM-DP-219, REV. 0

$222-S$ I A B O R A T OR Y

TANK 241-B-108, CORE 172

SDG 96001379

Contact L. L. Fritts

G U DE, cont.

Client TWRS

Tank 241-B-108

\section{U P I I C A T E}

2. A protocol factor (typically 2) times the average MDA as

a percent of the average result. This limit applies

when the results are close to the MDAs.

*

The RPD is underlined if it is greater than either limit.

* If specified by the lab, the second limit column is replaced by the Difference Error Ratio (DER), which is the absolute value of the difference of the results divided by the quadratic sum of their one sigma errors, the same errors as used in the first limit.

Except for differences due to rounding, the DER is the same as the RPD divided by the first RPD limit with the limit scaled to 1 sigma.

* The DER is underlined if it is greater than the sigma factor, typically 2 or 3 , shown in the header for the first RPD limit.

REPORT GUIDES

Page 10

SUMMARY DATA SECTION

Page 44
$\mathrm{Lab}$ id $222-\mathrm{S}$

Protocol SST

Version 1.0

Form DVD-RG

Version 3.08

Report date $02 / 24 / 97$ 


\section{$222-S$ I A B ORA TOR Y}

TANR 241-B-108, CORE 172

SDG 96001379

Contact L. I. Fritts
R E P O R T G U I D E

Client TWRS

Tank 241-B-108

\section{A T R I S P I R E}

The Matrix Spike Report shows all results, recoveries and primary supporting information for one Matrix spike and associated original sample.

The following notes apply to this report:

* All fields in common with the Data sheet Report have similar usage. This applies both to the spiked and original sample data. Refer to the Data sheet Report Guide for details.

If the spike has data for a TEST and the lab did not do this test to the Original, the Original's RESULTs are underlined.

* An amount ADDED is the lab's value for the actual amount spiked into the spike sample with its ERROR an estimate of the error of this amount.

An amount is underlined if its ratio to the corresponding RDL is outside protocol specified limits.

* REC (Recovery) is the spike RESULT minus the original RESULT divided by ADDED expressed as a percent.

* The first, computed limits for the recovery reflect:

1. The errors of the two REsuLTs, including those introduced by rounding them prior to printing.

If the limits are labeled (TOTAL), they include preparation error in the result. If labeled (COUNT), they do not.

2. The error of ADDED.

3. A lab specified, per analyte bias. The bias changes the center of the computed limits.

* The second limits are protocol defined upper and lower QC limits for the recovery.

\section{G I O U P 966001379}

\section{REPORT GUIDES}

Page 11

SUMMARY DATA SECTION

Page 45
Lab id $222-S$

Protocol SST

Version 1.0

Form DVD-RG

version 3.08

Report date $02 / 24 / 97$ 
HNF-SD-WM-DP-219, REV. 0

222 - S I A B ORA TOR Y

TANK 241-B-108, CORE 172

SDG 96001379

Contact L. L. Fritts

G UIDE, cont.

Client TWRS

Tank 241-B-108

\section{A T I X S P I K E}

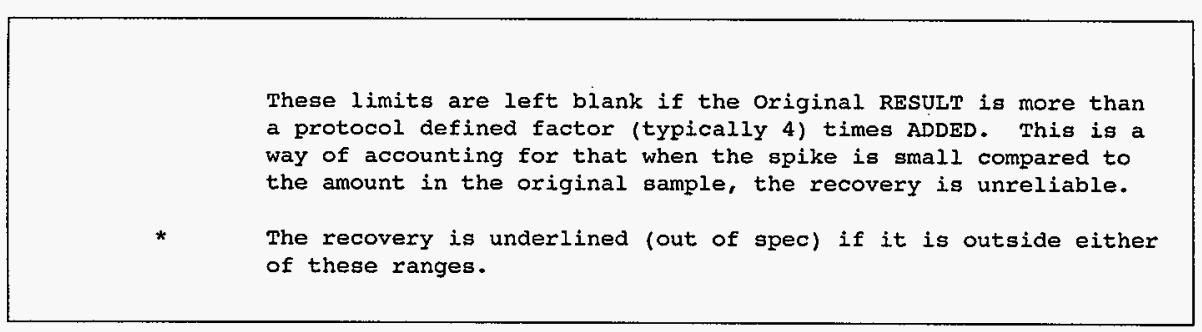

Group 96001379

REPORT GUIDES

Page 12

SUMMARY DATA SECTION

Page 46
Lab id $222-s$

Protocol SST

Version 1.0

Form DVD-RG

Version 3.08

Report date $02 / 24 / 97$ 
$222-S$ I A B O R A T O R Y

SDG 96001379

Contact $\underline{\text { L. L. Fritts }}$
TANK 241-B-108, CORE 172

RE POR T G UI D E
Client TWRS

Tank 241-B-108

\section{METHOD S U M A R Y}

The Method Summary Report has two tables. One shows up to five results measured using one method. The other has performance data for the method. There is one report for each TEST, as used on the Data sheet Report.

The following notes apply to this report:

* Each table is subdivided into sections, one for each preparation batch. A preparation batch is a group of aliquots prepared at roughly the same time in one work area of the lab using the same method.

There should be Lab control Sample and Method Blank results in each preparation batch since this close correspondence makes the QC meaningful. Depending on lab policy, Duplicates need not occur in each batch since they QC sample dependencies such as matrix effects.

* The RAW TEST column shows the test code used in the raw data to identify a particular analysis if it is different than the test code in the header of the report. This occurs in special cases due to method specific details about how the lab labels work.

The Lab sample or Planchet ID combined with the (Raw) Test Code and Suffix uniquely identify the raw data for each analysis.

* If a result is less than both its MDA and RDL, it is replaced by just ' $U$ ' on this report. If it is greater than or equal to the RDI but less than the MDA, the result is shown with a ' $U$ ' flag.

The $\mathrm{J}$ and $\mathrm{X}$ flags are as on the data sheet.

* Non-U results for Method Blanks are underlined to indicate possible contamination of other samples in the preparation batch. The Method Blank Report has supporting data.

* Lab control sample and Matrix Spike results are shown as: ok, No data, LOW or HIGH, with the last two underlined. 'No data' means no amount ADDED was specified. 'LOW' and 'HIGH'

G I 96001379

\section{REPORI GUIDES}

Page 13

SUMMARY DATA SECTION

Page 47
Lab id 222-S

Protocol SST

Version 1.0

Form DVD-RG

Version 3.08

Report date $\underline{02 / 24 / 97}$ 
HNF-SD-WM-DP-219, REV. O

$222-S$ I A B O R A T OR Y

TANK 241-B-108, CORE 172

SDG 96001379

Contact L. L. Fritts
GUIDE, cont.

Client TWRS

Tank 241-B-108

\section{METHOD S U M M R Y}

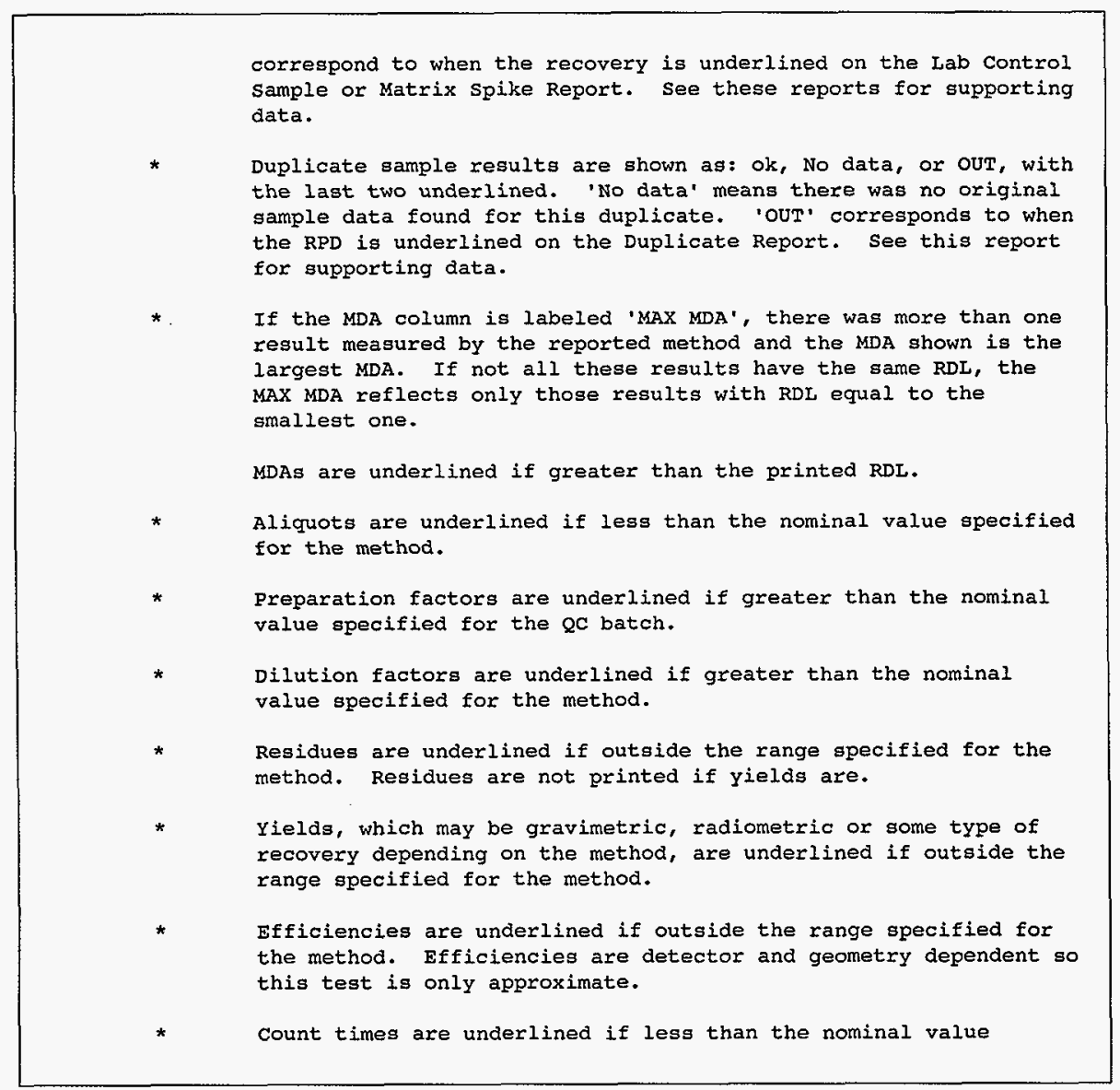

G r u p 960001379

\section{REPORT GUIDES}

Page 14

\section{SUMAARY DATA SECTION}

Page 48
Lab id 222-S

Protocol SST

Version 1.0

Form DVD-RG

version 3.08

Report date $02 / 24 / 97$ 
HNF-SD-WM-DP-219, REV. 0

$222-S$ I A B O R A T OR Y

TANK 241-B-108, CORE 172

SDG 96001379

Contact L. L. Fritts

G UIDE, cont.

Client TWRS

Tank 241-B-108

\section{METHOD S UMMARY}

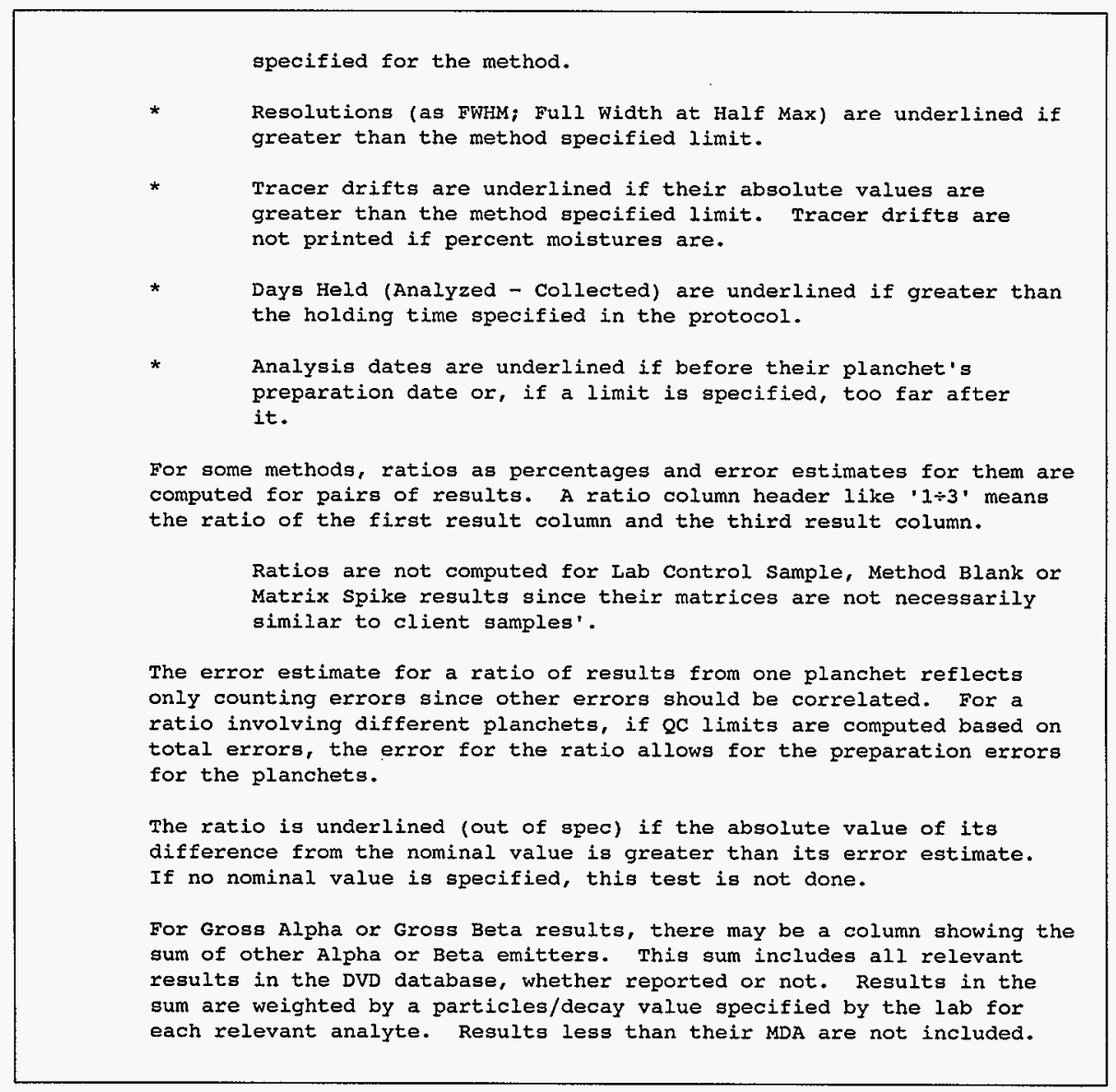

GIOU P 96001379

REPORT GUIDES

Page 15

SUMMARY DATA SECTION

Page 49
Lab id 222-S

Protocol SST

Version 1.0

Form DVD-RG

Version 3.08

Report date $02 / 24 / 97$ 
HNF-SD-WM-DP-219, REV.O

$222-S$ I A BOR A T OR Y

TANK 241-B-108, CORE 172

SDG 96001379

Contact I. L. Fritts

GUIDE, cont.

Client TWRS

Tank $241-\mathrm{B}-108$

\section{METHOD $\mathrm{S}$ U M M R Y}

No sums are computed for Lab Control, Method Blank or Matrix Spike samples since their various planchets may not be physically related.

If a ratio of total isotopic to Gross Alpha or Beta is shown, the error for the ratio reflects both the error in the Gross result and the sum, as square root of sum of squares, of the errors in the isotopic results.

For total elemental uranium or thorium results, there may be a column showing the total weight computed from associated isotopic results. Ignoring results less than their MDAs, this is a weighted sum of the isotopic results. The weights depend on the molecular weight and half-life of each isotope so as to convert activities (decays) to weight (atoms).

If a ratio of total computed to measured elemental uranium or thorium is shown, the error for the ratio reflects the errors in all the measurements.

G I O U P 960001379

REPORT GUIDES

Page 16

SUMMARY DATA SECTION

Page 50
Lab id $222-s$

Protocol SST

Version 1.0

Form DVD-RG

Version 3.08 


\section{$222-S$ I A B O R A T O R Y TANR 241-B-108, CORE 173}

SDG 96001380

Contact I. I. Fritts
Client TWRS

Tank 241-B-108

S U M M A R Y D A T A S E C T I O N

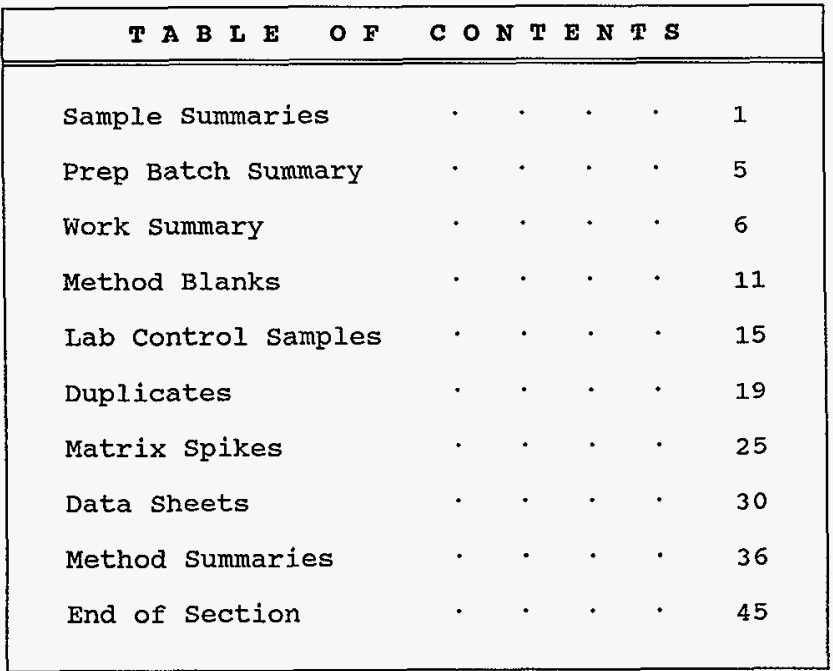
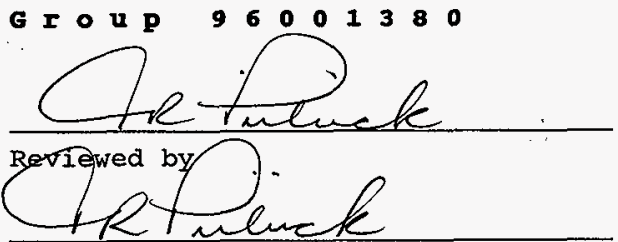

Approved by
Lab id $222-\mathrm{S}$

Protocol SST

Version 1.0

Form DVD-TOC

Version 3.08

Report date $\underline{02 / 24 / 97}$ 
HNF-SD-WM-DP-219, REV. 0

\section{$222-S$ LABORATORY}

TANK 241-B-108, CORE 173

SDG 96001380

Contact L. L. Fritts

SAMPLE SUMMARY

Client TWRS

Tank 241-B-108

\begin{tabular}{|c|c|c|c|c|c|c|}
\hline CLIENT SAMPLE ID & LOCATIOH & & MATRIX & LEVEL & $\begin{array}{l}\text { LAB } \\
\text { SAMPLE ID }\end{array}$ & RECE IVED \\
\hline B108 c173 COMP Fusion & R: 3 s: core Composite C & FUSION & SOLID & & s96T005487 & $10 / 17 / 96 \quad 12: 05$ \\
\hline B108 c173 Comp Fusion Re & R: 3 s: Core Composite C & FUSION & SOLID & & s97T000002 & $01 / 06 / 97 \quad 11: 37$ \\
\hline B108 c173 Comp Fusion Re & $\mathrm{R}: 3 \mathrm{~s}$ : Core Composite $\mathrm{C}$ & FUSION & SOLID & & S97T000002D & \\
\hline B108 c173 Comp Fusion Re & R: 3 S: Core Composite C & FUSION & SOLID & & s97T000002s & \\
\hline B108 c173 COMP Fusion-DU & R: 3 S: Core Composite C & FUSION & SOLID & & \$96T0054870 & \\
\hline B108 C173 COMP Fusion-sP & R: 3 s: Core Composite C & FUSION & SOLID & & \$96r005487s & \\
\hline B108 c173s1 LH Fusion & $\mathrm{R}: 3 \mathrm{~S}: 1 \mathrm{C}: 173$ & FUSION & SOLID & & $\$ 96 T 005477$ & $10 / 17 / 96 \quad 06: 42$ \\
\hline B108 c173s1 LH Fusion-DU & $R: 3$ s: 1 C: 173 & FUSION & SOLID & & S96T0054770 & \\
\hline B108 c173S1 LH Fusion-SP & $\mathrm{R}: 3 \mathrm{~s}: 1 \mathrm{C}: 173$ & FUSION & SOLID & & \$96T005477s & \\
\hline B108 C173s1 UH Fusion & $\mathrm{R}: 3 \mathrm{~s}: 1 \mathrm{C}: 173$ & FUSION & SOLID & & s96r005468 & $10 / 17 / 96 \quad 06: 25$ \\
\hline B108 C173S1 UH Fusion-DU & $\mathrm{R}: 3 \mathrm{~s}: 1 \mathrm{C}: 173$ & FUSION & SOLID & & S96T005468D & \\
\hline B108 C173s2 LH Fusion & $R: 3$ s: 2 C: 173 & FUSION & SOLID & & s96T005478 & $10 / 17 / 96 \quad 06: 42$ \\
\hline B108 C173s2 LH Fusion Re & $R: 3 \mathrm{~s}: 2 \mathrm{C}: 173$ & FUSION & SOLID & & \$97T000001 & $01 / 06 / 97 \quad 11: 37$ \\
\hline B108 c173s2 LH Fusion Re & R: 3 s: 2 C: 173 & FUSION & SOLID & & S97T000001D & \\
\hline B108 C173s2 LH Fusion Re & $R: 3 \mathrm{~s}: 2 \mathrm{C}: 173$ & FUSION & SOLID & & s977000001s & \\
\hline B108 c173s2 LH Fusion-DU & R: 3 S: 2 C: 173 & FUSION & SOL ID & & S96T0054780 & \\
\hline B108 C173S2 LH Fusion-SP & R: 3 s: 2 C: 173 & FUSION & SOLID & & S96T005478S & \\
\hline Method Blank & & & SOLID & & B14361-2 & \\
\hline Method Blank & & & SOLID & & B $14725-2$ & \\
\hline Method Blank & & & SOLID & & B15416-2 & \\
\hline Method Blank & & & SOLID & & B15952-2 & \\
\hline Method Blank & & & SOLID & & B16015-2 & \\
\hline Method Blank & & & SOLID & & $\mathrm{B} 16017-2$ & \\
\hline Method Blank & & & SOLID & & B16019-2 & \\
\hline Method Blank & & & SOLID & & B16256-2 & \\
\hline Method Blank & & & SOLID & & B16258-2 & \\
\hline Method Blank & & & SOLID & & B16349-2 & \\
\hline Method Blank & & & SOLID & & B16362-2 & \\
\hline Method Blank & & & SOL ID & & B16409-2 & \\
\hline Lab Control Sample & & & SOLID & & $\$ 14361-1$ & \\
\hline Lab Control Sample & & & SOLID & & s14725-1 & \\
\hline Lab Control Sample & & & SOLID & & $\$ 15416-1$ & \\
\hline Lab Control Sample & & & SOLID & & $\$ 15952-1$ & \\
\hline Lab Control Sample & & & SOLID & & $s 16015-1$ & \\
\hline Lab Control Sample & & & SOL. ID & & s16017-1 & \\
\hline Lab Control Sample & & & SOLID & & s16019-1 & \\
\hline Lab Control Sample & & & SOL ID & & $\$ 16256-1$ & \\
\hline Lab Control Sample & & & SOL.ID & & $s 16258-1$ & \\
\hline Lab Control Sample & & & SOLID & & $\$ 16349-1$ & \\
\hline
\end{tabular}

\section{Group 96001380}

SAMPLE SUMUARY

Page :

SUMAYARY DATA SECTIOH

Page 1
Lab id 222-s

Protocol SST

Version 1.0

Form DVD-Cs

Version 3.08

Report date $\underline{02 / 24 / 97}$ 
HNF-SD-WM-DP-219, REV. 0

\section{2 -S LABORATORY}

TANK 241-B-108, CORE 173

SDG 96001380

Contact L. L. Fritts

SAMPLE SUMMARY, cont.

Client TWRS

Tank 241-B-108

\begin{tabular}{|c|c|c|c|c|c|c|}
\hline CLIENT SAMPLE ID & LOCATION & MATRIX & LEVEL & $\begin{array}{l}\text { LAB } \\
\text { SAMPLE ID }\end{array}$ & PRIORITY COLLECTED & RECE IVED \\
\hline Lab Control Sample & & SOLID & & $s 96362-1$ & & \\
\hline Lab control Sample & & SOLID & & $s 16409-1$ & & \\
\hline
\end{tabular}

\section{Group 96001380}

SAMPLE SUMAARY

Page 2

SUMAARY DATA SECTION

Page 2
Lab id 222-s

Protocol SST

Version 1.0

Form DVD-CS

Version 3.08

Report date $02 / 24 / 97$ 


\section{$222-S$ IABORATORY}

SDG 96001380

Contact L. L. Fritts
TANK 241-8-108, CORE 173

QC SUMMARY
Client TWRS

Tank 241-8-108

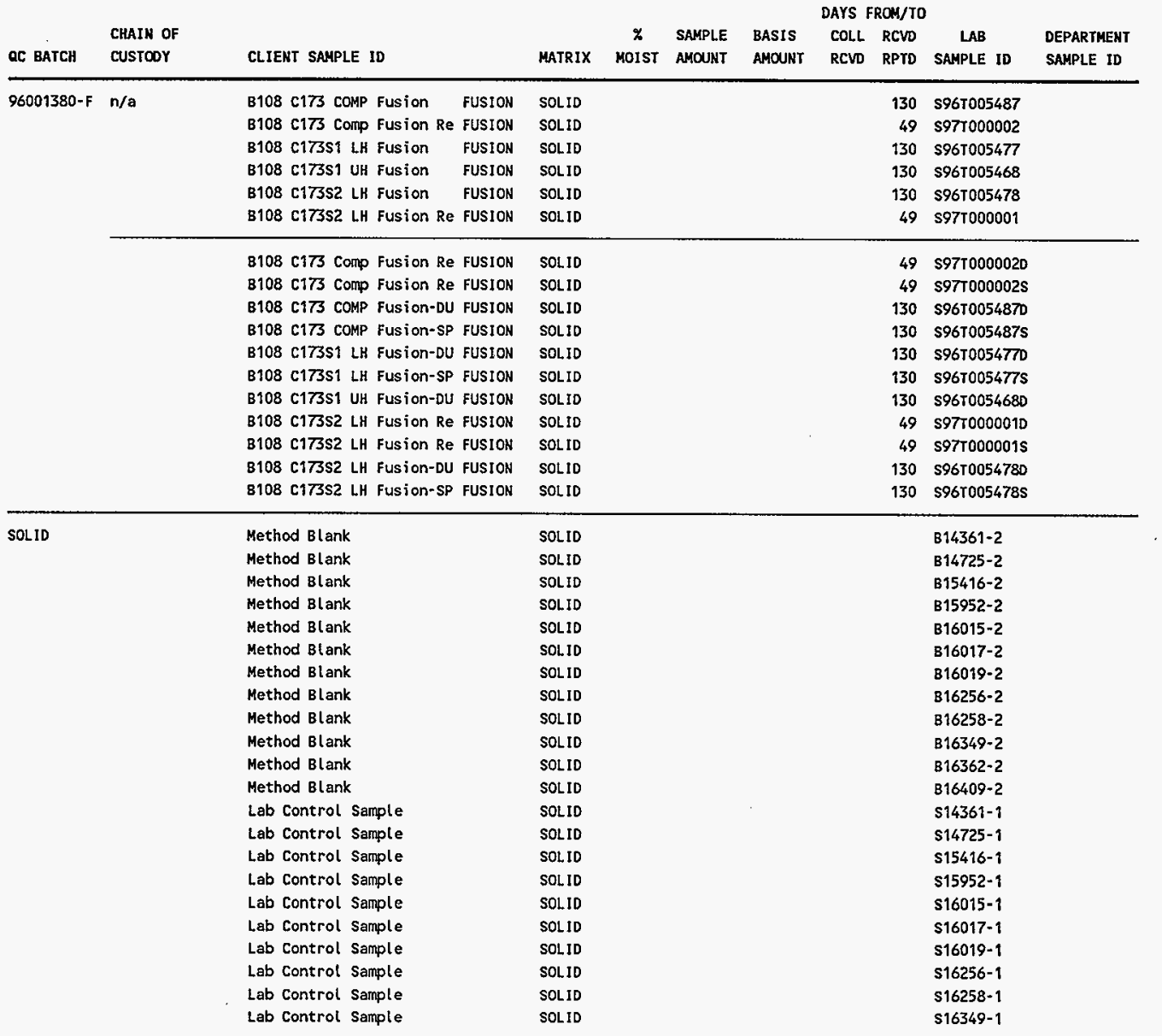

\section{Group 96001380}

QC SUMMARY

Page 1

SUMMARY DATA SECTION

Page 3

Lab id 222-S

Protocol SST

Version 1.0

Form DVD-OS

Version 3.08

Report date $02 / 24 / 97$ 
HNF-SD-WM-DP-219, REV. 0

SDG 96001380

Contact L. L. Fritts
222-S LABORATORY

TANK 241-B-108, CORE 173

QC SUMMARY, cont.
Client TWRS

Jank 241-B-108

\begin{tabular}{|c|c|c|c|c|c|c|c|c|c|c|}
\hline \multirow[b]{2}{*}{ OC BATCH } & \multirow[b]{2}{*}{$\begin{array}{l}\text { CHAIN OF } \\
\text { CUSTODY }\end{array}$} & \multirow[b]{2}{*}{ CLIENT SAMPLE ID } & \multirow[b]{2}{*}{ MATRIX } & \multicolumn{7}{|c|}{ DAYS FROH/TO } \\
\hline & & & & $\underset{\text { MOIST }}{\%}$ & $\begin{array}{l}\text { SAMPLE } \\
\text { AMOUNT }\end{array}$ & $\begin{array}{l}\text { BASIS } \\
\text { AMOUNT }\end{array}$ & $\begin{array}{l}\text { COLL. } \\
\text { RCVD }\end{array}$ & $\begin{array}{l}\text { RCVD } \\
\text { RPTD }\end{array}$ & $\begin{array}{c}\text { LAB } \\
\text { SAMPLE ID }\end{array}$ & $\begin{array}{l}\text { DEPARTMENT } \\
\text { SAMPLE ID }\end{array}$ \\
\hline & & Lab Control Sample & SOLID & & & & & & $S 16362-1$ & . \\
\hline & & Lab Controt Sample & SOLID & & & & & & s16409-1 & \\
\hline
\end{tabular}

\section{Group 96001380}

OC SUMALARY

Page 2

SUMAARY DATA SECTION

Page 4
Lab id 222-S

Protocol SST

Version 1.0

Form DVD-QS

Version 3.08

Report date $02 / 24 / 97$ 
HNF-SD-WM-DP-219, REV. 0

\section{2 -S LABORATORY}

TANK 241-B-108, CORE 173

SDG 96001380

Contact L. L. Fritts

PREP BATCH SUMMARY

Client TWRS

Tank $241-\mathrm{B}-108$

\begin{tabular}{|c|c|c|c|c|c|c|c|c|c|c|c|c|}
\hline \multirow[b]{2}{*}{ TEST } & \multirow[b]{2}{*}{ MATRIX } & \multirow[b]{2}{*}{ METHOD } & \multirow{2}{*}{$\begin{array}{l}\text { PREPARATION } \\
\text { BATCH }\end{array}$} & ERROR & \multicolumn{2}{|c|}{$\longrightarrow$} & \multicolumn{2}{|c|}{ PLANCHETS } & \multicolumn{3}{|c|}{ ANALYZED } & \multirow{2}{*}{$\begin{array}{l}\text { QUALI- } \\
\text { FIERS }\end{array}$} \\
\hline & & & & $2 \sigma \%$ & CLIENT & MORE & RE & BLANK & LCS & DUP/ORIG & MS/ORIG & \\
\hline \multicolumn{13}{|c|}{ Gas Proportional Counting } \\
\hline \multirow[t]{3}{*}{ SR } & SOLID & Strontium-89/90 & 96012589 & 15.0 & 1 & & & 1 & 1 & $1 / 1$ & & \\
\hline & & & 96012650 & 15.0 & $\uparrow$ & & & 1 & 1 & $1 / 1$ & & \\
\hline & & & 97000108 & 15.0 & 1 & & & 1 & 1 & $1 / 1$ & & \\
\hline \multicolumn{13}{|c|}{ Gas Proportional Counting } \\
\hline \multirow[t]{2}{*}{$A B$} & SOLID & Alpha Analysis & 96011318 & 15.0 & 1 & & & 1 & 1 & $1 / 1$ & $1 / 1$ & \\
\hline & & & 97000273 & 15.0 & 1 & & & 1 & 1 & $1 / 1$ & $1 / 1$ & \\
\hline \multirow[t]{2}{*}{ AT } & SOLID & Alpha Analysis & 96010903 & 15.0 & 2 & & & 1 & 1 & $2 / 2$ & $1 / 1$ & \\
\hline & & & 97000208 & 15.0 & 1 & & & 1 & 1 & $1 / 1$ & $1 / 1$ & \\
\hline TB & SOLID & Beta Analys is & 96012646 & 15.0 & 1 & & & 1 & 1 & $1 / 1$ & $1 / 1$ & \\
\hline \multirow{5}{*}{$\begin{array}{l}\text { Gamm: } \\
\text { GEA }\end{array}$} & a Energy & alysis & & & & & & & & & & \\
\hline & SOLID & Gama Spectroscopy & 96012029 & 15.0 & 1 & & & 1 & 1 & $1 / 1$ & & \\
\hline & & & 96012648 & 15.0 & 3 & & & 1 & 1 & $3 / 3$ & & \\
\hline & & & 97000110 & 15.0 & 1 & & & 1 & 1 & $1 / 1$ & & \\
\hline & & & 97000227 & 15.0 & 1 & & & 1 & 1 & $1 / 1$ & & \\
\hline
\end{tabular}

Duplicates and Matrix Spikes are those with original (client) sample in this Sample Detivery Group.

Blank and LCS planchets are those in the same preparation batch as some client, Duplicate or spike sample.

\section{Group 96001380}

PREP BATCH SUMMARY

Page 1

SUMMARY DATA SECTION

Page 5
Lab id 222-S

Protocol SST

Version $\mathbf{3 . 0}$

Form DVD-PBS

Version 3.08

Report date $02 / 24 / 97$ 


\section{$222-S$ LABORATORY}

TANK 241-B-108, CORE 173

WORK SUMMARY
Client TWRS

Tank 241-B-108

\begin{tabular}{|c|c|c|c|c|c|c|c|c|c|}
\hline $\begin{array}{l}\text { CLIENT SAMPLE IO } \\
\text { LOCATION } \\
\text { CUSTOOY }\end{array}$ & MATRIX & $\begin{array}{l}\text { LAB SAMPLE ID } \\
\text { COLLECTED } \\
\text { RECEIVED }\end{array}$ & PLANCHET & TEST & $\begin{array}{l}\text { SUF- } \\
\text { FIX }\end{array}$ & ANAL,YZED & REVIEWEO & BY & MEIHOO \\
\hline $\begin{array}{l}\text { B108 C173 COMP Fusion } \\
\text { R: } 3 \text { S: Core Composite C } \\
\text { n/a }\end{array}$ & $\begin{array}{l}\text { FUSION } \\
\text { SOLID }\end{array}$ & $\begin{array}{r}596 r 005487 \\
10 / 17 / 96\end{array}$ & $\begin{array}{l}14725-4 \\
15416-3 \\
15952-4\end{array}$ & $\begin{array}{l}A B \\
\text { GEA } \\
S R\end{array}$ & $\begin{array}{l}01 \\
01 \\
02\end{array}$ & $\begin{array}{l}11 / 08 / 96 \\
12 / 16 / 96 \\
01 / 06 / 97\end{array}$ & & $\begin{array}{l}\text { SLF } \\
\text { PPB } \\
\text { SAC }\end{array}$ & $\begin{array}{l}\text { Alpha Analysis } \\
\text { Gamma Spectroscopy } \\
\text { Strontium- } 89 / 90\end{array}$ \\
\hline $\begin{array}{l}\text { B108 C173 Comp Fusion Re } \\
\text { R: } 3 \text { s: Core Composite C } \\
\text { n/a }\end{array}$ & $\begin{array}{l}\text { FUSION } \\
\text { SOLID }\end{array}$ & $\begin{array}{r}\$ 971000002 \\
01 / 06 / 97\end{array}$ & $\begin{array}{l}16409-4 \\
16258-5 \\
16256-4\end{array}$ & $\begin{array}{l}A B \\
G E A \\
S R\end{array}$ & 01 & $\begin{array}{l}01 / 29 / 97 \\
01 / 22 / 97 \\
01 / 22 / 97\end{array}$ & & $\begin{array}{l}\text { SLF } \\
\text { PPB } \\
\text { SAC }\end{array}$ & $\begin{array}{l}\text { Alpha Analysis } \\
\text { Gamma Spectroscopy } \\
\text { Stront ium- } 89 / 90\end{array}$ \\
\hline $\begin{array}{l}\text { B108 C173 Comp Fusion Re } \\
\text { R: } 3 \text { s: Core Composite C }\end{array}$ & $\begin{array}{l}\text { FUSION } \\
\text { SOLID }\end{array}$ & $\begin{array}{r}\text { S97T000002D } \\
01 / 06 / 97\end{array}$ & $\begin{array}{l}16409-5 \\
16258-6 \\
16256-5\end{array}$ & $\begin{array}{l}\text { AB } \\
\text { GEA } \\
\text { SR }\end{array}$ & 01 & $\begin{array}{l}01 / 29 / 97 \\
01 / 22 / 97 \\
01 / 22 / 97\end{array}$ & & $\begin{array}{l}\text { SLF } \\
\text { PPB } \\
\text { SAC }\end{array}$ & $\begin{array}{l}\text { Alpha Analysis } \\
\text { Gamma Spectroscopy } \\
\text { Stront ium- } 89 / 90\end{array}$ \\
\hline $\begin{array}{l}\text { B108 C173 Comp Fusion Re } \\
R=3 \text { S: Core Composite C }\end{array}$ & $\begin{array}{l}\text { FUSION } \\
\text { SOL.ID }\end{array}$ & $\begin{array}{r}\text { s97T000002s } \\
01 / 06 / 97\end{array}$ & $16409-6$ & $A B$ & 01 & $01 / 29 / 97$ & & SLF & Alpha Analysis \\
\hline $\begin{array}{l}\text { B108 C173 COMP Fusion-DU } \\
\text { R: } 3 \text { S: Core Composite C } \\
\text {. }\end{array}$ & $\begin{array}{l}\text { FUSION } \\
\text { SOLID }\end{array}$ & $\begin{array}{r}59670054870 \\
10 / 17 / 96\end{array}$ & $\begin{array}{l}14725-5 \\
15416-4 \\
15952-5\end{array}$ & $\begin{array}{l}A B \\
G E A \\
S R\end{array}$ & $\begin{array}{l}01 \\
02\end{array}$ & $\begin{array}{l}11 / 08 / 96 \\
12 / 16 / 96 \\
01 / 06 / 97\end{array}$ & & $\begin{array}{l}\text { SLF } \\
\text { PPB } \\
\text { SAC }\end{array}$ & $\begin{array}{l}\text { Alpha Analysis } \\
\text { Gamma Spectroscopy } \\
\text { strontium- } 89 / 90\end{array}$ \\
\hline $\begin{array}{l}\text { B108 c173 COMP Fusion-SP } \\
\text { R: } 3 \text { S: Core Composite C }\end{array}$ & $\begin{array}{l}\text { FUSION } \\
\text { SOLID }\end{array}$ & $\begin{array}{r}\text { S96T005487S } \\
10 / 17 / 96\end{array}$ & $14725-6$ & $A B$ & 01 & $11 / 08 / 96$ & & SLF & Alpha Analysis \\
\hline $\begin{array}{l}\text { B108 C173s } 1 \text { LH Fusion } \\
\text { R: } 3 \text { S: } 1 \text { C: } 173 \\
\text { n/a }\end{array}$ & $\begin{array}{l}\text { FUSION } \\
\text { SOLID }\end{array}$ & $\begin{array}{r}\text { S96T005477 } \\
10 / 17 / 96\end{array}$ & $\begin{array}{l}14361-4 \\
16019-5 \\
16017-4 \\
16015-4\end{array}$ & $\begin{array}{l}\text { AT } \\
\text { GEA } \\
\text { SR } \\
\text { TB }\end{array}$ & & $\begin{array}{l}10 / 30 / 96 \\
01 / 09 / 97 \\
01 / 07 / 97 \\
01 / 09 / 97\end{array}$ & & $\begin{array}{l}\text { SLF } \\
\text { PPB } \\
\text { SAC } \\
\text { SLF }\end{array}$ & $\begin{array}{l}\text { Alpha Analysis } \\
\text { Gamma Spectroscopy } \\
\text { Strontium- } 89 / 90 \\
\text { Beta Analysis }\end{array}$ \\
\hline $\begin{array}{l}\text { B108 C173s } 1 \text { LA fusion-DU } \\
\text { R: } 3 \mathrm{~s}: 1 \mathrm{C}: 173\end{array}$ & $\begin{array}{l}\text { FUSION } \\
\text { SOLID }\end{array}$ & $\begin{array}{r}\text { S96T0054770 } \\
10 / 17 / 96\end{array}$ & $\begin{array}{l}14361-5 \\
16019-6 \\
16017-5 \\
16015-5\end{array}$ & $\begin{array}{l}\text { AT } \\
\text { GEA } \\
\text { SR } \\
\text { TB }\end{array}$ & & $\begin{array}{l}10 / 30 / 96 \\
01 / 09 / 97 \\
01 / 07 / 97 \\
01 / 09 / 97\end{array}$ & & $\begin{array}{l}\text { SLF } \\
\text { PPB } \\
\text { SAC } \\
\text { SLF }\end{array}$ & $\begin{array}{l}\text { Alpha Analysis } \\
\text { Gamma Spectroscopy } \\
\text { Strontium- } 89 / 90 \\
\text { Beta Analysis }\end{array}$ \\
\hline $\begin{array}{l}\text { B108 C173s } 1 \text { LH Fusion-SP } \\
\text { R: } 3 \text { S: } 1 \text { C: } 173\end{array}$ & $\begin{array}{l}\text { FUSION } \\
\text { SOLID }\end{array}$ & $\begin{array}{r}\text { s96T005477s } \\
10 / 17 / 96\end{array}$ & $16015-6$ & TB & & $01 / 09 / 97$ & & SLF & Beta Anatysis \\
\hline
\end{tabular}

\section{Group 96001380}

WORK SUMMARY

Page 1

SUMMARY DATA SECTION

Page 6
Lab id 222-s

Protocol SST

Version 1.0

Form DVD-CWS

Version 3.08

Report date $02 / 24 / 97$ 
HNF-SD-WM-DP-219, REV. 0

22 -S LABORATORY

TANK 241-B-108, CORE 173

SDG 96001380

Contact L. L. Fritts
WORK SUMMARY, cOnt.
Client TWRS

Tank 241-B-108

\begin{tabular}{|c|c|c|c|c|c|c|c|c|c|}
\hline $\begin{array}{l}\text { CLIENT SAMPLE ID } \\
\text { LOCATION } \\
\text { CUSTODY }\end{array}$ & MATRIX & $\begin{array}{l}\text { LAB SAMPLE ID } \\
\text { COLLECTED } \\
\text { RECEIVED }\end{array}$ & PLANCHET & IEST & $\begin{array}{l}\text { SUF- } \\
\text { FIX }\end{array}$ & AKALYZED & REVIEWED & BY & METHOD \\
\hline $\begin{array}{l}\text { B108 c173s1 UH fusion } \\
R: 3 \text { S: } 1 \text { C: } 173 \\
n / a\end{array}$ & $\begin{array}{l}\text { FUSION } \\
\text { SOLID }\end{array}$ & $\begin{array}{l}\$ 96 r 005468 \\
10 / 17 / 96\end{array}$ & $16019-3$ & GER & & $01 / 09 / 97$ & & PPB & Gama Spectroscopy \\
\hline $\begin{array}{l}\text { B108 C173s1 UH Fusion-DU } \\
\text { R: } 3 \text { S: } 1 \text { C: } 173\end{array}$ & $\begin{array}{l}\text { FUSION } \\
\text { SOLID }\end{array}$ & $\begin{array}{l}\text { \$96T0054680 } \\
10 / 17 / 96\end{array}$ & $16019-4$ & GEA & & $01 / 09 / 97$ & & PPB & Gamma Spectroscopy \\
\hline $\begin{array}{l}\text { B108 C173S2 LH Fusion } \\
\mathrm{R}: 3 \mathrm{~s}: 2 \mathrm{C}: 173 \\
\mathrm{n} / \mathrm{a}\end{array}$ & $\begin{array}{l}\text { FUSION } \\
\text { SOLID }\end{array}$ & $\begin{array}{r}5961005478 \\
10 / 17 / 96\end{array}$ & $\begin{array}{l}14361-6 \\
16019-7\end{array}$ & $\begin{array}{l}\text { AT } \\
\text { GEA }\end{array}$ & & $\begin{array}{l}10 / 30 / 96 \\
01 / 10 / 97\end{array}$ & & $\begin{array}{l}\text { SLF } \\
\text { PPB }\end{array}$ & $\begin{array}{l}\text { Alpha Analysis } \\
\text { Garma Spectroscopy }\end{array}$ \\
\hline $\begin{array}{l}\text { B108 c173s2 LH Fusion Re } \\
\text { R: } 3 \text { S: } 2 \text { C: } 173 \\
\mathrm{n} / \mathrm{a}\end{array}$ & $\begin{array}{l}\text { FUSION } \\
\text { SOLID }\end{array}$ & $\begin{array}{l}\text { s977000001 } \\
01 / 06 / 97\end{array}$ & $\begin{array}{l}16349-4 \\
16362-3\end{array}$ & $\begin{array}{l}\text { AT } \\
\text { GEA }\end{array}$ & $\begin{array}{l}01 \\
01\end{array}$ & $\begin{array}{l}01 / 26 / 97 \\
01 / 28 / 97\end{array}$ & & $\begin{array}{l}\text { SLF } \\
\text { LLF }\end{array}$ & $\begin{array}{l}\text { Alpha Analysis } \\
\text { Gamma Spectroscopy }\end{array}$ \\
\hline $\begin{array}{l}\text { B108 C173s2 LH Fusion Re } \\
\text { R: } 3 \text { S: } 2 \text { C: } 173\end{array}$ & $\begin{array}{l}\text { FUSION } \\
\text { SOLID }\end{array}$ & $\begin{array}{l}\text { S97T0000010 } \\
01 / 06 / 97\end{array}$ & $\begin{array}{l}16349-5 \\
16362-4\end{array}$ & $\begin{array}{l}\text { AT } \\
\text { GEA }\end{array}$ & 01 & $\begin{array}{l}01 / 26 / 97 \\
01 / 28 / 97\end{array}$ & & $\begin{array}{l}\text { SLF } \\
\text { LL.F }\end{array}$ & $\begin{array}{l}\text { Alpha Analysis } \\
\text { Ganma Spectroscopy }\end{array}$ \\
\hline $\begin{array}{l}\text { B108 C173s2 LH Fusion Re } \\
\text { R: } 3 \text { S: } 2 \text { C: } 173\end{array}$ & $\begin{array}{l}\text { FUSION } \\
\text { SOLIO }\end{array}$ & $\begin{array}{l}\text { s977000001s } \\
01 / 06 / 97\end{array}$ & $16349-6$ & AT & 01 & $01 / 26 / 97$ & & SLF & Alpha Analysis \\
\hline $\begin{array}{l}\text { B108 C173s2 LH Fusion-DU } \\
R: 3 \text { s: } 2 \text { C: } 173\end{array}$ & $\begin{array}{l}\text { FUSION } \\
\text { SOLID }\end{array}$ & $\begin{array}{r}\$ 9670054780 \\
10 / 17 / 96\end{array}$ & $\begin{array}{l}14361-7 \\
16019-8\end{array}$ & $\begin{array}{l}\text { AT } \\
\text { GEA }\end{array}$ & & $\begin{array}{l}10 / 30 / 96 \\
01 / 10 / 97\end{array}$ & & $\begin{array}{l}\text { SLF } \\
\text { PPB }\end{array}$ & $\begin{array}{l}\text { Alpha Analysis } \\
\text { Gamma Spectroscopy }\end{array}$ \\
\hline $\begin{array}{l}\text { B108 C173\$2 LH fusion-SP } \\
\text { R: } 3 \text { S: } 2 \text { C: } 173\end{array}$ & $\begin{array}{l}\text { FUSION } \\
\text { SOLID }\end{array}$ & $\begin{array}{r}\text { s96r005478s } \\
10 / 17 / 96\end{array}$ & $14361 \cdot 8$ & AT & & $10 / 30 / 96$ & & SLF & Alpha Analysis \\
\hline Method Blank & SOLID & $B 14361-2$ & $14361-2$ & AT & & $10 / 30 / 96$ & & SLF & Alpha Analysis \\
\hline Method Blank & SOLID & B14725-2 & $14725-2$ & $A B$ & & $11 / 08 / 96$ & & SLF & Alpha Analysis \\
\hline
\end{tabular}

\section{Group 96001380}

WORK SUMMARY

Page 2

SUNAARY DATA SECTION

Page 7

Lab id 222-s

Protocol SST

version 1.0

Form DVD-CWS

Version 3.08

Report date $02 / 24 / 97$ 
HAF-SD-WM-DP-219, REV.O

222-S LABORATORY

TANK 241-8-108, CORE 173

WORK SUMMARY, cOnt.
Client TWRS

Tank 241-B-108
CLIENT SAMPLE ID LOCATION cusroor

Method Blank MATRIX Priority

LAB SAMPLE ID COLLECTED

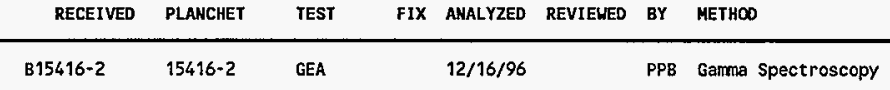

SOL.1D

Method Blank B15952-2 15952-2 SR

SOLID

Method Blank B16015-2 $16015-2$

SOLID

Method Btank B16017-2 16017-2 SR

SOLID

Method Blank 816019-2 16019-2 GEA

SOLID

Method Blank

$$
\text { - }
$$

Method Blank

\section{Method Blank}

Method Blank

SOL ID

SOLID

B16256-2 16256-2 SR

B16258-2 $16258-2 \quad$ GEA

816349-2 16349-2 AT

SOLID

Method Blank

816362-2 16362-2 GEA

SOLID

Method Blank

$816409-2 \quad 16409-2 \quad A B$

SOLID$$
\text { Lat }
$$

Lab Control Sample

SOL ID

\$14725-1 $14725-1 \quad A B$

\$15416-1 15416-1

GEA

SOLID

\section{Group 96001380}

WORK SUAMARY

Page 3

SUMALARY DATA SECTION

Page 8
Lab id 222-S

Protocol SST

Version 1.0 Form DVD-CWS

Version 3.08 Report date $02 / 24 / 97$ 
HNF-SD-WM-DP-219, REV. O

222 -S IABORATORY

TANK 241-8-108, CORE 173

SDG 96001380

Contact L. L. Fritts
WORK SUMMARY, cont.
Client TWRS

Tank 241-B-108
CLIENT SAMPLE ID LOCATION CustooY

Lab Control Sample
LAB SAMPLE ID

COLLECTEO

RECEIVED

S15952-1 15952-1 SR

SOLID

Lab Control Sample

\$16015-1

16015-1 TB

TB

$01 / 09 / 97$

SUF-

TEST FIX ANALYZED REVIEUED BY METHOD

01/06/97 SAC strontium $-89 / 90$

SOLID

Lab control Sample

S16017-1 $16017-1 \quad$ SR

$01 / 07 / 97$

SAC Strontium- $89 / 90$

SOLID

Lab Control Sample

\$16019-1 96019-9 GEA

$01 / 09 / 97$

PPB Gamma Spectroscopy

SOLID

Lab Control Sample

S16256-1 16256-1 SR

$01 / 22 / 97$

SAC Strontium- $89 / 90$

SOL. ID

Lab Control Sample

$\$ 16258-1 \quad 16258-1 \quad$ GEA

$01 / 22 / 97$

PPB Gamma Spectroscopy

SOLID

Lab Control Sample

\$16349-1 16349-1 AT

$01 / 26 / 97$

SLF Alpha Analysis

SOLID

Lab Control Sample

\$16362-1

16362-1

GEA

$01 / 28 / 97$

LLF Gamma Spectroscopy

SOLID

Lab Control Sample

S16409-1

16409-1

AB

$01 / 29 / 97$

SOL. ID

\section{Group 96001380}

WORK SUMMARY

Page 4

SUMAARY DATA SECTION

Page 9
Lab id 222-s

Protocol SST

Version 1.0 Form DVD-CWS

Version 3.08 Report date $\underline{02 / 24 / 97}$ 
HNF-SD-WM-DP-219, REV. 0

222-S LABORATORY

TANK 241-B-108, CORE 173

SDG 96001380

Contact L. L. Fritts

WORK SUMMARY, cOnt.

Client TWRS

Tank 241-B-108

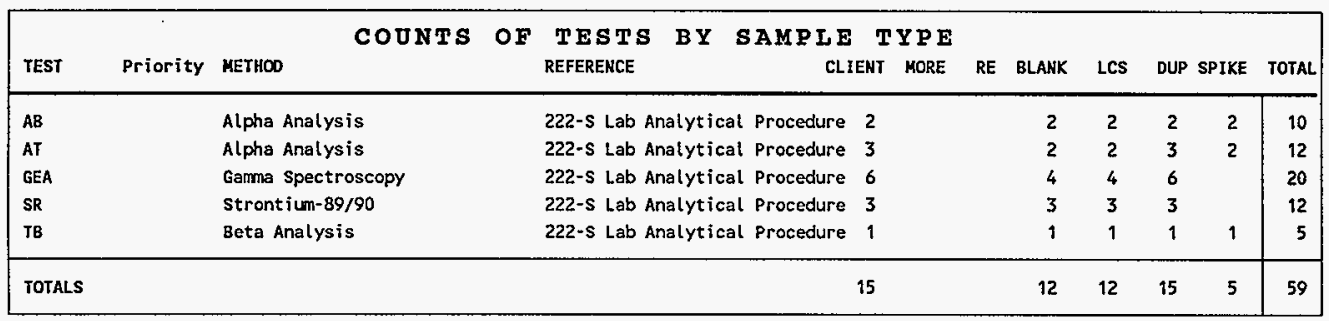

\section{Group 96001380}

HORK SUMMARY

Page 5

SUMAARY DATA SECTIOK

Page 10
Lab id 222-S

Protocol SST Version 1.0 Form DVD-CWS

Version 3.08 Report date 02/24/97 
HNF-SD-WM-DP-219, REV. 0

$222-S$ LABORATORY

TARK 241-B-108, CORE 173

SDG 96001380

Contact L. L. Fritts

BIANKS

Client IWRS

Tank 24?-B-108

\begin{tabular}{|c|c|c|c|c|c|c|c|c|}
\hline \multicolumn{2}{|c|}{$\begin{array}{l}\text { Lab sample id B14361-2 } \\
\text { Dept sample id }\end{array}$} & \multicolumn{3}{|c|}{$\begin{array}{l}\text { Client sample id } \\
\text { Material/Matrix }\end{array}$} & \multicolumn{2}{|c|}{ Method Blank } & \multicolumn{2}{|r|}{ SOLID } \\
\hline ANALYTE & CAS NO & $\begin{array}{r}\text { RESULT } \\
\text { uCi/g }\end{array}$ & $\begin{array}{c}2 \sigma \text { TPU } \\
\%\end{array}$ & $\begin{array}{c}\text { MDA } \\
\mathrm{UCi} / \mathrm{g}\end{array}$ & $\begin{array}{c}\mathrm{RDL} \\
\mathrm{uCi} / \mathrm{g}\end{array}$ & $\begin{array}{l}\text { QUALI- } \\
\text { FIERS }\end{array}$ & TEST & PREP BATCH \\
\hline Total Alpha & $12587-46-1$ & 408003 & & 78103 & & 1 & AT & 96010903 \\
\hline
\end{tabular}

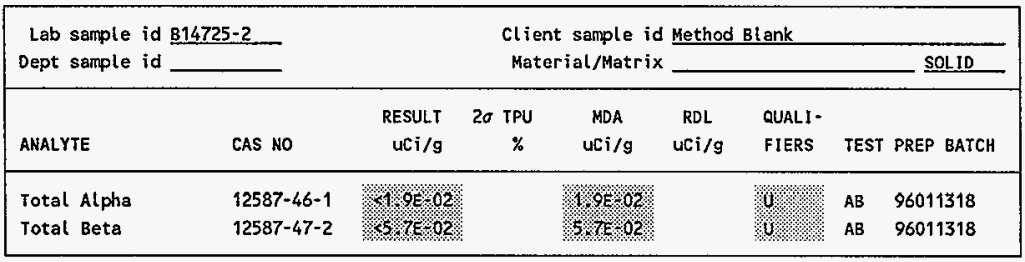

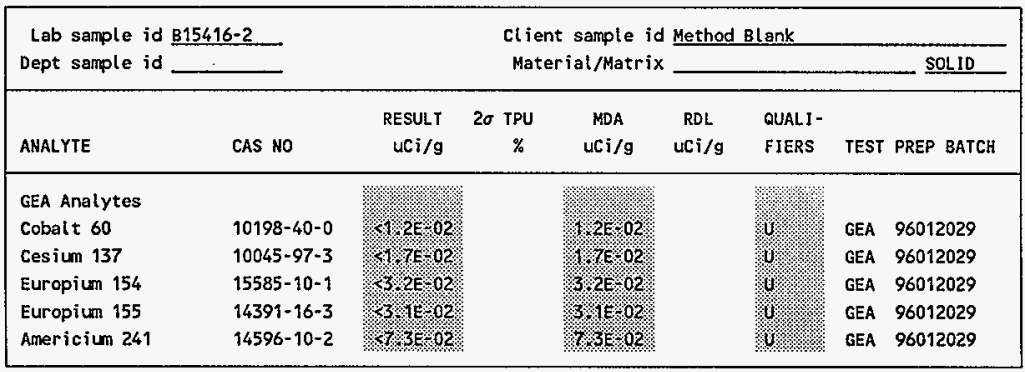

\section{Group 96001380}

\author{
BLANKS \\ Page 1 \\ SUMATARY DATA SECIION
}

Page 11
Lab id 222-s

Protocol SST

Version 1.0

Form DVD-BLANK

Version 3.08

Report date $02 / 24 / 97$ 
222-S LABORATORY

TANK 241-B-108, CORE 173

BLANRS
Client TWRS

Tank 241-B-108

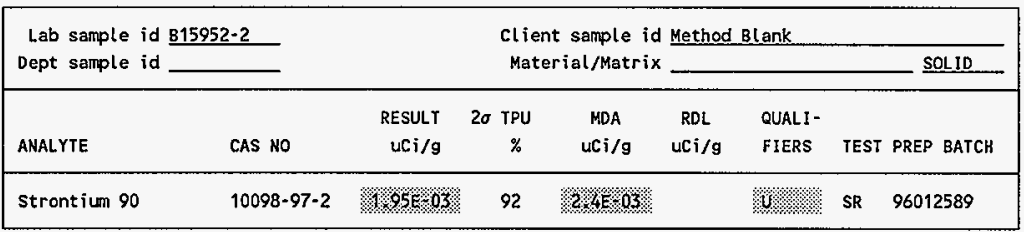

\begin{tabular}{|c|c|c|c|c|c|c|c|c|}
\hline \multicolumn{2}{|c|}{$\begin{array}{l}\text { Lab sample id B16015-2 } \\
\text { Dept sample id }\end{array}$} & \multicolumn{3}{|c|}{$\begin{array}{l}\text { Client sample id } \\
\text { Material/Matrix }\end{array}$} & \multicolumn{2}{|c|}{ Method Blank } & \multicolumn{2}{|r|}{ SOL ID } \\
\hline ANALYTE & CAS NO & $\begin{array}{r}\text { RESULT } \\
\text { UCi/g }\end{array}$ & $\begin{array}{c}2 \sigma \text { TPU } \\
\%\end{array}$ & $\begin{array}{c}\text { MDA } \\
\mathrm{uCi} / \mathrm{g}\end{array}$ & $\begin{array}{c}\text { RDL } \\
\text { UCi/g }\end{array}$ & $\begin{array}{l}\text { QUALI - } \\
\text { FIERS }\end{array}$ & TEST & PREP BATCH \\
\hline Total Beta & $12587-47-2$ & $3.32 E 002$ & 42 & Ko 602 & & & T8 & 96012646 \\
\hline
\end{tabular}

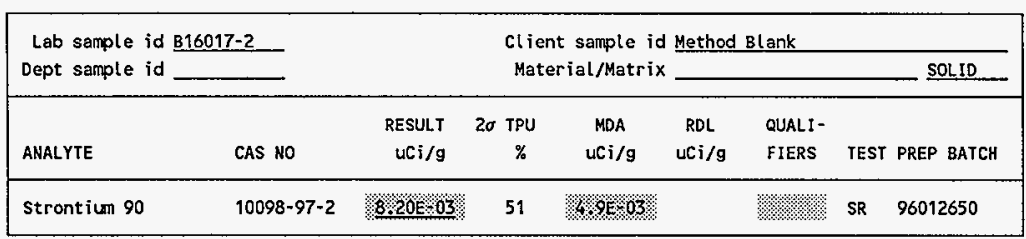

Group 96001380

BLANKS

Page 2

SUMLARY DATA SECTIOH

Page 12
Lab id 222-S

Protocol SST

Version 1.0

Form DVD-BLANK

Version 3.08

Report date $02 / 24 / 97$ 
HNF-SD-WM-DP-219, REV. O

222 -S LABORATORY

TANK 241-B-108, CORE 173

SDG 96001380

Contact L.L. Fritts

BLANKS

Ct ient TWRS

Tank 241-B-108

\begin{tabular}{|c|c|c|c|c|c|c|c|c|}
\hline \multirow{2}{*}{\multicolumn{2}{|c|}{$\begin{array}{l}\text { Lab sample id B16019-2 } \\
\text { Dept sample id }\end{array}$}} & \multirow{2}{*}{\multicolumn{5}{|c|}{ Client sample id Method Blank. }} & \multirow{2}{*}{\multicolumn{2}{|c|}{- SOL1D }} \\
\hline & & & & & & & & \\
\hline ANALYTE & CAS NO & $\begin{array}{r}\text { RESULT } \\
\text { UCi/g }\end{array}$ & $\begin{array}{c}2 \sigma \text { TPU } \\
\%\end{array}$ & $\begin{array}{l}\text { MDA } \\
\mathrm{UCi} / \mathrm{g}\end{array}$ & $\begin{array}{l}\text { RDL } \\
U C i / g\end{array}$ & $\begin{array}{l}\text { QUAL I- } \\
\text { FIERS }\end{array}$ & TEST & PREP BATCH \\
\hline \multicolumn{9}{|l|}{ GEA Analytes } \\
\hline Cobalt 60 & $10198-40-0$ & $1 \times 6 \mathrm{E} 02$ & & \%6\% & & u & GEA & 96012648 \\
\hline Cesium 137 & $10045-97-3$ & Y $3 \times 3502$ & & $3 \times 302$ & & \% & GEA & 96012648 \\
\hline Europium 154 & $15585-10-1$ & 5.0002 & & $50 \mathrm{OE} 02$ & & 0 & GEA & 96012648 \\
\hline Europitum 155 & $14391-16-3$ & $\times 4 \times 02$ & & $45 \mathrm{E} 02$ & & 6 & GEA & 96012648 \\
\hline Americium 241 & $14596-10-2$ & \%8.28 02 & & 8.2102 & & 6 & GEA & 96012648 \\
\hline
\end{tabular}

\begin{tabular}{|c|c|c|c|c|c|c|c|c|}
\hline \multirow{2}{*}{\multicolumn{2}{|c|}{$\begin{array}{l}\text { Lab sample id B16256-2 } \\
\text { Dept sample id }\end{array}$}} & \multicolumn{7}{|c|}{ client sample id Method Blank } \\
\hline & & \multicolumn{3}{|c|}{ Material/Matrix } & & & & SOLID \\
\hline ANALYTE & CAS NO & $\begin{array}{l}\text { RESULT } \\
\text { UCi/g }\end{array}$ & $\begin{array}{c}2 \sigma \mathrm{TPU} \\
\%\end{array}$ & $\begin{array}{c}\mathrm{MDA} \\
\mathrm{UC} i / \mathrm{g}\end{array}$ & $\begin{array}{c}\text { RDL } \\
\mathrm{uCi} / \mathrm{g}\end{array}$ & $\begin{array}{l}\text { QUALI* } \\
\text { FIERS }\end{array}$ & IESI & PREP BATCH \\
\hline Strontium 90 & $10098-97-2$ & KreEor & 34 & Y 3103 & & \%+x & SR & 97000108 \\
\hline
\end{tabular}

\begin{tabular}{|c|c|c|c|c|c|c|c|c|}
\hline \multicolumn{2}{|c|}{$\begin{array}{l}\text { Lab sample id B16258-2 } \\
\text { Dept sample id }\end{array}$} & \multicolumn{5}{|c|}{$\begin{array}{l}\text { Client sample id Method Blank } \\
\text { Material/Matrix }\end{array}$} & \multicolumn{2}{|r|}{ SOLID } \\
\hline ANALYTE & CAS NO & $\begin{array}{l}\text { RESULT } \\
\text { UCi } / \mathrm{g}\end{array}$ & $\begin{array}{c}2 \sigma \mathrm{TPU} \\
\%\end{array}$ & $\begin{array}{l}\text { MDA } \\
\text { uCi/g }\end{array}$ & $\begin{array}{c}\text { ROL } \\
\text { UCi } / g\end{array}$ & $\begin{array}{l}\text { QUALI - } \\
\text { FIERS }\end{array}$ & TEST & PREP BATCH \\
\hline $\begin{array}{l}\text { GEA Analytes } \\
\text { Cobalt } 60 \\
\text { Cesium } 137 \\
\text { Europium } 154 \\
\text { Europium } 155 \\
\text { Anericiun } 241\end{array}$ & $\begin{array}{l}10198-40-0 \\
10045-97-3 \\
15585-10-1 \\
14391-16-3 \\
14596-10-2\end{array}$ & 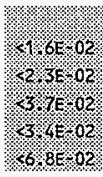 & & 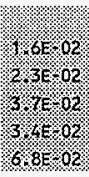 & & W & $\begin{array}{l}\text { GEA } \\
\text { GEA } \\
\text { GEA } \\
\text { GEA } \\
\text { GEA }\end{array}$ & $\begin{array}{l}97000110 \\
97000110 \\
97000110 \\
97000110 \\
97000110\end{array}$ \\
\hline
\end{tabular}

\section{Group 96001380}

\author{
BLANKS \\ Page 3 \\ SUMMARY DATA SECTION \\ Page 13
}

Lab id 222-S

Protocol SST

Version 1.0

Form DVD-BLANK

Version 3.08

Report date 02/24/97 
HNF-SD-WURA-DP-219, REV.O

\section{2 -S LABORATORY}

TANK 241-B-108, CORE 173

SDG 96001380

Contact L. L. Fritts

BLANRS

Client TWRS

Tank $241-8-108$

\begin{tabular}{|c|c|c|c|c|c|c|c|c|}
\hline \multicolumn{2}{|c|}{$\begin{array}{l}\text { Lab sample id B16349-2 } \\
\text { Dept sample id }\end{array}$} & \multicolumn{3}{|c|}{$\begin{array}{l}\text { client sample id } \\
\text { Material/Matrix }\end{array}$} & \multicolumn{2}{|c|}{ Method B lank } & \multicolumn{2}{|r|}{ SOLID } \\
\hline ANALYTE & CAS NO & $\begin{array}{r}\text { RESULT } \\
\mathrm{UCi} / \mathrm{g}\end{array}$ & $\begin{array}{c}2 \sigma \text { TPU } \\
\%\end{array}$ & $\begin{array}{l}\text { MDA } \\
\text { uCi/g }\end{array}$ & $\begin{array}{c}\text { RDL } \\
\mathrm{uCi} / \mathrm{g}\end{array}$ & $\begin{array}{l}\text { QUAL I- } \\
\text { FIERS }\end{array}$ & TEST & PREP BATCH \\
\hline Total Alpha & $12587-46-1$ & 432003 & & $3 \times 2 \mathrm{E}<3$ & & o & AT & 97000208 \\
\hline
\end{tabular}

\begin{tabular}{|c|c|c|c|c|c|c|c|c|}
\hline \multirow{2}{*}{\multicolumn{2}{|c|}{$\begin{array}{l}\text { Lab sample id B16362-2 } \\
\text { Dept sample id }\end{array}$}} & \multicolumn{7}{|c|}{ client sample id Method Blank } \\
\hline & & & Mat & al/Matri & & & 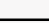 & - SOLID \\
\hline ANALYTE & CAS NO & $\begin{array}{r}\text { RESULT } \\
\text { uCi/g }\end{array}$ & $\begin{array}{c}2 \sigma \text { TPU } \\
\%\end{array}$ & $\begin{array}{l}\text { MDA } \\
\mathrm{uCi} / \mathrm{s}\end{array}$ & $\begin{array}{l}\text { RDL. } \\
\text { uCi/g }\end{array}$ & $\begin{array}{l}\text { QUAL I - } \\
\text { FIERS }\end{array}$ & TEST & PREP BATCH \\
\hline GEA Analytes & & & & & & & & \\
\hline Cobalt 60 & $10198-40-0$ & 3 11002 & & 302 & & 霖 & GEA & 97000227 \\
\hline Cesium 137 & $10045-97-3$ & 123002 & & 25102 & & \% & GEA & 97000227 \\
\hline Europium 154 & $15585-10-1$ & 183682 & & 3.6502 & & 19 & GEA & 97000227 \\
\hline Europium 155 & $14391-16-3$ & 3.30002 & & $80 \mathrm{O} 02$ & & 89 & GEA & 97000227 \\
\hline Americium 241 & $14596-10-2$ & $\times 8 \times 2=0$ & & $8 \times 2 \times 2$ & & 2. & GEA & 97000227 \\
\hline
\end{tabular}

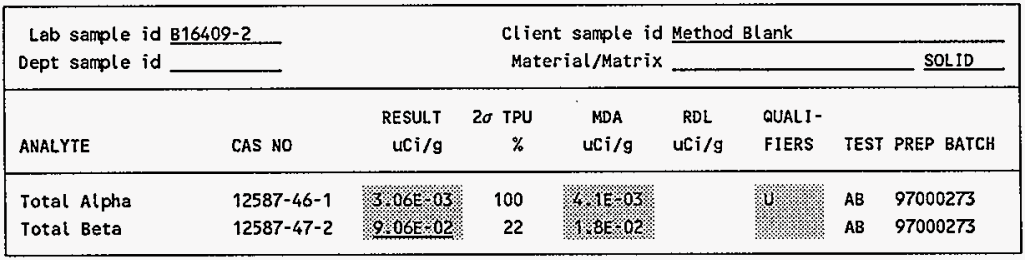

\section{Group 96001380}

\author{
BLANKS \\ Page 4 \\ SUMMARY DATA SECTIOH
}

Page 14
Lab id 222-5

Protocol SST

Version 1.0

Form DVD-BLANK

Version 3.08

Report date $\underline{02 / 24 / 97}$ 
222-S LABORATORY

TANK 241-B-108, CORE 173

SDG 96001380

Contact L. L. Fritts
IAB CONTROL SAMPLES
Client TWRS

Tank 241-8-108
Lab sample id s14361-1

Dept sample id

Dept sanple id

\begin{tabular}{|c|c|c|c|c|c|c|c|c|c|c|c|c|}
\hline ANALYTE & $\begin{array}{l}\text { RESULT } \\
\text { UCi/g }\end{array}$ & $\begin{array}{c}2 \sigma \text { TPU } \\
\%\end{array}$ & $\begin{array}{c}\text { MDA } \\
\text { UCi/g }\end{array}$ & $\begin{array}{c}\text { RDL } \\
\text { uCi } / g\end{array}$ & $\begin{array}{l}\text { QUALI - } \\
\text { FIERS }\end{array}$ & IEST & $\begin{array}{l}\text { ADDED } \\
\mathrm{uCi} / \mathrm{g}\end{array}$ & $\begin{array}{c}2 \sigma E R R \\
\%\end{array}$ & $\begin{array}{r}\text { REC } \\
\%\end{array}$ & $\begin{array}{l}3 \sigma \text { LMTS } \\
\text { (TOTAL) }\end{array}$ & $\begin{array}{l}\text { PROTOCOL } \\
\text { LIMITS }\end{array}$ & PREP BATCH \\
\hline Total Alpha & $8 / 8 E_{02}$ & 16 & $3 \% 6 \%$ & & \% & AT & \%oL & 5.0 & so & $80-120$ & 70,430 & 96010903 \\
\hline
\end{tabular}

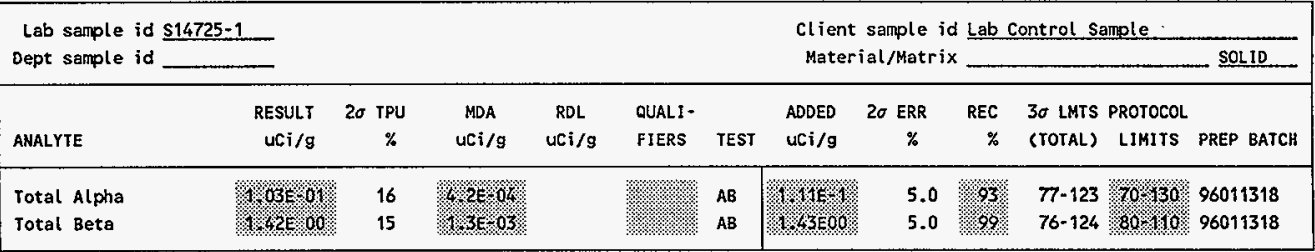

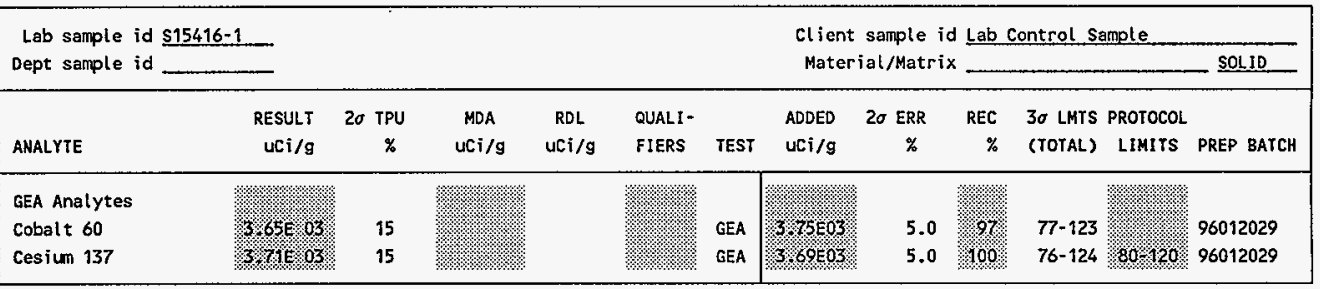

\section{Group 96001380}

LAB CONTROL SAMPLES

Page 1

SUMARARY DATA SECTION

Page 15

Client sample id Lab Control Sample SOLID

\author{
Material/Matrix
} $-$ 
HNF-SD-WRA-DP-219, REV. 0

$222-S$ IABORATORY

TANK 241-B-108, CORE 173

SDG 96001380

Contact L. L. Fritts
LAB CONTROL SAMPLES
Client IWRS

Tank 241-B-108

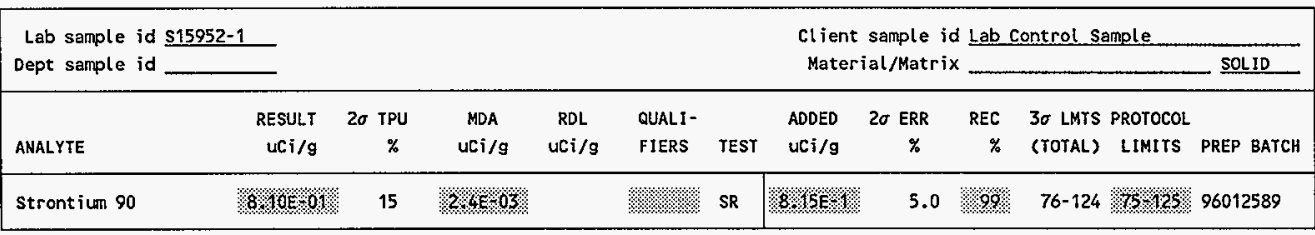

\begin{tabular}{|c|c|c|c|c|c|c|c|c|c|c|c|c|}
\hline \multirow{3}{*}{$\begin{array}{l}\text { Lab sample id } \\
\text { Dept sample id }\end{array}$} & $\$ 16015-1$ & & & & \multicolumn{8}{|c|}{ client sample id Lab control Sample } \\
\hline & & & & & & & Mater & ial/Matri & & 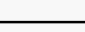 & 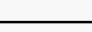 & - SOLID \\
\hline & RESULT & $2 \sigma$ TPU & MDA & RDL & QUALI - & & ADDED & $2 \sigma$ ERR & REC & 3o LMTS & PROTOCOL & \\
\hline ANALYTE & $\mathrm{uCi} / \mathrm{g}$ & $\%$ & $\mathbf{u c i} / \mathrm{g}$ & uci/g & FIERS & TEST & $\mathrm{uCi} / \mathrm{g}$ & $\%$ & $\%$ & (TOTAL) & LIMITS & PREP ВATCH \\
\hline Total Beta & $1 \% 41000$ & 15 & Yoso & & B & ТВ & $1 \% 30 \mathrm{~B} 00$ & 5.0 & 08 & $74-126$ & $80 \% 10$ & 96012646 \\
\hline
\end{tabular}

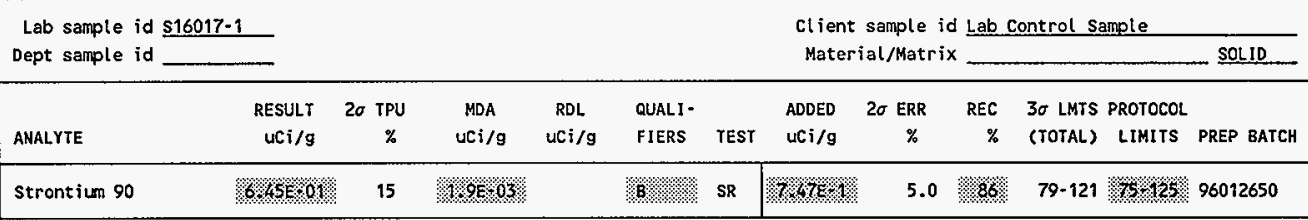

\section{Group 96001380}

LAB CONTROL SAMPLES

Page 2

SLRMARY DATA SECIION

Page 16
Lab id 222-s

Protocol SST

Version 1.0

Form DVD-LCS

Version 3.08

Report date $02 / 24 / 97$ 
HNF-SD-WMI-DP-219, REV. 0

222-S IABORATORY

TANK 241-8-108, CORE 173

SOG 96001380

Contact L. L. Fritts

IAB CONTROI SAMPLES

Client IWRS

Tank 241-B-108

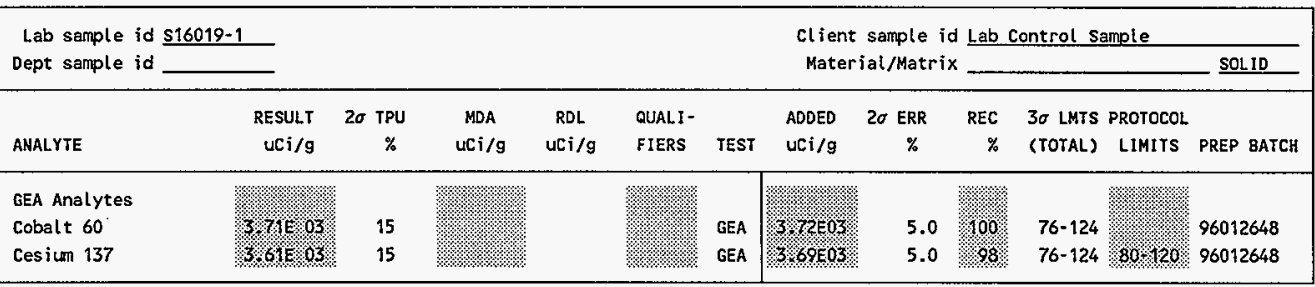

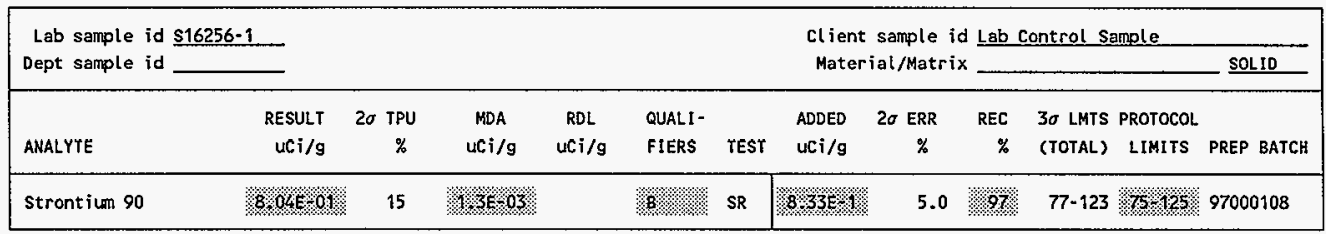

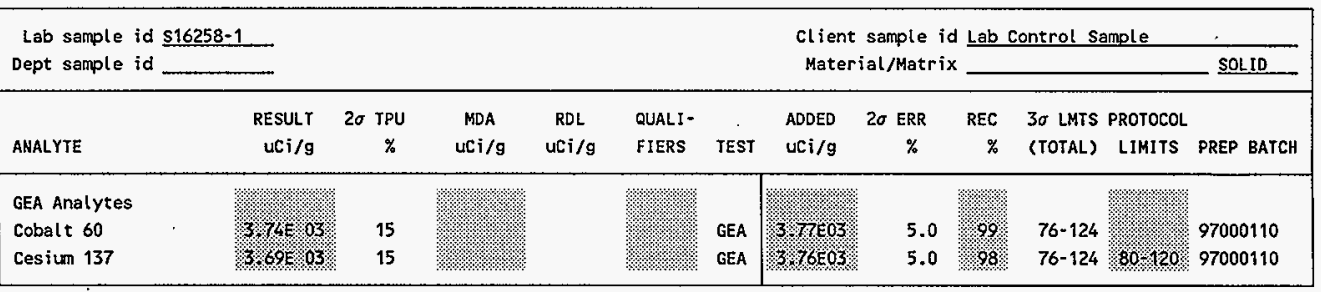

\section{Group 96001380}

LAB CONTROL SAMPLES

Page 3

SUMMARY DATA SECTION

Page 17
Lab id 222-s

Protocol SST

Version 1.0

Form DVD-LCS.

Version 3.08 Report date $02 / 24 / 97$ 
HNF-SD-WM-DP-219, REV. 0

$222-S$ LABORATORY

TANK 249-B-108, CORE 173

DUPIICATE

Client TWRS

Tank 241-8-108

Contact L. L. Fritts DUPLICATE

Lab sample id 596T005468D

ORIGINAL

Dept sample id

Lab sample id \$96T005468

Dept sample id

Received 10/17/96
Client sample id B108 C17351 UH Fusion

Location/Matrix R: 3 s: 1 C: 173

Collected

Chain of custody id $n / a$

\begin{tabular}{|c|c|c|c|c|c|c|c|c|c|c|c|c|c|}
\hline ANALYTE & $\begin{array}{c}\text { DUPL ICATE } \\
\text { uCi/g }\end{array}$ & $\begin{array}{c}2 \sigma \text { TPU } \\
x\end{array}$ & $\begin{array}{c}\text { MDA } \\
\mathrm{uCi} / \mathrm{g}\end{array}$ & $\begin{array}{c}\text { RDL. } \\
\mathrm{uCi} / \mathrm{g}\end{array}$ & $\begin{array}{l}\text { QUALI- } \\
\text { FIERS }\end{array}$ & TEST & $\begin{array}{l}\text { ORIGINAL } \\
\text { UCi/g }\end{array}$ & $\begin{array}{c}2 \sigma \mathrm{TPU} \\
\%\end{array}$ & $\begin{array}{c}\text { MDA } \\
u C i / g\end{array}$ & $\begin{array}{l}\text { QUALI- } \\
\text { FIERS }\end{array}$ & $\begin{array}{r}\text { RPD } \\
\%\end{array}$ & $\begin{array}{r}3 \sigma \\
\text { TOT }\end{array}$ & $\begin{array}{l}\text { PROT } \\
\text { LIMIT }\end{array}$ \\
\hline GEA Analytes & & & & & & & & & & & & & \\
\hline Cobalt 60 & \% & & r. $8 \mathrm{E} .02$ & & 棌 & GEA & $1 / 80.02$ & & K8 802 & $\mathbf{U}$ & & & \\
\hline Cesiun 137 & 22901 & 15 & 1008 oom & & 8 & GEA & 200001 & 15 & 0.0000 & & 0 & 32 & 20 \\
\hline Europium 154 & 15.0102 & & 159002 & & \% & GEA & $5 \% 9102$ & & 5.91 .02 & U & 8 & & \\
\hline Europium 155 & K1 & & 150,01 & & o & GEA & $41 \% 5401$ & & $65 \mathrm{E} 01$ & U & 4 & & \\
\hline Americium 241 & $\times 3,21001$ & & $325 \times 01$ & & 4 & GEA & 632001 & & $320 \% 0$ & $\mathbf{u}$ & \% & & \\
\hline
\end{tabular}

\section{Group 96001380}

DUPLICATES

Page 1

SUMMARY DATA SECTIOH

Page 19
Lab id 222-s Protocol SST Version 1.0 Form DVD-DUP Version 3.08 Report date $02 / 24 / 97$ 
HNF-SD-WM-DP-219, REV. 0

\section{2 - S IABORATORY}

TANK 241-B-108, CORE 173

DUPLICATE

Client IWRS

Tank 241-B-108

ORIGINAL

Lab sample id \$96T005477

Dept sample id Received 10/17/96
Client sample id B108 C173S1 LH fusion Location/Matrix R: 3 s: 1 C: 173 FUSIOH Collected

Chain of custody id $n / a$

\begin{tabular}{|c|c|c|c|c|c|c|c|c|c|c|c|c|c|}
\hline ANALYTE & $\begin{array}{l}\text { DUPLICATE } \\
\text { UCi/g }\end{array}$ & $\begin{array}{c}2 \sigma \text { TPU } \\
\%\end{array}$ & $\begin{array}{c}\text { MDA } \\
\mathrm{uCi} / \mathrm{g}\end{array}$ & $\begin{array}{c}\text { RDL } \\
\mathrm{uCi} / \mathrm{g}\end{array}$ & $\begin{array}{l}\text { QUALI- } \\
\text { FIERS }\end{array}$ & TEST & $\begin{array}{l}\text { ORIGINAL } \\
u C i / g\end{array}$ & $\begin{array}{c}2 \sigma \text { TPU } \\
x\end{array}$ & $\begin{array}{c}\text { MDA } \\
\mathrm{uCi} / \mathrm{g}\end{array}$ & $\begin{array}{l}\text { QUALI- } \\
\text { FIERS }\end{array}$ & $\begin{array}{r}\text { RPD } \\
\boldsymbol{x}\end{array}$ & $\begin{array}{r}30 \\
\text { TOT }\end{array}$ & $\begin{array}{r}\text { PROT } \\
\text { LIHIT }\end{array}$ \\
\hline Total Atpha & 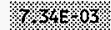 & 98 & 178003 & & W & AT & Wo8E 02 & 62 & 78003 & & 38 & 162 & 172 \\
\hline Total Beta & 1.t20 00 & 15 & $160 \% 2$ & & B & TB & $2033 \mathrm{E} 01$ & 15 & $1 \% 60.02$ & B & 16 & 32 & 20 \\
\hline Strontium 90 & 409800 & 15 & $3.3 E 03$ & & 8 & SR & \%rae 00 : & 15 & 3.8003 & B & 6 & 32 & 20 \\
\hline GEA Analytes & & & & & & & & & & & & & \\
\hline Cobalt 60 & \% $3 \mathrm{E} / 02$ & & 1. $30 \% 2$ & & 0 & GEA & $4 \times 3 \mathrm{E}=2$ & & \% 3602 & U & \% & & \\
\hline Cesium 137 & $106 \mathrm{1} .01$ & 15 & 10.01 & & & GEA & W & 15 & $00 \mathrm{~K} 00$ \% & & 5 & 32 & $20 \%$ \\
\hline Europium 154 & $3 \times 3 \times 02$ & & $3 \times 1602$ & & 80 & GEA & $3 \times 7+02$ & & $3 \% 702$ & U & 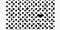 & & \\
\hline Europium 155 & \$2E 01 & & 13.2 01 . & & 4 & GEA & $1 \times 1 / 20 \times 1$ & & $3 \% 2501$ & $u$ & 棛 & & \\
\hline Americium 241 & $426 \mathrm{E} \% 1$ & & 260001 & & 40 & GEA & $2 \times 260 \% 0$ & & 2.601 & u & \%) & & \% \\
\hline
\end{tabular}

\section{Group 96001380}

DUPLICATES

Page 2

SUMMARY DATA SECTION

Page 20
Lab id 222-s

Protocol SST

Version 1.0

Form DVD-DUP

Version 3.08

Report date $02 / 24 / 97$ 
HNF-SD-WM-DP-219, REV. O

\section{$222-S$ LABORATORY}

TANK 241-8-108, CORE 173

DUPLICATE

SDG 96001380

Contact L. L. Fritts DUPLIICATE

Lab sample id 596T005478D

Dept sample id
ORIGINAL

Lab sample id \$96T005478

Dept sample id

Received 10/17/96
Client IWRS

Tank 241-8-108

Client sample id B108 C173\$2 LH Fusion FUSION Location/Matrix R: 3 S: 2 C: 173 SOLID Collected

Chain of custody id n/a

\begin{tabular}{|c|c|c|c|c|c|c|c|c|c|c|c|c|c|}
\hline ANALYTE & $\begin{array}{c}\text { DUPLICATE } \\
\text { UCI/g }\end{array}$ & $\begin{array}{c}2 \sigma \mathrm{TPU} \\
\%\end{array}$ & $\begin{array}{c}\text { MDA } \\
\text { uCi/g }\end{array}$ & $\begin{array}{c}\text { ROL } \\
\mathrm{uCi} / \mathrm{g}\end{array}$ & $\begin{array}{l}\text { QUALI- } \\
\text { FIERS }\end{array}$ & TEST & $\begin{array}{l}\text { ORIGINAL } \\
\text { UCI/g }\end{array}$ & $\begin{array}{c}2 \sigma \text { TPU } \\
\%\end{array}$ & $\begin{array}{c}\text { MDA } \\
\mathrm{uCi} / \mathrm{g}\end{array}$ & $\begin{array}{l}\text { QUALI- } \\
\text { FIERS }\end{array}$ & $\begin{array}{r}-\mathrm{RPD} \\
x\end{array}$ & $\begin{array}{r}3 \sigma \\
\text { TOT }\end{array}$ & $\begin{array}{r}\text { PROT } \\
\text { LIMIT }\end{array}$ \\
\hline $\begin{array}{l}\text { Total Alpha } \\
\text { GEA Analytes } \\
\text { Cobalt } 60 \\
\text { Cesium } 137 \\
\text { Europium } 154 \\
\text { Europium } 155 \\
\text { Anericium } 241\end{array}$ & 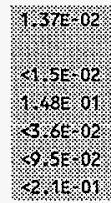 & 80 & 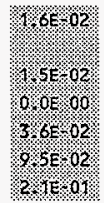 & & 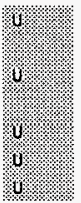 & $\begin{array}{l}\text { AT } \\
\text { GEA } \\
\text { GEA } \\
\text { GEA } \\
\text { GEA } \\
\text { GEA }\end{array}$ & 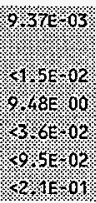 & 110 & 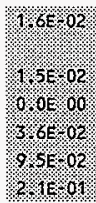 & $\begin{array}{l}\mathbf{U} \\
\mathbf{U} \\
\mathbf{U}\end{array}$ & 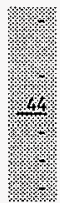 & 33 & 20 \\
\hline
\end{tabular}

\section{Group 96001380}

DUPLICATES

Page 3

SUMAARY DATA SECIION

Page 21
Lab id 222-S

Protocol SST

Version 1.0 Form DVD-DUP

Version 3.08 Report date $02 / 24 / 97$ 
HNF-SD-WM-DP-219, REV. O

222 -S LABORATORY

TANK 241-B-108, CORE 173

DUPIICATE

Client TWRS

Tank 241-B-108

Contact L. L. Fritts DUPLICATE

Lab sample id $\$ 9670054870$

Dept sample id
ORIGIMAL

Lab sample id \$96T005487

Dept sample id Received 10/17/96
Client sample id B108 C173 COMP Fusion FUSION Location/Matrix R: 3 s: Core Composite C SOLID Collected

chain of custody id $n / a$

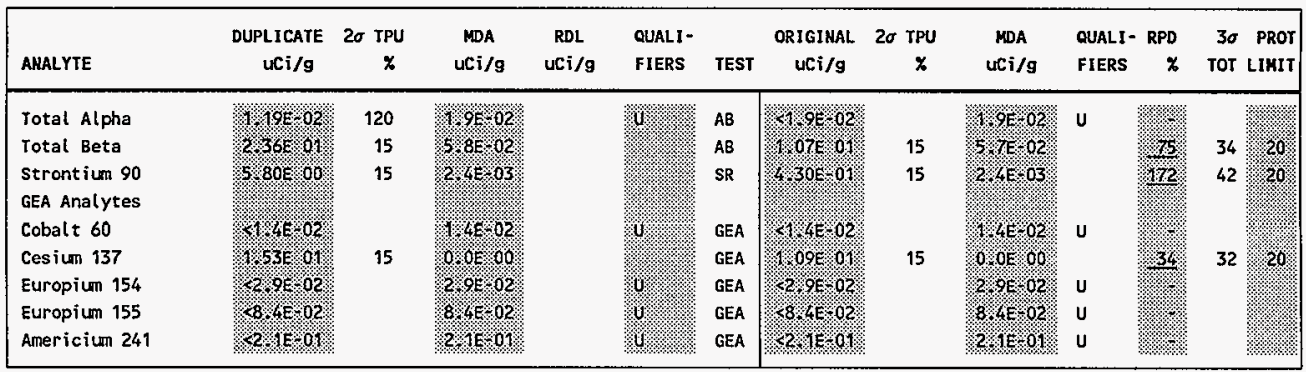

\section{Group 96001380}

DUPLICATES

Page 4

SUMMARY DATA SECIION

Page 22
Lab id 222-S

Protocol SST

Version 1.0

Form DVD-DUP

Version 3.08

Report date $\underline{02 / 24 / 97}$ 
HNF-SD-WM-DP-219, REV. 0

222 -S LABORATORY

TANK 241-B-108, CORE 173

DUPLICATE

Client TWRS

Tank $24 i-\mathrm{B}-108$

OR IGIMAL

Lab sample id $\underline{\text { s97T000001 }}$

Dept sample id Received 01/06/97
Client sample id B108 C17352 LH Fusion Re FUSION Location/Matrix R: 3 S: 2 C: 173 SOLID

Chain of custody id $n / a$

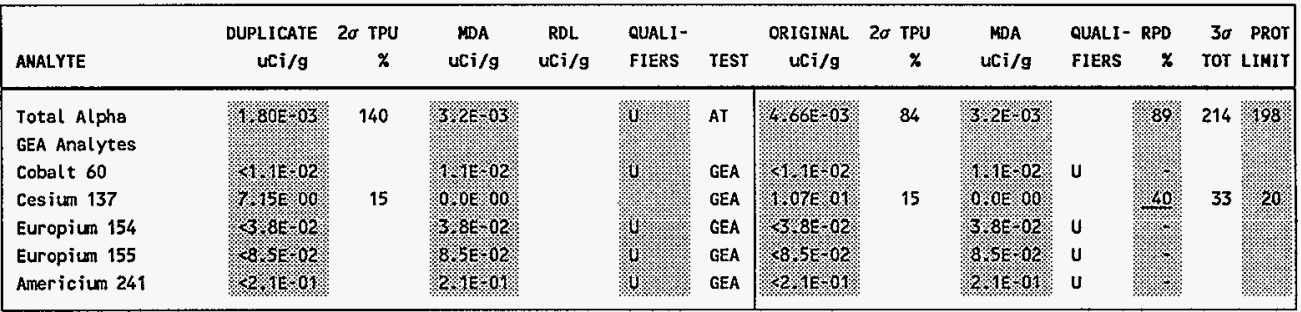

\section{Group 96001380}

DUPLICATES

Page 5

SUMMARY DATA SECTIOM

Page 23
Lab id 222-5 Protocol SST Version 1.0 Form DVD-DUP Version 3.08 Report date $02 / 24 / 97$ 
HNF-SD-WM-DP-249, REV. 0

222-S LABORATORY

TANK 241-B-108, CORE 173

DUPIICATE

SDG 96001380

Contact L. L. Fritts DUPLICATE

Lab sample id $\mathbf{5 9 7 7 0 0 0 0 0 2 D}$ Dept sample id
ORIGINAL.

Lab sample id \$97T000002. Dept sample id Received 01/06/97
Client TWRS

Tank 241-B-108

Client sample id B108 c173 Comp fusion Re FUSION Location/Matrix R: 3 S: Core Composite C SOLID Collected

Chain of custody id $\mathrm{n} / \mathrm{a}$

\begin{tabular}{|c|c|c|c|c|c|c|c|c|c|c|c|c|c|}
\hline ANALYYTE & $\begin{array}{l}\text { DUPLICATE } \\
\text { uCi/g }\end{array}$ & $\begin{array}{c}2 \sigma \mathrm{TPU} \\
\%\end{array}$ & $\begin{array}{c}\text { MDA } \\
\text { uCi/g }\end{array}$ & $\begin{array}{c}\text { RDL } \\
\mathrm{uCi} / \mathrm{g}\end{array}$ & $\begin{array}{l}\text { QUALI- } \\
\text { FIERS }\end{array}$ & TEST & $\begin{array}{c}\text { ORIGINAL } \\
\text { UCi/g }\end{array}$ & $\begin{array}{c}2 \sigma \text { IPU } \\
\%\end{array}$ & $\begin{array}{c}\text { MDA } \\
\mathrm{UCi} / \mathrm{g}\end{array}$ & $\begin{array}{l}\text { OUALI - } \\
\text { FIERS }\end{array}$ & $\begin{array}{r}\text { RPD } \\
x\end{array}$ & $\begin{array}{l}3 \sigma \\
\text { Tot }\end{array}$ & $\begin{array}{l}\text { PROT } \\
\text { LIMIT }\end{array}$ \\
\hline Total Alpha & $1 \times 7 \%=2$ & 36 & 1403 & & & $\mathbf{A B}$ & $10310 \%$ & 38 & Y & & S & 78 & 61 \\
\hline Total Beta & $2,4100 \%$ & 15 & $148 \mathrm{E} 02$ & & B & $\mathbf{A B}$ & 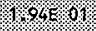 & 15 & $8 \times 8012$ & B & 22 & 32 & 20 \\
\hline Strontium $९ 0$ & 3.800 .00 & 15 & $5 \% 2=03$ & & 8 & SR & 326000 & 15 & 5.303 & B & 15 & 32 & 20 而 \\
\hline GEA Analytes & & & & & & & & & & & & & \\
\hline Cobalt 60 & 14. & & K5 $02 \%$ & & \% & GEA & 153E & & $143 \mathrm{~g} 02$ & $\mathbf{U}$ & 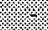 & & \\
\hline Cesium 137 & K5 201 & 15 & $000 \times 0$ & & & GEA & 1258.01 & 15 & O OE & & 19 & 32 & 20 \\
\hline Europium 154 & 3.5802 & & $8,5 \mathrm{E} 02$ & & y & GEA & $435 \mathrm{eq} 02$ & & 3.5002 & $\mathrm{U}$ & & & \\
\hline Europium 155 & 8.9602 & & $8 \% 02$ & & $\%$ & GEA & 8.9102 & & $8 \% \mathrm{E}^{\circ}$ & $\mathrm{U}$ & $\%$ & & \\
\hline Americium 241 & $2 \times 3401$ & & $203101 \%$ & & \% & GEA & 2,3601 & & $23 \% 0$ & $\mathrm{u}$ & \% & & 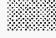 \\
\hline
\end{tabular}

Loc: Riser: 3 Seg: Core Composite Core: 173
Samp: B108 C173 Comp Fusion Reprep

Loc: Riser: 3 Seg: Core Composite Core: 173

\section{Group 96001380}

\section{DUPLICATES}

Page 6

SUMMARY DATA SECTION

Page 24
Lab id 222-s

Protocol SST

Version 1.0

Form DVD-DUP

Version $\underline{3.08}$ Report date $\underline{02 / 24 / 97}$ 
HNF-SD-WM-DP-219, REV. 0

222 -S IABORATORY

TANK 241-B-108, CORE 173

MATRIX SPIKE

SDG 96001380

Contact L.L. Fritts MATRIX SPIKE

Lab sample id $\$ 96 \mathrm{T0054775}$

Dept sample id
Dept sample id

Received $10 / 17 / 96$
Client TWRS

Tank 241-B-108

ORIGINAL

Client sample id B108 C173S1 LH Fusion Location/Matrix R: 3 S: 1 C: 173 Fusion Collected Chain of custody id n/a

\begin{tabular}{|c|c|c|c|c|c|c|c|c|c|c|c|c|}
\hline ANALYTE & $\begin{array}{l}\text { SPIKE } \\
\text { UCi/g }\end{array}$ & $\begin{array}{c}2 \sigma \text { TPU } \\
\%\end{array}$ & $\begin{array}{c}\text { MDA } \\
\mathrm{uCi} / \mathrm{g}\end{array}$ & $\begin{array}{c}\mathrm{RDL} \\
\mathrm{uCi} / \mathrm{g}\end{array}$ & $\begin{array}{l}\text { QUALI- } \\
\text { FIERS }\end{array}$ & TEST & $\begin{array}{l}\text { AODED } \\
\mathrm{uCi} / \mathrm{g}\end{array}$ & $\begin{array}{c}2 \sigma \text { ERR } \\
\%\end{array}$ & $\begin{array}{l}\text { ORIGINAL. } \\
\text { uCi/g }\end{array}$ & $\begin{array}{c}2 \sigma \mathrm{TPU} \\
\mathrm{x}\end{array}$ & $\begin{array}{r}\text { REC 3o LMTS } \\
\% \text { (TOTAL) }\end{array}$ & $\begin{array}{l}\text { PROTOCOL } \\
\text { LIMITS }\end{array}$ \\
\hline Total Beta & 968500 & & & & B & $\mathrm{TB}$ & $687 \mathrm{gal}$ & 5.0 & $283=01$ & 15 & 603 65-135 & 35425 \\
\hline
\end{tabular}

\section{Group 96001380}

MATRIX SPIKES

Page 1

SUMYARY DATA SECTION

Page 25
Lab id $222-\mathrm{s}$

Protocol SST

Version 1.0

Form DVD-MS

Version 3.08

Report date $02 / 24 / 97$ 
HNF-SD-WM-DP-219, REV. 0

\section{2 -S LABORATORY}

TAHK 241-B-108, CORE 173

MATRIX SPIRE

SDG 96001380

Contact L. L. Fritts MATRIX SPIKE

Lab sample id 596T005478S

Dept sample id
ORIGIMAL

Lab sample id $\$ 96$ T005478 Dept sample id Received $10 / 17 / 96$
Client TWRS

Tank 241-8-108

Ct ient sample id B108 C173S2 LH Fusion

Location/Matrix R: 3 S: 2 C: 173 FUSION Collected chain of custody id $n / a$

\begin{tabular}{|c|c|c|c|c|c|c|c|c|c|c|c|c|}
\hline ANALYIE & $\begin{array}{l}\text { SPIKE } \\
\text { uxi/g }\end{array}$ & $\begin{array}{c}2 \sigma \text { TPU } \\
x\end{array}$ & $\begin{array}{c}\text { MDA } \\
\text { uCi/g }\end{array}$ & $\begin{array}{c}\mathrm{RDL} \\
\mathrm{uCi} / \mathrm{g}\end{array}$ & $\begin{array}{l}\text { QUALI - } \\
\text { FIERS }\end{array}$ & TEST & $\begin{array}{l}\text { ADDED } \\
\mathrm{uCi} / \mathrm{g}\end{array}$ & $\begin{array}{c}2 \sigma \text { ERR } \\
\%\end{array}$ & $\begin{array}{c}\text { ORIGIMAL } \\
\text { uCi/g }\end{array}$ & $\begin{array}{c}2 \sigma \text { TPU } \\
x\end{array}$ & $\begin{array}{r}\text { REC } 3 \sigma \text { LHTS } \\
\% \text { (TOTAL) }\end{array}$ & $\begin{array}{l}\text { PROTOCOL } \\
\text { LIMITS }\end{array}$ \\
\hline Total Alpha & 1 736 & & 1\% & & & AT & 360001 & 5.0 & $9 \times 78 \times 03$ & 110 & 108 75-125 & 75425 \\
\hline
\end{tabular}

\section{Group 96001380}

MATRIX SPIKES

Page 2

SUMMARY DATA SECTION

Page 26
Lab id 222-s

Protocol SST

Version 1.0

Form DVD-NS

Version 3.08 Report date $\underline{02 / 24 / 97}$ 
HNF-SD-WM-DP-219, REV. 0

\section{2 -S LABORATORY}

TANK 241-B-108, CORE 173

MATRIX SPIKE

SDG 96001380

Contact L. L. Fritts MATRIX SPIKE

Lab sample id \$96T0054875

Dept sample id
ORIGINAL.

Lab sample id \$96T005487

Dept sample id Recejved 10/17/96
Client TWRS

Tank 241-B-108

Client sample id B108 C173 COMP Fusion Fusion Location/Matrix R: 3 S: Core Composite C SOLID Collected

Chain of custody id $\underline{n} / \mathrm{a}$

\begin{tabular}{|c|c|c|c|c|c|c|c|c|c|c|c|c|}
\hline ANALYTE & $\begin{array}{l}\text { SPIKE } \\
\mathrm{uCi} / \mathrm{g}\end{array}$ & $\begin{array}{c}2 \sigma \text { TPU } \\
\%\end{array}$ & $\begin{array}{c}\text { MDA } \\
\text { uCi/g }\end{array}$ & $\begin{array}{c}\text { RDL } \\
\mathrm{uCi} / \mathrm{g}\end{array}$ & $\begin{array}{l}\text { QUALI- } \\
\text { FIERS }\end{array}$ & TEST & $\begin{array}{l}\text { ADDED } \\
\mathrm{UCi} / \mathrm{g}\end{array}$ & $\begin{array}{c}2 \sigma \text { ERR } \\
\%\end{array}$ & $\begin{array}{c}\text { ORIGINAL } \\
\text { UCi/g }\end{array}$ & $\begin{array}{c}2 \sigma \text { IPU } \\
\\
\%\end{array}$ & $\begin{array}{r}\text { REC } 3 \sigma \text { LHTS } \\
* \text { (TOTAL) }\end{array}$ & $\begin{array}{l}\text { PROTOCOL } \\
\text { LIMITS }\end{array}$ \\
\hline Total Alpha & 29109 & & & & & $A B$ & Wronol & 5.0 & & & $7582-118$ & 75125 \\
\hline Total Beta & 787501 & & & & $\%$ \% & $A B$ & $733=01$ & 5.0 & $1 / 070 \times 0$ & 15 & 93 74-126 & $75 \% 25$ \\
\hline
\end{tabular}

\section{Group 96001380}

\section{MATRIX SPIKES}

Page 3

SUMHARY DATA SECTION

Page 27
Lab id 222-S

Protocol SST

Version 1.0

Form DVD-MS

Version 3.08

Report date $\underline{02 / 24 / 97}$ 


\section{2 -S LABORATORY}

IANK 241-8-108, CORE 173

MATRIX SPIKE

SDG 96001380

Contact L. L. Fritts MATRIX SPIKE

Lab sample id 5977000001s

Dept sample id
ORIGINAL

Lab sample id S97T000001 Dept sample id Received 01/06/97
Client TURS

Tank $241-8-108$

Client sample id B108 C173S2 LH Fusion Re FUSION Location/Matrix R: 3 s: 2 C: 173 SOLID collected

Chain of custody id $\mathrm{n} / \mathrm{a}$

\begin{tabular}{|c|c|c|c|c|c|c|c|c|c|c|c|c|}
\hline AKALYTE & $\begin{array}{l}\text { SPIKE } \\
\text { UEi/g }\end{array}$ & $\begin{array}{c}2 \sigma \text { TPU } \\
\\
\chi\end{array}$ & $\begin{array}{c}\text { MDA } \\
\mathrm{uCj} / \mathrm{g}\end{array}$ & $\begin{array}{c}\text { RDL } \\
\text { uCi/g }\end{array}$ & $\begin{array}{l}\text { QUALI - } \\
\text { FIERS }\end{array}$ & TEST & $\begin{array}{l}\text { ADDEO } \\
\mathrm{UCi} / \mathrm{g}\end{array}$ & $\begin{array}{c}2 \sigma \text { ERR } \\
\%\end{array}$ & $\begin{array}{l}\text { ORIGINAL } \\
\text { UCi/g }\end{array}$ & $\begin{array}{c}2 \sigma \mathrm{TPU} \\
\%\end{array}$ & $\begin{array}{r}\text { REC 3o LMTS } \\
\times \text { (TOTAL) }\end{array}$ & $\begin{array}{l}\text { PROTOCOL } \\
\text { LIHITS }\end{array}$ \\
\hline Total Alpha & $1 \% 15 \mathrm{E} 01$ & & & & & AT & 167604 & 5.0 & $606 \mathrm{E} 03$ & 84 & $6583-117$ & $25 \% 125$ \\
\hline
\end{tabular}

Group 96001380

MATRIX SPIKES

Page 4

SUMAARY DATA SECTION

Page 28
Lab id 222-s

Protocol SST

Version 1.0 Form DVD-MS

Version 3.08

Report date $02 / 24 / 97$ 


\section{2 -S LABORATORY}

TANK 241-B-108, CORE 173

MATRIX SPIRE

ORIGINAL

Lab sample id S97T000002. Dept sample id Received 01/06/97
Lab sample id $\$ 97 T 000002 S$

Dept sample id
L. L. Fritts

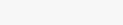

Client TWRS

Tank $241-8-108$
Ctient sample id B108 c173 Comp Fusion Re FUSION Location/Matrix R: 3 s: Core Composite C SOLID collected Chain of custody id n/a

Client $\frac{\text { TWRS }}{\text { Tank }} \frac{241-B-108}{\text { Ctient sample id B108 c173 Comp Fusion Re FUSION }}$
Location/Matrix R: 3 S: Core Composite C SOLID
Collected
Chain of custody id n/a

\begin{tabular}{|c|c|c|c|c|c|c|c|c|c|c|c|c|}
\hline ANALYTE & $\begin{array}{l}\text { SPIKE } \\
\text { uCi/g }\end{array}$ & $\begin{array}{c}2 \sigma \text { IPU } \\
\chi\end{array}$ & $\begin{array}{c}\text { MDA } \\
\text { uCi/g }\end{array}$ & $\begin{array}{c}\text { RDL } \\
\text { UCi/g }\end{array}$ & $\begin{array}{l}\text { QUALI- } \\
\text { FIERS }\end{array}$ & TEST & $\begin{array}{l}\text { ADDED } \\
\text { UCi/g }\end{array}$ & $\begin{array}{c}2 \sigma \text { ERR } \\
\chi\end{array}$ & $\begin{array}{c}\text { ORIGINAL } \\
\text { UCi/g }\end{array}$ & $\begin{array}{c}2 \sigma \text { TPU } \\
\%\end{array}$ & $\begin{array}{r}\text { REC } 3 \sigma \text { LMTS } \\
\% \text { (TOTAL) }\end{array}$ & $\begin{array}{l}\text { PROTOCOL } \\
\text { LIMITS }\end{array}$ \\
\hline $\begin{array}{l}\text { Total Alpha } \\
\text { Total Beta }\end{array}$ & 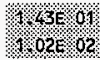 & & & & & $\begin{array}{l}A B \\
A B\end{array}$ & $\begin{array}{l}\text { W501 } \\
64401\end{array}$ & $\begin{array}{l}5.0 \\
5.0\end{array}$ & $133 \mathrm{E} 02$ & $\begin{array}{l}38 \\
15\end{array}$ & $\begin{array}{l}80-120 \\
68-132\end{array}$ & 35,125 \\
\hline
\end{tabular}

\section{Group 96001380}

MATRIX SPIKES

Page 5

SUMMARY DATA SECTION

Page 29
Lab id 222-s

Protocol SST

Version 1.0 Form DVD-MS

Version 3.08

Report date $\underline{02 / 24 / 97}$ 
HNF-SD-WM-DP-219, REV. 0

\section{$222-S$ I A B OR A T OR Y TANK 241-B-108, CORE 173}

D A T A S H E T

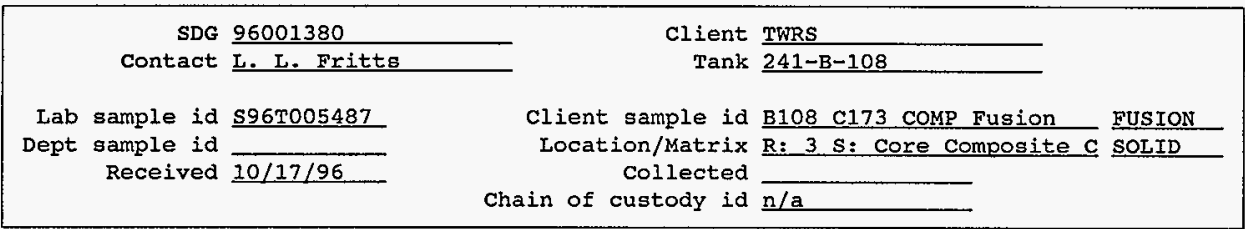

\begin{tabular}{|c|c|c|c|c|c|c|c|}
\hline ANALYTE & CAS No & $\begin{array}{r}\text { RESULT } \\
\text { uCi/g }\end{array}$ & $\underset{8}{2 \sigma} \underset{8 P U}{5}$ & $\begin{array}{c}\mathrm{MDA} \\
\mathrm{uCi} / \mathrm{g}\end{array}$ & $\begin{array}{c}\mathrm{RDL} \\
\mathrm{uCi} / \mathrm{g}\end{array}$ & $\begin{array}{l}\text { QUALI- } \\
\text { FIERS }\end{array}$ & TEST \\
\hline Total Alpha & $12587-46-1$ & $4.4 .9 \mathrm{E}=02$ & & $1.9 \mathrm{~B}-02$ & & Wu & $A B$ \\
\hline Total Beta & $12587-47-2$ & 1.078 .01 & 15 & $5=71=02$ & & & $\mathrm{AB}$ \\
\hline Strontium 90 & $10098-97-2$ & $4.30 \mathrm{~B} \quad 01$ & 15 & $2.4=-03$ & & & $\mathrm{SR}$ \\
\hline GEA Analytes & & & & & & & \\
\hline Cobalt 60 & $10198-40-0$ & $21 \times 4 \mathrm{E}=02$ & & $1 / 4 \mathrm{~B} \cdot 02$ & & u & GEA \\
\hline Cesium 137 & $10045-97-3$ & $1 / 09 \mathrm{P} \times 01$ & 15 & $0.0 \mathrm{~B}: 00$ & & & GEA \\
\hline Europium 154 & $15585-10-1$ & $2 \times 2.95=2$ & & $2.9 \mathrm{E}=02$ & & \% & GEA \\
\hline Europium 155 & $14391-16-3$ & $8.6 .4 \mathrm{~F}=02$ & & $8.44=02$ & & v & GEA \\
\hline Americium 241 & $14596-10-2$ & $12.1-1.03$ & & $2.112 \cdot 0.1$ & & 6 & GEA \\
\hline
\end{tabular}

Loc: Riser: $3 \mathrm{seg}$ : Core Composite Core: 173

G I U P 96001380

DATA SHEETS

Page 1

SUMMARY DATA SECTION

Page 30
Lab id 222-s

Protocol SST

Version 1.0 Form DVD-DS

Version 3.08 Report date $02 / 24 / 97$ 
$222-S$ I A B O R A T O R Y TANK 241-B-108, CORE 173

D A T A SH E T
B108. C173 comp Ris ton fre.

\section{SDG 95001380}

Contact L. L. Fritts

Lab sample id s97T000002

Dept sample id

Received $01 / 06 / 97$
Client TWRS

Tank 241-B-108

Client sample id B108 C173 Comp Fusion Re FUSION

Location/Matrix R: 3 s: Core Composite C SOLID Collected

Chain of custody id $\mathrm{n} / \mathrm{a}$

\begin{tabular}{|c|c|c|c|c|c|c|c|}
\hline ANALYTE & CAS NO & $\begin{array}{r}\text { RESULT } \\
\mathrm{uCi} / \mathrm{g}\end{array}$ & $\begin{array}{c}2 \sigma \text { TPU } \\
8\end{array}$ & $\begin{array}{c}\text { MDA } \\
\text { uCi/g }\end{array}$ & $\begin{array}{c}\mathrm{RDL} \\
\mathrm{uCi} / \mathrm{g}\end{array}$ & $\begin{array}{l}\text { QUALI- } \\
\text { FIERS }\end{array}$ & TEST \\
\hline Total Alpha & $12587-46-1$ & $1.33 \mathrm{~B} / 02$. & 38 & $1 / 1 \mathrm{E}-03$ & & \% & $A B$ \\
\hline Total Beta & $12587-47-2$ & $3.94 \mathrm{t} .01$ & 15 & $1.8 \mathrm{E}=02$ & & B & $A B$ \\
\hline strontium 90 & $10098-97-2$ & $3.65 \mathrm{~B} \times 00$. & 15 & $5.3 \mathrm{E}=03$ & & 数 & SR \\
\hline GEA Analytes & & ?: & & & & & \\
\hline Cobalt 60 & $10198-40-0$ & $4 / 1,3 \mathrm{~B}=02$ : & & $1 / 3 \mathrm{E}-02 \%$ & & 0. & GEA \\
\hline Cesium 137 & $10045-97-3$ & $1.25 \mathrm{~B} \cdot 0$. & 15 & $0.00 \%$ & & & GEA \\
\hline Europium 154 & $15585-10-1$ & $\times 3 / 5 \mathrm{~F}=02$ & & $3.5 \mathrm{~F}-02$. & & 紧 & GEA \\
\hline Europium 155 & $14391-16-3$ & 18. 98.02 & & $8 \cdot 95-02$ & & : & GEA \\
\hline Americium 241 & $14596-10-2$ & $3 \times 2 \times 1+01$ & & $2.3 \mathrm{~B}-01$ & & $10 \%$ & GEA \\
\hline
\end{tabular}

Samp: B108 C173 Comp Fusion Reprep

Loc: Riser: 3 seg: Core Composite core: 173

G I U P 96001380

DATA SHEETS

Page 2

SUMMARY DATA SECTION

Page 31
Lab id 222-s

Protocol SST

Version 1.0

Form DVD-DS

Version 3.08

Report date $02 / 24 / 97$ 
$222-$ S I A B O R A T O R Y TANR 241-B-108, CORE 173

SDG 96001380

Contact L. L. Fritts

Lab sample id $\mathbf{5 9 6 T 0 0 5 4 7 7}$

Dept sample id

Received $10 / 17 / 96$
Client TWRS

Tank 241-B-108

Client sample id B108 C173S1 LH Fusion

Location/Matrix R: 3 S: 1 C: 173 Collected

Chain of custody id $n / a$
FUSION

SOLID

\begin{tabular}{|c|c|c|c|c|c|c|c|}
\hline ANALYTE & CAS NO & $\begin{array}{r}\text { RESULT } \\
\mathrm{uCi} / \mathrm{g}\end{array}$ & $\underset{\frac{8}{8}}{2 \sigma}$ & $\begin{array}{c}\text { MDA } \\
\mathrm{uCi} / \mathrm{g}\end{array}$ & $\begin{array}{c}\text { RDL } \\
\mathrm{uCi} / \mathrm{g}\end{array}$ & $\begin{array}{l}\text { QUALI- } \\
\text { FIERS }\end{array}$ & TEST \\
\hline Total Alpha & $12587-46-1$ & $108 \mathrm{E}-02$ & 62 & W $8 \mathrm{E}=03$ & & $3 x$ & AT \\
\hline Total Beta & $12587-47-2$ & $2 \times 83 \mathrm{~B} \times 0.1$ & 15 & 1. $6 \mathrm{E}$ & & B. & TB \\
\hline Strontium 90 & $10098-97-2$ & $4=1 \% \mathrm{~B}=00$ & 15 & $3.3=03$ & & B. & $\mathbf{S R}$ \\
\hline GEA Analytes & & & & & & & \\
\hline Cobalt 60 & $10198-40-0$ & \% $1,3 \mathrm{~B}-02$ & & $1 / 3 \mathrm{P}-02$. & & $6 \%$ & GEA \\
\hline Cesium 137 & $10045-97-3$ & $1 \times 14 \mathrm{~b}$ 和 & 15 & $1 \% 0 \mathrm{E}$ & & m. & GEA \\
\hline Buropium 154 & $15585-10-1$ & 3. 3.7. 1.02 & & $3.7 \mathrm{E}-02$ & & 3 & GEA \\
\hline Europium 155 & $14391-16-3$ & $4+2 \mathrm{~F}=01$ & & $1.28=01$ & & jo. & GEA \\
\hline Americium 241 & $14596-10-2$ & $1 / 2.6 \mathrm{~F}: 0$ & & $226 \mathrm{p}=01$ : & & 10 & GEA \\
\hline
\end{tabular}

G I O u p 96001380

\section{DATA SHEETS}

Page 3

SUMMARY DATA SECTION

Page 32
Lab id 222-S

Protocol SST

Version 1.0

Form DVD-DS

Version 3.08

Report date $\underline{02 / 24 / 97}$ 
WNF-SD-WM-DP-219, REV. 0

\section{2 - S LABORATORY}

TANR 241-B-108, CORE 173

DA T A S H E T

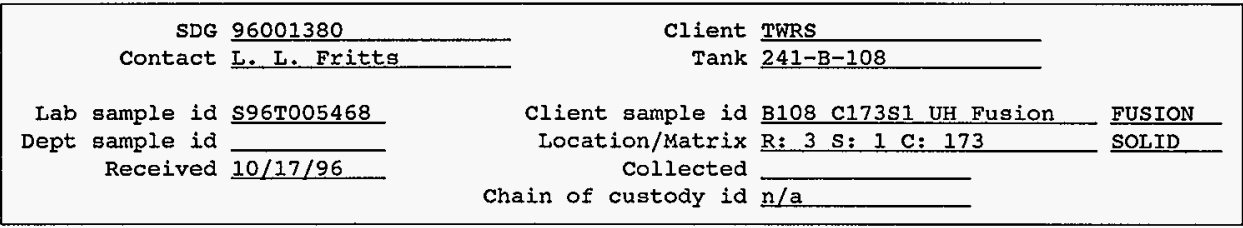

\begin{tabular}{|c|c|c|c|c|c|c|c|}
\hline ANALYTE & CAS No & $\begin{array}{r}\text { RESULT } \\
\mathrm{uCi} / \mathrm{g}\end{array}$ & $\begin{array}{c}20 \text { TPU } \\
8\end{array}$ & $\begin{array}{c}\mathrm{MDA} \\
\mathrm{uCi} / \mathrm{g}\end{array}$ & $\begin{array}{c}\mathrm{RDL} \\
\mathrm{uC} \dot{\mathrm{L}} / \mathrm{g}\end{array}$ & $\begin{array}{l}\text { QUALI- } \\
\text { FIERS }\end{array}$ & TEST \\
\hline $\begin{array}{l}\text { GEA Analytes } \\
\text { Cobalt } 60\end{array}$ & $10198-40-0$ & 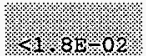 & & 1/. $8 \mathrm{~B}=02$ & & ux & GEA \\
\hline Cesium 137 & $10045-97-3$ & $2.30 \mathrm{~F} .01 \%$ & 15 & $040 \mathrm{~F}=00$ & & & GEA \\
\hline Europium 154 & $15585-10-1$ & $5 \% 9=02$. & & $5.9=02$ & & JU & GEA \\
\hline Europium 155 & $14391-16-3$ & $11 \% 5 \mathrm{E}, 01$ & & $1.5 \mathrm{E}-01$ & & U & GEA \\
\hline Americium 241 & $14596-10-2$ & $302 \mathrm{D}-01$. & & $3 \times 2 \mathrm{E}=01$ & & $1 \%$ & GEA \\
\hline
\end{tabular}

GIOUP 96001380

DATA SHEETS

Page 4

SULMARY DATA SECTION

Page 33
Lab id 222-S

Protocol SST

Version 1.0

Form DVD-DS

Version 3.08

Report date $02 / 24 / 97$ 


\section{$222-S$ I A B ORATOR Y} TANK 241-B-108, CORE 173

\section{A T A $\mathrm{S}$ H E E T}

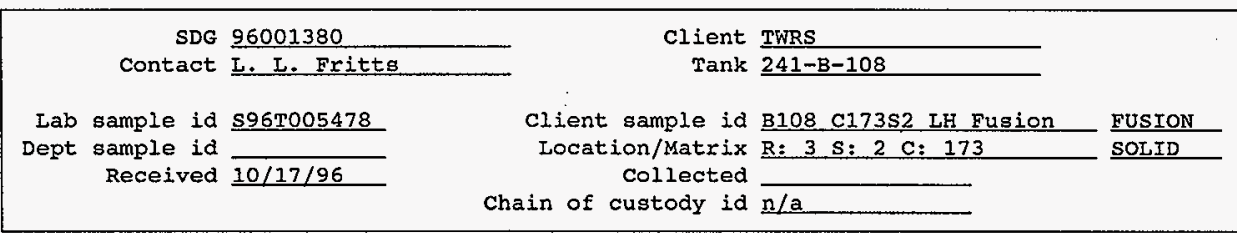

\begin{tabular}{|c|c|c|c|c|c|c|c|}
\hline ANALYTE & CAS NO & $\begin{array}{r}\text { RESULT } \\
\mathrm{uCi} / \mathrm{g}\end{array}$ & $\begin{array}{c}2 \sigma \text { TPU } \\
\&\end{array}$ & $\begin{array}{c}\mathrm{MDA} \\
\mathrm{uCi} / \mathrm{g}\end{array}$ & $\begin{array}{c}\text { RDL } \\
\mathrm{uCi} / \mathrm{g}\end{array}$ & $\begin{array}{l}\text { QUALI- } \\
\text { FIERS }\end{array}$ & TEST \\
\hline $\begin{array}{l}\text { Total Alpha } \\
\text { GEA Analytes }\end{array}$ & $12587-46-1$ & $9.37 \%$ & 110 & $1+6 \mathrm{P}=02$ & & (y) & $\mathrm{AT}$ \\
\hline Cobalt 60 & $10198-40-0$ & $1.5 \mathrm{~B}=02$ & & $1 / 5 \mathrm{~F}-02$, & & 0 & GEA \\
\hline Cesium 137 & $10045-97-3$ & $9.48 \mathrm{H}: 00$ & 15 & $0.0 \mathrm{O} 0.0$. & & & GEA \\
\hline Europium 154 & $15585-10-1$ & $4.68=02$ & & $3.6 \mathrm{~B}-02$. & & 6 & GEA \\
\hline Europium 155 & $14391-16-3$ & $69.5 \mathrm{p}=02$ & & $9.5 \mathrm{~B}-02$ & & xu & GEA \\
\hline Americium 241 & $14596-10-2$ & $22<15-08$ & & $2 \cdot 1=6.01$ & & 椾 & $G E A$ \\
\hline
\end{tabular}

G I U p 96001380

DATA SHEETS

$$
\text { Page } 5
$$

SUMMARY DATA SECTION

Page 34
Lab id 222-s

Protocol SST

Version 1.0

Form DVD-DS

Version 3.08

Report date $\underline{02 / 24 / 97}$ 
HNF-SD-WM-DP-219, REV.0

$222-S$ LA B O R A T OR Y TANK 241-B-108, CORE 173

D A A S E E T

SDG 96001380

Contact L. L. Fritts

Lab sample id $\mathbf{5 9 7 T 0 0 0 0 0 1}$

Dept sample id Received $01 / 06 / 97$
Client TWRS

Tank 241-B-108

Client sample id B108 C173S2 LH Fusion Re FUSION

Location/Matrix R: 3 s: 2 C: 173 Collected

Chain of custody id $\mathrm{n} / \mathrm{a}$

\begin{tabular}{|c|c|c|c|c|c|c|c|}
\hline ANALYTE & CAS NO & $\begin{array}{r}\text { RESULT } \\
\text { uCi/g }\end{array}$ & $\begin{array}{c}2 \sigma \text { TPU } \\
8\end{array}$ & $\begin{array}{c}\mathrm{MDA} \\
\mathrm{uCi} / \mathrm{g}\end{array}$ & $\begin{array}{c}\mathrm{RDL} \\
\mathrm{uCi} / \mathrm{g}\end{array}$ & $\begin{array}{l}\text { QUALI- } \\
\text { FIERS }\end{array}$ & $\operatorname{Tes} T$ \\
\hline $\begin{array}{l}\text { Total Alpha } \\
\text { GEA Analytes }\end{array}$ & $12587-46-1$ & $466 \mathrm{~F} \% \mathrm{O}$ & 84 & $\sqrt[3]{3.29} \times 03$ & & & AT \\
\hline Cobalt 60 & $10198-40-0$ & $1 / 2.18 .02$ & & $1.1=02$ & & 0. & GEA \\
\hline Cesium 137 & $10045-97-3$ & $1.098 \% 01$. & 15 & 0.0100. & & & GEA \\
\hline Europium 154 & $15585-10-1$ & $4.3 .8 \mathrm{~B}=02$ & & $3.8 \mathrm{E}-02$ & & $\%$ & GEA \\
\hline Buropium 155 & $14391-16-3$ & $18.6 \mathrm{E}=02$. & & $8.5 \mathrm{~F}=02 \%$ & & 10 & GEA \\
\hline Americium 241 & $14596-10-2$ & $42,11,01$ & & $2.1 \mathrm{~F}-01$ & & $\mathrm{U}$ & GEA \\
\hline
\end{tabular}

Samp: B108 C173S2 LH Fusion Reprep

G I U P 96001380

DATA SHEETS

Page 6

SUMMARY DATA SECTION

Page 35
Lab id 222-s

Protocol SST

Version 1.0 Form DVD-DS

Version 3.08

Report date $\underline{02 / 24 / 97}$ 
HNF-SD-WM-DP-219, REV. 0

\section{2 -S IABORATORY}

TANK 241-8-108, CORE 173

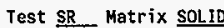
SDG 96001380

Contact L. L. Fritts
METHOD SUMMARY

STRONTIUM-89/90

GAS PROPORTIONAL COUNTING
Client TWRS

Tank 241-B-108

\section{RES ULTS \\ CLIENT SAMPLE ID}

LAB RAM SUF-

SAMPLE ID TEST FIX PLANCHET Strontiun 90

Preparation batch 96012589

B108 C173 coMP Fusion fu 5961005487

B108 C173 COMP Fusion-DU 59670054870

Method Blank

B15952-2

Lab Control Sample

515952-1

$\begin{array}{lll}02 & 15952-4 & \text { Vof } \\ 02 & 15952-5 & 001 \\ 15952-2 & \text { \% } \\ & 15952-1 & \end{array}$

Preparation batch 96012650

B108 C173S1 LH Fusion FU S96T0054T7

B108 C17351 LH Fusion-DU 596T0054770

Method Btank

B16017-2

Lab Control Sample

si6017-1

$16017-4$

$16017-5$

$16017-2$

$16017-1$

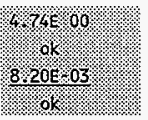

Preparation batch 97000108

B108 c173 Comp Fusion Re s97T000002

B108 c173 comp fusion Re $\$ 9710000020$

Method Blank

B16256-2

Lab Control Sample

$\mathbf{s} 16256-1$

\begin{tabular}{|c|c|}
\hline $16256-4$ & $3 \times 26100$ \\
\hline $16256-5$ & \%k \\
\hline $16256-2$ & $1,7,2002$ \\
\hline $16256-1$ & \% \\
\hline
\end{tabular}

Noninat values and linits from method

RD1.s (UCi/g)

\section{Group 96001380}

METHOD SUMMARIES

Page 1

SUMAARY DATA SECTION

Page 36

Lab id 222-s

Protocol SST

Version 1.0

Form DVD-CMS

Version 3.08

Report date $02 / 24 / 97$ 
HNF-SD-WM-DP-219, REV. 0

\section{2-S LABORATORY}

TANK 241-B-108, CORE 173

\author{
METHOD SUMMARY \\ STRONTIUM-89/90 \\ GAS PROPORTIONAL COUNTING
}

Cl ient TWRS

Tank 241-B-108

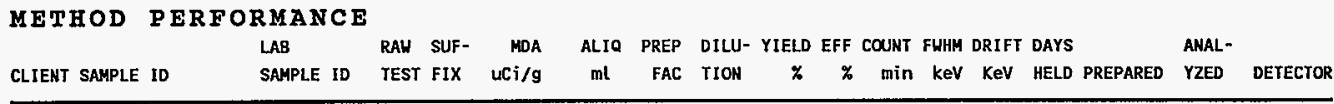

Preparation batch $96012589 \quad 2 \sigma$ prep error $15.0 \%$ Reference

\section{2 $15.0 \%$ Reference} B108 c173 comp fusion Fu S96T005487

B108 C173 CONP Fusion-DU S96T0054870

Method Blank

B15952-2

Lab Control Sample

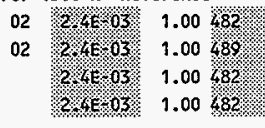

\begin{tabular}{|c|c|}
\hline 1.00 & 90 \\
\hline 1.00 & 90 \\
\hline 1.00 & 89 \\
\hline 1.00 & 89 \\
\hline
\end{tabular}

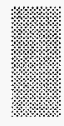

$10 / 26 / 96 \quad 01 / 06$ WB2687010

$10 / 26 / 96 \quad 01 / 06$ WB2687010 $01 / 06 / 97$ WB2687010 $01 / 06 / 97$ WB2687010
Preparation batch $960126502 \sigma$ prep error $15.0 \%$ Reference B108 c173s1 LH Fusion fu S96T005477 B108 C173\$1 LH Fusion-DU \$96T0054770 Method Blank

B $16017-2$

Lab Control Sample

\$16017-1

1.00
1.00
$1.00 \% 60 \%$
$1.00 \% 61 \%$

$10 / 26 / 96 \quad 01 / 07$ WB2687010

$10 / 26 / 96 \quad 01 / 07$ WB2687010

$01 / 07 / 97$ WB2687010 $01 / 07 / 97$ WB2687010

Preparation batch $97000108 \quad 2 \sigma$ prep error $15.0 \times$ Reference

B108 c 173 Comp fusion Re $\$ 977000002$

B108 C173 Comp Fusion Re S97T000002D

Method Blank

B16256-2

Lab Control Sample

s16256-1

\begin{tabular}{|c|c|}
\hline $5 x^{3} \mathrm{E}^{2} 03$ & 0.250493 \\
\hline $5 \times 2203$ & 0.250492 \\
\hline 5 S. 03 & 0.250493 \\
\hline $1 \% 3203$ & 1.00493 \\
\hline
\end{tabular}

$\begin{array}{ll}1.00 \\ 1.00 \% 90 \% \\ 1.00 \% 92 \\ 1.00 & 42 \\ 91 \% & 42\end{array}$

$01 / 15 / 97 \quad 09 / 22$ WB2781112 $01 / 15 / 97 \quad 01 / 22$ WB2781112 $01 / 22 / 97$ WB2781112 $01 / 22 / 97$ WB2781112

Nominal values and $L$ imits from method

0.100

30-105

10

$20-55$

PROCEDURES REFERENCE 222-s Lab Analytical Procedure

L0-160-103 Core Segment Extrusion Process and Sample Preparation, rev 17

LA-549-141 Fusion with Alkali Metal Hydroxide, rev 40

LA-220-101 High level strontium $89 / 90$ in aqueous samples, rev 41

LA-508-11NB Operation of the [Tennelec LB-5500 ( $n=0, A-5)$, LB-1000 ( $n=1, A-3)$, Gama Products $(n=4, A-2)]$ Alpha/Beta Counting Systems
AVERAGES \pm 2 SD

FOR 12 SAMPLES
MDA $3.3 \mathrm{E}-03 \pm 2.9 \mathrm{E}-03$

YIELD $86 \pm 18$

EFFICIENCY 41 _.

\section{Group 96001380}

\section{METHOD SUMHARIES}

Page 2

SLAMARY DATA SECTION

Page 37
Lab id $222 \cdot 5$

Protocol SST

Version 1.0

Form DVD-CMS

Version 3.08

Report date $02 / 24 / 97$ 
HNF-SD-WM-DP-219, REV.O

\section{$222-S$ IABORATORY}

TANK 241-8-108, CORE 173

Test $\mathrm{AB}$ Matrix SOLID

SDG 96001380

Contact L. L. Fritts

\section{METHOD SUMMARY}

ALPHA ANALYSIS

GAS PROPORTIONAL COUNTIHG
Client TWRS

Tank 241-B-108

\section{RESUITS}

CLIENT SAMPLE ID

LAB RAW SUF-

1: Total

2: Total

3: Sum, Alpha 4: Sum, Beta RESULT RATIOS (\%)

SAMPLE ID TEST FIX PLANCHET Alpha

Beta

Emitters

Emitters $\quad 3 \div 1 \quad 2 \sigma \quad 4 \div 2 \quad 2 \sigma$

\section{Preparation batch 96011318}

B108 C173 COMP Fusion FU S96T005487

B108 C173 COAP Fusion-DU S96T005487D

B108 C173 COMP fusion-SP 59670054875

Method Blank

B14725-2

Lab control Sample

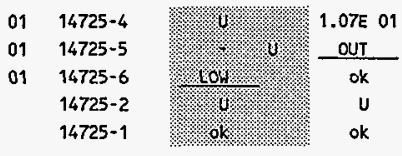

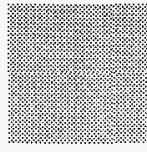

$1.09 E 01$

$1.53 \mathrm{E} 01$

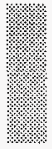

102

65

21

14
Preparation batch 97000273

B108 c173 Comp Fusion Re s97T000002

B108 c173 Comp fusion Re 59710000020

B108 c173 Comp fusion Re s97t000002s

Method Blank

B16409-2

Lab Control Sample

s16409-1
$01 \quad 16409-4$

01 16409-5

01 16409-6

16409-2

16409-1

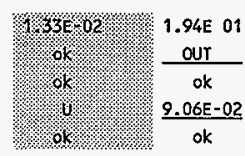

$1.25 E 01$

$1.52 E 01$

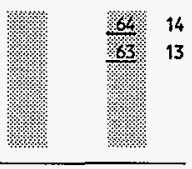

80

\section{Group 96001380}

METHOO SUMMARIES

page 3

SUMALAY DATA SECTION

Page 38
Lab id 222-s

Protocol SST

Version 1.0

Form DVD-CMS

Version 3.08

Report date $02 / 24 / 97$ 
HNF-SD-WM-DP-219, REV. 0

\section{2 -S IABORATORY}

TANK 241-B-108, CORE 173

Test $\underline{A B}$ _ Matrix SOLID

SDG 96001380

Contact L. L. Fritts

\section{METHOD SUMMARY \\ ALPHA ANALYSIS \\ GAS PROPORTIONAL COUHTING}

Client TWRS

Tank 241-B-108

\begin{tabular}{|c|c|c|c|c|c|c|c|c|c|c|c|c|c|c|c|c|}
\hline LETHOD PERFO & $\begin{array}{l}\text { RMANCE } \\
\text { LAB } \\
\text { SAMPLE ID }\end{array}$ & $\begin{array}{l}\text { RAU } \\
\text { TEST }\end{array}$ & $\begin{array}{l}\text { SUF- } \\
\text { FIX }\end{array}$ & $\begin{array}{l}\text { MAX MDA } \\
u C i / g\end{array}$ & $\begin{array}{c}\text { ALIO } \\
m L\end{array}$ & $\begin{array}{r}\text { PREP } \\
\text { FAC }\end{array}$ & $\begin{array}{l}\text { DILU- } \\
\text { TION }\end{array}$ & $\begin{array}{c}\text { RESID } \\
\text { mg }\end{array}$ & $\begin{array}{r}\text { EFF } \\
\%\end{array}$ & $\begin{array}{r}\text { COUHT } \\
\text { min }\end{array}$ & $\begin{array}{r}\text { FUHM } \\
\text { keV }\end{array}$ & $\begin{array}{l}\text { DRIFT } \\
\mathrm{KeV}\end{array}$ & $\begin{array}{l}\text { DAYS } \\
\text { HELD }\end{array}$ & PREPARED & $\begin{array}{l}\text { ANAL- } \\
\text { YZED }\end{array}$ & DETECTOR \\
\hline Preparation batch 9601131 & $2 \sigma$ pre & ep er & ror 1 & $5.0 \% \operatorname{Re}$ & ference & & & & & & & & & & & \\
\hline B108 C173 COMP Fusion FU & s96T005487 & & 01 & 5,2102 & 0.250 & 482 & 11.0 & & 45 & 30 & & & & $10 / 26 / 96$ & $11 / 08$ & WB27807 \\
\hline 8108 C173 COMP Fusion-DU & $596 \mathrm{~T} 0054870$ & & 01 & $58 \mathrm{E} 02$ & 0.250 & 389 & 11.0 & & 45 & 30 & & & & $10 / 26 / 96$ & $11 / 08$ & WB27807 \\
\hline 8108 c173 COMP Fusion-SP & $5967005487 \mathrm{~s}$ & & 01 & & 0.250 & 482 & 1.00 & & 45 & $30 \%$ & & & & $10 / 26 / 96$ & $11 / 08$ & UB27807 \\
\hline Method Blank & B14725-2 & & & 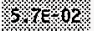 & 0.250 & 48 & 11.0 & & 45 & 30 & & & & \multicolumn{2}{|c|}{$11 / 08 / 96$} & $\mathrm{WB27807}$ \\
\hline Lab Control Sample & $\$ 14725-1$ & & & 103103 & 1.00 & 482 & 1.00 & k & 45 & $30 \%$ & & & & \multicolumn{2}{|c|}{$11 / 08 / 96$} & WB27807 \\
\hline
\end{tabular}

Preparation batch $97000273 \quad 2 \sigma$ prep error $15.0 \%$ Reference 8108 C173 Comp Fusion Re 5971000002 B108 c173 Comp Fusion Re $597 T 0000020$ B108 c173 Comp Fusion Re $\$ 9710000025$ Method Blank B16409-2

Lab Control Sample s16409-1

\begin{tabular}{|c|c|c|}
\hline 01 & (x) 802 & 0.100493 \\
\hline & 1.81 .02 & $0.100 \mathrm{Kg2}$ \\
\hline & & 0.100493 \\
\hline & $8 \mathrm{E}, 02$ & 0.100493 \\
\hline & K8E 0 & 1.00 \\
\hline
\end{tabular}

$1.00 \%$
1.00
1.00
1.00
$1.00 \%$

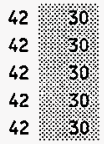

$01 / 15 / 97 \quad 01 / 29 \quad$ WB27806 $01 / 15 / 97 \quad 01 / 29 \quad$ WB27806 $01 / 15 / 97 \quad 01 / 29 \quad$ WB27806 $01 / 29 / 97$ W827806 $01 / 29 / 97$ WB27806
PROCEDURES REFERENCE 222-S Lab Analytical Procedure

L0-160-103 Core Segment Extrusion Process and Sample Preparation, rev 17

LA-549-149 Fusion with Alkali Metal Hydroxide, rev 40

LA-508-101 Alpha/Beta in liquid samples, rev 42

LA-508-11NA Operation of the [Tennelec LB-5500 ( $n=0, A-5)$, L.B-1000 ( $n=1, A-3)$, Gama Products $(n=4, A-2)]$ Alpha/Beta Counting Systems
AVERAGES \pm 2 SD

MDA $\underline{2.9 E-02} \pm \underline{4.9 E-02}$ FOR 10 SAMPLES

EFFICIENCY $44 \pm \frac{3.2}{3.2}$

\section{Group 96001380}

METHOD SUMMARIES

Page 4

SUMMARY DATA SECTION

Page 39
Lab id 222-s

Protocol SST

Version 1.0

Form DVD-CMS

Version 3.08

Report date $\underline{02 / 24 / 97}$ 
HNF-SD-WM-DP-219, REV.O

\section{2-S IABORATORY}

Test AT Matrix SOLID

SDG 96001380

Contact L.L. Fritts
TANK 241-B-108, CORE i73

METHOD SUMMARY

ALPHA ANALYSIS

GAS PROPORTIONAL COUNTIHG client TWRS

Tank 241-B-108

\section{RESULTS}

CLIENT SAMLE ID

LAB RAH SUF-

1: Total 2: Sum, Alpha

RESULT RATIO (\%)

SAMPLE ID TEST FIX PLANCHET

Alpha

Emitters

$2 \div 1 \quad 2 \sigma$

Preparation batch 96010903

B108 C173s! LH Fusion FU \$967005477

B108 C173S1 LH Fusion-DU S96T0054770

B108 Ci73\$2 LH Fusion FU $\$ 967005478$

B108 ci73s2 LH Fusion-DU 596T005478D

B108 C17352 LH Fusion-SP \$96T005478S

Method Blank

B14361-2

Lab Control Sample

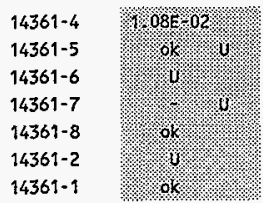

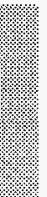

Preparation batch 97000208

B108 C173s2 LH Fusion Re $\$ 97 T 000001$

B108 C173S2 LH Fusion Re \$97T000001D

B108 C173S2 LH Fusion Re \$97T000001S

Method Blank

B16349-2

Lab Control Sample

s16349-1

$\begin{array}{ll}01 & 16349-4 \\ 01 & 16349-5 \\ 01 & 16349-6 \\ & 16349-2 \\ & 16349-1\end{array}$

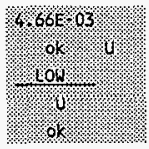

RDLs (UCi/g)

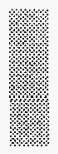

80

\section{Group 96001380}

\section{METHOO SUMMARIES}

Page 5

SUMAARY DATA SECTION

Page 40
Lab id 222-s

Protocol SST

version 1.0

Form DVD-CHS

version 3.08

Report date $\underline{02 / 24 / 97}$ 
HNF-SD-WM-DP-219, REV. 0

\section{2 -S LABORATORY}

TANK 241-B-108, CORE 173

\author{
METHOD SUMMARY \\ ALPHA aNALYSIS \\ GAS PROPORTIONAL COUNTING
}

Client TWRS

Tank $241-8-108$

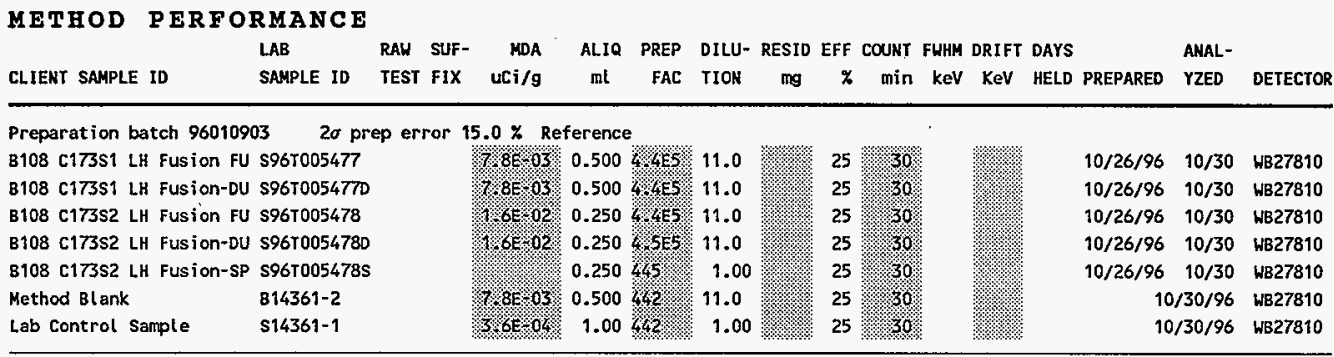

\section{Preparation batch $970002082 \sigma$ prep error $15.0 \%$ Reference} B108 c173s2 LH Fusion Re 597T000001 B108 C173S2 LH Fusion Re S97T000001D B108 c173s2 LH Fusion Re $\$ 97$ T000001S Method Blank B16349-2

Lab Control Sample \$16349-1

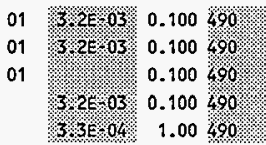

1.00
1.00
1.00
1.00
1.00

27
27
27
27
27

$01 / 15 / 97 \quad 01 / 26 \quad$ WB26872

$01 / 15 / 97 \quad 01 / 26 \quad W B 26872$

$01 / 15 / 97 \quad 01 / 26 \quad$ WB26872

$01 / 26 / 97$ WB26872 $01 / 26 / 97$ WB26872

Noninal values and limits from method

0.100

30

$20-55$

PROCEDURES REFERENCE 222-s Lab Analytical Procedure

10-160-103 Core Segment Extrusion Process and Sample Preparation, rev 17

LA-549-141 Fusion with Alkali Metal Hydroxide, rev 40 LA-508-101A Alpha in liquid samples, rev 42

LA-508-11NA Operation of the ITennelec $L B-5500(n=0, A-5)$, LB-1000 ( $n=1, A-3)$, Gamma Products $(n=4, A-2)]$ Alpha/Beta Counting Systems
AVERAGES \pm 2 SD

MDA $\underline{6.6 E-03} \pm \underline{1.1 E-02}$

FOR 12 SAMPLES

\section{Group 96001380}

METHOO SUMMARIES

Page 6

SUMMARY DATA SECTION

Page 41
Lab id 222-s

Protocol SST

Version 1.0

Form DVD-CMS

Version 3.08 Report date $02 / 24 / 97$ 
HNF-SD-WM-DP-219, REV.0

\section{2-S IABORATORY}

TANK 241-B-108, CORE 173

Test $\underline{\text { IB }}$ Matrix SOLID

SDG 96001380

Contact L. L. Fritts

\section{METHOD SUMMARY \\ BETA ANALYSIS \\ GAS PROPORTIONAL COUNTING}

Client TWRS

Tank 241-B-108

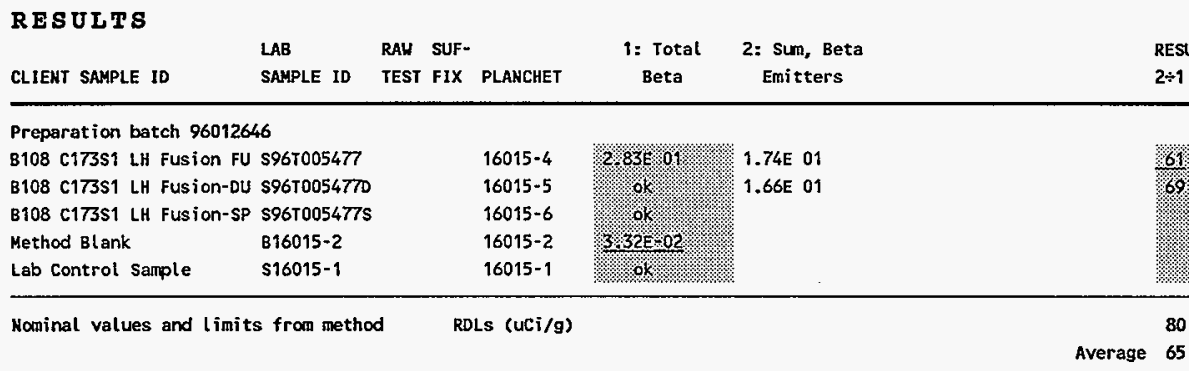

\section{METHOD PERFORMANCE}

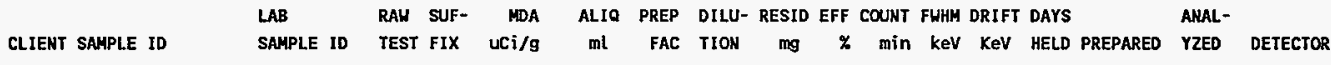

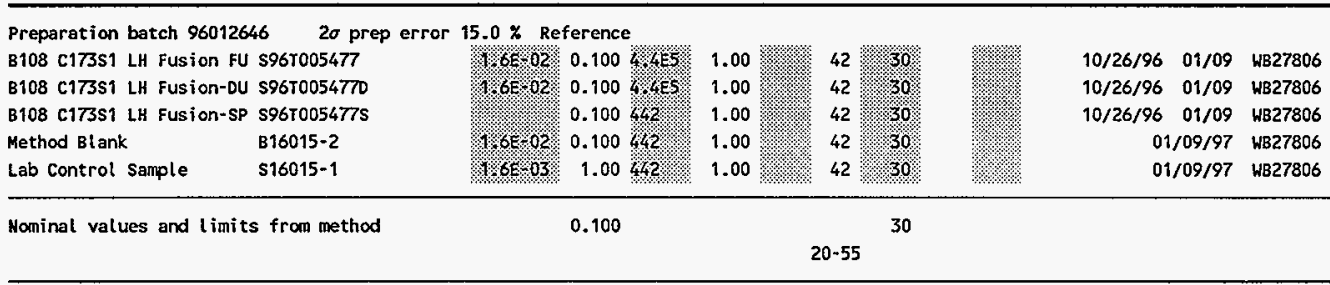

PROCEDURES REFERENCE 222-S Lab Analytical Procedure

L0-160-103 Core Segment Extrusion Process and Sample

Preparation, rev 17

LA-549-141 Fusion with Alkali Metal Hydroxide, rev 40

LA-508-101B Beta in liquid samples, rev 42

LA-508-11NB Operation of the [Tennelec LB-5500 ( $n=0, A-5)$, LB-1000 (n=1, A-3), Gamma Products $(n=4, A-2)]$

Alpha/8eta Counting Systems
AVERAGES \pm 2 SD

MDA $1.2 E-02 \pm 1.4 E-02$

FOR 5 SAMPLES
13

15

80 
HASF-SD-WR-DP-219, REV. O

\section{2-S LABORATORY}

TANK 241-B-108, CORE 173

Test GEA Matrix SOLID SDG 96001380

Contact L. L. Fritts
METHOD SUMMARY

GAMMA SPECTROSCOPY

GAMMA ENERGY ANALYSIS
Client TWRS

Tank 241-B-108

\section{RESULTS}

CLIENT SAMPLE ID

LAB RAU SUF-

SAMPLE ID TEST FIX PLANCHET Cobalt 60 Cesium 137 Europium 154 Europium 155

Anericium

241
Preparation batch 96012029

B108 1173 COMP Fusion FU S96T005487

B108 C173 COMP Fusion-DU 596T0054870

Method Blank

Lab Control Sample
815416-2

s15416-1

$01 \begin{array}{r}15416-3 \\ 15416-4 \\ 15416-2 \\ 15416-1\end{array}$

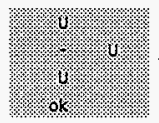

$1.09 E 01$

$\frac{\text { QUT }}{\mathrm{U}}$

ok

1.74E 01

16019-5

16019-6

16019-3

16019-4

16019-7

16019-8

16019-2

16019-1

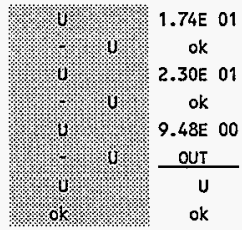

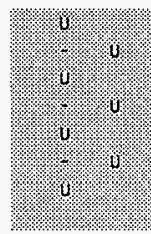

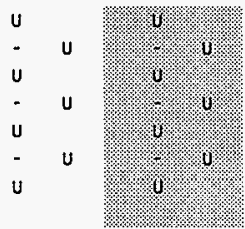

Preparation batch 97000110

B108 C173 Comp Fusion Re $\$ 977000002$

B108 C173 Comp fusion Re $\$ 9770000020$

Method Blank

B16258-2

Lab Control sample

s16258-1
16258-5

16258-6

16258-2

16258-1

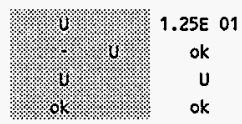

ok
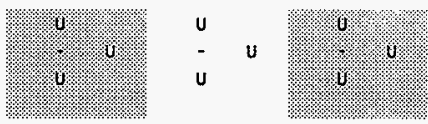

Preparation batch 97000227

B108 C173s2 LH Fusion Re S97T000001

B108 C173s2 L.H fusion Re S97T000001D

Method Blank

B16362-2

Lab Control Sample

s16362-1

$01 \quad \begin{array}{r}16362-3 \\ 16362-4 \\ 16362-2 \\ 16362-1\end{array}$

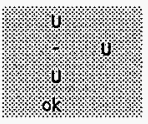

\section{Group 96001380}

METHOO SUMALARIES

Page 8

SLRMARY DATA SECTION

Page 43

\begin{tabular}{c} 
1.07E 01 \\
OUT \\
\hline$U$ \\
ok
\end{tabular}

ok
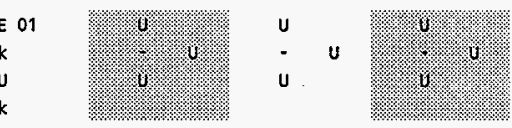

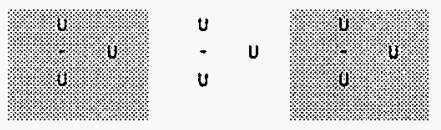

1.

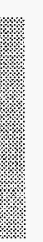


HNF-SD-WM-DP-219, REV.O

$222-S$ LABORATORY

Test GEA Matrix SOLID SDG 96001380 Contact L. L. Fritts
TANK 241-B-108, CORE 173

METHOD SUMMARY

GAMMA SPECTROSCOPY

GAMHA ENERGY ANALYSIS
Client TWRS

Tank 241-B-108

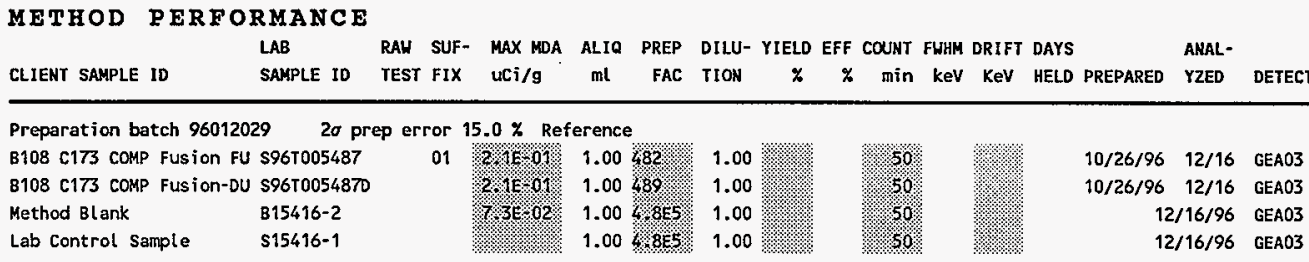

Preparation batch $96012648 \quad 2 \sigma$ prep error $15.0 \%$ Reference B108 C173\$1 LH Fusion FU \$96T005477 B108 C173S1 LH Fusion-DU $596 \mathrm{T0054770}$ B108 C173S1 UH Fusion FU \$96T005468 B108 C173s1 UH Fusion-DU S96T0054680 8108 C173s2 LH Fusion FU $596 \mathrm{~T} 005478$ B108 C173s2 LH Fusion-DU 59670054780 Method Blank B16019-2 Lab Control Sample

\begin{tabular}{|c|c|}
\hline $26=0 \%$ & $1.00 \%$ VES \\
\hline 2680 & $1.00 \% 4$ \\
\hline 3.2001 & $1.00 \% 865$ \\
\hline 325,01 & $1.00 \% 7,5$ \\
\hline KE & 1.004455 \\
\hline 211501 & $1.00 \% 5$ \\
\hline $8 \% 2=02$ & 1.00 /\% \\
\hline & 1.003805 \\
\hline
\end{tabular}

1.00

1.00

1.00

1.00

1.00

1.00

1.00

1.00

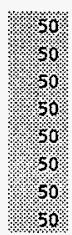

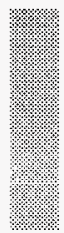

10/26/96 01/09 GEA02 10/26/96 01/09 GEA02 10/26/96 01/09 GEA02 10/26/96 01/09 GEA02 $10 / 26 / 96 \quad 01 / 10$ GEA02 10/26/96 01/10 GEA02 01/09/97 GEA02 01/09/97 GEA02

Preparation batch $97000110 \quad 2 \sigma$ prep error $15.0 \%$ Reference B108 c173 Comp Fusion Re 597 T000002 B108 c173 Comp Fusion Re 59710000020 Method 8lank B16258-2 Lab control samole \$16258-1

\begin{tabular}{|c|c|}
\hline $2 \times 301$ & $1.00 \%$ \\
\hline $2 \times 35 \%$ & 1.00892 \\
\hline $68 \mathrm{E} \% 2$ & $1.00 \% 955$ \\
\hline & $1.00 \% 95$ \\
\hline
\end{tabular}

1.00
1.00
1.00
1.00

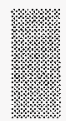

01/15/97 01/22 GEA03

$01 / 15 / 97$ 01/22 GEA03 01/22/97 GEA03 $01 / 22 / 97$ GEA03

Preparation batch $97000227 \quad 2 \sigma$ prep error $15.0 \%$ Reference $\begin{array}{ll}\text { B108 C173S2 LH Fusion Re } \$ 971000001 \\ \text { B108 c173s2 LH Fusion Re } \$ 97 T 0000010 \\ \text { Method 8lank } & \text { B16362-2 } \\ \text { Lab control Sample } & \mathbf{S 1 6 3 6 2 - 1}\end{array}$

01 Vivol: $1.00490 \%$

2.1501: $1.0040 \%$

8.2002

1.006905

1.00

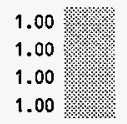

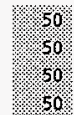

$01 / 15 / 97 \quad 01 / 28$ GEA03

$01 / 15 / 97 \quad 01 / 28$ GEA03

01/28/97 GEA03 $01 / 28 / 97$ GEA03

\section{Group 96001380}

\section{METHCD SUMMARIES}

Page 9

SUMMARY DATA SECTION

Page 44
Lab id 222-s

Protocol SST

Version 1.0

Form DVD-CMS

Version 3.08

Report date $\underline{02 / 24 / 97}$ 
HNF-SD-WA-DP-219, REV. 0

22 2-S LABORATORY

TANK 241-8-108, CORE 173

Test GEA Matrix

SDG 96001380

METHOD SUMMARY, cOnt.

GAMMA SPECTROSCOPY

Client IWRS

Contact L. L. Fritts

GAMA ENERGY ANALYSIS

PROCEDURES REFERENCE 222-S Lab Analytical Procedure

L0-160-103 Core Segment Extrusion Process and Sample

Preparation, rev 17

1A-549-141 Fusion with Alkali Metal Hydroxide, rev 40

LA-548-121 Preparation of Sample Mounts for Gama Energy Analysis, rev 41

LA-508-162 Ganma Energy Analysis - the Genie System, rev 11
AVERAGES \pm 2 SD

FOR 16 SAMPLES
Tank 241-8-108

FOR 16 SAYPLES

MDA 2.0E-01 $\pm 1.6 E-01$ YIELD

$\pm$

\section{Group 96001380}

METHOO SUMUARIES

Page 10

SLMALARY DATA SECTION

Page 45
Lab id 222-s

Protocol SST

Version 1.0

Form OVD-CNS

version 3.08

Report date $02 / 24 / 97$ 
HNF-SD-WM-DP-219, REV. 0

SAMPLE DATA SUMMARY 
HNF-SD-WM-DP-219, REV. 0

\section{THIS PAGE INTENTIONALLY LEFT BLANK}


Table 3. Data Sumary Table

CORE NUMBER: 172

SEGMENT \#: Field Blank

SEGMENT PORTION: Field Blank

\begin{tabular}{|c|c|c|c|c|c|c|c|c|c|c|c|c|}
\hline Sample\# & R A\# & Analyte & Unit & Standard \% & Blank & Result & Duplicate & Average & RPO \% & Spk Rec \% & Det Limit & Count Err\% \\
\hline S96T005463 & & DSC Exotherm Dry Calculated & Joules/g Dry & $n / a$ & $n / a$ & $0.00 \mathrm{e}+00$ & $0.00 \mathrm{e}+00$ & $0.00 \mathrm{e}+00$ & 0.00 & $n / a$ & $n / a$ & $n / a$ \\
\hline 5967005463 & & DSC Exotherm on Perkin Elmer & Joules/g & 100.8 & $n / a$ & $0.00 \mathrm{e}+00$ & $0.00 \mathrm{e}+00$ & $0.00 \mathrm{e}+00$ & 0.00 & $n / a$ & $n / a$ & $n / a$ \\
\hline 5967005463 & & Specific Gravity & Sp.G. & 99.75 & $\mathrm{n} / \mathrm{a}$ & 2.270 & 2.298 & 2.284 & 9.23 & $n / a$ & $7.00 \mathrm{e}-03$ & $n / a$ \\
\hline 5967005463 & & $\%$ Water by TGA using Mettler & $\%$ & 99.81 & $\mathrm{n} / \mathrm{a}$ & 99.42 & 99.69 & 99.56 & 0.27 & $n / a$ & $n / a$ & $n / a$ \\
\hline 5967005463 & D & Silver-ICP-Acid Dil. & $\mathrm{ug} / \mathrm{mL}$ & 98.60 & $<1.00 \mathrm{e}-02$ & $<7.00 \mathrm{e}-02$ & $<7.00 \mathrm{e}-2$ & $n / a$ & $n / a$ & $n / a$ & $7.00 \mathrm{e}-02$ & $n / a$ \\
\hline 5967005463 & $D$ & Aluminium-ICP-Acid Dil. & ug/mL & 97.40 & $<5.00 e-02$ & 1.850 & 1.780 & 1.815 & 3.86 & $n / a$ & $3.50 e-01$ & $n / a$ \\
\hline 5967005463 & D & Arsenic-ICP-Acid Dit. & $\mathrm{ug} / \mathrm{mL}$ & 103.6 & $<1.00 \mathrm{e}-01$ & $<7.00 \mathrm{e}-01$ & $<7.00 \mathrm{e}-1$ & $n / a$ & $\mathrm{n} / \mathrm{a}$ & $n / 3$ & $7.00 \mathrm{e}-01$ & $n / a$ \\
\hline S96T005463 & $D$ & Boron-1CP-Acid Dit. & ug/mL & 99.80 & $<5.00 \mathrm{e}-02$ & 2.080 & 2.000 & 2.040 & 3.92 & $\mathrm{n} / \mathrm{a}$ & $3.50 \mathrm{e}-01$ & $n / a$ \\
\hline 5961005463 & $\frac{L}{D}$ & Barium-ICP-Acid Dil. & $\mathrm{ug} / \mathrm{mL}$ & 98.40 & $<5.00 \mathrm{e}-02$ & $<3.50 \mathrm{e}-01$ & $<3.50 \mathrm{e}-1$ & $n / \mathbf{a}$ & $n / a$ & $n / a$ & $3.50 \mathrm{e}-01$ & $n / a$ \\
\hline 5967005463 & $\frac{10}{10}$ & Berylli ium-ICP-Acid Dil. & $\mathrm{ug} / \mathrm{mL}$ & 102.8 & $<5.00 \mathrm{e}-03$ & $<3.50 \mathrm{e}-02$ & $<3.50 \mathrm{e}-2$ & $n / a$ & $n / a$ & $n / 3$ & $3.50 \mathrm{e}-02$ & $n / a$ \\
\hline S96T005463 & $\bar{D}$ & Bismuth-ICP-Acid Dil. & $\mathrm{ug} / \mathrm{mL}$ & 101.6 & $<1.00 e-01$ & $<7.00 \mathrm{e}-01$ & $<7.00 \mathrm{e}-1$ & $n / a$ & $\mathrm{n} / \mathrm{a}$ & $n / 8$ & $7.00 \mathrm{e}-01$ & $n / a$ \\
\hline $596 T 005463$ & $D$ & Calcium-ICP-Acid Dil. & $\mathrm{ug} / \mathrm{mL}$ & 99.20 & $<1.00 \mathrm{e}-01$ & 17.00 & 17.00 & 17.00 & 0.00 & $n / a$ & $7.00 e-01$ & $n / a$ \\
\hline S96r005463 & D & Cadmium-1CP-Acid Dil. & $\mathrm{ug} / \mathrm{mL}$ & 100.4 & $<5.00 \mathrm{e}-03$ & $<3.50 \mathrm{e}-02$ & $<3.50 \mathrm{e}-2$ & $n / a$ & $n / a$ & $n / a$ & $3.50 e-02$ & $n / a$ \\
\hline 5967005463 & $D$ & Cerium-ICP-ACid Dil. & $\mathrm{ug} / \mathrm{mL}$ & 98.60 & $<1.00 e-01$ & $<7.00 \mathrm{e}=01$ & $<7.00 e^{-1}$ & $n / a$ & $\mathrm{n} / \mathrm{a}$ & $n / a$ & $7.00 \mathrm{e}-01$ & $n / a$ \\
\hline S96T005463 & D & Cobalt-ICP-Acid Dil. & $\underline{u g} / \mathrm{mL}$ & 100.6 & $<2.00 \mathrm{e}-02$ & $<1.40 \mathrm{e}-01$ & $\leq 1,40 \mathrm{e}^{-1}$ & $n / a$ & $\mathrm{n} / \mathrm{a}$ & $n / a$ & $1.40 \mathrm{e}-01$ & $n / a$ \\
\hline 5967005463 & $\mathrm{D}$ & Chromi um-ICP-Acid Dil. & $\mathrm{ug} / \mathrm{mL}$ & 100.6 & $<1.00 \mathrm{e}-02$ & $<7.00 e-02$ & $<7.00 e-2$ & $n / a$ & $\mathrm{n} / \mathrm{a}$ & $n / a$ & $7.00 \mathrm{e}-02$ & $n / a$ \\
\hline S96T005463 & $D$ & Copper-ICP-Acid Dil. & ug $/ \mathrm{mL}$ & 104.2 & $<1.00 \mathrm{e}-02$ & $<7.00 \mathrm{e}-02$ & $<7.00 \mathrm{e}-2$ & $n / a$ & $n / a$ & $n / a$ & $7.00 \mathrm{e}-02$ & $n / 2$ \\
\hline S96T005463 & D & Iron-ICP-Acid Dil. & $\mathrm{ug} / \mathrm{mL}$ & 100.0 & $<5.00 \mathrm{e}-02$ & $<3.50 e-01$ & $<3.50 \mathrm{e}-1$ & $n / a$ & $n / a$ & $n / a$ & $3.50 \mathrm{e}-01$ & $n / a$ \\
\hline $596 T 005463$ & $D$ & Potassium-ICP-Acid Dil. & $4 \mathrm{~g} / \mathrm{mL}$ & 100.4 & $<5.00 \mathrm{e}-01$ & 3.560 & 4.280 & 3.920 & 18.4 & $n / a$ & 3.500 & $n / a$ \\
\hline S96T005463 & D & Lanthanum-ICP-Acid Dil. & $\mathrm{ug} / \mathrm{mL}$ & 100.2 & $<5.00 \mathrm{e}-02$ & $<3.50 \mathrm{e}-01$ & $<3.50 \mathrm{e}-1$ & $n / a$ & $n / a$ & $n / a$ & $3.50 \mathrm{e}-01$ & $n / a$ \\
\hline $596 T 005463$ & D & Lithium-1CP-Acid Dil. & ug $/ \mathrm{mL}$ & 100.4 & $<1.00 \mathrm{e}-02$ & $<7.00 \mathrm{e}-02$ & $<7.00 \mathrm{e}-2$ & $\mathrm{n} / \mathrm{a}$ & n/a & $n / a$ & $7.00 \mathrm{e}-02$ & $n / a$ \\
\hline 5967005463 & D & Magnesium-ICP-Acid Dil. & $\mathrm{ug} / \mathrm{mL}$ & 96.20 & $<1.00 \mathrm{e}-01$ & 3.630 & 3.580 & 3.605 & 1.39 & $n / a$ & $7.00 \mathrm{e}-01$ & $\mathrm{n} / \mathrm{a}$ \\
\hline \$96T005463 & $D$ & Manganese-ICP-Acid Dil. & $\mathrm{ug} / \mathrm{mL}$ & 97.80 & $<1.00 \mathrm{e}-02$ & $<7.00 \mathrm{e}-02$ & $<7.00 \mathrm{e}-2$ & $n / a$ & $\mathrm{n} / \mathrm{a}$ & $n / a$ & $7.00 \mathrm{e}-02$ & $n / a$ \\
\hline 5967005463 & $D$ & Molybodenum-ICP-Acid Dil. & $\mathrm{ug} / \mathrm{mL}$ & 100.6 & $<5.00 \mathrm{e}-02$ & $<3.50 \mathrm{e}-01$ & $<3.50 \mathrm{e}-1$ & $n / a$ & $n / a$ & $n / a$ & $3.50 \mathrm{e}-01$ & $n / a$ \\
\hline S96T005463 & $D$ & Sodium-ICP-Acid Dil. & $\mathrm{ug} / \mathrm{mL}$ & 96.40 & $<1.00 \mathrm{e}-01$ & 56.30 & 55.50 & 55.90 & 1.43 & $n / a$ & $7.00 \mathrm{e}-01$ & $n / a$ \\
\hline S9670 & D & Neodymium-ICP-Acid Dil. & $\mathrm{ug} / \mathrm{mL}$ & 100.4 & $<1.00 e-01$ & $<7.00 \mathrm{e}-01$ & $<7.00 \mathrm{e}-1$ & $n / a$ & $n / a$ & $n / a$ & $7.00 \mathrm{e}-01$ & $n / a$ \\
\hline 5463 & D & Nickel-ICP-Acid Dil. & $\mathrm{ug} / \mathrm{mL}$ & 100.4 & $<2.00 e-02$ & $<1.40 \mathrm{e}-01$ & $<1.40 \mathrm{e}-1$ & $n / a$ & $n / a$ & $\mathrm{n} / \mathrm{a}$ & $1.40 \mathrm{e}-01$ & $n / a$ \\
\hline S96T0 & $\bar{D}$ & Phosphorus-ICP-Acid Dil, & $\mathrm{ug} / \mathrm{mL}$ & 99.60 & $<2.00 \mathrm{e}-01$ & 1.400 & $<1,40 \mathrm{e} 0$ & $n / a$ & $n / a$ & $\mathrm{n} / \mathrm{a}$ & 1.400 & $n / a$ \\
\hline 5967005463 & D & Lead-ICP-ACid Dil. & $\mathrm{ug} / \mathrm{mL}$ & 99.80 & $<1.00 e-01$ & $<7.00 \mathrm{e}-01$ & $<7.00 \mathrm{e}-1$ & $n / a$ & $n / a$ & $n / a$ & $7.00 \mathrm{e}-01$ & $n / a$ \\
\hline 5463 & $\frac{1}{D}$ & Sul fur-ICP-Acid Dil. & ug $/ \mathrm{mL}$ & 98.80 & $<1.00 \mathrm{e}-01$ & 8.650 & 8.890 & 8.770 & 2.74 & $\mathrm{n} / \mathrm{a}$ & $7.00 \mathrm{e}-01$ & $n / a$ \\
\hline $596 \mathrm{~T}$ & D & Ant imony-ICP-Acid Dil. & $\mathrm{Ug} / \mathrm{mL}$ & 95.80 & $<6.00 \mathrm{e}-02$ & $<4.20 \mathrm{e}-01$ & $<4.20 e^{-1}$ & $n / a$ & $n / a$ & $n / a$ & $4.20 \mathrm{e}-01$ & $n / a$ \\
\hline S96T005463 & D & Selenium-1CP-Acid Dil. & ug/mL & 97.20 & $<1.00 \mathrm{e}-01$ & $<7.00 \mathrm{e}-01$ & $<7.00 \mathrm{e}-1$ & $\mathrm{n} / \mathrm{a}$ & $n / a$ & $n / a$ & $7.00 \mathrm{e}-01$ & $\mathrm{n} / \mathrm{a}$ \\
\hline S96T005463 & D & silicon-ICP-Acid Dil. & $\mathrm{ug} / \mathrm{mL}$ & 99.80 & $<5.00 \mathrm{e}-02$ & 1.910 & 1.950 & 1.930 & 2.07 & $\mathrm{n} / \mathrm{a}$ & $3.50 e-01$ & $n / a$ \\
\hline $596 \mathrm{~T}$ & D & UM-ICP-RCId Dil. & $\mathrm{ug} / \mathrm{mL}$ & 97.20 & $<1.00 \mathrm{e}-01$ & $<7.00 \mathrm{e}-01$ & $<7.00 \mathrm{e}-1$ & $\mathrm{n} / \mathrm{a}$ & $n / a$ & $n / a$ & $7.00 e-01$ & $n / a$ \\
\hline 596T & D & IUm-ICP-ACId Dil. & $4 \mathrm{~g} / \mathrm{mL}$ & 97.60 & $<1.00 \mathrm{e}-02$ & $7.35 e-02$ & $7.35 \mathrm{e}-02$ & $7.35 e-02$ & 0.00 & $n / a$ & $7.00 \mathrm{e}-02$ & $\mathrm{n} / \mathrm{a}$ \\
\hline 5967005463 & 0 & Titaniun-ICP-Acid Dil. & ug $/ \mathrm{mL}$ & 97.00 & $<1.00 \mathrm{e}-02$ & $<7.00 \mathrm{e}^{-02}$ & $\leq 7.00 \mathrm{e}-2$ & $\mathrm{n} / \mathrm{a}$ & $\mathrm{n} / \mathrm{a}$ & $n / a$ & $7.00 \mathrm{e}-02$ & $n / a$ \\
\hline 5967005463 & $\bar{D}$ & Thallium-ICP-Acid Dit. & $\mathrm{Ug} / \mathrm{mL}$ & 96.80 & $<2.00 \mathrm{e}-01$ & 1.400 & $<1.40 \mathrm{e} 0$ & $\mathrm{n} / \mathrm{a}$ & $n / a$ & $n / a$ & 1.400 & $n / a$ \\
\hline S96T & 0 & Uranium-ICP-Acid Dil. & $\mathrm{ug} / \mathrm{mL}$ & 95.20 & $<5.00 e-01$ & 3.500 & $<3.50 \mathrm{e} 0$ & $n / a$ & $n / a$ & $n / a$ & 3.500 & $n / a$ \\
\hline 5961 & D & Vanadium-ICP-Acid Dil. & $\mathrm{ug} / \mathrm{mL}$ & 100.2 & $<5.00 e^{-02}$ & $<3.50 \mathrm{e}-01$ & $<3.50 \mathrm{e}-1$ & $\mathrm{n} / \mathrm{a}$ & $\mathrm{n} / \mathrm{a}$ & $n / a$ & $3.50 \mathrm{e}-01$ & $n / a$ \\
\hline 5961005463 & 0 & ZinC-ICP-ACid Dil. & $\mathrm{ug} / \mathrm{mL}$ & 101.6 & $\leq 1.00 \mathrm{e}-02$ & $<7.00 \mathrm{e}-02$ & $<7.00 \mathrm{e}-2$ & $\mathrm{n} / \mathrm{a}$ & $n / a$ & $\mathrm{n} / \mathrm{a}$ & $7.00 e-02$ & $\mathrm{n} / \mathrm{a}$ \\
\hline $596 \mathrm{TO}$ & $D$ & Zirconium-ICP-Acid Dil. & ug/mL & 98.20 & $<1.00 \mathrm{e}-02$ & $<7.00 \mathrm{e}-02$ & $<7.00 \mathrm{e}-2$ & $n / a$ & $n / a$ & $n / a$ & $7.00 \mathrm{e}=02$ & $n / a$ \\
\hline 463 & & Fluoride-IC-Dionex $4000 / 4$ & $4 \mathrm{~g} / \mathrm{mL}$ & 100.0 & $<1.20 e-02$ & $2.54 \mathrm{e}-01$ & $2.63 e-01$ & $2.59 e-01$ & 3.48 & $n / a$ & $1.20 \mathrm{e}-02$ & $n / a$ \\
\hline S96T005463 & & Chloride-1C-Dionex $4000 / 4500$ & $\mathrm{ug} / \mathrm{mL}$ & 94.05 & $<1.70 \mathrm{e}-02$ & 10.05 & 12.10 & 11.07 & 18.0 & $n / a$ & 1.717 & $n / a$ \\
\hline S96r005463 & & Nitrite-IC - Dionex $4000 / 4500$ & $\mathrm{ug} / \mathrm{mL}$ & 93.35 & $<1.08 \mathrm{e}-01$ & 19.70 & 19.80 & 19.75 & 0.51 & $n / a$ & $1.08 \mathrm{e}-01$ & $n / a$ \\
\hline
\end{tabular}




\begin{tabular}{|c|c|c|c|c|c|c|c|c|c|c|c|}
\hline Samole\# & A\# Analyte & Unit & Standard $\%$ & Blank & Result & Duplicatel & Average & RPD \% & Spk Rec \% & Det Limit & Count Err\% \\
\hline S961005463 & Bromide by Ion Chromatograph & ug/mL & 97.96 & $<1.25 e-01$ & $4.33 e-01$ & $4.33 e^{-011}$ & $4.33 \mathrm{e}-01$ & 0.00 & n/a & $1.25 e-01$ & $\mathrm{n} / \mathrm{a}$ \\
\hline S96r005463 & Nitrate by IC-Dionex $4000 / 4500$ & $\mathrm{ug} / \mathrm{mL}$ & 95.29 & $<1.39 \mathrm{e}-01$ & 51.09 & 51.40 & 51.25 & 0.59 & $n / a$ & $1.39 \mathrm{e}-01$ & $n / a$ \\
\hline S96T005463 & Phosphate-IC-Dionex $4000 / 4500$ & ug/aL & 93.57 & $<1.20 \mathrm{e}-01$ & $<1.20 \mathrm{e}-01$ & $1.51 \mathrm{e}-01$ & $n / a$ & $n / a$ & $n / a$ & $1.20 \mathrm{e}-01$ & $n / a$ \\
\hline S967005463 & Sulfate by IC-Dionex $4000 / 4500$ & $\mathrm{ug} / \mathrm{mL}$ & 96.99 & $<1.38 \mathrm{e}-01$ & 96.09 & 16.20 & 16.14 & 0.62 & $n / a$ & $1.38 \mathrm{e}-01$ & $n / a$ \\
\hline 5967005463 & Oxalate by IC-Dionex $4000 / 4500$ & $\mathrm{ug} / \mathrm{mL}$ & 102.5 & $<1.05 e-01$ & $<1.05 e^{-01}$ & $<1.05 \mathrm{e}-1$ & $n / a$ & $n / a$ & $n / a$ & $1.05 \mathrm{e}-01$ & $n / a$ \\
\hline 5961005463 & Alpha in liquid Samples & $\mathrm{uCt} / \mathrm{mL}$ & 78.79 & $<4.40 \mathrm{e}=06$ & $<3,96 \mathrm{e}-06$ & $<4.40 E-6$ & $\mathrm{n} / \mathrm{a}$ & $\mathrm{n} / \mathrm{a}$ & $n / a$ & $7.13 e-06$ & $5.00 \mathrm{E}+02$ \\
\hline
\end{tabular}


Table 3. Data Sumary Table

CORE NUMBER: 172

SEGMENT \#: 1

SEGMENT PORTION: U Upper Half of Segment

\begin{tabular}{|c|c|c|c|c|c|c|c|c|c|c|c|c|}
\hline Sample\# & $\left.R\right|_{\text {A\# }}$ & \#Analy & Unit & Standard \% & Blank & Resul t & Dupl icatel & Average & RPD * & Spk Rec \% & Det Limit & Count Err\% \\
\hline 5961005502 & & Bulk Density of Sample & $\mathrm{g} / \mathrm{mL}$ & $n / a$ & $\mathrm{n} / \mathrm{a}$ & 1.700 & $n / a$ & $\mathrm{n} / \mathrm{a}$ & $n / a$ & $\mathrm{n} / \mathrm{a}$ & $5.00 \mathrm{e}-01$ & $\mathrm{n} / \mathrm{a}$ \\
\hline 5967005505 & & DSC Exotherm using Mettler & Joules/g & 100.9 & $n / a$ & $0.00 \mathrm{e}+00$ & $0.00 \mathrm{e}+00$ & $0.00 \mathrm{e}+00$ & 0.00 & $n / a$ & $\mathrm{n} / \mathrm{a}$ & $n / a$ \\
\hline S96T005505 & & DSC Exotherm Dry Calculated & Joules/g Dry & $\mathrm{n} / \mathrm{a}$ & $\mathrm{n} / \mathrm{a}$ & $0.00 \mathrm{e}+00$ & $0.00 \mathrm{e}+00$ & $0.00 \mathrm{e}+00$ & 0.00 & $\mathrm{n} / \mathrm{a}$ & $\mathrm{n} / \mathrm{a}$ & $n / a$ \\
\hline S967005505 & & \% Water by TGA using Mettler & $\%$ & 100.3 & $n / a$ & 34.40 & 32.63 & 33.52 & 5.28 & $n / a$ & $\mathrm{n} / \mathrm{a}$ & n/a \\
\hline 5967005510 & $\mathbf{F}$ & Nickel-ICP-Fusion & ug/g & 99.201 & $8.60 \mathrm{e}-02$ & $5.55 \mathrm{e}+03$ & $4.87 \mathrm{e}+03$ & $5.27 \mathrm{e}+03$ & 13.1 & $n / a$ & 399.0 & $n / a$ \\
\hline 5967005510 & $\mathrm{~F}$ & Silver-ICP-Fusion & $\mathrm{ug} / \mathrm{g}$ & 98.00 & $<1.00 \mathrm{e}-02$ & $<2.00 \mathrm{e}+02$ & $<1.99 \mathrm{e} 2$ & $\mathrm{n} / \mathrm{a}$ & $n / a$ & $n / a$ & 200.0 & $n / a$ \\
\hline 5967005510 & $F$ & Aluminium-ICP-Fusion & $4 g / g$ & 96.60 & $<5.00 \mathrm{e}-02$ & $9.92 e+04$ & $9.89 \mathrm{e}+04$ & $9.90 e+04$ & 0.30 & $\mathrm{n} / \mathrm{a}$ & 996.0 & $n / a$ \\
\hline$\$ 967005510$ & $F$ & Arsenic-ICP-Fusion & ug/g & 101.6 & $<1.00 \mathrm{e}-01$ & $<2.00 e+03$ & $<1.99 \mathrm{e} 3$ & $n / a$ & $n / a$ & $n / a$ & $2.00 \mathrm{e}+03$ & $n / a$ \\
\hline 5961005510 & $F$ & Boron-ICP-Fusion & ug/g & 98.40 & $<5.00 e^{-02}$ & $<9.98 \mathrm{e}+02$ & $<9,97 \mathrm{e} 2$ & $\mathrm{n} / \mathrm{a}$ & $n / a$ & $n / a$ & 996.0 & $n / \mathbf{a}$ \\
\hline$\$ 96 \mathrm{~T} 005510$ & $F$ & Barium-ICP-Fusion & $49 / 9$ & 98.20 & $<5.00 e-02$ & $<9.98 \mathrm{e}+02$ & $<9.97 \mathrm{e} 2$ & $n / a$ & $n / a$ & $n / a$ & 996.0 & $n / a$ \\
\hline 5967005510 & $\dot{\mathrm{F}}$ & Beryllium-ICP-Fusion & ug/g & 102.0 & $<5.00 e^{-03}$ & $<\quad 99.80$ & $<9.97 \mathrm{e} 1$ & $\mathrm{n} / \mathrm{a}$ & $n / a$ & $n / a$ & 99.60 & $n / a$ \\
\hline S96T005510 & $F$ & Bismuth - ICP-Fusion & ug/g & 100.4 & $<1.00 e-01$ & $3.04 \mathrm{e}+03$ & $2.59 e+03$ & $2.82 \mathrm{e}+03$ & 16.0 & $n / a$ & $2.00 \mathrm{e}+03$ & $n / a$ \\
\hline $596 \mathrm{~T} 005510$ & $F$ & Calcium-ICP-Fusion & ug/g & 98.80 & $<1.00 \mathrm{e}-01$ & $<2.00 \mathrm{e}+03$ & $<1.99 \mathrm{e} 3$ & $\mathrm{n} / \mathrm{a}$ & $n / a$ & $n / a$ & $2.00 e+03$ & $n / a$ \\
\hline $596 \mathrm{~T} 005510$ & $F$ & Cadmium - ICP-Fusion & ug/g & 99.40 & $<5.00 \mathrm{e}-03$ & $<\quad 99.80$ & $<9.97 \mathrm{e} 1$ & $\mathrm{n} / \mathrm{a}$ & $n / a$ & $n / a$ & 99.60 & $n / a$ \\
\hline S96T005510 & $F$ & Cerium-ICP-Fusion & ug/g & 98,20 & $<1.00 \mathrm{e}-01$ & $<2.00 e+03$ & $<1.99 \mathrm{e} 3$ & $\mathrm{n} / \mathrm{a}$ & $\mathrm{n} / \mathrm{a}$ & $n / a$ & $2.00 \mathrm{e}+03$ & $n / a$ \\
\hline S96T005510 & $F$ & Cobalt -ICP-Fusion & ug/g & 99.80 & $<2.00 \mathrm{e} \cdot 02$ & $<3.99 \mathrm{e}+02$ & $<3.99 \mathrm{e} 2$ & $\mathrm{n} / \mathrm{a}$ & $n / a$ & $n / a$ & 399.0 & $n / a$ \\
\hline 5967005510 & $F$ & Chromium - ICP-Fusion & ug/g & 100.0 & $<1.00 e^{-02}$ & $5.00 e+02$ & 526.0 & 513.0 & 5.07 & $n / a$ & 200.0 & $n / a$ \\
\hline$\$ 967005510$ & $F$ & Copper -ICP-Fusion & ug/g & 103.4 & $<1.00 \mathrm{e}=02$ & $<2.00 \mathrm{e}+02$ & $<1.99 \mathrm{e} 2$ & $\mathrm{n} / \mathrm{a}$ & $n / a$ & $n / a$ & 200.0 & $n / a$ \\
\hline 5967005510 & $F$ & Iron-ICP-Fusion & $\mathrm{ug} / \mathrm{g}$ & 99.20 & $<5.00 e-02$ & $4.07 \mathrm{e}+03$ & $6.18 \mathrm{e}+03$ & $5.12 \mathrm{e}+03$ & 41.2 & $n / a$ & 996.0 & $n / a$ \\
\hline S96T005510 & $F$ & Lanthanum -ICP-Fusion & $\operatorname{lug} / 9$ & 99.80 & $<5.00 \mathrm{e}^{-02}$ & $<9.98 \mathrm{e}+02$ & $<9.97 \mathrm{e} 2$ & $n / a$ & $n / a$ & $n / a$ & 996.0 & $n / a$ \\
\hline 5967005510 & $F$ & Lithium-ICP-Fusion & $\operatorname{lug} / \mathrm{g}$ & 99.00 & $<1.00 e^{-02}$ & $<2.00 \mathrm{e}+02$ & $<1.99 \mathrm{e} 2$ & $\mathrm{n} / \mathrm{a}$ & $n / a$ & $n / a$ & 200.0 & $\mathrm{n} / \mathrm{a}$ \\
\hline $596 \mathrm{~T} 005510$ & $\mathrm{~F}$ & Magnesium -ICP-Fusion & ug/g & 95.00 & $<1,00 \mathrm{e}-01$ & $<2.00 \mathrm{e}+03$ & $<1.99 \mathrm{e} 3$ & $\mathrm{n} / \mathrm{a}$ & $n / a$ & $n / a$ & $2.00 \mathrm{e}+03$ & $n / a$ \\
\hline $596 \mathrm{T005510}$ & $F$ & Manganese - ICP-fusion & ug/g & 96,60 & $<1.00 e^{-02}$ & $<2.00 e+02$ & $<1.99 \mathrm{e} 2$ & $n / a$ & $n / a$ & $n / a$ & 200.0 & $n / a$ \\
\hline$\$ 967005510$ & $\mathbf{F}$ & Molybdenum - ICP-Fusion & $\mathrm{ug} / \mathrm{g}$ & 100.0 & $<5.00 \mathrm{e} \cdot 02$ & $<9.98 \mathrm{e}+02$ & $<9.97 \mathrm{e} 2$ & $n / a$ & $\mathrm{n} / \mathrm{a}$ & $n / a$ & 996.0 & $n / a$ \\
\hline 5967005510 & $F$ & Sodium -ICP-Fusion & $\mathrm{ug} / \mathrm{g}$ & 95.60 & 1.670 & $1.50 \mathrm{e}+05$ & $1.47 \mathrm{e}+05$ & $1.48 e+05$ & 2.02 & $\mathrm{n} / \mathrm{a}$ & $2.00 \mathrm{e}+03$ & $n / a$ \\
\hline 5967005510 & $F$ & Neodymium-ICP-Fusion & $\mathrm{ug} / \mathrm{g}$ & 99.60 & $<1.00 \mathrm{e}-01$ & $<2.00 \mathrm{e}+03$ & $<1.99 \mathrm{e} 3$ & $n / a$ & $n / a$ & $n / a$ & $2.00 \mathrm{e}+03$ & $n / a$ \\
\hline 5967005510 & F & Phosphiorus -ICP-Fusion & $\mathrm{ug} / \mathrm{g}$ & 99.60 & $<2.00 e^{-01}$ & $2.54 \mathrm{e}+04$ & $2.51 e+04$ & $2.52 \mathrm{e}+04$ & 1.19 & $n / a$ & $3.99 \mathrm{e}+03$ & $n / a$ \\
\hline S96T & $F$ & Lead-ICP-Fusion & $409 / 9$ & 98.80 & $<1.00 \mathrm{e}-01$ & $<2.00 \mathrm{e}+03$ & $<7.99 \mathrm{e} 3$ & $n / a$ & $n / a$ & $n / a$ & $2.00 \mathrm{e}+03$ & $n / a$ \\
\hline$\$ 967005510$ & $\mathbf{F}$ & Sulfur-ICP-fusion & ug/g & 98.00 & $<7.00 \mathrm{e}-01$ & $3.43 e+03$ & $3.49 \mathrm{e}+03$ & $3.46 \mathrm{e}+03$ & 1.73 & $\mathrm{n} / \mathrm{a}$ & $2.00 e+03$ & $n / a$ \\
\hline \$967005510 & $F$ & Antimony -ICP-Fusion & ug/a & 95.00 & $<6.00 e-02$ & $<1.200+03$ & $<1.20 \mathrm{e} 3$ & $\mathrm{n} / \mathrm{a}$ & $n / a$ & $n / a$ & $1.20 \mathrm{e}+03$ & $n / a$ \\
\hline$\$ 967005510$ & $F$ & Selenium-ICP-Fusion & $\lg / 9$ & 96.40 & $<1.00 \mathrm{e}-01$ & $<2.00 \mathrm{e}+03$ & $<1.99 \mathrm{e} 3$ & n/a & $n / a$ & $\mathrm{n} / \mathrm{a}$ & $2.00 \mathrm{e}+03$ & n/a \\
\hline S967005510 & F & Silicon-ICP-Fusion & $\mathrm{ug} / \mathrm{g}$ & 98.80 & $<5.00 e-02$ & $2.09 \mathrm{e}+03$ & $2.19 \mathrm{e}+03$ & $2.14 \mathrm{e}+03$ & 4.67 & $\mathrm{n} / \mathrm{a}$ & 996.0 & $n / a$ \\
\hline 5967005510 & F & Samarium-ICP-Fusion & $\mathrm{ug} / \mathrm{g}$ & 96.60 & $<1.00 \mathrm{e}-01$ & $<2.00 \mathrm{e}+03$ & $<1.99 \mathrm{e} 3$ & $n / a$ & $n / a$ & $n / a$ & $2.00 \mathrm{e}+03$ & $n / a$ \\
\hline$\$ 967005510$ & $F$ & Strontium -ICP-Fusion & $\mathrm{ug} / \mathrm{g}$ & 97.40 & $<1.00 e^{-02}$ & $<2,00 \mathrm{e}+02$ & $<1.99 \mathrm{e} 2$ & $n / a$ & $n / a$ & $n / a$ & 200.0 & $n / a$ \\
\hline$\$ 967005510$ & $\mathrm{~F}$ & Titanium-ICP-Fusion & $\mathrm{ug} / \mathrm{g}$ & 97.20 & $<1.00 e^{-02}$ & $<2.00 \mathrm{e}+02$ & $<1.99 \mathrm{e} 2$ & $n / a$ & $\mathrm{n} / \mathrm{a}$ & $n / a$ & 200.0 & $\mathrm{n} / \mathrm{a}$ \\
\hline S96TC & $F$ & Thallium-1CP-Fusion & $u g / 9$ & 96.40 & $<2.00 e-01$ & $<3.99 \mathrm{e}+03$ & $<3.99 \mathrm{e} 3$ & $\mathrm{n} / \mathrm{a}$ & $\mathrm{n} / \mathrm{a}$ & $n / a$ & $3.99 \mathrm{e}+03$ & $n / a$ \\
\hline 5967005510 & $F$ & Uranium-ICP-Fusion & $\operatorname{lig} / 9$ & 94.60 & $<5.00 \mathrm{e}-01$ & $<9.98 \mathrm{e}+03$ & $<9.97 \mathrm{e} 3$ & $\mathrm{n} / \mathrm{a}$ & $\mathrm{n} / \mathrm{a}$ & $\mathrm{n} / \mathrm{a}$ & $9.96 \mathrm{e}+03$ & $\mathrm{n} / \mathrm{a}$ \\
\hline 5967005510 & $F$ & Vanadium -ICP-Fusion & ug/g & 99.80 & $<5.00 \mathrm{e}-02$ & $<9.98 \mathrm{e}+02$ & $<9.97 \mathrm{e} 2$ & $n / a$ & $\mathrm{n} / \mathrm{a}$ & $n / a$ & 996.0 & $\mathrm{n} / \mathrm{a}$ \\
\hline 5967005510 & $\vec{F}$ & Zinc-1CP-fusion & $\mathrm{ug} / \mathrm{g}$ & 100.8 & $<1.00 \mathrm{e}-02$ & $<2.00 \mathrm{e}+02$ & $<1.99 \mathrm{e} 2$ & $\mathrm{n} / \mathrm{a}$ & $\mathrm{n} / \mathrm{a}$ & $\mathrm{n} / \mathrm{a}$ & 200.0 & $\mathrm{n} / \mathrm{a}$ \\
\hline S96TO & $F$ & Zirconium-ICP-Fusion & $\mathrm{ug} / \mathrm{g}$ & 97.60 & $<7.00 \mathrm{e}-02$ & $<2.00 \mathrm{e}+02$ & $<1.99 \mathrm{e} 2$ & $n / a$ & $n / a$ & $n / a$ & 200.0 & $n / a$ \\
\hline \$96T005510 & $F$ & Cobalt $t-60$ by GEA & $\mathrm{uCi} / \mathrm{g}$ & 98.72 & $<1.35 \mathrm{e}-02$ & $<1.18 \mathrm{e}-02$ & $<1.41 \mathrm{e}-2$ & $n / a$ & $\mathrm{n} / \mathrm{a}$ & $n / a$ & $1.20 \mathrm{e}-02$ & $n / a$ \\
\hline \$\%6TO & $F$ & Cesium- 137 by GEA & $\mathrm{UCi} / \mathrm{g}$ & 99.09 & $<2.89 \mathrm{e}-02$ & 24.70 & 25.10 & 24.90 & 1.61 & $n / a$ & $n / a$ & .960 \\
\hline S96T005510 & $F$ & Europium- 154 by GEA & $\mathrm{uCi} / \mathrm{g}$ & $\mathrm{n} / \mathrm{a}$ & $<3.45 \mathrm{e}-02$ & $<3.82 \mathrm{e}-02$ & $<3.32 \mathrm{e}-2$ & $n / a$ & $\mathrm{n} / \mathrm{a}$ & $\mathrm{n} / \mathrm{a}$ & $3.80 \mathrm{e}-02$ & $\mathrm{n} / \mathrm{a}$ \\
\hline 5967005510 & $F$ & 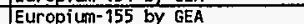 & $\mathrm{uCi} / \mathrm{g}$ & $\underline{n}$ & $<3.14 \mathrm{e}^{-02}$ & $<1.23 \mathrm{e}-01$ & $<1.20 \mathrm{e}-1$ & $n / a$ & $\mathrm{n} / \mathrm{a}$ & $n / a$ & $1.23 \mathrm{e}-01$ & $n / a$ \\
\hline
\end{tabular}




\begin{tabular}{|c|c|c|c|c|c|c|c|c|c|c|c|c|}
\hline mple\# & \begin{tabular}{l|l}
$\mathrm{R}$ & A\# \\
&
\end{tabular} & \#/Analyte & Unit & Standard \% & Blank & Result & Dupl icate & Average & RPD \% & Spk Rec $\%$ & Det Limit & Count Err\% \\
\hline S96T005510 & $\frac{n}{F}$ & Americium-241 by GEA & $u C i / g$ & $n / a$ & $<7.02 e-02$ & $<3.14 \mathrm{e}-01$ & $<3.08 \mathrm{e}-1$ & $n / a$ & $n / a$ & $n / a$ & $3.14 \mathrm{e}-01$ & $n / a$ \\
\hline $596 r 005513$ & $\bar{W}$ & Fluoride-1C-Dionex $4000 / 4500$ & $\mathrm{ug} / \mathrm{g}$ & 103.4 & $<1.20 e-02$ & $1.48 e+04$ & $1.24 \mathrm{e}+04$ & $1.36 \mathrm{e}+04$ & 17.6 & $n / a$ & 51.18 & $n / a$ \\
\hline S96T005513 & $\vec{W}$ & Chloride-IC-Dionex $4000 / 4500$ & $\operatorname{lng} / \mathrm{g}$ & 97.34 & $4.60 e-02$ & $1.76 \mathrm{e}+03$ & 865.0 & $1.31 \mathrm{e}+03$ & 68.2 & $n / a$ & 72.49 & $\mathrm{n} / \mathrm{a}$ \\
\hline S96T & $W$ & Nitrite-IC - Dionex $4000 / 4500$ & $u g / g$ & 96.30 & $<1.08 \mathrm{e}-01$ & $1.11 \mathrm{e}+04$ & $9.88 \mathrm{e}+03$ & $1.05 e+04$ & 11.6 & $n / a$ & 460.5 & $\mathrm{n} / \mathrm{a}$ \\
\hline S961 & $\mathbf{W}$ & Bromide by Ion Chromatograph & ug/g & 100.3 & $<1.25 e-01$ & $<5.33 \mathrm{e}+02$ & $<5.03 \mathrm{e} 2$ & $n / a$ & $n / a$ & $\mathrm{n} / \mathrm{a}$ & 533.0 & $n / a$ \\
\hline S96T005513 & $\bar{W}$ & Nitrate by IC-Dionex $4000 / 4500$ & ug/g & 97.98 & $<1.39 e-01$ & $7.76 \mathrm{e}+04$ & $6.91 e+04$ & $7.34 \mathrm{e}+04$ & 11.6 & $\mathrm{n} / \mathrm{a}$ & 592.6 & $\mathrm{n} / \mathrm{a}$ \\
\hline$\frac{0701005513}{5967005513}$ & 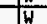 & Phosphate-IC-Dionex $4000 / 4500$ & $49 / 9$ & 95.22 & $<1.20 \mathrm{e}-01$ & $1.27 e+05$ & $1.05 \mathrm{e}+05$ & $1.16 \mathrm{e}+05$ & 19.0 & $n / a$ & 511.8 & $\mathrm{n} / \mathrm{a}$ \\
\hline S96T005513 & $\bar{W}$ & Sulfate by IC-Dionex $4000 / 4500$ & ug/g & 100.5 & $<1.38 \mathrm{e}-01$ & $8.21 \mathrm{e}+03$ & $6.85 e+03$ & $7.53 e+03$ & 18.1 & $n / a$ & 588.4 & \\
\hline 967005513 & $\bar{w}$ & Oxalate by IC-Dionex $4000 / 4500$ & $\mathrm{ug} / \mathrm{g}$ & 106.7 & $<1.05 e-01$ & $<4.48 \mathrm{e}+02$ & $<4.23 \mathrm{e} 2$ & $n / a$ & $\mathrm{n} / \mathrm{a}$ & $\mathrm{n} / \mathrm{a}$ & 447.7 & a \\
\hline
\end{tabular}

\begin{tabular}{|c|c|c|c|}
\hline Sample\# & R AA\# & Analyte & Unit \\
\hline S96T005503 & & Bulk Density of Sample & $\mathrm{g} / \mathrm{mL}$ \\
\hline S96T005506 & & DSC Exotherm using Mettler & Joules/g \\
\hline 5961005506 & & DSC Exotherm Dry Calculated & Joules/g Dry \\
\hline \$96T005506 & & $\%$ Water by TGA using Mettler & $\%$ \\
\hline S96T005511 & $\bar{F}$ & Uranium by Phosphorescence & $u g / g$ \\
\hline S96T005511 & $\mathbf{F}$ & U-Phosphorescence Inst. Error & \% Inst Error \\
\hline S96T005511 & $F$ & Strontium-89/90 High Level & $u C i / 9$ \\
\hline S96T005511 & $F$ & Nickel-1CP-Fusion & 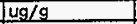 \\
\hline S96T005511 & $\mathbf{F}$ & Silver-ICP-Fusion & ug/g \\
\hline \$967005511 & $\mathbf{F}$ & Aluminiun -ICP-Fusion & $\underline{\mu g} / \mathrm{g}$ \\
\hline \$96T005511 & $\mathbf{F}$ & Arsenic-ICP-Fusion & ug $/ 9$ \\
\hline S96T005511 & $\mathbf{F}$ & Boron-ICP-Fusion & $u \mathrm{~g} / \mathrm{g}$ \\
\hline S96T005511 & $\mathbf{F}$ & Barium-ICP-Fusion & ug/g \\
\hline S96T005511 & $\mathbf{F}$ & Beryllium-1CP-Fusion & $4 \mathrm{gg} / \mathrm{g}$ \\
\hline S96T005511 & $F$ & Bismuth -ICP-Fusion & ug $/ \mathrm{g}$ \\
\hline S967005511 & $F$ & Calcium-ICP-Fusion & $4 \mathrm{~g} / \mathrm{g}$ \\
\hline \$96T005511 & $F$ & Cadmium-icp-Fusion & $u g / g$ \\
\hline S96T005511 & $F$ & Cerium-ICP-Fusion & $u g / g$ \\
\hline 59670055511 & $\mathbf{F}$ & Cobalt -ICP-Fusion & $\mathrm{ug} / \mathrm{g}$ \\
\hline S96T005511 & $F$ & Chromium-ICP-Fusion & ug/g \\
\hline S96T005511 & $F$ & Copper -ICP-Fusion & $\mathrm{ug} / \mathrm{g}$ \\
\hline $596 \mathrm{~T} 005511$ & $\vec{F}$ & Iron-ICP-Fusion & $\mathrm{ug} / \mathrm{g}$ \\
\hline \$967005511 & $\mathrm{F}$ & Lanthanum - ICP-Fusion & $u g / g$ \\
\hline 5967005511 & $\bar{F}$ & Lithium -ICP-Fusion & $\mathrm{ug} / \mathrm{g}$ \\
\hline S96T005511 & $\bar{F}$ & Magnesium-ICP-Fusion & $\mathrm{ug} / \mathrm{g}$ \\
\hline S96T005511 & $F$ & Manganese -ICP-Fusion & $u g / g$ \\
\hline S96T005511 & $F$ & Molybdenum -1CP-Fusion & $u g / g$ \\
\hline \$96T005511 & $\mathbf{F}$ & Sodium-ICP-Fusion & $\mathrm{ug} / \mathrm{g}$ \\
\hline$\$ 967005511$ & $F$ & Neodymium-1CP-Fusion & $\mathrm{ug} / \mathrm{g}$ \\
\hline 5967005511 & $\mathbf{F}$ & Phosphorus - ICP-Fusion & $u g / g$ \\
\hline S96T005511 & $F$ & Lead-ICP-Fusion & $\mathrm{ug} / \mathrm{g}$ \\
\hline S96T005511 & $F$ & sulfur-ICP-Fusion & $u g / g$ \\
\hline 5967005511 & $F$ & Antimony-1CP-Fusion & $u g / g$ \\
\hline S96T005511 & $\mathbf{F}$ & Selenium-ICP-Fusion & $u g / g$ \\
\hline S9670 & $\mathbf{F}$ & Silicon-ICP-Fusion & $\mathrm{ug} / \mathrm{g}$ \\
\hline 59690 & $F$ & Samarium-ICP-Fusion & $u g / \mathrm{g}$ \\
\hline S967005511 & $\mathbf{F}$ & Strontium-1CP-Fusion & $\mathrm{ug} / \mathrm{g}$ \\
\hline S96T00551 & & ium-ICP-Fusio & $\mathrm{ug} / \mathrm{g}$ \\
\hline
\end{tabular}

\begin{tabular}{|c|c|c|c|c|c|c|c|c|}
\hline standard \% & Blank & Result & Duplicate & Average & RPD \% & Spk Rec \% & Det Limit & Count Err\% \\
\hline$n / a$ & $n / a$ & 1.690 & $n / a$ & $n / a$ & $n / a$ & $n / a$ & $5.00 \mathrm{e}-01$ & $n / a$ \\
\hline 100.9 & $n / a$ & $0.00 \mathrm{e}+00$ & $0.00 \mathrm{e}+00$ & $0.00 e^{+00}$ & 0.00 & $n / a$ & $n / a$ & $n / a$ \\
\hline $\mathrm{n} / \mathrm{a}$ & $\mathrm{n} / \mathrm{a}$ & $0.00 e+00$ & $0.00 e+00$ & $0.00 \mathrm{e}+00$ & 0.00 & $n / a$ & $\mathrm{n} / \mathrm{a}$ & $n / a$ \\
\hline 100.3 & $n / a$ & 34.10 & 34.68 & 34.39 & 1.69 & $n / a$ & $n / a$ & $\mathrm{n} / \mathrm{a}$ \\
\hline 110.2 & 4.580 & $1.28 \mathrm{e}+03$ & $1.26 \mathrm{e}+03$ & $1.27 \mathrm{e}+03$ & 1.57 & 111.7 & 1.950 & $1.31 E+00$ \\
\hline 3.22 & 2.310 & 1.310 & 1.330 & 1.320 & 1.33 & $\mathrm{n} / \mathrm{a}$ & $n / a$ & $\mathrm{n} / \mathrm{a}$ \\
\hline 100.6 & $2.00 \mathrm{e}-03$ & $4.25 e-01$ & $3.88 \mathrm{e}-01$ & $4.06 \mathrm{e}-01$ & 9.10 & $\mathrm{n} / \mathrm{a}$ & $3.00 \mathrm{e}-03$ & $3.28 \mathrm{E}+00$ \\
\hline 99.20 & $8.60 e-02$ & $1.59 \mathrm{e}+03$ & $1.64 \mathrm{e}+03$ & $1.62 \mathrm{e}+03$ & 3.10 & 99.60 & 435.0 & $n / a$ \\
\hline 98.00 & $<7.00 \mathrm{e}-02$ & $<2.16 e+02$ & $<2.34 \mathrm{e} 2$ & $n / a$ & $n / a$ & 92.10 & 216.0 & $\mathrm{n} / \mathrm{a}$ \\
\hline 96.60 & $<5.00 e-02$ & $9.61 \mathrm{e}+03$ & $1.01 \mathrm{e}+04$ & $9.86 \mathrm{e}+03$ & 4.97 & 96.50 & $9.08 \mathrm{e}+03$ & $n / a$ \\
\hline 101.6 & $<1.00 \mathrm{e}-01$ & $<2.16 \mathrm{e}+03$ & $<2.34 \mathrm{e} 3$ & $n / a$ & $n / a$ & 103.0 & $2.16 \mathrm{e}+03$ & $\mathrm{n} / \mathrm{a}$ \\
\hline 98.40 & $<5.00 \mathrm{e}-02$ & $<1.08 \mathrm{e}+03$ & $<1.17 \mathrm{e}^{3}$ & $\mathrm{n} / \mathrm{a}$ & $n / a$ & 97.80 & $1.08 \mathrm{e}+03$ & $n / a$ \\
\hline 98.20 & $<5.00 \mathrm{e}-02$ & $<1.08 \mathrm{e}+03$ & $<1.17 \mathrm{e} 3$ & $\mathrm{n} / \mathrm{a}$ & $n / a$ & 95.90 & $1.08 \mathrm{e}+03$ & $\mathrm{n} / \mathrm{a}$ \\
\hline 102.0 & $<5.00 \mathrm{e}-03$ & $<1.08 \mathrm{e}+02$ & $<1.17 \mathrm{e} 2$ & $n / a$ & $n / a$ & 99.90 & 108.0 & $n / a$ \\
\hline 100.4 & $<1.00 \mathrm{e}-01$ & $<2.16 \mathrm{e}+03$ & $<2.34 \mathrm{e} 3$ & $\mathrm{n} / \mathrm{a}$ & $n / a$ & 105.0 & $2.16 \mathrm{e}+03$ & $n / a$ \\
\hline 98.80 & $<1.00 \mathrm{e}-01$ & $<2.16 \mathrm{e}+03$ & $<2.34 \mathrm{e} 3$ & $n / a$ & $n / a$ & 100.0 & $2.16 \mathrm{e}+03$ & $n / a$ \\
\hline 99.40 & $<5.00 \mathrm{e}-03$ & $<1.08 \mathrm{e}+02$ & $<1.17 \mathrm{e} 2$ & $\mathrm{n} / \mathrm{a}$ & $n / a$ & 99.40 & 108.0 & $n / a$ \\
\hline 98.20 & $<1.00 e-01$ & $<2.16 \mathrm{e}+03$ & $<2.34 \mathrm{e} 3$ & $\mathrm{n} / \mathrm{a}$ & $n / a$ & 97.80 & $2.16 \mathrm{e}+03$ & $n / a$ \\
\hline 99.80 & $<2.00 \mathrm{e}-02$ & $<4.33 e+02$ & $<4.69 \mathrm{e} 2$ & $n / a$ & $n / a$ & 99.60 & 435.0 & $n / a$ \\
\hline 100.0 & $<1.00 \mathrm{e}-02$ & $2.74 \mathrm{e}+02$ & 296.0 & 285.0 & 7.72 & 99.90 & 216.0 & $n / a$ \\
\hline 103.4 & $<1.00 \mathrm{e}-02$ & $<2.16 e+02$ & $<2.34 \mathrm{e} 2$ & $n / a$ & $n / a$ & 102.0 & 216.0 & $n / a$ \\
\hline 99.20 & $<5.00 e-02$ & $<1.08 \mathrm{e}+03$ & $<1.17 e^{3}$ & $n / a$ & $n / a$ & 103.0 & $1.08 \mathrm{e}+03$ & $n / a$ \\
\hline 99.80 & $45.00 \mathrm{e}-02$ & $<1.08 \mathrm{e}+03$ & $<1.17 \mathrm{e}^{3}$ & $\mathrm{n} / \mathrm{a}$ & $\mathrm{n} / \mathrm{a}$ & 98.80 & $1.08 \mathrm{e}+03$ & $n / a$ \\
\hline 99.00 & $<1.00 \mathrm{e}-02$ & $<2.16 \mathrm{e}+02$ & $<2.34 \mathrm{e} 2$ & $n / a$ & $n / a$ & 96.50 & 216.0 & $n / a$ \\
\hline 95.00 & $<1.00 \mathrm{e}-01$ & $<2.16 \mathrm{e}+03$ & $<2.34 \mathrm{e}^{3}$ & $\mathrm{n} / \mathrm{a}$ & $n / a$ & 95.40 & $2.16 \mathrm{e}+03$ & $n / a$ \\
\hline 96.60 & $<1.00 \mathrm{e}-02$ & $<2.16 \mathrm{e}+02$ & $<2.34 \mathrm{e} 2$ & $\mathrm{n} / \mathrm{a}$ & $n / a$ & 94.60 & 216.0 & $n / a$ \\
\hline 100.0 & $<5.00 \mathrm{e}-02$ & $<1.08 \mathrm{e}+03$ & $<1.17 \mathrm{e} 3$ & $\mathrm{n} / \mathrm{a}$ & $n / a$ & 100.0 & $1.08 \mathrm{e}+03$ & $\mathrm{n} / \mathrm{a}$ \\
\hline 95.60 & 1.670 & $2.30 \mathrm{e}+05$ & $2.30 \mathrm{e}+05$ & $2.30 \mathrm{e}+05$ & 0.00 & 102.0 & $2.16 \mathrm{e}+03$ & $n / a$ \\
\hline 99.60 & $<1.00 \mathrm{e}-01$ & $<2.16 \mathrm{e}+03$ & $<2.34 \mathrm{e}^{3}$ & $\mathrm{n} / \mathrm{a}$ & $n / a$ & 98.10 & $2.16 \mathrm{e}+03$ & $n / a$ \\
\hline 99.60 & $<2.00 e^{-01}$ & $8.93 e+03$ & $1.29 \mathrm{e}+04$ & $1.09 \mathrm{e}+04$ & 36.4 & 99.90 & $4.35 e+03$ & $n / a$ \\
\hline 98.80 & $<1.00 e^{-01}$ & $<2.16 \mathrm{e}+03$ & $<2.34 \mathrm{e}^{3}$ & $n / a$ & $n / a$ & 101.0 & $2.16 \mathrm{e}+03$ & $n / a$ \\
\hline 98.00 & $<1.00 \mathrm{e}-01$ & $4.80 \mathrm{e}+04$ & $4.35 e+04$ & $4.58 \mathrm{e}+04$ & 9.84 & 100.0 & $2.16 \mathrm{e}+03$ & $n / a$ \\
\hline 95.00 & $<6.00 \mathrm{e}-02$ & $<7.30 \mathrm{e}+03$ & $<1.41 \mathrm{e}^{3}$ & $n / a$ & $n / a$ & 95.40 & $1.30 \mathrm{e}+03$ & $n / a$ \\
\hline 96.40 & $<1.00 \mathrm{e}-01$ & $<2.16 e+03$ & $<2.34 \mathrm{e}^{3}$ & $n / a$ & $n / a$ & 101.0 & $2.16 \mathrm{e}+03$ & $n / a$ \\
\hline 98.80 & $<5.00 \mathrm{e}-02$ & $1.42 \mathrm{e}+03$ & $1.50 \mathrm{e}+03$ & $1.46 \mathrm{e}+03$ & 5.48 & 99.90 & $1.08 \mathrm{e}+03$ & $n / a$ \\
\hline 96.60 & $<1.00 \mathrm{e}-01$ & $<2.16 e+03$ & $<2.34 \mathrm{e}^{3}$ & $n / a$ & $\mathrm{n} / \mathrm{a}$ & 95.30 & $2.16 \mathrm{e}+03$ & $\mathrm{n} / \mathrm{a}$ \\
\hline 97.40 & $<1.00 \mathrm{e}-02$ & $<2.16 \mathrm{e}+02$ & $<2.34 \mathrm{e} 2$ & $\mathrm{n} / \mathrm{a}$ & $n / a$ & 95.70 & 216.0 & $n / a$ \\
\hline 97.20 & $<1.00 e-02$ & $<2.16 \mathrm{e}+02$ & $<2.34 \mathrm{e} 2$ & $n / a$ & $n / a$ & 96.60 & 216.0 & $n / a$ \\
\hline
\end{tabular}




\begin{tabular}{|c|c|c|c|c|c|c|c|c|c|c|c|c|}
\hline Sample\# & R|A\# & Analyte & Unit & Standard \% & Blank & Result & Dupl icate & Average & $\mathrm{RPD} \%$ & Spk Rec \% & Det Limit & Count Err\% \\
\hline S967005511 & $F$ & Thallium-ICP-Fusion & $\mathrm{ug} / \mathrm{g}$ & 96.40 & $<2.00 \mathrm{e}-01$ & $<4.33 \mathrm{e}+03$ & $<4.69 \mathrm{e} 3$ & $n / a$ & $n / a$ & 94.90 & $4.35 e+03$ & $n / a$ \\
\hline 5967005511 & $F$ & Uranium-ICP-Fusion & $49 / 9$ & 94.60 & $<5.00 \mathrm{e}-01$ & $<1.08 \mathrm{e}+04$ & $<1.17 \mathrm{e} 4$ & $\mathrm{n} / \mathrm{a}$ & $n / a$ & 91.00 & $1.08 \mathrm{e}+04$ & $\mathrm{n} / \mathrm{a}$ \\
\hline 5967005511 & $F$ & Vanadium-ICP-Fusion & $49 / 9$ & 99.80 & $\leqslant 5.00 \mathrm{e}-02$ & $<1.08 \mathrm{e}+03$ & $<1.17 \mathrm{e}^{3}$ & $\mathrm{n} / \mathrm{a}$ & $\mathrm{n} / \mathrm{a}$ & 99.00 & $1.08 \mathrm{e}+03$ & $n / a$ \\
\hline 5967005511 & $F$ & Zinc-ICP-Fusion & ug/g & 100.8 & $<1.00 \mathrm{e}-02$ & $<2.16 \mathrm{e}+02$ & $<2.34 \mathrm{e} 2$ & $n / a$ & $\mathrm{n} / \mathrm{a}$ & 101.0 & 216.0 & $\mathrm{n} / \mathrm{a}$ \\
\hline S967005511 & $F$ & Zirconium -ICP-fusion & ug $/ 9$ & 97.60 & $<1.00 \mathrm{e}-02$ & $<2.16 \mathrm{e}+02$ & $<2.34 \mathrm{e} 2$ & $n / a$ & $\mathrm{n} / \mathrm{a}$ & 96.60 & 216.0 & $n / a$ \\
\hline S96T005511 & $F$ & Cobal $t-60$ by GEA & $\mathrm{uCi} / \mathrm{g}$ & 98.72 & $<1.35 e^{-02}$ & $<1.21 e-02$ & $<1.70 e-2$ & $n / a$ & $n / a$ & $n / a$ & $1.20 \mathrm{e}-02$ & $n / a$ \\
\hline 5967005511 & $F$ & Cesium- 137 by GEA & $\mathrm{UCi} / \mathrm{g}$ & 99.09 & $<2.89 e-02$ & $7.63 \mathrm{e}=02$ & 39.00 & 19.54 & 3.28 & $\mathrm{n} / \mathrm{a}$ & $n / a$ & $4.03 e+01$ \\
\hline S96T005511 & $F$ & Europium- 154 by GEA & $\mathrm{uCi} / \mathrm{g}$ & $n / a$ & $<3.45 \mathrm{e}-02$ & $<3.90 \mathrm{e}=02$ & $<2.91 \mathrm{e}-2$ & $\mathrm{n} / \mathrm{a}$ & $n / a$ & $\mathrm{n} / \mathrm{a}$ & $3.90 e-02$ & $n / a$ \\
\hline 5961005511 & $\bar{F}$ & Europium- 155 by GEA & $\mathrm{uCi} / \mathrm{g}$ & $n / a$ & $<3.14 \mathrm{e}-02$ & $<1.56 \mathrm{e}-01$ & $<1.63 \mathrm{e}-1$ & $\mathrm{n} / \mathrm{a}$ & $n / a$ & $\mathrm{n} / \mathrm{a}$ & $1.56 e-01$ & $n / a$ \\
\hline S96T005511 & $F$ & Americium-241 by GEA & $\mathrm{uCi} / \mathrm{g}$ & $n / a$ & $<7.02 e-02$ & $<4.09 \mathrm{e}-01$ & $<4.17 \mathrm{e}-1$ & $\mathrm{n} / \mathrm{a}$ & $n / a$ & $\mathrm{n} / \mathrm{a}$ & $4.09 e-01$ & $n / a$ \\
\hline S961005511 & $F$ & Beta of Solid Sample & $\mathrm{uCi} / \mathrm{g}$ & 109.5 & $<7.38 \mathrm{e}-02$ & 41.40 & 35.90 & 38.65 & 14.2 & 95.36 & $1.70 e-02$ & $4.24 E-01$ \\
\hline 5961005511 & $\bar{F}$ & Alpha of Digested Solid & $\mathrm{uCi} / \mathrm{g}$ & 85.28 & $<1.69 e-03$ & $3.99 e-03$ & $3.84 \mathrm{e}-03$ & $3.92 \mathrm{e}-03$ & 3.83 & 45.40 & $4.00 \mathrm{e}-03$ & $9.19 E+01$ \\
\hline 5967005514 & $\mathbf{W}$ & Filuoride-IC-Dionex $4000 / 4500$ & $\mathrm{ug} / \mathrm{g}$ & 103.4 & $<1.20 \mathrm{e}-02$ & $3.79 \mathrm{e}+04$ & $4.09 \mathrm{e}+04$ & $3.94 e+04$ & 7.61 & 106.4 & 231.1 & $n / a$ \\
\hline 5961005514 & W & Chloride-IC-Dionex $4000 / 4500$ & ug $/ g$ & 97.34 & $4.60 \mathrm{e}-02$ & $1.78 \mathrm{e}+03$ & $1.88 \mathrm{e}+03$ & $1.83 \mathrm{e}+03$ & 5.46 & 93.04 & 327.4 & $n / a$ \\
\hline 5967005514 & W & Nitrite-IC - Dionex $4000 / 4500$ & ug/g & 96.30 & $<1.08 \mathrm{e}-01$ & $2.23 \mathrm{e}+04$ & $2.40 \mathrm{e}+04$ & $2.310+04$ & 7.34 & 93.35 & $2.08 e+03$ & $\mathrm{n} / \mathrm{a}$ \\
\hline 5961005514 & $\mathbf{W}$ & Bromide by Ion Chromatograph & $\log / g$ & 96.27 & $<12.60$ & $<9.77 e+02$ & $<1.06 \mathrm{e} 3$ & $n / a$ & $\mathrm{n} / \mathrm{a}$ & 90.32 & 977.4 & $n / a$ \\
\hline 5961005514 & W & Mitrate by IC-Dionex $4000 / 4500$ & $49 / 9$ & 97.98 & $<1.39 e-01$ & $1.51 \mathrm{e}+05$ & $1.62 \mathrm{e}+05$ & $1.57 \mathrm{e}+05$ & 7.03 & 106.9 & $2.68 \mathrm{e}+03$ & $n / a$ \\
\hline 5961005514 & $\mathbf{W}$ & Phosphate-IC-Dionex $4000 / 4500$ & $\mathrm{ug} / \mathrm{g}$ & 95.22 & $<1.20 \mathrm{e}-01$ & $4.09 e+04$ & $3.60 \mathrm{e}+04$ & $3.84 \mathrm{e}+04$ & 12.7 & 92.28 & $2.31 \mathrm{e}+03$ & $n / a$ \\
\hline 5961005514 & $\hat{\mathbf{W}}$ & Sulfate by IC-Dionex $4000 / 4500$ & ug/g & 100.5 & $<1,38 \mathrm{e}-01$ & $1.49 \mathrm{e}+05$ & $1.69 \mathrm{e}+05$ & $1.59 \mathrm{e}+05$ & 12.6 & 106.3 & $2.66 \mathrm{e}+03$ & $n / a$ \\
\hline 5967005574 & W & oxalate by IC-Dionex $4000 / 4500$ & $49 / 9$ & 106.7 & $<1.05 \mathrm{e}-01$ & $<2.02 e+03$ & $<2.20 \mathrm{e} 3$ & $n / a$ & $n / a$ & 103.4 & $2.02 e+03$ & $n / a$ \\
\hline 5961005519 & $\bar{I}$ & Silver -ICP-H2O Dig/ACid & ug/g & 97.40 & $\leq 1.00 \mathrm{e}-02$ & $<\quad 19.60$ & $<2.13 \mathrm{e} 1$ & $n / a$ & $n / a$ & 98.70 & 19.60 & $\bar{n} / \mathrm{a}$ \\
\hline 5967005519 & $I$ & Aluminium-ICP-H2O Dig/ACid & $09 / 9$ & 100.0 & $3.39 e-01$ & $1.11 \mathrm{e}+03$ & $1.15 \mathrm{e}+03$ & $1.13 \mathrm{e}+03$ & 3.54 & 97.50 & 97.90 & $n / a$ \\
\hline S96r005519 & I & Arsenic -ICP-H2O Dig/Acid & ug/g & 103.6 & $<1.00 \mathrm{e}-01$ & $<1.96 e+02$ & $<2.13 \mathrm{e} 2$ & $\mathrm{n} / \mathrm{a}$ & $n / a$ & 105.0 & 196.0 & $n / a$ \\
\hline 5967005519 & $I$ & Boron-ICP-H2O Dig/ACid & ug/g & 101.0 & 3.570 & $2.18 e+03$ & $2.37 e+03$ & $2.28 \mathrm{e}+03$ & 8.35 & 103.0 & 97.90 & $n / a$ \\
\hline S967005519 & $\bar{I}$ & Barium-ICP-H2O Dig/ACid & ug/g & 103.6 & $<5.00 \mathrm{e}-02$ & 97.90 & $<1.06 \mathrm{e} 2$ & $n / a$ & $n / a$ & 103.0 & 97.90 & $n / a$ \\
\hline S967005519 & I & Beryllium -ICP-H2O Dig/ACid & ug/g & 107.4 & $<5.00 e-03$ & 9.790 & $<1.06 \mathrm{e} 1$ & $n / a$ & $n / a$ & 102.0 & 9.790 & $n / a$ \\
\hline $596 r 005519$ & I & Bismuth - ICP-H2O Dig/ACid & ug/g & 105.8 & $<1.00 \mathrm{e}-01$ & $<1.96 e+02$ & $<2.13 e^{2}$ & $n / a$ & $\mathrm{n} / \mathrm{a}$ & 109.0 & 196.0 & $n / a$ \\
\hline 5967005519 & I & Calcjum-ICP-H2O Dig/Acid & $4 g / 9$ & 98.00 & $<1.00 \mathrm{e}-01$ & $<1.96 \mathrm{e}+02$ & $<2.13 \mathrm{e} 2$ & $n / a$ & $\mathrm{n} / \mathrm{a}$ & 98.70 & 196.0 & $n / a$ \\
\hline S96T & I & Cadmium -ICP-H2O Dig/ACid & $\mathrm{ug} / \mathrm{g}$ & 98.20 & $<5.00 \mathrm{e}-03$ & 9.790 & $<1.06 \mathrm{e} 1$ & $\mathrm{n} / \mathrm{a}$ & $n / a$ & 101.0 & 9.790 & $n / a$ \\
\hline \$96T & I & Cerium-ICP-H2O Dig/Acid & $\mathrm{ug} / \mathrm{g}$ & 107.8 & $<1.00 e-01$ & $<1.96 \mathrm{e}+02$ & $<2.13 \mathrm{e} 2$ & $\mathrm{n} / \mathrm{a}$ & $n / a$ & 102.0 & 196.0 & n/a \\
\hline S967005519 & I & Cobalt -ICP-H2O Dig/ACid & $\mathrm{ug} / \mathrm{g}$ & 99.40 & $<2.00 \mathrm{e}-02$ & 59.70 & 70.70 & 65.20 & 16.9 & 102.0 & 39.20 & $n / a$ \\
\hline 5519 & I & Chromitm - ICP-H2O Dig/Acid & $\mathrm{ug} / \mathrm{g}$ & 96.00 & $<7.00 \mathrm{e}-02$ & $2.54 \mathrm{e}+02$ & 281.0 & 267.5 & 10.1 & 99.50 & 19.60 & $n / a$ \\
\hline 5519 & I & Copper -ICP-H2O Dig/ACid & ug/g & 104.0 & $<1.00 \mathrm{e}-02$ & 19.60 & $<2.13 \mathrm{e} 1$ & $\mathrm{n} / \mathrm{a}$ & $n / a$ & 104.0 & 19.60 & $n / a$ \\
\hline 5519 & I & Iron-ICP-H2O Dig/ACId & ug/g & 98.00 & $<5.00 \mathrm{e}-02$ & 97.90 & $\leq 1.06 \mathrm{e} 2$ & $n / a$ & $n / a$ & 96.00 & 97.90 & $n / a$ \\
\hline S96T0 & II & Potassium -ICP-H2O Dig/ACid & $\mathrm{ug} / \mathrm{g}$ & 103.8 & $<5.00 e-01$ & $1.40 \mathrm{e}+03$ & $1.79 \mathrm{e}+03$ & $\mathrm{e}+03$ & 24.5 & 116.0 & 979.0 & $n / a$ \\
\hline 5961 & I & Lanthanum $-I C P-H 2 O \mathrm{Dig} /$ Acid & ug/g & 99.60 & $<5.00 \mathrm{e}-02$ & 97.90 & $<1.06 \mathrm{e} 2$ & $n / a$ & $n / a$ & 100.0 & 97.90 & $n / a$ \\
\hline S96T & I & Lithium - ICP-H2O Dig/Acid & $\mathrm{ug} / \mathrm{g}$ & 102.4 & $<1.00 \mathrm{e}-02$ & 19.60 & $<2.13 \mathrm{e} 1$ & $n / a$ & $n / a$ & 96.80 & 19.60 & $n / a$ \\
\hline 5967005519 & I & Magnesium - ICP-H2O Dig/Acid & ug/g & 96.60 & $<1.00 e-01$ & $<1.96 \mathrm{e}+02$ & $<2.13 \mathrm{e} 2$ & $\mathrm{n} / \mathrm{a}$ & $n / a$ & 98.90 & 196.0 & $n / a$ \\
\hline S967 & I & Manganese -ICP-H2O Dig/ACid & ug/g & 97.40 & $<7.00 \mathrm{e}-02$ & $<19.60$ & $<2.13 \mathrm{el}$ & $\mathrm{n} / \mathrm{a}$ & $n / a$ & 101.0 & 19.60 & $n / a$ \\
\hline 5961 & I & Holybdenum - ICP-H2O Dig/Acid & $\mathrm{ug} / \mathrm{g}$ & 100.8 & $<5.00 \mathrm{e}-02$ & 97.90 & $<1.06 \mathrm{e} 2$ & $n / a$ & $\mathrm{n} / \mathrm{a}$ & 104.0 & 97.90 & $\mathrm{n} / \mathrm{a}$ \\
\hline 5961 & I & Sodium-ICP-H2O Dig/Acid & ug/g & 108.2 & 5.150 & $2.16 \mathrm{e}+05$ & $2.23 \mathrm{e}+05$ & $2.20 \mathrm{e}+05$ & 3.19 & 118.0 & 196.0 & $\mathrm{n} / \mathrm{a}$ \\
\hline S96T005519 & $\mathrm{I}$ & Neodymi um $-I \mathrm{CP}-\mathrm{H} 20 \mathrm{Dig} /$ Acid & ug $/ 9$ & 100.8 & $<1.00 \mathrm{e}-01$ & $<1.96 \mathrm{e}+02$ & $<2.13 \mathrm{e} 2$ & $n / a$ & $n / a$ & 99.10 & 196.0 & $n / a$ \\
\hline S96TO & I & Nickel -ICP-H2O Dig/Acid & $u g / g$ & 97.60 & $<2.00 e-02$ & 39.20 & $<4.25 \mathrm{e} 1$ & $n / a$ & $n / a$ & 101.0 & 39.20 & $n / a$ \\
\hline $596 \mathrm{~T}$ & I & Phosphorus - ICP-H2O Dig/Acid & ug/g & 105.8 & $<2.00 \mathrm{e}-01$ & $1.42 \mathrm{e}+04$ & $1.23 \mathrm{e}+04$ & $1.32 e+04$ & 14.3 & 140.0 & 392.0 & $n / a$ \\
\hline 5519 & I & Lead -ICP-H2O Dig/Acid & ung/g & 96.40 & $<1.00 \mathrm{e}-01$ & $<1.960+02$ & $<2.13 \mathrm{e} 2$ & $n / a$ & $n / a$ & 104.0 & 196.0 & $n / a$ \\
\hline 5967005519 & I & Sulfur -ICP-H2O Dig/ACid & $\mathrm{ug} / \mathrm{g}$ & 100.8 & $<1.00 \mathrm{e}-01$ & $4.79 \mathrm{e}+04$ & $5.44 \mathrm{e}+04$ & $5.12 \mathrm{e}+04$ & 12.7 & 230.0 & 196.0 & $n / a$ \\
\hline S96T0 & I & Antimony - ICP-H2O Dig/Acid & ug/g & 101.6 & $<6.00 e-02$ & $<1.17 e+02$ & $<1.28 \mathrm{e} 2$ & $n / a$ & $n<a$ & 99.50 & 117.0 & $n / a$ \\
\hline S96T & I & Selenium - ICP-H2O Dig/Acid & $4 \mathrm{~g} / \mathrm{g}$ & 101.8 & $<1.00 e-01$ & $<1.96 \mathrm{e}+02$ & $<2.13 \mathrm{e} 2$ & $n / a$ & $n / a$ & 107.0 & 196.0 & $n / a$ \\
\hline $596 \mathrm{~T}$ & $\overline{\mathrm{I}}$ & Silicon - ICP-H2O Dig/Acid & ug/g & 99.20 & 7.280 & $1.68 \mathrm{e}+04$ & $1.85 e+04$ & $1.76 \mathrm{e}+04$ & 9.63 & 140.0 & 97.90 & $n / a$ \\
\hline 5967005519 & I & Samarium - ICP-H2O Dig/Acid & $4 \mathrm{~g} / \mathrm{g}$ & 104.6 & $<1.00 \mathrm{e}-01$ & $<1.96 \mathrm{e}+02$ & $<2.13 \mathrm{e} 2$ & $n / a$ & $n / a$ & 106.0 & 196.0 & $n / a$ \\
\hline $596 T 005519$ & $\bar{I}$ & Strontium - ICP-H2O Dig/ACid & ug/g & 103.4 & $<1.00 \mathrm{e}-02$ & 19.60 & $<2.13 \mathrm{e} 1$ & $n / a$ & $n / a$ & 104.0 & 19.60 & $n / a$ \\
\hline
\end{tabular}




\begin{tabular}{|c|c|c|c|c|c|c|c|c|c|c|c|c|}
\hline Sample\# & $\mathbf{R} \mid$ A\# & Analyte & Unit & Standard $\%$ & Blank & Result & Dupl icate & Average & RPD \% & Spk Rec \% & Det Limit & Count Err\% \\
\hline 5961005519 & I & Titanium-ICP-H2O Dig/ACid & $4 \mathrm{~g} / \mathrm{g}$ & 97.80 & $<1.00 \mathrm{e}-02$ & 19.60 & $<2.13 e 1$ & $n / a$ & $n / a$ & 99.00 & 19.60 & $n / a$ \\
\hline S96T005519 & I & Thallium - ICP-H2O Dig/Acid & $\mathrm{ug} / \mathrm{g}$ & 99.00 & $<2.00 \mathrm{e}-01$ & $<3.92 \mathrm{e}+02$ & $<4.25 \mathrm{e} 2$ & $\mathrm{n} / \mathrm{a}$ & $n / a$ & 99.10 & 392.0 & $n / a$ \\
\hline \$96T005519 & I & Uranium - ICP-H20 Dig/ACid & ug/g & 105.0 & $<5.00 \mathrm{e}=01$ & $<9.79 e+02$ & $<1.06 \mathrm{e} 3$ & $n / a$ & $n / a$ & 111.0 & 979.0 & $n / a$ \\
\hline S967005519 & I & Vanadium -ICP-H2O Dig/ACid & ug/g & 100.4 & $<5.00 \mathrm{e}=02$ & 97.90 & $<1.06 \mathrm{e} 2$ & $\mathrm{n} / \mathrm{a}$ & $n / a$ & 103.0 & 97.90 & $n / a$ \\
\hline \$96T005519 & I & Zinc -ICP-H2O Dig/Acid & ug/g & 100.6 & $2.30 \mathrm{e}-02$ & 19.60 & 42.70 & $n / a$ & $n / a$ & 105.0 & 19.60 & $\mathrm{n} / \mathrm{a}$ \\
\hline 967005519 & & Zirconium -ICP-H20 Dig/Acid & $u g / g$ & 101.0 & $<1.00 \mathrm{e}-02$ & 19.60 & $<2.13 \mathrm{e} 1$ & $n / a$ & $n / a$ & 103.0 & 19.60 & $n / a$ \\
\hline
\end{tabular}

\begin{tabular}{|c|c|c|c|c|c|c|c|c|c|c|c|c|}
\hline Sample\# & $\mathbf{R}$ A\# & \#Analyte & Unit & Standard \% & Blank & Resul $t]$ & Ouplicete & Average & RPD \% & Spk Rec \% & Det Limit & ount Err\% \\
\hline S96ro05523 & & DSC Exotherm Dry Calculated & Joules/g Dry & $n / a$ & $n / a$ & $0.00 \mathrm{e}+00$ & $0.00 \mathrm{e}+00$ & $0.00 \mathrm{e}+00$ & 0.00 & n/a & $n / a$ & $n / a$ \\
\hline S96T0055523 & & DSC Exotherm on Perkin Elmer & Joules/g & 97.47 & $n / a$ & $0.00 \mathrm{e}+00$ & $0.00 \mathrm{e}+00$ & $0.00 \mathrm{e}+00$ & 0.00 & $\mathrm{n} / \mathrm{a}$ & $n / a$ & $\mathrm{n} / \mathrm{a}$ \\
\hline S96T005523 & & Specific Gravity & Sp.G. & 99.67 & $n / a$ & 1.387 & 1.374 & 1.381 & 0.94 & n/a & $1.00 \mathrm{e}-03$ & $n / a$ \\
\hline S9670055523 & & $\%$ Water by JGA on Perkin Elmer & $\%$ & 98.73 & $\mathrm{n} / \mathrm{a}$ & 78.50 & 79.39 & 78.94 & 1.13 & $\mathrm{n} / \mathrm{a}$ & $n / a$ & $n / a$ \\
\hline S96T005523 & $\mathrm{D}$ & Silver-ICP-Acid Dil. & ug/mL & 98.60 & $\leq 1.00 \mathrm{e}-02$ & 12.40 & 12.70 & 12.55 & 2.39 & 91.60 & 4.010 & $\mathrm{n} / \mathrm{a}$ \\
\hline S96T005523 & D & Aluminium-ICP-Acid Dil. & $\mathrm{ug} / \mathrm{mL}$ & 97.40 & $<5.00 \mathrm{e}-02$ & $8.82 \mathrm{e}+02$ & 915.0 & 898.5 & 3.67 & 115.0 & 20.10 & $\mathrm{n} / \mathrm{a}$ \\
\hline S96T005523 & D & Arsenic-ICP-Acid Dil. & ug/mL & 103.6 & $<1.00 \mathrm{e}-01$ & $<40.10$ & $<4.01 \mathrm{e} 1$ & $n / a$ & $n / a$ & 105.0 & 40.10 & $n / a$ \\
\hline S96ro0 55523 & D & Boron-ICP-Acid Dil. & $\mathrm{ug} / \mathrm{mL}$ & 99.80 & $<5.00 \mathrm{e}-02$ & 20.30 & 21.60 & 20.95 & 6.21 & 99.10 & 20.10 & $n / a$ \\
\hline 59670055523 & D & Barium-ICP-Acid Dil. & $\mathrm{ug} / \mathrm{mL}$ & 98.40 & $<5.00 \mathrm{e}-02$ & 20.70 & $<2.01 \mathrm{e} 1$ & $n / a$ & $n / a$ & 99.00 & 20.10 & $n / a$ \\
\hline S96T005523 & D & Beryllium-ICP-Acid Dil. & $\mathrm{ug} / \mathrm{mL}$ & 102.8 & $<5.00 e-03$ & 2.000 & $<2,0000$ & n/a & $\mathrm{n} / \mathrm{a}$ & 101.0 & 2.000 & $\mathrm{n} / \mathrm{a}$ \\
\hline 5967005523 & D & Bismuth-ICP-Acid Dil. & $4 \mathrm{~g} / \mathrm{mL}$ & 107.6 & $<1.00 \mathrm{e}-01$ & 40.10 & $\leq 4.01 \mathrm{e} 1$ & $n / a$ & $\mathrm{n} / \mathrm{a}$ & 104.0 & 40.10 & $n / a$ \\
\hline 5967005523 & D & Calcium-ICP-Acid Dil. & ug $/ \mathrm{mL}$ & 99.20 & $<1.00 \mathrm{e}-01$ & 40.70 & $<4.01 \mathrm{e} 1$ & n/a & n/a & 101.0 & 40.10 & $n / a$ \\
\hline S96T005523 & D & Cadmilun-ICP-Acid Dil. & $\mathrm{ug} / \mathrm{mL}$ & 100.4 & $<5.00 \mathrm{e}-03$ & 2.000 & $<2.00 \mathrm{e} 0$ & $n / a$ & $n / a$ & 100.0 & 2.000 & $n / a$ \\
\hline S96r005523 & D & Cerium-ICP-ACid Dil. & $.4 \mathrm{~g} / \mathrm{mL}$ & 98.60 & $<1.00 \mathrm{e}-01$ & $<\quad 40.10$ & $<4.01 \mathrm{e} 1$ & n/a & $n / a$ & 100.0 & 40.10 & $n / a$ \\
\hline S96T005523 & D & Cobalt-ICP-Acid Dil. & $\mathrm{ug} / \mathrm{mL}$ & 900.6 & $<2.00 \mathrm{e}-02$ & 8.020 & $<8.02 \mathrm{e} 0$ & $n / a$ & $n / a$ & 100.0 & 8.020 & $n / a$ \\
\hline S96T005523 & $D$ & Chromium-ICP-Acid Dil. & $\mathrm{ug} / \mathrm{mL}$ & 100.6 & $<7.00 \mathrm{e}-02$ & $5.91 \mathrm{e}+02$ & 602.0 & 596.5 & 1.84 & 111.0 & 4.010 & $n / a$ \\
\hline S96T005523 & D & Copper-ICP-Acid Dil. & $\mathrm{ug} / \mathrm{mL}$ & 104.2 & $<1.00 \mathrm{e}-02$ & 4.070 & $<4.01 \mathrm{e} 0$ & $\mathrm{n} / \mathrm{a}$ & $n / a$ & 105.0 & 4.010 & $n / a$ \\
\hline S96T0055523 & D & Iron-ICP-Acid Dil. & $4 \mathrm{~g} / \mathrm{mL}$ & 100.0 & $<5.00 \mathrm{e}=02$ & 20.10 & $<2.01 \mathrm{e} 1$ & $\mathrm{n} / \mathrm{a}$ & $\mathrm{n} / \mathrm{a}$ & 99.40 & 20.10 & $n / a$ \\
\hline 5967005523 & $\bar{D}$ & Potassium-ICP-Acid Dil. & $\mathrm{ug} / \mathrm{mL}$ & 100.4 & $<5.00 \mathrm{e}-01$ & $3.16 \mathrm{e}+03$ & $3.31 \mathrm{e}+03$ & $3.24 \mathrm{e}+03$ & 4.64 & 164.0 & 200.0 & $n / a$ \\
\hline 5967005523 & D & Lanthanum-ICP-Acid Dil. & $\mathrm{ug} / \mathrm{mL}$ & 900.2 & $<5.00 \mathrm{e}-02$ & $<\quad 20.10$ & $<2.01 \mathrm{e} 1$ & $\mathrm{n} / \mathrm{a}$ & $n / a$ & 102.0 & 20.10 & $n / a$ \\
\hline S96T005523 & D & Lithium-ICP-Acid Dil. & $4 \mathrm{~g} / \mathrm{mL}$ & 100.4 & $<1.00 \mathrm{e}-02$ & 4.010 & $<4.01 \mathrm{e} 0$ & $n / a$ & $n / a$ & 103.0 & 4.010 & $n / a$ \\
\hline S96T005523 & D & Magnesiem-ICP-Acid Dil. & ug/irL & 96.20 & $<1.00 \mathrm{e}-01$ & 40.10 & $<4.01 \mathrm{e} 1$ & $n / a$ & $\mathrm{n} / \mathrm{a}$ & 95.70 & 40.10 & $n / a$ \\
\hline S96T005523 & D & Manganese-ICP-Acid Dil. & $\mathrm{ug} / \mathrm{mL}$ & 97.80 & $<1.00 e^{-02}$ & 4.010 & $<4.01 \mathrm{eO}$ & $n / a$ & $\mathrm{n} / \mathrm{a}$ & 95.60 & 4.070 & $n / a$ \\
\hline $596 T 005523$ & D & Molybdenum-ICP-Acid Díl. & $\mathrm{ug} / \mathrm{mL}$ & 100.6 & $<5.00 \mathrm{e}-02$ & 35.30 & 35.20 & 35.25 & 0.28 & 102.0 & 20.10 & $\mathrm{n} / \mathrm{a}$ \\
\hline 5967005523 & $\bar{D}$ & Sodium-ICP-Acid Dil. & $4 \mathrm{~g} / \mathrm{mL}$ & 96.40 & $<1.000=01$ & $1.72 \mathrm{e}+05$ & $1.76 \mathrm{e}+05$ & $1.74 \mathrm{e}+05$ & 2.30 & $3.55 \mathrm{e}+03$ & 40.10 & $n / a$ \\
\hline S96T005523 & D & Neodymi um-ICP-Acid Dil. & $\mathrm{ug} / \mathrm{mL}$ & 100.4 & $<1.00 \mathrm{e}-01$ & 40.10 & $<4.01 \mathrm{e1}$ & $\mathrm{n} / \mathrm{a}$ & $n / a$ & 102.0 & 40.10 & $n / a$ \\
\hline S96T005523 & $\mathrm{D}$ & Nickel-ICP-Acid Dil. & ug/mL & 100.4 & $<2.00 \mathrm{e}-02$ & 8.020 & $<8.02 \mathrm{e} 0$ & $n / a$ & $n / a$ & 99.00 & 8.020 & $n / a$ \\
\hline S96T005523 & D & Phosphorus-ICP-Acid Dil. & $\mathrm{ug} / \mathrm{mL}$ & 99.60 & $<2.00 \mathrm{e}-01$ & $1.16 \mathrm{e}+03$ & $1.26 \mathrm{e}+03$ & $1.21 \mathrm{e}+03$ & 8.26 & 124.0 & 80.20 & $\mathrm{n} / \mathrm{a}$ \\
\hline 5967005523 & D & Lead-ICP-Acid Dil. & ug/mL & 99.80 & $<1.00 \mathrm{e}-01$ & $<\quad 40.10$ & $<4.01 \mathrm{e} 1$ & n/a & $n / a$ & 102.0 & 40.10 & $n / a$ \\
\hline 5967005523 & $D$ & Sulfur-ICP-Acid Dil. & $\mathrm{ug} / \mathrm{mL}$ & 98.80 & $<1.00 e^{-01}$ & $4.69 \mathrm{e}+03$ & $4.82 e+03$ & $4.76 \mathrm{e}+03$ & 2.73 & 179.0 & 40.10 & $n / a$ \\
\hline 5967005523 & $D$ & Antimony-ICP-ACid Dil. & $\mathrm{ug} / \mathrm{mL}$ & 95.80 & $<6.00 e-02$ & 24.10 & $<2.41 \mathrm{e} 1$ & $n / a$ & $n / a$ & 98.10 & 24.10 & $n / a$ \\
\hline S96T005523 & $D$ & Selenium-ICP-ACid Dil. & ug $/ \mathrm{mL}$ & 97.20 & $<1.00 \mathrm{e}-01$ & 40.10 & $<4.01 \mathrm{e} 1$ & $\mathrm{n} / \mathrm{a}$ & $\mathrm{n} / \mathrm{a}$ & 108.0 & 40.10 & $n / a$ \\
\hline \$967005523 & D & Silicon-ICP-Acid Dil. & $\operatorname{lug} / \mathrm{mL}$ & 99.80 & $<5.00 \mathrm{e}-02$ & 59.50 & 82.00 & 70.75 & 31.8 & 121.0 & 20.10 & $n / a$ \\
\hline 5967005523 & D & Samarium-ICP-Acid Dit. & $\mathrm{ug} / \mathrm{mL}$ & 97.20 & $<7.00 e-01$ & 40.10 & $<4.01 \mathrm{e} 1$ & $n / a$ & $n / a$ & 97.90 & 40.10 & $\mathrm{n} / \mathrm{a}$ \\
\hline S96T005523 & D & Strontium-ICP-ACid Dil. & $\mathrm{ug} / \mathrm{mL}$ & 97.60 & $<1.00 \mathrm{e}-02$ & 4.010 & $<4.01 \mathrm{e} 0$ & $n / a$ & $n / a$ & 98.40 & 4.010 & $n / a$ \\
\hline 5967005523 & D & Titanilum-ICP-ACid Dil. & $\mathrm{ug} / \mathrm{mL}$. & 97.00 & $<7.00 \mathrm{e}=02$ & 4.010 & $<4.01 \mathrm{e} 0$ & $\mathrm{n} / \mathrm{a}$ & $\mathrm{n} / \mathrm{a}$ & 100.0 & 4.010 & $\mathrm{n} / \mathrm{a}$ \\
\hline 5967005523 & D & Thall ium-ICP-Acid Dil. & $\mathrm{ug} / \mathrm{mL}$ & 96.80 & $<2.00 \mathrm{e}-01$ & 80.20 & $\leq 8.02 \mathrm{e} 1$ & $n / a$ & $n / a$ & 95.50 & 80.20 & $n / a$ \\
\hline 5967005523 & D & Uraniun-ICP-Acid Dil. & $\mathrm{ug} / \mathrm{mL}$ & 95.20 & $<5.00 e^{-01}$ & $<2.000+02$ & $<2.00 \mathrm{e} 2$ & $n / a$ & $n / a$ & 98.00 & 200.0 & $n / a$ \\
\hline S96T005523 & D & Vanadium-1CP-Acid Dil. & $\mathrm{Lug} / \mathrm{mL}$ & 100.2 & $<5.00 e^{-02}$ & 20.10 & $\leq 2.01 \mathrm{e} 1$ & $n / a$ & $n / a$ & 101.0 & 20.10 & $n / a$ \\
\hline S967005523 & D & Zinc-ICP-Acid Dil. & $\mathrm{Lg} / \mathrm{mL}$ & 101.6 & $<1.00 \mathrm{e}-02$ & 4.010 & $<4.01 \mathrm{e0}$ & $n / a$ & $n / a$ & 103.0 & 4.010 & $n / a$ \\
\hline S96T005523 & D & Zirconium-1CP-Acid Dil & $\mathrm{ug} / \mathrm{mL}$ & 98.20 & $<9.00 \mathrm{e}-02$ & 4.010 & $<4.01 \mathrm{e} 0$ & $n / a$ & $n / a$ & 98.90 & 4.010 & $n / a$ \\
\hline
\end{tabular}




\begin{tabular}{|c|c|c|c|c|c|c|c|c|c|c|c|c|}
\hline Sample\# & R A\# & Analyte & Unit & Standard \% & Blank & Result & Duplicate & Average & RPD \% & Spk Rec \% & Det Limit & Count Err\% \\
\hline \$967005523 & & Fluoride-IC-Dionex $4000 / 4500$ & ug $/ \mathrm{mL}$ & 102.7 & $<1.20 \mathrm{e}^{-02}$ & $5.94 \mathrm{e}+02$ & $<1.22 \mathrm{e} 2$ & $\mathrm{n} / \mathrm{a}$ & $n / a$ & 77.97 & 122.4 & $n / a$ \\
\hline 5967005523 & & Chloride-ic-Dionex $4000 / 4500$ & $\mathrm{ug} / \mathrm{mL}$ & 95.70 & $2.00 \mathrm{e}=02$ & $4.79 \mathrm{e}+03$ & $4.38 \mathrm{e}+03$ & $4.58 \mathrm{e}+03$ & 8.94 & 87.34 & 173.4 & $n / a$ \\
\hline$\$ 967005523$ & & Nitrite-IC - Dionex $4000 / 4500$ & ug/mL. & 95.38 & $<1.08 \mathrm{e}-01$ & $5.43 e+04$ & $5.31 e+04$ & $5.37 e+04$ & 2.23 & 93.35 & $1.10 \mathrm{e}+03$ & $n / a$ \\
\hline S96T005523 & & Bromide by Ion Chromatograph & $\mathrm{ug} / \mathrm{mL}$ & 99.49 & $<1.25 e-01$ & $<1.28 \mathrm{e}+03$ & $<1.28 \mathrm{e} 3$ & $n / a$ & $\mathrm{n} / \mathrm{a}$ & 87.27 & $1.28 \mathrm{e}+03$ & $\mathrm{n} / \mathrm{a}$ \\
\hline 5967005523 & & Nitrate by IC-Dionex $4000 / 4500$ & $\mathrm{ug} / \mathrm{mL}$ & 98.15 & $<1.39 \mathrm{e}-01$ & $3.75 \mathrm{e}+05$ & $3.78 \mathrm{e}+05$ & $3.77 \mathrm{e}+05$ & 0.80 & 82.49 & $1.42 \mathrm{e}+03$ & $\mathrm{n} / \mathrm{a}$ \\
\hline S96T005523 & & Phosphate-1C-Dionex $4000 / 4500$ & $\mathrm{ug} / \mathrm{mL}$ & 95.77 & $<1.20 e-01$ & $2.74 \mathrm{e}+03$ & $3.22 \mathrm{e}+03$ & $2.98 \mathrm{e}+03$ & 16.1 & 92.83 & $1.22 \mathrm{e}+03$ & $n / a$ \\
\hline 5967005523 & & Sulfate by IC-Dionex $4000 / 4500$ & $\mathrm{ug} / \mathrm{mL}$ & 100.8 & $<1.38 \mathrm{e}-01$ & $1.38 \mathrm{e}+04$ & $1.34 \mathrm{e}+04$ & $1.36 \mathrm{e}+04$ & 2.94 & 99.37 & $7.41 \mathrm{e}+03$ & $n / a$ \\
\hline 5967005523 & & Oxalate by IC-Dionex $4000 / 4500$ & $\mathrm{ug} / \mathrm{mL}$ & 106.1 & $<1.05 e-01$ & $<1.07 \mathrm{e}+03$ & $<1.07 \mathrm{e} 3$ & $n / a$ & $\mathrm{n} / \mathrm{a}$ & 103.0 & $1.07 \mathrm{e}+03$ & $n / a$ \\
\hline S96T005523 & & Alpha in Liquid Samples & $\mathrm{uCi} / \mathrm{mL}$ & 113.0 & $<5.78 \mathrm{e}-03$ & $<5.04 e-03$ & $<2.85 \mathrm{E}-3$ & $\mathrm{n} / \mathrm{a}$ & $n / a$ & 98.33 & $7.00 \mathrm{e}-03$ & $5.00 E+02$ \\
\hline
\end{tabular}


Table 3. Data Sumary Table B-108

CORE NUMBER: 172

SEGMENT \#: 2

SEGMENT PORTION: L LOWer Half of Segment

\begin{tabular}{|c|c|c|c|c|c|c|c|c|c|c|c|c|}
\hline Sample\# & R A\# & Analyte & Unit & Standard \% & Blank & Result & Duplicate & Average & RPD \% & Spk Rec \% & Det Limit & Count Err\% \\
\hline S96T005507 & & DSC Exotherm using Mettler & Joules/g & 100.9 & $n / a$ & $0.00 \mathrm{e}+00$ & $0.00 \mathrm{e}+00$ & $0.00 e+00$ & 0.00 & $n / a$ & $n / a$ & $n / a$ \\
\hline$\$ 967005507$ & & DSC Exotherm Dry Calculated & joules/g Dry & $n / a$ & $n / a$ & $0.00 \mathrm{e}+00$ & $0.00 \mathrm{e}+00$ & $0,000+00$ & 0.00 & $n / a$ & $n / a$ & $n / a$ \\
\hline$\$ 967005507$ & & $\%$ Water by IGA using Metcler & $\%$ & 100.3 & $\mathrm{n} / \mathrm{a}$ & 18.54 & 20.12 & 19.33 & 8.17 & $\mathrm{n} / \mathrm{a}$ & $n / a$ & $\mathrm{n} / \mathrm{a}$ \\
\hline S967005512 & $F$ & Nickel -ICP-Fusion & ug/g & 99.20 & $8.60 e-02$ & $1.78 \mathrm{e}+03$ & $2.84 \mathrm{e}+03$ & $2.31 e+03$ & 45.9 & $n / a$ & 418.0 & $n / a$ \\
\hline$\$ 967005512$ & $F$ & Silver-ICP-Fusion & ug/g & 98.00 & $<1.00 \mathrm{e}-02$ & $3.56 \mathrm{e}+02$ & $<2.02 \mathrm{e} 2$ & $\mathrm{n} / \mathrm{a}$ & $\mathrm{n} / \mathrm{a}$ & $\mathrm{n} / \mathrm{a}$ & 209.0 & $\mathrm{n} / \mathrm{a}$ \\
\hline S967005512 & $F$ & Aluminium-ICP-Fusion & $49 / 9$ & 96.60 & $<5.00 \mathrm{e}-02$ & $8.53 \mathrm{e}+03$ & $8.76 \mathrm{e}+03$ & $8.64 e+03$ & 2.66 & $n / a$ & $1.05 \mathrm{e}+03$ & $n / a$ \\
\hline S96T005512 & $F$ & Arsenic-ICP-Fusion & ug $/ \mathrm{g}$ & 101.6 & $<1.00 \mathrm{e}-01$ & $<2.09 \mathrm{e}+03$ & $<2.02 \mathrm{e} 3$ & $n / a$ & $n / a$ & $\mathrm{n} / \mathrm{a}$ & $2.09 \mathrm{e}+03$ & $\mathrm{n} / \mathrm{a}$ \\
\hline S96T005512 & $\mathrm{F}$ & Boron-1CP-Fusion & ug/g & 98.40 & $<5.00 \mathrm{e}-02$ & $<1.05 \mathrm{e}+03$ & $<1.01 \mathrm{e} 3$ & $\mathrm{n} / \mathrm{a}$ & $n / a$ & $n / a$ & $1.05 e+03$ & $n / a$ \\
\hline S967005512 & $\mathbf{F}$ & Barium - ICP-Fusion & ug/g & 98.20 & $<5.00 e-02$ & $<1.05 e+03$ & $<1.01 \mathrm{e} 3$ & $\mathrm{n} / \mathrm{a}$ & $n / a$ & $n / a$ & $1.05 \mathrm{e}+03$ & $\mathrm{n} / \mathrm{a}$ \\
\hline 5967005512 & $F$ & Beryllium-ICP-Fusion & ug $/ 9$ & 102.0 & $<5.00 \mathrm{e}-03$ & $<1.05 \mathrm{e}+02$ & $<1.01 \mathrm{e}^{2}$ & $\mathrm{n} / \mathrm{a}$ & $n / a$ & $n / a$ & 105.0 & $\mathrm{n} / \mathrm{a}$ \\
\hline$\$ 967005512$ & $F$ & Bismuth -ICP-Fusion & $\mathrm{ug} / \mathrm{g}$ & 100.4 & $<1.00 \mathrm{e}-01$ & $<2.09 \mathrm{e}+03$ & $<2.02 \mathrm{e} 3$ & $\mathrm{n} / \mathrm{a}$ & $\mathrm{n} / \mathrm{a}$ & $n / a$ & $2.09 e+03$ & $\mathrm{n} / \mathrm{a}$ \\
\hline$\$ 967005512$ & $\mathbf{F}$ & Calcium-ICP-Fusion & ug/g & 98.80 & $<1.00 \mathrm{e}-01$ & $<2.09 \mathrm{e}+03$ & $<2.02 \mathrm{e} 3$ & $n / a$ & $n / a$ & $n / a$ & $2.09 \mathrm{e}+03$ & $n / a$ \\
\hline 5967005512 & $F$ & Cadmiun -ICP-Fusion & $4 \mathrm{~g} / \mathrm{g}$ & 99.40 & $<5.00 e-03$ & $<1.05 \mathrm{e}+02$ & $<1.01 \mathrm{e} 2$ & $\mathrm{n} / \mathrm{a}$ & $n / a$ & $n / a$ & 105.0 & $\mathrm{n} / \mathrm{a}$ \\
\hline 5967005512 & $F$ & Cerium-ICP-Fusion & ug/g & 98.20 & $<1.00 \mathrm{e}-01$ & $<2.09 \mathrm{e}+03$ & $<2.02 \mathrm{e} 3$ & $n / a$ & $n / a$ & $n / a$ & $2.09 e+03$ & $n / a$ \\
\hline 5967005512 & $F$ & Cobalt - ICP-Fusion & $\mathrm{ug} / \mathrm{g}$ & 99.80 & $<2.00 \mathrm{e}-02$ & $<4.19 \mathrm{e}+02$ & $<4.03 \mathrm{e} 2$ & $\mathrm{n} / \mathrm{a}$ & $n / a$ & $n / a$ & 418.0 & $\mathrm{n} / \mathrm{a}$ \\
\hline \$96T005512 & $\bar{F}$ & Chromium-ICP-Fusion & ug/g & 100.0 & $<1.00 \mathrm{e}-02$ & $<2.09 \mathrm{e}+02$ & $<2.02 \mathrm{e} 2$ & $n / a$ & $n / a$ & $\mathrm{n} / \mathrm{a}$ & 209.0 & $n / a$ \\
\hline 5967005512 & $F$ & Copper -ICP-Fusion & $49 / 9$ & 103.4 & $<1.00 \mathrm{e}-02$ & $<2.09 \mathrm{e}+02$ & $<2.02 \mathrm{e} 2$ & $n / a$ & $\mathrm{n} / \mathrm{a}$ & $n / a$ & 209.0 & $n / a$ \\
\hline$\$ 967005512$ & $\mathbf{F}$ & Iron-ICp-Fusion & ug/g & 99.20 & $<5.00 \mathrm{e}-02$ & $\leq 1.05 \mathrm{e}+03$ & $<1.01 \mathrm{e} 3$ & $n / a$ & $\mathrm{n} / \mathrm{a}$ & $n / a$ & $1.05 e+03$ & $n / a$ \\
\hline 5967005512 & $F$ & Lanthanum - ICP-Fusion & tug/g & 99.80 & $<5.00 e^{-02}$ & $<1.05 \mathrm{e}+03$ & $<1.01 \mathrm{e} 3$ & $n / a$ & $n / a$ & $n / a$ & $1.05 \mathrm{e}+03$ & $\mathrm{n} / \mathrm{a}$ \\
\hline$\$ 967005512$ & $F$ & Lithium-ICP-Fusion & ug $/ 9$ & 99.00 & $<1.00 \mathrm{e}-02$ & $<2.09 \mathrm{e}+02$ & $<2.02 \mathrm{e} 2$ & $n / a$ & $n / a$ & $n / a$ & 209.0 & $n / a$ \\
\hline$\$ 967005512$ & $F$ & Magnesium-ICP-Fusion & $49 / 9$ & 95.00 & $<1.00 e^{-01}$ & $<2.09 \mathrm{e}+03$ & $<2.02 \mathrm{e} 3$ & $n / a$ & $\mathrm{n} / \mathrm{a}$ & $n / a$ & $2.09 \mathrm{e}+03$ & $\mathrm{n} / \mathrm{a}$ \\
\hline$\$ 967005512$ & $F$ & Manganese-ICP-Fusion & ug/g & 96.60 & $<1.00 \mathrm{e}-02$ & $<2.09 \mathrm{e}+02$ & $<2.02 \mathrm{e} 2$ & $n / a$ & $\mathrm{n} / \mathrm{a}$ & $\mathrm{n} / \mathrm{a}$ & 209.0 & $n / a$ \\
\hline 5967005512 & $F$ & Molybodenum - ICP-Fusion & ug/g & 100.0 & $<5.00 \mathrm{e}-02$ & $<1.05 \mathrm{e}+03$ & $<1.01 \mathrm{e} 3$ & $n / a$ & $\mathrm{n} / \mathrm{a}$ & $n / a$ & $1.05 \mathrm{e}+03$ & $n / a$ \\
\hline 5967005512 & $F$ & Sodium-ICP-Fusion & ug $/ g$ & 95.60 & 1.670 & $2.98 \mathrm{e}+05$ & $2.97 e+05$ & $2.98 \mathrm{e}+05$ & 0.34 & $\mathrm{n} / \mathrm{a}$ & $2.09 e+03$ & $\mathrm{n} / \mathrm{a}$ \\
\hline 5967005512 & $F$ & Neodymium-1CP-Fusion & ug/g & 99.60 & $<1.00 \mathrm{e}-01$ & $<2.09 \mathrm{e}+03$ & $<2.02 \mathrm{e} 3$ & $\mathrm{n} / \mathrm{a}$ & $\mathrm{n} / \mathrm{a}$ & $n / a$ & $2.09 \mathrm{e}+03$ & $n / a$ \\
\hline 5967005512 & $F$ & Phosphorus -ICP-Fusion & ug/g & 99.60 & $<2.00 e-01$ & $2.30 \mathrm{e}+04$ & $1.19 \mathrm{e}+04$ & $1.74 e+04$ & 63.6 & $n / a$ & $4.18 \mathrm{e}+03$ & $n / a$ \\
\hline 5967005512 & $\mathbf{F}$ & Lead -1CP-Fusion & ug $/ \mathrm{g}$ & 98.80 & $<1.00 \mathrm{e}-01$ & $<2.09 \mathrm{e}+03$ & $<2.02 \mathrm{e} 3$ & $\mathrm{n} / \mathrm{a}$ & $\mathrm{n} / \mathrm{a}$ & $n / a$ & $2.09 \mathrm{e}+03$ & $n / a$ \\
\hline S96\%O & $\mathbf{F}$ & Sulfur - ICP-Fusion & ug/g & 98.00 & $<1.00 \mathrm{e}-01$ & $8.48 \mathrm{e}+04$ & $9.44 \mathrm{e}+04$ & $8.96 e+04$ & 10.7 & $n / a$ & $2.09 e+03$ & $n / a$ \\
\hline S96T005512 & $F$ & Ant imony -ICP-Fusion & ug/g & 95.00 & $<6.00 \mathrm{e}-02$ & $<1.26 \mathrm{e}+03$ & $<1.21 \mathrm{e}$ & $\mathrm{n} / \mathrm{a}$ & $\mathrm{n} / \mathrm{a}$ & $n / a$ & $1.25 e+03$ & $n / a$ \\
\hline \$967005512 & $F$ & Selenium-ICP-Fusion & $4 \mathrm{~g} / \mathrm{g}$ & 96.40 & $<1.00 e-01$ & $<2.09 \mathrm{e}+03$ & $<2.02 \mathrm{e} 3$ & $\mathrm{n} / \mathrm{a}$ & $n / a$ & $n / a$ & $2.09 \mathrm{e}+03$ & $\mathrm{n} / \mathrm{a}$ \\
\hline S967005512 & $F$ & Silicon-ICP-Fusion & $4 \mathrm{~g} / \mathrm{g}$ & 98.80 & $<5.00 \mathrm{e}-02$ & $<1.05 e+03$ & $<1.01 \mathrm{e} 3$ & $n / a$ & $\mathrm{n} / \mathrm{a}$ & $n / a$ & $1.05 e+03$ & $\mathrm{n} / \mathrm{a}$ \\
\hline S96TC & $F$ & Samarium - ICP-Fusion & $4 g / g$ & 96.60 & $<1.00 \mathrm{e}-01$ & $<2.09 \mathrm{e}+03$ & $<2.02 \mathrm{e} 3$ & $\mathrm{n} / \mathrm{a}$ & $n / a$ & $n / a$ & $2.09 e+03$ & $\mathrm{n} / \mathrm{a}$ \\
\hline S96r005512 & $\mathbf{F}$ & Strontium -ICP-Fusion & $\mathrm{ug} / \mathrm{g}$ & 97.40 & $<1.00 e^{-02}$ & $<2.09 \mathrm{e}+02$ & $\leq 2.02 \mathrm{e} 2$ & $\mathrm{n} / \mathrm{a}$ & $\mathrm{n} / \mathrm{a}$ & $n / a$ & 209.0 & $n / a$ \\
\hline S96T005512 & $F$ & Iitanium-ICP-Fusion & ug/g & 97.20 & $<1.00 \mathrm{e}-02$ & $<2.09 \mathrm{e}+02$ & $\leq 2.02 \mathrm{e} 2$ & $n / a$ & $\mathrm{n} / \mathrm{a}$ & $n / a$ & 209.0 & $n / a$ \\
\hline 5961005512 & $\mathbf{F}$ & Thall ium-ICP-Fusion & $49 / 9$ & 96.40 & $<2.00 e-01$ & $<4.19 \mathrm{e}+03$ & $<4.03 \mathrm{e} 3$ & $\mathrm{n} / \mathrm{a}$ & $n / a$ & $\mathrm{n} / \mathrm{a}$ & $4.18 \mathrm{e}+03$ & $n / a$ \\
\hline S96T0 & $\mathbf{F}$ & Uraniun-ICP-Fusion & ug/g & 94.60 & $55.00 \mathrm{e}-01$ & $<1.05 \mathrm{e}+04$ & $\leq 1.01 \mathrm{e}^{4}$ & $n / a$ & $n / a$ & $\mathrm{n} / \mathrm{a}$ & $1.05 e+04$ & $n / a$ \\
\hline 5967005512 & $\mathbf{F}$ & Vanadium-ICP-Fusion & $\mathrm{ug} / \mathrm{g}$ & 99.80 & $<5.00 \mathrm{e}-02$ & $<1.05 e+03$ & $<1.01 \mathrm{e} 3$ & $n / a$ & $n / a$ & $\mathrm{n} / \mathrm{a}$ & $1.05 \mathrm{e}+03$ & $n / a$ \\
\hline S967005512 & $\mathbf{F}$ & Zine-ICP-Fusion & w9/9 & 100.8 & $<1.00 \mathrm{e}-02$ & $<2.09 \mathrm{e}+02$ & $<2.02 \mathrm{e} 2$ & $n / a$ & $n / a$ & $n / a$ & 209.0 & $n / a$ \\
\hline S967005512 & $\mathbf{F}$ & Zirconium-ICP-Fusion & ug/g & 97.60 & $<1.00 \mathrm{e}-02$ & $<2.09 \mathrm{e}+02$ & $<2.02 \mathrm{e} 2$ & $\mathrm{n} / \mathrm{a}$ & $n / a$ & $n / a$ & 209.0 & $n / a$ \\
\hline 5967005512 & $F$ & Cobalt -60 by GEA & UCi/g & 97.36 & $<1.15 e^{-02}$ & $<1.12 \mathrm{e}-02$ & $<8.63 \mathrm{e}-3$ & $\mathrm{n} / \mathrm{a}$ & $\mathrm{n} / \mathrm{a}$ & $n / a$ & $1.10 \mathrm{e}-02$ & $\mathrm{n} / \mathrm{a}$ \\
\hline 512 & $f$ & Cesium- 137 by GEA & $\mathrm{uCi} / \mathrm{g}$ & 97.83 & $<3.00 \mathrm{e}-02$ & 15.62 & 19.60 & 17.61 & 22.7 & $\mathrm{n} / \mathrm{a}$ & $n / a$ & 1.25 \\
\hline$\$ 967005512$ & $F$ & Europium- 154 by GEA & uci $/ \mathrm{g}$ & $n / a$ & $<2.81 \mathrm{e}-02$ & $<4.46 \mathrm{e}-02$ & $<4.25 \mathrm{e}-2$ & $n / a$ & $n / a$ & $n / a$ & $4.50 e-02$ & $n / a$ \\
\hline 5967005512 & & Europium- 155 by GEA & $\mathrm{uCj} / \mathrm{g}$ & $n / a$ & $<3.42 \mathrm{e}-02$ & $<9.91 \mathrm{e}-02$ & $<1.07 \mathrm{e}-1$ & $\mathrm{n} / \mathrm{a}$ & $\mathrm{n} / \mathrm{a}$ & $n / a$ & $9.90 \mathrm{e}-02$ & $\mathrm{n} / \mathrm{a}$ \\
\hline 5967005512 & $\mathbf{F}$ & Americium-241 by $G$ & $=$ & & $<8$. & $<2$. & $<2$. & 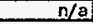 & $n / a$ & $n / a$ & $2.63 e-01$ & $n / a$ \\
\hline
\end{tabular}




\begin{tabular}{|c|c|c|c|c|c|c|c|c|c|c|c|c|}
\hline Sample\# & $\left.R\right|_{A H}$ & \# Analyte & Unit & Standard \% & Blank & Result & Dupl icate & Average & RPD \% & Spk Rec \% & Det Limit & ount Err\% \\
\hline S96T005512 & $F$ & Alpha of Digested Solid & $u C i / g$ & 98.70 & $<3.16 \mathrm{e}-03$ & $3.85 e-03$ & $4.42 e^{-03}$ & $4.13 e-03$ & 13.8 & $n / a$ & $2.00 e-03$ & $63 \mathrm{E}+01$ \\
\hline 5967005515 & $\bar{W}$ & Fluoride-1C-Dionex $4000 / 4500$ & $\mathrm{ug} / \mathrm{g}$ & 87.29 & $<1.270$ & $5.89 e+04$ & $7.02 \mathrm{e}+04$ & $6.46 \mathrm{e}+04$ & 17.5 & 129.0 & 249.9 & $\mathrm{n} / \mathrm{a}$ \\
\hline \$967005515 & $W$ & Chloride-IC-Dionex $4000 / 4500$ & $\mathrm{ug} / \mathrm{g}$ & 101.8 & $<1.720$ & $5.33 e+02$ & 887.0 & 709.9 & 49.9 & 113.9 & 353.9 & $n / a$ \\
\hline S967005515 & W & Nitrite-IC - Dionex $4000 / 4500$ & ug/g & 103.9 & $<10.90$ & $9.65 \mathrm{e}+03$ & $1.02 \mathrm{e}+04$ & $9.93 e+03$ & 5.54 & 120.5 & $2.25 e+03$ & $n / a$ \\
\hline 5967005515 & W & Bromide by Ion Chromatograph & ug/g & 96.27 & $<12.60$ & $<1.06 \mathrm{e}+03$ & $<7.03 \mathrm{e}^{3}$ & $n / a$ & $n / a$ & 110.9 & $1.06 \mathrm{e}+03$ & $n / a$ \\
\hline$\$ 967005515$ & $\omega$ & Nitrate by IC-Dionex $4000 / 4500$ & $\mathrm{wg} / \mathrm{g}$ & 100.8 & $<14.00$ & $7.24 \mathrm{e}+04$ & $7.72 \mathrm{e}+04$ & $7.48 \mathrm{e}+04$ & 6.42 & 120.0 & $2.89 \mathrm{e}+03$ & $n / a$ \\
\hline$\$ 967005515$ & I & Phosphate-IC-D ionex $4000 / 4500$ & ug/g & 93,38 & $<12.10$ & $9.33 \mathrm{e}+05$ & $3.77 \mathrm{e}+04$ & $8.54 e+04$ & 112 & 126.3 & $2.50 \mathrm{e}+03$ & $n / 8$ \\
\hline 5967005515 & W & Sul fate by IC-0ionex $4000 / 4500$ & $40 / 9$ & 100.2 & $<13.90$ & $2.90 \mathrm{e}+05$ & $3.23 e+05$ & $2.67 \mathrm{e}+05$ & 42.4 & 130.7 & $2.87 \mathrm{e}+03$ & $n / a$ \\
\hline 5967005515 & W & Oxalate by IC-Dionex $4000 / 4500$ & ug/g & 102.1 & $<10.60$ & $<2.19 \mathrm{e}+03$ & $<2.14 \mathrm{e}^{\mathrm{s}}$ & $n / a$ & $n / a$ & 118.1 & $2.19 \mathrm{e}+03$ & $n / a$ \\
\hline
\end{tabular}


CORE NUMBER: 173

SEGMENT \#: Core Composite

SEGMENT PORTION: Core Composite

\begin{tabular}{|c|c|c|c|c|c|c|c|c|c|c|c|c|c|}
\hline & Samole\# & Aft & Analyte & Unit & Standard \% & Blank & Result & Duplicate & Average & RPD \% & Spk Rec \% & Det Limit & Count Err\% \\
\hline & S96T005483 & & Bulk Density of Sample & $\mathrm{g} / \mathrm{mL}$ & $n / a$ & $n / a$ & 1.790 & $n / a$ & $n / a$ & $n / a$ & $n / a$ & $5.00 \mathrm{e}-01$ & $\mathrm{n} / \mathrm{a}$ \\
\hline & \begin{tabular}{|l|} 
s96T005485 \\
\end{tabular} & & DSC Exotherm using Mettler & Joules/g & 95.61 & $\mathrm{n} / \mathrm{a}$ & $0.00 \mathrm{e}+00$ & $0.00 \mathrm{e}+00$ & $0.00 \mathrm{e}+00$ & 0.00 & $n / a$ & $n / a$ & $n / a$ \\
\hline & \$96T005485 & & DSC Exotherm Dry Calculated & Joules/g Dry & $n / a$ & $\mathrm{n} / \mathrm{a}$ & $0.00 e+00$ & $0.00 \mathrm{e}+00$ & $0.00 \mathrm{e}+00$ & 0.00 & $\mathrm{n} / \mathrm{a}$ & $n / a$ & $n / a$ \\
\hline & S96T005485 & & $\%$ water by TGA using Mettler & $\%$ & 99.09 & $n / a$ & 39.65 & 30.95 & 35.30 & 24.6 & $n / a$ & $n / a$ & $n / a$ \\
\hline & \begin{tabular}{|l}
57060005485 \\
5967005485
\end{tabular} & & itc by Acid/coulometry & ug/g & 100.2 & 4.200 & $1.51 \mathrm{e}+03$ & $1.26 \mathrm{e}+03$ & $1.38 \mathrm{e}+03$ & 18.1 & 96.00 & 5.000 & $n / a$ \\
\hline & S96T005485 & & Toc by Persulfate/Coulometry & $u g / g$ & 93.67 & 3.500 & $3.39 \mathrm{e}+02$ & 294.0 & 316.5 & 14.2 & 92.60 & 40.00 & $n / a$ \\
\hline & S96T005487 & $F$ & Uranium by Phosphorescence & ug/g & 107.7 & 104.0 & $8.09 \mathrm{e}+02$ & $1.12 \mathrm{e}+03$ & 964.5 & 32.2 & 91.80 & 1.780 & $1.24 \mathrm{E}+00$ \\
\hline & S96T005487 & $F$ & U-Phosphorescence Inst. Error & $\%$ Inst Error & 3.50 & 1.150 & 1.240 & 1.540 & 1.390 & 1.54 & $n / a$ & $\mathrm{n} / \mathrm{a}$ & $n / a$ \\
\hline & S96T005487 & F & Stront ium-89/90 High Level & uCi $/ \mathrm{g}$ & 99,41 & $2.00 \mathrm{e}-03$ & $4.30 \mathrm{e}-01$ & 5.800 & 3.115 & 172 & $n / a$ & $2.00 \mathrm{e}-03$ & $2.29 E+00$ \\
\hline & S96T005487 & $F$ & Nickel -ICP-Fusion & $y g / g$ & 100.0 & 1.220 & $1.53 \mathrm{e}+03$ & $1.58 \mathrm{e}+03$ & $1.56 \mathrm{e}+03$ & 3.22 & 96.20 & 386.0 & $\mathrm{n} / \mathrm{a}$ \\
\hline & S96T005487 & $\mathrm{F}$ & Silver-ICp-Fusion & $\mathrm{ug} / \mathrm{g}$ & 99,00 & $<9.00 \mathrm{e}-02$ & $<1.93 e+02$ & $<1.96 \mathrm{e} 2$ & $n / a$ & $\mathrm{n} / \mathrm{a}$ & 87.30 & 193.0 & $\mathrm{n} / \mathrm{a}$ \\
\hline & \$96T005487 & $\bar{F}$ & Aluminium -ICP-Fusion & ug/a & 95.80 & $<5.00 \mathrm{e}-02$ & $1.97 \mathrm{e}+04$ & $7.23 \mathrm{e}+04$ & $4.60 \mathrm{e}+04$ & 114 & 85.10 & 964.0 & $\mathrm{n} / \mathrm{a}$ \\
\hline & \$96T005487 & $F$ & Arsenic -ICP-Fusion & $\mathrm{ug} / \mathrm{g}$ & 101.0 & $<1.00 \mathrm{e}-01$ & $<1.93 e+03$ & $<1.96 \mathrm{e} 3$ & $n / a$ & $n / a$ & 95.90 & $.93 e+03$ & $\mathrm{n} / \mathrm{a}$ \\
\hline & \begin{tabular}{|l} 
S96T005487 \\
\end{tabular} & $F$ & Boron-ICP-Fusion & ug/g & 97.80 & $<5.00 \mathrm{e}-02$ & $<9.64 e+02$ & $<9.78 \mathrm{e} 2$ & $n / a$ & $n / a$ & 88.70 & 964.0 & $\mathrm{n} / \mathrm{a}$ \\
\hline & S96T005487 & $\mathrm{F}$ & Barium-ICP-Fusion & $\mathrm{ug} / \mathrm{g}$ & 97.60 & $<5.00 \mathrm{e}-02$ & $<9.64 e+02$ & $<9.78 \mathrm{e} 2$ & $n / a$ & $n / a$ & 88.20 & 964.0 & $n / a$ \\
\hline \multirow{29}{*}{$\begin{array}{l}\infty \\
6\end{array}$} & \$96T005487 & $F$ & Beryllium -ICP-Fusion & $\mathrm{ug} / \mathrm{g}$ & 100.2 & $<5.00 e^{-03}$ & $<96.40$ & $<9.78 \mathrm{e} 1$ & $n / a$ & $n / a$ & 90.70 & 96.40 & $\mathrm{n} / \mathrm{a}$ \\
\hline & \$96T005487 & $F$ & Bismuth -ICP-Fusion & ug/g & 100.6 & $<1.00 \mathrm{e}-07$ & $<1.93 e+03$ & $<1.96 \mathrm{e} 3$ & $n / a$ & $\mathrm{n} / \mathrm{a}$ & 102.0 & $1.93 e+03$ & $\mathrm{n} / \mathrm{a}$ \\
\hline & \$96T005487 & $F$ & Calcium-ICP-Fusion & $\mathrm{ug} / \mathrm{g}$ & 101.0 & $<1.00 \mathrm{e}-01$ & $<7.93 e+03$ & $<1.96 \mathrm{e} 3$ & $n / a$ & $n / a$ & 99.40 & $1.93 \mathrm{e}+03$ & $\mathrm{n} / \mathrm{a}$ \\
\hline & S96T005487 & $F$ & Cadmium -ICP-Fusion & ug/g & 100.2 & $<5.00 \mathrm{e}-03$ & $<96.40$ & $<9.78 \mathrm{e} 1$ & $n / a$ & $\mathrm{n} / \mathrm{a}$ & 94.90 & 96.40 & $n / a$ \\
\hline & \$96T005487 & $F$ & Cerium-ICP-Fusion & $\mathrm{ug} / \mathrm{g}$ & 98.40 & $<1.00 \mathrm{e}-01$ & $\leq 1.93 \mathrm{e}+03$ & $<1.96 \mathrm{e} 3$ & $n / a$ & $\mathrm{n} / \mathrm{a}$ & 91.30 & $1.93 \mathrm{e}+03$ & $n / a$ \\
\hline & \$96T005487 & $F$ & Cobalt -ICP-Fusion & ug/g & 101.4 & $<2.00 \mathrm{e}-02$ & $<3.85 \mathrm{e}+02$ & $<3.91 \mathrm{e} 2$ & $n / a$ & $n / a$ & 96.40 & 386.0 & $\mathrm{n} / \mathrm{a}$ \\
\hline & \begin{tabular}{|l|}
967005487 \\
\end{tabular} & $F$ & Chromium -ICP-Fusion & $u g / g$ & 101.0 & $<1.00 \mathrm{e}-02$ & $<1.93 e+02$ & $<1.96 \mathrm{e} 2$ & $n / a$ & $n / a$ & 96.50 & 193.0 & $\mathrm{n} / \mathrm{a}$ \\
\hline & S96T005487 & $F$ & Copper-ICP-Fusion & $\mathrm{ug} / \mathrm{g}$ & 102.2 & $<1.00 \mathrm{e}-02$ & $<1.93 e+02$ & $<1.96 \mathrm{e} 2$ & $n / a$ & $n / a$ & 91.70 & 193.0 & $\mathrm{n} / \mathrm{a}$ \\
\hline & \$96T005487 & $\mathrm{F}$ & Iron-ICP-Fusion & ug/g & 99.60 & $<5.00 \mathrm{e}-02$ & $<9.64 \mathrm{e}+02$ & $3.29 \mathrm{e}+03$ & $n / a$ & $n / a$ & 93.70 & 964.0 & $\mathrm{n} / \mathrm{a}$ \\
\hline & \$96T005487 & $F$ & Lanthanum - ICP-Fusion & ug/g & 100.0 & $<5.00 \mathrm{e}-02$ & $<9.64 \mathrm{e}+02$ & $<9.78 \mathrm{e} 2$ & $n / a$ & $n / a$ & 90.90 & 964.0 & $n / a$ \\
\hline & $596 T 005487$ & F & Lithium-ICP-Fusion & ug/g & 96.80 & $<1.00 \mathrm{e}-02$ & $<1.93 e+02$ & $<1.96 \mathrm{e} 2$ & $\mathrm{n} / \mathrm{a}$ & $n / a$ & 85.20 & 193.0 & $\mathrm{n} / \mathrm{a}$ \\
\hline & \$96T005487 & F & Magnesium-ICP-Fusion & ug/g & 94.00 & $<1.00 \mathrm{e}-01$ & $<1.93 e+03$ & $<1.96 \mathrm{e}$ & $n / a$ & $\mathrm{n} / \mathrm{a}$ & 87.70 & $1.93 e+03$ & $\mathrm{n} / \mathrm{a}$ \\
\hline & 596T005487 & $F$ & Manganese -ICP-Fusion & ug/g & 97.20 & $<1.00 \mathrm{e}-02$ & $<1.93 \mathrm{e}+02$ & $<1.96 \mathrm{e} 2$ & $\mathrm{n} / \mathrm{a}$ & $n / a$ & 90.30 & 193.0 & $\mathrm{n} / \mathrm{a}$ \\
\hline & S96T & $F$ & Molybdenum - ICP-Fusion & $\mathrm{ug} / \mathrm{g}$ & 101.0 & $<5.00 \mathrm{e}-02$ & $<9.64 \mathrm{e}+02$ & $<9.78 \mathrm{e} 2$ & $n / a$ & $\mathrm{n} / \mathrm{a}$ & 95.60 & 964.0 & $\mathrm{n} / \mathrm{a}$ \\
\hline & 5967005487 & $F$ & Sodium - ICP-Fusion & $\mathrm{ug} / \mathrm{g}$ & 94.80 & 7.510 & $2.34 \mathrm{e}+05$ & $1.67 \mathrm{e}+05$ & $2.00 \mathrm{e}+05$ & 33.4 & 38.60 & $1.93 e+03$ & $n / a$ \\
\hline & $\$ 961005487$ & $F$ & Neodymium -ICP-Fusion & $\mathrm{ug} / \mathrm{g}$ & 99.60 & $<9.00 \mathrm{e}-01$ & $<7.93 e+03$ & $<1.96 \mathrm{e} 3$ & $\mathrm{n} / \mathrm{a}$ & $\mathrm{n} / \mathrm{a}$ & 89.00 & $1.93 \mathrm{e}+03$ & $n / a$ \\
\hline & \$961005487 & $F$ & Phosphorus -ICP-Fusion & ug/g & 98.40 & $<2.00 e-01$ & $5.62 \mathrm{e}+04$ & $3.52 \mathrm{e}+04$ & $4.57 e+04$ & 46.0 & 86.50 & $3.86 \mathrm{e}+03$ & $n / a$ \\
\hline & S96T005487 & $\mathrm{F}$ & Lead-ICP-Fusion & $\mathrm{ug} / \mathrm{g}$ & 100.4 & $<1.00 \mathrm{e}-01$ & $<1.93 e+03$ & $<1.96 \mathrm{e} 3$ & $n / a$ & $\mathrm{n} / \mathrm{a}$ & 97.70 & $1.93 \mathrm{e}+03$ & $n / a$ \\
\hline & $\$ 96 \mathrm{~T} 005487$ & F & Sulfur-ICP-Fusion & $\mathrm{ug} / \mathrm{g}$ & 98.20 & $<1.00 \mathrm{e}-01$ & $2.44 \mathrm{e}+04$ & $1.24 \mathrm{e}+04$ & $1.84 \mathrm{e}+04$ & 65.2 & 91.60 & $1.93 \mathrm{e}+03$ & $n / a$ \\
\hline & S96T005487 & $F$ & Antimony -ICP-Fusion & ug/g & 96.00 & $<6.00 e^{-02}$ & $<1.16 \mathrm{e}+03$ & $<1.17 \mathrm{e} 3$ & $n / a$ & $\mathrm{n} / \mathrm{a}$ & 91.10 & $1.16 \mathrm{e}+03$ & $n / a$ \\
\hline & $\$ 967005487$ & F & Selenium-ICP-Fusion & $\mathrm{ug} / \mathrm{g}$ & 95.00 & $<1.00 \mathrm{e}-01$ & $<1.93 e+03$ & $<1.96 \mathrm{e} 3$ & $n / a$ & $n / a$ & 93.30 & $1.93 \mathrm{e}+03$ & $n / a$ \\
\hline & $\$ \$ 967005487$ & $F$ & silicon-ICP-Fusion & ug/g & 96.80 & $<5.00 \mathrm{e}-02$ & $2.60 \mathrm{e}+03$ & $1.45 e+03$ & $2.02 e+03$ & 56.8 & 93.40 & 964.0 & $\mathrm{n} / \mathrm{a}$ \\
\hline & 5965005487 & $\dot{F}$ & Samarium-ICP-Fusion & ug/g & 96.60 & $<1.00 e-01$ & $<1.93 \mathrm{e}+03$ & $<1.96 \mathrm{e}$ & $n / a$ & $\mathrm{n} / \mathrm{a}$ & 92.00 & $9.93 \mathrm{e}+03$ & $n / a$ \\
\hline & 596T005487 & $\sqrt{F}$ & Strontiun -ICP-Fusion & ug/s & 97.00 & $<1.00 \mathrm{e}-02$ & $\leq 1.93 \mathrm{e}+02$ & $\leq 1.96 \mathrm{e} 2$ & $n / a$ & $\mathrm{n} / \mathrm{a}$ & 87.40 & 193.0 & $n / a$ \\
\hline & S96T005487 & $F$ & Titanium-1CP-Fusion & ug/g & 97.40 & $<1.00 e-02$ & $<1.93 e+02$ & $<1.96 \mathrm{e} 2$ & $n / a$ & $n / a$ & 89.60 & 193.0 & $n / a$ \\
\hline & 5967005487 & $F$ & Thallium-ICP-Fusion & $\mathrm{ug} / \mathrm{g}$ & 94.60 & $<2.00 \mathrm{e}-01$ & $<3.85 \mathrm{e}+03$ & $<3.91 \mathrm{e} 3$ & $n / a$ & $n / a$ & 93.30 & $3.86 \mathrm{e}+03$ & $n / a$ \\
\hline & S96r005487 & $F$ & Üranitum-ICP-Fusion & ug/g & 94.90 & $<5.00 \mathrm{e}-01$ & $<9.64 e+03$ & $<9.78 \mathrm{e} 3$ & $n / a$ & $n / a$ & 93.50 & $9.64 \mathrm{e}+03$ & $n / a$ \\
\hline & $\$ 967005487$ & $F$ & Vanadium -ICP-Fusion & ug/g & 100.4 & $<5,00 \mathrm{e}-02$ & $<9.64 \mathrm{e}+02$ & $<9.78 \mathrm{e} 2$ & $n / a$ & $n / a$ & 94.70 & 964.0 & $n / a$ \\
\hline & & & & 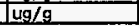 & & $<1.00 \mathrm{e}=02$ & $<1.938$ & $<1.96 \mathrm{e} 2$ & $n / a$ & $n / a$ & 99.50 & 193.0 & $n / a$ \\
\hline
\end{tabular}




\begin{tabular}{|c|c|c|c|c|c|c|c|c|c|c|c|c|}
\hline Sample\# & R A\# & \#Analyte & Unit & Standard \% & Blank & Result $t$ & Duplicate & Average & RPD \% & Spk Rec \% & Det Limit & Count Err\% \\
\hline 5967005487 & $\mathbf{F}$ & Zirconium-ICP-Fusion & $\log / 9$ & 98.60 & $<1.00 \mathrm{e}-02$ & $<1.93 \mathrm{e}+02$ & $<1.96 \mathrm{e} 2$ & $n / a$ & $n / a$ & 91.10 & 193.0 & $n / a$ \\
\hline S96T005487 & $F$ & Cobal $t-60$ by GEA & $\mathrm{uCi} / \mathrm{g}$ & 97.49 & $<1.15 \mathrm{e}-02$ & $<1.41 \mathrm{e}-02$ & $<1.18 \mathrm{e}-2$ & $n / a$ & n/a & $\mathrm{n} / \mathrm{a}$ & $1.40 \mathrm{e} \cdot 02$ & $n / a$ \\
\hline$\$ 967005487$ & $F$ & Cesium-137 by GEA & $\mathrm{uCi} / \mathrm{g}$ & 100.5 & $<1.72 \mathrm{e}-02$ & 10.89 & 15.30 & 13.10 & 33.6 & $\mathrm{n} / \mathrm{a}$ & $n / a$ & 1.45 \\
\hline $596 T 005487$ & $\bar{F}$ & Europium- 154 by GEA & $\mathrm{uCi} / \mathrm{g}$ & $n / a$ & $<3.21 \mathrm{e}-02$ & $<2.89 \mathrm{e}=02$ & $<3.76 \mathrm{e}-2$ & n/a & $\mathrm{n} / \mathrm{a}$ & $\mathrm{n} / \mathrm{a}$ & $2.90 \mathrm{e}-02$ & $n / a$ \\
\hline$\$ 967005487$ & F & Europium- 155 by GEA & $\mathrm{uCi} / \mathrm{g}$ & $\mathrm{n} / \mathrm{a}$ & $<3.11 \mathrm{e}-02$ & $\angle 8.40 \mathrm{e}-02$ & $\leq 1.00 \mathrm{e}-1$ & $n / a$ & $n / a$ & $n / a$ & $8.40 \mathrm{e}-02$ & $n / a$ \\
\hline S96T005487 & $F$ & Americium- 241 by GEA & $\mathrm{uCi} / \mathrm{g}$ & $\mathrm{n} / \mathrm{a}$ & $<7.26 \mathrm{e}-02$ & $<2.10 \mathrm{e}-01$ & $<2.47 \mathrm{e}-1$ & $n / a$ & $\mathrm{n} / \mathrm{a}$ & $\mathrm{n} / \mathrm{a}$ & $2.10 \mathrm{e}-01$ & $n / a$ \\
\hline S967005487 & F & Alpha of Digested Solid & $\mathrm{uCi} / \mathrm{g}$ & 92,64 & $<1.24 \mathrm{e}^{-02}$ & $<1.24 \mathrm{e}-02$ & $1.19 e-02$ & $n / a$ & $n / a$ & 74.37 & $1.90 \mathrm{e}-02$ & \\
\hline$\$ 967005487$ & $F$ & Beta of Solid Sample & $\mathrm{uCi} / \mathrm{g}$ & 99.66 & $<2.81 \mathrm{e}-02$ & 10.70 & 23.60 & 17.15 & 75.2 & 92.76 & $5.70 \mathrm{e}-02$ & $1.63 E+00$ \\
\hline $596 T 005488$ & $W$ & Fluoride-IC-Dionex $4000 / 4500$ & $\mathrm{ug} / \mathrm{g}$ & 110.7 & $<1.20 e^{-02}$ & $7.92 \mathrm{e}+04$ & $4.56 \mathrm{e}+04$ & $3.24 \mathrm{e}+04$ & 81.5 & 181.4 & 235.7 & $\mathrm{n} / \mathrm{a}$ \\
\hline $596 T 005488$ & W & Chioride-IC-Dionex $4000 / 4500$ & ug $/ 9$ & 95.06 & $2.70 e^{-02}$ & $8.84 \mathrm{e}+03$ & $1.14 \mathrm{e}+03$ & $4.99 \mathrm{e}+03$ & 154 & 43.92 & 333.9 & $\mathrm{n} / \mathrm{a}$ \\
\hline S96T005488 & $\frac{\pi}{4}$ & Mitrite-IC - Dionex $4000 / 4500$ & $u g / g$ & 95.93 & $<1.08 \mathrm{e}-01$ & $5.33 \mathrm{e}+03$ & $1.05 \mathrm{e}+04$ & $7.92 \mathrm{e}+03$ & 65.3 & 96.67 & $2.12 e+03$ & $n / a$ \\
\hline 5961005488 & W & Bromide by Ion Chromatograph & $u g / g$ & 98.64 & $<1.25 e-01$ & $<2.46 \mathrm{e}+03$ & $<2.44 \mathrm{e} 3$ & $\mathrm{n} / \mathrm{a}$ & $\mathrm{n} / \mathrm{a}$ & 102.0 & $2.46 \mathrm{e}+03$ & $\mathrm{n} / \mathrm{a}$ \\
\hline \$96T005488 & $\bar{\omega}$ & Nitrate by IC-Dionex $4000 / 4500$ & ug/g & 97.64 & $<1.39 \mathrm{e}-01$ & $1.94 \mathrm{e}+04$ & $4.05 e+04$ & $2.99 \mathrm{e}+04$ & 70.5 & 102.9 & $2.73 \mathrm{e}+03$ & $\mathrm{n} / \mathrm{a}$ \\
\hline \$9670 & $\mathbf{H}$ & Phosphate-IC-D ionex $4000 / 4500$ & $\mathrm{ug} / \mathrm{g}$ & 94.30 & $<1.20 \mathrm{e}-01$ & $1.30 \mathrm{e}+05$ & $7.64 \mathrm{e}+04$ & $1.03 \mathrm{e}+05$ & 51.9 & 150.7 & $2.36 \mathrm{e}+03$ & $n / a$ \\
\hline 5967005488 & $\mathrm{H}$ & Sul fate by IC-Dionex $4000 / 4500$ & $u g / g$ & 99.05 & $<1.38 \mathrm{e}-01$ & $2.25 e+04$ & $1.65 \mathrm{e}+05$ & $9.37 \mathrm{e}+04$ & 152 & 102.4 & $2.71 \mathrm{e}+03$ & $n / a$ \\
\hline S967005488 & $\ddot{\mathrm{H}}$ & Oxalate by IC-Dionex $4000 / 4500$ & ug/g & 704.2 & $<1.05 \mathrm{e}-01$ & $<2.06 \mathrm{e}+03$ & $<2.05 \mathrm{e} 3$ & $\mathrm{n} / \mathrm{a}$ & $n / a$ & 107.6 & $2.06 e+03$ & $\mathrm{n} / \mathrm{a}$ \\
\hline S967005489 & ! & Silver-ICP-H2O Dig/Acid & ug/g & 100.8 & $<1.00 e-02$ & 12.50 & 14.20 & 13.35 & 12.7 & 102.0 & 4.000 & $n / a$ \\
\hline \$96T005489 & 1 & Aluminium - ICP-H2O Dig/ACid & $u g / g$ & 99.80 & $3.01 e-01$ & $2.30 \mathrm{e}+02$ & 816.0 & 523.0 & 112 & 101.0 & 20.00 & $n / a$ \\
\hline$\$ 967005489$ & I & Arsenic - ICP-H2O Dig/Acid & $\mathrm{ug} / \mathrm{g}$ & 104.2 & $<1,00 \mathrm{e}-01$ & $<39.90$ & $<3.97 \mathrm{e} 1$ & $n / a$ & $\mathrm{n} / \mathrm{a}$ & 109.0 & 40.00 & $n / a$ \\
\hline$\$ 967005489$ & 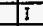 & Boron -ICP-H2O Dig/ACid & ug/g & 102.4 & 4.400 & $5.58 \mathrm{e}+02$ & $1.60 e+03$ & $1.08 \mathrm{e}+03$ & 96.6 & 103.0 & 20.00 & $n / a$ \\
\hline$\$ 967005489$ & $I$ & Barium -ICP-H2O Dig/ACid & $\mathrm{ug} / \mathrm{g}$ & 102.2 & $<5.00 e^{-02}$ & 20.00 & $<1.98 \mathrm{e} 1$ & $\mathrm{n} / \mathrm{a}$ & $\mathrm{n} / \mathrm{a}$ & 101.0 & 20.00 & $\mathrm{n} / \mathrm{a}$ \\
\hline 5967005489 & I & Beryllium -ICP-H20 Dig/ACid & ug/g & 104.4 & $<5.00 e-03$ & 2.000 & $<9.98 \mathrm{e} 0$ & $\mathrm{n} / \mathrm{a}$ & $n / a$ & 104.0 & 2.000 & $n / a$ \\
\hline S96T005489 & 1 & Bismuth - ICP-H2O Dig/ACid & $\operatorname{lug} / 9$ & 103.0 & $<1.00 \mathrm{e}^{-01}$ & 39.90 & $<3.97 \mathrm{e} 1$ & $\mathrm{n} / \mathrm{a}$ & $n / a$ & 108.0 & 40.00 & $\mathrm{n} / \mathrm{a}$ \\
\hline$\$ 96 T 005489$ & I & Calcium-ICP-H2O Dig/ACid & $49 / 9$ & 99.80 & $<1.00 \mathrm{e}-01$ & 39.90 & $<3.97 \mathrm{e} 1$ & $\mathrm{n} / \mathrm{a}$ & $\mathrm{n} / \mathrm{a}$ & 107.0 & 40.00 & $\mathrm{n} / \mathrm{a}$ \\
\hline S96T005489 & 1 & Cadmium - ICP-H2O Dig/ACid & ug/g & 101.6 & $<5.00 \mathrm{e}-03$ & 2.000 & $<1.98 \mathrm{eO}$ & $n / a$ & $\mathrm{n} / \mathrm{a}$ & 104.0 & 2.000 & $n / a$ \\
\hline S96T005489 & I & Cerium-ICP-H2O Dig/Acid & ug/g & 102.4 & $<1.00 e^{-01}$ & 39.90 & $<3.97 \mathrm{e} 1$ & $n / a$ & $n / a$ & 104.0 & 40.00 & $n / a$ \\
\hline 5961005489 & I & Cobalt - ICP-H2O Dig/ACid & ug/g & 102.0 & $<2.00 \mathrm{e}-02$ & 7.990 & $<7.93 \mathrm{e} 0$ & $\mathrm{n} / \mathrm{a}$ & $\mathrm{n} / \mathrm{a}$ & 103.0 & 7.980 & $n / a$ \\
\hline S96T & I & Chromium - ICP-H2O Dig/ACid & $\mathrm{ug} / \mathrm{g}$ & 102.0 & $<1.00 e^{-02}$ & 73.00 & 128.0 & 100.5 & 54.7 & 104.0 & 4.000 & $\mathrm{n} / \mathrm{a}$ \\
\hline $596 \mathrm{~T}$ & $I$ & Copper - ICP-H2O Dig/Acid & ug $/ 9$ & 107.6 & $<1.00 \mathrm{e}-02$ & 3.990 & $<3.97 \mathrm{e} 0$ & $n / a$ & $n / a$ & 106.0 & 4.000 & $n / a$ \\
\hline S96T005489 & 1 & Iron-ICP-H2O Dig/ACid & ug $/ \mathrm{g}$ & 101.8 & $<5.00 \mathrm{e}-02$ & 20.00 & $<1.98 \mathrm{e} 1$ & $\mathrm{n} / \mathrm{a}$ & $\mathrm{n} / \mathrm{a}$ & 102.0 & 20.00 & $\mathrm{n} / \mathrm{a}$ \\
\hline$\$ 96 r 005489$ & 1 & Potassium $-1 \mathrm{CP}-\mathrm{H} 2 \mathrm{O}$ Dig/Acid & $\mathrm{ug} / \mathrm{g}$ & 97.80 & $<5.00 e-01$ & $3.29 \mathrm{e}+02$ & 611.0 & 470.0 & 60.0 & 114.0 & 200.0 & $\mathrm{n} / \mathrm{a}$ \\
\hline S96T & I & Lanthanum $-\mathrm{ICP}-\mathrm{H} 20$ Dig/Acid & ug/g & 104.0 & $<5.00 e^{-02}$ & 20.00 & $<1.98 \mathrm{e} 1$ & $n / a$ & $n / a$ & 104.0 & 20.00 & $n / a$ \\
\hline $596 \mathrm{TO}$ & 1 & Lithium - ICP-H2O Dig/ACid & $4 \mathrm{~g} / \mathrm{g}$ & 104.8 & $<1.00 \mathrm{e}-02$ & 3.990 & $<3.97 \mathrm{e} 0$ & $\mathrm{n} / \mathrm{a}$ & $n / a$ & 105.0 & 4.000 & $n / a$ \\
\hline S96T005489 & I & Magnesium - ICP-H2O Dig/ACid & $u g / g$ & 98.60 & $<1.00 \mathrm{e}-01$ & 39.90 & $<3,97 \mathrm{e} 1$ & $\mathrm{n} / \mathrm{a}$ & $\mathrm{n} / \mathrm{a}$ & 98.00 & 40.00 & $\mathrm{n} / \mathrm{a}$ \\
\hline $596 \mathrm{TO}$ & I & Manganese -ICP-H2O Dig/ACid & ug/g & 98.80 & $<1.00 \mathrm{e}^{-02}$ & 3.990 & $<3.97 \mathrm{e} 0$ & $n / a$ & $\mathrm{n} / \mathrm{a}$ & 98.60 & 4.000 & $n / a$ \\
\hline S967 & I & Molybdenum -ICP-H2O Dig/ACid & $\mathrm{ug} / \mathrm{g}$ & 102.4 & $<5.00 e-02$ & 20.00 & $<1.98 \mathrm{e} 1$ & $n / a$ & $n / a$ & 106.0 & 20.00 & $n / a$ \\
\hline $596 \mathrm{TO}$ & I & Sodium-ICP-H2O Dig/ACid & $\mathrm{ug} / \mathrm{g}$ & 101.6 & 6.170 & $1.78 \mathrm{e}+05$ & $2.06 e+05$ & $1.92 \mathrm{e}+05$ & 14.6 & $-1.920 \mathrm{e} 1$ & 40.00 & $n / a$ \\
\hline 5961005489 & I & Neodymium - ICP-H2O Dig/Acid & $\mathrm{ug} / \mathrm{g}$ & 104.6 & $<1.00 e-01$ & 39.90 & $<3.97 \mathrm{e} 1$ & $n / a$ & $\mathrm{n} / \mathrm{a}$ & 104.0 & 40.00 & $\mathrm{n} / \mathrm{a}$ \\
\hline S96T0 & I & Nickel -ICP-H2O Dig/ACid & ug $/ \mathrm{g}$ & 101.2 & $<2.00 e-02$ & 7.990 & $<7.93 \mathrm{e} 0$ & $n / a$ & $n / a$ & 104.0 & 7.980 & $n / a$ \\
\hline$\$ 96 \mathrm{~T}$ & I & Phosphorus - ICP-H2O Dig/ACid & $\mathrm{ug} / \mathrm{g}$ & 104.4 & $<2.00 e^{-01}$ & $6.37 \mathrm{e}+04$ & $2.75 e+04$ & $4.56 \mathrm{e}+04$ & 79.4 & $-5.620 \mathrm{e} 1$ & 79.80 & $n / a$ \\
\hline S96T0 & $I$ & Lead-1CP-H2O Dig/ACid & ug/g & 101.2 & $<1.00 \mathrm{e}-01$ & 39.90 & $33.97 \mathrm{e} 1$ & $\mathrm{n} / \mathrm{a}$ & $n / a$ & 104.0 & 40.00 & $n / a$ \\
\hline 5967005489 & I & Sulfur -ICP-H2O Dig/ACId & ug/g & 100.8 & $<1.00 \mathrm{e}-01$ & $7.55 e+03$ & $5.65 e+04$ & $3.20 \mathrm{e}+04$ & 153 & 95.60 & 40.00 & $n / a$ \\
\hline S96T0 & I & Antimony $-1 \mathrm{CP}-\mathrm{H} 20 \mathrm{Dig} / \mathrm{Acid}$ & ug $/ \mathrm{g}$ & 97.20 & $<6.00 e^{-02}$ & 24.00 & $<2.38 \mathrm{e} 1$ & $n / a$ & $\mathrm{n} / \mathrm{a}$ & 101.0 & 24.00 & $n / a$ \\
\hline S96T & $I$ & Selenium - ICP-H2O Dig/Acid & ug/g & 98.60 & $<1.00 \mathrm{e}-01$ & 39.90 & $<3.97 \mathrm{e} 1$ & $n / a$ & $\mathrm{n} / \mathrm{a}$ & 99.40 & 40.00 & $\mathrm{n} / \mathrm{a}$ \\
\hline$\$ 961005489$ & $\mathrm{I}$ & Silicon-ICP-HLO Dig/ACid & $49 / 9$ & 98.20 & 1.950 & $1.75 e+03$ & $9.24 \mathrm{e}+04$ & $7.08 \mathrm{e}+03$ & 151 & 136.0 & 20.00 & $n / a$ \\
\hline$\$ 967005489$ & I & Samarium-ICP-H2O Dig/Acid & ug/g & 100.8 & $<1.00 \mathrm{e}-01$ & 39.90 & $<3.97 \mathrm{e}$ & $n / a$ & $n / a$ & 101.0 & 40.00 & $\mathrm{n} / \mathrm{a}$ \\
\hline 59670 & $i$ & Strontium-ICP-H2O Dig/ACid & ug/g & 101.4 & $\leq 1.00 e-02$ & 3.990 & $<3.97 \mathrm{e} 0$ & $n / a$ & $n / a$ & 101.0 & 4.000 & $\mathrm{n} / \mathrm{a}$ \\
\hline 5967 & I & TitaniLm-ICP-H2O Dig/ACid & $4 \mathrm{~g} / \mathrm{g}$ & 100.0 & $<1.00 \mathrm{e}-02$ & 3.990 & $<3.97 \mathrm{e} 0$ & $n / a$ & $n / 2$ & 100.0 & 4.000 & $\mathrm{n} / \mathrm{a}$ \\
\hline 5967005489 & I & Thallium - ICP-H2O Dig/Acid & $\operatorname{lug} / 9$ & 98.20 & $<2.00 e-01$ & 79.90 & $<7.93 \mathrm{e} ?$ & $n / a$ & $n / a$ & 99.70 & 79.80 & $\mathrm{n} / \mathrm{a}$ \\
\hline$\$ 967005489$ & $I$ & Uranium $-1 \mathrm{CP}-\mathrm{H} 2 \mathrm{D} \mathrm{Dig} / \mathrm{Acid}$ & $\mathrm{ug} / \mathrm{g}$ & 98.60 & $<5.00 \mathrm{e}-01$ & $<2.00 \mathrm{e}+02$ & $<1.98 \mathrm{e} 2$ & $\mathrm{n} / \mathrm{a}$ & $n / a$ & 98.50 & 200.0 & $\mathrm{n} / \mathrm{a}$ \\
\hline 61005489 & I & Vanadium - ICP-H2O Dig/Acid & lug/g & 102.4 & $<5.00 e^{-02}$ & 20.00 & $<1.98 \mathrm{e} 1$ & $\mathrm{n} / \mathrm{a}$ & $n / a$ & 104.0 & 20.00 & $\mathrm{n} / \mathrm{a}$ \\
\hline
\end{tabular}




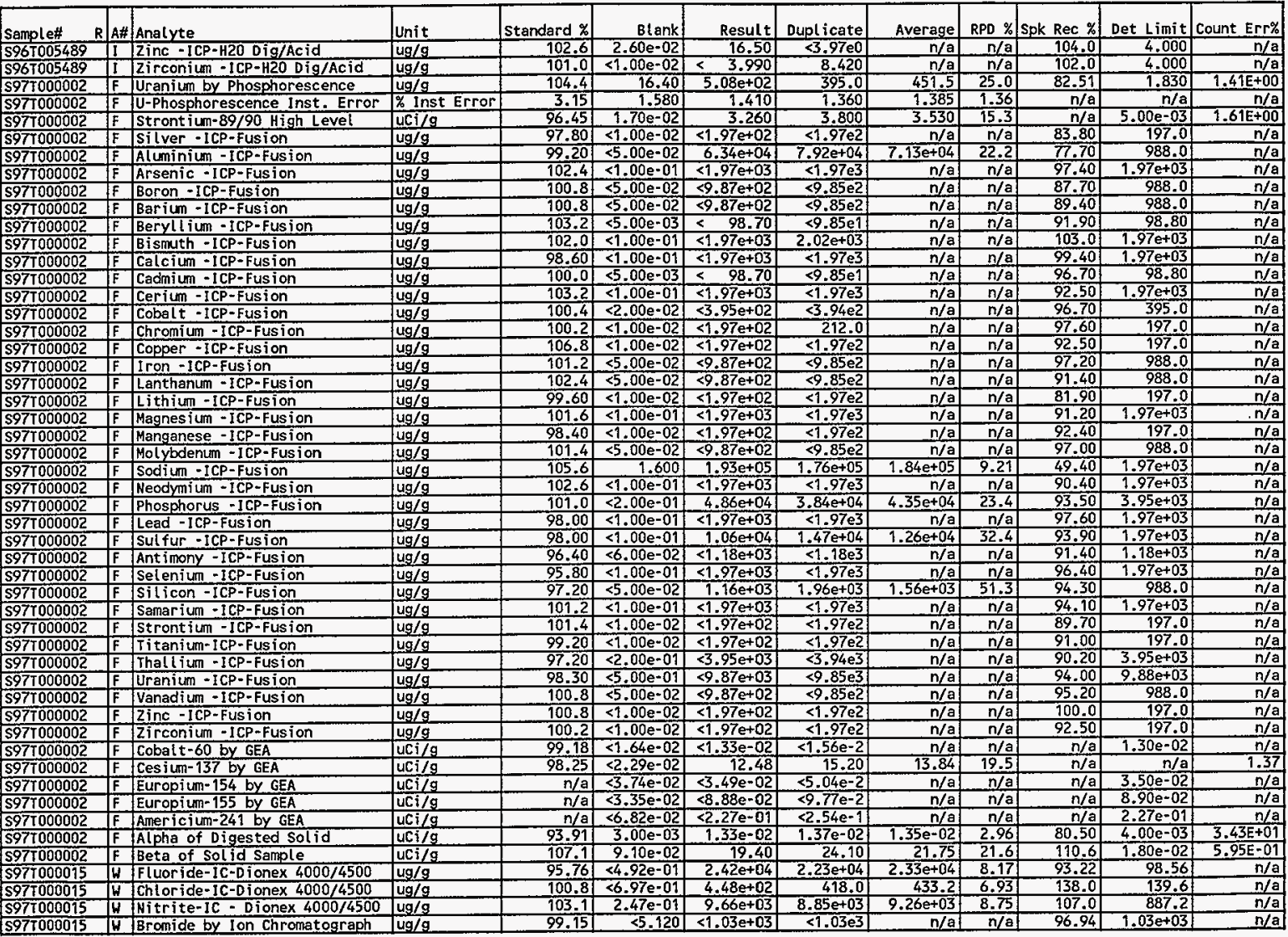




\begin{tabular}{|c|c|c|c|c|c|c|c|c|c|c|c|c|}
\hline Sample\# & $\mathbf{R} \mid \mathrm{At}$ & \#Analyte & Unit & Standard \% & Blank & Result & Dupl icate & Average & RPD $\%$ & Spk Rec \% & Det Limit & nt Err\% \\
\hline S97T000015 & $w$ & Nitrate by IC-Dionex $4000 / 4500$ & $\mathrm{ug} / \mathrm{g}$ & 100.0 & $<5.700$ & $3.48 \mathrm{e}+04$ & $3.11 \mathrm{e}+04$ & $3.29 \mathrm{e}+04$ & 11.2 & 106.1 & $1.14 \mathrm{e}+03$ & $n / a$ \\
\hline S97T000015 & $\bar{W}$ & Phosphate-IC-Dionex $4000 / 4500$ & ug/g & 100.0 & $<4.920$ & $1.44 \mathrm{e}+05$ & $1.38 \mathrm{e}+05$ & $1.41 \mathrm{e}+05$ & 4.26 & 109.4 & 985.6 & $n / a$ \\
\hline S97T000015 & $\ddot{w}$ & Sulfate by [C-Dionex $4000 / 4500$ & ug/g & 99.84 & $<5.660$ & $4.02 e+04$ & $3.47 \mathrm{e}+04$ & $3.74 \mathrm{e}+04$ & 14.7 & 106.0 & $1.13 \mathrm{e}+03$ & $\mathrm{n} / \mathrm{a}$ \\
\hline $\begin{array}{l}\text { S97T000015 } \\
\end{array}$ & $\pi$ & Oxalate by IC-Dionex $4000 / 4500$ & ug/9 & 100.9 & $<4.300$ & $<8.63 \mathrm{e}+02$ & $<8.62 \mathrm{e} 2$ & $n / a$ & $\mathrm{n} / \mathrm{a}$ & 100.0 & 862.6 & $\mathrm{n} / \mathrm{a}$ \\
\hline S97T000016 & $\bar{I}$ & Silver-ICP-H2O Dig/Acid & $\mathrm{ug} / \mathrm{g}$ & 98.40 & $<1.00 \mathrm{e}-02$ & 13.60 & 12.50 & 13.05 & 8.43 & 98.60 & 6.180 & $n / a$ \\
\hline S97T000016 & $\bar{I}$ & Aluminium -ICP-H2O Oig/Acid & $\mathrm{ug} / \mathrm{g}$ & 99.00 & $1.74 \mathrm{e}-01$ & $8.49 \mathrm{e}+02$ & 785.0 & 817.0 & 7.83 & 96.50 & 30.90 & $\mathrm{n} / \mathrm{a}$ \\
\hline S97T000016 & I & Arsenic -iCP-H2O Dig/Acid & ug/a & 103.8 & $\leq 1.00 \mathrm{e}-01$ & $<61.70$ & $<6.17 \mathrm{e} 1$ & $n / a$ & $\mathrm{n} / \mathrm{a}$ & 104.0 & 61.80 & $n / a$ \\
\hline 5977000016 & I & Boron - ICP-H2O Dig/ACid & ug/g & 101.2 & 2.230 & $8.51 \mathrm{e}+02$ & 838.0 & 844.5 & 1.54 & 99.60 & 30.90 & $\mathrm{n} / \mathrm{a}$ \\
\hline 5977000016 & I & Barium - ICP-H2O Dig/ACid & $u g / g$ & 102.6 & $<5.00 \mathrm{e}-02$ & 30.90 & $<3.08 \mathrm{e} 1$ & $\mathrm{n} / \mathrm{a}$ & $n / a$ & 102.0 & 30.90 & $n / a$ \\
\hline S97T000016 & $I$ & Beryllium -ICP-H2O Dig/Acid & $u g / g$ & 102.4 & $<5.00 \mathrm{e}-03$ & 3.090 & $<3.08 \mathrm{e} 0$ & $\mathrm{n} / \mathrm{a}$ & $\mathrm{n} / \mathrm{a}$ & 102.0 & 3.090 & $\bar{n} / a$ \\
\hline S97r000016 & It & Bismuth - ICP-H2O Dig/Acid & $4 \mathrm{~g} / \mathrm{g}$ & 102.2 & $<1.00 \mathrm{e}-01$ & 61.70 & $<6.17 \mathrm{e} 1$ & $\mathrm{n} / \mathrm{a}$ & $\mathrm{n} / \mathrm{a}$ & 110.0 & 61.80 & $n / a$ \\
\hline S97T000016 & I & Calcium-iCP-H2O Dig/Acid & $u g / g$ & 97.60 & $<1.00 e-01$ & 70.70 & $<6.17 \mathrm{e} 1$ & $n / a$ & $\mathrm{n} / \mathrm{a}$ & 91.60 & 61.80 & $\mathrm{n} / \mathrm{a}$ \\
\hline S97T000016 & $I$ & Cadmium - ICP-H2O Dig/Acid & $\operatorname{lug} / \mathrm{g}$ & 100.4 & $<5.00 \mathrm{e}-03$ & 3.090 & $<3.08 \mathrm{e} 0$ & $n / \theta$ & $n / a$ & 101.0 & 3.090 & 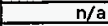 \\
\hline \$97T000016 & $\mid \frac{1}{I}$ & Cerium-ICP-H2O Dig/Acid & $49 / 9$ & 100.4 & $<1.00 \mathrm{e}-01$ & 61.70 & $<6.17 \mathrm{e} 1$ & $\mathrm{n} / \mathrm{a}$ & $\mathrm{n} / \mathrm{a}$ & 102.0 & 61.80 & $n / a$ \\
\hline S97T000016 & I & Cobalt -ICP-H2O Dig/Acid & $\operatorname{lng} / 9$ & 101.2 & $<2.00 \mathrm{e}-02$ & 12.30 & $<1.23 \mathrm{e} 1$ & $n / a$ & $\mathrm{n} / \mathrm{a}$ & 102.0 & 12.30 & $n / a$ \\
\hline 5971000016 & I & Chromium -ICP-H2O Dig/Acid & $\mathrm{ug} / \mathrm{g}$ & 99.40 & $<1.00 \mathrm{e}-02$ & $1.26 \mathrm{e}+02$ & 117.0 & 121.5 & 7.41 & 100.0 & 6.180 & $n / a$ \\
\hline S97T000016 & II & Copper - ICP-H2O Dig/Acid & $\operatorname{lug} / 9$ & 107.4 & $<7.00 e^{-02}$ & $<\quad 6.170$ & $<6.17 e 0$ & $\mathrm{n} / \mathrm{a}$ & $n / a$ & 107.0 & 6.180 & $n / a$ \\
\hline S971000016 & I & Iron-ICP-H2O Dig/Acid & ug $/ 9$ & 100.2 & $<5.00 \mathrm{e}-02$ & 30.90 & $<3.08 \mathrm{e} 1$ & $\mathrm{n} / \mathrm{a}$ & $n / a$ & 103.0 & 30.90 & $n / a$ \\
\hline S97T000016 & 1 & Potassium -ICP-H2O Dig/ACid & ug $/ 9$ & 106.6 & $<5.00 \mathrm{e}-01$ & $7.47 \mathrm{e}+02$ & 628.0 & 687.5 & 17.3 & 80.20 & 309.0 & $n / a$ \\
\hline S97T000016 & I & Lanthanum -ICP-H2O Dig/Acid & ug/g & 102.0 & $<5.00 \mathrm{e}-02$ & $<30.90$ & $<3.08 \mathrm{e} 1$ & $n / a$ & $\mathrm{n} / \mathrm{a}$ & 103.0 & 30.90 & $\mathrm{n} / \mathrm{a}$ \\
\hline S97T000016 & 1 & Lithium $-I C P-H 2 O D i g / A C i d$ & ug/g & 101.2 & $<1.00 e-02$ & 6.170 & $<6,17 \mathrm{e} 0$ & $n / a$ & n/a & 107.0 & 6.180 & $n / a$ \\
\hline 597T000016 & I & Hagnes ium-ICP-H2O Dig/Acid & ug/g & 101.2 & $<1.00 \mathrm{e}-01$ & 61.70 & $<6.17 \mathrm{e} 1$ & $\mathrm{n} / \mathrm{a}$ & $n / a$ & 100.0 & 61.80 & $\mathrm{n} / \mathrm{a}$ \\
\hline S97T000016 & 1 & Manganese $-I C P-H 2 O D i g / A c i d$ & $\mathrm{ug} / \mathrm{g}$ & 97.60 & $<1.00 \mathrm{e}-02$ & 6.170 & $<6.17 \mathrm{e} 0$ & $\mathrm{n} / \mathrm{a}$ & $n / a$ & 96.90 & 6.180 & $n / a$ \\
\hline$\$ 971000016$ & I & Molybdenum -ICP-H2O Dig/Acid & ug/g & 101.2 & $<5.00 \mathrm{e}-02$ & 30.90 & $<3.08 \mathrm{e} 1$ & $\mathrm{n} / \mathrm{a}$ & $n / a$ & 104.0 & 30.90 & $\mathrm{n} / \mathrm{a}$ \\
\hline S97T000016 & I & Sodium -ICP-H2O Dig/ACid & ug/g & 104.4 & 3.640 & $1.74 \mathrm{e}+05$ & $1.68 \mathrm{e}+05$ & $1.71 \mathrm{e}+05$ & 3.51 & $-5.100 \mathrm{e} 2$ & 61.80 & $n / a$ \\
\hline$\$ 971000016$ & I & Neodymilim - ICP-H20 Dig/Acid & ug/g & 102.0 & $<1.00 \mathrm{e}-01$ & $<61.70$ & $<6.17 \mathrm{e} 1$ & $\mathrm{n} / \mathrm{a}$ & $n / a$ & 104.0 & 61.80 & $\mathrm{n} / \mathrm{a}$ \\
\hline 5977000016 & $I$ & Nickel -ICP-H2O Dig/ACid & ug/g & 100.6 & $<2.00 \mathrm{e}-02$ & 12.30 & $<1.23 \mathrm{e} 1$ & $n / a$ & $n / a$ & 100.0 & 12.30 & $n /$ \\
\hline S977000016 & I & Phosphorus - ICP-H2O Dig/Acid & ug/g & 103.2 & $<2.00 \mathrm{e}-01$ & $4.77 \mathrm{e}+04$ & $4.77 \mathrm{e}+04$ & $4.77 e+04$ & 0.00 & $-2.590 \mathrm{e} 1$ & 123.0 & $\mathrm{n} / \mathrm{a}$ \\
\hline S97T000016 & I & Lead - ICP-H2O Dig/ACid & $\mathrm{ug} / \mathrm{g}$ & 97.60 & $<1.00 \mathrm{e}-01$ & 61.70 & $<6.17 \mathrm{e} 1$ & $\mathrm{n} / \mathrm{a}$ & $n / a$ & 101.0 & 61.80 & $\mathrm{n} / \mathrm{a}$ \\
\hline 5971000016 & \begin{tabular}{|l} 
\\
\end{tabular} & Sulfur -ICP-H2O Dig/Acid & ug/g & 99.60 & $<1.00 \mathrm{e}-01$ & $1.42 \mathrm{e}+04$ & $1.29 \mathrm{e}+04$ & $1.36 \mathrm{e}+04$ & 9.59 & 63.60 & 61.80 & $\mathrm{n} / \mathrm{a}$ \\
\hline 5971000016 & I & Ant imony -ICP-H2O Dig/Acid & $\mathrm{ug} / \mathrm{g}$ & 96.00 & $<6.00 \mathrm{e}-02$ & 37.00 & $<3.70 \mathrm{e} 1$ & $n / a$ & $n / a$ & 97.20 & 36.90 & $n / a$ \\
\hline 5971000016 & I & Selenium -ICP-H2O Dig/ACId & ug/g & 94.20 & $<1.00 \mathrm{e}-01$ & 61.70 & $<6.17 \mathrm{e} 1$ & $\mathrm{n} / \mathrm{a}$ & $\mathrm{n} / \mathrm{a}$ & 93.80 & 61.80 & $n / a$ \\
\hline S9710 & I & Silicon-ICP-H2O Dig/ACid & ug $/ \mathrm{g}$ & 97.20 & $5.92 e-01$ & $4.25 \mathrm{e}+03$ & $4.23 e+03$ & $4.24 \mathrm{e}+03$ & 0.47 & 153.0 & 30.90 & $n / a$ \\
\hline S97T000016 & I & Samarium-ICP-H2O Dig/Acid & ug/g & 100.2 & $<1.00 e-01$ & 61.70 & $<6.17 \mathrm{e} 1$ & $\mathrm{n} / \mathrm{a}$ & $n / a$ & 301.0 & 61.80 & $\mathrm{n} / \mathrm{a}$ \\
\hline 5977000016 & II & Strontiun -ICP-H20 Dig/ACid & $49 / 9$ & 101.0 & $<1.00 \mathrm{e}-02$ & 6.170 & $<6.17 \mathrm{e} 0$ & $n / a$ & $\mathrm{n} / \mathrm{a}$ & 101.0 & 6.180 & $n / a$ \\
\hline 5977000016 & I & Iitanium-ICP-H2O Dig/Acid & $\mathrm{ug} / \mathrm{g}$ & 99.00 & $<1.00 e^{-02}$ & 6.170 & $<6.17 \mathrm{e} 0$ & $\mathrm{n} / \mathrm{a}$ & $\mathrm{n} / \mathrm{a}$ & 99.40 & 6.180 & $n / a$ \\
\hline s97ro & I & Thallium - ICP-H20 Dig/Acid & ug/g & 97.00 & $<2.00 \mathrm{e}-01$ & $<1.23 \mathrm{e}+02$ & $<1.23 \mathrm{e} 2$ & $n / a$ & $n / a$ & 95.00 & 123.0 & $\mathrm{n} / \mathrm{a}$ \\
\hline 5977000016 & II & Uranium - ICP-H2O Dig/ACid & ug/g & 98.00 & $<5,00 e-01$ & $<3.09 \mathrm{e}+02$ & $<3.08 \mathrm{e} 2$ & $\mathrm{n} / \mathrm{a}$ & $n / a$ & 100.5 & 309.0 & $\mathrm{n} / \mathrm{a}$ \\
\hline$\$ 977000016$ & II & Vanadium - ICP-H2O Dig/Acid & ug/g & 100.8 & $<5.00 \mathrm{e}-02$ & 30.90 & $<3.08 \mathrm{e} 1$ & $n / a$ & $n / a$ & 103.0 & 30.90 & $n / a$ \\
\hline 5977000016 & II & Zinc-ICP-H2O Dig/ACid & $\mathrm{ug} / \mathrm{g}$ & 101.6 & $<1.00 \mathrm{e}-02$ & 6.170 & $<6.17 \mathrm{e} 0$ & $n / a$ & $n / a$ & 104.0 & 6.180 & $\mathrm{n} / \mathrm{a}$ \\
\hline S977000016 & & Zirconium -ICP-H2O Dig/Acid & $\mathrm{ug} / \mathrm{g}$ & 100.2 & $<1.00 e-02$ & 6.170 & $<6.17 \mathrm{e} 0$ & $n / a$ & $n / a$ & 101.0 & 6.180 & $n / a$ \\
\hline
\end{tabular}


SEGMENT PORTION: U UPper Half of Segment

\begin{tabular}{|c|c|c|c|c|c|c|c|c|c|c|c|c|}
\hline samole\# & A\# & Analyte & Unit & Standard $\%$ & $B$ lank & Result & Duplicate & Average & RPD \% & Spk Rec $\%$ & Det Limit & Count Err\% \\
\hline S96T005465 & & Bulk Density of Sample & $\mathrm{g} / \mathrm{mL}$ & $n / a$ & $\mathrm{n} / \mathrm{a}$ & 1.910 & $n / a$ & $\mathrm{n} / \mathrm{a}$ & $n / a$ & $n / a$ & $5.00 e^{-01}$ & $n / a$ \\
\hline \begin{tabular}{|l}
39070005466 \\
596005
\end{tabular} & & DSC Exotherm using Mettler & Joules/g & 96.31 & $n / a$ & $0.00 e+00$ & $0.00 e+00$ & $0.00 \mathrm{e}+00$ & 0.00 & $\mathrm{n} / \mathrm{a}$ & $\mathrm{n} / \mathrm{a}$ & $n / a$ \\
\hline S96r0005466 & & DSC Exotherm Dry Calculated & Joules/g Dry & $n / a$ & n/a & $0.00 \mathrm{e}+00$ & $0.00 \mathrm{e}+00$ & $0.00 \mathrm{e}+00$ & 0.00 & $\mathrm{n} / \mathrm{a}$ & $n / a$ & $n / a$ \\
\hline$\$ 967005466$ & & $\%$ Water by TGA using Mettler & $\%$ & 99.70 & $n / a$ & 18.38 & 18.34 & 18.36 & 0.22 & $n / a$ & $n / a$ & $n / a$ \\
\hline$\frac{1901007400}{5967005468}$ & $F$ & Nickel -ICP-Fusion & ug/g & 100.0 & 1.220 & $7.83 \mathrm{e}+03$ & $3.67 \mathrm{e}+03$ & $5.75 \mathrm{e}+03$ & 72.3 & 97.00 & 384.0 & $\mathrm{n} / \mathrm{a}$ \\
\hline$\frac{5701009400}{5967005468}$ & $\frac{1}{F}$ & Silver-ICP-Fusion & $u g / g$ & 99.00 & $<1.00 e-02$ & $<1.92 \mathrm{e}+02$ & $<1.88 \mathrm{e} 2$ & $n / a$ & $n / a$ & 90.80 & 192.0 & $n / a$ \\
\hline 5967005468 & $F$ & Aluminium-ICP-Fusion & $u g / 9$ & 95.80 & $<5.00 \mathrm{e}-02$ & $2.10 \mathrm{e}+05$ & $2.31 \mathrm{e}+05$ & $2.20 \mathrm{e}+05$ & 9.52 & 93.80 & 960.0 & $\mathrm{n} / \mathrm{a}$ \\
\hline \$967005468 & $F$ & Arsenic-ICP-fusion & $\frac{u g / g}{u g / g}$ & 101.0 & $<1.00 \mathrm{e}-01$ & $<1.92 \mathrm{e}+03$ & $\leq 1.88 \mathrm{e} 3$ & $n / a$ & $n / a$ & 100.0 & $1.92 \mathrm{e}+03$ & $n / a$ \\
\hline$\frac{3901003400}{5967005468}$ & $F$ & Boron-ICP-Fusion & $u g / 9$ & 97.80 & $55.00 e-02$ & $<9.60 \mathrm{e}+02$ & $<9.42 \mathrm{e} 2$ & $n / a$ & n/a & 93.40 & 960.0 & $\mathrm{n} / \mathrm{a}$ \\
\hline \begin{tabular}{|l}
3701007400 \\
$596 T 005468$
\end{tabular} & $\mathrm{~F}$ & Barium -ICP-Fusion & $u g / g$ & 97.60 & $5.00 \mathrm{e}-02$ & $8.60 \mathrm{e}+02$ & $<9.42 \mathrm{e} 2$ & $n / a$ & $n / a$ & 92.20 & 960.0 & $n / a$ \\
\hline S967005468 & $F$ & Beryltium-ICP-Fuston & ug/g & 100.2 & $<5.00 \mathrm{e}-03$ & 96.00 & $<9.42 \mathrm{e} 1$ & $\mathrm{n} / \mathrm{a}$ & $\mathrm{n} / \mathrm{a}$ & 95.60 & 96.00 & $\mathrm{n} / \mathrm{a}$ \\
\hline$\frac{1901002400}{596 T 005468}$ & $F$ & Bismuth -ICP-Fusion & ugs/g & 100.6 & $<1.00 e-01$ & $2.46 \mathrm{e}+03$ & $2.36 \mathrm{e}+03$ & $2.41 \mathrm{e}+03$ & 4.15 & 97.40 & $1.92 e+03$ & $\mathrm{n} / \mathrm{a}$ \\
\hline S96T005468 & F & Calcium-ICP-Fusion & $\operatorname{ug} / 9$ & 101.0 & $<1.00 e-01$ & $<1.92 \mathrm{e}+03$ & $<1.88 \mathrm{e} 3$ & $n / a$ & $n / a$ & 101.0 & $7.92 \mathrm{e}+03$ & n/a \\
\hline 5961005468 & $F$ & Cadmium-ICP-Fusion & $u g / 9$ & 100.2 & $<5.00 \mathrm{e}-03$ & $<96.00$ & $<9.42 \mathrm{e} 1$ & $n / a$ & n/a & 97.60 & 96.00 & $\mathrm{n} / \mathrm{a}$ \\
\hline S967005468 & $F$ & Cerium-ICP-Fusion & $\mathrm{ug} / \mathrm{g}$ & 98.40 & $<7.00 \mathrm{e}-01$ & $<1.92 \mathrm{e}+03$ & $<1.88 \mathrm{e} 3$ & n/a & $n / a$ & 94.70 & $9.92 \mathrm{e}+03$ & $n / a$ \\
\hline 5967005468 & $F$ & Cobalt -ICP-Fusion & $\log / 9$ & 101.4 & $<2.00 \mathrm{e}-02$ & $<3.84 \mathrm{e}+02$ & $<3.77 \mathrm{e}$ & $n / a$ & $n / a$ & 98.50 & 384.0 & $n / a$ \\
\hline$\frac{3701007400}{5967005468}$ & $F$ & Chromium-ICP-Fusion & $\mathrm{ug} / \mathrm{g}$ & 101.0 & $<1.00 \mathrm{e}-02$ & $2.08 \mathrm{e}+02$ & 243.0 & 225.5 & 15.5 & 98.80 & 192.0 & $\mathrm{n} / \mathrm{a}$ \\
\hline 5961005468 & $F$ & Copper-ICP-Fusion & $09 / 9$ & 102.2 & $<1.00 \mathrm{e}-02$ & $<1.92 \mathrm{e}+02$ & $<1.88 \mathrm{e} 2$ & $\mathrm{n} / \mathrm{a}$ & $n / a$ & 97.40 & 192.0 & $n / a$ \\
\hline S96T005468 & $F$ & Iron-ICP-Fusion & $\mathrm{ug} / \mathrm{g}$ & 99.60 & $<5.00 e-02$ & $9.43 e+03$ & $1.67 \mathrm{e}+03$ & $1.55 \mathrm{e}+03$ & 15.5 & 96.10 & 960.0 & $\mathrm{n} / \mathrm{a}$ \\
\hline$\frac{5701005400}{5967005468}$ & F & Lanthanum - ICP-Fusion & $\operatorname{lug} / 9$ & 100.0 & $<5.00 \mathrm{e}-02$ & $<9.60 \mathrm{e}+02$ & $<9.42 \mathrm{e} 2$ & $\mathrm{n} / \mathrm{a}$ & $n / a$ & 95.60 & 960.0 & $\mathrm{n} / \mathrm{a}$ \\
\hline S96T005468 & $\mathrm{F}$ & Lithium-ICP-Fusion & $u g / g$ & 96.80 & $<1.00 \mathrm{e}-02$ & $<1.92 e+02$ & $<1.88 \mathrm{e} 2$ & $\mathrm{n} / \mathrm{a}$ & $\mathrm{n} / \mathrm{a}$ & 91.30 & 192.0 & $\mathrm{n} / \mathrm{a}$ \\
\hline S96T005468 & $\mathrm{F}$ & Magnesium-ICP-Fusion & $u g / 9$ & 94.00 & $<7.00 \mathrm{e}-01$ & $<1.92 \mathrm{e}+03$ & $<7.88 \mathrm{e}^{3}$ & $n / a$ & $n / a$ & 89.80 & $1.92 \mathrm{e}+03$ & $n / a$ \\
\hline \$96T005468 & $F$ & Manganese -ICP-Fusion & $\frac{\log / g}{\operatorname{ug} / g}$ & 97.20 & $<1.00 e-02$ & $<7.92 \mathrm{e}+02$ & $<1.88 \mathrm{e} 2$ & n/a & $n / a$ & 93.40 & 192.0 & $n / a$ \\
\hline 5967005468 & $F$ & Molybdenum - ICP-Fusion & $u g / 9$ & 101.0 & $<5.00 \mathrm{e} \cdot 02$ & $<9.60 \mathrm{e}+02$ & $<9.42 \mathrm{e} 2$ & $n / a$ & $n / a$ & 98.40 & 960.0 & $n / a$ \\
\hline$\$ 9967005468$ & $\mathbf{F}$ & Sodium-ICP-Fusion & $4 \mathrm{~g} / \mathrm{g}$ & 94.80 & 1.510 & $8.67 \mathrm{e}+04$ & $7.54 \mathrm{e}+04$ & $8,10 e+04$ & 13.9 & 88.40 & $1.92 e+03$ & $\mathrm{n} / \mathrm{a}$ \\
\hline \$967005468 & $\mathrm{F}$ & Neodymiun -ICP-Fusion & $u g / 9$ & 99.60 & $<1.00 \mathrm{e}^{-01}$ & $<1.92 \mathrm{e}+03$ & $<1.88 \mathrm{e} 3$ & $n / a$ & $n / a$ & 95.10 & $1.92 \mathrm{e}+03$ & $\mathrm{n} / \mathrm{a}$ \\
\hline 5967005468 & $F$ & Phosphorus-ICP-Fusion & $\mathrm{ug} / \mathrm{g}$ & 98.40 & $<2.00 \mathrm{e}-01$ & $5.69 \mathrm{e}+03$ & $<3.77 \mathrm{e}^{3}$ & $n / a$ & $n / a$ & 98.20 & $3.84 \mathrm{e}+03$ & $n / a$ \\
\hline \begin{tabular}{|l}
$5907005+00$ \\
$596 T 005468$
\end{tabular} & $\mathrm{~F}$ & Lead -ICP-Fusion & $u g / g$ & 100.4 & $<1.00 \mathrm{e}-01$ & $<1.92 \mathrm{e}+03$ & $<1.88 \mathrm{e} 3$ & $n / a$ & $n / a$ & 99.807 & $1.92 \mathrm{e}+03$ & $n / a$ \\
\hline S96T005468 & $\mathrm{F}$ & Sulfur-ICP-Fusion & $u g / g$ & 98.20 & $<7.00 \mathrm{e}-07$ & $1.98 \mathrm{e}+03$ & $2.25 \mathrm{e}+03$ & $2.12 \mathrm{e}+03$ & 12.8 & 97.90 & $1.92 \mathrm{e}+03$ & $\bar{n} / \mathrm{a}$ \\
\hline S967005468 & $\vec{F}$ & Antimony-ICP-Fusion & ug/g & 96.00 & $<6.00 \mathrm{e}-02$ & $<1.15 e+03$ & $<1.13 \mathrm{e} 3$ & $n / a$ & $n / a$ & 93.50 & $1.15 \mathrm{e}+03$ & $n / a$ \\
\hline S96T005468 & $F$ & Selenium-ICP-Fusion & $\log / g$ & 95.00 & $<1.00 \mathrm{e}-01$ & $<1.92 \mathrm{e}+03$ & $<7.88 \mathrm{e} 3$ & $\mathrm{n} / \mathrm{a}$ & $\mathrm{n} / \mathrm{a}$ & 98.50 & $1.92 \mathrm{e}+03$ & $\overline{n / a}$ \\
\hline S967005468 & $F$ & Silicon-ICP-Fusion & $u g / 9$ & 96.80 & $<5.00 e-02$ & $9.74 e+02$ & $1.10 \mathrm{e}+03$ & $1.04 \mathrm{e}+03$ & 12.2 & 96.20 & 960.0 & $n / a$ \\
\hline S967005468 & $F$ & Samarium -ICP-fusion & $u g / g$ & 96.60 & $<1.00 \mathrm{e}-01$ & $<1.92 \mathrm{e}+03$ & $<1.88 \mathrm{e}^{3}$ & $n / a$ & n/a & 93.80 & $1.92 \mathrm{e}+03$ & $n / a$ \\
\hline S967005468 & $\mathrm{F}$ & stront fum -ICP-Fusion & $\mathrm{ug} / \mathrm{g}$ & 97.00 & $<7.00 \mathrm{e}-02$ & $<1.92 \mathrm{e}+02$ & $\leq 1.88 \mathrm{e} 2$ & $n / a$ & $\mathrm{n} / \mathrm{a}$ & 92.40 & 192.0 & $n / a$ \\
\hline \begin{tabular}{|l}
967005468 \\
\end{tabular} & $F$ & Iitanium-ICP-Fusion & ug/g & 97.40 & $<7.00 \mathrm{e}-02$ & $<7.92 \mathrm{e}+02$ & $<1.88 \mathrm{e} 2$ & $n / a$ & $\mathrm{n} / \mathrm{a}$ & 94.50 & 192.0 & $n / a$ \\
\hline S96T005468 & $\tilde{F}$ & Thallium-ICP-Fusion & $\mathrm{ug} / \mathrm{g}$ & 94.60 & $<2.00 \mathrm{e}-01$ & $<3.84 \mathrm{e}+03$ & $<3.77 \mathrm{e} 3$ & $n / a$ & $n / a$ & 90.70 & $3.84 \mathrm{e}+03$ & $n / a$ \\
\hline 5967005468 & F & Uranium-ICP-Fusion & ug/g & 94.90 & $<5.00 \mathrm{e} \cdot 01$ & $<9.60 \mathrm{e}+03$ & $<9.42 \mathrm{e} 3$ & $n / a$ & $n / a$ & 94.50 & $9.60 \mathrm{e}+03$ & $n / a$ \\
\hline S967005468 & $F$ & Vanadium-ICP-Fusion & $40 / 9$ & 100.4 & $<5.00 e-02$ & $<9.60 \mathrm{e}+02$ & $<9.42 \mathrm{e} 2$ & $n / a$ & $\mathrm{n} / \mathrm{a}$ & 97.80 & 960.0 & $n / a$ \\
\hline S96T005468 & F & Zinc-ICP-Fusion & $u g / g$ & 102.0 & $<1.00 \mathrm{e}-02$ & $2.01 \mathrm{e}+02$ & 213.0 & 207.0 & 5.80 & 101.0 & 192.0 & $n / a$ \\
\hline$\$ 96 T 005468$ & $\mathbf{F}$ & Zirconium -ICP-Fusion & $4 \mathrm{~g} / \mathrm{g}$ & 98.60 & $<1,00 \mathrm{e}-02$ & $<1.92 \mathrm{e}+02$ & $<1,88 \mathrm{e} 2$ & $n / a$ & $n / a$ & 95.00 & 192.0 & $n / a$ \\
\hline \$96T005468 & $F$ & Cobalt -60 by GEA & $u C i / g$ & 99.75 & $<1.65 e-02$ & $<.75 e-02$ & $<1.35 e-2$ & $n / a$ & $n / a$ & $\mathrm{n} / \mathrm{a}$ & $80 \mathrm{e}-02$ & $n / a$ \\
\hline$\$ 967005468$ & $\bar{F}$ & Cesium- 137 by GEA & uCi/g & 98.05 & $<3.33 \mathrm{e}-02$ & 23.00 & 22.90 & 22.95 & 0.44 & $n / a$ & $n / a$ & 1.11 \\
\hline$\$ 967005468$ & $F$ & Europium-154 by GEA & $u C i / g$ & $\mathrm{n} / \mathrm{a}$ & $<5.01 \mathrm{e}-02$ & $<5.94 \mathrm{e}-02$ & $<5.51 e^{-2}$ & $n / a$ & $n / a$ & $\mathrm{n} / \mathrm{a}$ & $5.90 e-02$ & $\mathrm{n} / \mathrm{a}$ \\
\hline 5967005468 & $\frac{5}{F}$ & Europium-155 by GEA & $\mathrm{uCi} / \mathrm{g}$ & n/a & $<4.31 \mathrm{e}-02$ & $<1.48 \mathrm{e}-01$ & $<1.47 e^{-1}$ & $n / a$ & $n / a$ & $n / a$ & $1.48 \mathrm{e}-01$ & $n / a$ \\
\hline
\end{tabular}




\begin{tabular}{|c|c|c|c|c|c|c|c|c|c|c|c|c|}
\hline mple\# & $R$ A\# & Analyte & Unit & Standard \% & Blank & Result & Duplicate & Average & RPD \% & Spk Rec \% & Det Limit & Count Err\% \\
\hline 5967005468 & $F$ & Americium-241 by GEA & uci $/ 9$ & $n / a$ & $<8,16 \mathrm{e}=02$ & $<3.16 \mathrm{e}-01$ & $<3.09 e^{-1}$ & $n / a$ & $\mathrm{n} / \mathrm{a}$ & $n / a$ & $3.16 e-01$ & $n / a$ \\
\hline$\$ 967005469$ & î & Fluoride-1C-0ionex $4000 / 4500$ & $\mathrm{ug} / \mathrm{g}$ & 102.2 & $<1.20 \mathrm{e}-02$ & $2.32 e+03$ & $2.01 e+03$ & $2.16 \mathrm{e}+03$ & 14.3 & 95.59 & 12.80 & $n / a$ \\
\hline 5967005469 & W & Chloride-IC-Dionex $4000 / 4500$ & $\log / 9$ & 103.4 & $<1.70 \mathrm{e}-02$ & $9.08 \mathrm{e}+02$ & 879.0 & 893.6 & 3.25 & 104.2 & 18.14 & $\mathrm{n} / \mathrm{a}$ \\
\hline S96T0 & 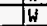 & Nitrite-IC - Dionex $4000 / 4500$ & $\log / 9$ & 101.5 & $<7.08 \mathrm{e}-01$ & $1.44 \mathrm{e}+04$ & $1.38 \mathrm{e}+04$ & $1.41 \mathrm{e}+04$ & 4.26 & 103.3 & 715.2 & $\mathrm{n} / \mathrm{a}$ \\
\hline S96T & $W$ & Bromide by Ion Chromatograph & ug/g & 99.49 & $<1.25 \mathrm{e}-01$ & $1.36 \mathrm{e}+02$ & $\leq 1.36 \mathrm{e} 2$ & $n / a$ & $n / a$ & 100.2 & 133.4 & $n / a$ \\
\hline S96T & $W$ & Nitrate by $1 \mathrm{c}-0$ ionex $4000 / 4500$ & ug/g & 100.8 & $1.94 \mathrm{e}-01$ & $5.28 \mathrm{e}+04$ & $5.18 \mathrm{e}+04$ & $5.23 e+04$ & 1.91 & 103.4 & 148.3 & $n / a$ \\
\hline 69 & $\mathrm{~W}$ & Phosphate-IC-Dionex $4000 / 4500$ & ug/g & 98.71 & $<1.20 \mathrm{e}=01$ & $1.53 \mathrm{e}+04$ & $1.32 e+04$ & $1.43 \mathrm{e}+04$ & 14.7 & 109.4 & 128.0 & $n / a$ \\
\hline S96T & W & Sulfate by $1 \mathrm{C}-0$ ionex $4000 / 4500$ & ug/g & 102.2 & $<1.38 \mathrm{e}-01$ & $5.91 \mathrm{e}+03$ & $5.72 e+03$ & $5.81 \mathrm{e}+03$ & 3.27 & 108.9 & 147.2 & $n / a$ \\
\hline 1. & & Oxalate by IC-Dionex $4000 / 4500$ & 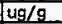 & 106.9 & $\leq 1.05 \mathrm{e}-01$ & $1.41 \mathrm{e}+02$ & 139.0 & 139.8 & 1.43 & 105.3 & 112.0 & $n / a$ \\
\hline
\end{tabular}
5967005469 W Oxalate by IC-Dionex $4000 / 4500$ ug/g

\begin{tabular}{|c|c|c|c|c|c|c|c|c|c|c|c|c|}
\hline mple\#\# & $\mathbf{R} \mid$ A\# & Analyte & Unit & standard $\%$ & Blank & Result & Dupl icate & Average & RPD \% & Spk Rec \% & Det Limit & Count Err\% \\
\hline 5967005471 & & Bulk Density of Sample & $\mathrm{g} / \mathrm{mL}$ & $\mathrm{n} / \mathrm{a}$ & $\mathrm{n} / \mathrm{a}$ & 1.740 & $n / a$ & $n / a$ & $n / a$ & $n / a$ & $5.00 e^{-01}$ & $n / a$ \\
\hline S96T005473 & & DSC Exotherm using Mettler & Joules/g & 96.31 & $\mathrm{n} / \mathrm{a}$ & $0.00 \mathrm{e}+00$ & $0.00 \mathrm{e}+00$ & $0.00 \mathrm{e}+00$ & 0.00 & $n / a$ & $n / a$ & $n / a$ \\
\hline S967005473 & & DSC Exotherm Dry Calculated & Joules/g Dry & $\mathrm{n} / \mathrm{a}$ & $\mathrm{n} / \mathrm{a}$ & $0.00 \mathrm{e}+00$ & $0.00 \mathrm{e}+00$ & $0.00 \mathrm{e}+00$ & 0.00 & $n / a$ & $\mathrm{n} / \mathrm{a}$ & $\mathrm{n} / \mathrm{a}$ \\
\hline 5967005473 & & $\%$ Water by TGA using Mettler & $\%$ & 99.70 & $\mathrm{n} / \mathrm{a}$ & 34.86 & 36.85 & 35.86 & 5.55 & $\mathrm{n} / \mathrm{a}$ & $n / a$ & $n / a$ \\
\hline S96T005477 & $F$ & Üranium by Phosphorescence & $u g / g$ & 109.3 & $5.93 e-05$ & $1.49 \mathrm{e}+03$ & $1.47 \mathrm{e}+03$ & $1.48 \mathrm{e}+03$ & 1.35 & 111.3 & 1.640 & $1.28 \mathrm{E}+00$ \\
\hline \$967005477 & $F$ & U-Phosphorescence Inst. Error & $\%$ Inst Error & 3.24 & 1.160 & 1.280 & 1.240 & 1.260 & 1.24 & $n / a$ & $n / a$ & $\mathrm{n} / \mathrm{a}$ \\
\hline$\$ 967005477$ & $F$ & Strontiun- $89 / 90$ High Level & uci $/ g$ & 86.39 & $8.00 \mathrm{e} \cdot 03$ & 4.740 & 4.090 & 4.415 & 14.7 & $\mathrm{n} / \mathrm{a}$ & $3.00 \mathrm{e}-03$ & $8.29 E-01$ \\
\hline 5967005477 & $F$ & Nickel -ICP-Fusion & $\mathrm{ug} / \mathrm{g}$ & 100.0 & 1.220 & $5.02 \mathrm{e}+03$ & $3.76 \mathrm{e}+03$ & $4.39 \mathrm{e}+03$ & 28.7 & $\mathrm{n} / \mathrm{a}$ & 354.0 & $\mathrm{n} / \mathrm{a}$ \\
\hline 5967005477 & $F$ & Silver-ICP-fusion & $4 \mathrm{~g} / \mathrm{g}$ & 99.00 & $<1.00 e^{-02}$ & $<1.77 e+02$ & $<1.78 \mathrm{e} 2$ & $\mathrm{n} / \mathrm{a}$ & $n / a$ & $n / a$ & 177.0 & $n / a$ \\
\hline 5967005477 & $F$ & Aluminium-1CP-Fusion & $\mathrm{ug} / \mathrm{g}$ & 95.80 & $<5.00 \mathrm{e}-02$ & $5: 34 e+04$ & $4.96 \mathrm{e}+04$ & $5.15 e+04$ & 7.38 & $n / a$ & 884.0 & $n / a$ \\
\hline 5967005477 & $F$ & Arsenic-ICP-Fusion & $\mathrm{ug} / \mathrm{g}$ & 101.0 & $<1.00 \mathrm{e}-01$ & $<1.77 e+03$ & $<1.78 \mathrm{e} 3$ & $n / a$ & $n / a$ & $\mathrm{n} / \mathrm{a}$ & $1.77 \mathrm{e}+03$ & $\mathrm{n} / \mathrm{a}$ \\
\hline 5967005477 & $F$ & Boron-1CP-Fusion & ug/g & 97.80 & $<5.00 e^{-02}$ & $<8.84 \mathrm{e}+02$ & $<8.88 \mathrm{e} 2$ & $n / a$ & $n / a$ & $n / a$ & 884.0 & $n / a$ \\
\hline 5967005477 & $\mathrm{~F}$ & Barium -ICP-fusion & ug $/ 9$ & 97.60 & $<5.00 \mathrm{e}-02$ & $<8.84 \mathrm{e}+02$ & $<8.88 \mathrm{e} 2$ & $\mathrm{n} / \mathrm{a}$ & $\mathrm{n} / \mathrm{a}$ & $\mathrm{n} / \mathrm{a}$ & 884.0 & $\mathrm{n} / \mathrm{a}$ \\
\hline 5967005477 & $f$ & Beryllium-ICP-Fusion & ug $/ \mathrm{g}$ & 100.2 & $<5.00 \mathrm{e}-03$ & $<\quad 88.40$ & $<8.88 \mathrm{e} 1$ & $\mathrm{n} / \mathrm{a}$ & $n / a$ & $\mathrm{n} / \mathrm{a}$ & 88.40 & $n / a$ \\
\hline 5967005477 & $F$ & Bismuth -ICP-Fusion & $\mathrm{ug} / \mathrm{g}$ & 100.6 & $<1.00 \mathrm{e}-01$ & $2.10 \mathrm{e}+03$ & $2.01 \mathrm{e}+03$ & $2.06 \mathrm{e}+03$ & 4.38 & $\mathrm{n} / \mathrm{a}$ & $9.77 \mathrm{e}+03$ & $n / a$ \\
\hline 5967005477 & $F$ & Calcium-ICP-Fusion & $49 / 9$ & 101.0 & $<1.00 e^{-01}$ & $<1.77 \mathrm{e}+03$ & $<1.78 \mathrm{e} 3$ & $n / a$ & $n / a$ & $n / a$ & $1.77 \mathrm{e}+03$ & $n / a$ \\
\hline S967005477 & $F$ & Cadmium -ICP-Fusion & ug/g & 100.2 & $<5.00 e^{-03}$ & $<\quad 88.40$ & $<8.88 \mathrm{e} 1$ & $n / a$ & $n / a$ & $\mathrm{n} / \mathrm{a}$ & 88.40 & $n / a$ \\
\hline 5967005477 & $F$ & Cerium-ICP-Fusion & ug/g & 98.40 & $<9.00 \mathrm{e}-01$ & $<1.77 \mathrm{e}+03$ & $<1.78 \mathrm{e} 3$ & $n / a$ & $n / a$ & $n / a$ & $1.77 e+03$ & $n / a$ \\
\hline 5967005477 & $F$ & Cobalt-ICP-Fusion & ug/g & 101.4 & $<2.00 \mathrm{e}-02$ & $<3.54 \mathrm{e}+02$ & $<3.55 \mathrm{e} 2$ & $\mathrm{n} / \mathrm{a}$ & $\mathrm{n} / \mathrm{a}$ & $\mathrm{n} / \mathrm{a}$ & 354.0 & $\mathrm{n} / \mathrm{a}$ \\
\hline 5967005477 & $F$ & Chromium -ICP-Fusion & ug $/ 9$ & 101.0 & $<1.00 \mathrm{e}-02$ & $1.83 \mathrm{e}+02$ & 187.0 & 185.0 & 2.16 & $n / a$ & 177.0 & $n / a$ \\
\hline$\$ 967005477$ & $F$ & Copper -ICP-Fusion & ug/g & 102.2 & $<1.00 \mathrm{e}-02$ & $<1.77 \mathrm{e}+02$ & $<1.78 \mathrm{e} 2$ & $\mathrm{n} / \mathrm{a}$ & $n / a$ & $\mathrm{n} / \mathrm{a}$ & 177.0 & $\mathrm{n} / \mathrm{a}$ \\
\hline S96T005477 & $F$ & Iron-ICP-Fusion & $\operatorname{ug} / \mathrm{g}$ & 99.60 & $<5.00 \mathrm{e}-02$ & $<8.84 \mathrm{e}+02$ & $<8.88 \mathrm{e}$ & $n / a$ & $n / a$ & $n / a$ & 884.0 & $n / a$ \\
\hline S96T005477 & $F$ & Lanthanum -ICP-Fusion & $\mathrm{ug} / \mathrm{g}$ & 100.0 & $<5.00 \mathrm{e}-02$ & $<8.84 e+02$ & $<8.88 \mathrm{e} 2$ & $n / a$ & $n / a$ & $\mathrm{n} / \mathrm{a}$ & 884.0 & $n / a$ \\
\hline S967005477 & $F$ & Lithium -ICP-Fusion & ug $/ 9$ & 96.80 & $<7.00 \mathrm{e}-02$ & $<1.77 \mathrm{e}+02$ & $<1.78 e 2$ & $n / a$ & $n / a$ & $\mathrm{n} / \mathrm{a}$ & 177.0 & $n / a$ \\
\hline S96T005477 & $\bar{F}$ & Magnesium-ICP-fusion & ug/g & 94.00 & $<7.00 \mathrm{e}=01$ & $<1.77 \mathrm{e}+03$ & $<1.78 \mathrm{e} 3$ & $\mathrm{n} / \mathrm{a}$ & $n / a$ & $\mathrm{n} / \mathrm{a}$ & $77 \mathrm{e}+03$ & $n / a$ \\
\hline \$967005477 & $F$ & Manganese -ICP-Fusion & $49 / 9$ & 97.20 & $<1.00 \mathrm{e}-02$ & $<1.77 \mathrm{e}+02$ & $<1.78 \mathrm{e} 2$ & $n / a$ & $\mathrm{n} / \mathrm{a}$ & $n / a$ & 177.0 & $n / a$ \\
\hline 5477 & $F$ & Molybodenum -1CP-Fusion & $\mathrm{ug} / \mathrm{g}$ & 101.0 & $<5.00 \mathrm{e}-02$ & $<8.84 \mathrm{e}+02$ & $<8.88 \mathrm{e} 2$ & $n / a$ & $n / a$ & $\mathrm{n} / \mathrm{a}$ & 884.0 & $n / a$ \\
\hline 5967005477 & $F$ & Sodium -ICP-Fusion & ug/g & 94.80 & 1.510 & $1.62 \mathrm{e}+05$ & $1.79 \mathrm{e}+05$ & $1.70 \mathrm{e}+05$ & 9.97 & $n / a$ & $.77 \mathrm{e}+03$ & $\mathrm{n} / \mathrm{a}$ \\
\hline 5967005477 & $F$ & Neodymium-ICP-Fusion & $\mathrm{ug} / \mathrm{g}$ & 99.60 & $<1.00 \mathrm{e}-01$ & $<1.77 \mathrm{e}+03$ & $<1.78 \mathrm{e} 3$ & $n / a$ & $\mathrm{n} / \mathrm{a}$ & $\mathrm{n} / \mathrm{a}$ & $.77 \mathrm{e}+03$ & $n / a$ \\
\hline 5967005477 & $F$ & Phosphorus -1CP-Fusion & ug/g & 98.40 & $<2,00 \mathrm{e}-01$ & $3.40 \mathrm{e}+04$ & $3.82 \mathrm{e}+04$ & $3.61 e+04$ & 11.6 & $n / a$ & $3.54 e+03$ & $n / a$ \\
\hline S96T005477 & $F$ & Lead -ICP-Fusion & ug/g & 100.4 & $<7.00 e-01$ & $<1.77 \mathrm{e}+03$ & $<1.78 \mathrm{e} 3$ & $n / a$ & $n / a$ & $\mathrm{n} / \mathrm{a}$ & $1.77 \mathrm{e}+03$ & $n / a$ \\
\hline 5967005477 & $F$ & Sulfur - ICP-fusion & ug $/ 9$ & 98.20 & $<1.00 \mathrm{e}-01$ & $1.55 \mathrm{e}+04$ & $1.48 \mathrm{e}+04$ & $1.52 \mathrm{e}+04$ & 4.62 & $\mathrm{n} / \mathrm{a}$ & $1.77 \mathrm{e}+03$ & $n / a$ \\
\hline 5967005477 & $F$ & Antimony -ICP-Fusion & ug $/ \mathrm{g}$ & 96.00 & $<6.00 \mathrm{e}-02$ & $<1.06 \mathrm{e}+03$ & $<1.07 \mathrm{e} 3$ & $n / a$ & $n / a$ & $n / a$ & $1.06 \mathrm{e}+03$ & $n / a$ \\
\hline 5967005477 & $F$ & Selenium-ICP-Fusion & ug/g & 95.00 & $<1.00 e^{-01}$ & $<1.77 \mathrm{e}+03$ & $<1.78 \mathrm{e} 3$ & $n / a$ & $n / a$ & $\mathrm{n} / \mathrm{a}$ & $1.77 \mathrm{e}+03$ & $n / a$ \\
\hline$\$ 967005477$ & $F$ & Silicon-ICP-Fusion & $4 \mathrm{~g} / \mathrm{g}$ & 96.80 & $<5.00 \mathrm{e}^{-02}$ & $1.76 \mathrm{e}+03$ & $1.76 \mathrm{e}+03$ & $1.76 \mathrm{e}+03$ & 0.00 & $\mathrm{n} / \mathrm{a}$ & 884.0 & $n / a$ \\
\hline 5961005477 & $F$ & Samarium-ICP-Fusion & ug/g & 96.60 & $<7.00 \mathrm{e}-01$ & $<1.77 \mathrm{e}+03$ & $<1.78 \mathrm{e} 3$ & $n / a$ & $n / a$ & $\mathrm{n} / \mathrm{a}$ & $1.77 \mathrm{e}+03$ & $n / a$ \\
\hline 5967005477 & $F$ & Strontium -ICP-Fusion & ug/g & 97.00 & $<1.00 e^{-02}$ & $<1.77 \mathrm{e}+02$ & $<1.78 \mathrm{e} 2$ & $n / a$ & $n / a$ & $\mathrm{n} / \mathrm{a}$ & 177.0 & $n / a$ \\
\hline S96T005477 & $F$ & Titanium-ICP-Fusion & $\mathrm{ug} / \mathrm{g}$ & 97.40 & $<1.00 \mathrm{e}^{-02}$ & $<1.77 e+02$ & $<1.78 \mathrm{e} 2$ & $n / a$ & $n / a$ & $n / a$ & 177.0 & $n / a$ \\
\hline
\end{tabular}




\begin{tabular}{|c|c|c|c|c|c|c|c|c|c|c|c|c|}
\hline Sample\# & Rla\# & Analyte & Unit & Standard \% & Blank & Result & Dupl icate & Average & RPD \% & Spk Rec \% & Det Limit & Count Err\% \\
\hline 5967005477 & $F$ & Thaltium-ICP-Fusion & $\mathrm{ug} / \mathrm{g}$ & 94.60 & $\leq 2,00 \mathrm{e}-01$ & $<3.54 \mathrm{e}+03$ & $<3.55 \mathrm{e} 3$ & $\mathrm{n} / \mathrm{a}$ & $n / a$ & $n / a$ & $3.54 \mathrm{e}+03$ & $n / a$ \\
\hline 5967005477 & $F$ & Úranium-ICP-Fusion & $40 / 9$ & 94.90 & $<5.00 \mathrm{e}-01$ & $<8.84 \mathrm{e}+03$ & $<8.88 \mathrm{e} 3$ & n/a & $n / a$ & $\mathrm{n} / \mathbf{a}$ & $8.84 \mathrm{e}+03$ & $n / 8$ \\
\hline $596 T 005477$ & $F$ & Vanadium-ICP-Fustion & $4 \mathrm{~g} / \mathrm{g}$ & 100.4 & $5.00 \mathrm{e}-02$ & $<8.84 \mathrm{e}+02$ & $<8.88 \mathrm{e} 2$ & $n / a$ & $n / a$ & $n / a$ & 884.0 & $\mathrm{n} / \mathrm{a}$ \\
\hline 9961005477 & $F$ & Zine-1CP-Fusion & ug/g & 102.0 & $\leq 1.00 \mathrm{e}-02$ & $<1.77 \mathrm{e}+02$ & $<1.78 \mathrm{e} 2$ & $n / a$ & $n / a$ & $n / a$ & 177.0 & $n / a$ \\
\hline 5967005477 & $\vec{F}$ & Zirconjum-ICP-Fusion & $\mathrm{ug} / \mathrm{g}$ & 98.60 & $<1.00 \mathrm{e}-02$ & $<1.77 \mathrm{e}+02$ & $<1.78 \mathrm{e} 2$ & $n / a$ & $n / a$ & $n / a$ & 177.0 & $n / a$ \\
\hline 5967005477 & $\mathbf{F}$ & Cobalt -60 by GEA & UCi/g & 99.75 & $<1.65 \mathrm{e}-02$ & $<1.27 \mathrm{e}-02$ & $<1.19 e-2$ & $\mathrm{n} / \mathrm{a}$ & n/a & $n / a$ & $1.30 \mathrm{e}-02$ & $n / a$ \\
\hline 5967005477 & $\mathbf{F}$ & Cesium-137 by GEA & $\mathrm{uCi} / \mathrm{g}$ & 98.05 & $<3.33 \mathrm{e}-02$ & 17.42 & 16.60 & 17.01 & 4.71 & $\mathrm{n} / \mathrm{a}$ & $n / a$ & 1.21 \\
\hline S96T005477 & $\mathbf{F}$ & Europium-154 by GEA & $\mathrm{uCj} / \mathrm{g}$ & $n / a$ & $55.01 \mathrm{e}-02$ & $<3.66 \mathrm{e}-02$ & $<3.56 \mathrm{e}-2$ & $n / a$ & $n / a$ & $\mathrm{n} / \mathrm{a}$ & $3.70 \mathrm{e}-02$ & $n / a$ \\
\hline 5967005477 & $\mathbf{F}$ & Europium-155 by GEA & $\mathrm{uCi} / \mathrm{g}$ & $n / a$ & $<4.31 \mathrm{e}-02$ & $<1.24 \mathrm{e}-01$ & $\leq 1.23 \mathrm{e}-1$ & $n / a$ & $\mathrm{n} / \mathrm{a}$ & $n / a$ & $1.24 \mathrm{e}-01$ & $\mathrm{n} / \mathrm{a}$ \\
\hline S96r005477 & $\mathbf{F}$ & Americium-241 by GEA & $\mathrm{uCi} / \mathrm{g}$ & $n / 8$ & $<8.16 e-02$ & $<2.59 e-01$ & $<2.49 \mathrm{e}-1$ & $n / a$ & $\mathrm{n} / \mathrm{a}$ & $\mathrm{n} / \mathrm{a}$ & $2.59 \mathrm{e}-01$ & $n / a$ \\
\hline$\$ 967005477$ & $\bar{F}$ & Beta of solid Sample & uCi/g & 107.8 & $3.30 \mathrm{e}-02$ & 28.30 & 24.20 & 26.25 & 15.6 & 102.6 & $1.60 \mathrm{e}-02$ & $4.66 \mathrm{E}-01$ \\
\hline 5967005477 & $\mathbf{F}$ & Alpha of Digested Solid & $\mathrm{uCi} / \mathrm{g}$ & 80.09 & $<4.50 \mathrm{e}-03$ & $9.08 \mathrm{e}-02$ & $7.34 \mathrm{e}-03$ & $9.07 e-03$ & 38.1 & $n / a$ & $8.00 \mathrm{e}-03$ & $6.04 \mathrm{E}+01$ \\
\hline 5967005479 & $W$ & Fluoride-IC-Dionex $4000 / 4500$ & $\mathrm{ug} / \mathrm{g}$ & 102.2 & $<1.20 \mathrm{e}-02$ & $2.69 \mathrm{e}+04$ & $2.68 \mathrm{e}+04$ & $2.69 \mathrm{e}+04$ & 0.37 & $\mathrm{n} / \mathrm{a}$ & .89 .87 & $n / a$ \\
\hline 5967005479 & W & Chloride-1C-Dionex $4000 / 4500$ & ug $/ 9$ & 103.4 & $<1.70 \mathrm{e}-02$ & $8.98 \mathrm{e}+02$ & 894.0 & 855.8 & 9.81 & $\mathrm{n} / \mathrm{a}$ & 127.3 & $n / a$ \\
\hline 5967005479 & $\mathbf{W}$ & Nitrite-IC - Dionex $4000 / 4500$ & $\mathrm{ug} / \mathrm{g}$ & 101.5 & $<1.08 \mathrm{e}-01$ & $1.28 \mathrm{e}+04$ & $1.07 \mathrm{e}+04$ & $1.17 \mathrm{e}+04$ & 17.9 & $n / a$ & 808.9 & $n / a$ \\
\hline \$96T005479 & $\mathbf{W}$ & Bromide by lon Chromatograph & $\mathrm{ug} / \mathrm{g}$ & 99.49 & $<1.25 \mathrm{e}-01$ & $<9.36 \mathrm{e}+02$ & $<9.35 \mathrm{e} 2$ & $n / a$ & $\mathrm{n} / \mathrm{a}$ & $n / a$ & 936.0 & $n / a$ \\
\hline S96T005479 & $\mathbf{W}$ & Witrate by IC-Dionex $4000 / 4500$ & ug/a & 100.8 & $1.94 \mathrm{e}-01$ & $4.85 \mathrm{e}+04$ & $4.10 \mathrm{e}+04$ & $4.48 \mathrm{e}+04$ & 16.8 & $n / a$ & $1.04 \mathrm{e}+03$ & $n / a$ \\
\hline 5967005479 & $\mathbf{W}$ & Phosphate-IC-Dionex $4000 / 4500$ & ug/g & 98.71 & $<1.20 \mathrm{e}-01$ & $1.28 \mathrm{e}+05$ & $1.39 \mathrm{e}+05$ & $1.33 \mathrm{e}+05$ & 8.24 & $\mathrm{n} / \mathrm{a}$ & 898.7 & $n / a$ \\
\hline 5967005479 & $\mathbf{W}$ & Sulfate by JC-Dionex $4000 / 4500$ & $\mathrm{ug} / \mathrm{g}$ & 102.2 & $<1.38 \mathrm{e}-01$ & $6.54 \mathrm{e}+04$ & $5.99 \mathrm{e}+04$ & $6.26 \mathrm{e}+04$ & 8.78 & $n / a$ & $1.03 \mathrm{e}+03$ & $\mathrm{n} / \mathrm{a}$ \\
\hline 5967005479 & $W$ & Oxalate by IC-Dionex $4000 / 4500$ & ug/9 & 106.9 & $<1.05 e-01$ & $<7.86 \mathrm{e}+02$ & $<7.86 \mathrm{e} 2$ & $n / a$ & $n / a$ & $\mathrm{n} / \mathrm{a}$ & 786.4 & $\mathrm{n} / \mathrm{a}$ \\
\hline $596 T 005481$ & 1 & Silver -ICP-H2O Dig/Acid & $\mathrm{ug} / \mathrm{g}$ & 99.60 & $<1.00 e-02$ & 18.80 & $<1.87 \mathrm{e} 1$ & $n / a$ & $n / a$ & 98.40 & 18.80 & $n / a$ \\
\hline 5481 & $I$ & Aluminium -ICP-H2O Dig/Acid & $4 \mathrm{~g} / \mathrm{g}$ & 96.00 & $3.81 \mathrm{e}-01$ & $8.98 \mathrm{e}+02$ & 483.0 & 690.5 & 60.1 & 93.60 & 93.80 & $\mathrm{n} / \mathrm{a}$ \\
\hline 5967005481 & I & Arsenic -ICP-H2O Dig/Acid & $\mathrm{ug} / \mathrm{g}$ & 107.4 & $<1.00 \mathrm{e}-01$ & $<1.88 \mathrm{e}+02$ & $\leq 1.87 \mathrm{e} 2$ & $n / a$ & $n / a$ & 106.0 & 188.0 & $\sqrt{a}$ \\
\hline 5967005481 & $I$ & Boron -ICP-H 20 Dig/Acid & $\mathrm{ug} / \mathrm{g}$ & 100.0 & 4.430 & $1.30 \mathrm{e}+03$ & 774.0 & $1.01 \mathrm{e}+03$ & 58.2 & 100.0 & 93.80 & $n / a$ \\
\hline $596 T 005481$ & I & Barium-ICP-H2O Dig/ACid & $\mathrm{ug} / \mathrm{g}$ & 103.2 & $<5.00 \mathrm{e}-02$ & 93.80 & $9.37 \mathrm{e} 1$ & $\mathrm{n} / \mathrm{a}$ & $n / a$ & 102.0 & 93.80 & $n / a$ \\
\hline 596 T005481 & I & Beryllium-ICP-H2O Dig/Acid & ug/g & 103.2 & $<5.00 \mathrm{e}-03$ & 9.380 & $<9.37 \mathrm{e} 0$ & $\mathrm{n} / \mathrm{a}$ & $n / a$ & 103.0 & 9.380 & $n / a$ \\
\hline 5967005481 & I & Bismuth $-1 \mathrm{CP}-\mathrm{H} 20$ Dig/Acid & $\mathrm{ug} / \mathrm{g}$ & 110.6 & $<1.00 \mathrm{e}-01$ & $<1.88 \mathrm{e}+02$ & $<1.87 \mathrm{e} 2$ & $n / a$ & $n / a$ & 109.0 & 188.0 & $n / a$ \\
\hline S96TO & 1 & Calcium -ICP-H2O Dig/Acid & ug/g & 97.40 & $<1.00 \mathrm{e}-01$ & $<1.88 \mathrm{e}+02$ & $<1.87 \mathrm{e} 2$ & $\mathrm{n} / \mathrm{a}$ & $\mathrm{n} / \mathrm{a}$ & 98.00 & 188.0 & $\underline{n / a}$ \\
\hline $\mathrm{S} 96 \mathrm{TC}$ & I & Cadmiun - ICP-H2O Dig/Acid & $\mathrm{ug} / \mathrm{g}$ & 101.4 & $<5.00 \mathrm{e}-03$ & 9.380 & $<9.37 \mathrm{e} 0$ & $\mathrm{n} / \mathrm{a}$ & $n / a$ & 100.0 & 9.380 & $n / a$ \\
\hline $596 r 005481$ & $\mathrm{I}$ & Cerium-ICP-H2O Dig/Acid & $\mathrm{ug} / \mathrm{g}$ & 100.6 & $<1.00 \mathrm{e}-01$ & $<1.88 \mathrm{e}+02$ & $<1.87 \mathrm{e} 2$ & $n / a$ & $\mathrm{n} / \mathrm{a}$ & 95.90 & 188.0 & $\mathrm{n} / \mathbf{a}$ \\
\hline 5481 & $\mathrm{I}$ & Cobalt -ICP-H2O Dig/ACid & $\mathrm{ug} / \mathrm{g}$ & 101.8 & $<2.00 e^{-02}$ & 47.30 & 51.20 & 49.25 & 7.92 & 98.90 & 37.50 & $n / a$ \\
\hline 481 & I & Chromium - ICP-H2O Dig/ACid & ug/g & 98.60 & $<1.00 \mathrm{e}-02$ & $1.82 \mathrm{e}+02$ & 149.0 & 165.5 & 19.9 & 96.30 & 18.80 & n/a \\
\hline 5481 & $I$ & Copper -ICP-H2O Dig/Acid & $\mathrm{ug} / \mathrm{g}$ & 100.6 & $<1.00 \mathrm{e}-02$ & 18.80 & $<1.87 \mathrm{e} 1$ & $n / a$ & $\mathrm{n} / \mathrm{a}$ & 102.0 & 18.80 & $n / a$ \\
\hline 596T & I & Iron-ICP-H 20 Dig/Acid & ug/g & 100.8 & $<5.00 \mathrm{e}-02$ & 93.80 & $<9.37 \mathrm{e} 1$ & $n / a$ & $\mathrm{n} / \mathrm{a}$ & 98.30 & 93.80 & n/a \\
\hline $596 \mathrm{~T}$ & I & Potassium -ICP-H2O Dig/Acid & $4 g / g$ & 100.0 & $<5.00 e-01$ & $<9.38 \mathrm{e}+02$ & $<9.37 \mathrm{e} 2$ & $n / a$ & $n / a$ & 151.0 & 938.0 & $n / a$ \\
\hline S961 & I & Lanthanum - ICP-H2O Dig/Acid & ug/g & 99.60 & $<5.00 e-02$ & 93.80 & $<9.37 e 1$ & $n / a$ & $\mathrm{n} / \mathrm{a}$ & 99.50 & 93.80 & $\mathrm{n} / \mathrm{a}$ \\
\hline 596 & I & Lithium -ICP-H2O Dig/ACid & $49 / 9$ & 94.40 & $<1.00 e-02$ & 18.80 & $<1.87 \mathrm{e} 1$ & $n / a$ & $n / a$ & 92.90 & 18.80 & $n / a$ \\
\hline 5961005481 & I & Magnesium - ICP-H2O Dig/ACid & $\mathrm{ug} / \mathrm{g}$ & 103.2 & $<1.00 \mathrm{e}-01$ & $<1.88 \mathrm{e}+02$ & $<1.87 \mathrm{e} 2$ & $\mathrm{n} / \mathrm{a}$ & $n / a$ & 102.0 & 188.0 & $n / a$ \\
\hline 5481 & I & Manganese $-\mathrm{ICP}-\mathrm{H} 2 \mathrm{O} \mathrm{Dig} / \mathrm{Acid}$ & ug/9 & 100.4 & $<1.00 \mathrm{e}-02$ & 18.80 & $<1.87 \mathrm{e} 1$ & $\mathrm{n} / \mathrm{a}$ & $n / a$ & 99.80 & 18.80 & $n / a$ \\
\hline 5481 & I & Molybdenum - ICP-H2O Dig/Acid & $49 / 9$ & 103.6 & $<5.00 e-02$ & 93.80 & $<9.37 \mathrm{e} 1$ & $\mathrm{n} / \mathbf{a}$ & $\mathrm{n} / \mathrm{a}$ & 102.0 & 93.80 & $\mathrm{n} / \mathrm{a}$ \\
\hline 481 & I & Sodium - ICP-H2O Dig/ACid & $49 / 9$ & 98.80 & 6.210 & $1.74 \mathrm{e}+05$ & $1.69 \mathrm{e}+05$ & $2 \mathrm{e}+05$ & 2.92 & 62.90 & 188.0 & $n / a$ \\
\hline 5961005481 & I & Neodymium-ICP-H2O Dig/Acid & ug/g & 99.00 & $<7.00 e-01$ & $<1.88 \mathrm{e}+02$ & $<1.87 \mathrm{e} 2$ & $\mathrm{n} / \mathrm{a}$ & $n / a$ & 98.30 & 188.0 & $n / a$ \\
\hline 5961005481 & I & Wickel-ICP-H2O OIg/ACid & $\mathrm{ug} / \mathrm{g}$ & 100.2 & $<2.00 e-02$ & 37.50 & $<3.75 e 1$ & $n / a$ & $n / a$ & 100.0 & 37.50 & $n / a$ \\
\hline 5481 & I & Phosphorus - ICP-H2O Dig/ACid & ug/g & 106.0 & $<2.00 \mathrm{e}-01$ & $4.16 \mathrm{e}+04$ & $4.37 \mathrm{e}+04$ & $4.26 \mathrm{e}+04$ & 4.92 & 118.0 & 375.0 & $n / a$ \\
\hline 5481 & 1 & Lead-ICP-H2O Dig/Acid & $4 \mathrm{~g} / \mathrm{g}$ & 99.20 & $<1.00 e-01$ & $<1.88 \mathrm{e}+02$ & $<1.87 \mathrm{e} 2$ & $\mathrm{n} / \mathrm{a}$ & $n / a$ & 102.0 & 188.0 & $n / a$ \\
\hline S96T005481 & I & Sulfur-ICP-H2O Dig/Acid & $\mathrm{ug} / \mathrm{g}$ & 102.0 & $\leq 1.00 e-01$ & $2.07 \mathrm{e}+04$ & $1.86 \mathrm{e}+04$ & $1.96 \mathrm{e}+04$ & 10.7 & 113.0 & 188.0 & $\mathrm{n} / \mathrm{a}$ \\
\hline 5967005481 & I & Antimony - ICP-H2O Dig/Acid & ug/g & 111.6 & $<6.00 e-02$ & $<1.13 \mathrm{e}+02$ & $<1.12 \mathrm{e} 2$ & $n / a$ & $n / a$ & 98.40 & 113.0 & $n / a$ \\
\hline 5581 & I & Selenium -ICP-H2O Dig/Acid & ug/g & 84.20 & $<7.00 e-01$ & $<1.88 \mathrm{e}+02$ & $<1.87 \mathrm{e} 2$ & $\mathrm{n} / \mathrm{a}$ & $\mathrm{n} / \mathrm{a}$ & 79.90 & 188.0 & $\mathrm{n} / \mathrm{a}$ \\
\hline 5481 & I & Isilicon - ICP-H20 big/Acid & $\mathrm{ug} / \mathrm{g}$ & 98.00 & 4.480 & $8.32 \mathrm{e}+03$ & $2.19 e+03$ & $26 \mathrm{e}+03$ & 117 & 117.0 & 93.80 & $n / a$ \\
\hline 596T0 & $t$ & Samarium - ICP-H2O Dig/ACid & ug/g & 109.0 & $<1.00 e-01$ & $<7.88 \mathrm{e}+02$ & $<1.87 \mathrm{e} 2$ & $n / a$ & $n / a$ & 110.0 & 188.0 & $\mathrm{n} / \mathrm{a}$ \\
\hline 5967005481 & & Strontium - ICP-H 20 Dig/Acid & ug/g & 105.2 & $<1.00 e^{-02}$ & 8.80 & $<1.87 \mathrm{e} 1$ & $n / a$ & $n / a$ & 105.0 & 18.80 & $n / a$ \\
\hline
\end{tabular}




\begin{tabular}{|c|c|c|c|c|c|c|c|c|c|c|c|c|}
\hline Sample\# & R A\# & \#Anatyte & Unit & Standard \% & Blank & Result & Duplicate & Average & RPD $\%$ & Spk Rec $\%$ & Det Limit & Count Err\% \\
\hline 596T005481 & I & Titanium-ICP-H2O Dig/Acid & $4 \mathrm{~g} / \mathrm{g}$ & 97.00 & $<1.00 \mathrm{e}-02$ & 18.80 & $<1.87 \mathrm{e} 1$ & $n / a$ & $n / a$ & 95.90 & 18.80 & $\mathrm{n} / \mathrm{a}$ \\
\hline 5961005481 & I & That I ium -ICP-H20 Dig/Acid & $u g / g$ & 102.4 & $<2.00 \mathrm{e}-01$ & $<3.75 \mathrm{e}+02$ & $<3.75 \mathrm{e} 2$ & $\mathrm{n} / \mathrm{a}$ & $\mathrm{n} / \mathrm{a}$ & 96.30 & 375.0 & $n / a$ \\
\hline 596 & I & Uranium -ICP-H2O Dig/Acid & $\mathrm{ug} / \mathrm{g}$ & 105.0 & $<5.00 \mathrm{e}-07$ & $<9.38 \mathrm{e}+02$ & $<9.37 \mathrm{e} 2$ & $\mathrm{n} / \mathrm{a}$ & $n / a$ & 112.0 & 938.0 & $n / a$ \\
\hline $596 \mathrm{~T}$ & I & Vanadium -ICP-H2O Dig/Acid & $\mathrm{Lg} / \mathrm{g}$ & 103.2 & $<5,00 e-02$ & $<93.80$ & $<9.37 \mathrm{e} 1$ & $\mathrm{n} / \mathrm{a}$ & $n / a$ & 103.0 & 93.80 & $\mathrm{n} / \mathrm{a}$ \\
\hline 5967005481 & I & ZinC-ICP-HZO Dig/ACid & $u g / g$ & 104.0 & $<1.00 \mathrm{e}-02$ & 39.40 & $<1.87 \mathrm{e} 1$ & $n / a$ & $\mathrm{n} / \mathrm{a}$ & 103.0 & 18.80 & $n / a$ \\
\hline 5967005481 & I & Zirconium -ICP-H2O Dig/Acid & $\mathrm{ug} / \mathrm{g}$ & 104.0 & $<1.00 \mathrm{e}-02$ & 18.80 & $<1.87 \mathrm{e} 1$ & $\mathrm{n} / \mathrm{a}$ & $\mathrm{n} / \mathrm{a}$ & 105.0 & 18.80 & $\mathrm{n} / \mathrm{a}$ \\
\hline
\end{tabular}


Table 3. Data Summary rabłe

CORE NUMBER: 173

SEGMENT \#: 2

SEGMENT PORTION: L Lower Half of Segment

\begin{tabular}{|c|c|c|c|c|c|c|c|c|c|c|c|c|}
\hline Sample\# & R A AH & \#Analyte & Unit & Standard \% & Blank & Result $t$ & Duplicate & Average & RPD \% & Spk Rec \% & Det Limit & Count Err\% \\
\hline 5967005472 & & Butk Density of Sample & $\mathrm{g} / \mathrm{mt}^{\mathrm{t}}$ & $n / a$ & $\mathrm{n} / \mathrm{a}$ & 1.570 & $n / a$ & $n / a$ & $\mathrm{n} / \mathrm{a}$ & $n / a$ & $5.00 \mathrm{e}-01$ & $\mathrm{n} / \mathrm{a}$ \\
\hline $596 T 005474$ & & DSC Exotherm using Mettler & Joules/g & 95.61 & n/a & $0.00 \mathrm{e}+00$ & $0.00 \mathrm{e}+00$ & $0.00 \mathrm{e}+00$ & 0.00 & $\mathrm{n} / \mathrm{a}$ & $n / a$ & $n / a$ \\
\hline 5967005474 & & DSC Exotherm Dry Catculated & Joules/g Dry & $\mathrm{n} / \mathrm{a}$ & $n / a$ & $0.00 \mathrm{e}+00$ & $0.00 \mathrm{e}+00$ & $0.00 \mathrm{e}+00$ & 0.00 & $\mathrm{n} / \mathrm{a}$ & $n / a$ & $\mathrm{n} / \mathrm{a}$ \\
\hline 5967005474 & & \& Water by TGA using Mettler & $\%$ & 99.09 & $\mathrm{n} / \mathrm{a}$ & 44.53 & 43.03 & 43.78 & 3.43 & $\mathrm{n} / \mathrm{a}$ & $\mathrm{n} / \mathrm{a}$ & $n / a$ \\
\hline S961005478 & $F$ & Nickel-ICP-Fusion & ug/g & 100.0 & 1.220 & $2.52 \mathrm{e}+03$ & $2.41 \mathrm{e}+04$ & $1.33 \mathrm{e}+04$ & 762 & 95.30 & 356.0 & $n / a$ \\
\hline 5967005478 & $F$ & Sitver-ICP-Fusion & $49 / 9$ & 99,00 & $<1.00 \mathrm{e}-02$ & $\leq 1.78 \mathrm{e}+02$ & $<1.80 \mathrm{e} 2$ & $\mathrm{n} / \mathrm{a}$ & $\mathrm{n} / \mathrm{a}$ & 89.30 & 178.0 & $n / a$ \\
\hline$\$ 967005478$ & $F$ & Aluminium-ICP-Fusion & $49 / 9$ & 95.80 & $<5.00 \mathrm{e}-02$ & $1.63 e+04$ & $7.25 e+04$ & $4.44 \mathrm{e}+04$ & 127 & 89.80 & 888.0 & $n / a$ \\
\hline$\$ 967005478$ & $F$ & Arsenic -ICP-Fusion & $\mathrm{ug} / \mathrm{g}$ & 101.0 & $<7.00 e^{-01}$ & $<1.78 \mathrm{e}+03$ & $<1.80 \mathrm{e} 3$ & $n / a$ & $n / a$ & 98.60 & $1.78 \mathrm{e}+03$ & $n / a$ \\
\hline 5967005478 & $F$ & Boron -ICP-Fusion & $\mathrm{ug} / \mathrm{g}$ & 97.80 & $<5.00 \mathrm{e}-02$ & $<8.90 \mathrm{e}+02$ & $<9.01 \mathrm{e} 2$ & $\mathrm{n} / \mathrm{a}$ & $n / a$ & 91.50 & 888.0 & $n / a$ \\
\hline 5961005478 & $F$ & Barium -ICP-Fusion & $\mathrm{ug} / \mathrm{g}$ & 97.60 & $\angle 5.00 \mathrm{e}-02$ & $<8.90 \mathrm{e}+02$ & $\leq 9.01 \mathrm{e} 2$ & $n / a$ & $n / a$ & 90.90 & 888.0 & $n / a$ \\
\hline 5967005478 & $F$ & Beryllium-ICP-Fusion & ug $/ 9$ & 100.2 & $55.00 e-03$ & $<\quad 89.00$ & $<9.01 \mathrm{e} 1$ & $\mathrm{n} / \mathrm{a}$ & $n / a$ & 94.20 & 88.80 & $n / a$ \\
\hline 5967005478 & $F$ & Bismuth -ICP-Fusion & $\mathrm{ug} / \mathrm{g}$ & 100.6 & $<1.00 e-01$ & $<1.78 \mathrm{e}+03$ & $<1.80 e^{3}$ & $\mathrm{n} / \mathrm{a}$ & $n / a$ & 99.70 & $.78 \mathrm{e}+03$ & $n / a$ \\
\hline 5961005478 & $F$ & Calcium -ICP-Fusion & $\operatorname{lng} / 9$ & 101.0 & $<1.00 \mathrm{e}-01$ & $\leq 1.78 \mathrm{e}+03$ & $<1.80 \mathrm{e} 3$ & $\mathrm{n} / \mathrm{a}$ & n/a & 107.0 & $1.78 \mathrm{e}+03$ & $n / a$ \\
\hline 5961005478 & $F$ & Cadmitum-ICP-Fusion & $\mathrm{ug} / \mathrm{g}$ & 100.2 & $<5.00 \mathrm{e}-03$ & $<\quad 89.00$ & $<9.01 \mathrm{e} 1$ & $n / a$ & $n / a$ & 97.00 & 88.80 & $n / a$ \\
\hline 5961005478 & $\mathrm{~F}$ & Cerium-icP-fusion & ug/g & 98.40 & $<1.00 \mathrm{e}-01$ & $<1.78 \mathrm{e}+03$ & $<1.80 \mathrm{e} 3$ & $n / a$ & $n / a$ & 93.10 & $1.78 \mathrm{e}+03$ & $n / a$ \\
\hline 5967005478 & $F$ & Cobalt - ICP-Fusion & $\mathrm{ug} / \mathrm{g}$ & 101.4 & $<2.00 \mathrm{e}-02$ & $<3.56 e+02$ & $<3.60 \mathrm{e} 2$ & $n / a$ & $n / a$ & 98.70 & 356.0 & $n / a$ \\
\hline $596 \mathrm{~T} 005478$ & $F$ & Chromium -ICP-Fusion & ug/g & 101.0 & $<1.00 \mathrm{e}-02$ & $<1.78 \mathrm{e}+02$ & 196.0 & $n / a$ & $\mathrm{n} / \mathrm{a}$ & 98.60 & 178.0 & $n / a$ \\
\hline S96T005478 & $F$ & Copper-ICP-Fusion & ug/g & 102.2 & $<1.00 \mathrm{e}-02$ & $<1.78 \mathrm{e}+02$ & $<1.80 \mathrm{e} 2$ & $n / a$ & $n / a$ & 94.80 & 178.0 & $n / a$ \\
\hline S96T005478 & $F$ & Iron-ICP-Fusion & ug/9 & 99.60 & $55.00 \mathrm{e}-02$ & $<8.90 \mathrm{e}+02$ & $<9.01 \mathrm{e} 2$ & $n / a$ & n/a & 95.90 & 888.0 & $n / a$ \\
\hline 5961005478 & $F$ & Lanthanum -ICP-Fusion & ug/g & 100.0 & $<5.00 \mathrm{e}-02$ & $<8.90 \mathrm{e}+02$ & $<9.01 e_{2}$ & $n / a$ & $n / a$ & 93.70 & 888.0 & $n / a$ \\
\hline 15478 & $F$ & Lithium -ICP-Fusion & ug/g & 96.80 & $\angle 7.00 \mathrm{e}-02$ & $<1.78 \mathrm{e}+02$ & $<1.80 \mathrm{e} 2$ & $n / a$ & $n / a$ & 88.20 & 178.0 & $n / a$ \\
\hline 5967005478 & $F$ & Magnesium-ICP-Fusion & ug $/ 9$ & 94.00 & $<1,00 \mathrm{e}-01$ & $<1.78 \mathrm{e}+03$ & $<1.80 \mathrm{e} 3$ & $n / a$ & $n / a$ & 88.10 & $78 \mathrm{e}+03$ & $n / a$ \\
\hline 5967005478 & $\bar{F}$ & Manganese $-I C P-F u s i o n$ & ug/g & 97.20 & $<1.00 \mathrm{e}-02$ & $<1.78 \mathrm{e}+02$ & $<1.80 \mathrm{e} 2$ & $n / a$ & $n / a$ & 92.80 & 178.0 & $n / a$ \\
\hline 15478 & $F$ & Molybdenum - ICP-Fusion & $\mathrm{ug} / \mathrm{g}$ & 101.0 & $<5.00 \mathrm{e}-02$ & $<8.90 \mathrm{e}+02$ & $<9.01 e^{2}$ & $n / a$ & $n / a$ & 98.00 & 888.0 & $n / a$ \\
\hline 5478 & $F$ & Sodium-ICP-Fusion & $\mathrm{ug} / \mathrm{g}$ & 94.80 & 1.510 & $2.12 \mathrm{e}+05$ & $1.73 \mathrm{e}+05$ & $1.92 e+05$ & 20.3 & 89.70 & $78 \mathrm{e}+03$ & $\mathrm{n} / \mathrm{a}$ \\
\hline S96T005478 & $F$ & Neodymium-ICP-Fusion & $\mathrm{ug} / \mathrm{g}$ & 99.60 & $<1.00 \mathrm{e}-01$ & $<1.78 \mathrm{e}+03$ & $<1.80 e^{3}$ & $\mathrm{n} / \mathrm{a}$ & $n / a$ & 92.30 & $.78 \mathrm{e}+03$ & $n / a$ \\
\hline S96T005478 & $F$ & Phosphorys -ICP-Fusion & ug/g & 98.40 & $<2.00 e^{-01}$ & $5.42 \mathrm{e}+04$ & $2.21 e+04$ & $3.82 \mathrm{e}+04$ & 84.1 & 94.00 & $3.56 \mathrm{e}+03$ & $n / a$ \\
\hline S96T & $F$ & Lead -ICP-Fusion & $49 / 9$ & 100.4 & $<1.00 \mathrm{e}-01$ & $<1.78 \mathrm{e}+03$ & $<1.80 \mathrm{e}^{3}$ & $n / a$ & $n / a$ & 98.80 & $1.78 \mathrm{e}+03$ & $n / a$ \\
\hline S96TC & $F$ & Sulfur-ICP-Fusion & ug/g & 98.20 & $<1.00 \mathrm{e}=01$ & $2.12 \mathrm{e}+04$ & $3.42 \mathrm{e}+04$ & $2.77 \mathrm{e}+04$ & 46.9 & 98.60 & $1.78 \mathrm{e}+03$ & $\mathrm{n} / \mathrm{a}$ \\
\hline $596 T 005478$ & $F$ & Ant imony -ICP-Fusion & ug $\angle 9$ & 96.00 & $<6.00 \mathrm{e}-02$ & $<1.07 e+03$ & $<1.08 \mathrm{e} 3$ & $n / a$ & $n / a$ & 92.50 & $1.07 \mathrm{e}+03$ & $\mathrm{n} / \mathrm{a}$ \\
\hline S96T0 & $F$ & Selenium -ICP-Fusion & $\mathrm{ug} / \mathrm{g}$ & 95.00 & $<1.00 \mathrm{e}-01$ & $<1.78 \mathrm{e}+03$ & $<1.80 \mathrm{e} 3$ & $\mathrm{n} / \mathrm{a}$ & $n / a$ & 95.80 & $1.78 \mathrm{e}+03$ & $n / a$ \\
\hline 478 & $\mathrm{~F}$ & Silicon-ICP-Fusion & ug/g & 96.80 & $<5.00 \mathrm{e}-02$ & $1.08 \mathrm{e}+03$ & $1.12 e+03$ & $7.10 \mathrm{e}+03$ & 3.64 & 95.001 & 888.0 & $n / a$ \\
\hline 5478 & $F$ & Samarium-ICP-Fusion & ug/9 & 96.60 & $<1.00 \mathrm{e}-01$ & $<1.78 \mathrm{e}+03$ & $<1.80 \mathrm{e} 3$ & $\mathrm{n} / \mathrm{a}$ & $n / a$ & 93.50 & $1.78 \mathrm{e}+03$ & $n / a$ \\
\hline$\$ 967005478$ & $F$ & Strontium - ICP-Fusion & $\mathrm{ug} / \mathrm{g}$ & 97.00 & $<1.00 \mathrm{e}-02$ & $<1.78 \mathrm{e}+02$ & $<1.80 \mathrm{e} 2$ & $n<a$ & $n / a$ & 90.30 & 178.0 & $\mathrm{n} / \mathrm{a}$ \\
\hline \$96T005478 & $\mathrm{F}$ & Iitanium-ICP-Fusion & $\mathrm{ug} / \mathrm{g}$ & 97.40 & $<1.00 e-02$ & $<1.78 \mathrm{e}+02$ & $<1.80 \mathrm{e} 2$ & $n / a$ & $n / a$ & 92.80 & 178.0 & $\mathrm{n} / \mathrm{a}$ \\
\hline 5478 & $F$ & Thallium-ICP-Fusion & $4 g / 9$ & 94.60 & $<2.00 \mathrm{e}-01$ & $<3.56 \mathrm{e}+03$ & $<3.60 \mathrm{e} 3$ & $n / a$ & $n / a$ & 89.80 & $3.56 \mathrm{e}+03$ & $n / a$ \\
\hline 55478 & $F$ & Úraniuil -ICP-Fusion & $\mathrm{ug} / \mathrm{g}$ & 94.90 & $<5.00 \mathrm{e}-01$ & $<8.90 \mathrm{e}+03$ & $<9.01 e^{3}$ & $n / a$ & $n / a$ & 94.001 & $8.88 \mathrm{e}+03$ & $\mathrm{n} / \mathrm{a}$ \\
\hline$\$ 967005478$ & $\mathrm{~F}$ & Vanadium -ICP-Fusion & ug/g & 100.4 & $55.00 \mathrm{e}-02$ & $<8.90 \mathrm{e}+02$ & $\angle 9.01 \mathrm{e} 2$ & $n / a$ & $n / a$ & 97.001 & 888.0 & $n / a$ \\
\hline 5478 & $F$ & Zinc-ICP-Fusion & ug/g & 102.0 & $<1.00 \mathrm{e}-02$ & $<1.78 \mathrm{e}+02$ & $<1.80 \mathrm{e} 2$ & $n / a$ & $n / a$ & 101.0. & 178.0 & $n / a$ \\
\hline 6478 & F & Zirconium-1CP-Fusion & ug/g & 98.60 & $<1.00 \mathrm{e}-02$ & $<1.78 \mathrm{e}+02$ & $<1.80 \mathrm{e} 2$ & $n / a$ & $n / e$ & 93.70 & 178.0 & $\mathrm{n} / \mathrm{a}$ \\
\hline 35478 & $F$ & Alpha of Digested Solid & uci/g & 80.09 & $<4.50 \mathrm{e}-03$ & $9.37 \mathrm{e}-03$ & $1.37 \mathrm{e}-02$ & $1.15 \mathrm{e}-02$ & 37.5 & 108.1 & $1.60 \mathrm{e}-02$ & $1.06 \mathrm{E}+02$ \\
\hline S96T005480 & $\mathrm{H}$ & Fluoride-IC-Dionex $4000 / 4500$ & $\mathrm{ug} / \mathrm{g}$ & 110.7 & $<1.20 \mathrm{e}-02$ & $3.90 \mathrm{e}+04$ & $3.53 \mathrm{e}+04$ & $3.71 \mathrm{e}+04$ & 9.96 & $-3.750 \mathrm{e} 1$ & 86.14 & $n / a$ \\
\hline 5967005480 & $\mathrm{~W}$ & Chloride-IC-Dionex $4000 / 4500$ & ug/g & 95.06 & $2.70 \mathrm{e}-02$ & $4.57 \mathrm{e}+02$ & 528.0 & 492.7 & 14.4 & 98.86 & 122.1 & $\mathrm{n} / \mathrm{a}$ \\
\hline 480 & & Mitrite-IC - Dionex $4000 / 4500$ & $\mathrm{ug} / \mathrm{g}$ & 95.93 & $<1.08 e-01$ & $7.13 \mathrm{e}+03$ & $6.21 \mathrm{e}+03$ & $6.67 \mathrm{e}+03$ & 13.8 & 95.01 & 775.3 & $n / a$ \\
\hline
\end{tabular}




\begin{tabular}{|c|c|c|c|c|c|c|c|c|c|c|c|c|}
\hline Sample\# & $\mathbf{R} \mid$ A\# & \#Analyte & Unit & Standard \% & Blank & Result $t$ & Duplicate & Average & RPD \% & Spk Rec \% & Det Limit & Count Err\% \\
\hline 5967005480 & $\bar{W}$ & Bromide by Ion Chromatograph & $\operatorname{ug} / \mathrm{g}$ & 98.64 & $<1.25 \mathrm{e}=01$ & $<8.97 \mathrm{e}+02$ & $<9.23 \mathrm{e} 2$ & $\mathrm{n} / \mathrm{a}$ & $n / a$ & 100.3 & 897.5 & $\mathrm{n} / \mathrm{a}$ \\
\hline 5967005480 & Wu & Nitrate by IC-Dionex $4000 / 4500$ & $\log / 9$ & 97.64 & $<1.39 \mathrm{e}-01$ & $3.84 \mathrm{e}+04$ & $3.39 \mathrm{e}+04$ & $3.61 \mathrm{e}+04$ & 12.4 & 94.61 & 997.9 & $n / a$ \\
\hline$\$ 967005480$ & H & Phosphate-IC-Dionex $4000 / 4500$ & $40 / 9$ & 94.30 & $<1.20 \mathrm{e}-01$ & $2.14 \mathrm{e}+05$ & $1.87 \mathrm{e}+05$ & $2.01 \mathrm{e}+05$ & 13.5 & 28.86 & 861.4 & $\mathrm{n} / \mathrm{a}$ \\
\hline$\$ 967005480$ & H & Sulfate by IC-Dionex $4000 / 4500$ & $\mathrm{ug} / \mathrm{g}$ & 99.05 & $<1.38 \mathrm{e}-01$ & $7.21 \mathrm{e}+04$ & $6.57 e+04$ & $6.89 e+04$ & 9.29 & 87.64 & 990.6 & $\mathrm{n} / \mathrm{a}$ \\
\hline$\$ 967005480$ & W & Oxalate by IC-Dionex $4000 / 4500$ & ug/g & 104.2 & $<1.05 e-01$ & $<7.54 \mathrm{e}+02$ & $<7.75 \mathrm{e} 2$ & $n / a$ & $\mathrm{n} / \mathrm{a}$ & 109.3 & 754.0 & $n / a$ \\
\hline S97T000001 & $F$ & Silver-ICP-Fusion & $\mathrm{ug} / \mathrm{g}$ & 97.80 & $<1.00 \mathrm{e}-02$ & $<9.96 \mathrm{e}+02$ & $<1.96 \mathrm{e} 2$ & $n / a$ & $n / a$ & 85.60 & 196.0 & $n / a$ \\
\hline S97T000001 & $F$ & Aluminium-1CP-Fusion & ug/9 & 99.20 & $<5.00 \mathrm{e}-02$ & $2.39 \mathrm{e}+04$ & $1.24 \mathrm{e}+04$ & $1.82 \mathrm{e}+04$ & 63.4 & 93.50 & 980.0 & $n / a$ \\
\hline S97T000001 & $F$ & Arsenic-ICP-Fusion & ug/g & 102.4 & $<1.00 \mathrm{e}-01$ & $<1.96 \mathrm{e}+03$ & $<1.96 \mathrm{e} 3$ & $n / 8$ & $\mathrm{n} / \mathrm{a}$ & 101.0 & $1.96 \mathrm{e}+03$ & $\mathrm{n} / \mathrm{a}$ \\
\hline S97T000001 & $F$ & Boron-ICP-Fusion & $\mathrm{ug} / \mathrm{g}$ & 100.8 & $<5.00 \mathrm{e}-02$ & $<9.80 \mathrm{e}+02$ & $<9.80 \mathrm{e} 2$ & $n / a$ & $n / a$ & 95.50 & 980.0 & $\mathrm{n} / \mathrm{a}$ \\
\hline S971000001 & $F$ & Barium-ICP-Fusion & $\mathrm{ug} / \mathrm{g}$ & 100.8 & $<5.00 \mathrm{e}-02$ & $<9.80 \mathrm{e}+02$ & $\angle 9.80 \mathrm{e} 2$ & $n / a$ & $\mathrm{n} / \mathrm{a}$ & 94.30 & 980.0 & $\mathrm{n} / \mathrm{a}$ \\
\hline S97T000001 & $F$ & Beryllium-ICP-Fusion & $\operatorname{lug} / 9$ & 103.2 & $<5.00 \mathrm{e}-03$ & 98.00 & $<9.80 \mathrm{e}$ & $\mathrm{n} / \mathrm{a}$ & $\mathrm{n} / \mathrm{a}$ & 97.70 & 98.00 & $n / a$ \\
\hline$\$ 97 T 000001$ & $\mathrm{~F}$ & Bismuth-ICP-Fusion & ug/g & 102.0 & $<7.00 \mathrm{e}-01$ & $<1.96 \mathrm{e}+03$ & $<1.96 \mathrm{e} 3$ & $n / a$ & $\mathrm{n} / \mathrm{a}$ & 105.0 & $1.96 \mathrm{e}+03$ & $n / a$ \\
\hline S97T000001 & $F$ & Calcium-ICP-Fusion & ug $/ 9$ & 98.60 & $<1.00 e-01$ & $<1.96 \mathrm{e}+03$ & $<1.96 \mathrm{e} 3$ & $n / a$ & $n / a$ & 98.70 & $1.96 \mathrm{e}+03$ & $n / a$ \\
\hline S97T000001 & $F$ & Cadmium-ICP-fusion & $\mathrm{ug} / \mathrm{g}$ & 100.0 & $<5.00 \mathrm{e}-03$ & $<98.00$ & $<9.80 \mathrm{e1}$ & $\mathrm{n} / \mathrm{a}$ & $n / a$ & 97.90 & 98.00 & $n / a$ \\
\hline S97T000001 & $F$ & Cerium-ICP-Fusion & $u g / g$ & 103.2 & $<1.00 \mathrm{e}-01$ & $<1.96 \mathrm{e}+03$ & $<1.96 \mathrm{e} 3$ & $n / a$ & $n / a$ & 99.60 & $1.96 e+03$ & $n / a$ \\
\hline S97T000001 & $F$ & Cobalt - ICP-Fusion & ug/g & 100.4 & $<2.00 \mathrm{e}-02$ & $<3.92 \mathrm{e}+02$ & $<3.92 \mathrm{e} 2$ & $n / a$ & $n / a$ & 98.00 & 392.0 & $n / a$ \\
\hline S97T000001 & $F$ & Chromium - ICP-Fusion & $\mathrm{ug} / \mathrm{g}$ & 100.2 & $<1,00 \mathrm{e}-02$ & $<1.96 \mathrm{e}+02$ & $<1.96 \mathrm{e} 2$ & $n / a$ & $n / a$ & 99.10 & 196.0 & $n / a$ \\
\hline S97T000001 & $F$ & Copper - ICP-Fusion & ug/g & 106.8 & $<1,00 \mathrm{e}-02$ & $<1.96 \mathrm{e}+02$ & $<1.96 \mathrm{e} 2$ & $\mathrm{n} / \mathrm{a}$ & $\mathrm{n} / \mathrm{a}$ & 100.0 & 196.0 & $\mathrm{n} / \mathrm{a}$ \\
\hline $597 T 000001$ & $F$ & Iron-ICP-Fusion & $\mathrm{ug} / \mathrm{g}$ & 101.2 & $<5.00 \mathrm{e}-02$ & $<9.80 \mathrm{e}+02$ & $<9.80 \mathrm{e} 2$ & $n / a$ & $\mathrm{n} / \mathrm{a}$ & 98.60 & 980.0 & $n / a$ \\
\hline 5977000001 & $\bar{F}$ & Lanthanum -ICP-Fusion & $\mathrm{ug} / \mathrm{g}$ & 102.4 & $<5.00 \mathrm{e}-02$ & $<9.80 \mathrm{e}+02$ & $<9.80 \mathrm{e} 2$ & $n / a$ & $n / a$ & 97.20 & 980.0 & $n / a$ \\
\hline S97T000001 & $F$ & Lithium-ICP-Fusion & $\mathrm{ug} / \mathrm{g}$ & 99.60 & $<1.00 \mathrm{e}-02$ & $<1.96 \mathrm{e}+02$ & $<1.96 \mathrm{e} 2$ & $n / a$ & $\mathrm{n} / \mathrm{a}$ & 91.30 & 196.0 & $n / a$ \\
\hline S97T000001 & $\mathrm{F}$ & Magnesium -ICP-Fusion & ug/g & 101.6 & $<1.00 \mathrm{e}-01$ & $<1.96 \mathrm{e}+03$ & $<1.96 \mathrm{e} 3$ & $\mathrm{n} / \mathrm{a}$ & $\mathrm{n} / \mathrm{a}$ & 98.00 & $1.96 \mathrm{e}+03$ & $n / a$ \\
\hline 5977000001 & $\mathrm{~F}$ & Manganese-ICP-Fusion & $\mathrm{ug} / \mathrm{g}$ & 98.40 & $<1.00 \mathrm{e}-02$ & $<1.96 \mathrm{e}+02$ & $<1.96 \mathrm{e} 2$ & $n / a$ & $\mathrm{n} / \mathrm{a}$ & 94.30 & 196.0 & $n / a$ \\
\hline 5977000001 & $F$ & Molybdenum -ICP-Fusion & ug/g & 101.4 & $<5.00 \mathrm{e}-02$ & $<9.80 \mathrm{e}+02$ & $<9.80 \mathrm{e} 2$ & $\mathrm{n} / \mathrm{a}$ & $n / a$ & 99.80 & 980.0 & $\mathrm{n} / \mathrm{a}$ \\
\hline$\$ 977000001$ & $F$ & Sodium-ICP-Fusion & $\operatorname{lug} / 9$ & 105.6 & 1.600 & $2.44 \mathrm{e}+05$ & $2.46 \mathrm{e}+05$ & $2.45 e+05$ & 0.82 & 83.90 & $1.96 \mathrm{e}+03$ & $n / a$ \\
\hline$\$ 977000001$ & $F$ & Neodymium -ICP-fusion & ug/g & 102.6 & $<1.00 \mathrm{e}=01$ & $<1.96 \mathrm{e}+03$ & $<1.96 \mathrm{e} 3$ & $\mathrm{n} / \mathrm{a}$ & $n / a$ & 97.00 & $1.96 e+03$ & $\mathrm{n} / \mathrm{a}$ \\
\hline 5977000001 & $\bar{F}$ & Phosphorus -ICP-Fusion & $\operatorname{ug} / \mathrm{g}$ & 101.0 & $<2.00 \mathrm{e}-01$ & $5.72 \mathrm{e}+04$ & $6.73 e+04$ & $6.22 e+04$ & 16.2 & 104.0 & $3.92 \mathrm{e}+03$ & $n / a$ \\
\hline 5971000001 & $F$ & Lead-ICP-Fusion & ug/g & 98.00 & $<1.00 \mathrm{e}-01$ & $<1.96 \mathrm{e}+03$ & $<1.96 \mathrm{e} 3$ & $\mathrm{n} / \mathrm{a}$ & $\mathrm{n} / \mathrm{a}$ & 99.90 & $1.96 \mathrm{e}+03$ & $n / a$ \\
\hline S $97 \mathrm{TIC}$ & $F$ & Sulfur -1CP-Fusion & ug/g & 98.00 & $<1.00 \mathrm{e}-01$ & $2.14 \mathrm{e}+04$ & $1.43 e+04$ & $1.78 \mathrm{e}+04$ & 39.8 & 98.60 & $1.96 \mathrm{e}+03$ & $n / a$ \\
\hline$\$ 971000001$ & $F$ & Antimony - ICP-Fusion & ug/g & 96.40 & $<6.00 \mathrm{e}-02$ & $<1.18 e+03$ & $<1.18 \mathrm{e} 3$ & $n / a$ & $\mathrm{n} / \mathrm{a}$ & 94.70 & $1.18 \mathrm{e}+03$ & $n / a$ \\
\hline 5977000001 & F & Selenium-ICP-Fusion & $\mathrm{ug} / \mathrm{g}$ & 95.80 & $<1.00 \mathrm{e}-01$ & $<1.96 \mathrm{e}+03$ & $<1.96 \mathrm{e}^{3}$ & $n / a$ & $\mathrm{n} / \mathrm{a}$ & 96.40 & $1.96 \mathrm{e}+03$ & $n / a$ \\
\hline$\$ 971000001$ & $F$ & Silicon-ICP-Fusion & ug/g & 97.20 & $<5.00 \mathrm{e}-02$ & $1.01 \mathrm{e}+03$ & $<9.80 \mathrm{e} 2$ & $n / a$ & $\mathrm{n} / \mathrm{a}$ & 96.50 & 980.0 & $\mathrm{n} / \mathrm{a}$ \\
\hline S97TC & F & Samarium -ICP-Fusion & $\frac{0 g / 9}{402}$ & 101.2 & $<1.00 \mathrm{e}-01$ & $<1.96 \mathrm{e}+03$ & $<1.96 \mathrm{e} 3$ & $n / a$ & $n / a$ & 95.10 & $1.96 \mathrm{e}+03$ & $\mathrm{n} / \mathrm{a}$ \\
\hline 5977000001 & $\bar{F}$ & Strontium -ICP-Fusion & $\operatorname{ug} / \mathrm{g}$ & 101.4 & $<1.00 \mathrm{e}-02$ & $<1.96 \mathrm{e}+02$ & $<1.96 \mathrm{e} 2$ & $n / a$ & $n / a$ & 95.70 & 196.0 & $\mathrm{n} / \mathrm{a}$ \\
\hline S97T000001 & $F$ & Titanium-ICP-Fusion & $\mathrm{ug} / \mathrm{g}$ & 99.20 & $<1.00 \mathrm{e}-02$ & $<1.96 \mathrm{e}+02$ & $<1.96 \mathrm{e} 2$ & $n / a$ & $\mathrm{n} / \mathrm{a}$ & 94.80 & 196.0 & $n / a$ \\
\hline 5971 & $F$ & Thallium-ICP-Fustion & $4 \mathrm{~g} / \mathrm{g}$ & 97.20 & $<2.00 \mathrm{e}-07$ & $<3.92 \mathrm{e}+03$ & $<3.92 \mathrm{e} 3$ & $\mathrm{n} / \mathrm{a}$ & $\mathrm{n} / \mathrm{a}$ & 92.70 & $3.92 \mathrm{e}+03$ & $\mathrm{n} / \mathrm{a}$ \\
\hline S97TO & $F$ & Uranium-ICP-Fusion & $\mathrm{ug} / \mathrm{g}$ & 98.30 & $<5.00 \mathrm{e}-01$ & $<9.80 \mathrm{e}+03$ & $<9.80 \mathrm{e} 3$ & $\mathrm{n} / \mathrm{a}$ & $\mathrm{n} / \mathrm{a}$ & 90.00 & $9.80 \mathrm{e}+03$ & $n / a$ \\
\hline \$97T000001 & $\vec{F}$ & Vanadium -ICP-Fusion & $49 / 9$ & 100.8 & $<5.00 \mathrm{e}-02$ & $<9.80 \mathrm{e}+02$ & $<9.80 \mathrm{e} 2$ & $n / a$ & $\mathrm{n} / \mathrm{a}$ & 97.70 & 980.0 & $\mathrm{n} / \mathrm{a}$ \\
\hline S97T000001 & $F$ & Zinc-ICP-Fusion & $\mathrm{ug} / \mathrm{g}$ & 100.8 & $<1.00 \mathrm{e}-02$ & $<1.96 e+02$ & $<1.96 \mathrm{e} 2$ & $\mathrm{n} / \mathrm{a}$ & $\mathrm{n} / \mathrm{a}$ & 101.0 & 196.0 & $n / a$ \\
\hline $597 \mathrm{TC}$ & $F$ & Zirconium-ICP-Fusion & $49 / 9$ & 100.2 & $<1.00 \mathrm{e}-02$ & $<1.96 \mathrm{e}+02$ & $<1.96 \mathrm{e} 2$ & $n / a$ & $\mathrm{n} / \mathrm{a}$ & 95.90 & 196.0 & $n / a$ \\
\hline S971 & $\bar{F}$ & Cobalt -60 by GEA & uci/g & 100.4 & $<1.13 e^{-02}$ & $<1.12 e-02$ & $<9.76 \mathrm{e}-3$ & $n / a$ & $\mathrm{n} / \mathrm{a}$ & $n / a$ & $1.10 \mathrm{e}-02$ & $n / a$ \\
\hline 5971000001 & $F$ & Cesium- 137 by GEA & uCi/g & 103.5 & $<2.29 \mathrm{e}-02$ & 10.68 & 7.150 & 8.915 & 39.8 & $\mathrm{n} / \mathrm{a}$ & $n / a$ & 1.47 \\
\hline S971000001 & $F$ & Europium- 154 by GEA & uCi/g & $n / a$ & $<3.55 e-02$ & $<3.84 \mathrm{e}-02$ & $<4.16 \mathrm{e}-2$ & $n / a$ & $n / a$ & $n / a$ & $3.80 \mathrm{e}-02$ & $n / a$ \\
\hline S9710 & $\bar{F}$ & Europium- 155 by GEA & $u C i / g$ & $\mathrm{n} / \mathrm{a}$ & $<2.98 \mathrm{e}-02$ & $<8.45 e-02$ & $<6.86 \mathrm{e}=2$ & $n / a$ & $\mathrm{n} / \mathrm{a}$ & $\mathrm{n} / \mathrm{a}$ & $8.50 e-02$ & $\mathrm{n} / \mathrm{a}$ \\
\hline 5977000001 & F & Americium-241 by GEA & $\mathrm{uCi} / \mathrm{g}$ & $n / a$ & $<8.16 \mathrm{e}-02$ & $<2.13 e-01$ & $<1.70 \mathrm{e}-1$ & $n / a$ & $\mathrm{n} / \mathrm{a}$ & $n / a$ & $2.13 e-01$ & $\mathrm{n} / \mathrm{a}$ \\
\hline S97T000001 & $F$ & Átpha of Digested Solid & uci/g & 101.3 & $<3.02 \mathrm{e}-03$ & $4.66 \mathrm{e}-03$ & $1.80 \mathrm{e}-03$ & $3.23 e-03$ & 88.5 & 65.46 & $3.00 \mathrm{e}-03$ & $8.21 E+01$ \\
\hline 5971000017 & W & Fluoride-IC-Dionex $4000 / 4500$ & $\mathrm{ug} / \mathrm{g}$ & 95.76 & $<4.92 \mathrm{e}+01$ & $3.31 \mathrm{e}+04$ & $3.46 \mathrm{e}+04$ & $3.39 \mathrm{e}+04$ & 4.43 & 88.81 & 97.66 & $\mathrm{n} / \mathrm{a}$ \\
\hline S971 & $W$ & Chlor ide-1C-D ionex $4000 / 4500$ & ug/g & 100.8 & $<6.97 e^{-011}$ & $3.12 e+02$ & 328.0 & 319.9 & 5.00 & 97.85 & 138.4 & $\mathrm{n} / \mathrm{a}$ \\
\hline 597100 & W & Nitrite-IC - Dionex $4000 / 4500$ & $\mathrm{ug} / \mathrm{g}$ & 103.1 & $2.47 e^{-07}$ & $5.080+03$ & $6.86 \mathrm{e}+03$ & $5.97 \mathrm{e}+03$ & 29.8 & 110.0 & 879.0 & $n / B$ \\
\hline $597 T 000017$ & $W$ & Bromide by ion Chromatograph & $\mathrm{ug} / \mathrm{g}$ & 99.15 & 5.120 & $<1.02 \mathrm{e}+03$ & $<1.01 \mathrm{e} 3$ & $n / a$ & $\mathrm{n} / \mathrm{a}$ & 97.62 & $1.02 \mathrm{e}+03$ & $n / a$ \\
\hline S97T000017 & tw & Nitrate by IC-Dionex $4000 / 4500$ & $\mathrm{ug} / \mathrm{g}$ & 100.0 & 55.700 & $2.36 \mathrm{e}+04$ & $3.26 \mathrm{e}+04$ & $2.81 \mathrm{e}+04$ & 32.0 & 99.33 & $1.13 e+03$ & $n / \theta$ \\
\hline
\end{tabular}




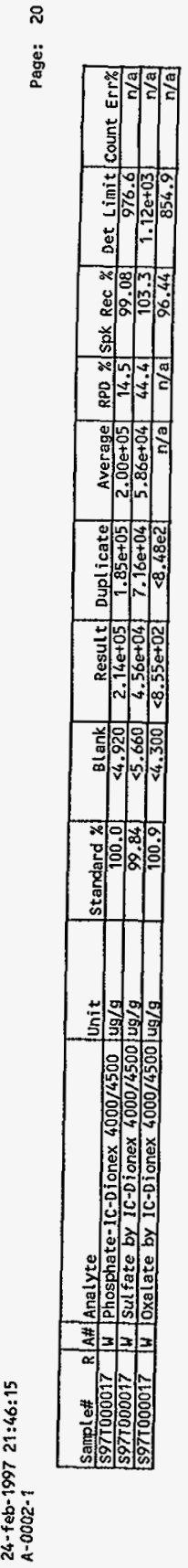


HNF-SD-WM-DP-219, REV. 0

CHAIN OF CUSTODY FORMS

142 
HNF-SD-WM-DP-219, REV. 0

THIS PAGE INTENTIONALLY LEFT BLANK 
Oct.11.1996_11:47AM__WHC 2225 LAB ROOM 2F BACKSIDE

No. 5792

P. $2 / 5$

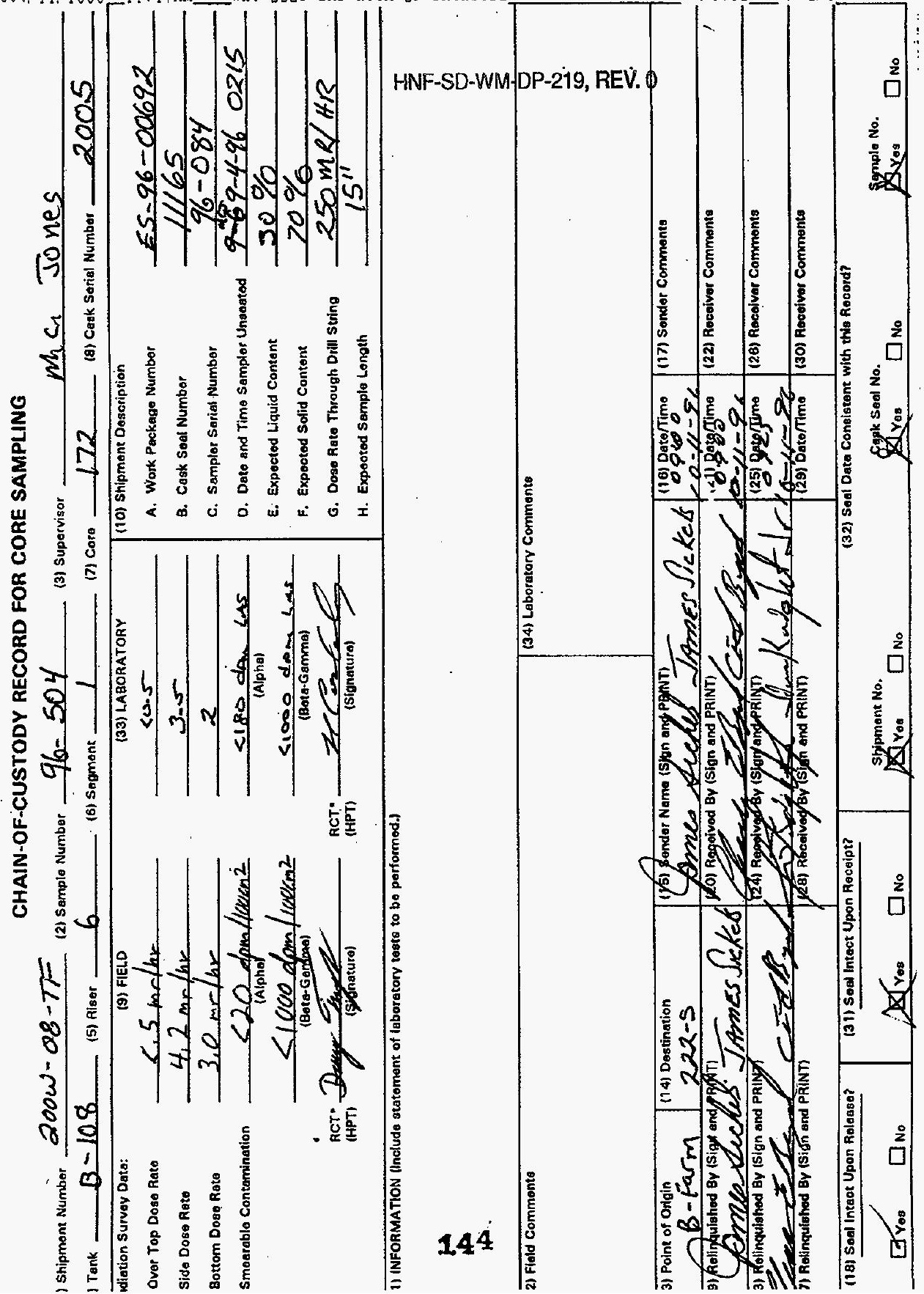




\section{CHAIN-OF-CUSTODY RECORD FOR CORE SAMPLING}

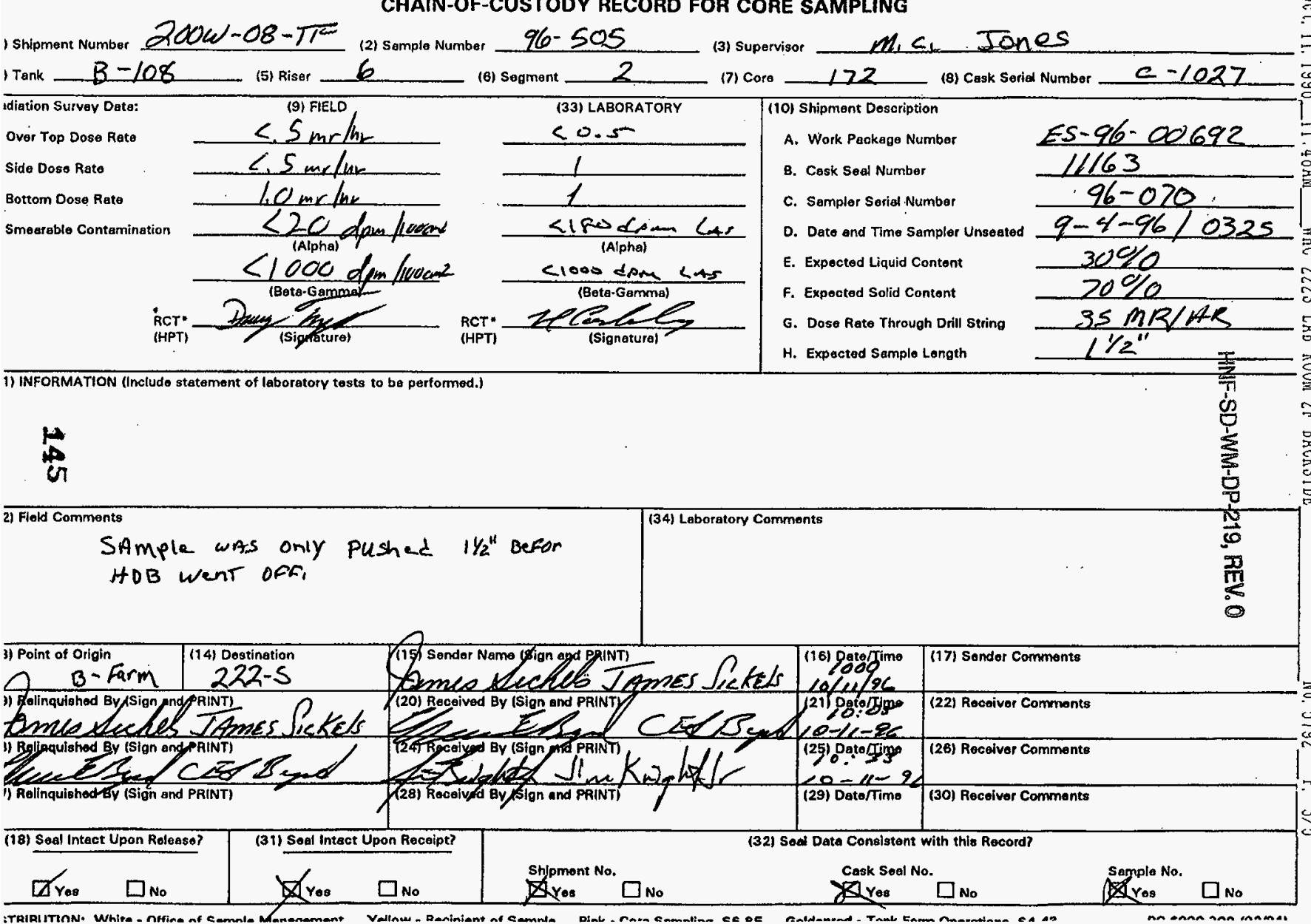


CHAIN-OF-CUSTODY RECORD FOR CORE SAMPLING

i shipment Number $200 \omega-08-T T$

(2) Sample Number $96-507$

(3) supervisor Mc. Tones

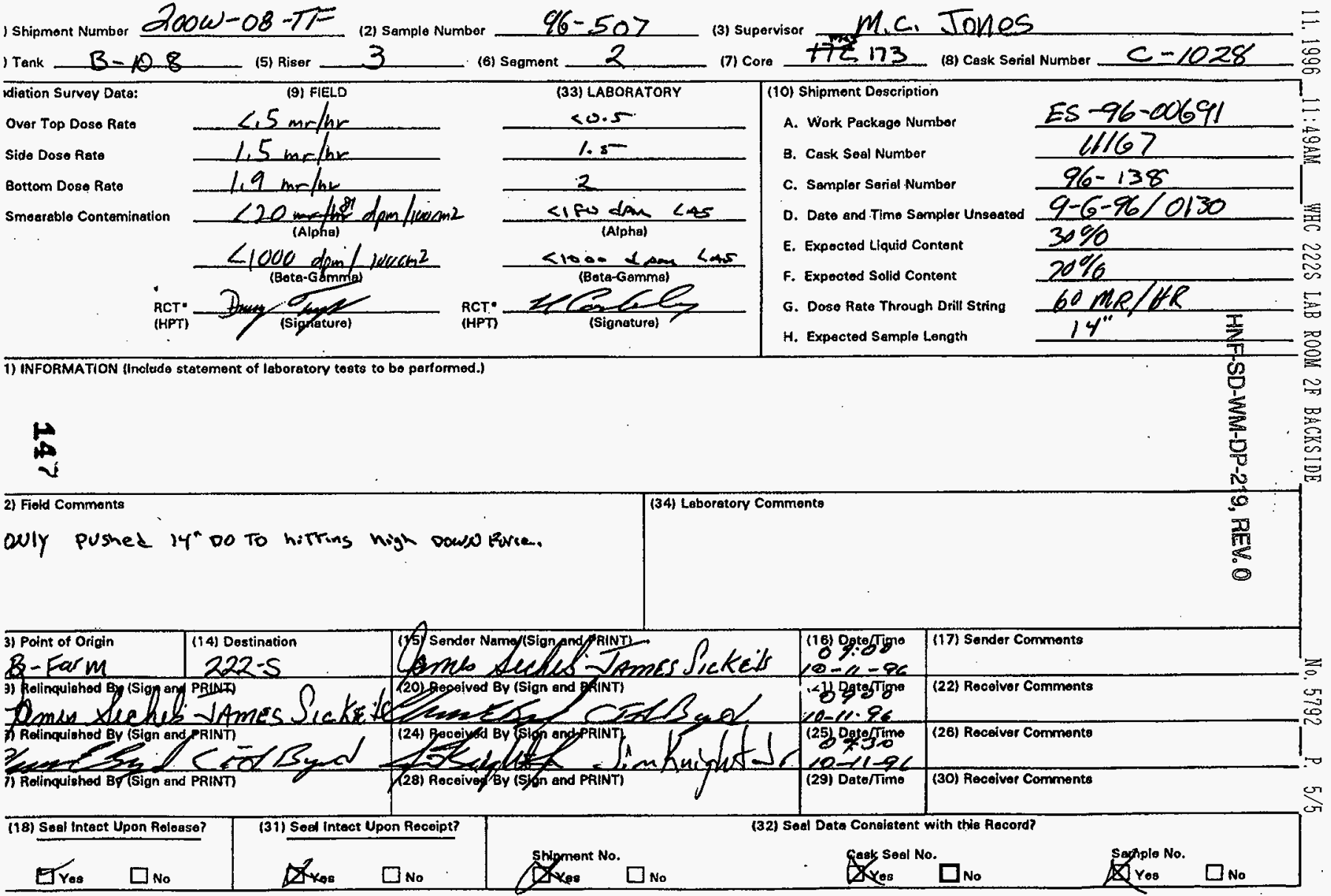


HNF-SD-WM-DP-219, REV. 0

\section{PHOTOGRAPHS}


HNF-SD-WM-DP-219, REV. 0

THIS PAGE INTENTIONALLY LEFT BLANK 
HNF-SD-WM-DP-219, REV 0

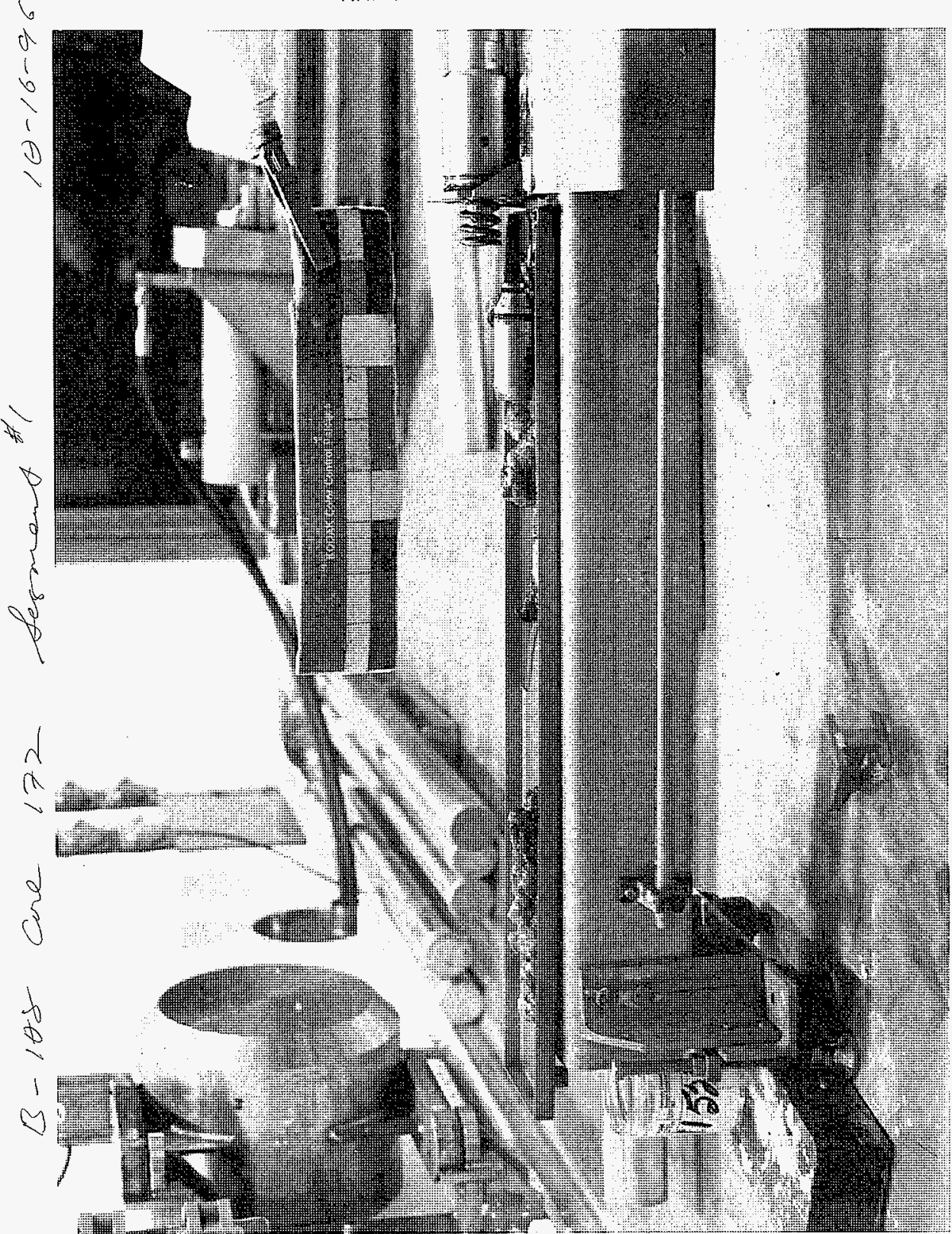




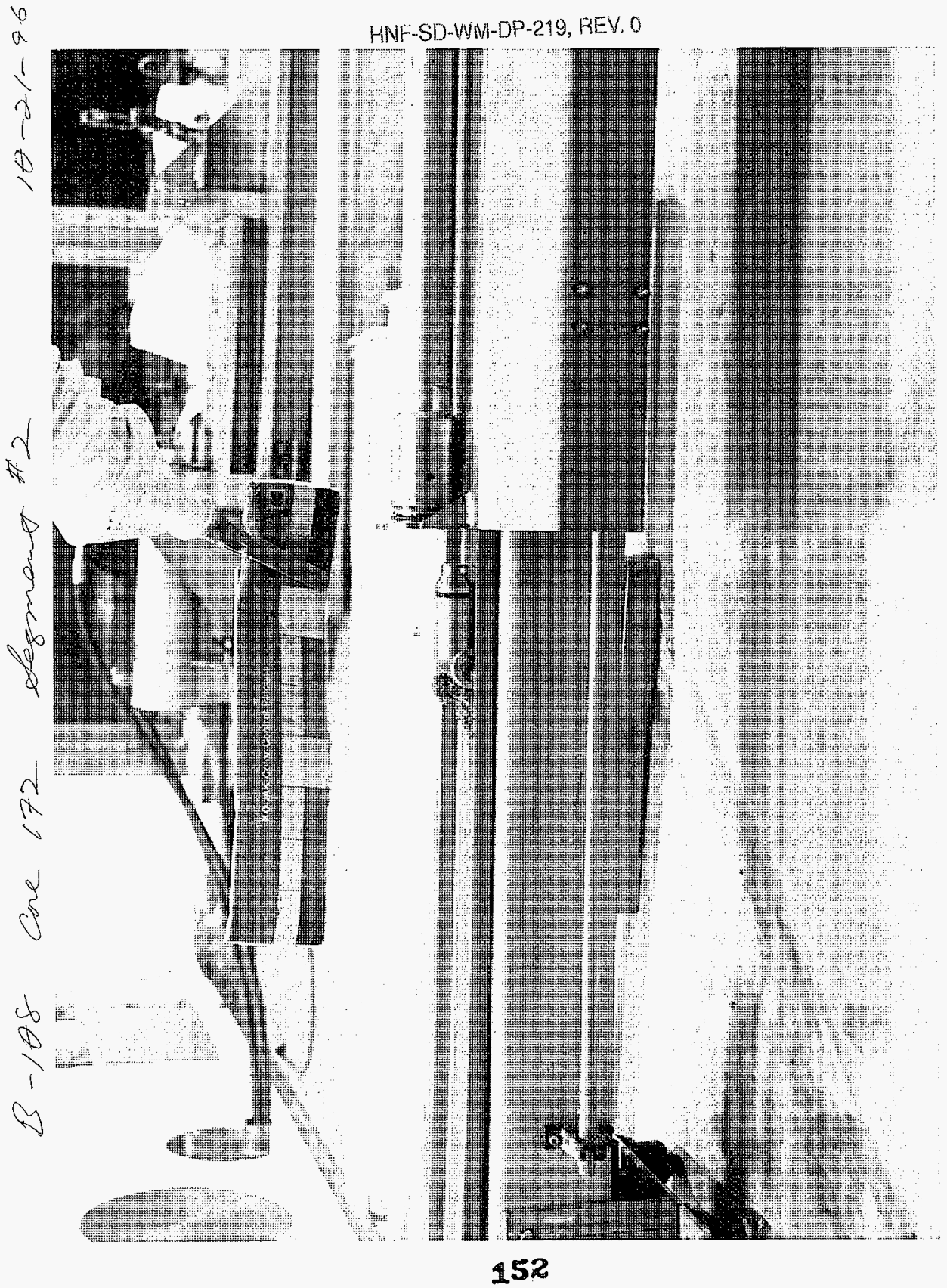


HNF-SO-WM-DP-219, REV.O

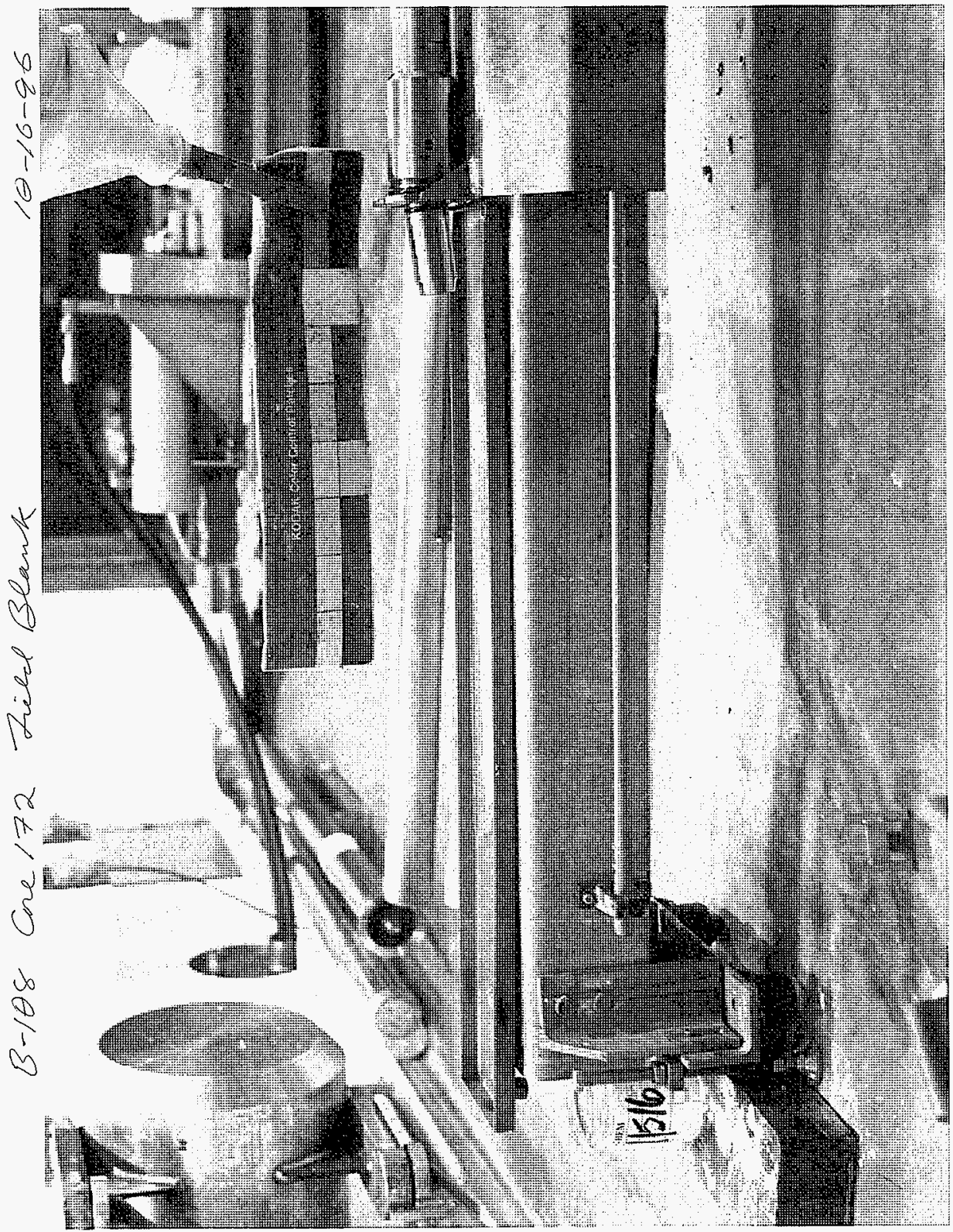


n

$$
1
$$

1
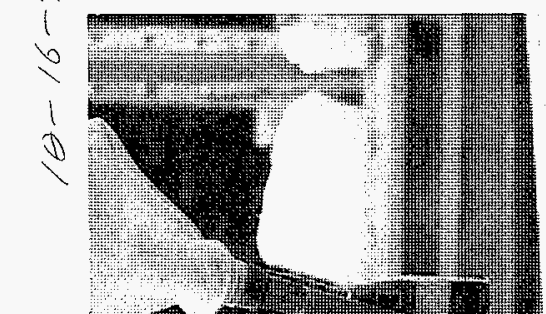

6

俥

i)
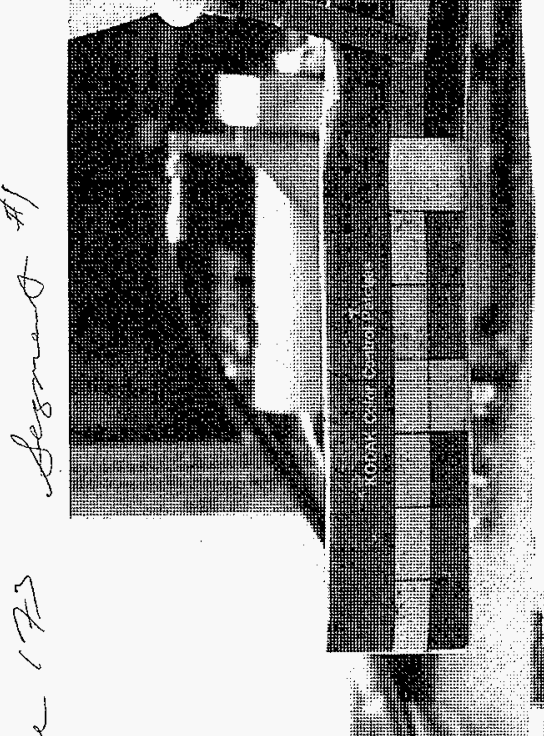

$\int$

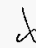

D

1

\%
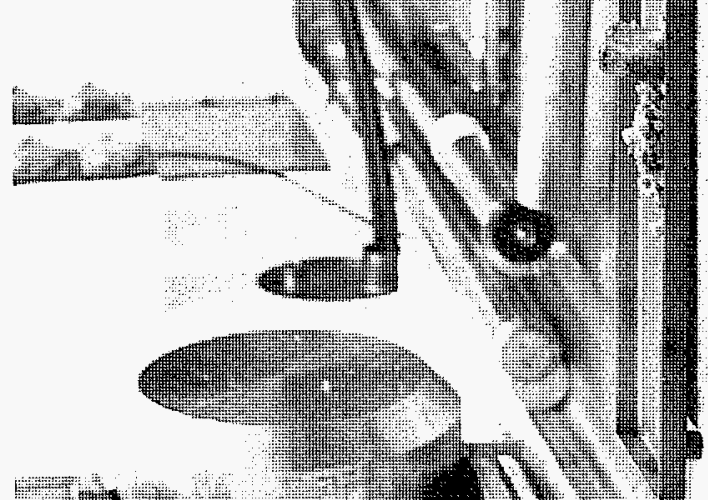

F

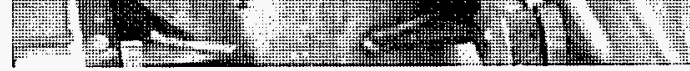

\section{REV.O}




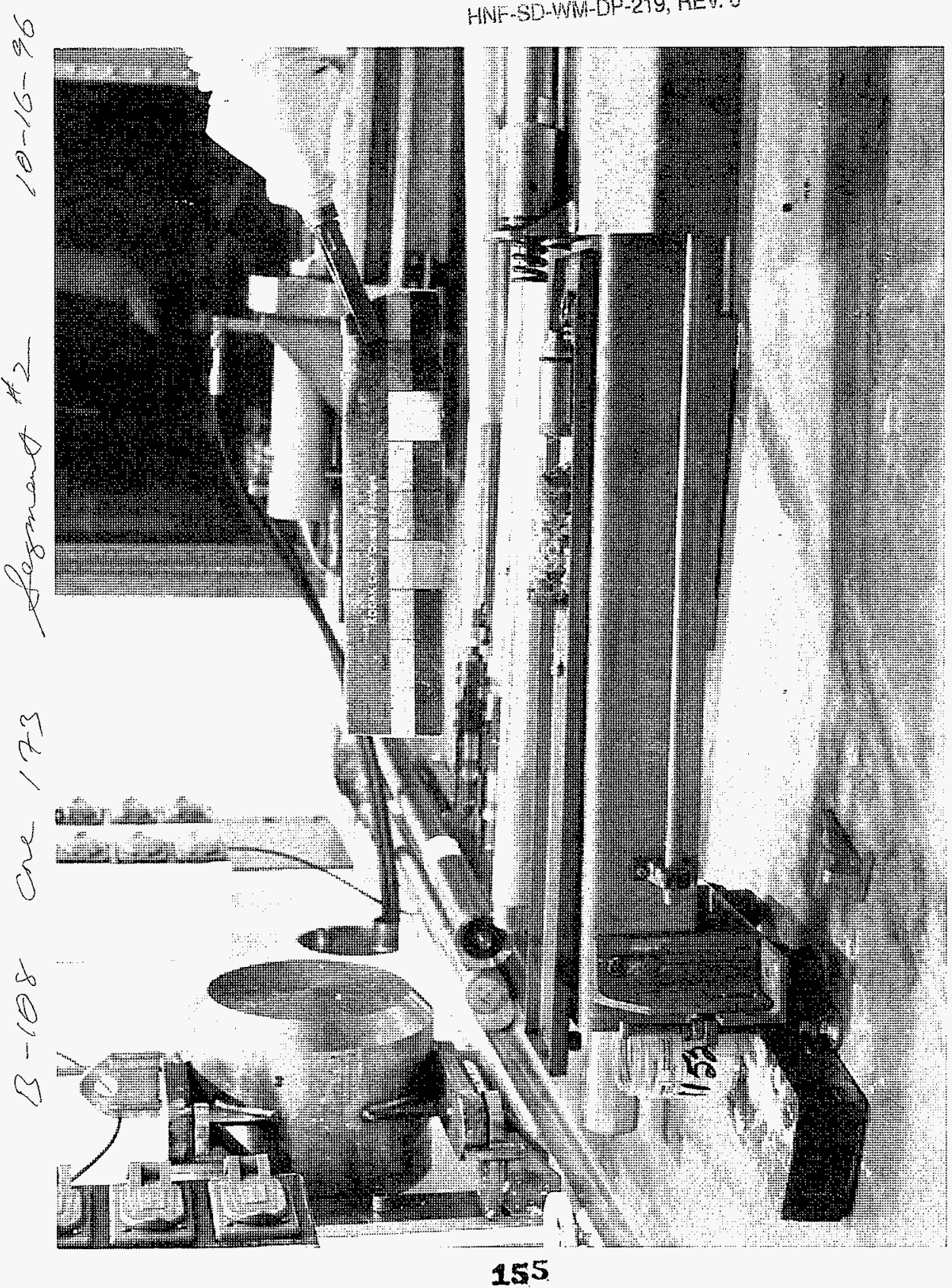


HNF-SD-WM-DP-219, REV. 0

CORE COMPOSITE WORKSHEETS 
HNF-SD-WM-DP-219, REV. 0

THIS PAGE INTENTIONALLY LEFT BLANK 


\section{Final Composite Calculation Sheet}

Date: $10 / 19 / 96$ Technician:

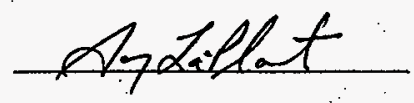

Total number of jars used to build the composite:

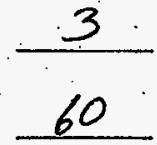

Composite Jar number:

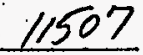

Jar size:

1) Sample from segment jar 11505 to composite jàr $=20.0$ grams

2) Sample from segment jar 11506 to composite jar $=\ldots 20.4$ grams

3) Sample from segment jar 11484 to composite jar $=.21 \% 0$ grams

4) Sathple from segment jar $\because$ to composite jar = grams

5) Sample from segment jar to composite jar = grams

6) Sarnple fromsegment jar to composite jar $=$ grams

7). Sample from seghent jar to composite jar $=$ grams

8) Sample from segment Siar __ to composite jar = grams

9) Sample from ségment jar

10) Sample from segment jar

11) Sample from segment jar to to composite jar : grams

12) Sample from segment jar A2. th Composite jar : grams

13). Sample from segment jar to compesite jar = grams

14) Sample fron segment jar - $\quad$ to composite jar = grms to composite jar grams

Total grams collected in composite jar \#11507 $=.61 .4$ grams. 
HNF-SD-WM-DP-219, REV.O

\section{Comoosite \% Calculation Sheet}

Date: $10-19-96$ Technician:

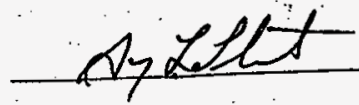

1) Segment jar $\# 1505$ : Segment jar $\# \frac{11505}{20.0 \text { grams from secment jar to composite jar }} \times 100=32,6$
61.4 Total gräms in composite jar

i2) Segment jar $\frac{i}{\pi} \not 306$ : $\frac{20.4 \text { grams from segment jar to composite jar }}{61.4 \text { Total grams in composite jar }} \times 100=33.2$

3) Segment jar $\frac{n}{*} 11484$ :

$\frac{21.0 \text { grams from segment jar to comoosite jar }}{61.4 \text { Total grams in }} \times 100=34.2 \%$

4) Segment jar $\frac{n}{i t}$

orams from seoment jar to compositè jar $\times 100=$ $\%$ Total grans in composite jar

5) Segmejt jar $\frac{n}{n t}$

aramis from seonent jar to composite jar $\times 100=$ 8

Fatal grams in composite jar

6) Segment jar.

Guams from segnent jar to comoosite jar $\times 100=$ Total grams in composite jar

7) Segment jar $\frac{12}{11}$

grams from seament jar to composite jar $\times 100=$ Total grans in composite jar

8) Segment jar $\frac{n}{\pi}$

9) Segment jar $\frac{n}{17}$

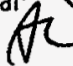

arams from seqment jar to conoosite jar $x$ Total grans in composite jar

10) Segment jar $\frac{n}{\pi}$

grams from seoment jar to composite jar $\times 100$ Tútât gi uñ̇ in composite jar. 
HNF - SD-WM-DP-219, REV. 0

EXTRUSION ANALYSIS 
HNF - SD-WM-DP-219, REV. 0

\section{THIS PAGE INTENTIONALLY LEFT BLANK}


worklistrpt Version 2.1 05/15/95 - - HNF-SD-WM-DP-249, REV. O

Page: $\quad 1$

10/15/96 14:29

LABCORE Data Entry Template for Worklist\# 14037

Analyst:
Method: LO-160-103 Rev/Mod 10
Worklist Comment: B-108 C172 FIELD BLANK RISER 6 EXTRUSION

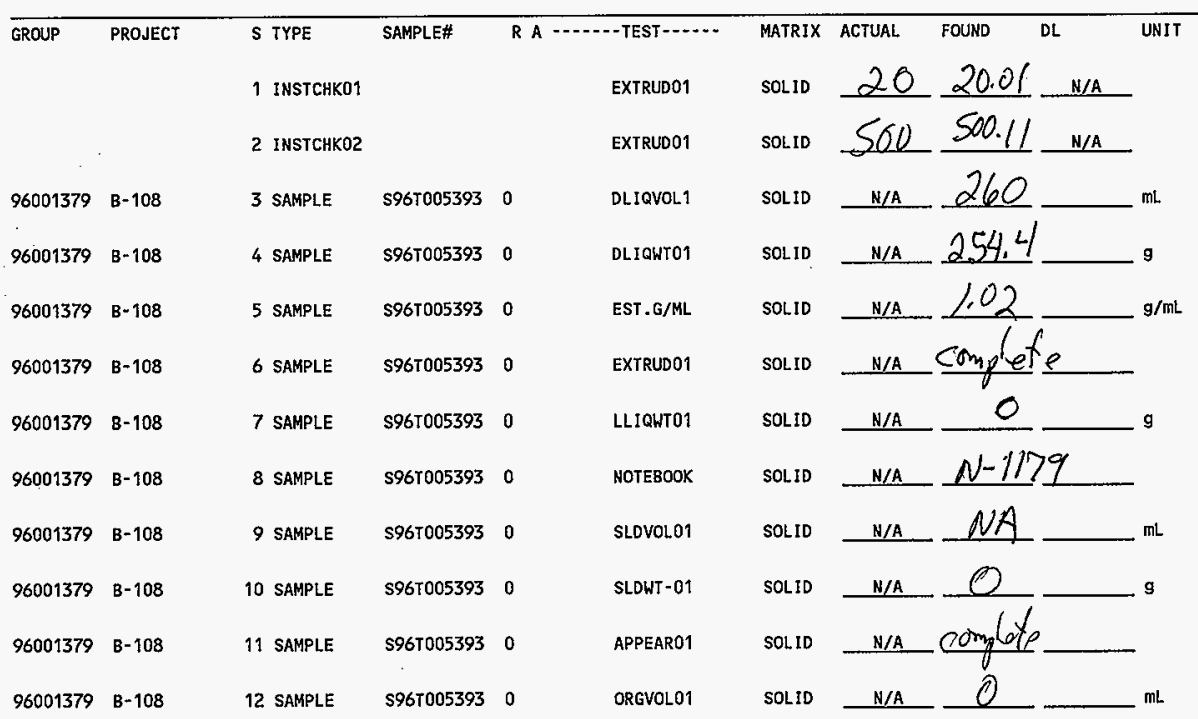

\section{Final page for worklist \# 14037}
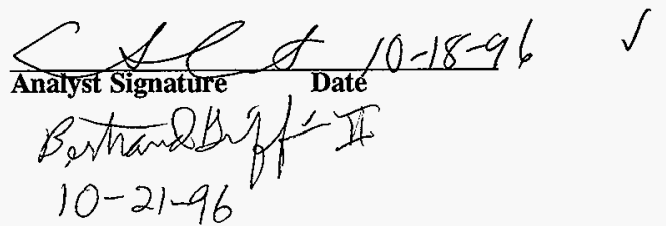

$$
\text { Posthandist } 10-21-96
$$

Data Entry Comments:

Units shown for $Q C$ (SPK \& STD) may not reflect the actual units. $D L=$ Detection Limit, $S=$ Worklist Slot Number, $R=$ Replicate Number, $A=$ Aliquot Code. 


\section{LABCORE Data Entry Template for Worklist\# 14038}

Analyst:

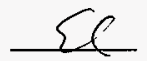

Instrument: BA000

Book \# NA

Method: LO-160-103 Rev/Mod

00

\section{Worklist Comment: B-108 C172 SEG \# 1 RISER 6 EXTRUSION}

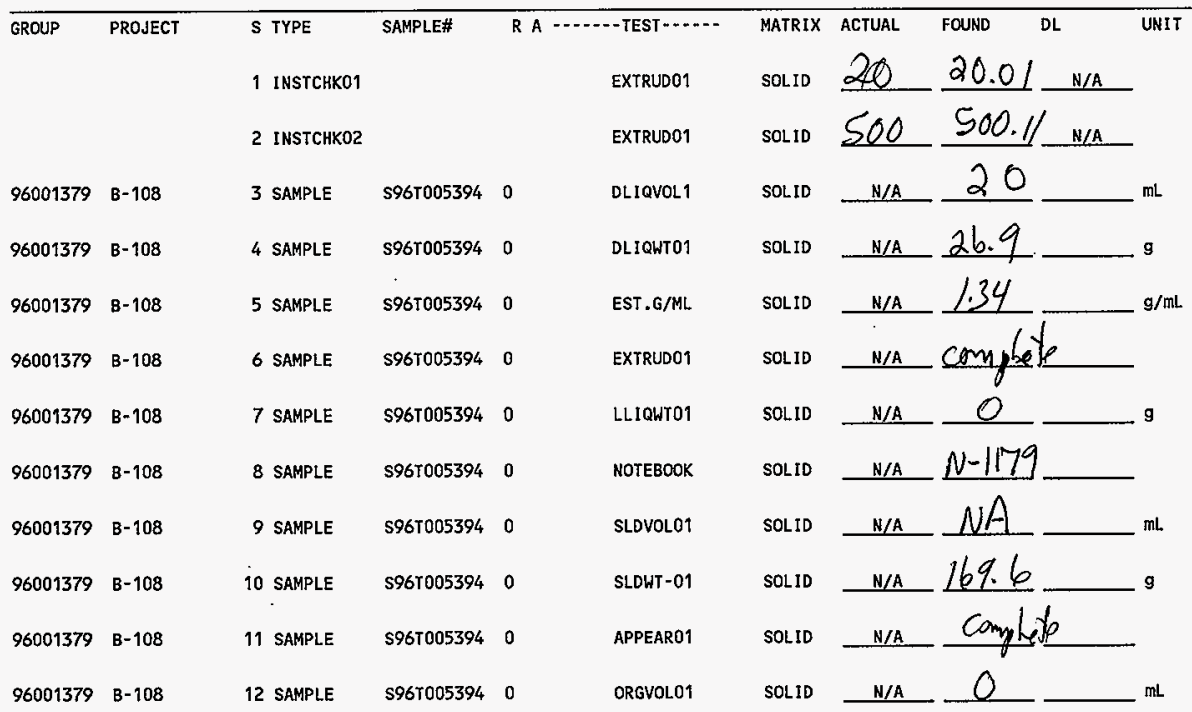

\section{Final page for worklist \# 14038}
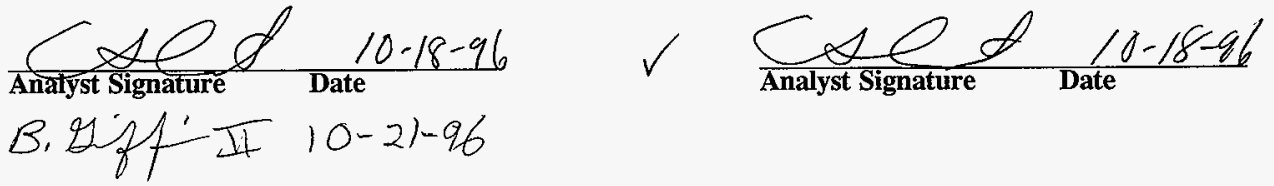

Data Entry Comments:

Units shown for $Q C$ (SPK \& STD) may not reflect the actual units. $D L=$ Detection Limit, $S=$ Worklist Slot Number, $R=$ Replicate Number, $A=$ Aliquot Code . 


Analyst: $\quad \sum C$ Instrument: BA000 Book \# NA

Method: LO-160-103 Rev/Mod Bo

Worklist Comment: B-108 C172 SEG \# 2 RISER 6 EXTRUSION

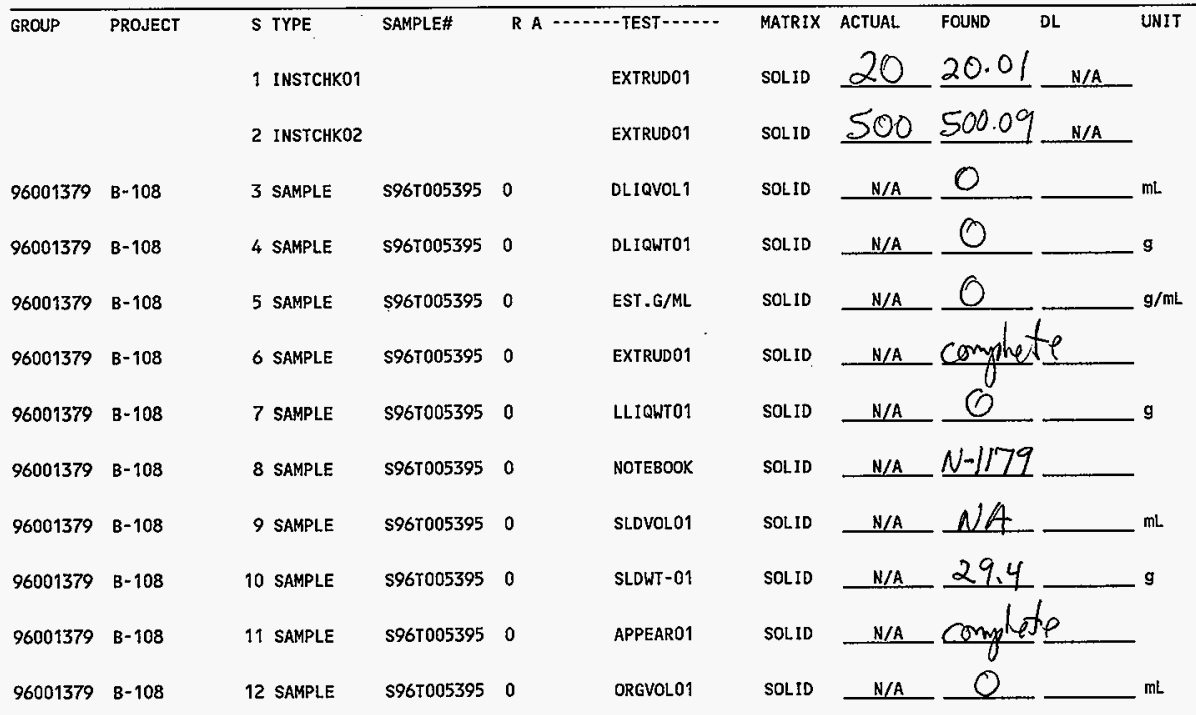

\section{Final page for worklist \# 14040}
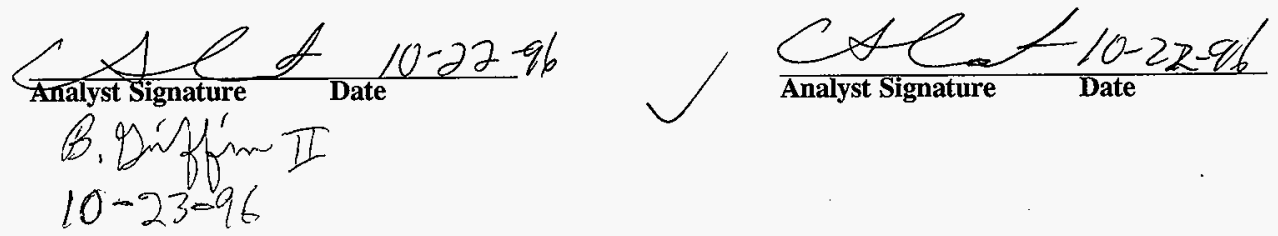

Data Entry Comments:

Units shown for $Q C$ (SPK \& STD) may not reflect the actual units. $D L=$ Detection Limit, $S=$ Worklist Slot Number, $R=$ Replicate Number, $A=$ Aliquot Code. 


\section{LABCORE Data Entry Template for Worklist\# 14042}

Analyst: $\quad \sum($ Instrument: BA000 _ Book \# NA

Method: LO-160-103 Rev/Mod BO

Worklist Comment: B-108 C173 SEG \# 1 RISER 3 EXTRUSION

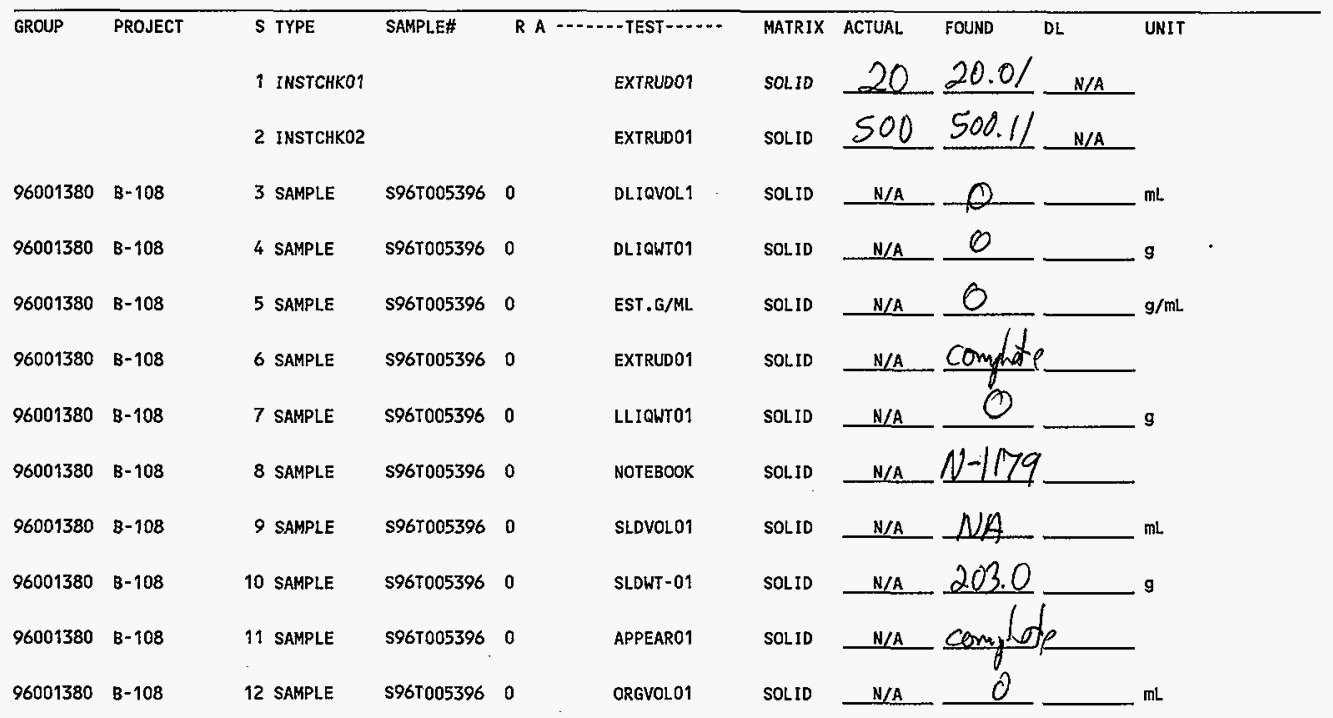

Final page for worklist \# 14042

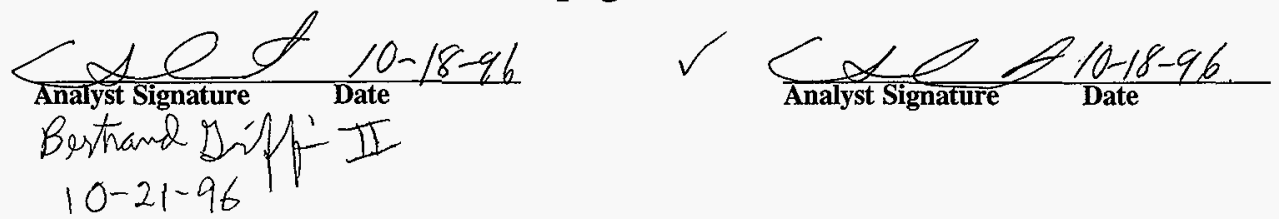

Data Entry Comments:

Units shown for $Q C$ (SPK \& STD) may not reflect the actual units. $D L=$ Detection Limit, $S=$ Worklist Slot Number, $R=$ Replicate Number, $A=$ Aliquot Code. 


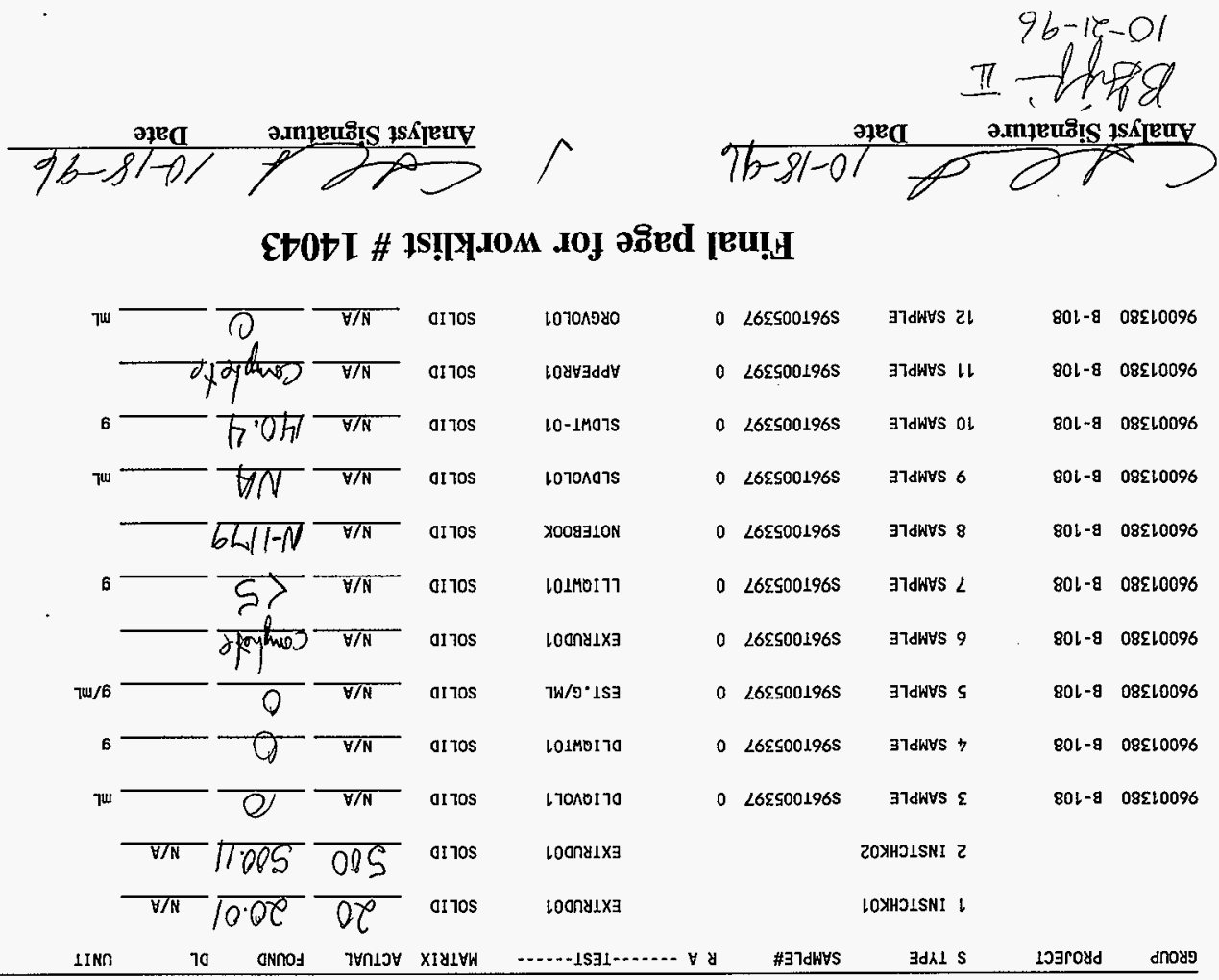

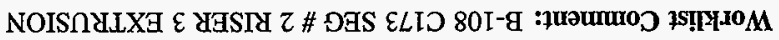

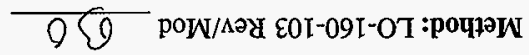
$\forall / \#$ yoog

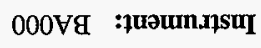
$\sqrt{3}$ :?Sא [EUV

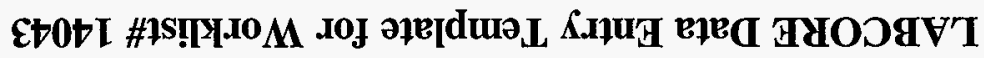


HNF-SD-WM-DP-219, REV. 0

SAMPLE PREPARATION 
HNF-SD-WM-DP-219, REV. 0

\section{THIS PAGE INTENTIONALLY LEFT BLANK}


Oct .26.1996 5:07PM WHC 222S LAB ROOM 2F BACKSIDE

No. $6313 \quad$ P. $1 / 6$ worklistrpt Version 2.1 05/15/95 HNF-SD-WM-DP-219, REV. 0

Page: $\quad l$ $10 / 17 / 9613: 15$

LABCORE Data Entry Template for Worklist\# 14104

Analyst: \&AL Instrument: Fus0i _ _ Book \# NA

Method: LA-549-141 Rev/Mod F.O

Worklist Comment: B-108 FUSION DIGEST C173S1,2,COMP lad

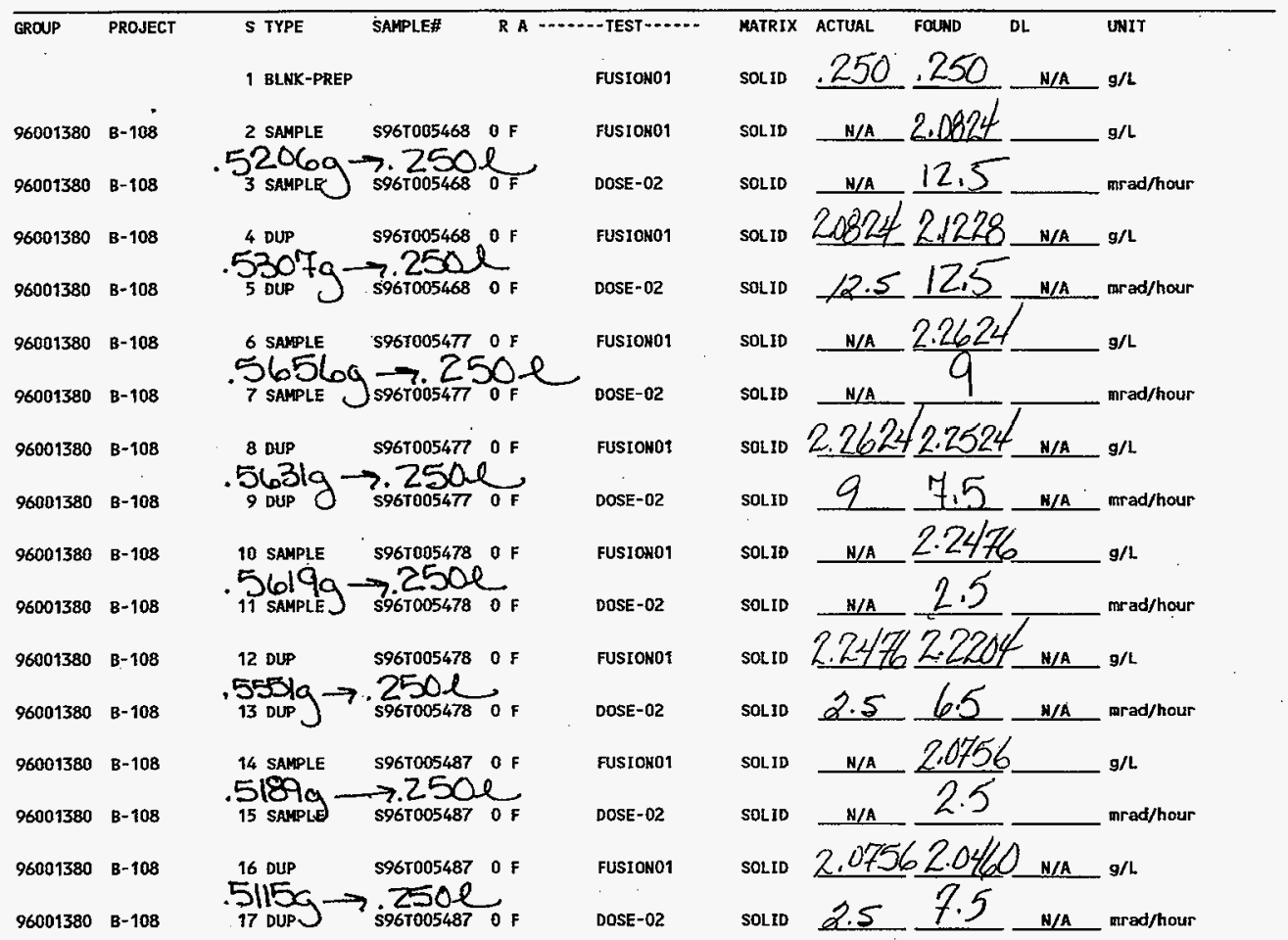

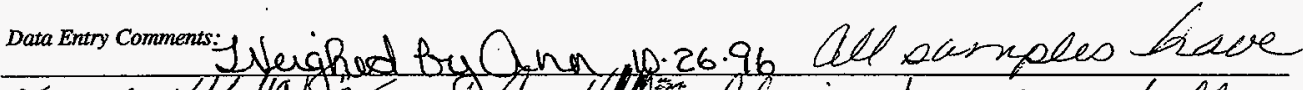
5 mb. IH HCL. Some. Ton H. Color is absent and all are clear.

Units shown for QC (SPK \& STD) m not reflect the a dull units. $D L=$ Detection Limit, $S=$ Worklist Slot Number, $\boldsymbol{R}=$ Replicate Number, $A=$ Aliquot Code.

169 
Oct. 28. 1996 5:08PM THC 222S LAB ROOM IF BACKSIDE

No. 6313 P. $2 / 6$

worklistrpt Version 2.1 05/15/95

HNF-SD-WMM-DP-219, REV. 0

Page: 2 $10 / 17 / 96$ 13:15

LABCORE Data Entry Template for Worklist\# 14104

GROUP PROJECT $\quad$ S TYPE SAMPLE\# RA -

Final page for worklist \# 14104

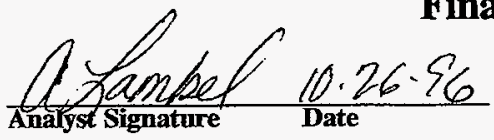

$\frac{\text { Sum bee }}{\text { Analyst Signature }}$ Date $/ 96$

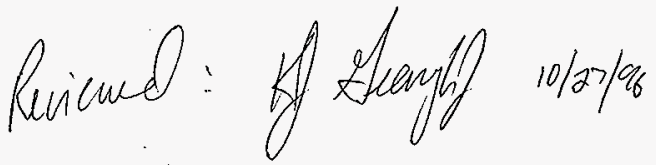

Datantry comments: Samples Save D\% solids. Samples were not hon zogenewe.

Units shown for QC (SPK \& STD) may not reflect the actual units. DL = Detection Limit, $S=$ Worklist Slot Number, $R=$ Replicate Number, $A=$ Aliquot Code.

170 
Analyst:

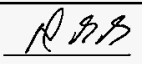

Instrument: FUS01

Book \#

Method: LA-549-141 Rev/Mod F-O

Worklist Comment: B-108 FUSION DIGEST C172S1,2 LH UH lad

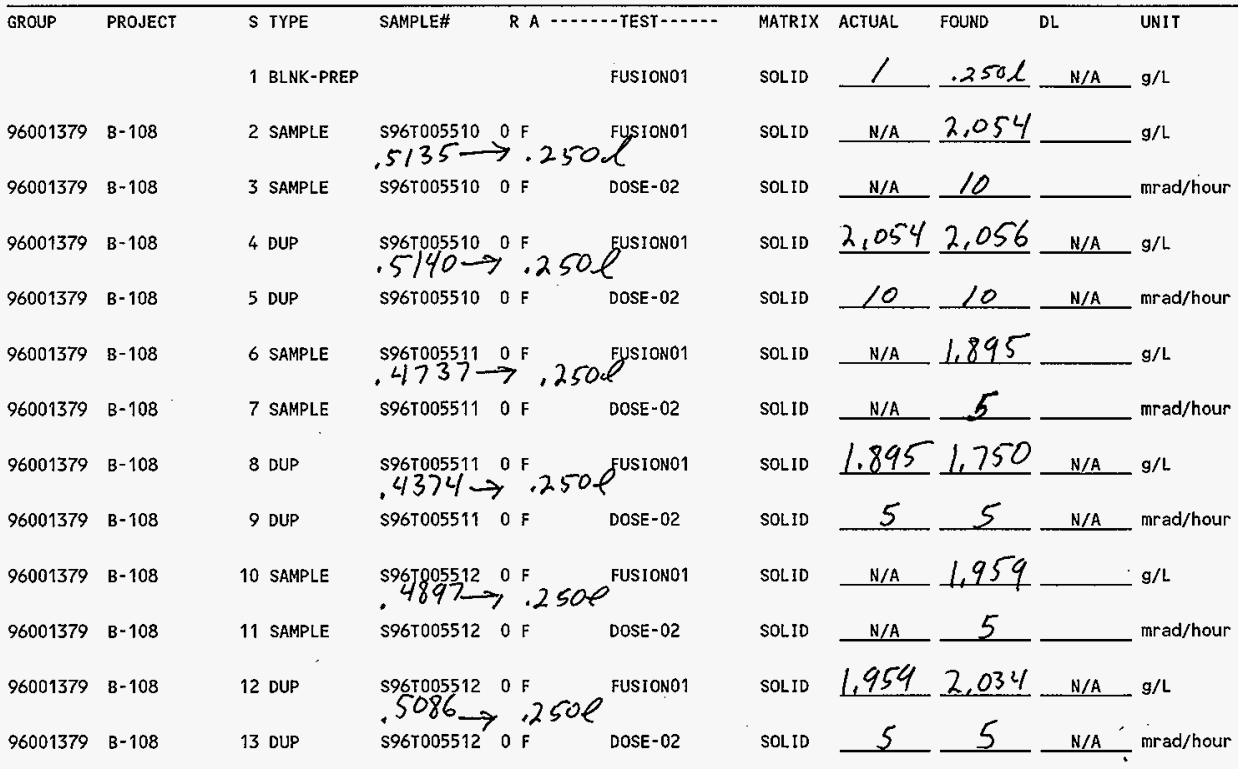

\section{Final page for worklist \# 14172}
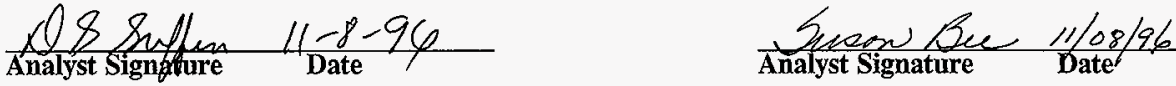

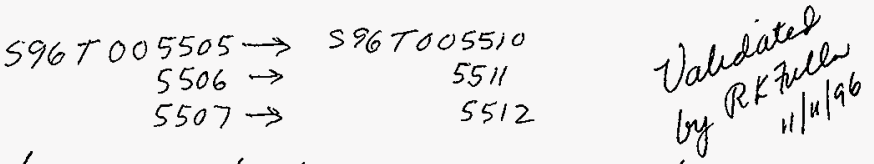

Data Entry Comments:

simples are not homogewous.; smple $55 / 2$ has lange pizees of solids.

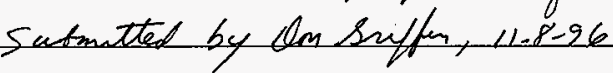

Units shown for QC (SPK \& STD) may not reflect the actual units. $D L=$ Detection Limit, $S=$ Worklist Slot Number,

$R=$ Replicate Number, $A=$ Aliquot Code. 


\section{LABCORE Completed Worklist Report for Worklist\# 16005}

Analyst: $a b c$

Instrument: FUS01

Book\#

Method:

Rev/Mod

Worklist Comment: B-108 C173S2LH \& COMP lad Repreps. Originals showed hi RPD

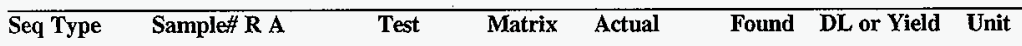

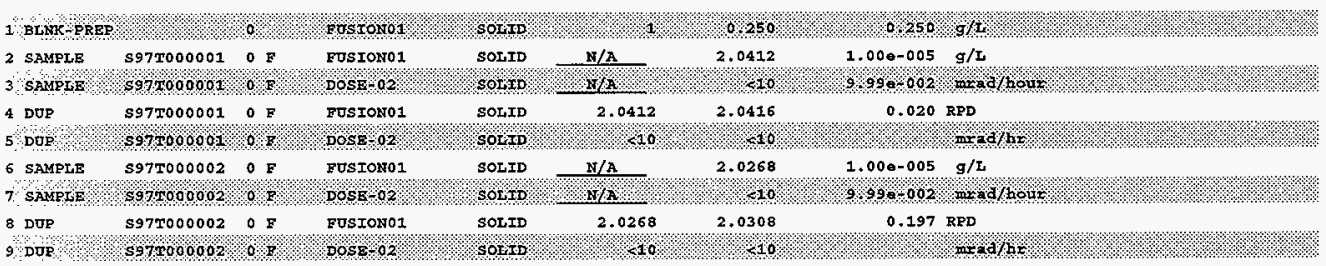

\section{Final page for worklist\# 16005}

Analyst Signature Date




Analyst: $\mathrm{CK}^{\mathrm{T}} \mathrm{VAM}$ Instrument: H2O01 __ Book \#_NA

Method: LA-504-101 Rev/Mod $\Sigma-O$

Worklist Comment: B-108 H2O DIGEST C173S1 UH,LH lad

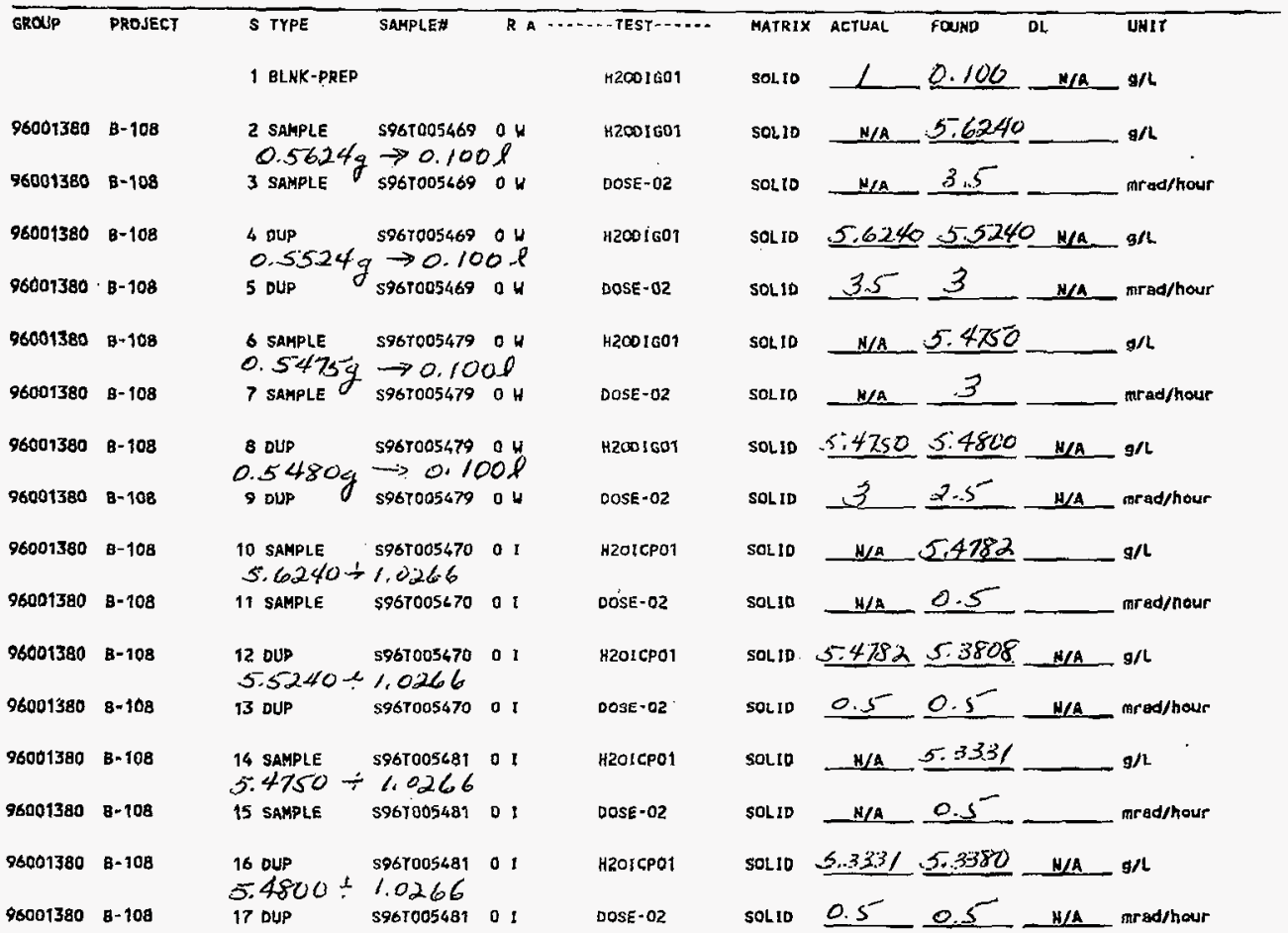

Data Entry Comments:

Units shown for $Q C$ (SPK \& STD) may not reflect the actual unirs, $D L=$ Detection Limit, $S=$ Worklist Slot Number, $R=$ Replicate Number, $A=$ Aliquot Code. 
HNF-SD-WH-DP-219, REV.0

Nerfistripi Versioñ $2.105 / 15 / 95$

10/28/96 15:32

\section{LABCORE Data Entry Template for Worklist\# 14105}

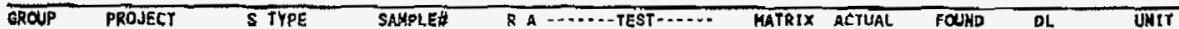

\section{PKFullaw Final page for worklist \# 14105}

$$
\text { for. }
$$

TA Muephy 10128196

Performed $10 / 28 / 96$

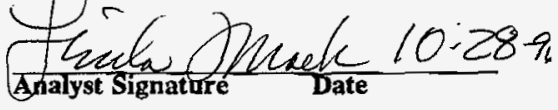

$$
\begin{aligned}
& 8967005466 \rightarrow 5469 \rightarrow 5470 \\
& 5473 \rightarrow 5479 \rightarrow 5481
\end{aligned}
$$

Revinuel:

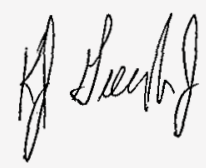

$$
10 / 28 / 96
$$

Daka Entry Comments:

Unlts shown for $Q C$ (SPK \& STD) may not refiect the actual units. $D L=$ Detecrion Limit, $S=$ Worklist Slot Number, $\boldsymbol{R}=$ Replicate Nimber, $\boldsymbol{A}=$ Aliquol Code. 
0, t. 26. $1996 \quad 5: 09 P M \quad$ WHC $222 S$ LAB ROOM $2 F$ BACKSIDE

No. $6313 \quad$ P. $5 / 6$

HNF-SD-WM-DP-219, REV. 0

worklistrpt Version $2.105 / 15 / 95$

$\checkmark$ 10/17/96 13:17

\section{LABCORE Data Entry Template for Worklist\# 14105}

Analyst: $\mathrm{DAM}$ Instrument: $\mathrm{H} 2001$

Method: LA-504-101 Rev/Mod

S19

Book \#N/A

Worklist Comment: B-108 H2O DIGEST C173S1 UH,LH lad

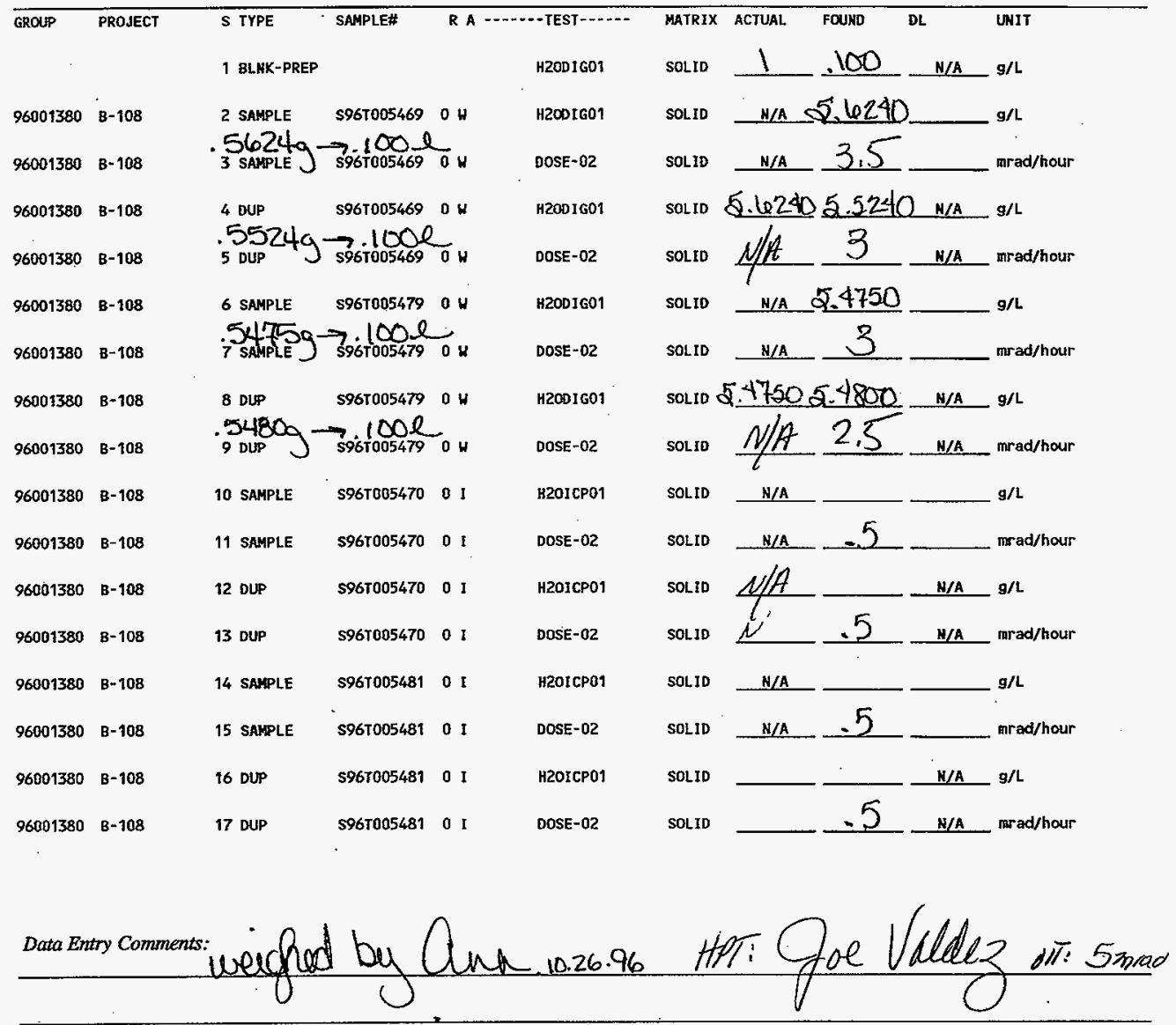

Units showin for $Q C$ (SPK \& STD) may not reflect the actual units. $D L=$ Detection Limit, $S=$ Worklist Slot Number, $R=$ Replicate Number, $A=$ Aliquot Code. 


\section{LABCORE Data Entry Template for Worklist\# 14105}

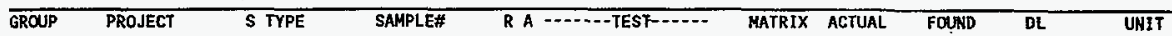

\section{Final page for worklist \# 14105}

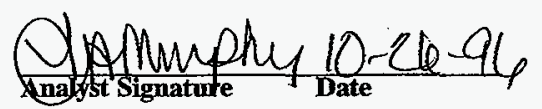

Amalyst Sigmature Date

Data Entry Comments:

Units shown for QC (SPK \& STD) may not reflect the actual units. $D L=$ Detection Limit, $S=$ Worklist Slot Number, $R=$ Replicate Number, $A=$ Aliquot Code. 


\section{LABCORE Data Entry Template for Worklist\# 14106}

Page: I

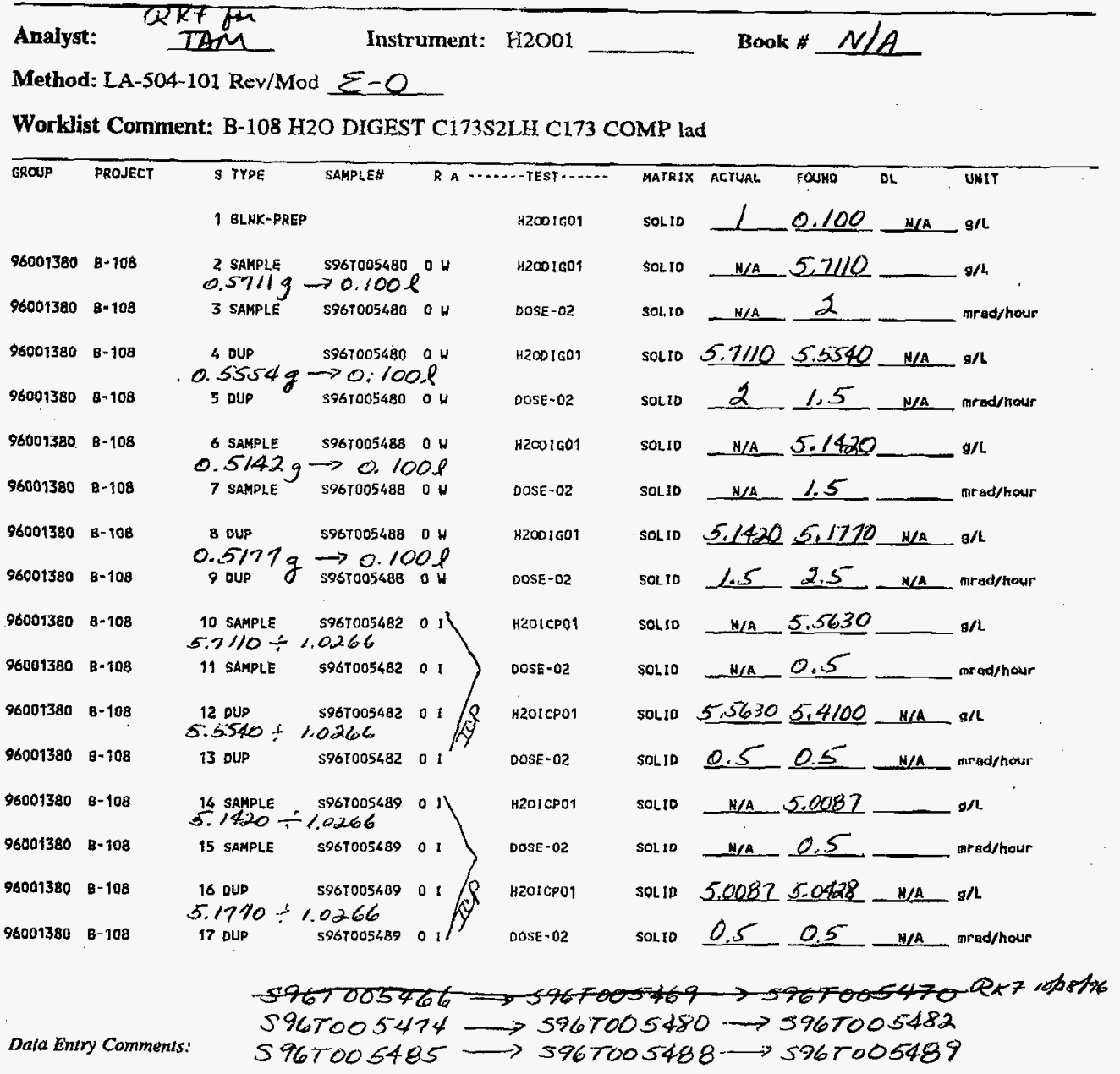

Utits shown for $Q C$ (SPK \& STD) may nor reflect the actual units. $D L=$ Detection Limi, $S=$ Worklist Slos Number. $R=$ Replicate Number, $A=$ Aliguot Code. 
Worklistrpt Version 2.1 05/15/95

HNF-SD-WM-DP-219, REV.0

Page: $\quad 2$

10/28/96\%14:18

LABCORE Data Entry Template for Worklist\# 14106

GROUP PROJECT S TYPE SAMPLEE $Q$ A -...-TEST..... HATRIX ACTUAL FOUHO OL UKIY

Zeith Fueler for Final page for worklist \# 14106

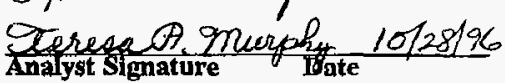

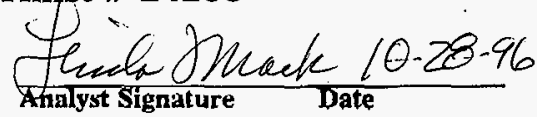

Reviemed: Hf Alughte

Data Entry Comments:

Units shown for $Q C$ (SPK \& STD) may not reflect the actual units. DL $=$ Detection Limit, $S=$ Worklist $\$$ Ilot Number, $\mathrm{R}=$ Replicare Number, $\mathrm{A}=$ Aliquot Code. 
0ot. 26. $1996 \quad 5: 08 \mathrm{PM}$

worklistrpt Version 2.1 05/15/95

10/17/96 13:18

\section{LABCORE Data Entry Template for Worklist\# 14106}

Analyst: $\mathrm{QAm}$ Instrument: $\mathrm{H} 2 \mathrm{OO1}$

Method: LA-504-101 Rev/Mod $\Sigma \cdot O$

Book \# $\frac{N / A}{1}$

Worklist Comment: B-108 H2O DIGEST C173S2LH C173 COMP lad

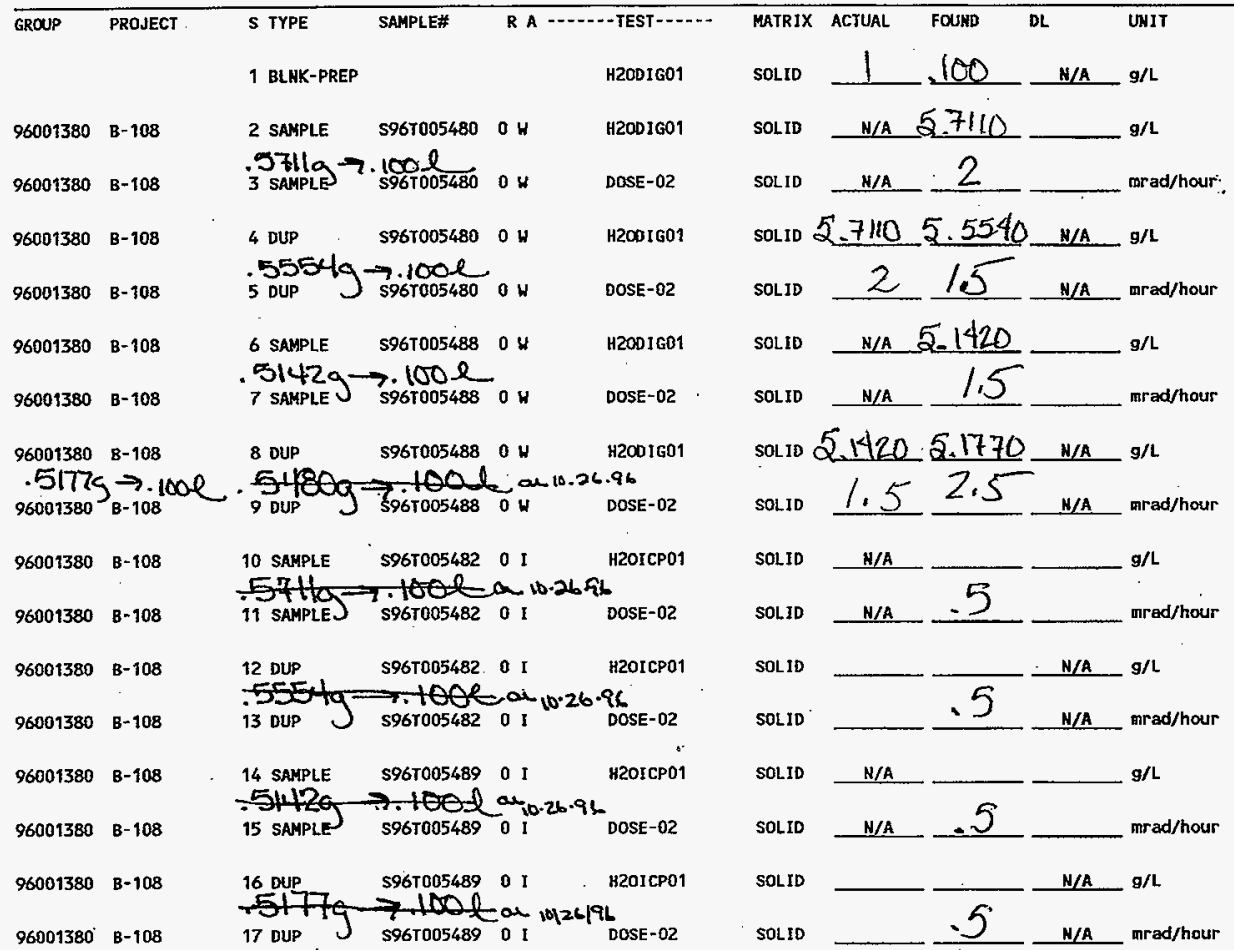

Data Entry comments: weighed by Aun:10.26.96 APT: qoe Whldez on:2.5mialla

Units shown for QC (SPK \& STD) may not reflect the actual units. $D L=$ Detection Limit, $S=$ Worklist Slot Number,

$R=$ Replicate Number, $A=$ Aliquot Code. 
Oci. 26. $1996 \quad 5: 08 \mathrm{PM}$

WHC 2225 LAB ROOM $2 \$$ BACKSIDE

No. 6313

P. $4 / 6$

worklistrpt Version 2.1 05/15/95

10/17/96 13:18

\section{LABCORE Data Entry Template for Worklist\# 14106}

\begin{tabular}{lllllll}
\hline GROUP PROJECT & S TYPE & SAMPLE\# & A $\cdots$ MATRIX ACTUAL FONND DL & UNIT
\end{tabular}

Final page for worklist \# 14106

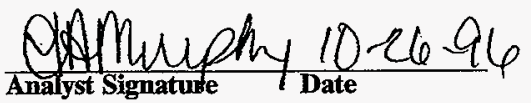

Analyst Signature Date

Units shown for QC (SPK \& STD) may not reflect the actual units. $D L=$ Detection Limit, $S=$ Worklist Slot Number, $R=$ Replicate Number, $A=$ Aliquot Code. 


\section{LABCORE Data Entry Template for Worklist\# 14173}

\section{Analyst: $\quad K N T$ Instrument: $\mathrm{H} 2 \mathrm{OO1}$ Book\# \\ Method: LA-504-101 Rev/Mod \\ Worklist Comment: B-108 H2ODIG H2OICP C172S1,2 UH LH lad}

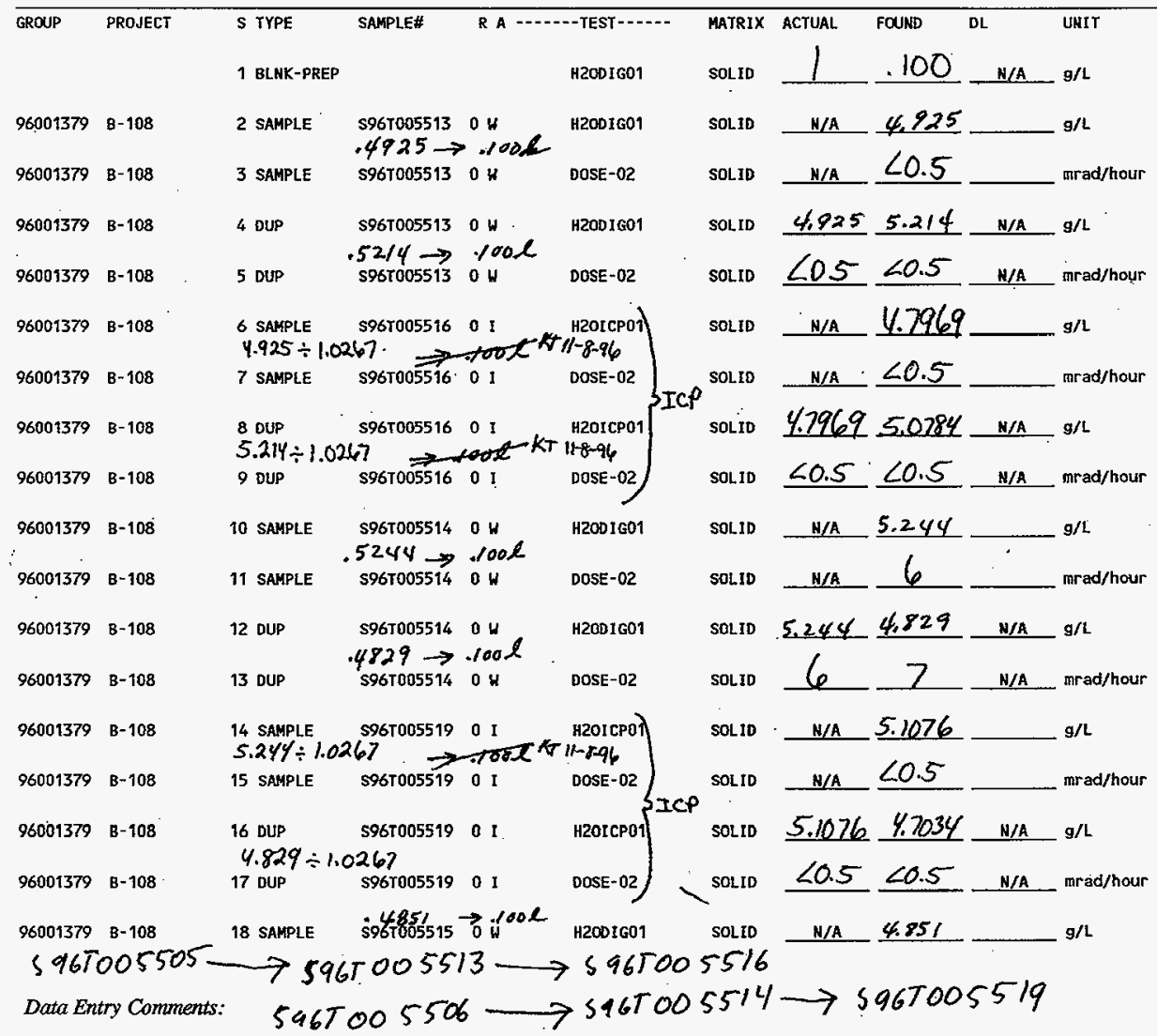

\section{samples wot homogenous.}

Units shown for QC (SPK \& STD) may not reflect the actual tunits. $D L=$ Detection Limit, $s=$ Worklist Slot Number,

$R=$ Replicate Number, $A=$ Aliquot Code.

\section{0}




\title{
LABCORE Completed Worklist Report for Worklist\# 16245
}

Analyst: $a b c$

\author{
Instrument: H2O01
}

Book\#

Method:

$\operatorname{Rev} / \mathrm{Mod}$

Worklist Comment: B-108 H2O DIGEST C173S2LH \& COMP lad

\begin{tabular}{lllllll}
\hline Seq Type & Sample\# R A & Test & Matrix Actual & Found DL or Yield Unit
\end{tabular}

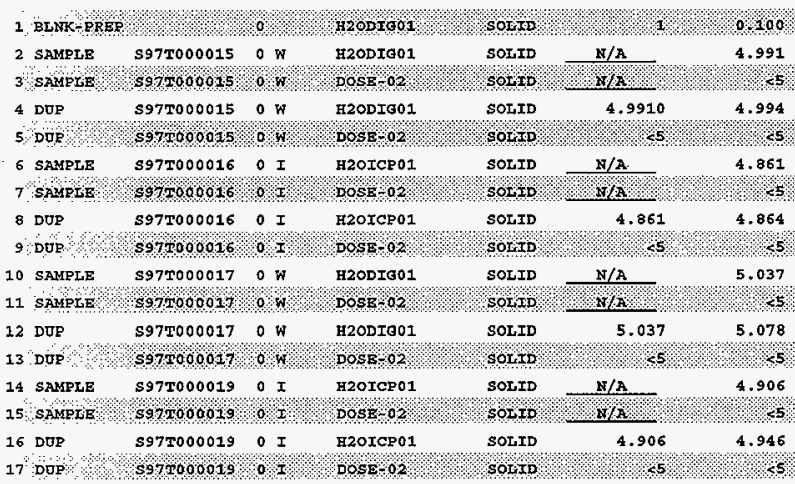

\section{Final page for worklist\# 16245}

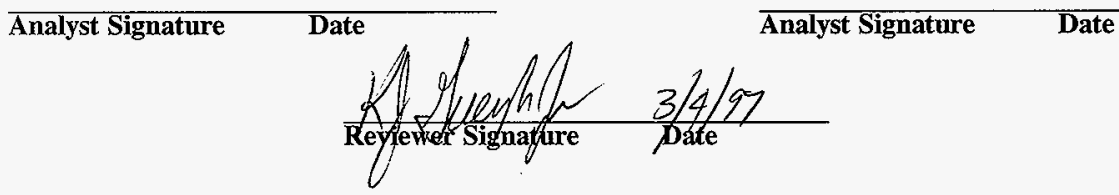


worklistrpt Version 2.1 05/15/95

HNF-SD-WMA-DP-219, REV. O

Page: 2 10/21/96.13:15

LABCORE Data Entry Template for Worklist\# 14173

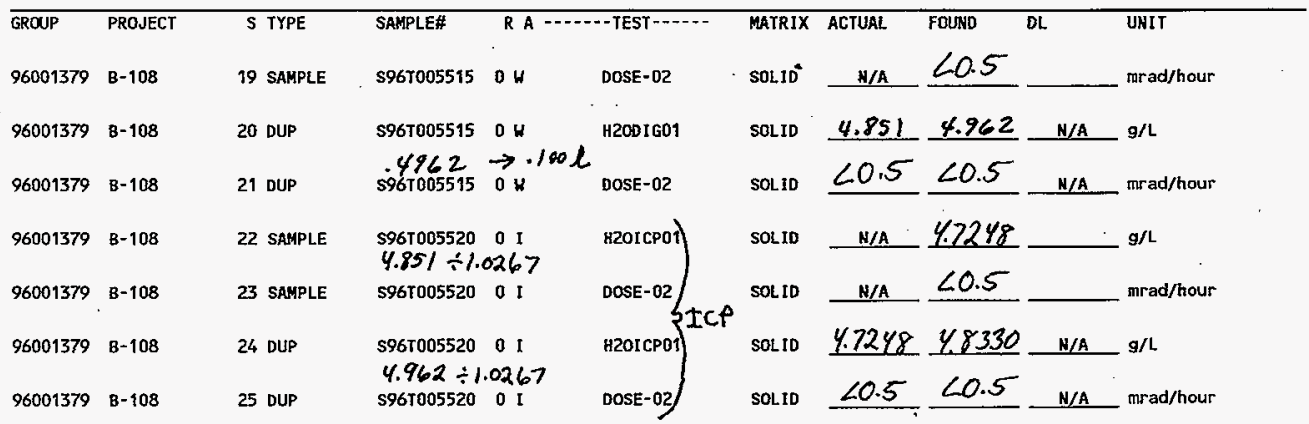

Final page for worklist \# 14173

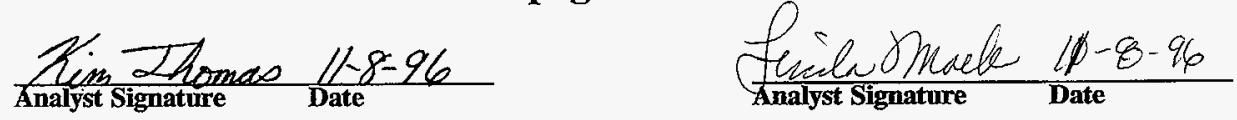

$$
596 T 005507 \longrightarrow 596 T 005515 \longrightarrow S 96 T 005520
$$

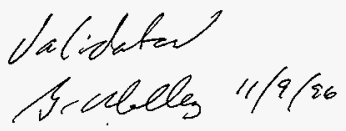

Data Entry Comments:

Units shown for $Q C$ (SPK \& STD) mon not reflect the actual units. DL = Detection Limit, $S=$ Worklist Slot Number, $R=$ Replicate Number, $A=$ Aliquot Code.

181

L I d co Lg on

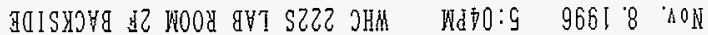


HNF-SD-WM-DP-219, REV. 0

BULK DENSITY WORKLISTS 
HNF-SD-WM-DP-219, REV. 0

\section{THIS PAGE INTENTIONALLY LEFT BLANK}




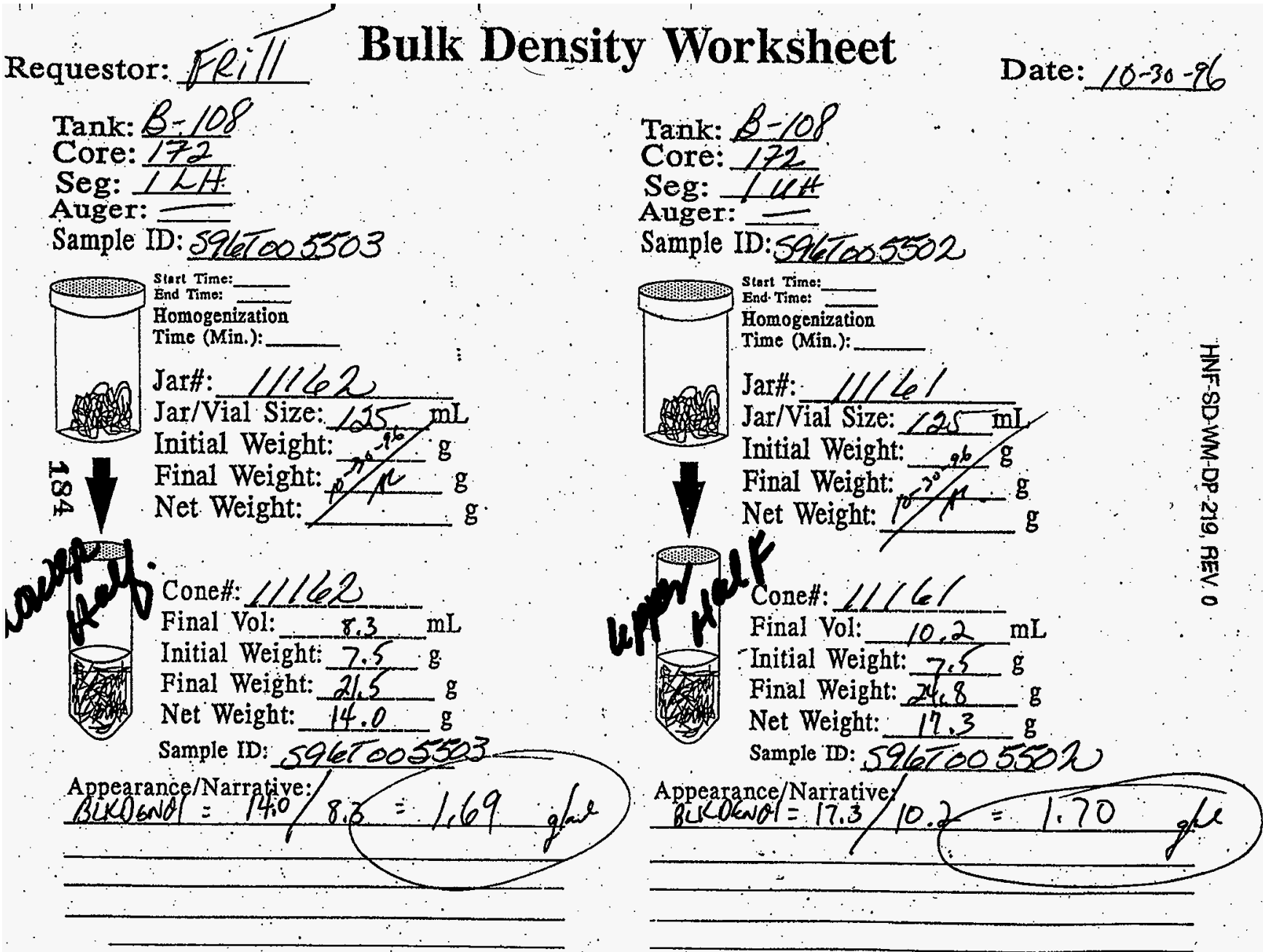


questor: $f R I T S$

\section{Bulk Density Worksheet}

Date: $10 / 18 / 96$

Tank: 6 cox

Core: $n 3$

Seg:

Auger:

Sample ID:

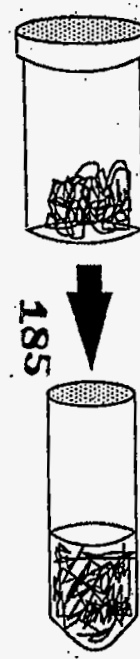

Start Time:

End Time:

Homogenization

Time (Min.):

Jar\#: $\quad 11159$

Jar/Vial Size: $125 \mathrm{ml}$

Initial Weight: $25 \% 2 \mathrm{~g}$

Final Weight:

$\mathrm{g}$

Net Weight: $\mathrm{g}$

Cone\#:

Final Vol:

59

Initial Weight: $7.45 \mathrm{~g}$

Final Weight: $24,69 \mathrm{~g}$

Net Weight: $17,24 \cdot \mathrm{g}$

Sample ID:

Appearance/Narrative: $17.24 \mathrm{gro}=1.91 \mathrm{~g} / \mathrm{mL}$
Tank:

Core:

Seg:

Auger:

Sample ID:

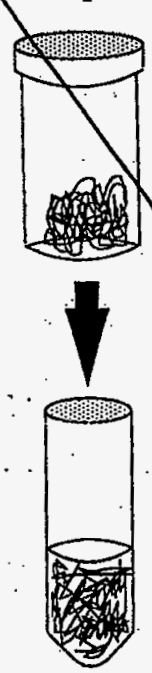

Start Time:

End Tims:

Homogenization

Time (Min.)

Jar\#:

Jar/Vial Size:__mL

Initial Weight:

Finat Weight:

Net Wight: _ _ $\mathrm{g}$

g.

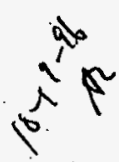

Conet:

Final Vol:

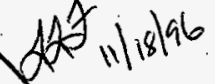

Initial Weight

Final Weight:

Net Weight:

Sample ID:

Appearance/Narrative: 
Requestor: Feitts

\section{Bulk Density Worksheet}

Tank: $\beta-108$

Core: 173

Seg: $\frac{1 \angle H}{1 \cdot \angle H}$

Auger:

Sample ID: 596 To05471

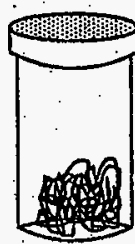

Start Tine:

End Timo:

Homogenization

Time (Min.):

Jar\#:

Jar/Vial Size: $125 \mathrm{ml}$

Initial Weight: $1872 \mathrm{~g}$

of $\begin{aligned} & \text { Final Weight: } \\ & \text { Net Weight: }\end{aligned}$

Conett: . ///58

Final vol: $7 . \quad \mathrm{mL}$

Initial Weight: $7,52 \mathrm{~g}$

Final Weight: $20.57 \mathrm{~g}$

Net Weight: $\frac{20.05}{13}$

Sample ID: 596T00 547/

Appearance/Narrative:

$13.05 / 7.5=17.4$ gim
Tank:

Core:

Seg:

Auger:

Sample ID:

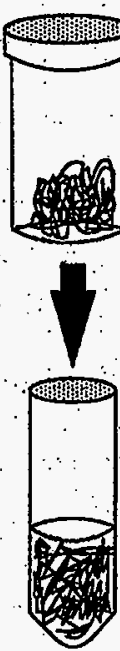

Stan Timo:

End Timo:

Homoreninstion

Time (Min.):

Jari:

Jar/Vial Size:

Initial Weight:

Final Weight:

Date: $18 / 12 / 96$

Net Weight:

Cone\#:

Final Vol:

Initial Weight:

Final Weight:

Net Weight:

Sample ID:

Appearance/Narrative:

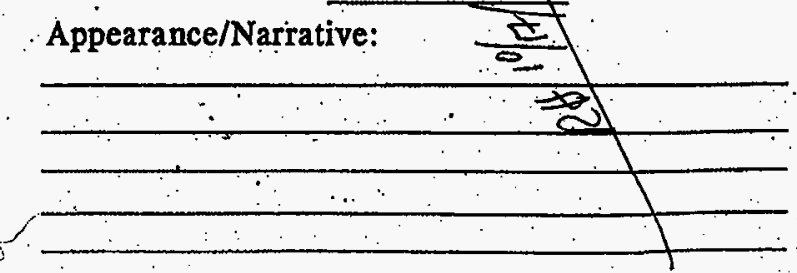


Requestor: Fiills

Tank: $\beta-108$

Core: 173

Seg: $\frac{214}{2}$

Auger:

Sample ID:S56Te05472
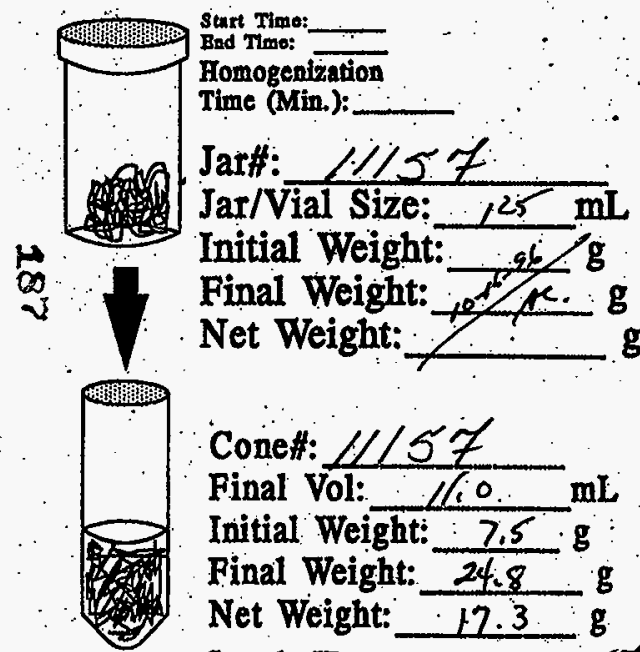

Conet:

Final vol: $/ / 10$. mL Initial Weight: $7.5 \mathrm{~g}$ Final Weight: Net Weight: $\frac{217}{13}$

Sample ID: 596T005472

Appearance/Narratiye:

$B C K D_{f} N \theta=77.3 \% 11.0$ 1.57

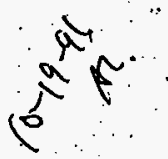

D10 NoT Pack

Congistency of stanple:
Tank:

Core:

Seg:

Auger:

Sample ID:

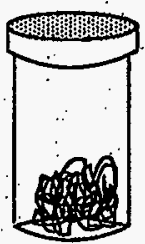

tart Tho:

End Timo

Homogetization

Time (Min):

Jait:

Jar/Vial Size: $\quad \mathbf{m L}$

Initial Weight:

Final Weight

Net Weight:

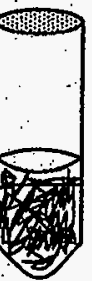

Coneff:

Final Vol:

Initial Weight:

Final Weight:

Net Weight:

Sample ID:

Appearance/Narirative:

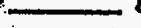

Date: $10-16-96$ 


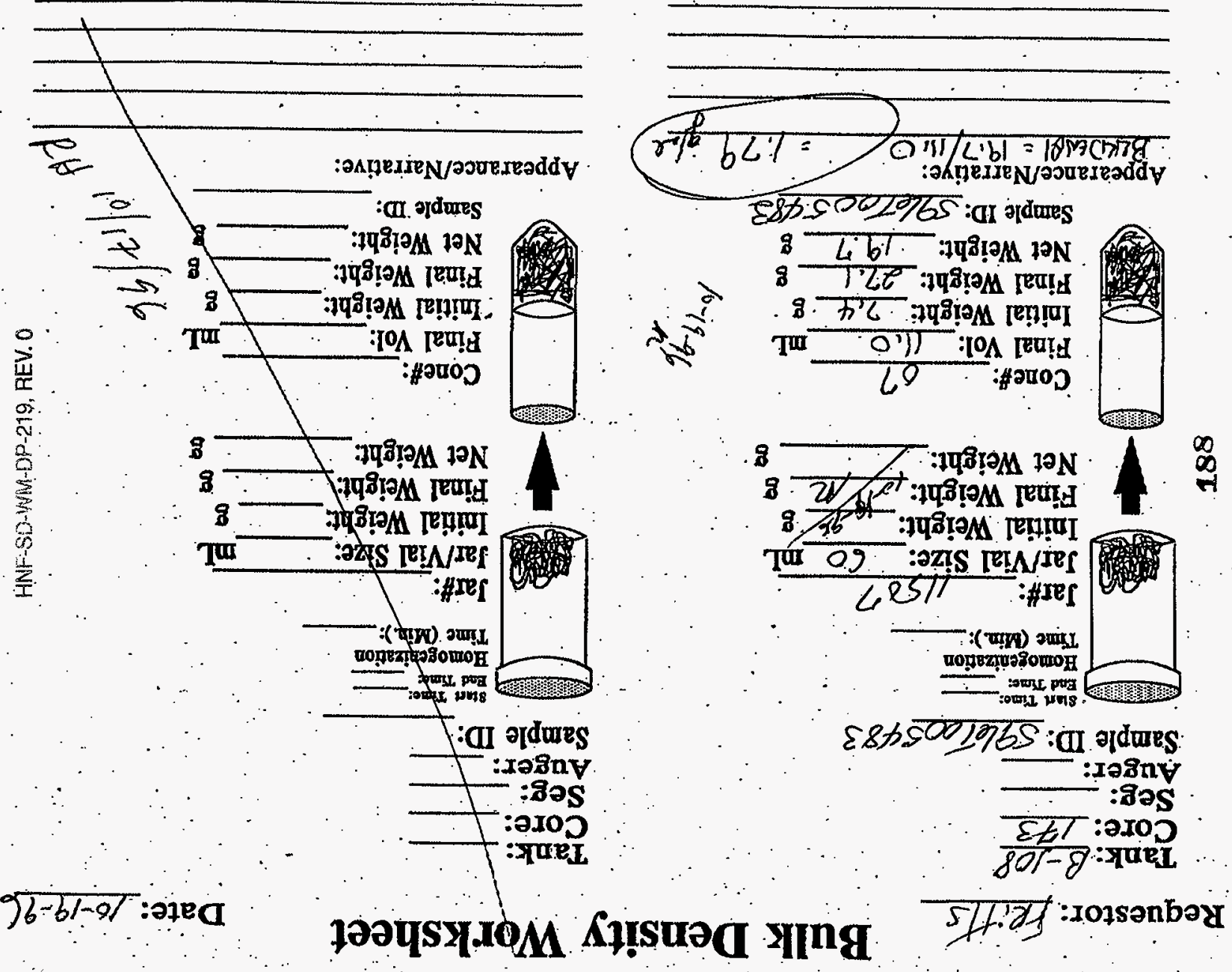


HNF-SD-WM-DP-219, REV. 0

INORGANIC ANALYSIS

189 
HNF-SD-WM-DP-219, REV. 0

THIS PAGE INTENTIONALLY LEFT BLANK 


\section{LABCORE-Data Entry Template for Worklist\# 14390}

Analyst: Jds Instrument: $D^{2}{ }^{2} 11$ Book\# $12 N 14 B$

Method: LA-514-113 Rev/Mod C-1

Worklist Comment: DSC-01 FOR B-108 PLEASE RUN UNDR N2 ～RTS!

\begin{tabular}{|c|c|c|c|c|c|c|c|c|c|c|}
\hline \multirow[t]{2}{*}{ GROUP } & PROJECT & $S$ TYPE & SAMPLE\# & & A $\quad \cdots . . . .$. TEST $-\ldots$ & MATRIX & ACTUAL & FOUND & DL & UNIT \\
\hline & & 1 STb & & & DSC-0? & SOLID & 28.45 & 27.4 & $\mathrm{~N} / \mathrm{A}$ & Joules/g \\
\hline 96001380 & $B-108$ & 2 SAMPLE & 5967005466 & 0 & DSC-01 & SOL.1D & N/A & $\theta$ & & Joules/g \\
\hline 96001380 & $B-108$ & 3 DUP & s967005466 & 0 & DSC-01 & SOLID & $\cdot \phi$ & & N/A & Joutes/s \\
\hline 96001380 & B-108 & 4 SAMPLE & s96r005473 & 0 & DSC-01 & SOLID & N/A & $(x$ & & Joules/g \\
\hline 96001380 & $B-108$ & 5 DUP & S96T005473. & 0 & DSC-01 & SOLIO & $\phi$ & $\not$ & N/A & Joules/g \\
\hline
\end{tabular}

Final page for worklist \# 14390

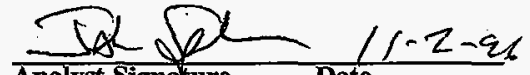

Analyst Signafure Date

Verifiad Nabiated iry

$P$ om dina

Valengava 11.12 .96

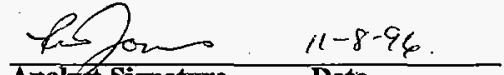

Analyst Signature Date

Data Entry Comments:

Units shown for $Q C$ (SPK \& STD) may not reflect the actual units. $D L=$ Detection Limit, $S=$ Worklist Slot Number,

$R=$ Replicate Number, $A=$ Aliquot Code. 
SIGNATURE BELOW REPRESENTS CHEMCAL TECHNOLOGIST/CHEMIST THAT

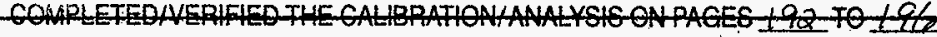

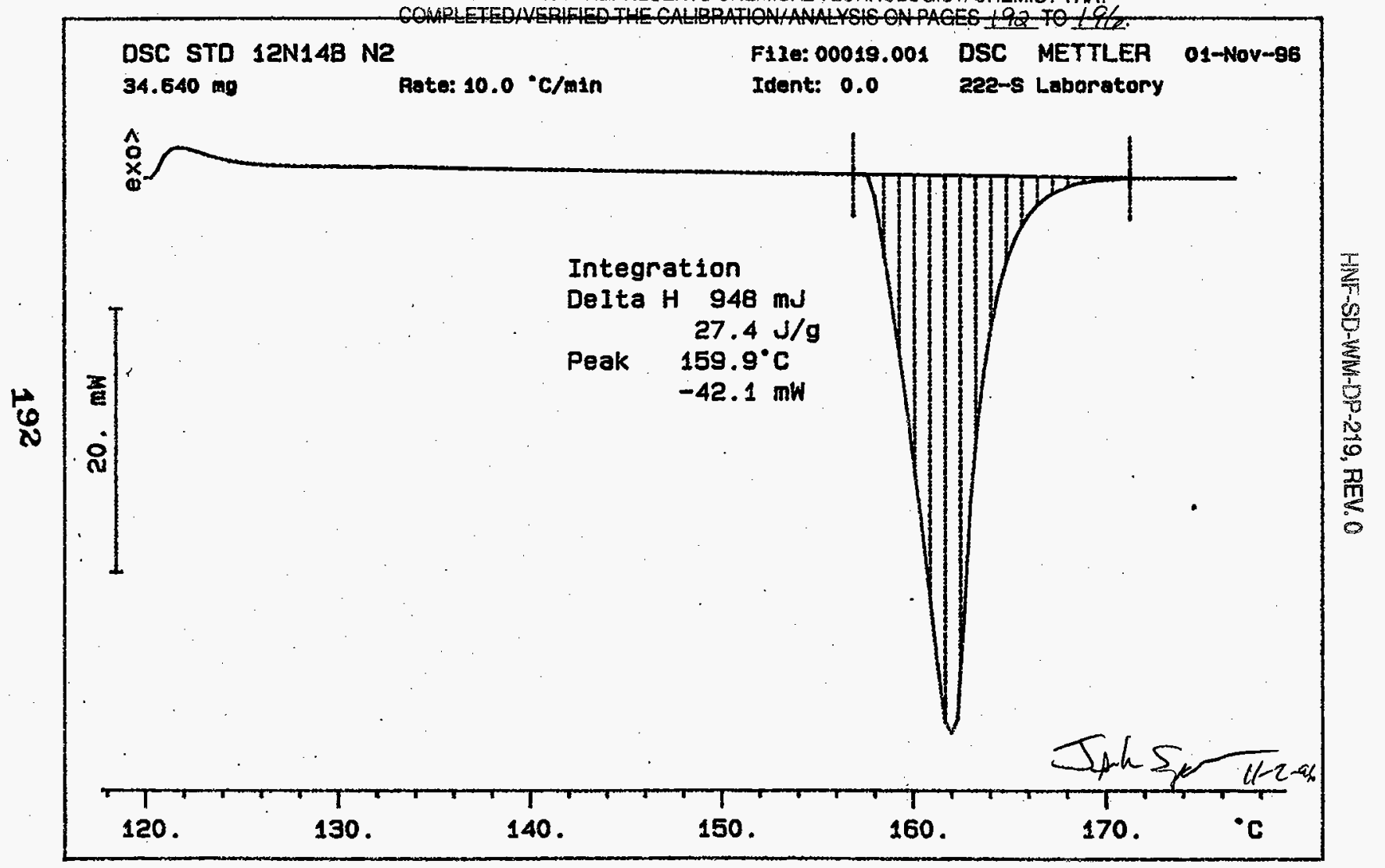


HNAF-SD-WW-DP-219, REV.O

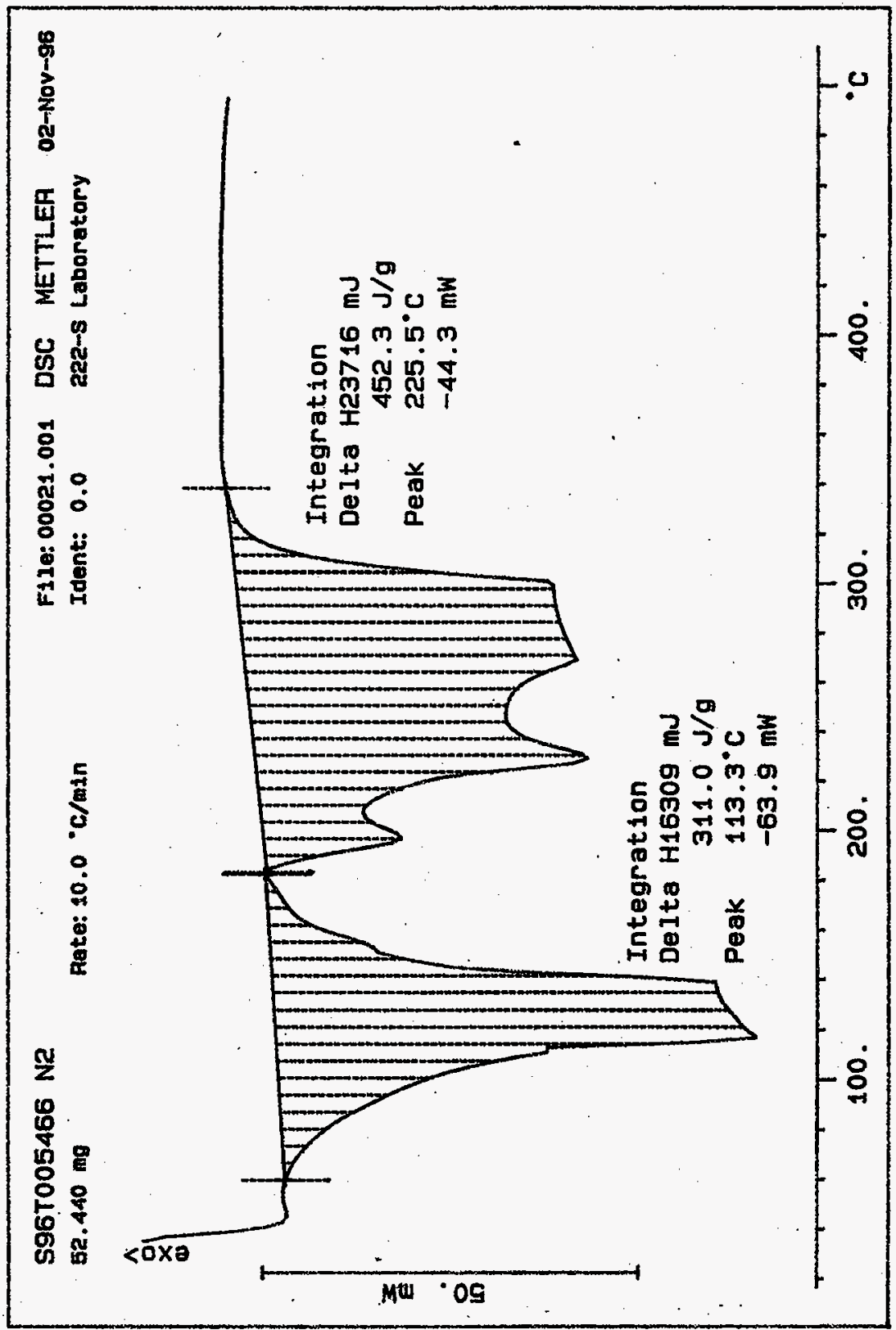

193 


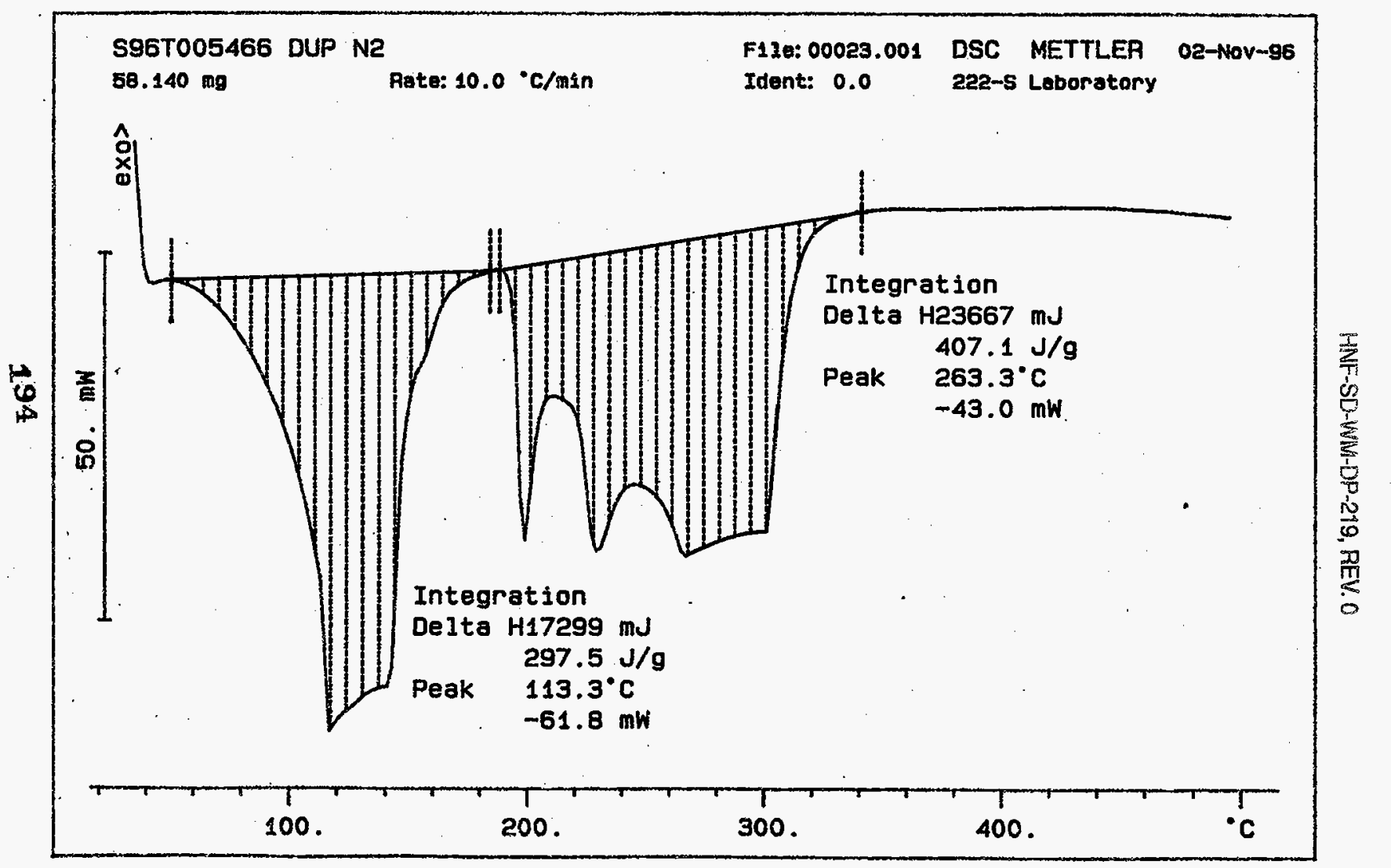




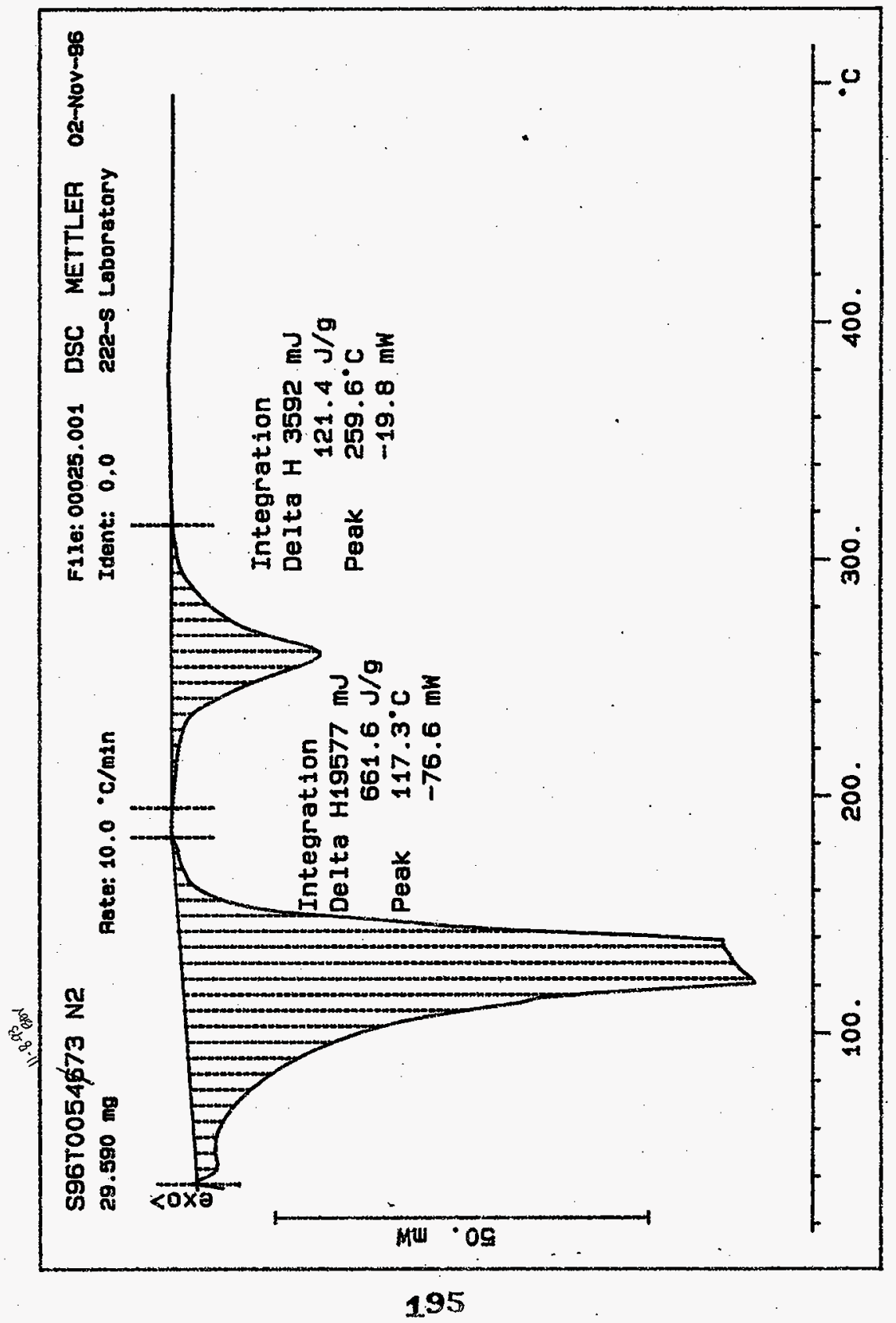


HNF-SD-MMM-DP-219, REV. 0

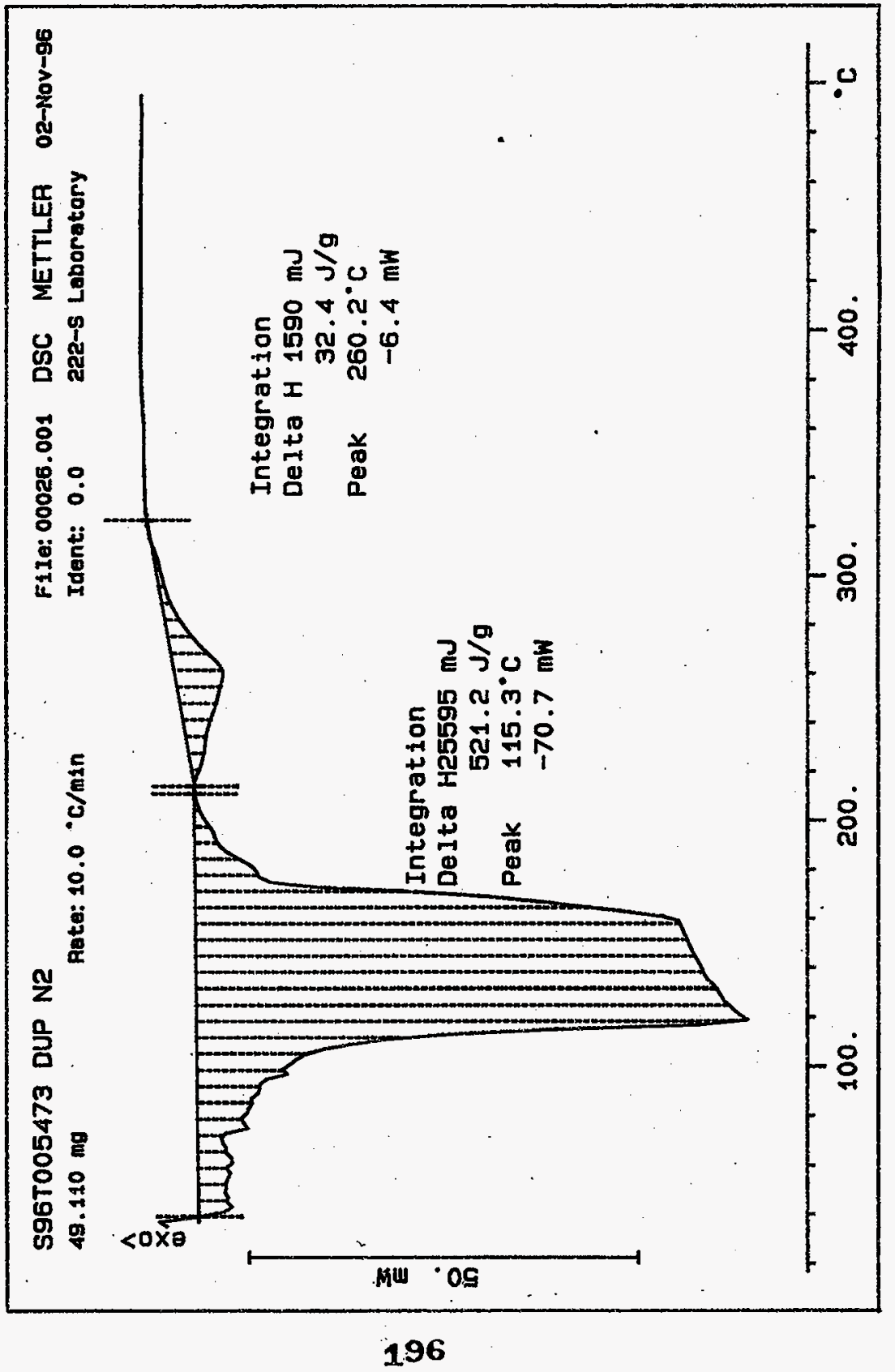


HNF-SD-WM-DP-219, REV .O

worklistrpt Version 2.1 05/15/95

Page: 1

10/28/96 14:26

LABCORE Data Entry Template for Worklist\# 14391

Analyst: $\quad \frac{K R M}{2} \quad$ Instrument: $\mathrm{DSCO}^{\circ} \perp \quad$ Book $\frac{\lg N / 4 B}{2}$

Method: LA-514-113 Rev/Mod C-1

Worklist Comment: DSC-01 FOR B-108 PLEASE RUN UNDER N2 . RTS!

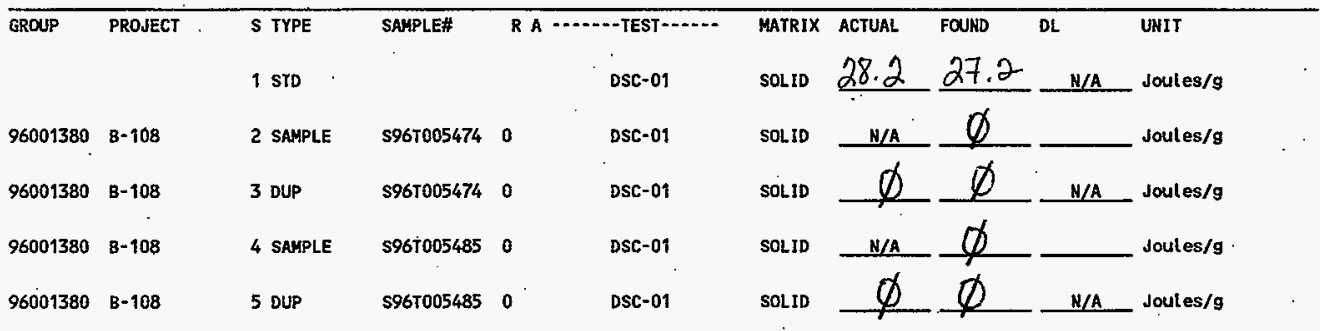

Final page for worklist \# 14391

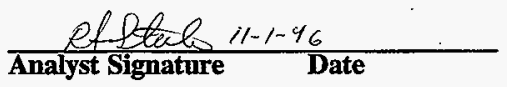

Verified/Validated by

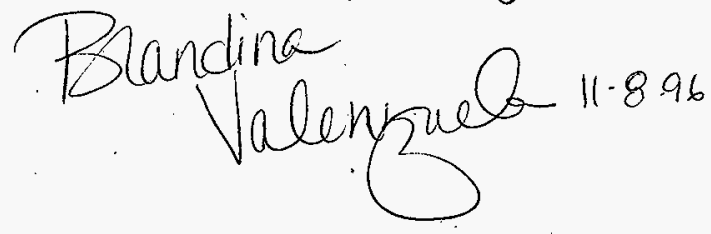

Data Entry Comments:

Units shown for $Q C$ (SPK \& STD) may not reflect the actual units. $D L=$ Detection Limit, $S=$ Worklist Slot Number, $R=$ Replicate Number, $A=$ Aliquot Code.

197 


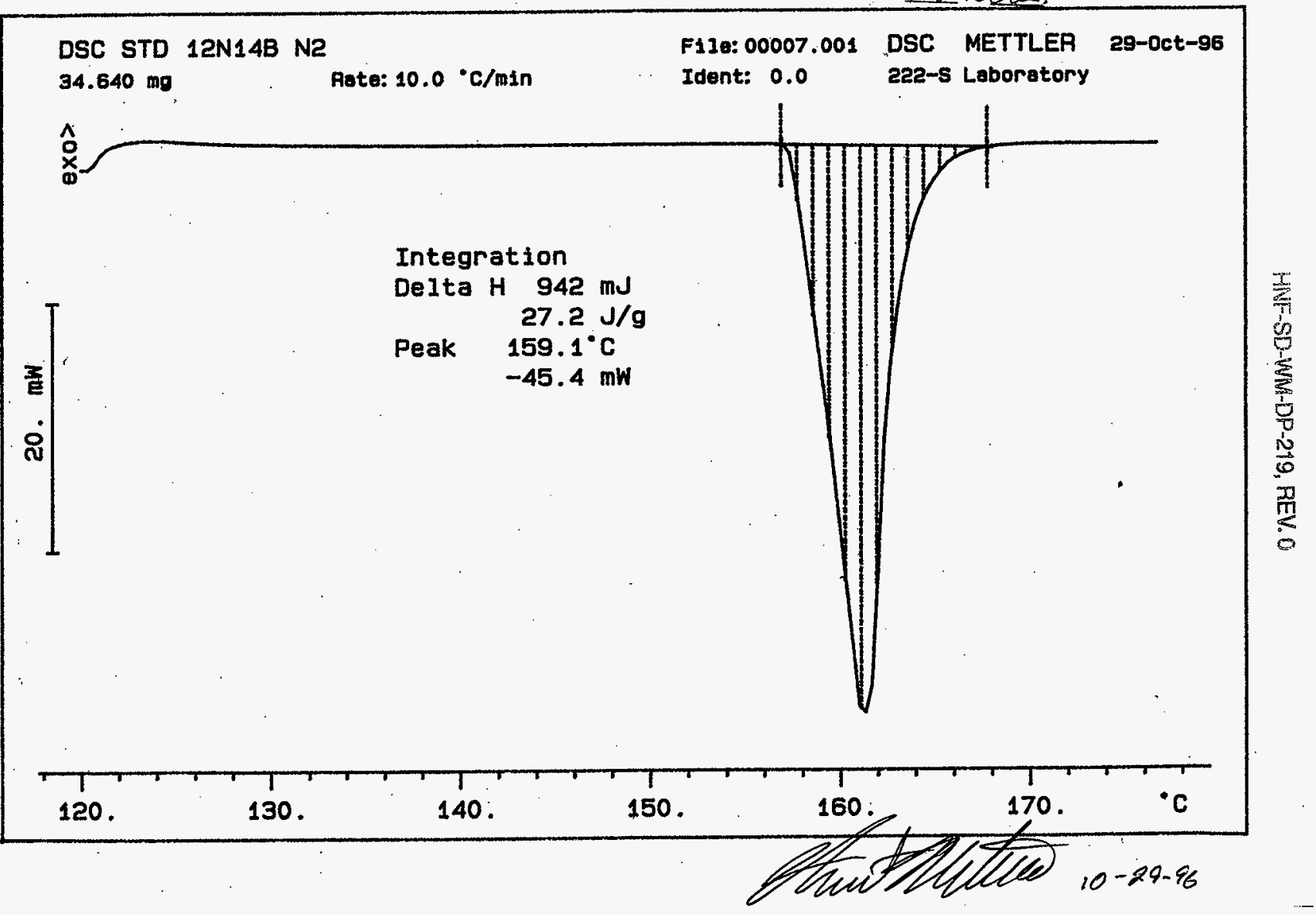




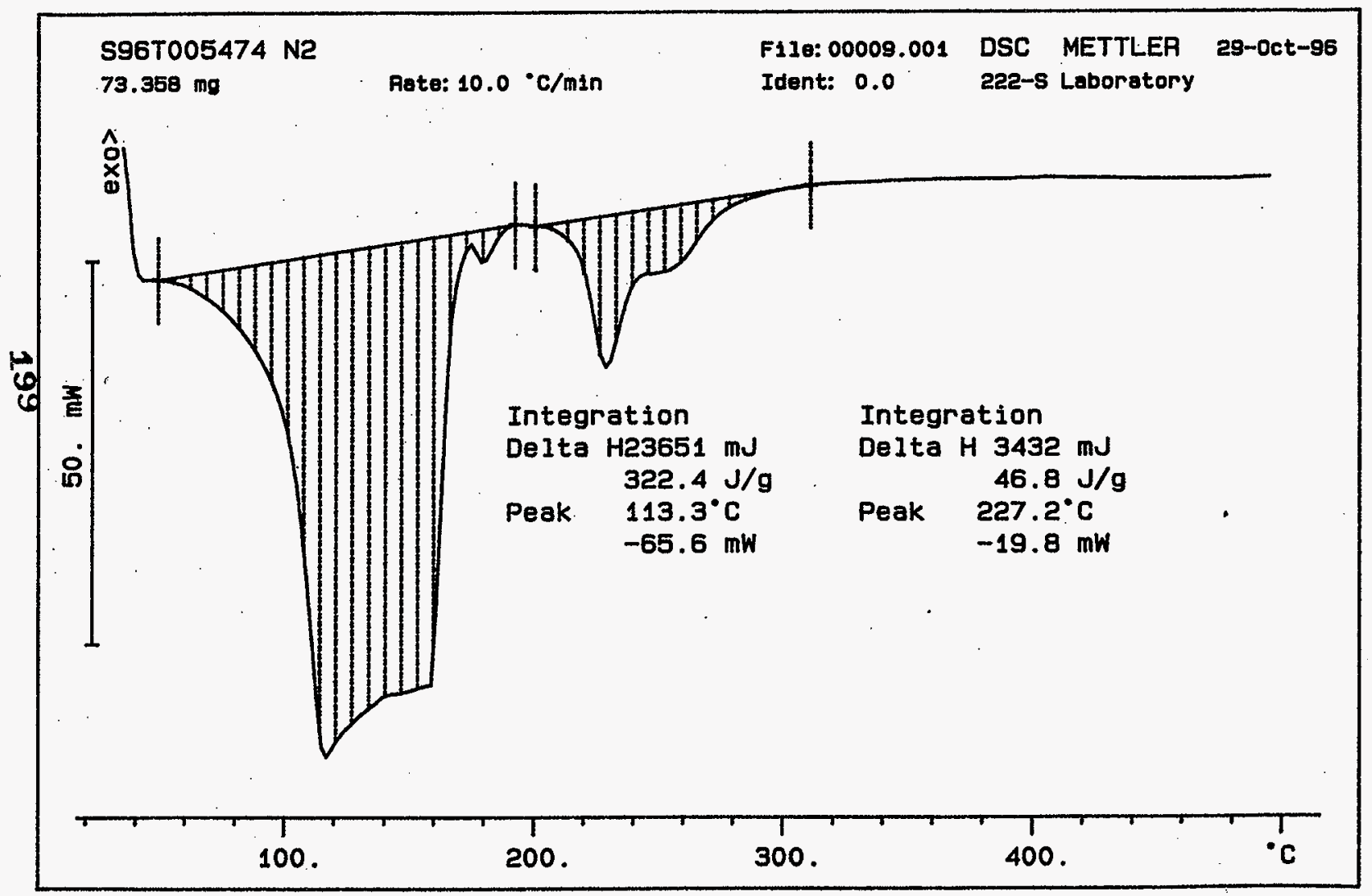


HNF-SD-WM-OP-219, REV.O

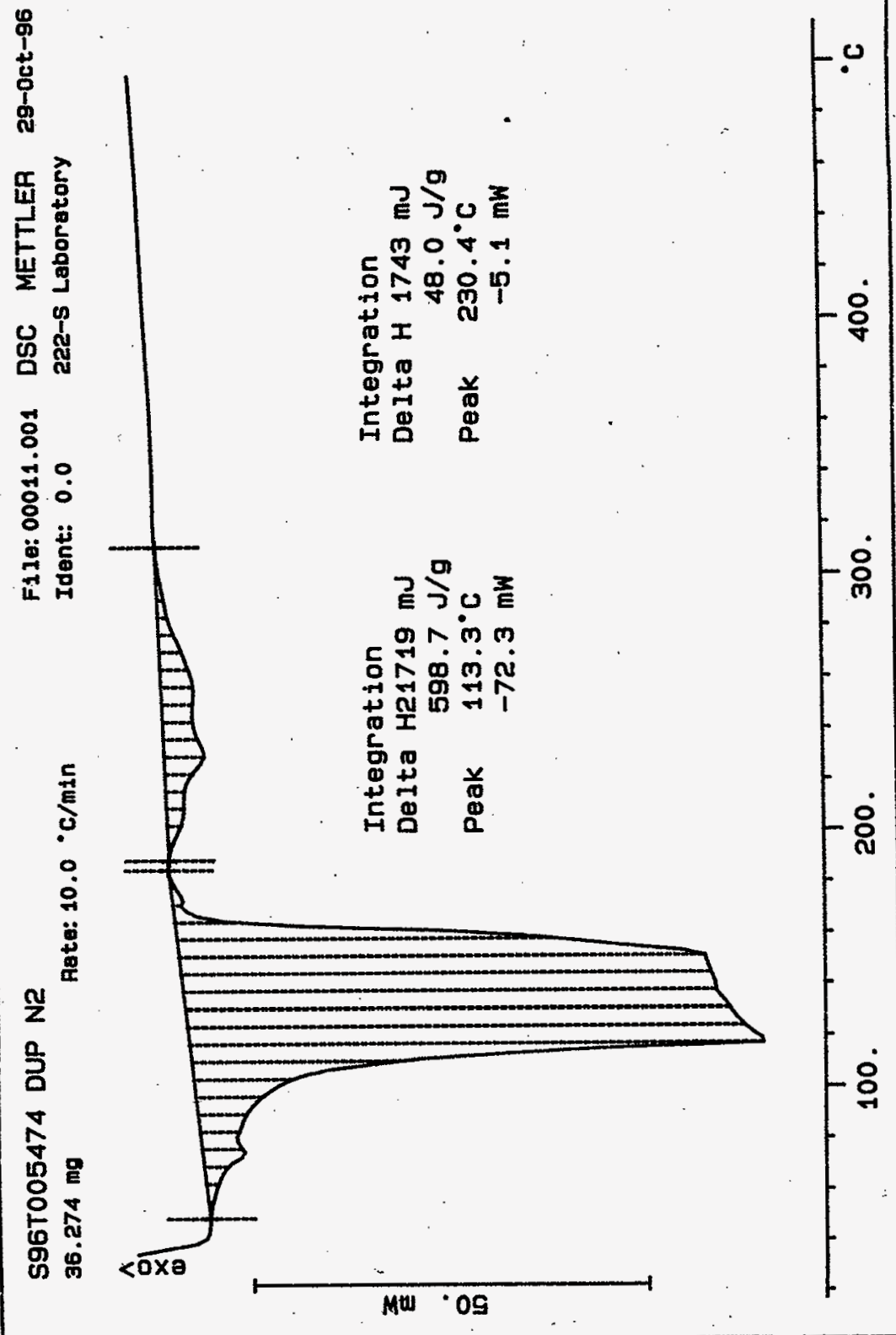




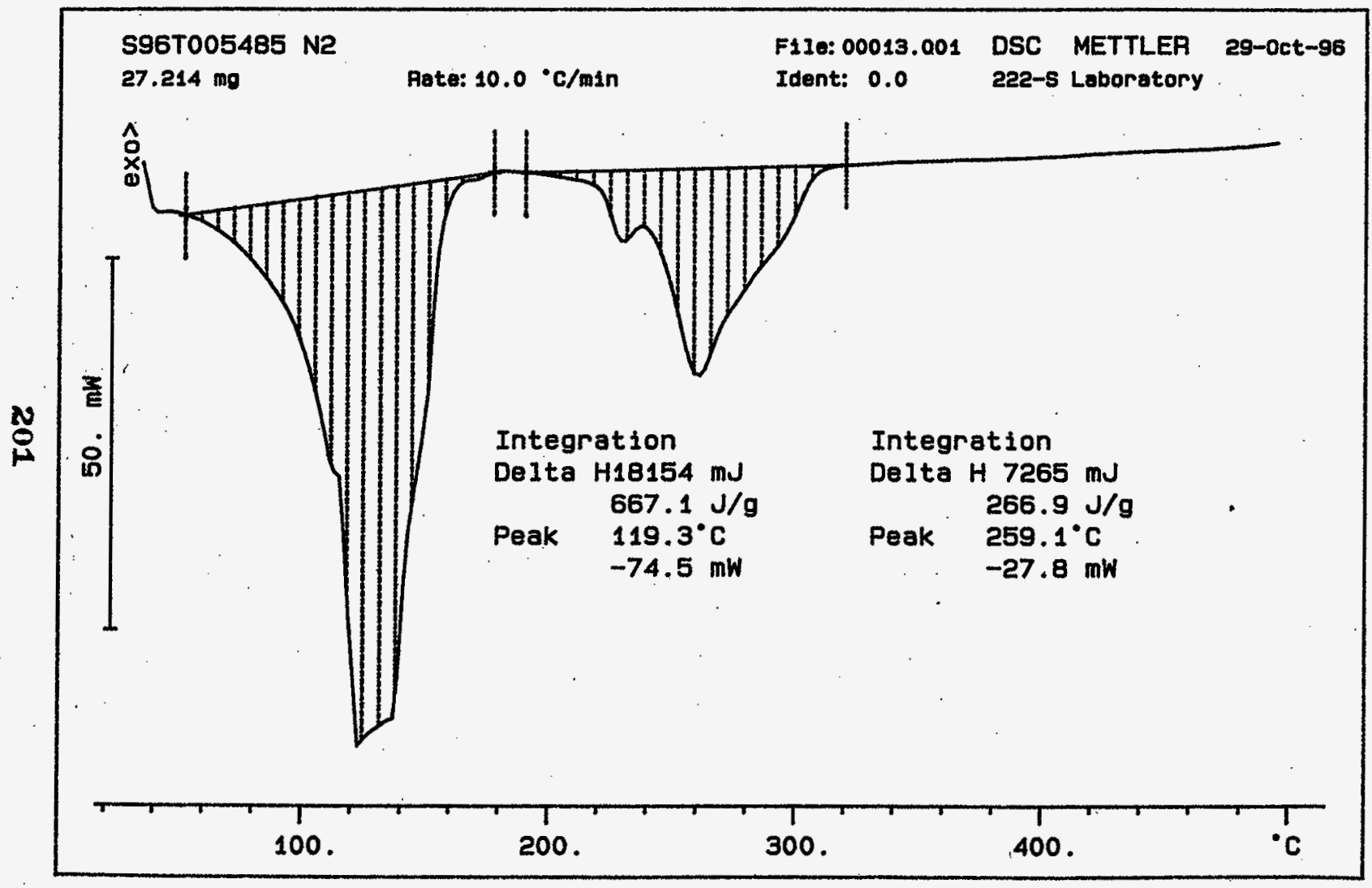




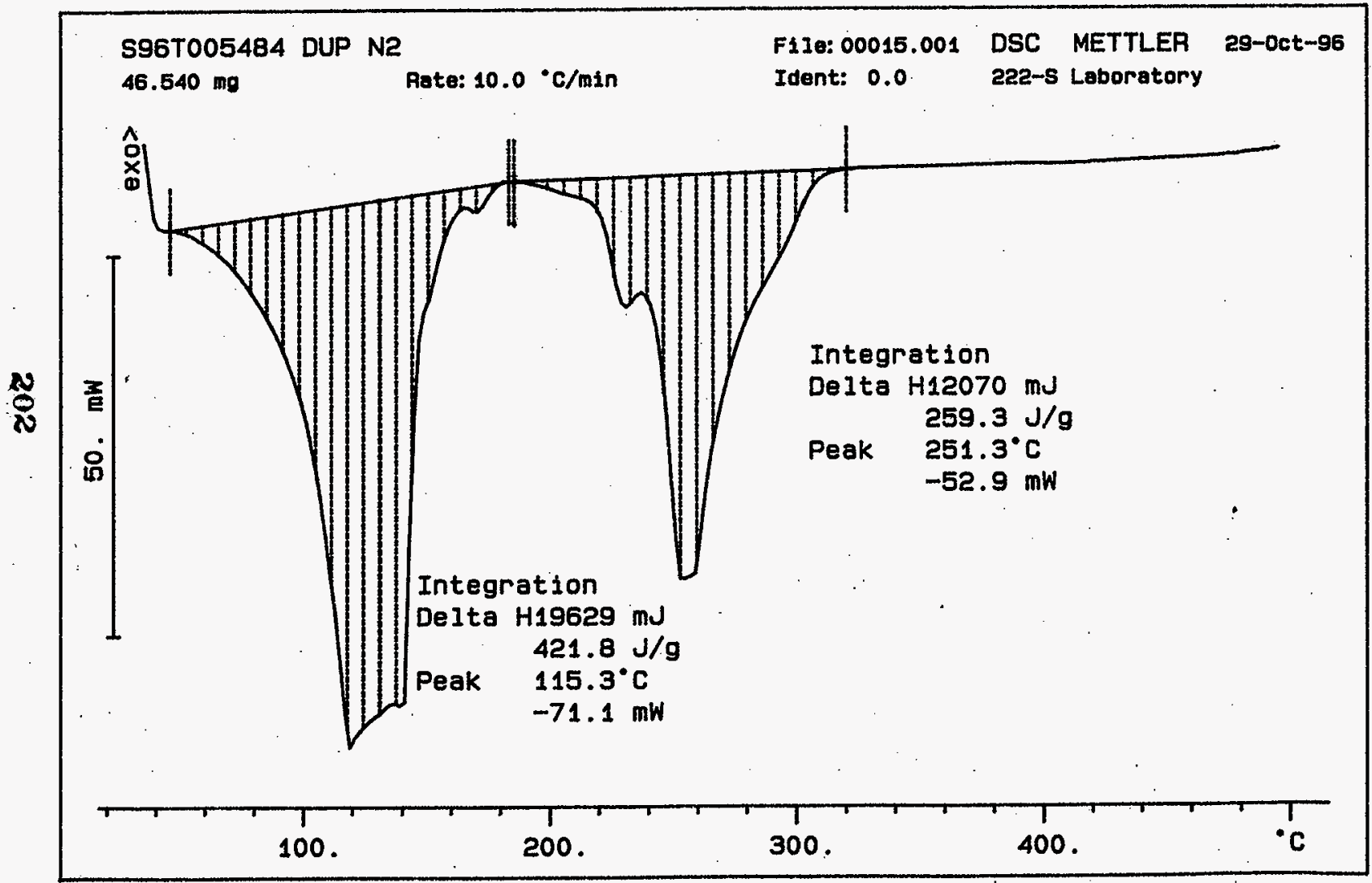




\section{LABCORE Data Entry Template for Worklist\# 14656}

Analyst: SMF Instrument: $\mathrm{DSCO} 3$ Book \# $12 \mathrm{~N} 14 \mathrm{~B}$

Method: LA-514-114 Rev/Mod D-O

Worklist Comment: Please run B-108 DSC under N2. bdv

\begin{tabular}{|c|c|c|c|c|c|c|c|c|c|c|}
\hline \multirow[t]{2}{*}{ GROUP } & PROJECT & $S$ TYPE & SAMPLE\# & R A & A $\cdots$ TEST $-1 . .$. & MATRIX & ACTUAL & FOUND & $\mathrm{DL}$ & UNIT \\
\hline & & 1 STD & & & DSC-03 & LIQUID & 28.45 & 28.68 & N/A & _ Joules/g \\
\hline 96001379 & $B-108$ & 2 SAMPLE & S96T005463 & 0 & DSC-03 & LIQUID & $\mathrm{N} / \mathrm{A}$ & $\varnothing$ & & Joules/g \\
\hline 96001379 & B-108 & 3 DUP & S96T005463 & 0 & DSC -03 & LIQUID & $\varnothing$ & $\varnothing$ & $\mathrm{N} / \mathrm{A}$ & Joules/g \\
\hline
\end{tabular}

\section{Final page for worklist \# 14656}

\section{Seattached or signatures)}

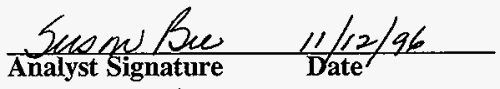

Verifid/Validated ly

Plandina.

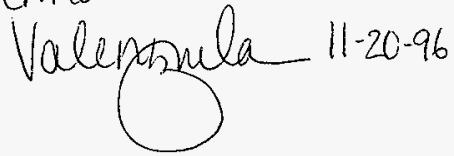

Data Entry Comments:

Units shown for $Q C$ (SPK \& STD) may not reflect the actual units. $D L=$ Detection Limit, $S=$ Worklist Slot Number,

$R=$ Replicate Number, $A=$ Aliquot Code. 
worklistrpt Version 2.1 05/15/\$5JNF-SD-WM-DP-219, REV.O

$\mathrm{cos}^{20}$

11/04/96 14:42

LABCORE Data Entry Template for Worklist\# 14656

Analyst:

EAt Instrument: DSCO

Book \#12N14B

Method: LA-514-113 Rev/Mod

Worklist Comment: Please run B-108 DSC under N2. bdv

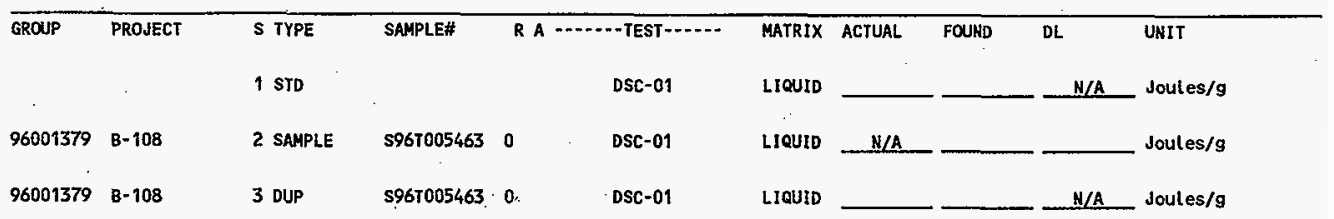

Final page for worklist \# 14656

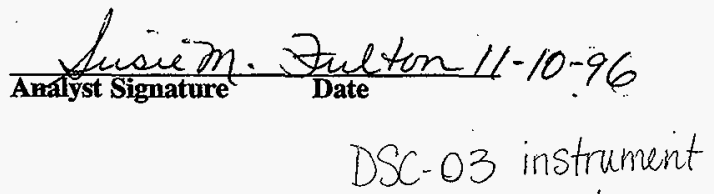

Analyst Signature Date

wow used.

11.12 .96

Blandina
Valenzuela

Data Entry Comments:

Units shown for $Q C$ (SPK \& STD) may not reflect the actual units. $D L=$ Detection Limit, $S=$ Worklist Slot Number, $R=$ Replicate Number, $A=$ Aliquot Code.

204 
Curve 1: DSC

F1le info: IND11090 s Sat Nor 9 17: 36: 361996

Sample Weight: 22.750 ig

12N14-B INDIUN AT 10C/MIN

SIGNATURE BELOW REPRESENTS CHEMICAL TECHNOLOGIST/CHEMIST THAT

COMPLETED/VERIFIED THE CALIBRATION/ANALYSIS ON PAGES 205 TO 207.

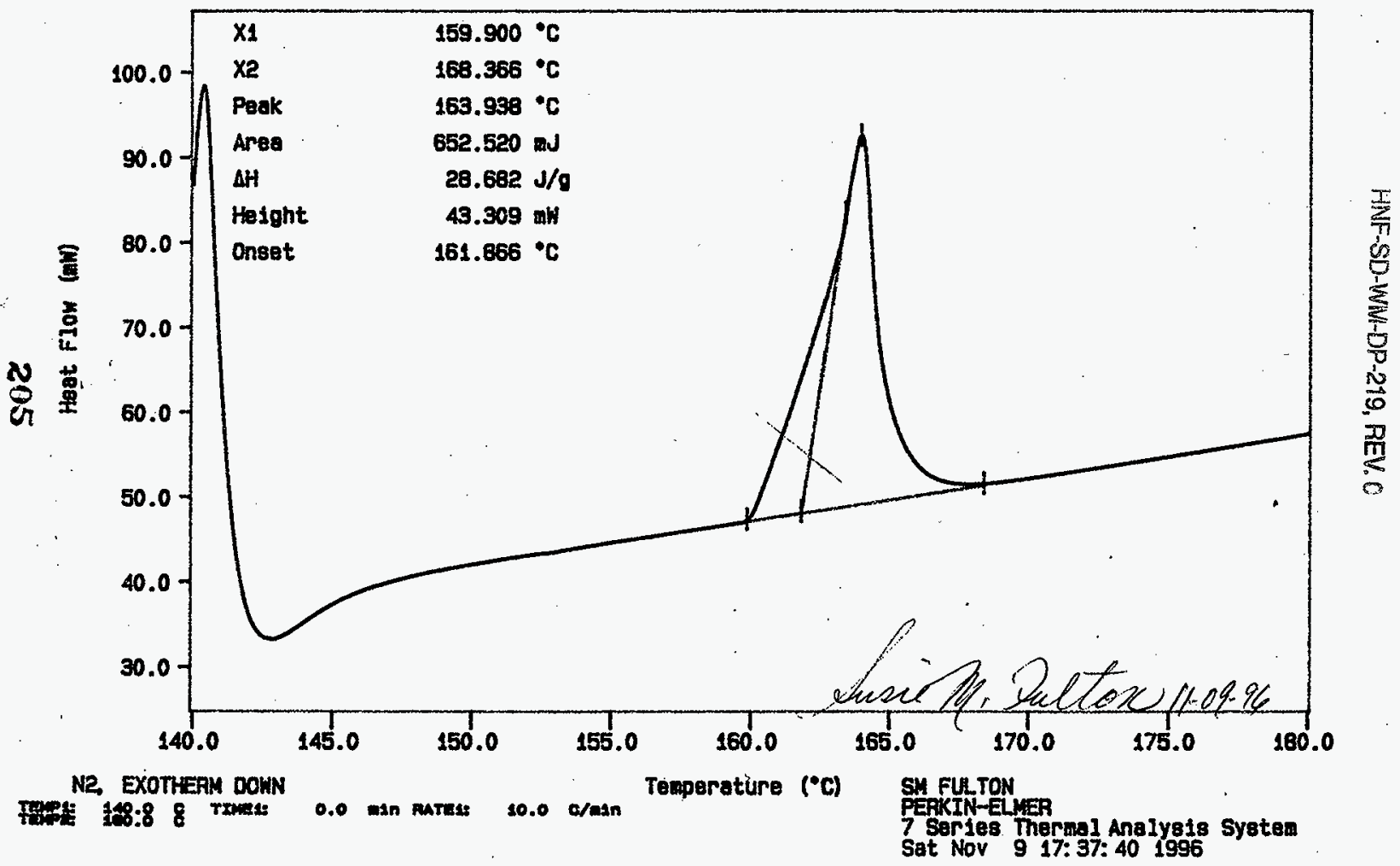


Curvo 1: DSC

File info: SAM110901 Sat Nor 9 20: 21: 511996

Sample Weight: 9.180

\$96T005463

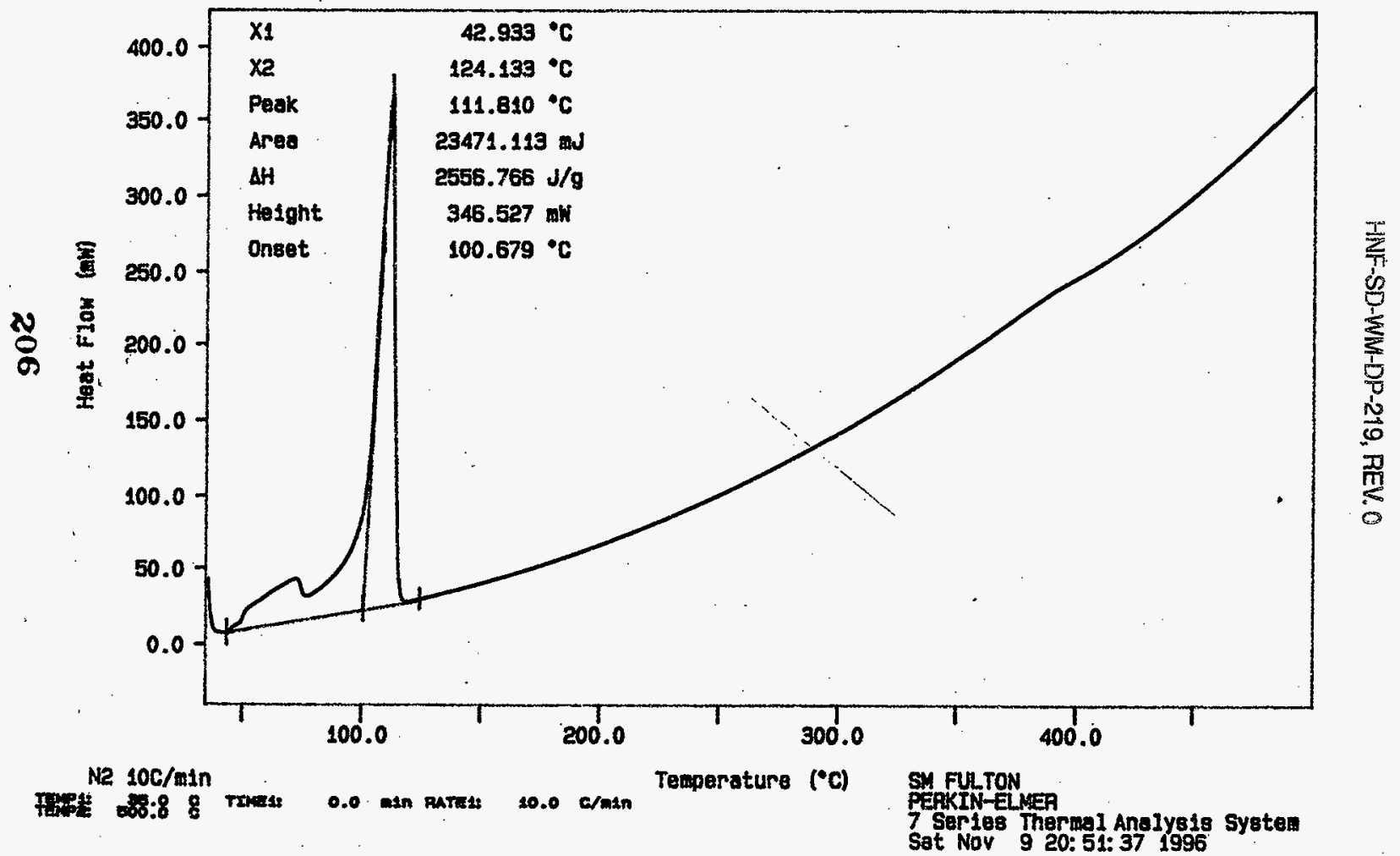


Curve i: DSC

Fłle info: SAM110902 Sat Nov 9 225 52 271996

Sanple Height: 9.650

ang

S96T005463 DUP

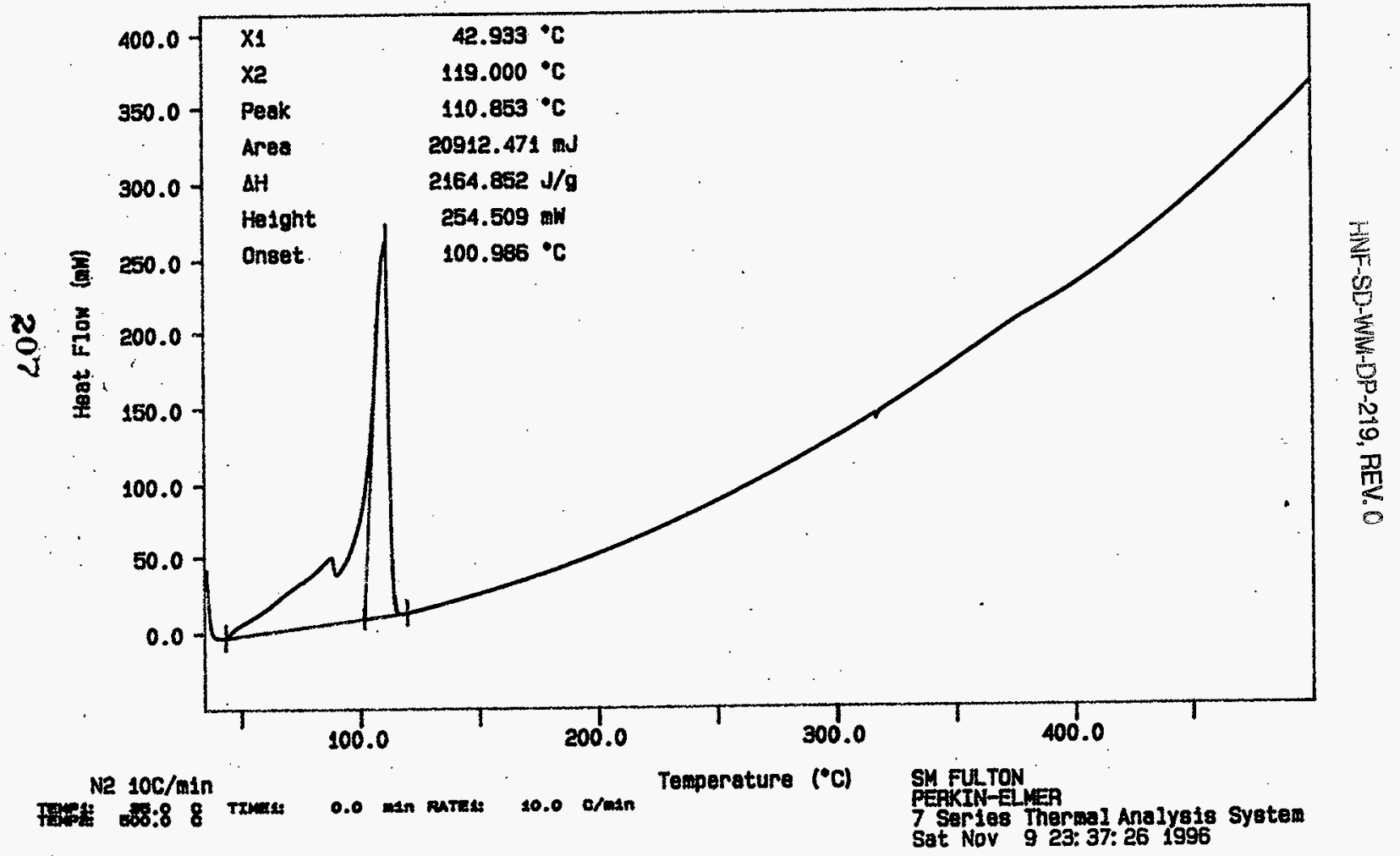




\section{LABCORE Data Entry Template for Worklist\# 14715}

Analyst: $\quad$ DCD $\quad$ Instrument: $\mathrm{DSCO}^{\circ} 3$ Book \# 12 N $14 \mathrm{~B}$

Method: LA-514-114 Rev/Mod D-O

Worklist Comment: B-108 DSC, RUN UNDER N2. RCJ

\begin{tabular}{|c|c|c|c|c|c|c|c|c|c|c|}
\hline \multirow[t]{2}{*}{ GROUP } & PROJECT & S TYPE & SAMPLE\# & R A & ........ TEST $\cdots$ & MATRIX & ACTUAL & FOUND & $\overline{D L}$ & UNIT \\
\hline & & 1 sto & & & DSC -03 & LIQUID & 28.45 & 27.73 & $N / A$ & Joules/g \\
\hline 96001379 & 8-108 & 2 SAMPLE & S96T005523 & 0 & DSC-03 & LIQUID & $N / A$ & $\phi$ & & Joules/g \\
\hline 96001379 & B-108 & 3 DUP & S96T005523 & 0 & DSC-03 & LIQUID & $\varnothing$ & $\varnothing$ & N/A & Joules/g \\
\hline
\end{tabular}

Final page for worklist \# 14715

\section{See attached or signatures}

Analyst Signature $11-20.96$

Verifiad/Vaidated by

Analyst Signature Date

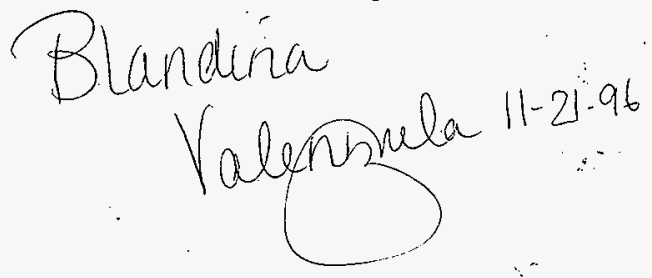

Data Entry Comments:

Units shown for $Q C$ (SPK \& STD) may not reflect the actual units. $D L=$ Detection Limit, $S=$ Worklist Slot Number, $R=$ Replicate Number, $A=$ Aliquot Code. 


\section{LABCORE Data Entry Template for Worklist\# 14715}

Analyst: $\quad \mathrm{DCD} \quad$ Instrument: $\mathrm{DSCO}^{\circ} \ldots$ Book \# $12 \mathrm{~N} 14 \mathrm{~B}$

Method: LA-514-113 Rev/Mod

Worklist Comment: B-108 DSC, RUN UNDER N2. RCJ

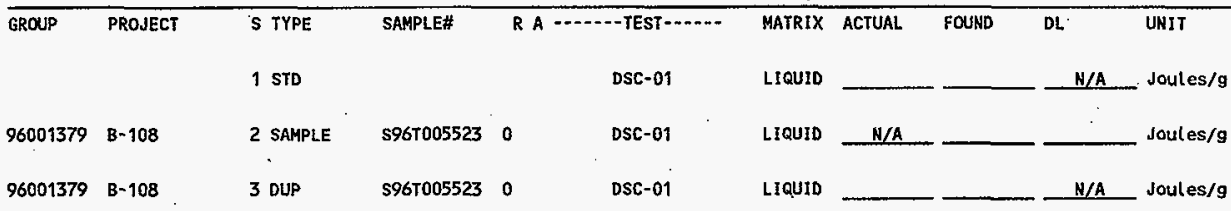

\section{Final page for worklist \# 14715}

\section{Daxid Cuncem 11-19-96}

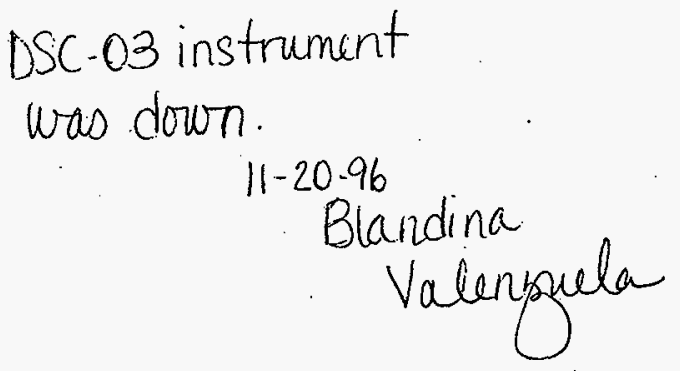

Data Entry Comments:

Units shown for $Q C$ (SPK \& STD) may not reflect the actual units. $D L=$ Detection Limit, $S=$ Worklist Slot Number, $R=$ Replicate Number, $A=$ Aliquot Code. 
Gurve 1: DSC

F11e info: IND111901 Tue Nov 19 14:49: 121996

sample Weight: B.580 ang

1EN14-B INDIUM AT 10C/MIN

SIGNATURE BELOW REPRESENTS CHEMICAL TECHNOLOGIST/CHEMIST THAT

CCMPLETED/VERIFIED THE CALIBRATION/ANALYSIS ON PAGES 210 TO2 $2 \%$

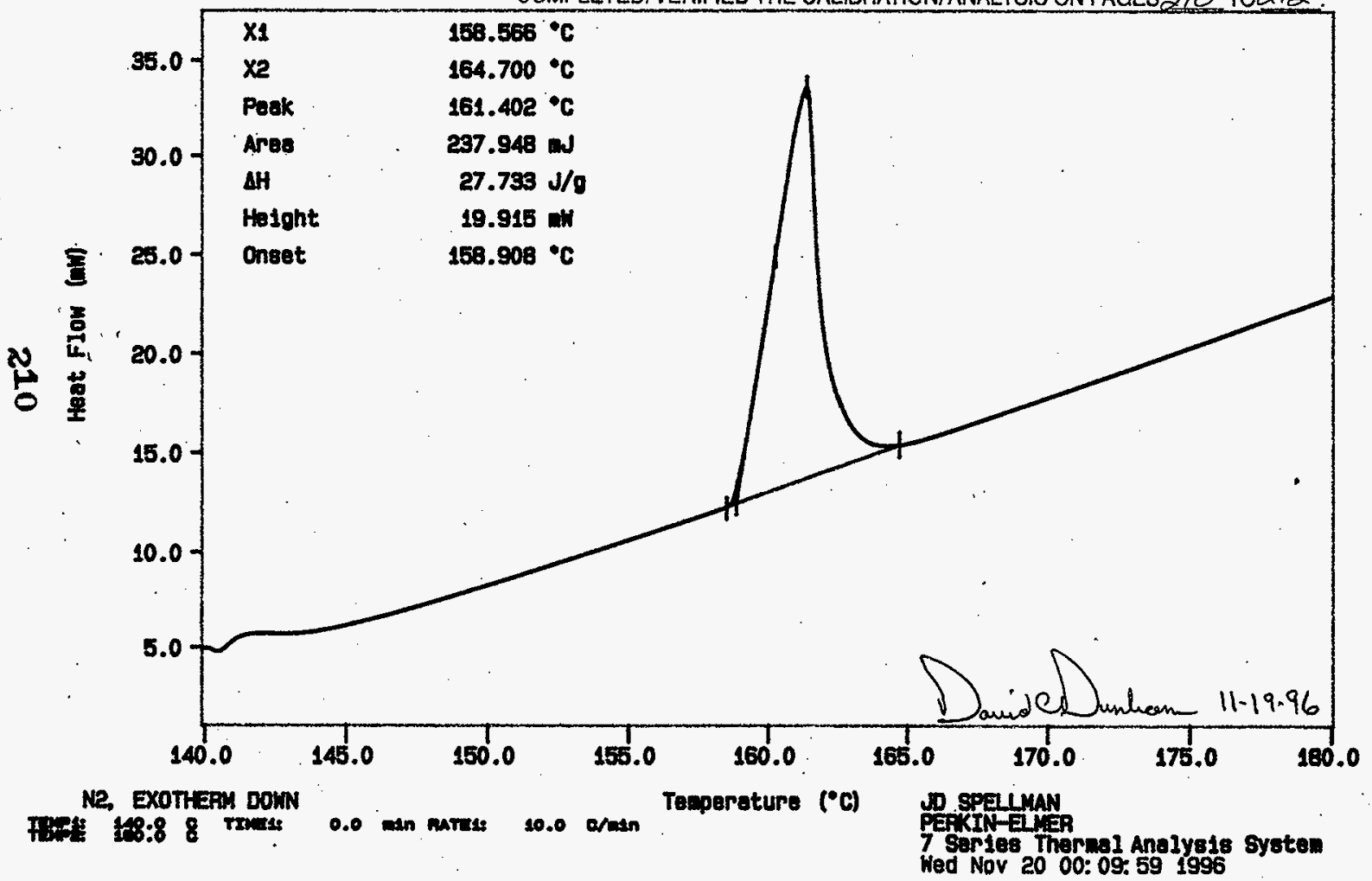


HNF-SD-WM-DP-219, REV. 0

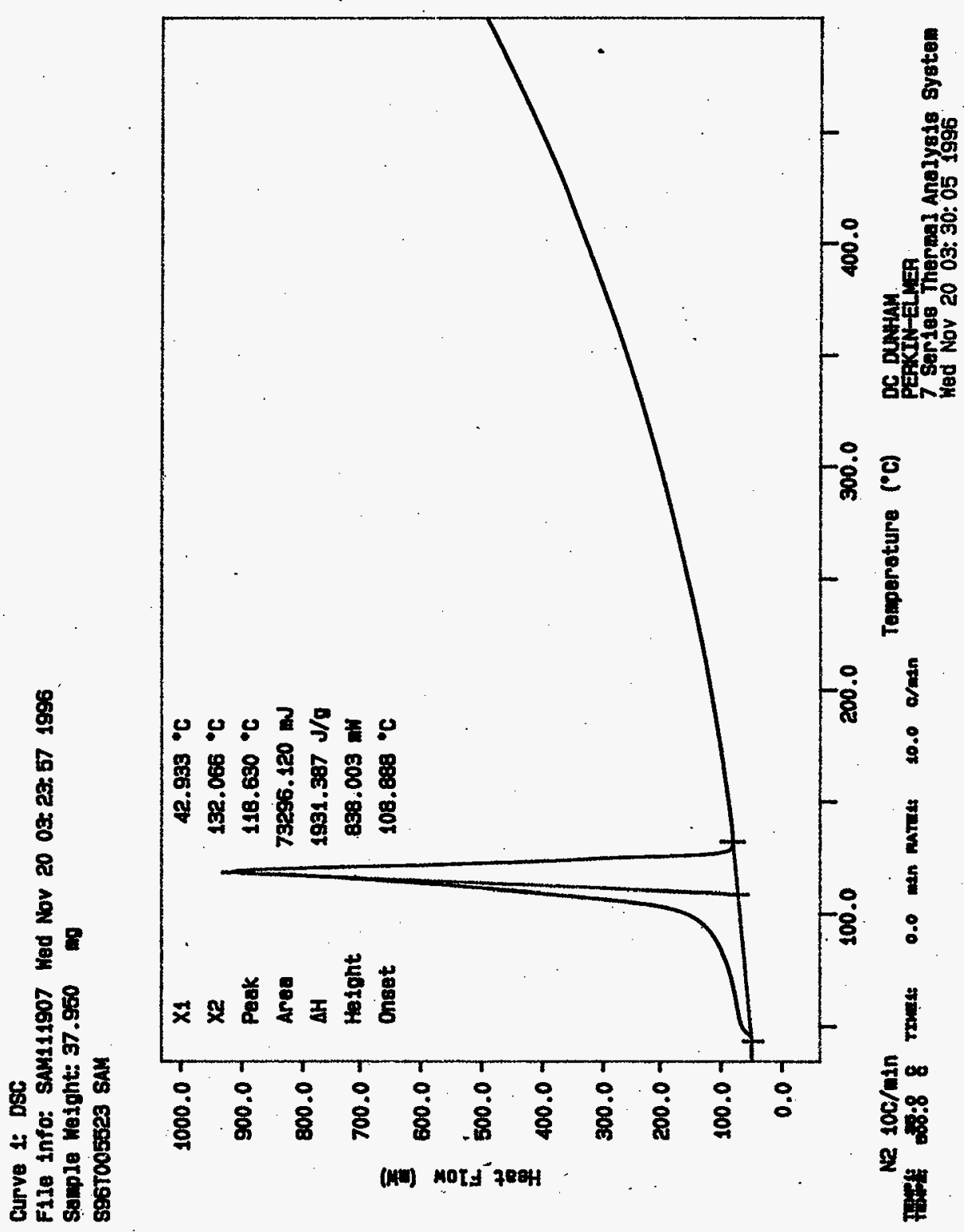

211 
Curve 1: DSC

File info: SAM111908 Med NoV 20 04: 25: 551996

Sauple Neight: 23.630

S96T005623 DUP

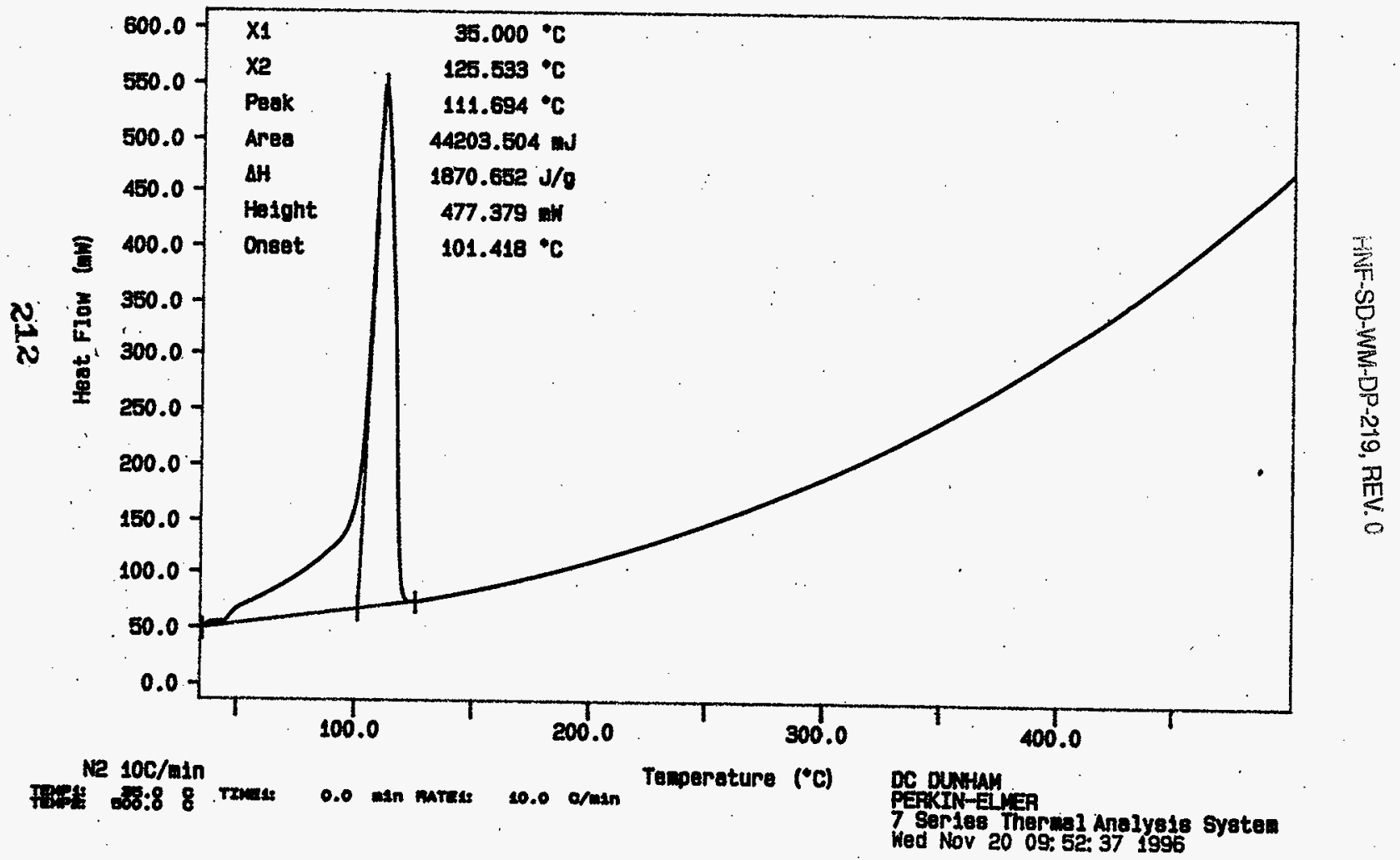




\section{LABCORE Data Entry Template for Worklist\# 14718}

Analyst: $D P B \quad$ Instrument: ${ }^{D S C} \quad 1 \quad$ Book $\# / 21 / 14-B$

Method: LA-514-113 Rev/Mod C -1

Worklist Comment: B-108 DSC, RUN UNDER N2. RCJ

\begin{tabular}{|c|c|c|c|c|c|c|c|c|c|c|}
\hline GROUP & PROJECT & $\begin{array}{l}\text { S TYPE } \\
\text { i STD }\end{array}$ & SAMPLE\# & & $\begin{array}{l}\text {-TEST }-. . . . . \\
\text { DSL-01 }\end{array}$ & $\begin{array}{l}\text { MATRIX } \\
\text { SOLID }\end{array}$ & $\begin{array}{l}\text { ACTUAL } \\
28.4^{5} \\
\end{array}$ & $\begin{array}{l}\text { Found } \\
28.7 \\
\end{array}$ & $\begin{array}{l}\text { DL } \\
\text { N/A } \\
\end{array}$ & $\begin{array}{l}\text { UNIT } \\
\text { Joules/g }\end{array}$ \\
\hline 96001379 & B- 108 & 2 SAMPLE & S96T005505 & 0 & DSC -01 & SOLID & H/A & $\not x$ & & Joules $/ g$ \\
\hline 96001379 & B-108 & 3 DUP & \$96T005505 & 0 & DSC-01 & SOLID & $\phi$ & $\varnothing$ & N/A & Joules/g \\
\hline 96001379 & B- 108 & 4 SNPLE & $\$ 967005506$ & 0 & DSC-01 & SOLID & $\mathrm{N} /$ & $\not 2$ & & _ Joules/g \\
\hline 96001379 & B- 108 & 5 DUP. & S96T005506 & 0 & DSC-01 & SOLID & $\varnothing$ & $\varnothing$ & N/A & Joules/g \\
\hline 96001379 & B- 108 & 6 SAMPLE & S967005507 & 0 & DSC-01 & SOLID & N/t & $\phi$ & & Joules/g \\
\hline 96001379 & $B-108$ & 7 DuP & \$96T005507 & 0 & DSC-01 & SOLID & $\theta$ & $\phi$ & $\mathrm{N} / \mathrm{A}$ & Joutes/g \\
\hline
\end{tabular}

\section{Final page for worklist \# 14718}

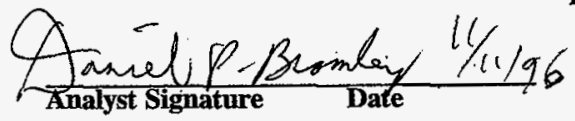

Verified/Validated by

Blandina Valentracla $11-12 \cdot 96$

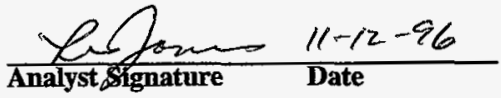

S967005505 thermograms display an endotherm consistant with Qluminum Hydroxide.

Data Entry Commens: S96 T005506 thermogians displey an endotherm consistant with Aluminum Hydroxide

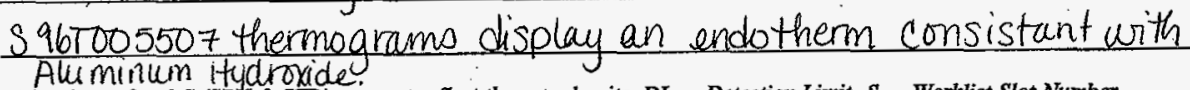

Units shown for $Q C$ (SPK \& STD) may not reflect the actual units. $D L=$ Detection Limit, $S=$ Worklist Stot Number,

$R=$ Replicate Number, $A \mp$ Aliquot Code. 
SIGNATURE BELOW REPRESENTS CHEMICAL TECHNOLOGIST/CHEMIST THAT

COUPLETEDIVERIFIED THE CALIBRATIONIANALYSIS ON PAGES 214 TO 230.

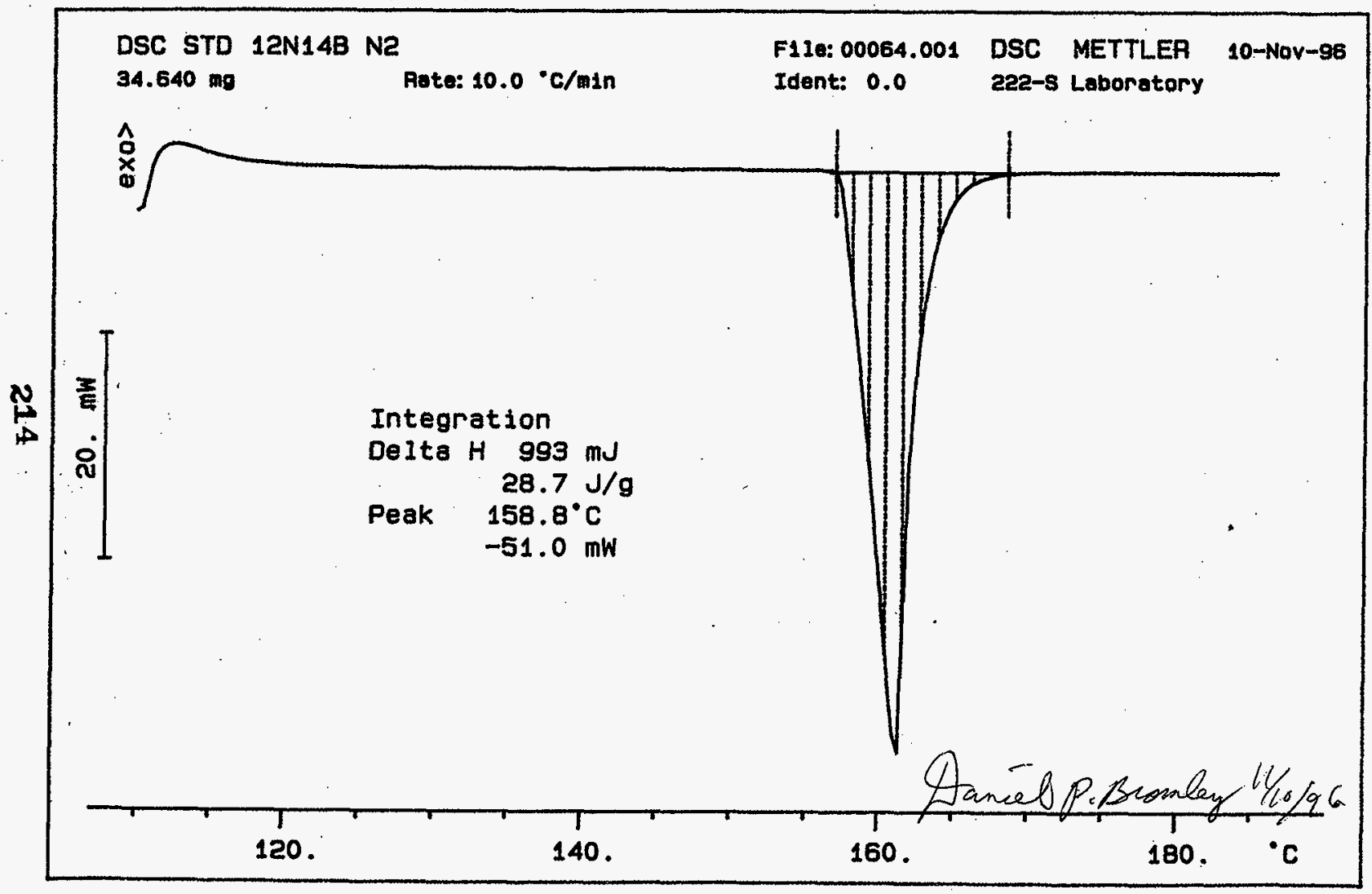


ANE-SD-WM-DP-219, REV. O

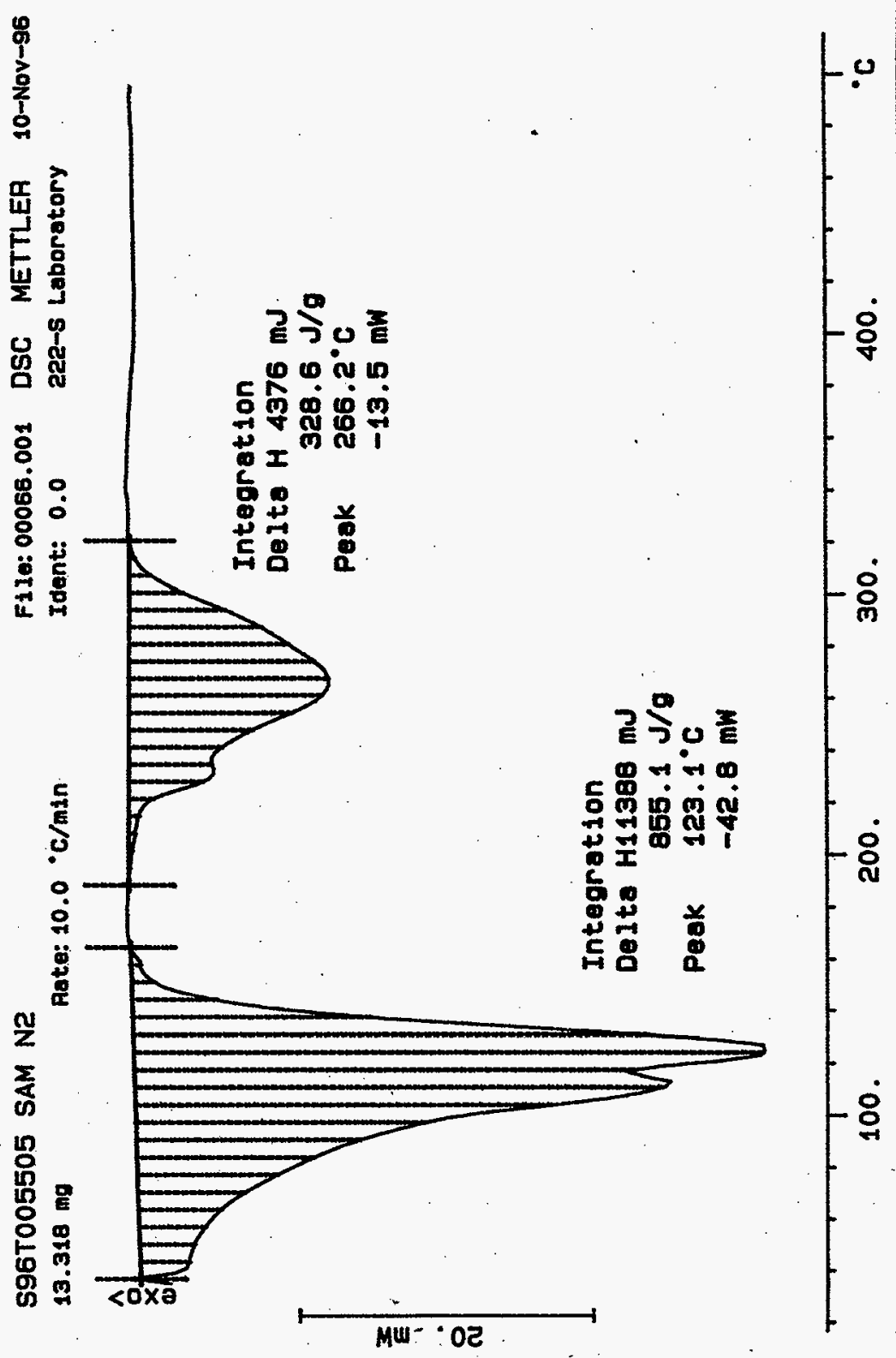




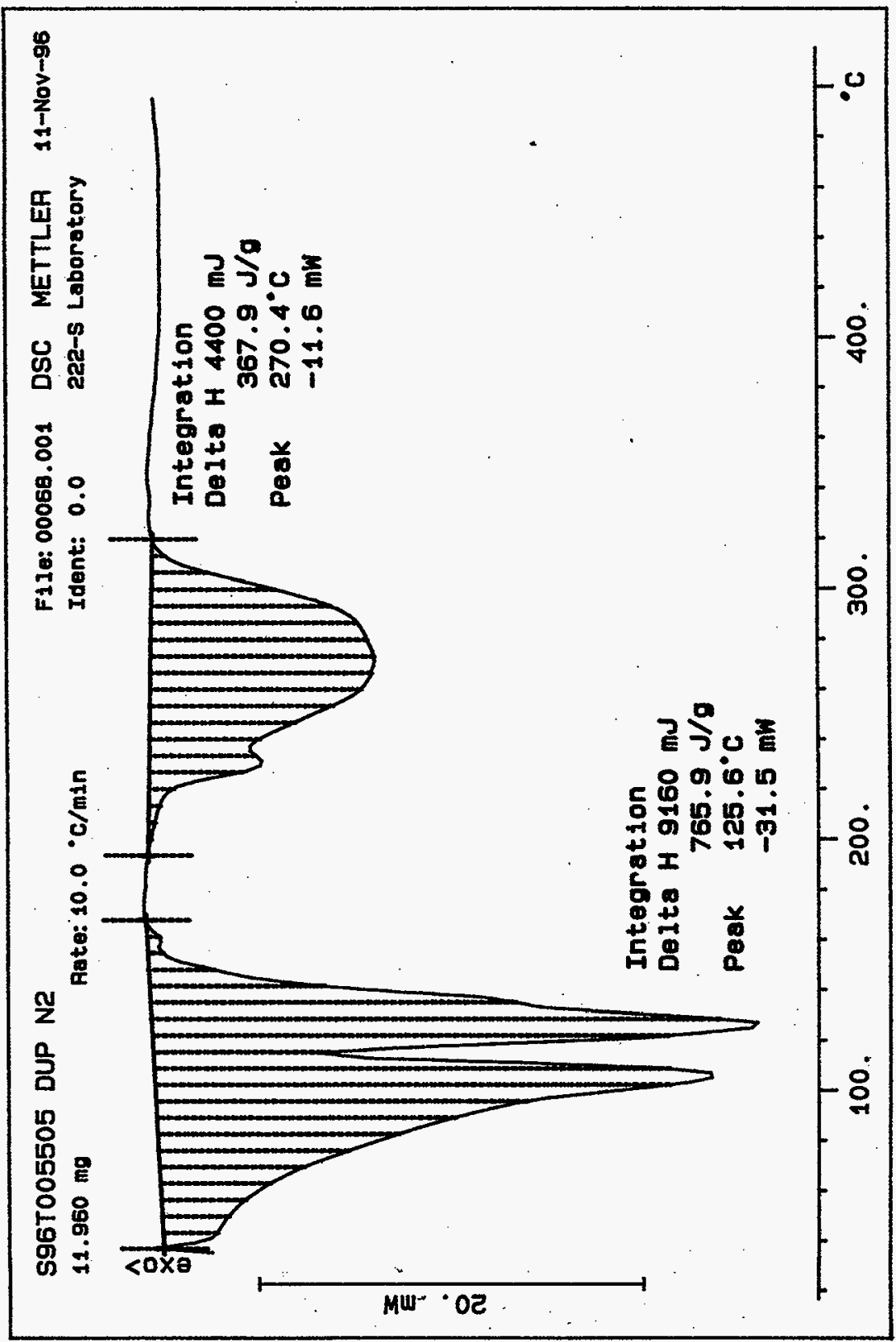


HNF-SD-WM-DP-219, REV.O

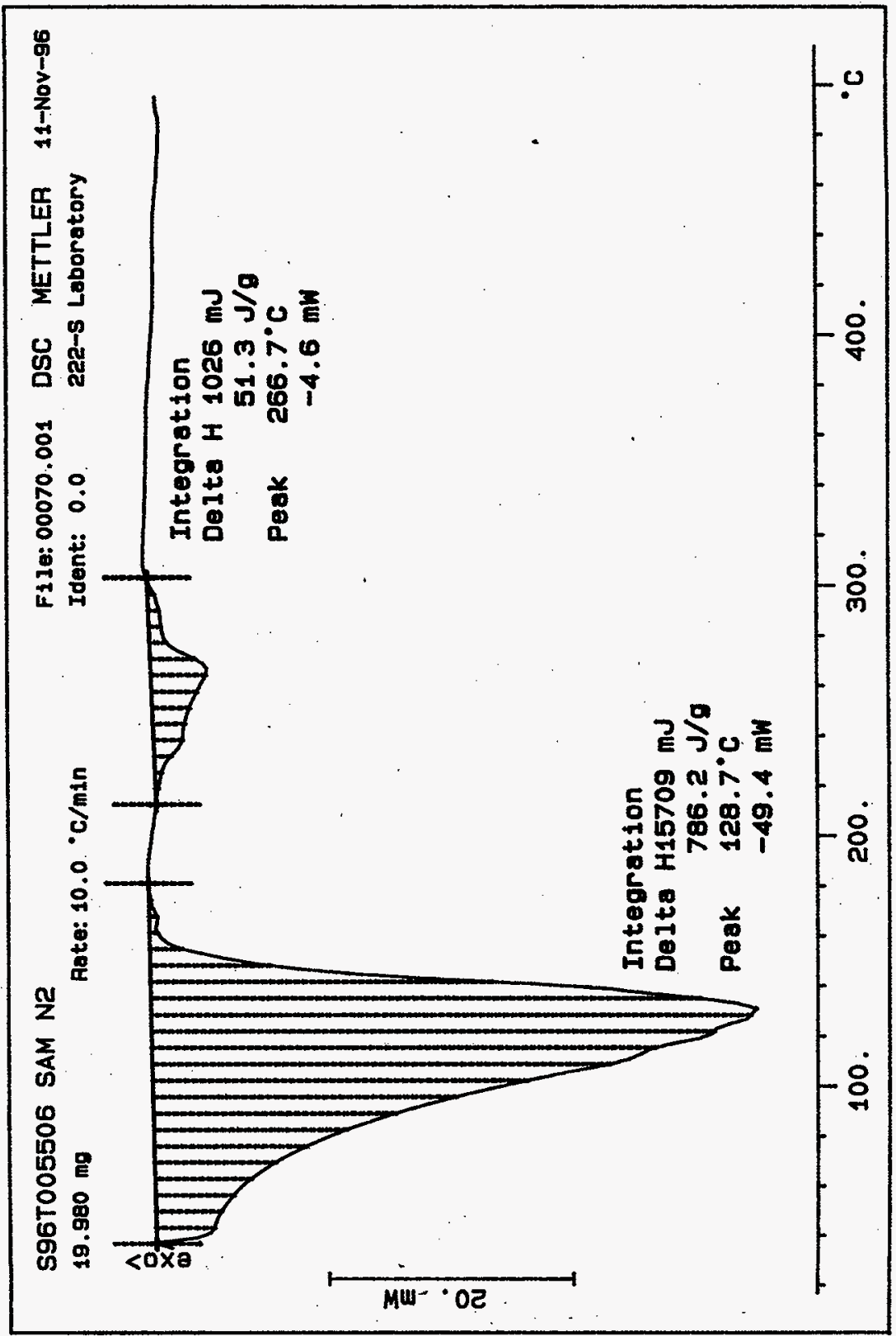


HNF-SD-WM-DP-219, REV. 0

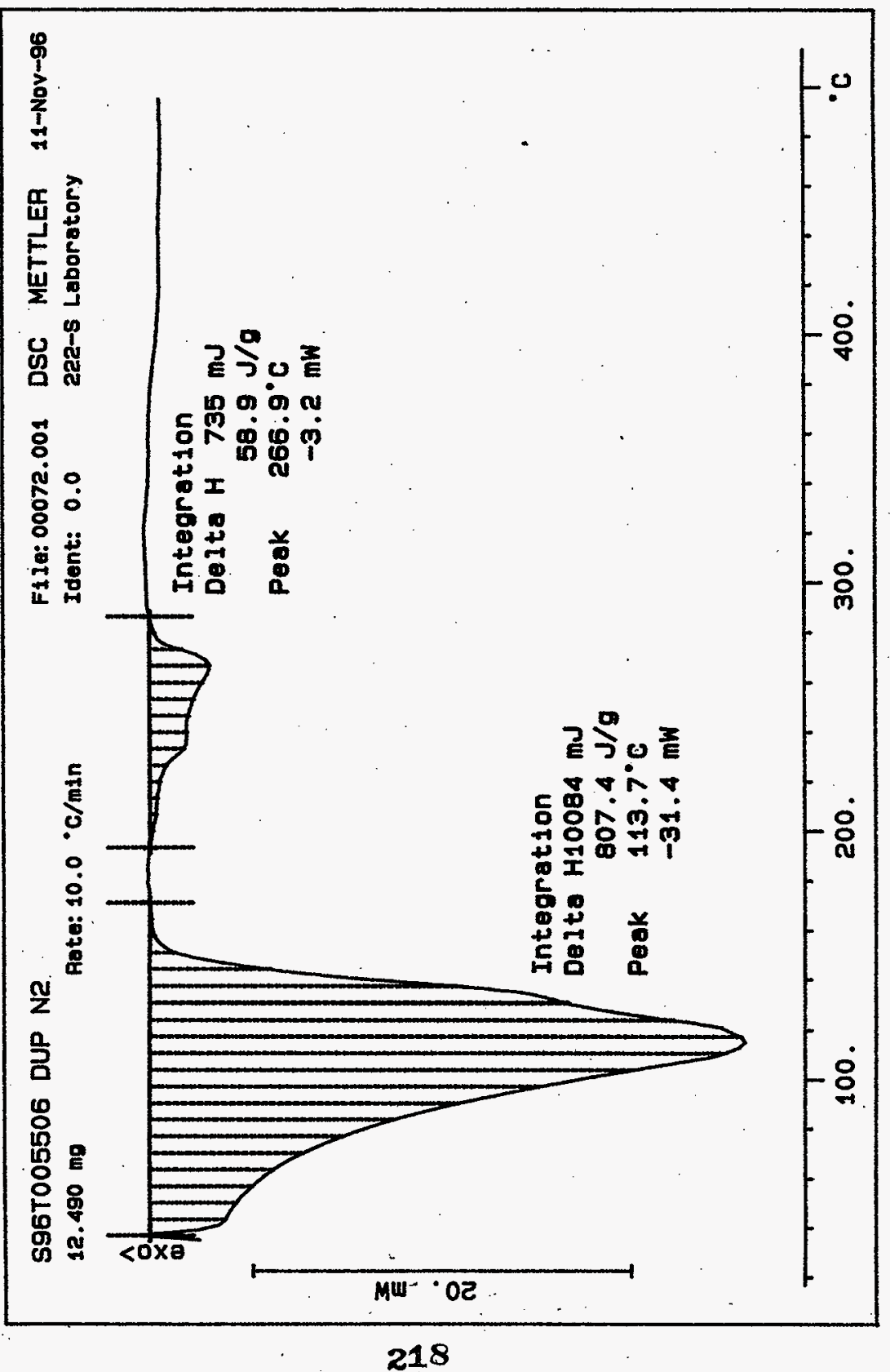




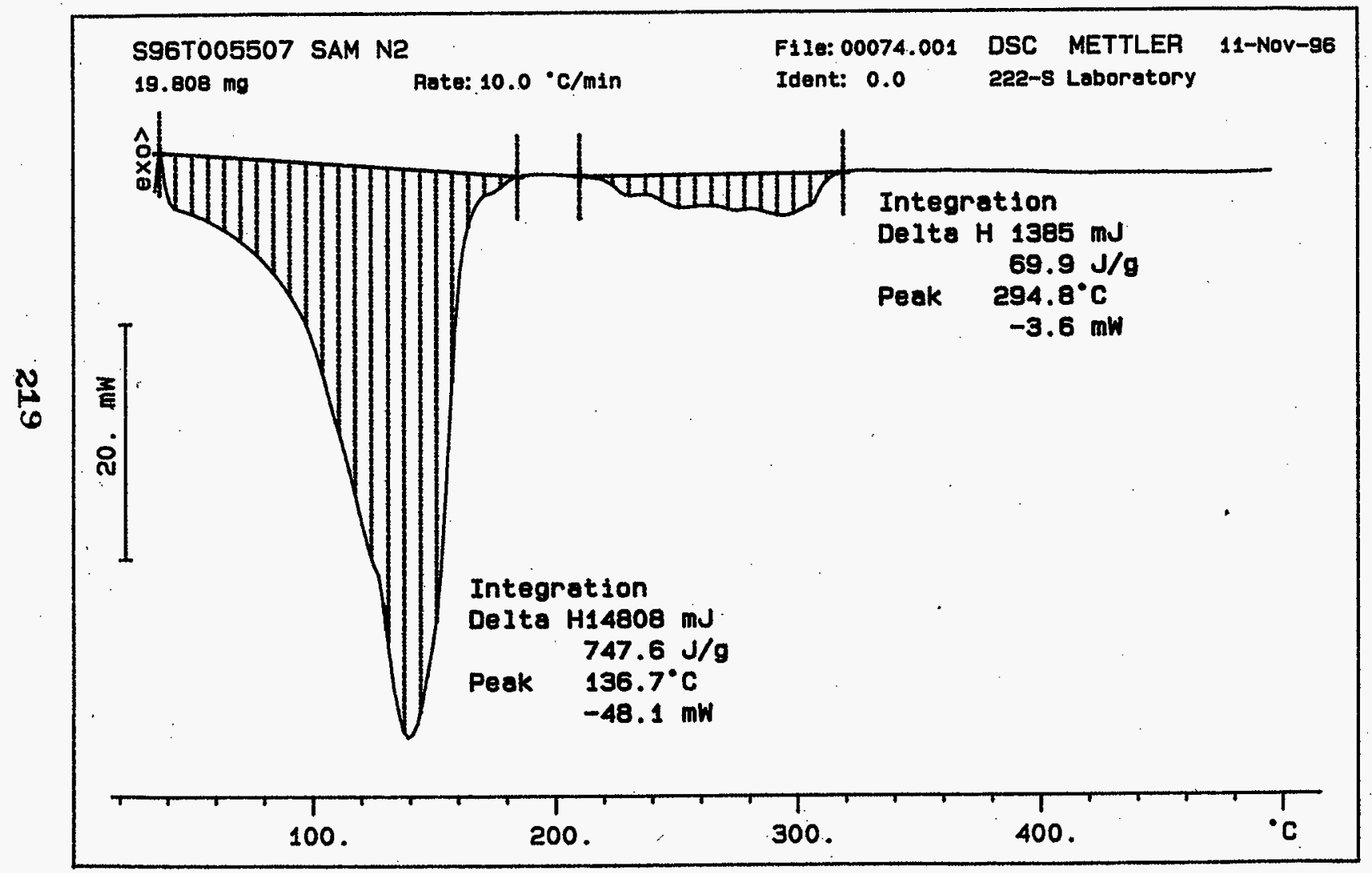




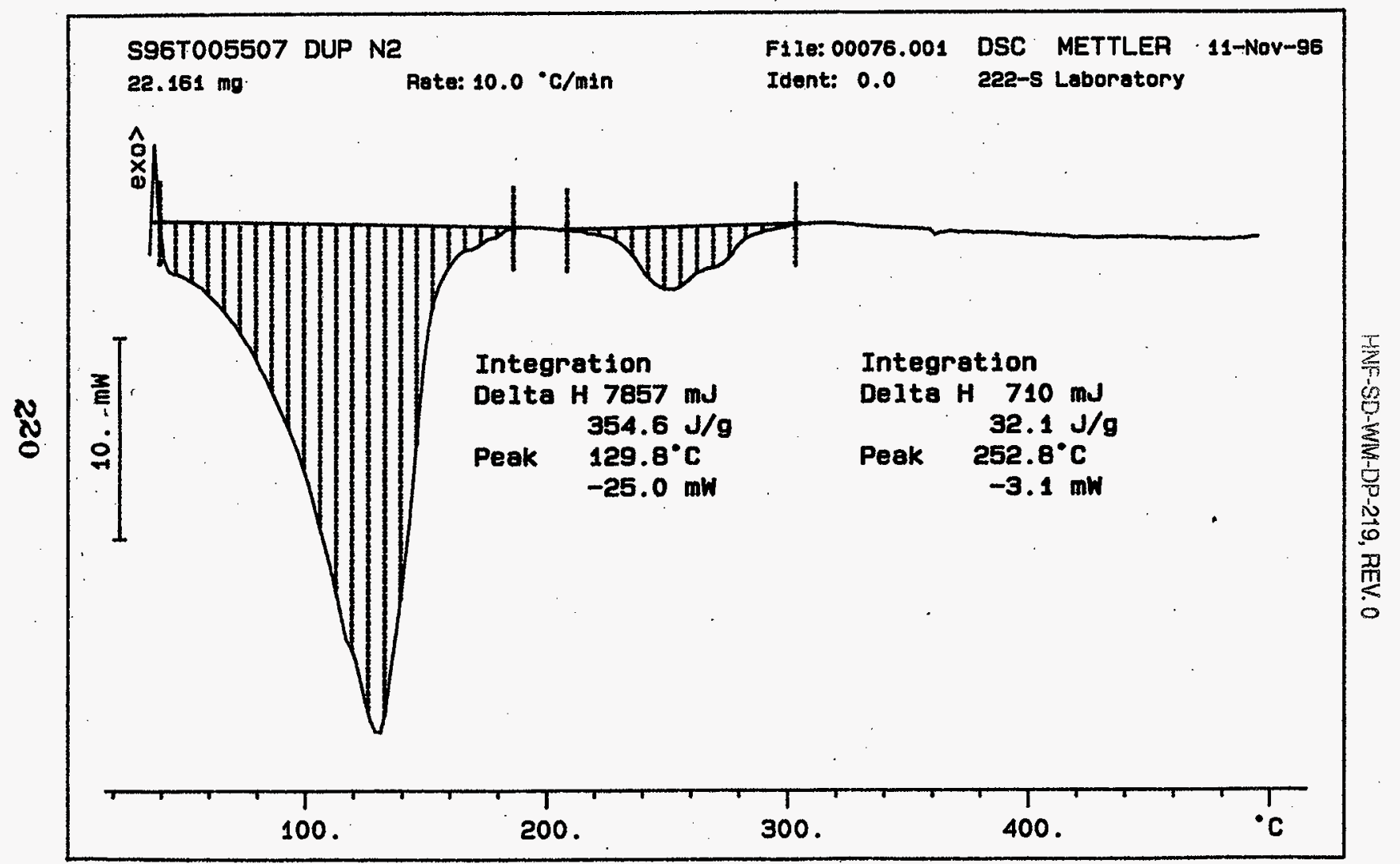




\section{LABCORE Data Entry Template for Worklist\# 14742}

Analyst: BOV Instrument: DSC01

Book \#

Method: LA-514-113 Rev/Mod

Worklist Comment: Dry DSC for B-108. bdv

\begin{tabular}{|c|c|c|c|c|c|c|c|c|c|c|}
\hline GROUP & PROJECT & S TYPE & SAMPLE\# & R A & --TEST $\cdots$ & MATRIX & ACTUAL & FOUND & $\mathrm{DL}$ & UNIT \\
\hline 96001379 & $B-108$ & $\uparrow$ SAMPLE & S96T005505 & 0 & DSC-02 & SOLID & N/A & $\varnothing$ & & Joules/g Dry \\
\hline 96001379 & $B-108$ & 2 DUP & S96T005505 & 0 & DSC-02 & SOLID & $\varnothing$ & $\varnothing$ & N/A & Joules/g Dry \\
\hline 96001379 & B-108 & 3 SAMPLE & S96T005506 & 0 & DSC-02 & SOLID & N/A. & $\notin$ & & Joules/g Dry \\
\hline 96001379 & $B-108$ & 4 DUP & \$96T005506 & 0 & DSC-02 & SOLID & $\varnothing$ & $\varnothing$ & N/A & Joules/g Dry \\
\hline 96001379 & $B-108$ & 5 SAMPLE & S96r005507 & 0 & DSC-02 & SOLID & N/A & $\phi$ & & Joules/g Dry \\
\hline 96001379 & B-108 & 6 DUP & $\$ 967005507$ & 0 & DSC-02 & SOLID & $\phi$ & $\phi$ & N/A & Joules/g Dry \\
\hline 96001380 & B-108 & 7 SAMPLE & $\$ 96 T 005473$ & 0 & DSC -02 & SOLID & $N / A$ & $\phi$ & & Joules/g Dry \\
\hline 96001380 & B- 108 & 8 DUP & 5967005473 & 0 & DSC-02 & SOLID & $\varnothing$ & $\varnothing$ & N/A. & - Joules/g Dry \\
\hline 96001380 & B-108 & 9 SAMPLE & S96T005474 & 0 & DSC-02 & SOLID & N/A & $\phi$ & & - Joules/g Dry \\
\hline 96001380 & B-108 & 10 DUP & S96T005474 & 0 & DSC-02 & SOLID & 0 & $\varnothing$ & N/A & Joules/g Dry \\
\hline 96001380 & $8-108$ & 11 SAMPLE & \$96T005485 & 0 & DSC-02 & SOLID & N/A & & & - Joules/g Dry \\
\hline 96001380 & $B-108$ & 12 DUP & S96T005485 & 0 & DSC-02 & SOLID & $\pi$ & $\psi$ & N/A & Joules/g Dry \\
\hline 96001379 & B- 108 & 13 SAMPLE & S96T005463 & 0 & DSC-02 & LIQUID & $\mathrm{N} / \mathrm{A}$ & $\phi$ & & - Joules/g Dry \\
\hline 96001379 & $B-108$ & 14 DUP & s967005463 & 0 & DSC-02 & LIQUID & $\phi$ & & N/A & _ Joules/g Dry \\
\hline 96001379 & B-108 & 15 SAMPLE & \$96T005523 & 0 & DSC-02 & LIQUID & N/A & $\emptyset$ & & - Joules/g Dry \\
\hline 96001379 & B- 108 & 16 DUP & \$96T005523 & 0 & DSC- 02 & LIQUID & $\phi$ & & $\mathrm{N} / \mathrm{A}$ & _ Joules/g Dry \\
\hline 96001380 & B-108 & 17 SAMPLE & S96T005466 & 0 & DSC-02 & SOLID & $N / A$ & & & _ Joules/g Dry \\
\hline 96001380 & $B-108$ & 18 DUP & S96T005466 & 0 & DSC-02 & SOLID & 6 & & $N / A$ & Joules/g Dry \\
\hline
\end{tabular}

Data Entry Comments:

Units shown for $Q C$ (SPK \& STD) may not reflect the actual units. $D L=$ Detection Limit, $S=$ Worklist Slot Number,

$R=$ Replicate Number, $A=$ Aliquot Code. 
R A $\cdots-$ TEST $\cdots+-$

MATREX ACTUAL

FOUND

UNIT

\section{Final page for worklist \# 14742}

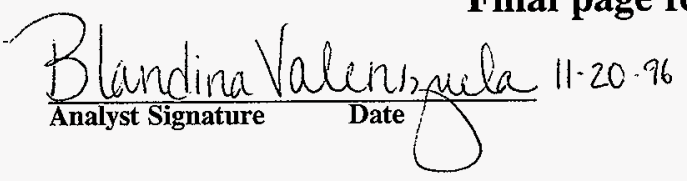

Analyst Signature Date

Units shown for $Q C$ (SPK \& STD) may not reflect the actual units. $D L=$ Detection Limit, $S=$ Worklist Slot Number, $R=$ Replicate Number, $A=$ Aliquot Code. 


\begin{tabular}{|c|c|c|c|}
\hline \multicolumn{4}{|c|}{ CALCULATED DRY DSC } \\
\hline SAMPLE NO. & DSC RESULT $(\mathrm{J} / \mathrm{g})$ & TGA RESULT (\% water) & DRY DSC RESULT \\
\hline S967005505 & $\varnothing$ & $\longrightarrow$ & $\varnothing$ \\
\hline 55050 & $\varnothing$ & $\longrightarrow$ & $\not$ \\
\hline 5506 & $\varnothing$ & - & $\varnothing$ \\
\hline $5506 \mathrm{D}$ & $\varnothing$ & $\ldots$ & $\varnothing$ \\
\hline 5507 & $\varnothing$ & $=$ & $\not$ \\
\hline $5507 D$ & $\varnothing$ & $=$ & $x$ \\
\hline 5473 & (D) $\quad 11 / 2 / 96$ & 14383 & 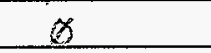 \\
\hline 54730 & $\phi \quad 11 / 2 / 96$ & 16383 & 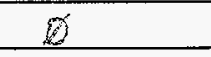 \\
\hline 5474 & $\phi$ & 43.78 & 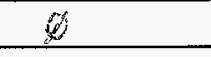 \\
\hline $5474 D$ & $\varnothing$ & 4.7 .78 & 0 \\
\hline 5485 & $\varnothing$ & 35.30 & $D$ \\
\hline 54850 & $\varnothing$ & 35.30 & $\varnothing$ \\
\hline 5463 & $\varnothing$ & 99.56 & $\phi$ \\
\hline $5463 D$ & $\varnothing$ & 99.56 & $\varnothing$ \\
\hline 5523 & $14715 \quad \varnothing$ & 14707 & $\varnothing$ \\
\hline 55230 & $14715 \quad \varnothing$ & 4707 & $\not \varnothing$ \\
\hline 5466 & $\varnothing$ & 14383. & $\emptyset$ \\
\hline $5466 D$ & $\varnothing$ & 14383 & $\varnothing$ \\
\hline & & & \\
\hline & & & \\
\hline & & & \\
\hline & & & \\
\hline
\end{tabular}




\section{LABCORE-Data Entry Template for Worklist\# 14377}

Analyst:

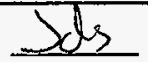

Instrument: TGA0

Book \# 82 N 8 A

Method: LA-560-112 Rev/Mod C.O

Worklist Comment: TGA-01 FOR B-108 PLEASE RUN UNDER N2

RTS!

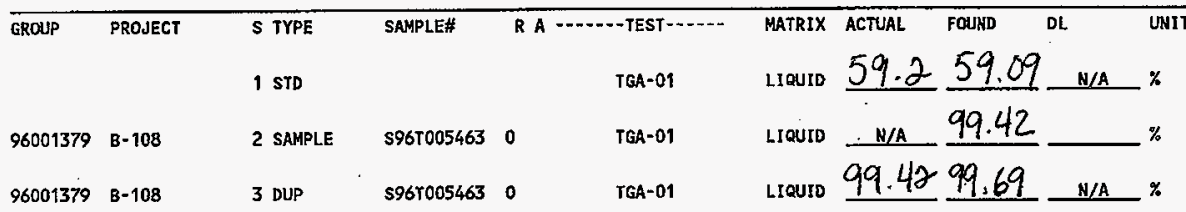

\section{Final page for worklist \# 14377}

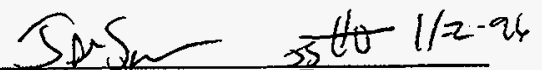

Analyst Signature

$\operatorname{lnm}($ ind $)$

Nalidatid by

Blandina

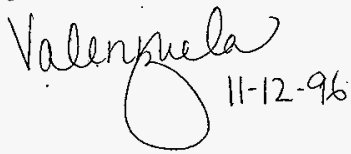

$\frac{26}{\text { Analyst Signature }} \frac{11-8-96}{\text { Date }}$

\section{Data Entry Comments:}

Units shown for $Q C$ (SPK \& STD) may not reflect the actual units. $D L=$ Detection Limit, $S=$ Worklist Slot Number, $R=$ Replicate Number, $A=$ Aliquot Code. 
SIGNATURE BELOW REPRESENTS CHEMICAL TECHNOLOGIST/CHEMIST THAT

COMPLEIEDIVERIEIED THE CAL BBRATIONLANALVSIS ONLAGES 225 TO 227

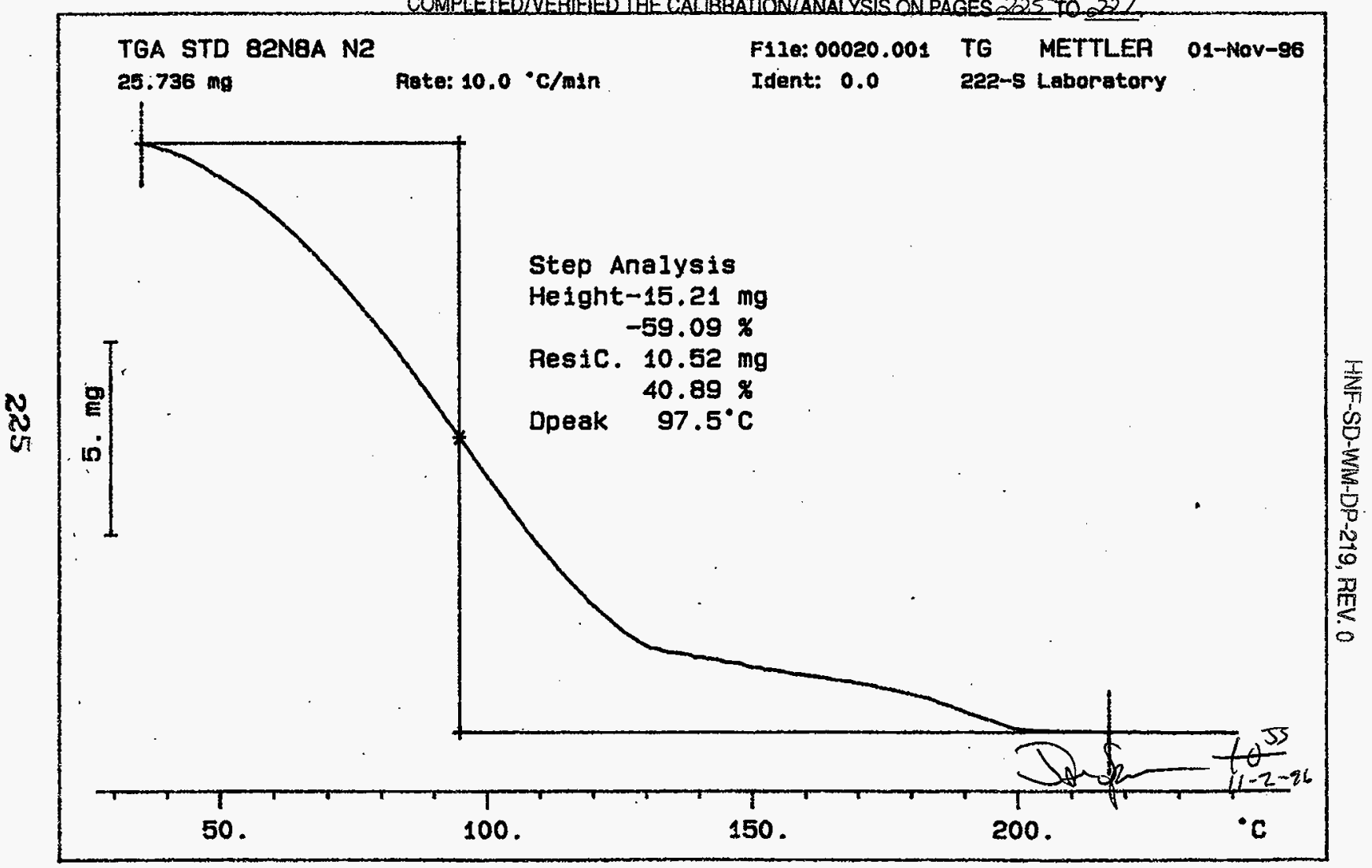


HNE-SO-WW-DP-219, REV.O

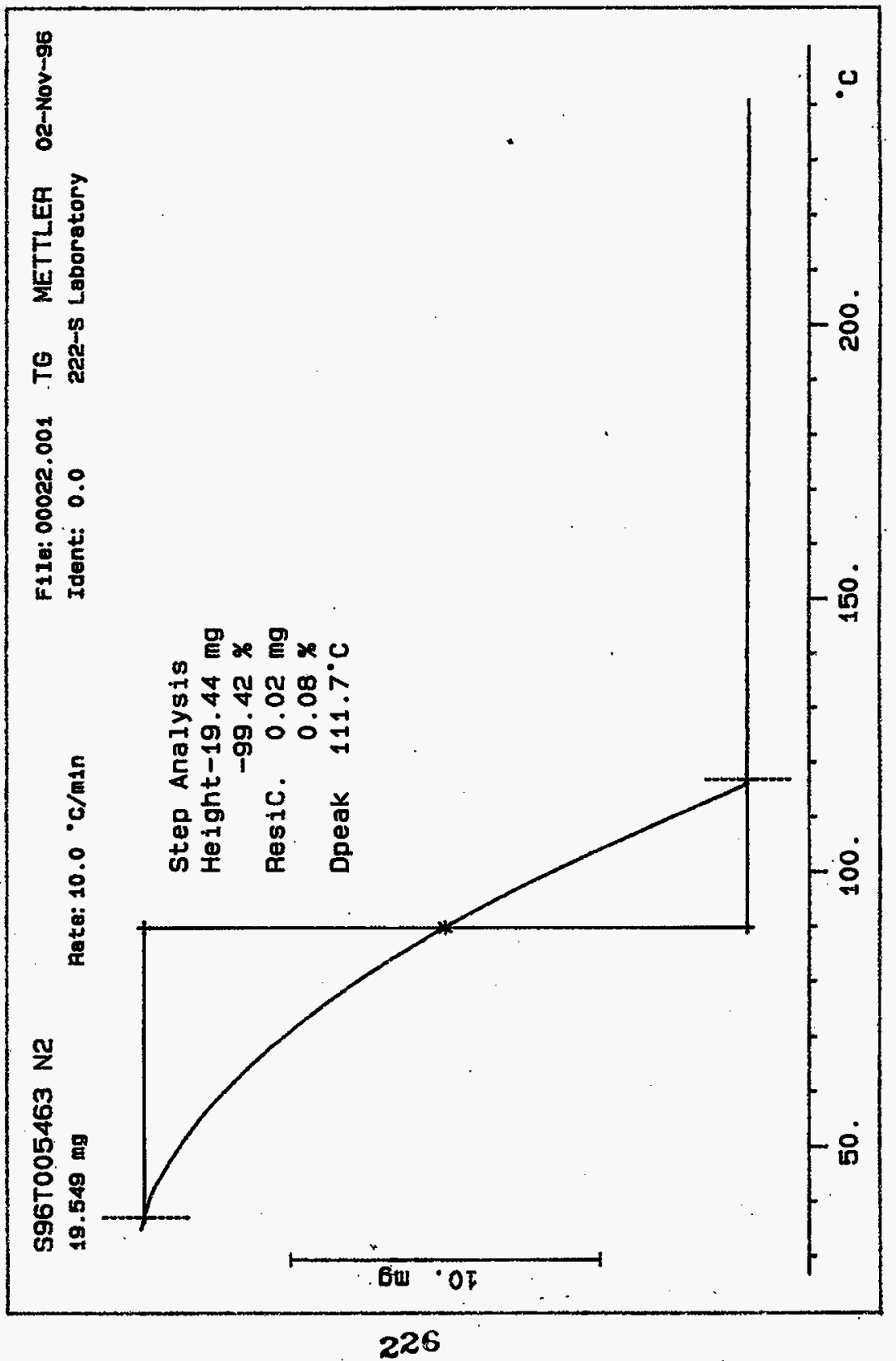




\section{LABCORE Data Entry 'Template for Worklist\# 14383}

Analyst:

Instrument: $\mathrm{TGAO}^{*}$

Book \#.82N8-17

Method: LA-560-112 Rev/Mod C-O

Worklist Comment: TGA-01 FOR B-108 PLEASE RUN UNDER N2

RTS

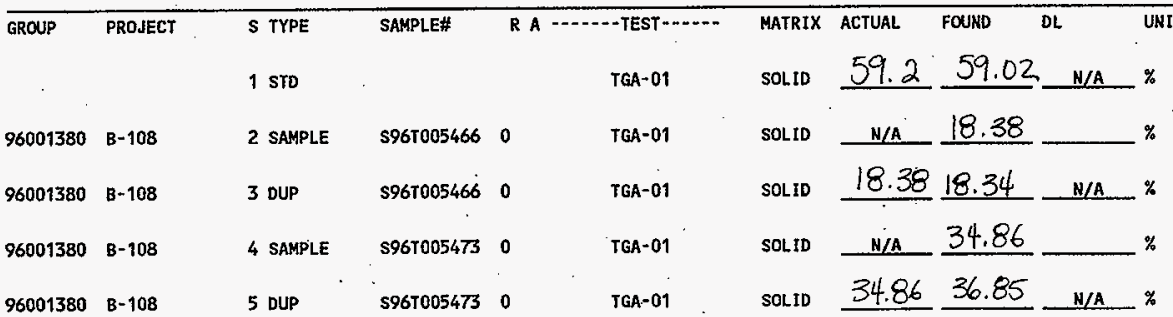

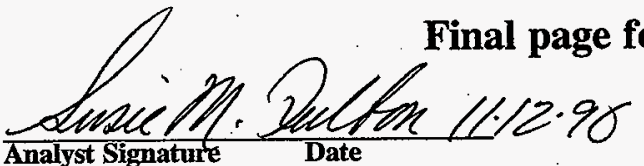

Analyst Signature Date

Analyst Signature Date

Data Entry Comments:

Units shown for QC (SPK \& STD) may not reflect the actual units. $D L=$ Detection Limit, $S=$ Worklist Slot Number,

$R=$ Replicate Number, $A=$ Aliquot Code. 


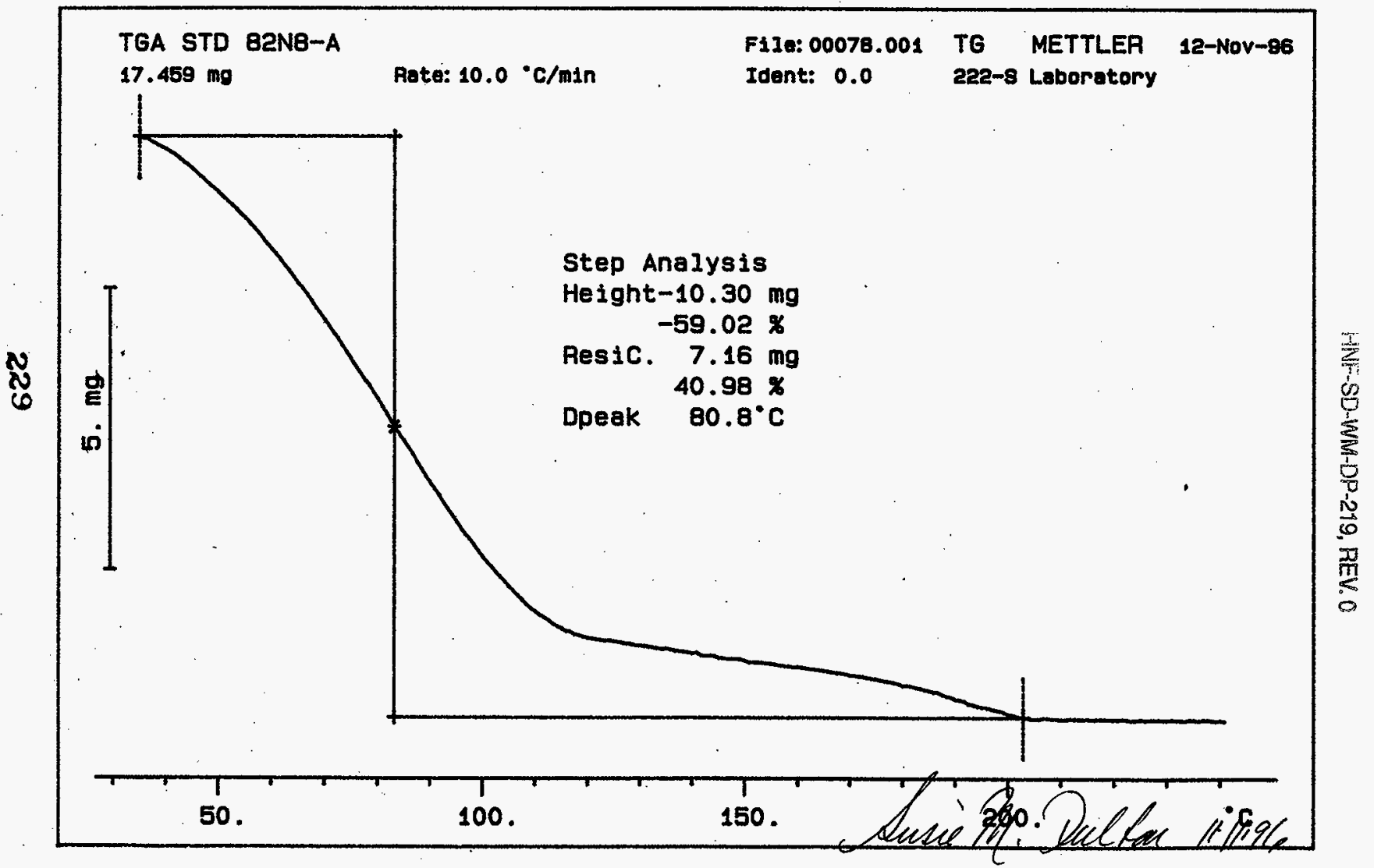




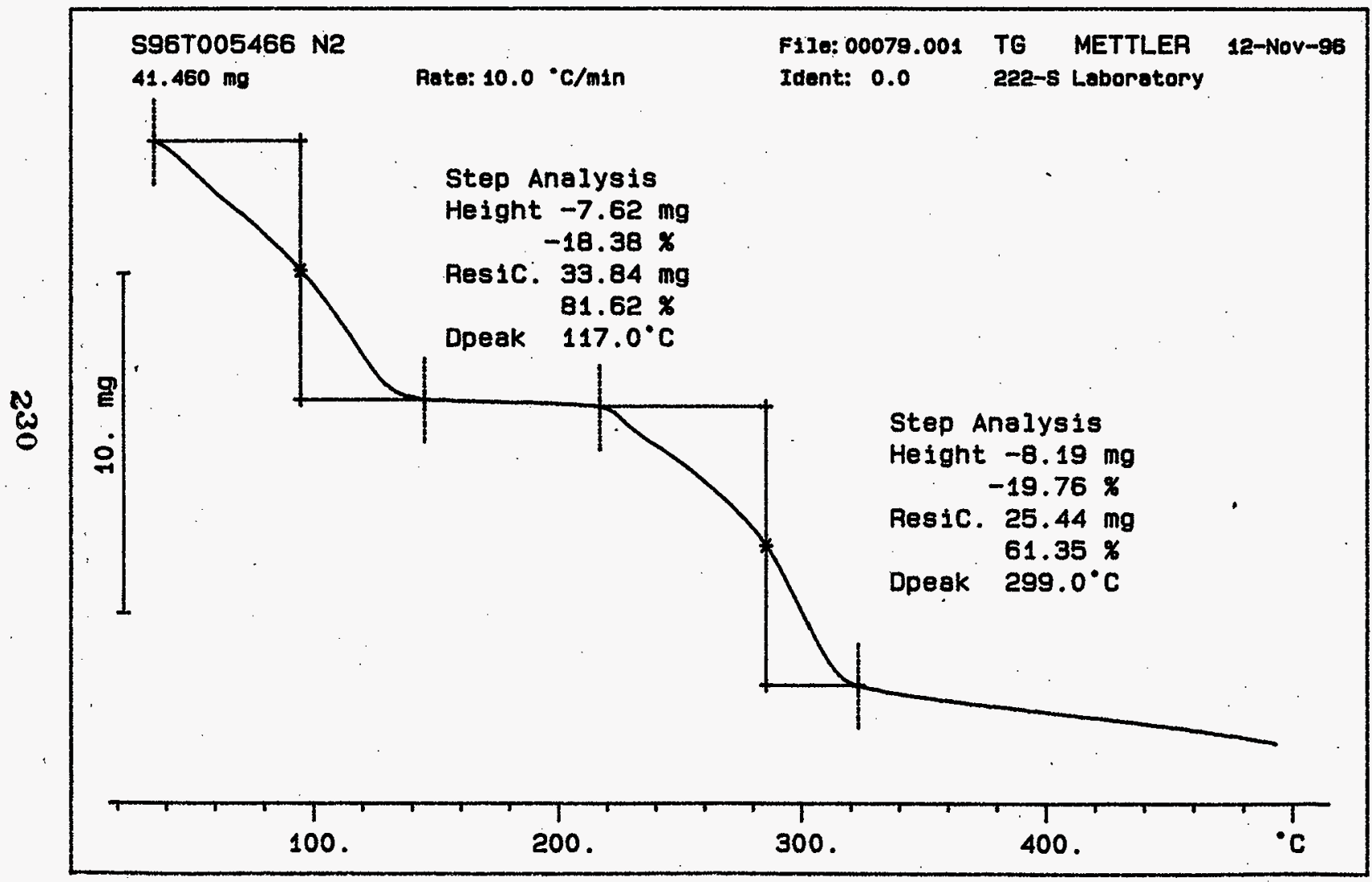




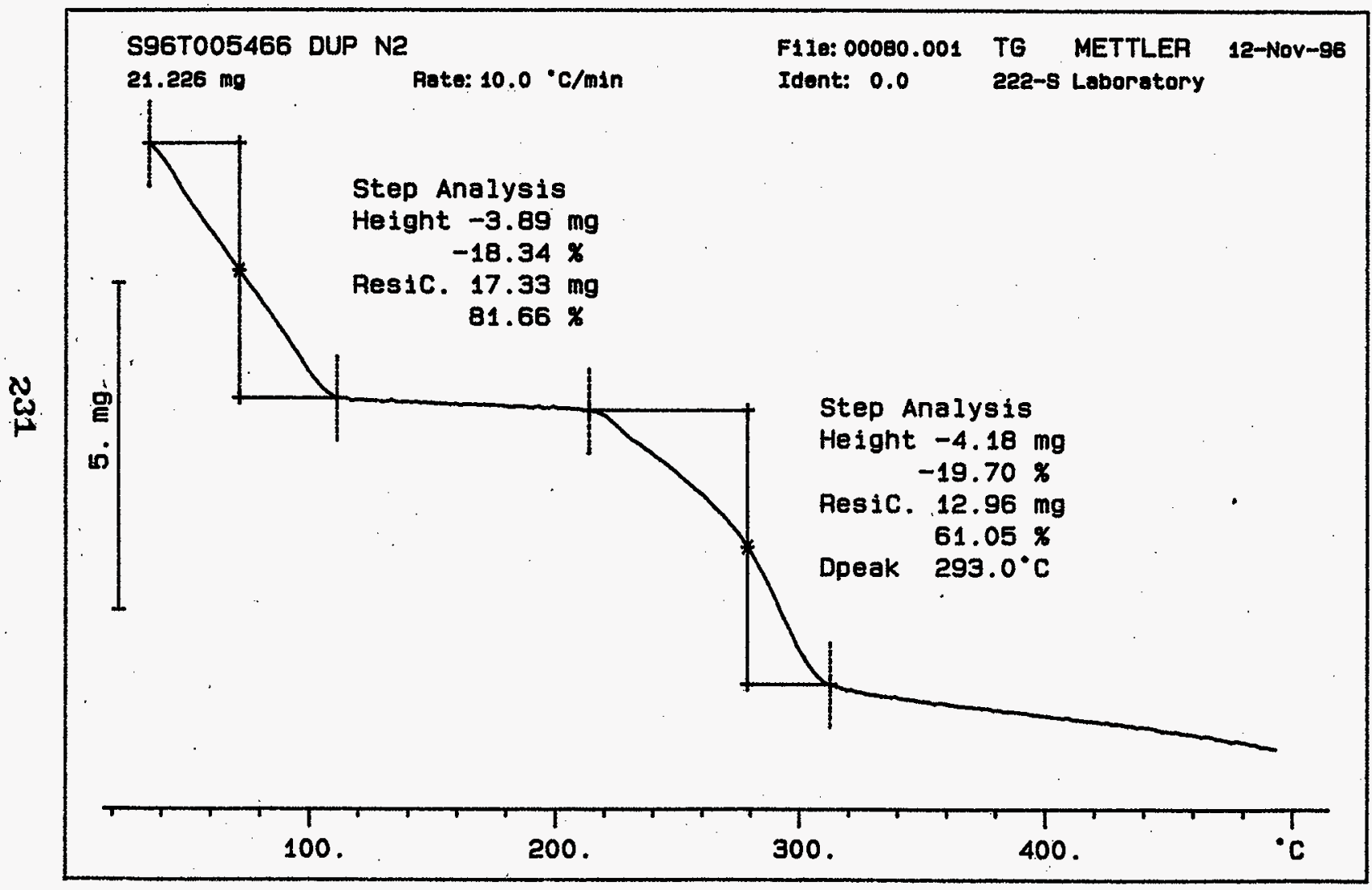




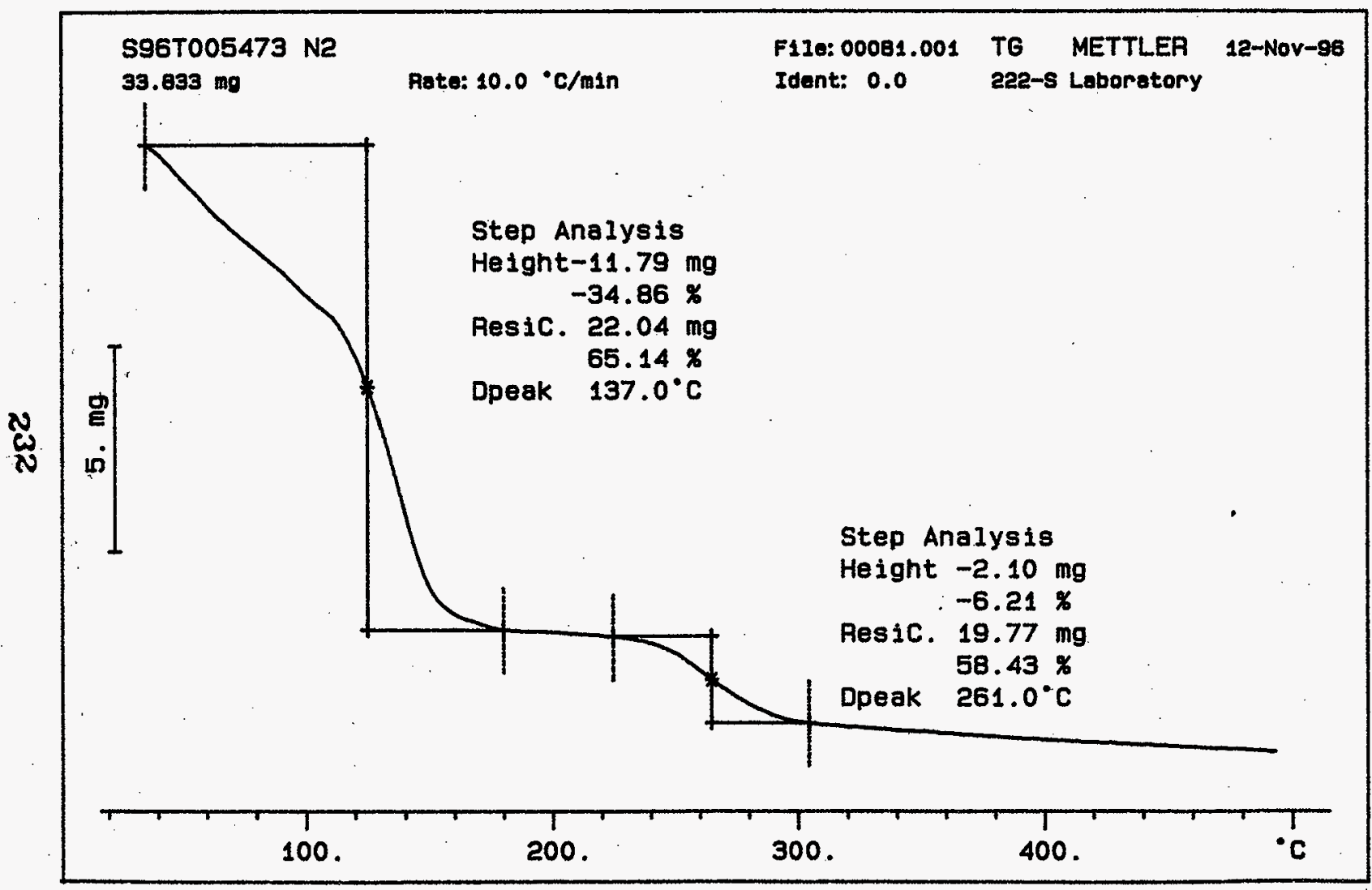




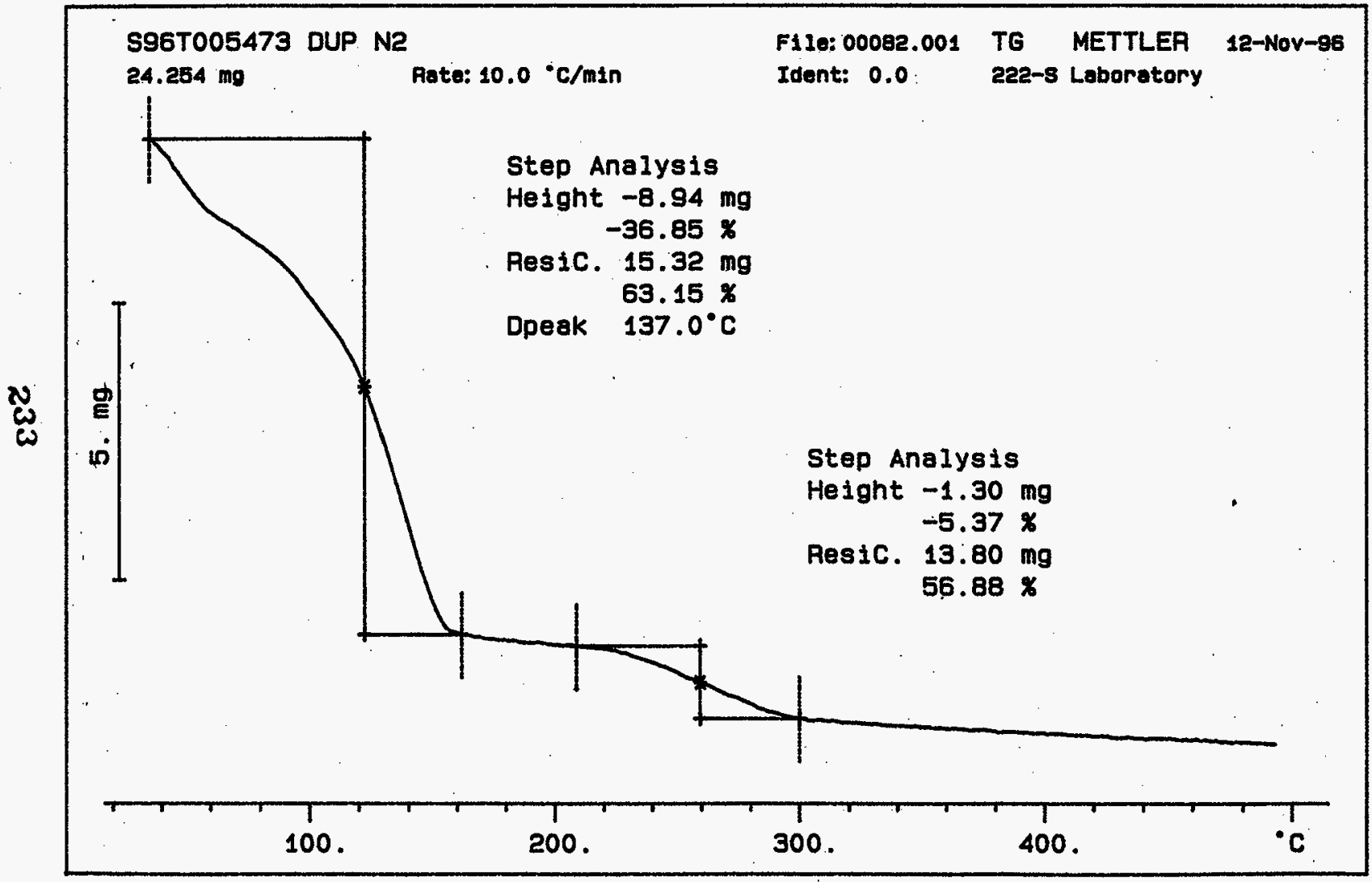




\section{LABCORE Data Entry Template for Worklist\# 14384}

Analyst: KRMI Instrument: TGA0 I

Method: LA-560-112 Rev/Mod C-O

Worklist Comment: TGA-01 FOR B-108 PLEASE RUN UNDER N2

RTS

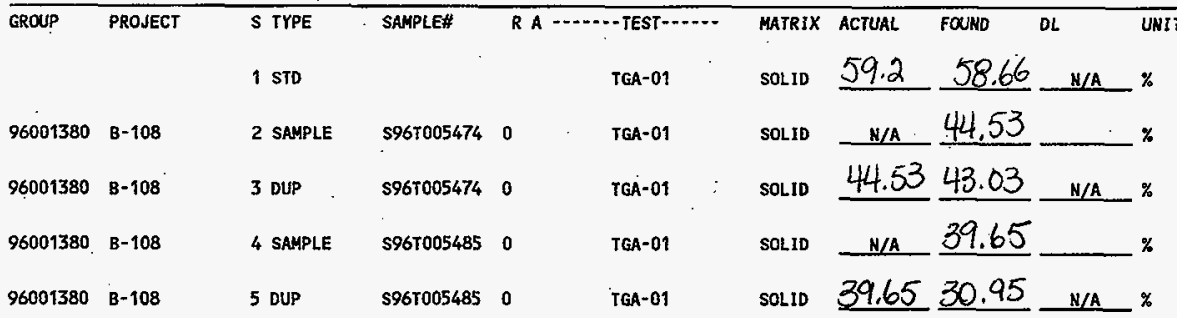
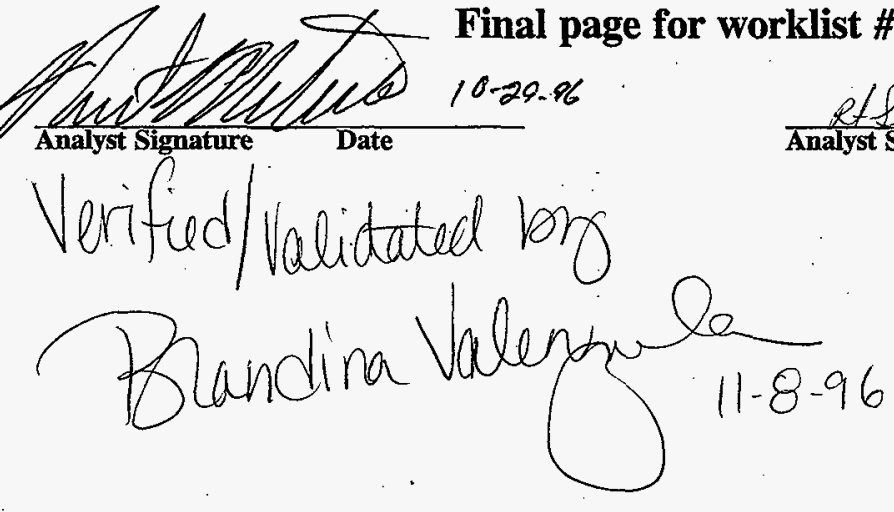

Data Entry Comments:

Units shown for $Q C$. (SPK \& STD) may not reflect the actual units. $D L=$ Detection Limit, $S=$ Worklist Slot Number,

$R=$ Replicate Number, $A=$ Aliquot Code. 
SIGNATURE BELOW AEPRESENTS CHEMICAL TECHNOLOGIST/CHEMIST THAT DATIONIANAIYSIS. ON PAGES 23510239

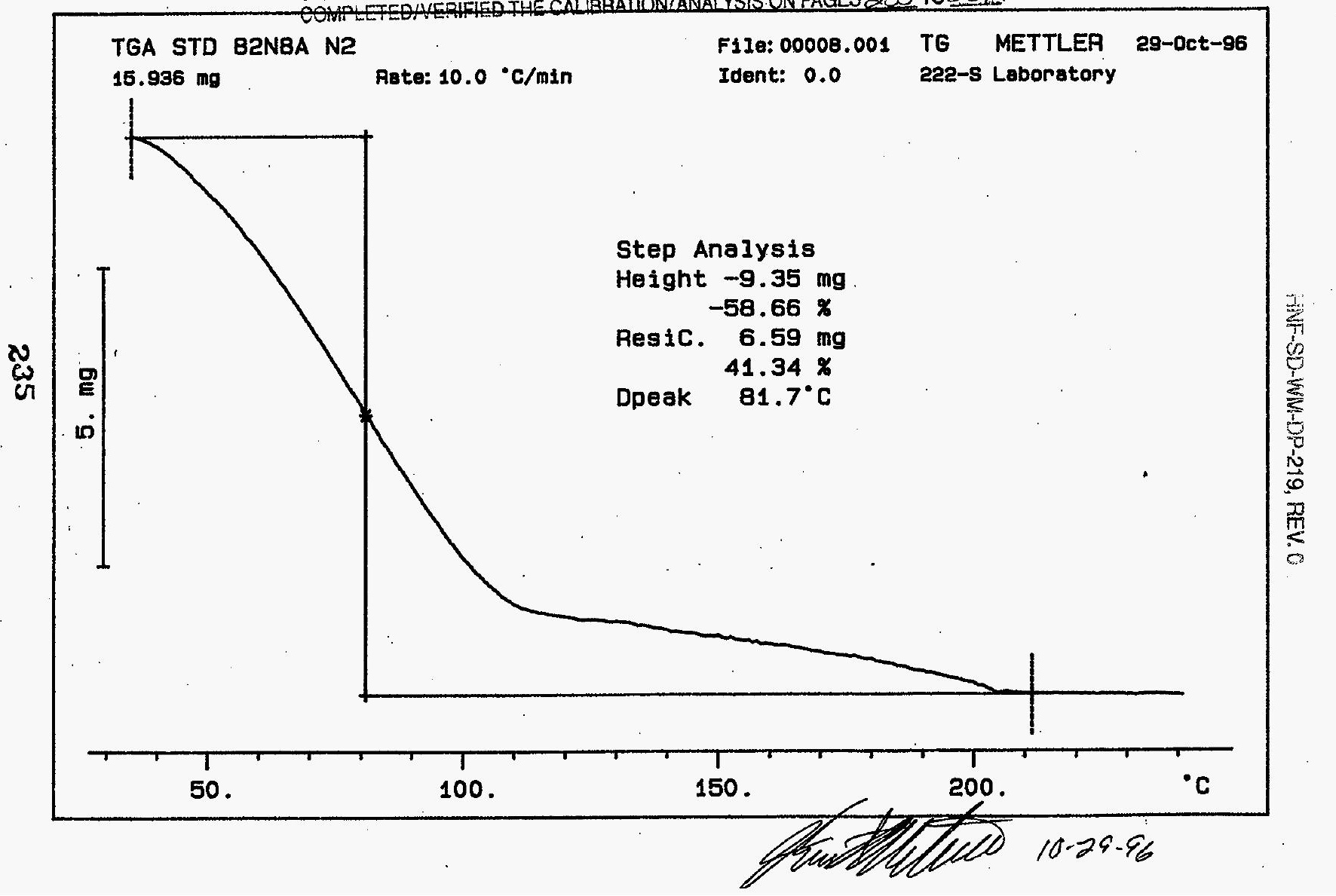




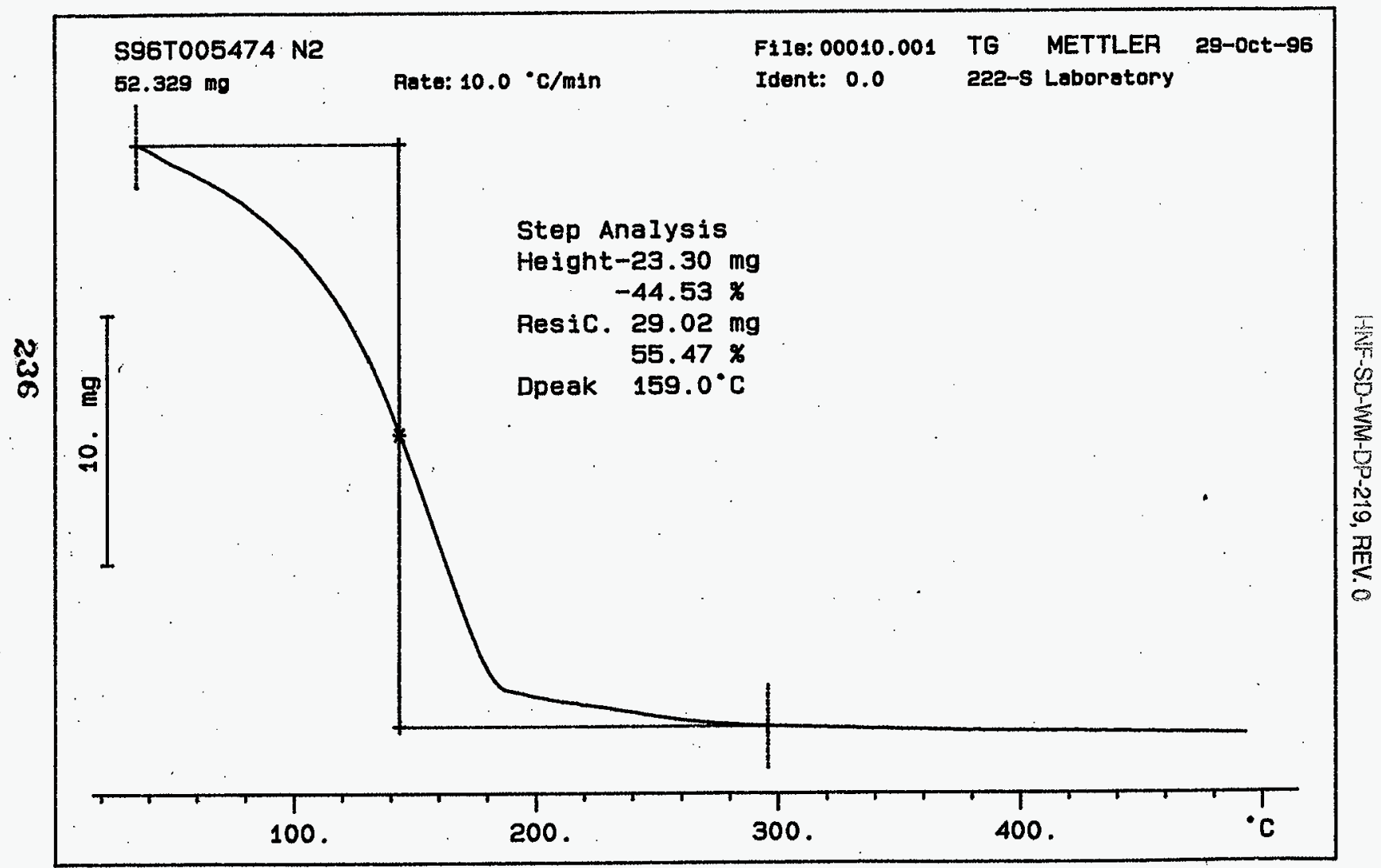




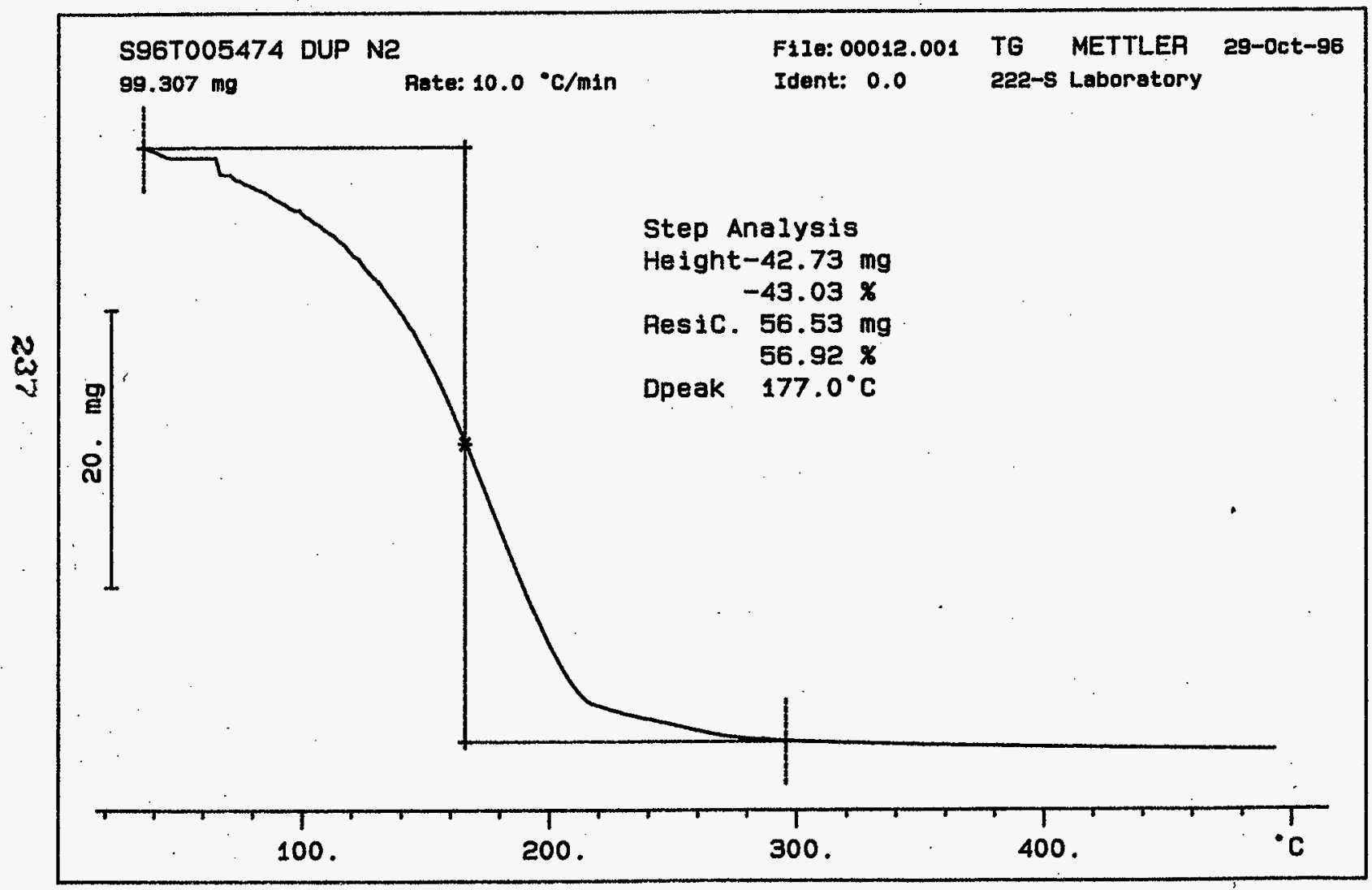




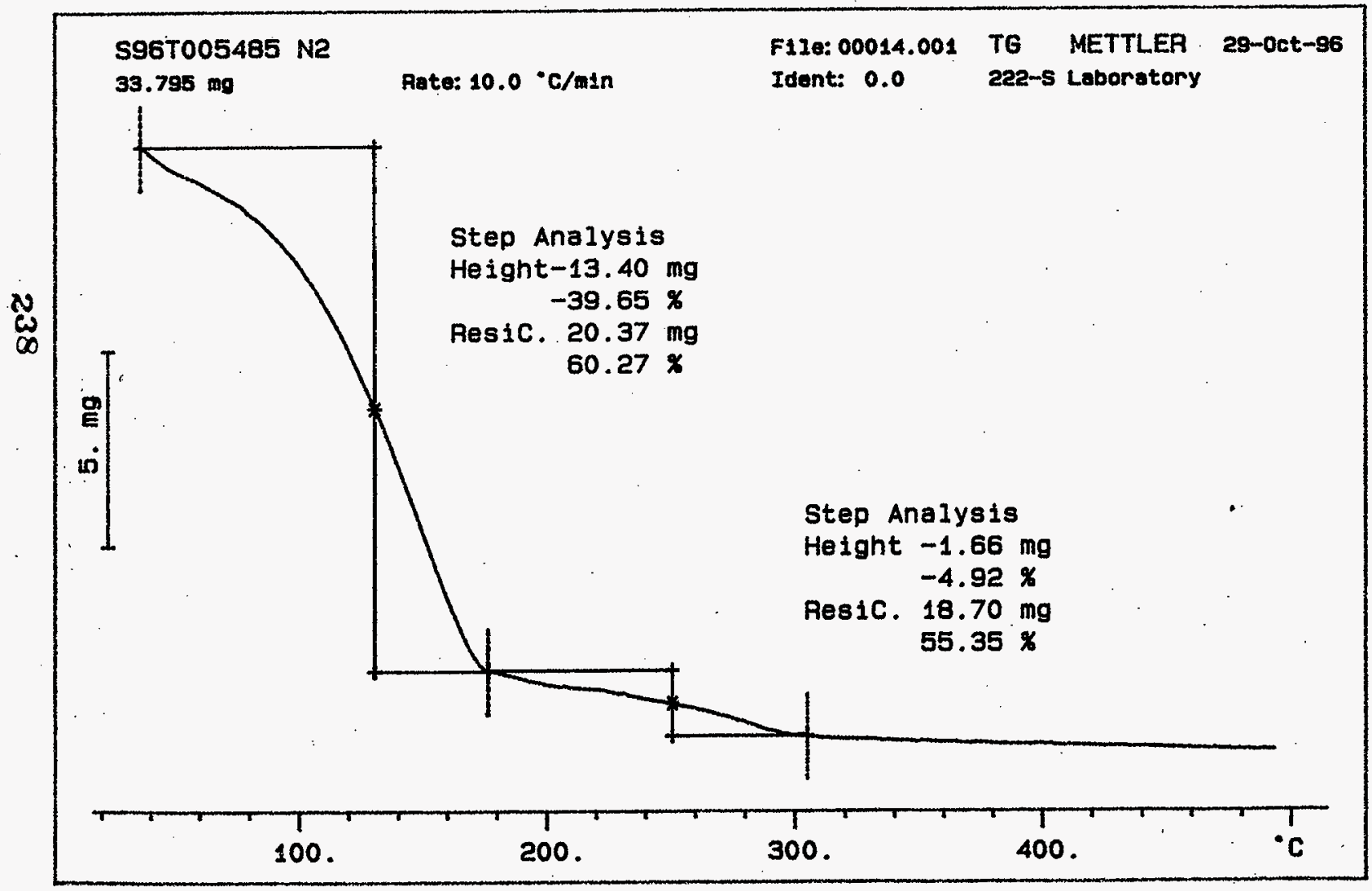




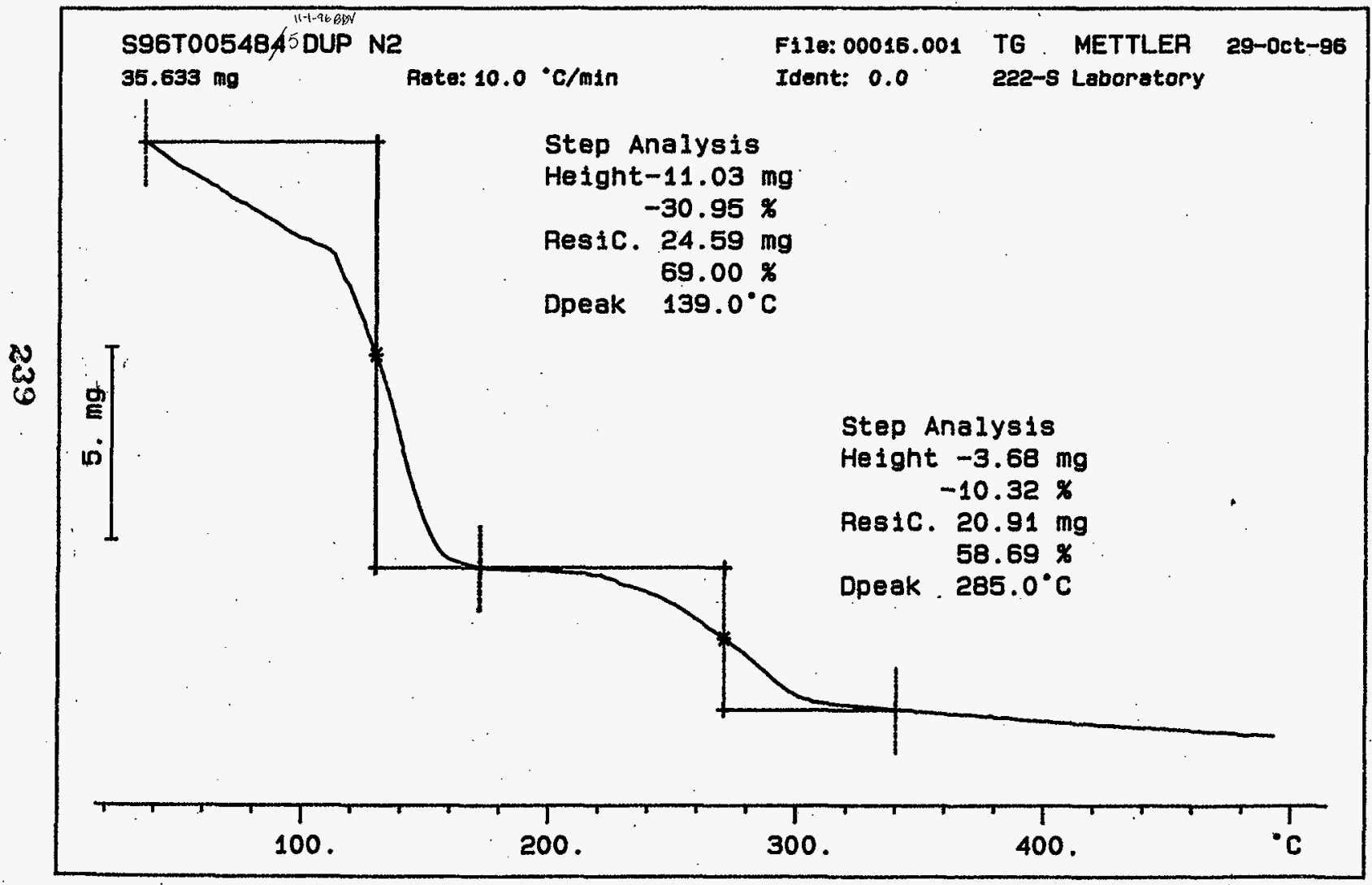




\section{LABCORE Data Entry Template for Worklist\# 14707}

Analyst: $\quad$ Instrument: TGA0 3 Book \# 82N8A

Method: LA-514-114 Rev/Mod D-O

Worklist Comment: B-108 TGA, RUN UNDER N2. RCJ

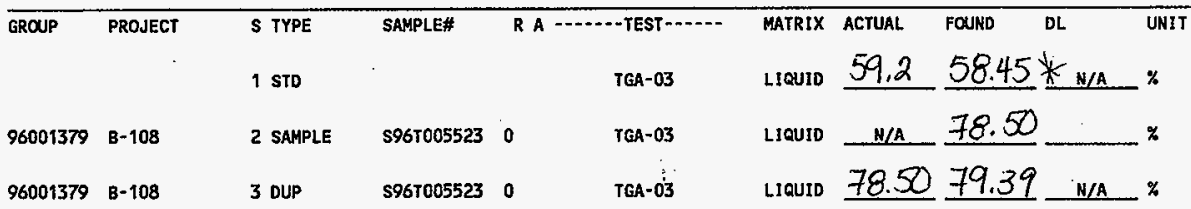

\section{Final page for worklist \# 14707}

Sel attached for signatures

Enalyst Signature fotel $11-20-96 ; \cdots \quad$ Analyst Signature

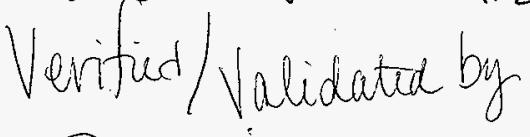

Powndina

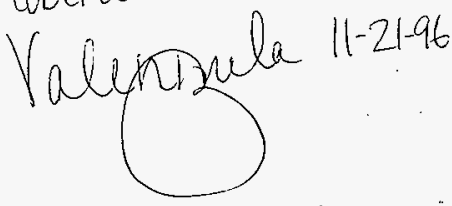

Data Entry Comments:

Units shown for $Q C$ (SPK \& STD) may not reflect the actual units. $D L=$ Detection Limit, $S=$ Worklist Slot Number, $R=$ Replicate Number, $A=$ Aliquot Code. 


\section{LABCORE Data Entry Template for Worklist\# 14707}

Analyst: $\quad D C D$

Instrument: TGAO

Book \# $82 N 8$ A

Method: LA-560-112 Rev/Mod

Worklist Comment: B-108 TGA, RUN UNDER N2. RCJ

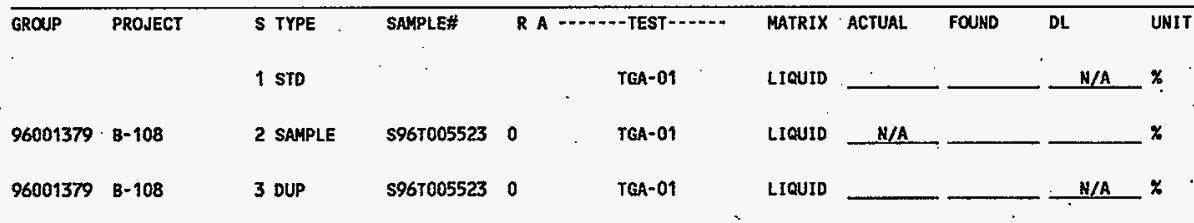

\section{Final page for worklist \# 14707}
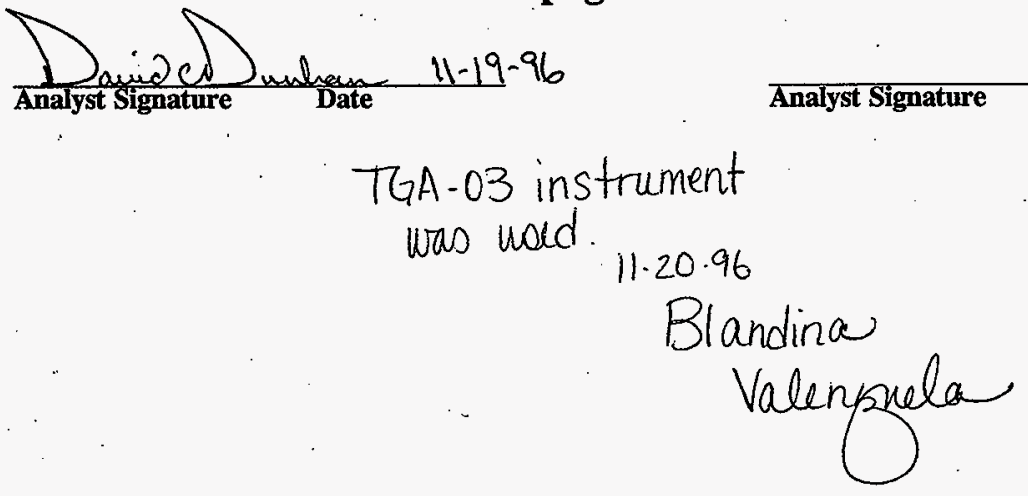

Data Entry Comments:

Units shown for QC (SPK \& STD) may not reflect the actual units. $D L=$ Detection Limit, $S=$ Worklist Slot Number, $R=$ Replicate Number, $A=$ Aliquot Code. 
Curve 1: TEA

F110 info: ter111901 Tue Nor 19 11: 16: 201996

Sorple Wedght: 20.479 m

TGA STD 8ENB-A

SIGNATURE BELOW REPRESENTS CHEMICAL TECHNOLOGIST/CHEMIST THAT

COMPLETED/VERIFIED THE CALIBRATION/ANALYSIS ON PAGES 242 TO 244.

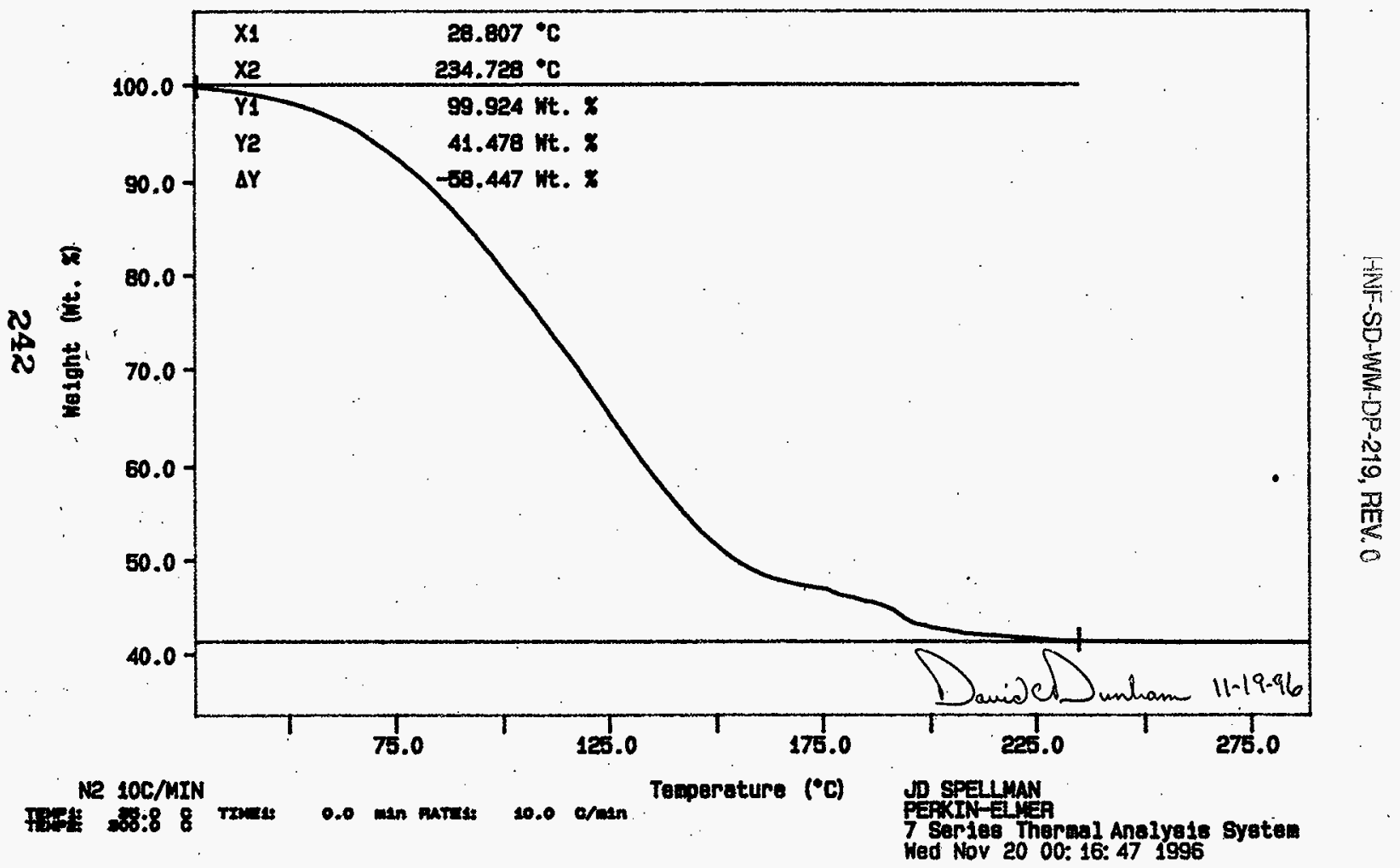




\section{Curve 1: TEA}

F110 Info: SAM114907 Ved Nov 20 03: 23: 191996

Sangle Hetght: 10.709 ag

S96T005523 SAM

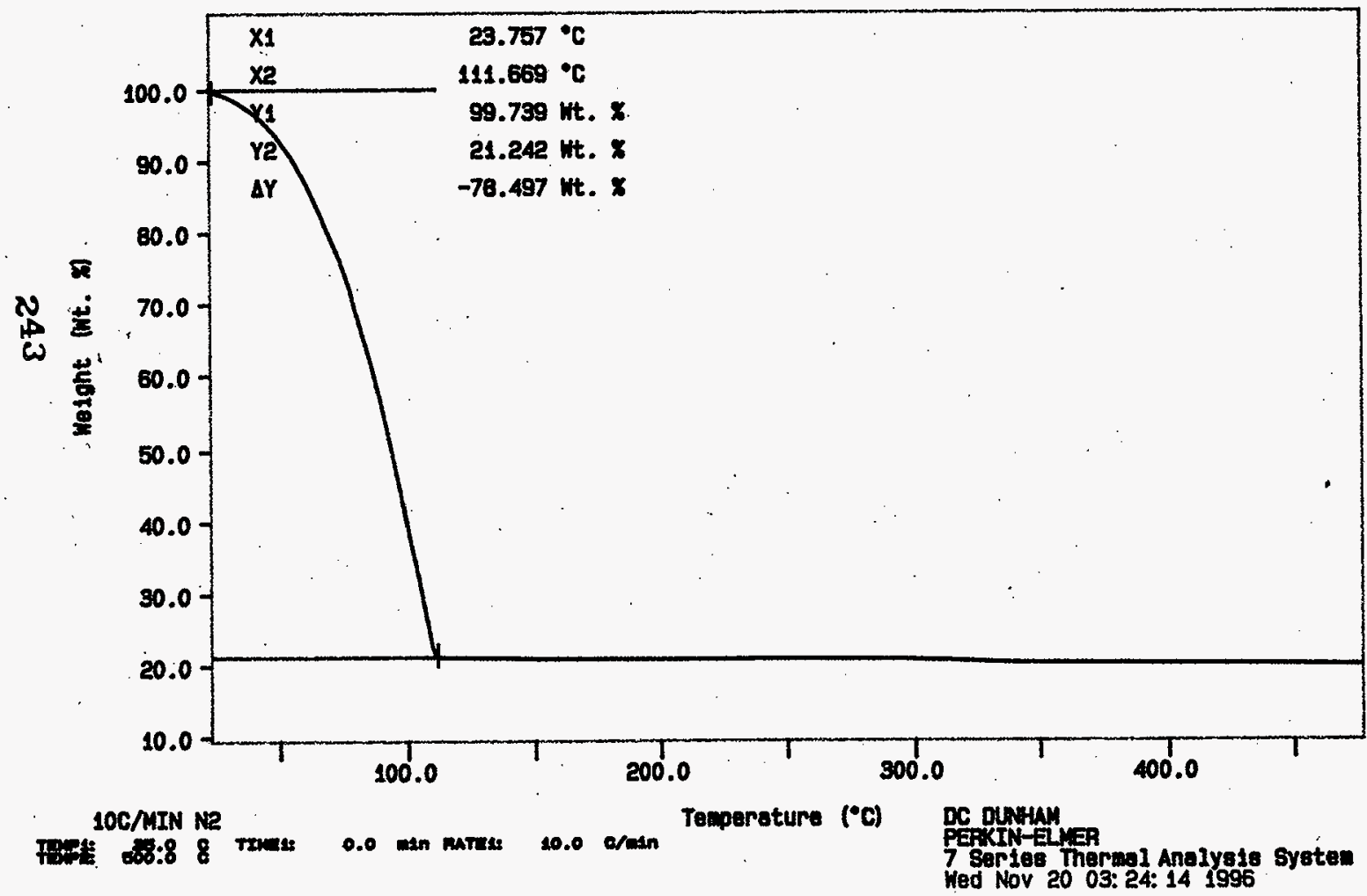


HNF-SD-WN-DP-219, REV.C

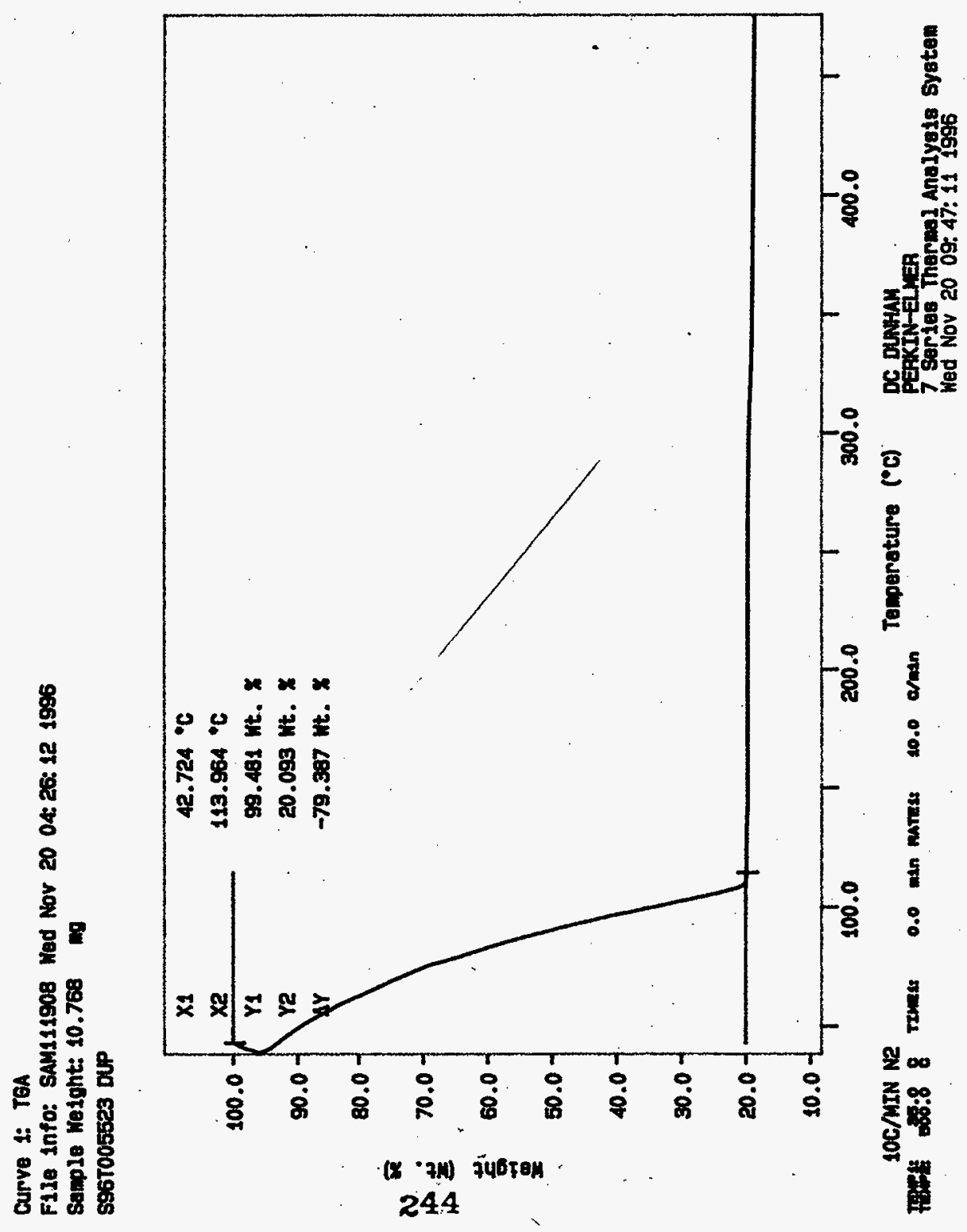




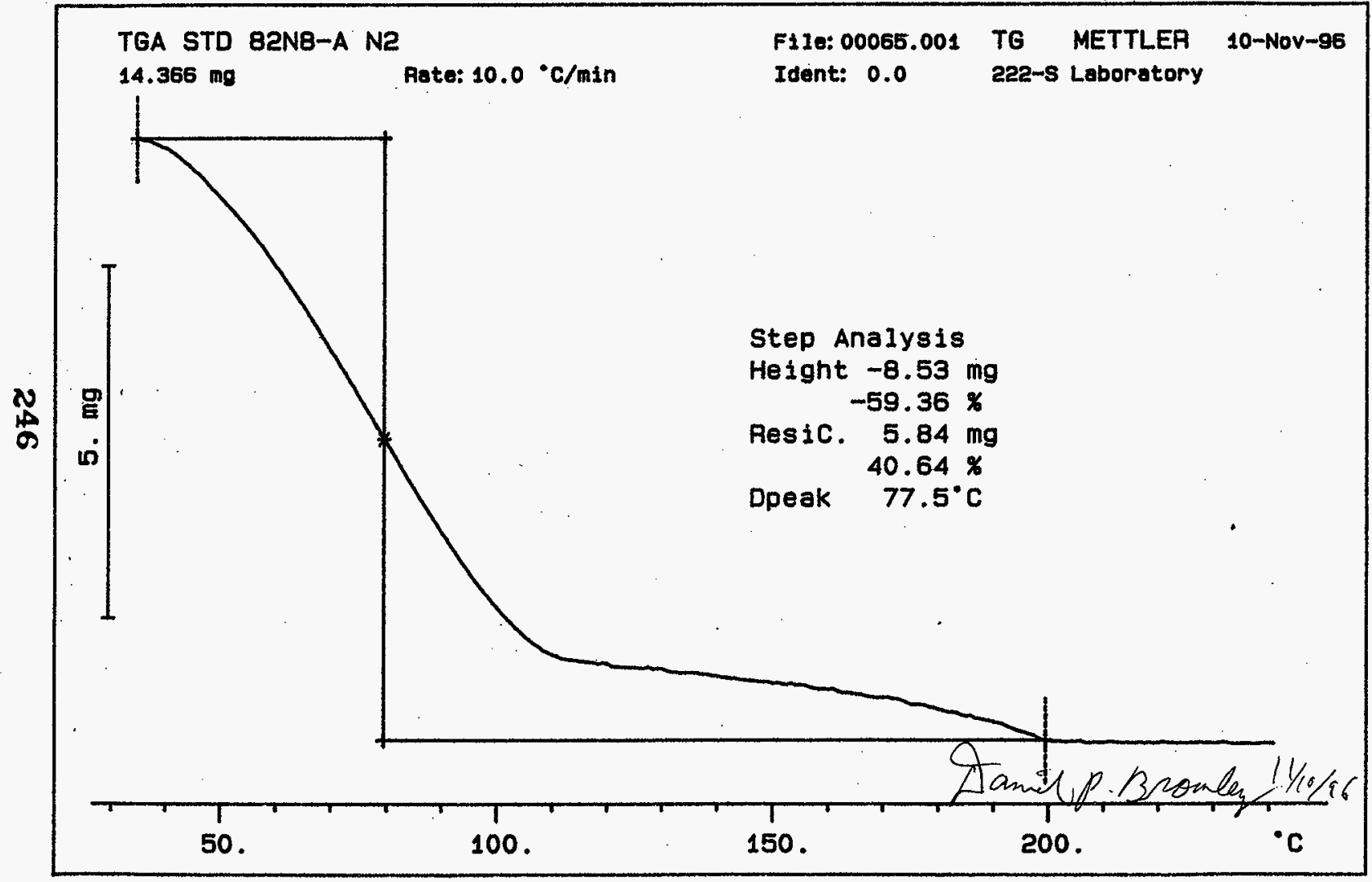




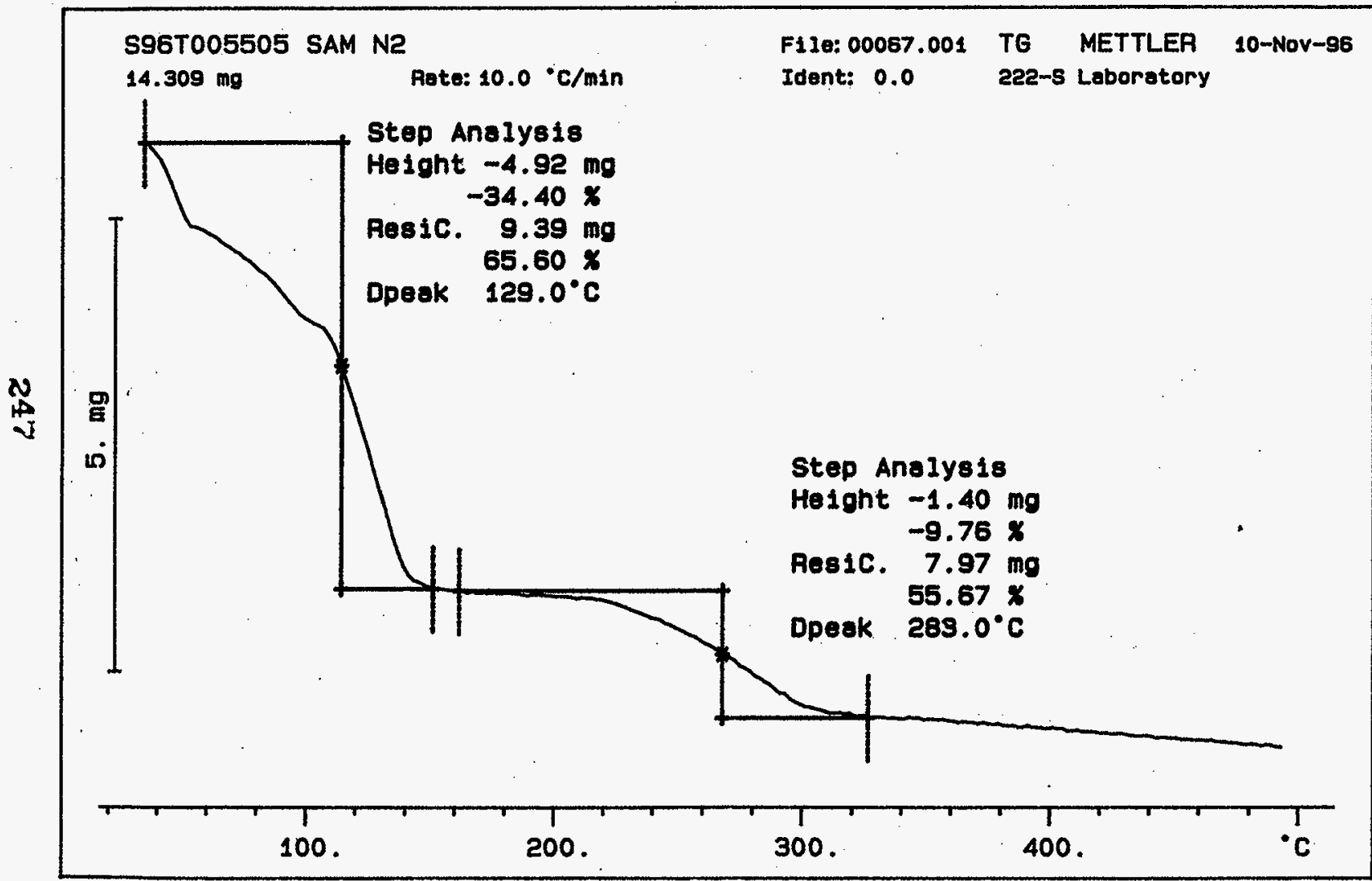




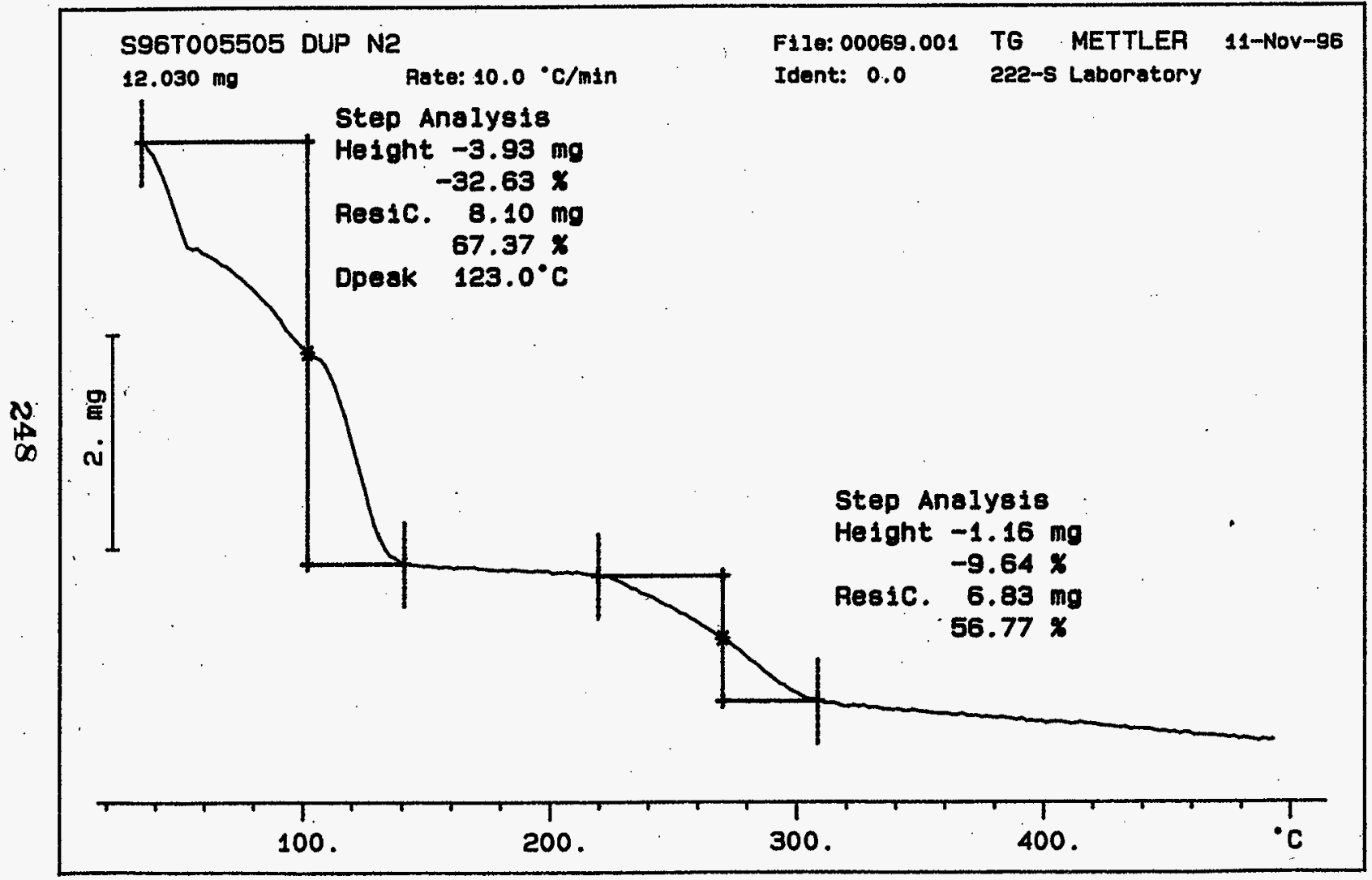




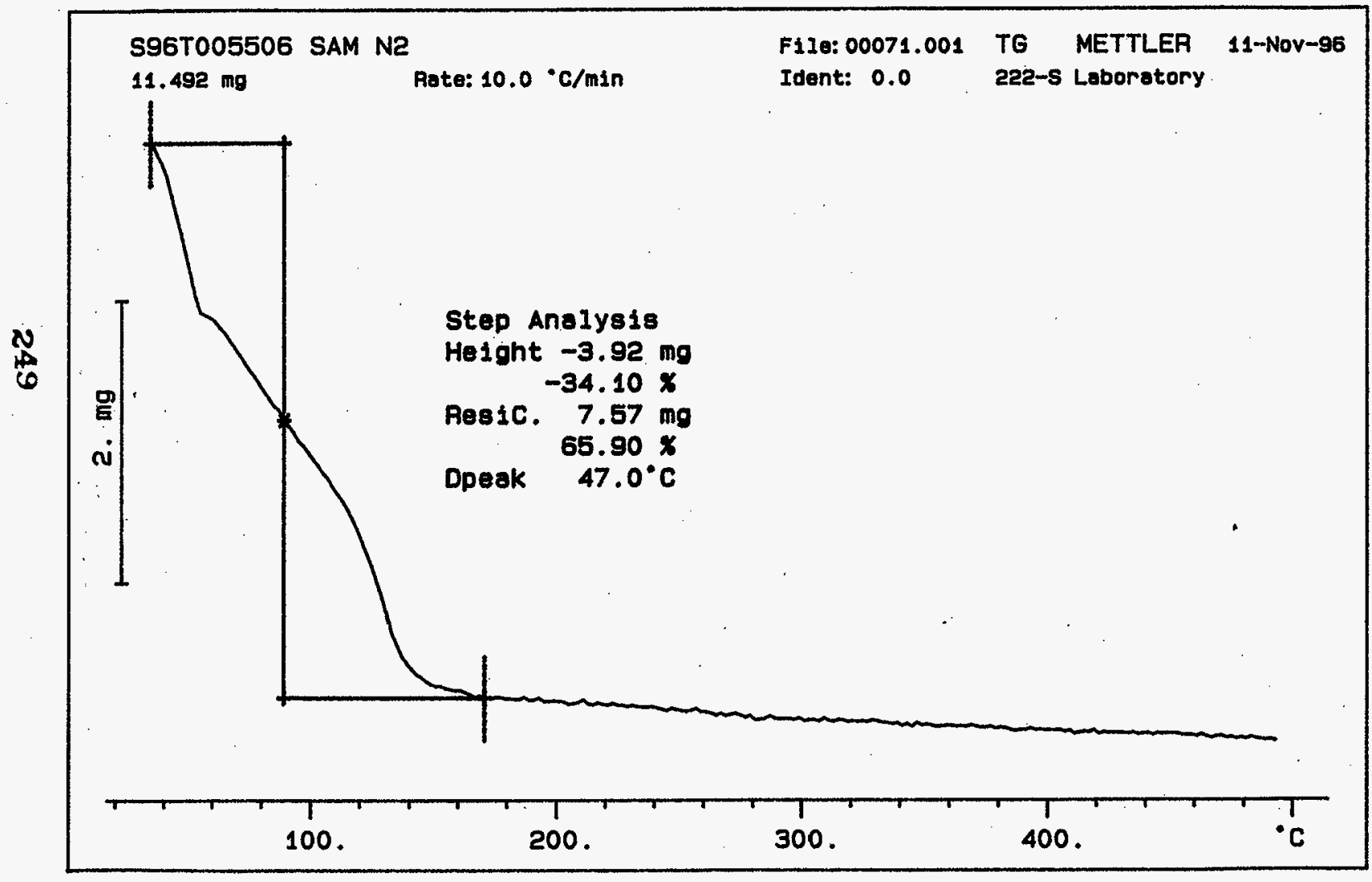




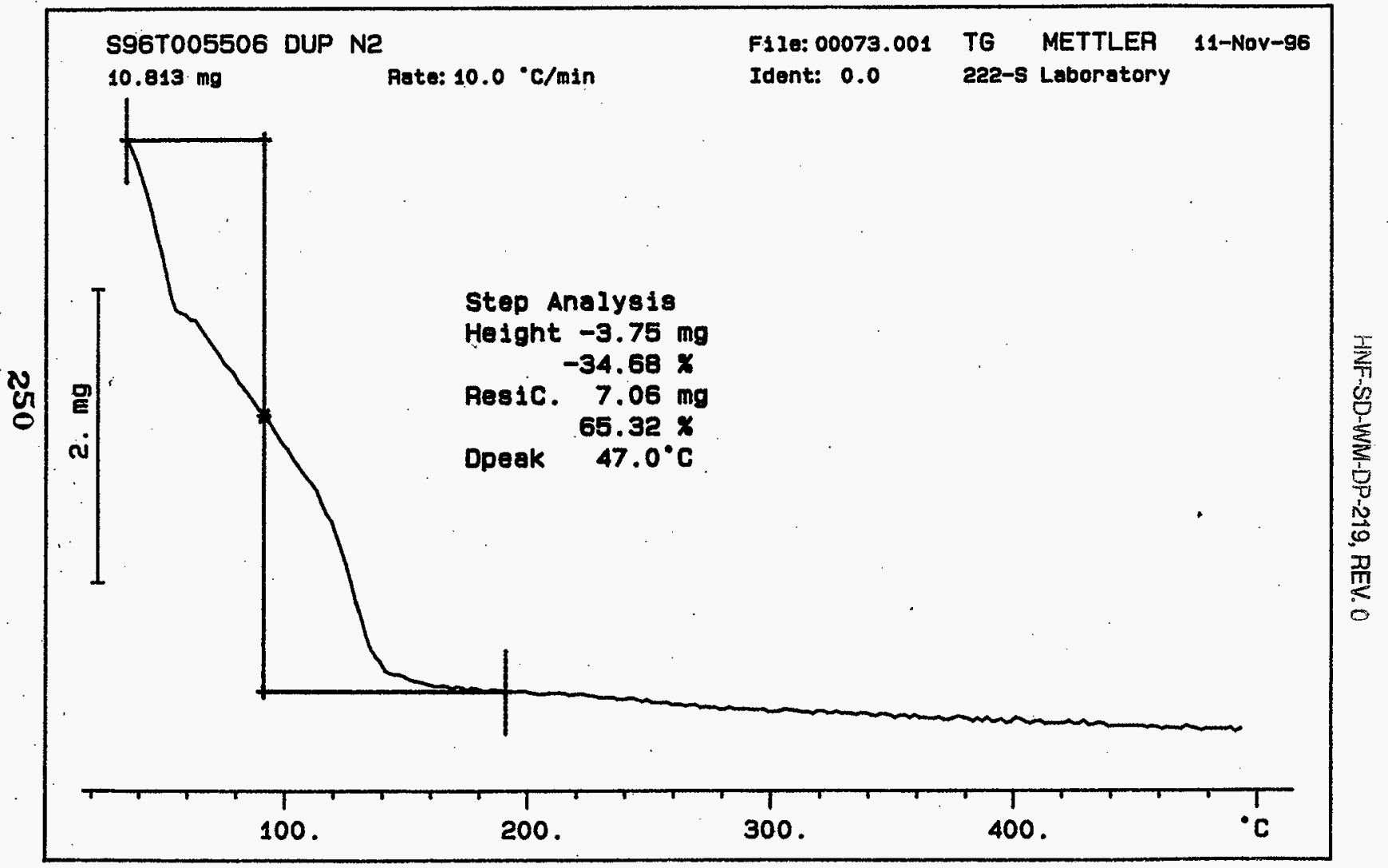




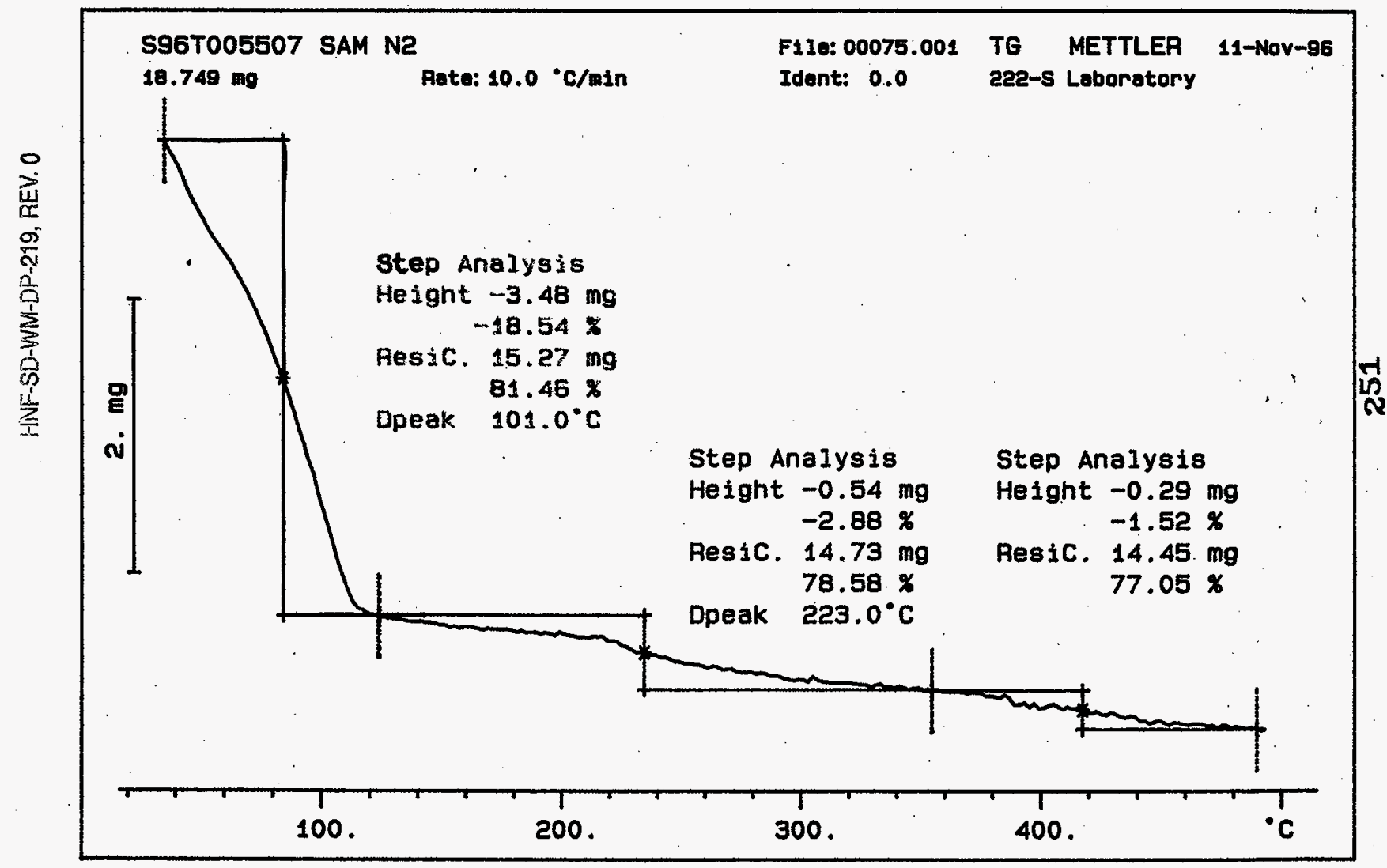




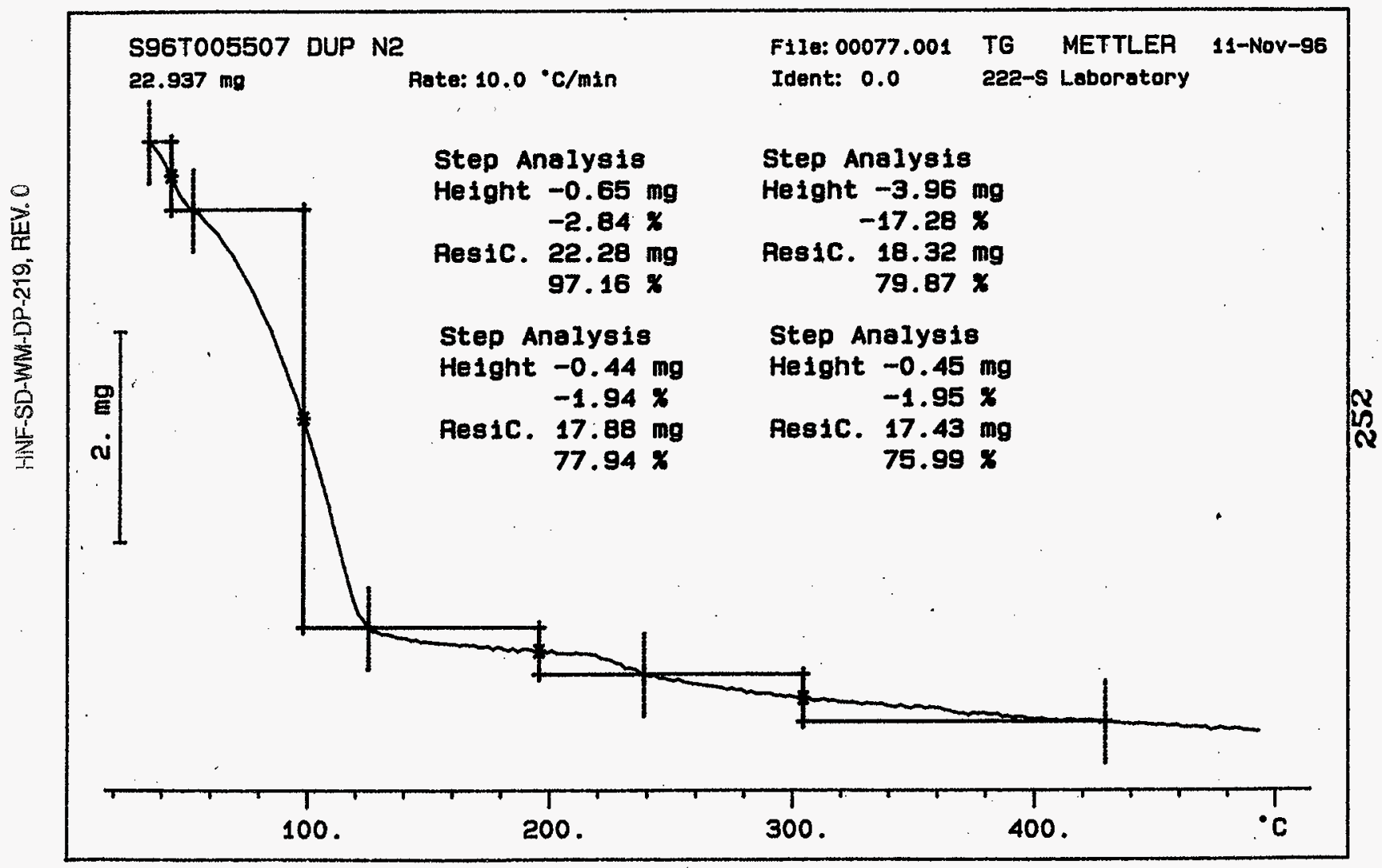




\section{LABCORE Data Entry Template for Worklist\# 14392}

Analyst: $\quad$ DCD Instrument: BA001

Book \# $133 \mathrm{~N} / 6-A$

Method: LA-510-112 Rev/Mod C-3

Worklist Comment: SPG-01 FOR AN-103 \& B-108 SAMPLES

RTS!

\begin{tabular}{|c|c|c|c|c|c|c|c|c|c|c|}
\hline GROUP & PROJECT & $\begin{array}{l}\text { S TYPE } \\
1 \text { STO }\end{array}$ & SAMPLE\# & RA & SPG 01 & $\begin{array}{l}\text { MATRIX } \\
\text { LIQUID }\end{array}$ & $\begin{array}{l}\text { ACTUAL } \\
1.398 \\
\end{array}$ & $\begin{array}{l}\text { FOUND } \\
1.394 \\
\end{array}$ & $\begin{array}{l}\mathrm{DL} \\
\mathrm{N} / \mathrm{A} \\
\end{array}$ & $\begin{array}{l}\text { UNIT } \\
\text { Sp.G. }\end{array}$ \\
\hline 96001318 & AN- 103 & 2 SAMPLE & $\$ 96 \mathrm{~T} 005406$ & 0 & SPG-01 & LIQUID & N/A & 1.016 & 0.010 & Sp.G. \\
\hline 96001318 & AN-103 & 3 DUP & \$96T005406 & 0 & SPG-01 & LIQUID & 1.016 & 1.056 & N/A & Sp.G. \\
\hline 96001339 & $A N-103$ & 4 SAMPLE & 5967005420 & 0 & SPG-01 & LIQUID & $\mathrm{N} / \mathrm{A}$ & 1.404 & 0.010 & Sp.G. \\
\hline 96001339 & $A N-103$ & 5 DUP & $\$ 967005420$ & 0 & SPG-01 & LIQUID & 1.404 & 1.402 & $N / A$ & Sp.G. \\
\hline 96001339 & AN-103 & 6 SAMPLE & \$96T005421 & 0 & SPG-01 & LIQUID & $\mathrm{N} / \mathrm{A}$ & 2.669 & 0.010 & sp.G. \\
\hline 96001339 & $A N-103$ & 7 DUP & 5967005421 & 0 & SPG-01 & LIQUID & 2.669 & 2.767 & $N / A$ & Sp. G. \\
\hline 96001379 & B- 108 & 8 SAMPLE & S96T005463 & 0 & SPG-01 & LIQUID & $N / A$ & 2.270 & 0.010 & Sp.G. \\
\hline 96001379 & B- 108 & 9 DUP & s96T005463 & 0 & SPG-01 & LIQUID & 2.270 & 2.298 & N/A & Sp.G. \\
\hline
\end{tabular}

Final page for worklist \# 14392

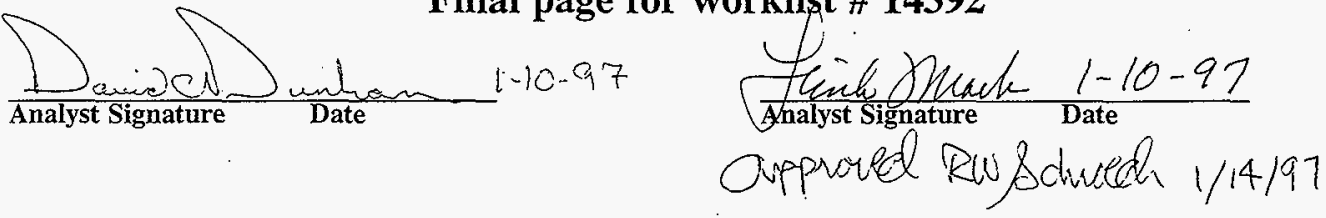

Data Entry Comments:

Units shown for QC (SPK \& STD) may not reflect the actual units. $D L=$ Detection Limit, $S=$ Worklist Slot Number, $R=$ Replicate Number, $A=$ Aliquot Code. 


\section{. SPECIFIC GRAVITY: LA-510-112 (C-3)}

\begin{tabular}{|c|c|c|}
\hline $\begin{array}{c}\text { WORKLIST } \\
\quad\end{array}$ & $\begin{array}{l}\text { ANALST } \\
\text { INITIALS } \\
\end{array}$ & $\begin{array}{r}\text { ANALYSIS } \\
\text { DATE } \\
\end{array}$ \\
\hline 14392 & $D C D$ & $1-10-97$ \\
\hline
\end{tabular}

\begin{tabular}{|c|c|c|}
\hline & $\begin{array}{l}\text { SAMPLE } \\
\text { STANDARD }\end{array}$ & Wouplicate. \\
\hline SAMPLE \# = & & STD \# $=133 \mathrm{~N} / 6 \mathrm{~A}$ \\
\hline $\begin{array}{l}\text { TARE WEIGHT (g) } \\
\text { GROSS WEIGHT (g) } \\
\text { VOL. of SOLUTTON (mL) }\end{array}$ & $\begin{array}{l}1.8459 \\
1.9512 \\
.07538 \\
\end{array}$ & \begin{tabular}{|c|} 
REPLICATE \\
$\frac{1.980 ?}{2.08857}$ \\
.07538 \\
\end{tabular} \\
\hline
\end{tabular}

ANALYSIS
TIME
INSTRUMENT
CODE

0.530

$\underbrace{\text { STMLLELICATE }}_{\text {STANDARO }}$

SAMPLE \# = S96 T00 5\% $1 / 63$

STD \# =

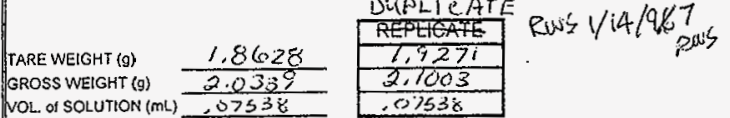

\begin{tabular}{|c|c|c|c|}
\hline $\boldsymbol{N}$ & $\begin{array}{l}\text { ZISAMPLE } \\
\text { STANDARD }\end{array}$ & 7]OUPLICATE & \\
\hline $\int_{i}$ SAMPLE\# = & S96T005 & $\begin{array}{l}\text { O6 STD \# } \\
\text { DUPLICATE } \\
\text { PHEAFE }\end{array}$ & $=$ \\
\hline $\begin{array}{l}\text { TARE WEIGHT (g) } \\
\text { GROSS WEIGHT (g) } \\
\text { VOL. of SOUTTION (mL }\end{array}$ & $\frac{1.8921}{1.9687}$ & $\begin{array}{l}1.9157 \\
1.9953 \\
.07538 \\
\end{array}$ & \\
\hline
\end{tabular}

区 STAMPLE

SAMPLE\# $=996 T 005420 . \quad$ STD\# =

\begin{tabular}{|c|c|}
\hline $\mathrm{MP}$ & $\begin{array}{l}\text { DUPLICATE } \\
\text { BEPUCATE }\end{array}$ \\
\hline 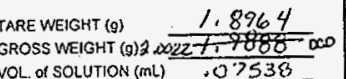 & \begin{tabular}{|l|}
$\frac{1.8831}{1.9888}$ \\
.07538 \\
\end{tabular} \\
\hline
\end{tabular}

\section{区 SATANLEARD}

SAMPLE \# $=596$ TOO542/

TARE WEIGHT $(g) \frac{1,907}{2,1089}$
STD \# =

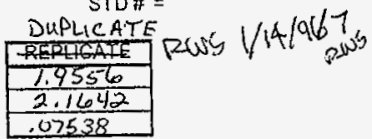

STD $\#=$
SAMPLE \# =

TARE WEIGHT (g)

GROSS WEIGHT (9)
VOL. of SOLUTiON (mL)

\section{SAMPLE \\ STANDARD}

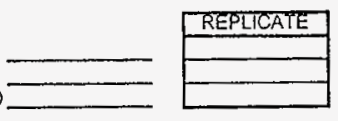

SAMPLE \# =

TARE WEIGHT (g)

VOL. of SOLUTION (mL)
GROSS WEIGHT (g)

STD \# =

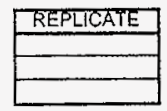

SAMPLE \# =

\section{SAMAPLE} STANDARO

TARE WEIGHT (g)

GROSS WEIGHT (g)

VOL. of SOLUTION (mL)
Juplicate

STO\# $=$

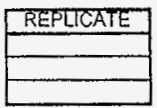




\section{SPECIFIC GRAVITY : LA-510-112 (C-3)}

\begin{tabular}{|c|c|c|c|}
\hline 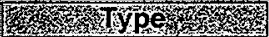 & & STD & REPLICATE \\
\hline STD & Gross Weight (W2) & 1.9512 & 2.0857 \\
\hline 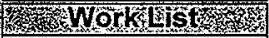 & Tare Weight (W1) & 1.8459 & 1.9809 \\
\hline 14392 & Weight of Solution (W2-W1) & 0.1053 & 0.1048 \\
\hline 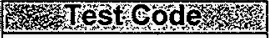 & Volume of Solution $\mu \mathrm{L}$ & 75.3800 & 75.3800 \\
\hline SPG-01 & Specific Gravity & 1.3969 & 1.3903 \\
\hline 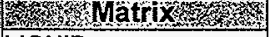 & Specific Gravity (Average) & 1.3936 & \\
\hline
\end{tabular}

LIQUID

Samplej

STD\# 133N16A

Onstrumenterode Gross Weight $(W 2)=W t$. of vial + cap + cotton + solution

BA001

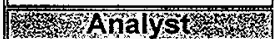

DCD

Wh

$01 / 10 / 97$

Tare Weight $(\mathrm{W} 1)=\mathrm{Wt}$. of vial + cap + cotton

Wrime

Specific Gravity $=[(W 2-W 1) * 1000 \mu \mathrm{L} / \mathrm{mL}] /[$ Vol. of Solution $\mu \mathrm{L} * 1.000 \mathrm{~g} / \mathrm{mL}]$

05:30 AM

v RESULT V

Specific Gravity Average =

1.394

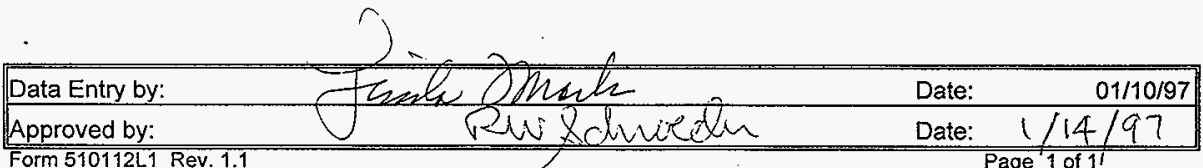




\section{SPECIFIC GRAVITY : LA-510-112 (C-3)}

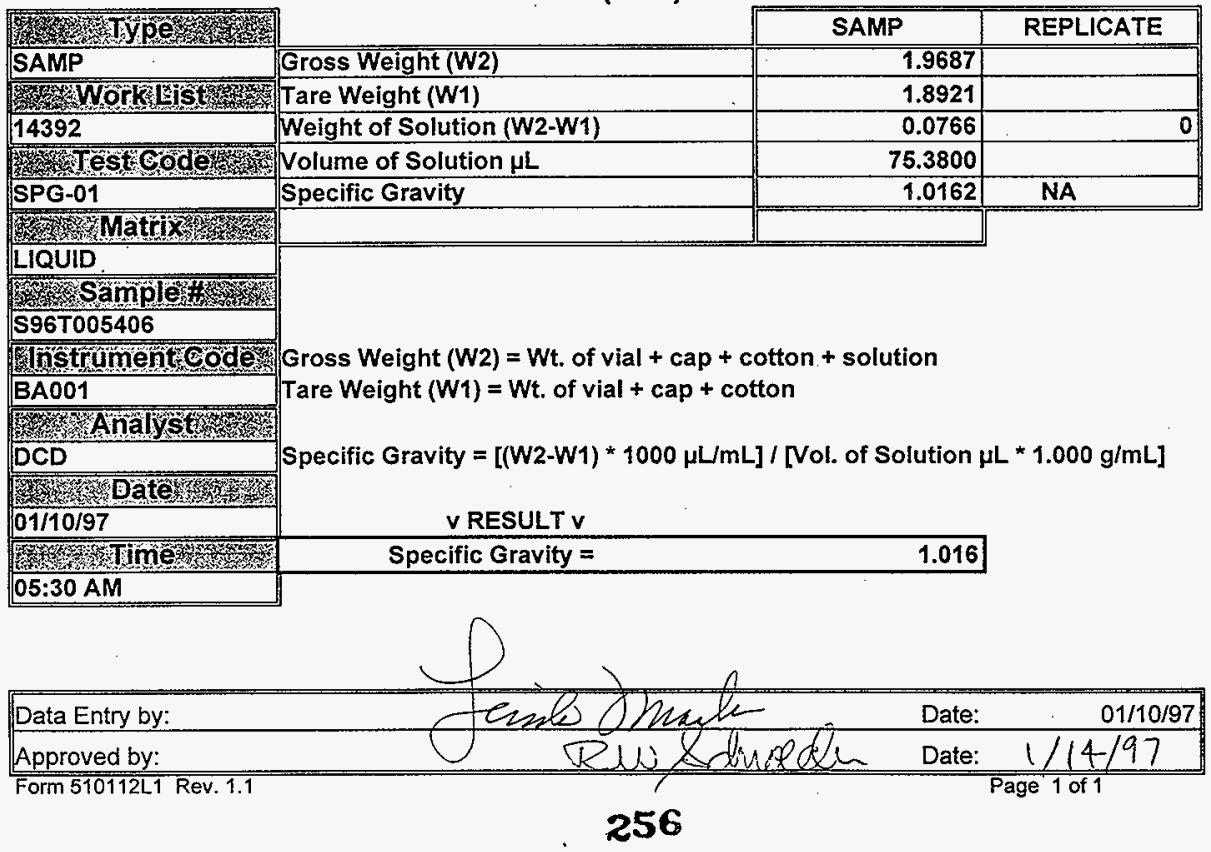


HINF-SD-WM-DP-219, REV.O

PLACE ANALYTICAL CARD IN BOX BELOW OR ATTACH TRAVELER

\section{SPECIFIC GRAVITY : LA-510-112 (C-3)}

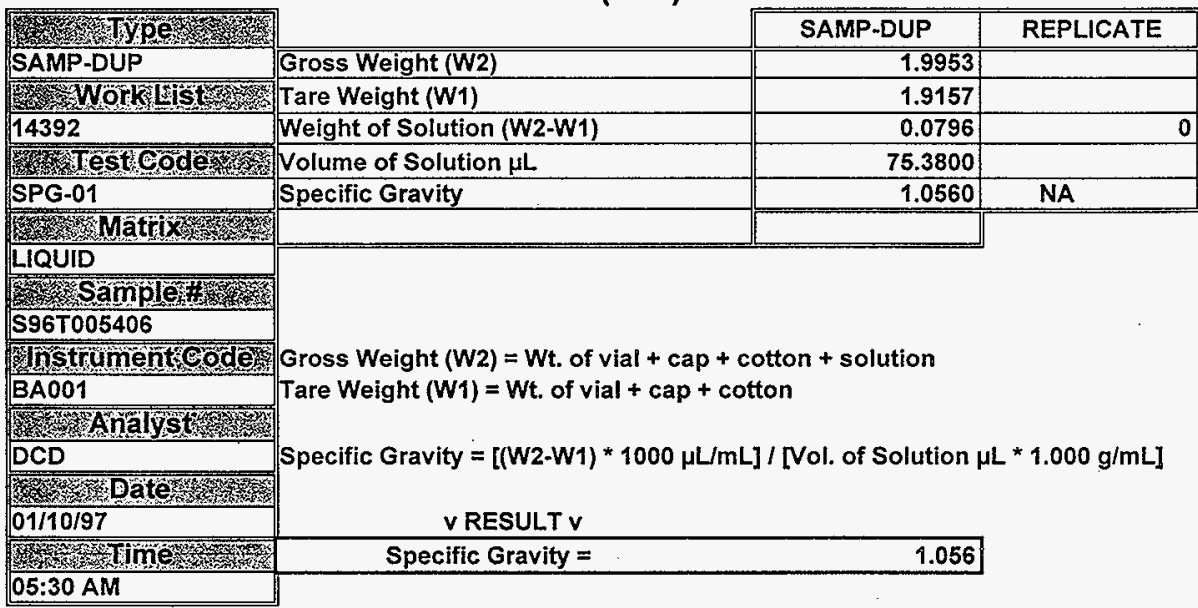

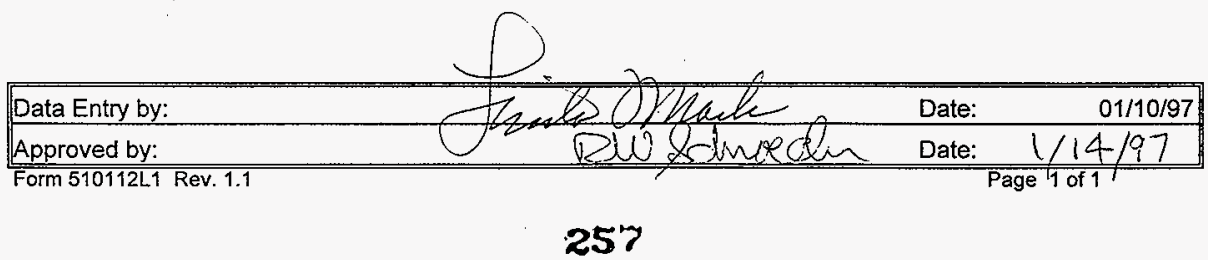




\section{SPECIFIC GRAVITY : LA-510-112 (C-3)}

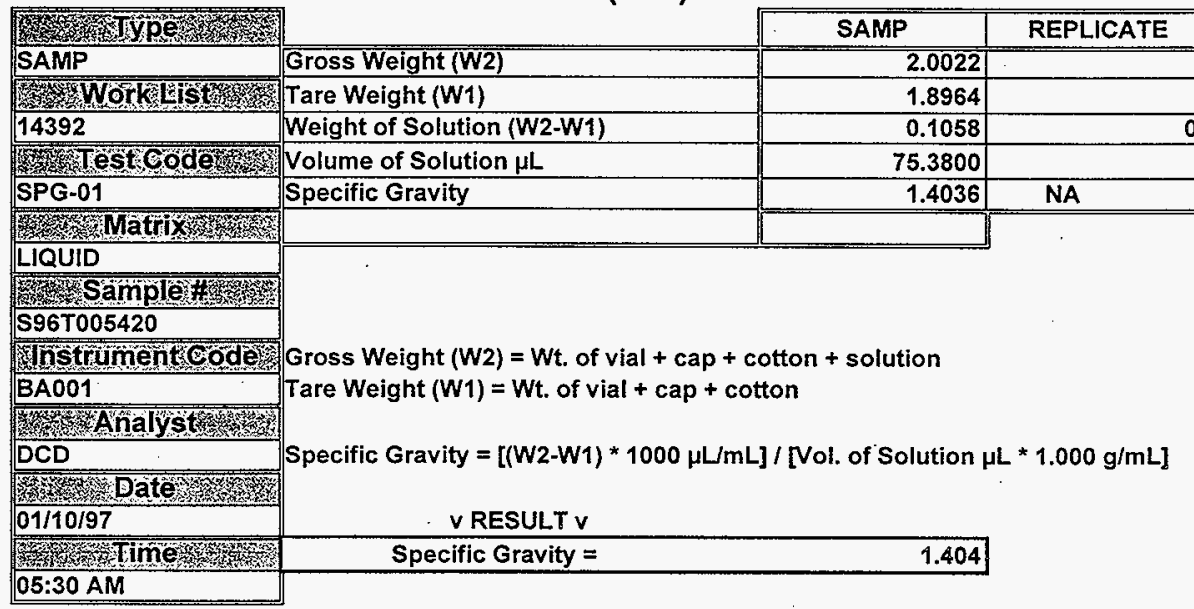

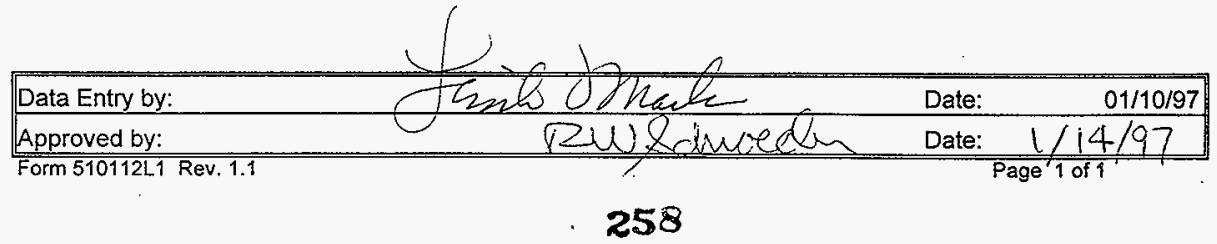




\section{SPECIFIC GRAVITY : LA-510-112 (C-3)}

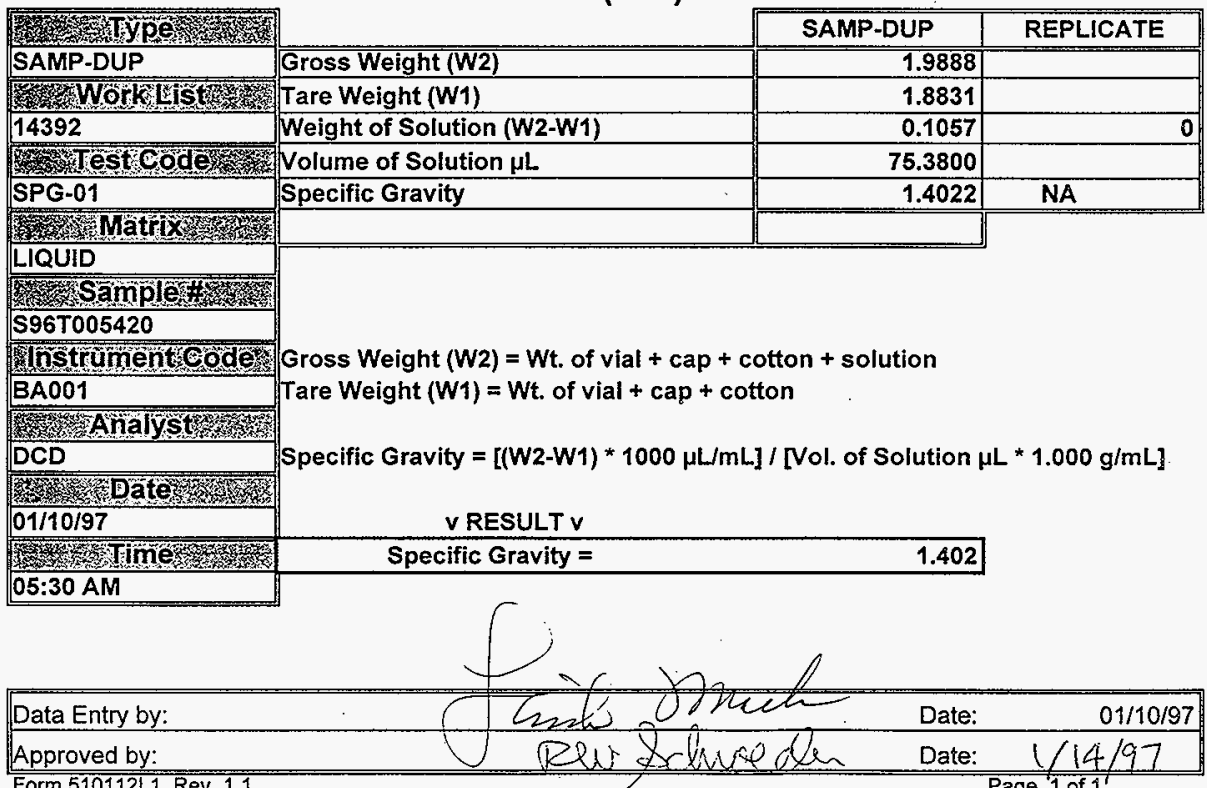




\section{SPECIFIC GRAVITY : LA-510-112 (C-3)}

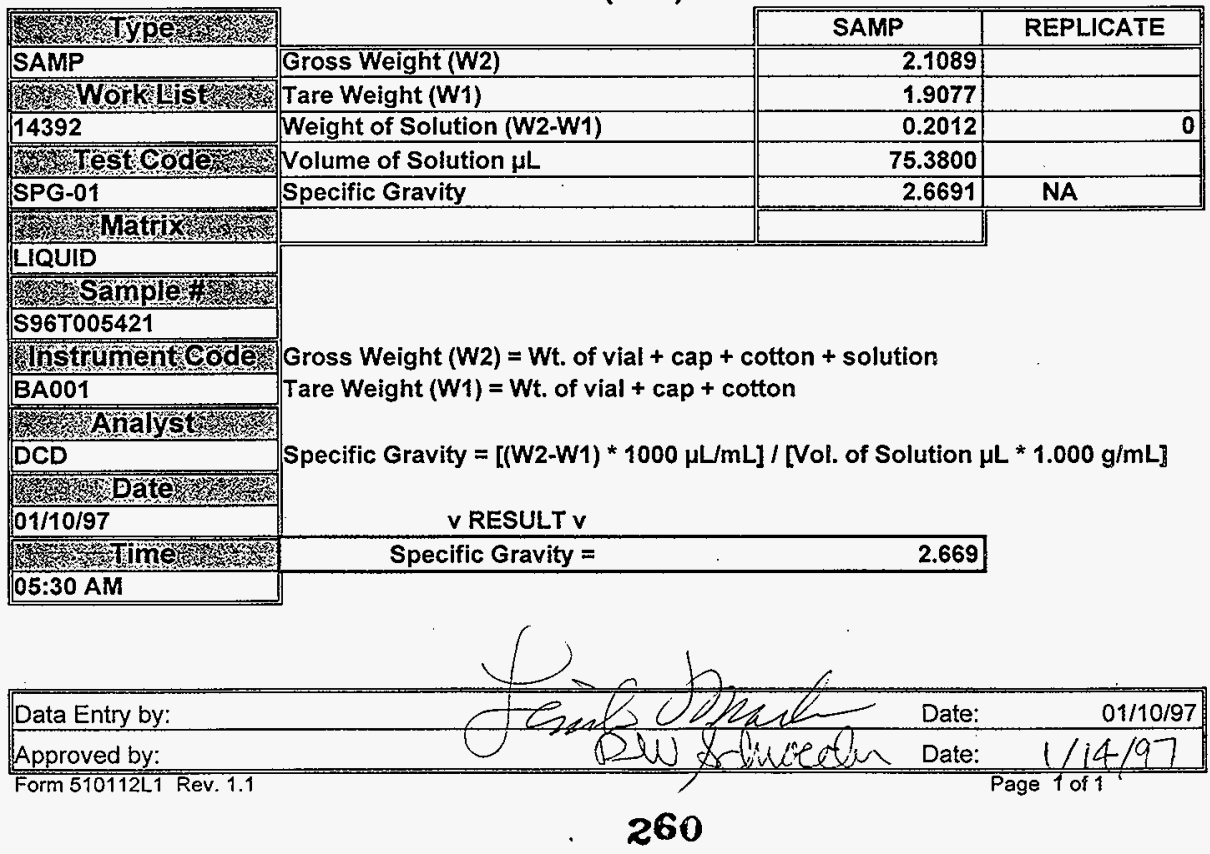




\section{SPECIFIC GRAVITY : LA-510-112 (C-3)}

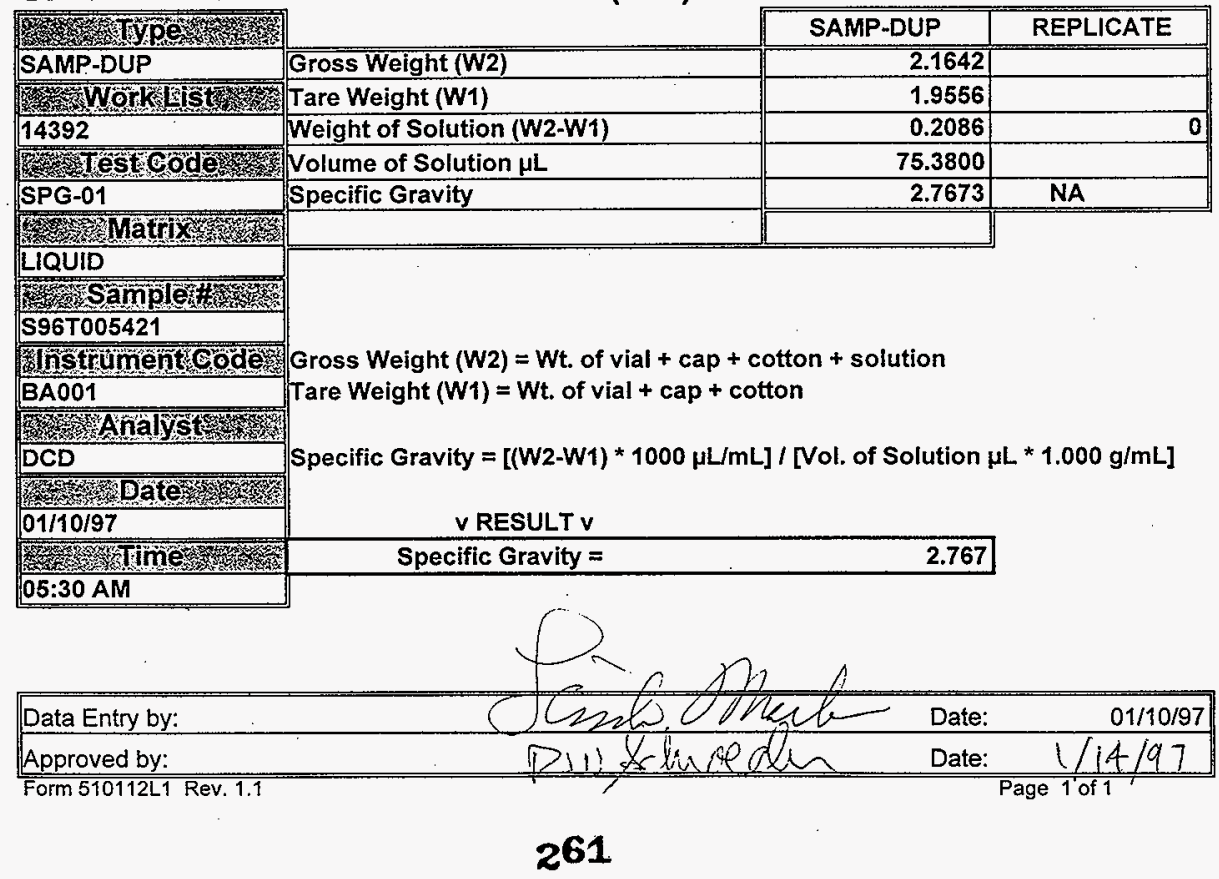


SPECIFIC GRAVITY : LA-510-112 (C-3)

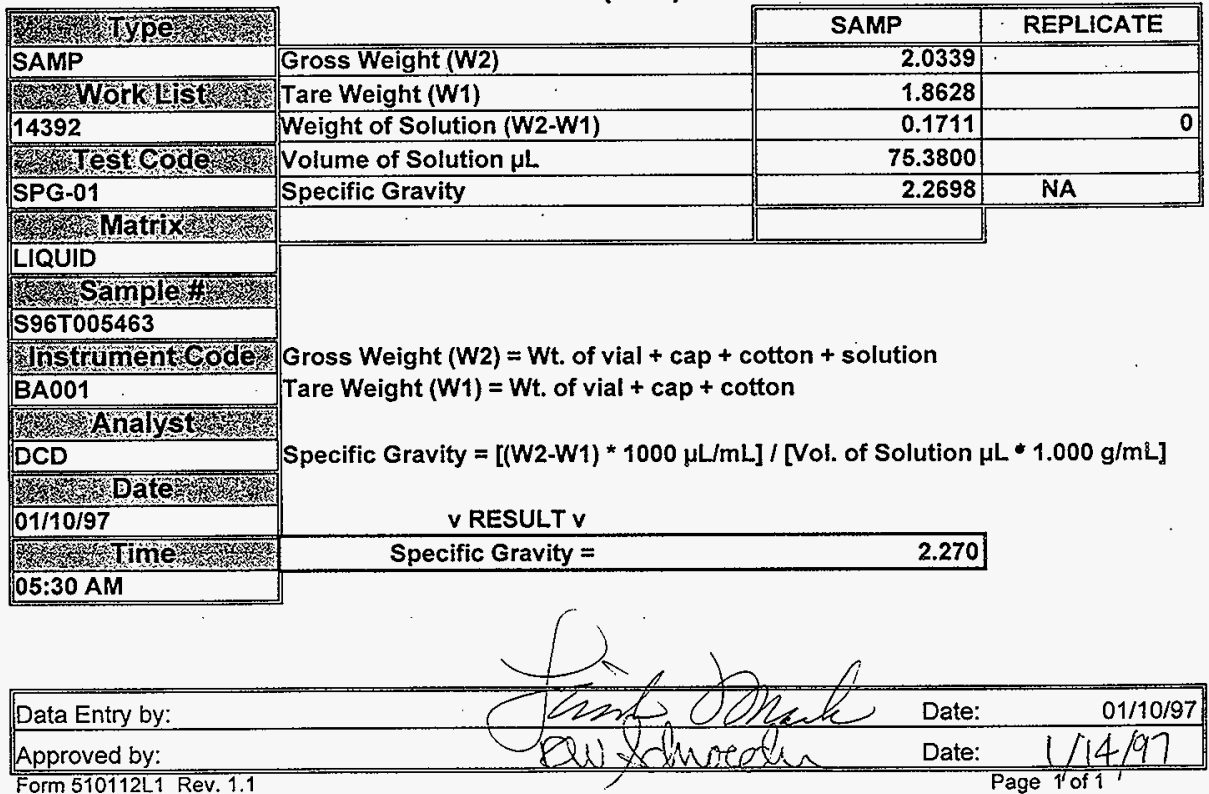




\section{SPECIFIC GRAVITY : LA-510-112 (C-3)}

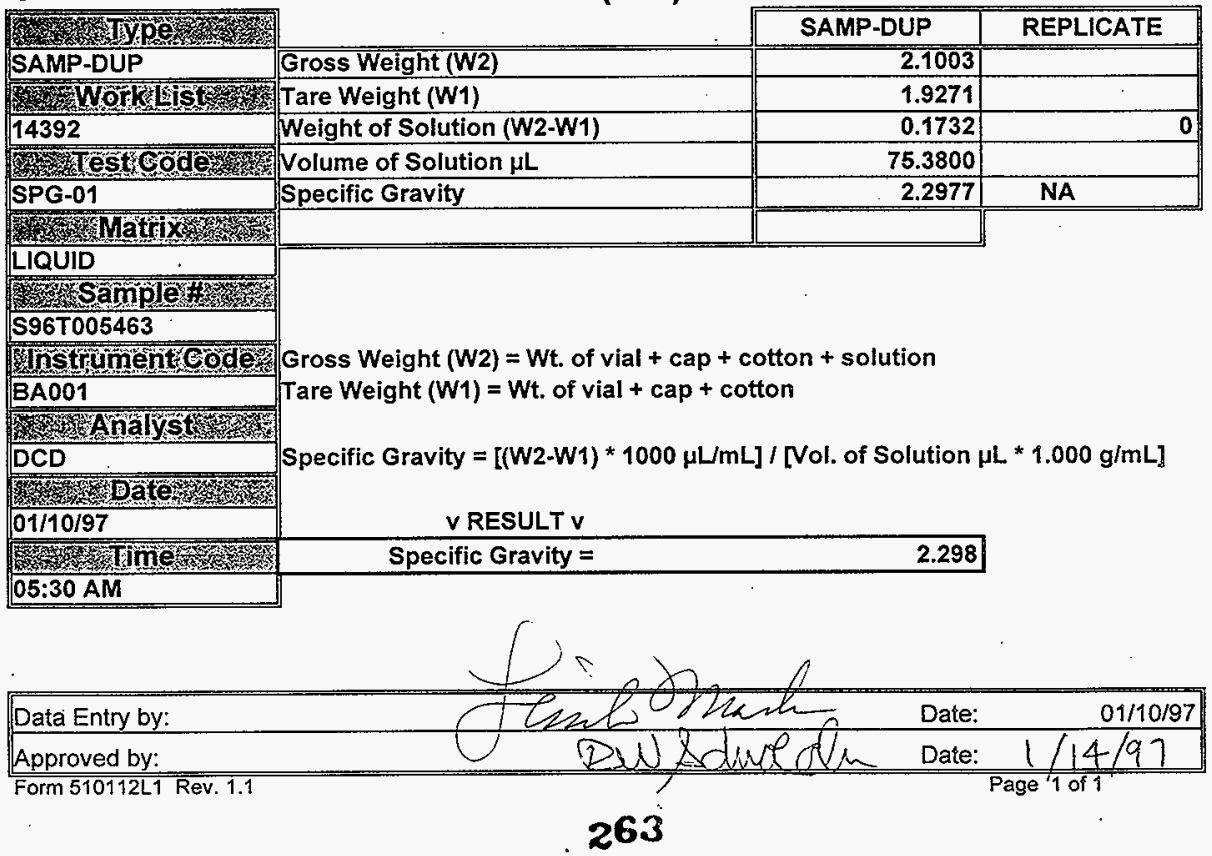


HNF-SD-WM-DP-219, REV. 0

worklistrpt Version $2.105 / 15 / 95$

11/04/96 14:39

\section{LABCORE Data Entry Template for Worklist\# 14659}

Page: $\quad 1$

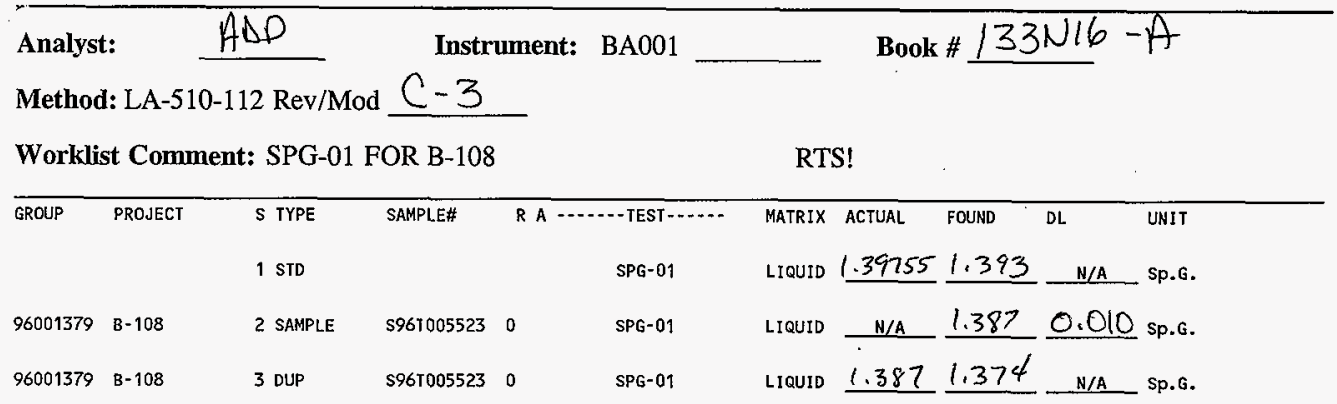

Final page for worklist \# 14659
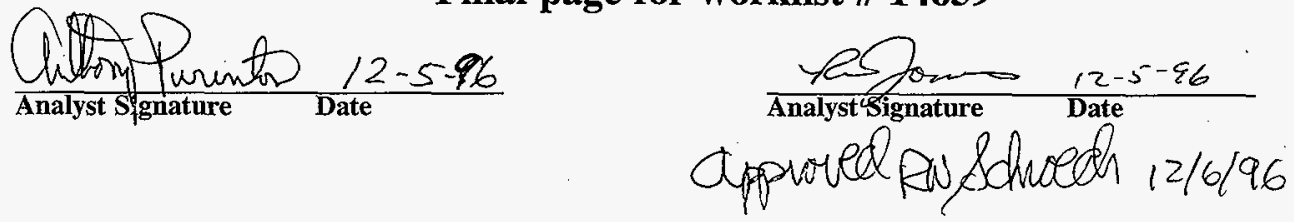

Data Entry Comments:

Units shown for $Q C$ (SPK \& STD) may not reflect the actual units. $D L=$ Detection Limit, $S=$ Worklist Slot Number, $R=$ Replicate Number, $A=$ Aliquot Code. 


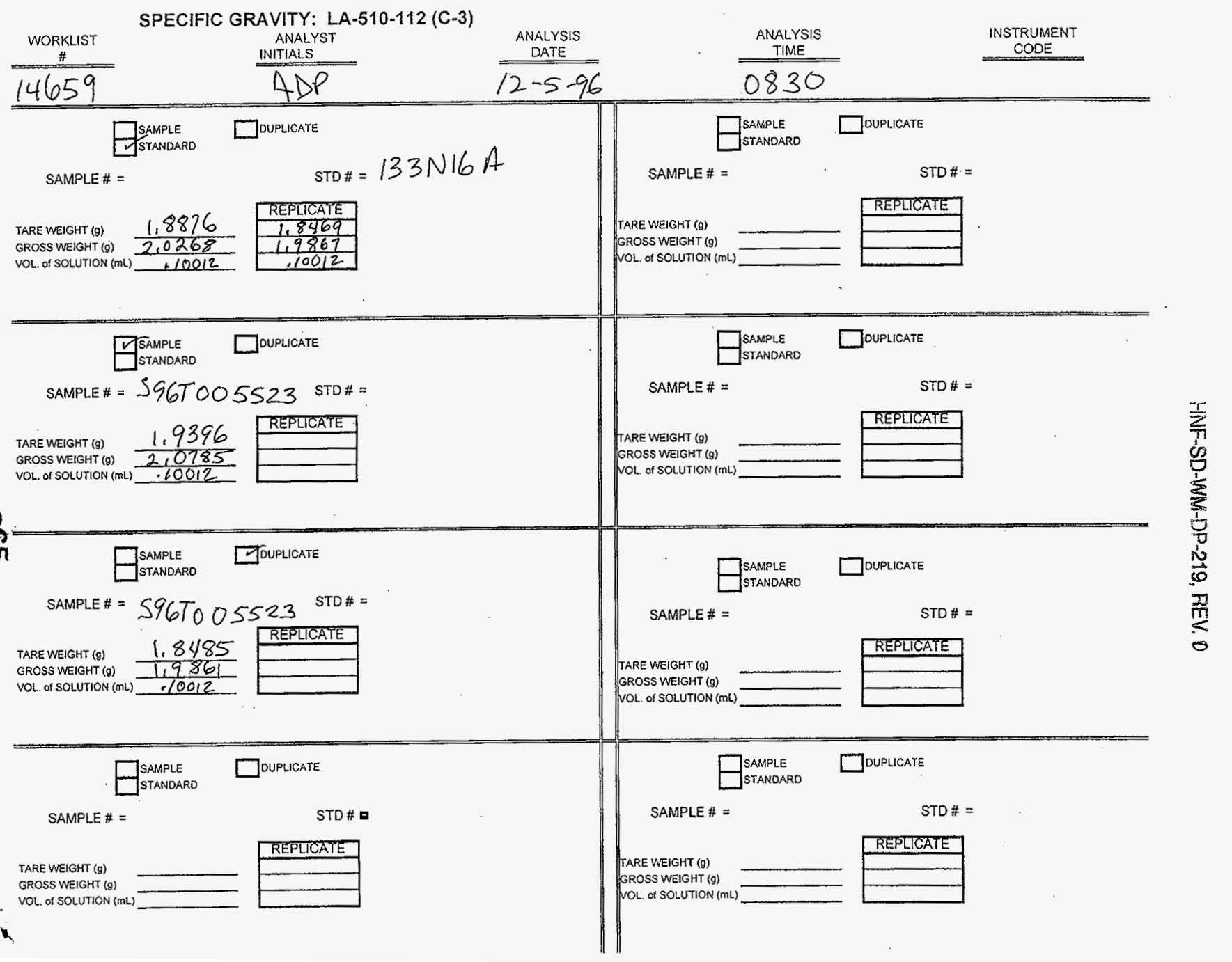


SPECIFIC GRAVITY : LA-510-112 (C-3)

\begin{tabular}{|c|c|c|c|}
\hline 2, & & STANDARD & STANDARD \\
\hline STANDARD & Gross Weight (W2) & 2.0268 & 1.9867 \\
\hline 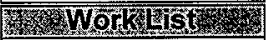 & Tare Weight (W1) & 1.8876 & 1.8469 \\
\hline 14659 & Weight of Solution (W2-W1) & 0.1392 & 0.1398 \\
\hline Sy & Volume of Solution $\mu \mathrm{L}$ & 100.1200 & 100.1200 \\
\hline SPG-01 & Specific Gravity & 1.3903 & 1.3963 \\
\hline 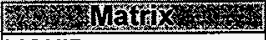 & Specific Gravity (Average) & 1.3933 & \\
\hline LIQUID & \multirow{9}{*}{\multicolumn{3}{|c|}{$\begin{array}{l}\text { Gross Weight }(W 2)=W t \text {. of vial + cap + cotton + solution } \\
\text { Tare Weight }(W 1)=W t \text {. of vial + cap + cotton } \\
\text { Specific Gravity }=[(W 2-W 1) * 1000 \mu L / \mathrm{mL}] / \text { Vol. of Solution } \mu \mathrm{L} * 1.000 \mathrm{~g} / \mathrm{mL}]\end{array}$}} \\
\hline 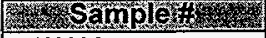 & & & \\
\hline 133N16A & & & \\
\hline Instimentgode & & & \\
\hline BA001 & & & \\
\hline 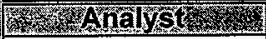 & & & \\
\hline ADP & & & \\
\hline ervatex & & & \\
\hline $12 / 05 / 96$ & & & \\
\hline Mne & Specific Gravity Average = & 1.393 & \multirow{2}{*}{ - } \\
\hline $08: 30 \mathrm{AM}$ & & & \\
\hline
\end{tabular}

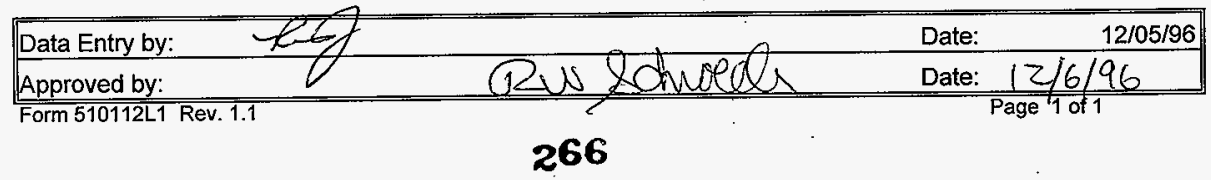




\section{SPECIFIC GRAVITY : LA-510-112 (C-3)}

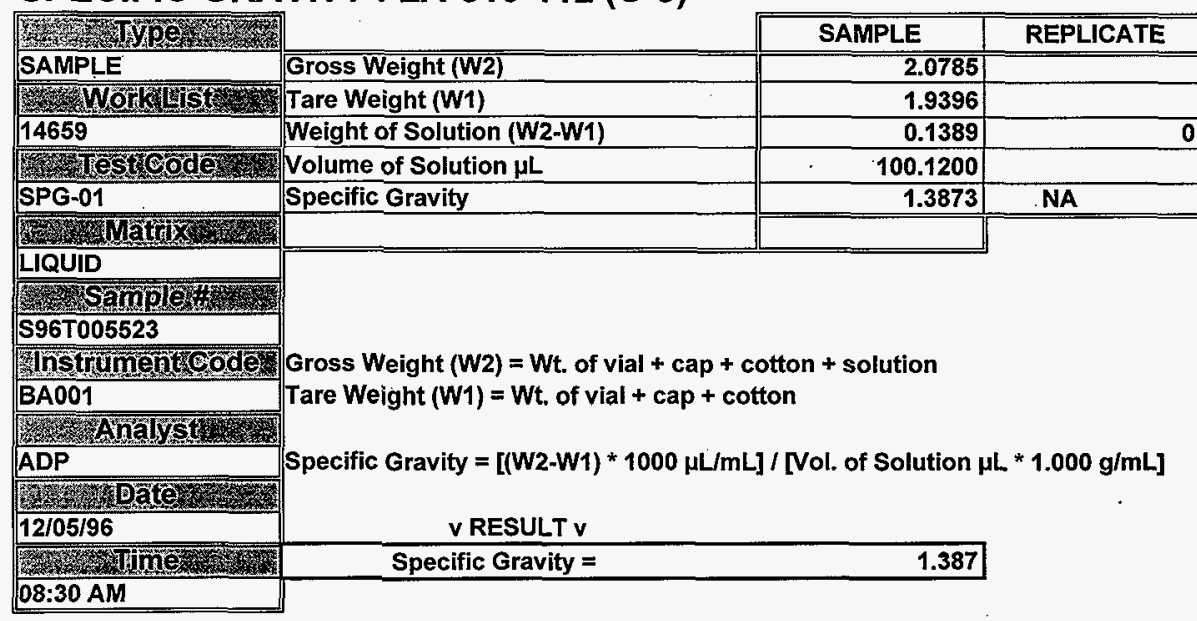

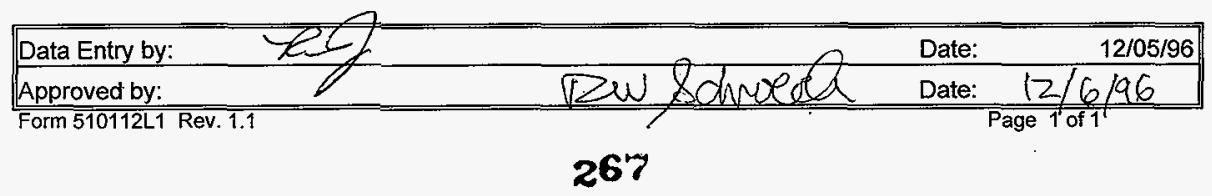




\section{SPECIFIC GRAVITY : LA-510-112 (C-3)}

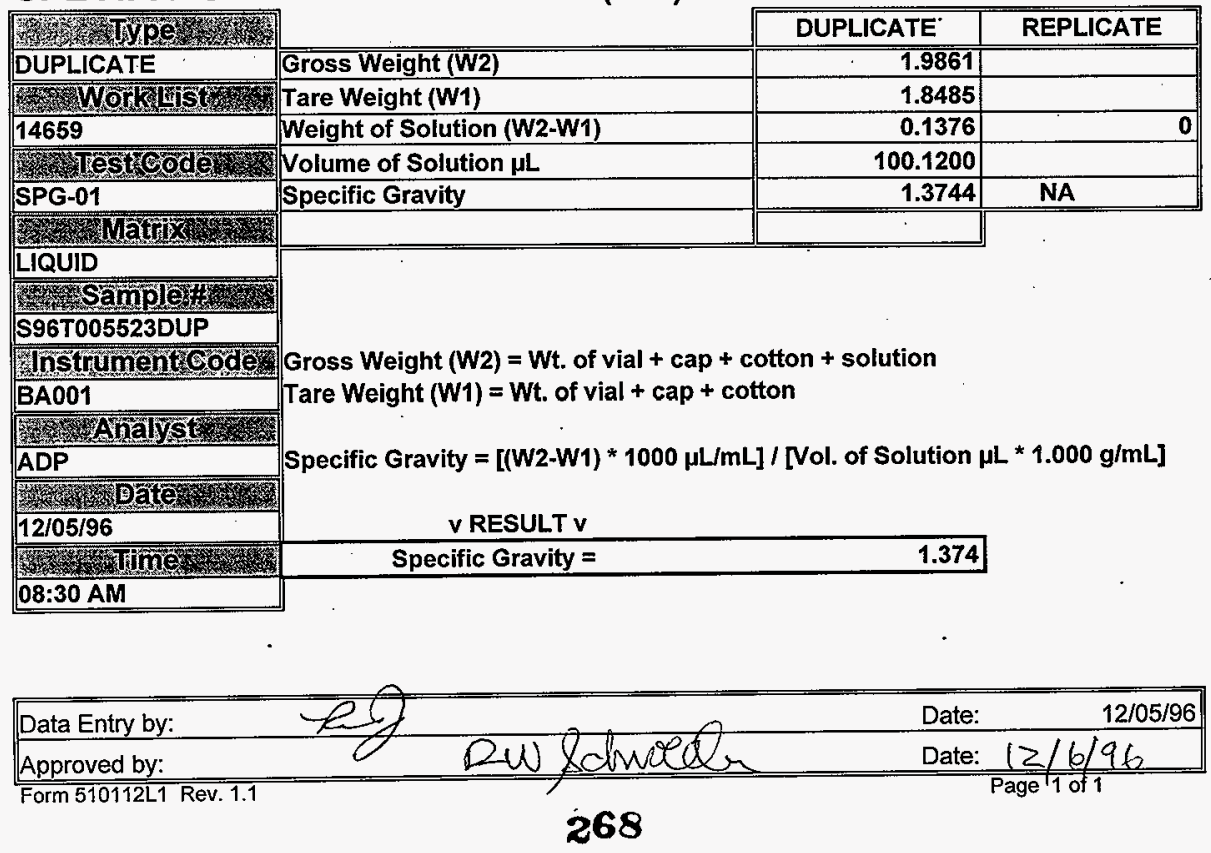




\section{LABCORE Completed Worklist Report for Worklist\# 14375}

Analyst: smf

Instrument: IC01

Book\# $36420 C$

Method: $\angle A-533-105 \operatorname{Rev} / \mathrm{Mod} D-1$

Worklist Comment: @IC-01 FOR B-108

RTS!

\begin{tabular}{|c|c|c|c|c|c|c|c|}
\hline Seq Type & Sample & $\# \mathbf{R} \mathbf{A}$ & & Test & Matrix & Actual & Found DL or Yield Unit \\
\hline $1 \mathrm{CeB}$ & & 0 & $8 \mathrm{sCl}_{0}$ & m & 00 & & $1 / 8002$ \\
\hline $1 \mathrm{ccB}$ & & 0 & $\triangle I C-Q C$ & $C L$ & $Q c$ & 1 & $<1.700-2$ \\
\hline $1 \mathrm{ccs}$ & & 0 & OIC OC & no2 & 00 & 1 & $01,080-1$ \\
\hline $1 \mathrm{CCB}$ & & 0 & $\triangle I C-Q C$ & $\mathbf{B R}$ & $9 c$ & 1 & $<1.250-1$ \\
\hline $10 \mathrm{cs}$ & & 0 & OIC 0 & $\mathrm{No3}$ & 60 & 1 & $1-390-1$ \\
\hline $1 \mathrm{ccB}$ & & $\therefore$ & $0 I C-Q C$ & PO4 & 25 & 1 & $<1.200-1$ \\
\hline $1 \mathrm{CAB}$ & & 0 & $\operatorname{scc} 00$ & 804 & $0 c$ & 1 & $<1<801$ \\
\hline $1 \mathrm{CCB}$ & & 0 & OIC-QC & OXAT_ATE2 & 80 & 1 & $<1.050-1$ \\
\hline $2 \mathrm{cct}$ & & 0 & $0 \mathrm{rct} 0 \mathrm{C}$ & $\mathrm{B}$ & 00 & $5 \% 0001$ & $5400+01 / 4,1000000 \%$ Recovory $/$ । \\
\hline $2 \mathrm{ccv}$ & & 0 & $Q I C-Q C$ & CL & $8 c$ & 7.90002 & $94.051 \%$ Recovery \\
\hline $200 v$ & & 0 & $0+\mathrm{C}-\mathrm{C}$ & no2 & 80 & 5.1002 & $93,346 \%$ Recovory \\
\hline $2 \mathrm{ccv}$ & & 0 & $B I C-Q C$ & BR & $\propto c$ & 5.89002 & $97.963 \%$ Recovery \\
\hline $2 \mathrm{Cov}$ & & 0 & $0=0-0$ & no3. & oc & 504002 & $2,95,286 / 200076 \mathrm{Y}$ \\
\hline $2 \mathrm{ccv}$ & & 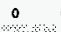 & $\operatorname{arc-QC}$ & PO4 & $8 c$ & $5.44 e_{002}$ & $93.566 \%$ Recovery \\
\hline 200 & & 0 & 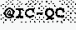 & sot & $0 \%$ & 6,3002 & $960890 \%$ ro-000-Y) \\
\hline $2 \mathrm{ccv}$ & & 0 & $Q I C-Q C$ & OXATATE2 & Qc & 5.25002 & $102.476 \%$ Recovery \\
\hline 3 SAMPDE & $\$ 960005463$ & 0 & $01001 \%$ & 102 & LPougd & W/A. & 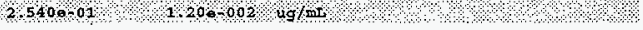 \\
\hline 3 SAMPLE & 5967005463 & 0 & QIC-01 & $\mathrm{CL}-02$ & LIQJID & $\mathbb{N} / \mathbf{A}$ & $1.717 \mathrm{ug} / \mathrm{mL}$ \\
\hline 3 SAMPIE & $806 \times 005463$ & 0 & $010-01$ & 10202 & LIQUID & a/A. & $0,100 \mathrm{ug} / \mathrm{mat}$ \\
\hline 3 SAMPLE & 5967005463 & 0 & QIC- 01 & BR-02 & IIQUID & $\mathrm{N} / \mathrm{A}$ & $0.125 \mathrm{ug} / \mathrm{mL}$ \\
\hline 3 SAMPXE & 5965005463 & 0 & $9 \mathrm{~s} 01$ & $\mathrm{NO}_{3}-02$ & $\mathrm{LSOUTD}$ & $3 / 2$ & $0.139 \mathrm{ug} / \mathrm{mr}$ \\
\hline 3 SAMPLE & 5967005463 & 0 & QIC- 01 & $P O 4-02$ & IIQUTD & $N / 2<$ & $0.120 \mathrm{ug} / \mathrm{rLI}_{\mathrm{s}}$ \\
\hline 3 SAMPSE & 5962005463 & 0 & $8+C 01$ & $504-02$ & $4 T Q M \times D$ & SIl A & $40-138$ og $/ \mathrm{mit}$ \\
\hline 3 SAMPLE & 5967005463 & 0 & QIC-01 & OXALATE2 & IIQVID & $n / A<$ & $0.105 \mathrm{ug} / \mathrm{mr}$ \\
\hline $4 \mathrm{pop}$ & 5967005468 & 0 & $0 x-01$ & $\mathrm{P} 02$ & 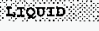 & 254001 & $2.63-01$ मे \\
\hline $4 \mathrm{DUP}$ & 5967005463 & 0 & $\operatorname{arc-01}$ & CL-02 & LIQUID & $1.010+01$ & $18.018 \mathrm{RPD}$ \\
\hline $4 \mathrm{pDP}$ & 596700563 & 0 & $6 \leq c+0$ & 10202 & TIOOND & $1,070+01$ & 0,506 RPD \\
\hline $4 \mathrm{DOP}$ & $596 \mathrm{T005463}$ & 0 & QIC-01 & BR-02 & LIQUID & $4.33 e-01$ & $0.000 \mathrm{RPD}$ \\
\hline $4 \mathrm{DOP}$ & 5969005468 & $\%$ & $0 x \mathrm{CO}_{01}$ & $\mathrm{No3}, 02$ & I $=0,0$ & $5110+01$ & $8140+01$ \\
\hline $4 \mathrm{DOP}$ & $596 \mathrm{T005463}$ & 0 & $\operatorname{QIC-01}$ & PO4-02 & LIQUTD & $<1.200-1$ & $1.51 e-01$ \\
\hline $4 \mathrm{pup}$ & 5965005463 & 6 & $8 C_{01}$ & 80400 & OLQLID & $1,630+01$ & $1 / 62001$ \\
\hline 4 DUP & $\$ 96 \mathrm{T005463}$ & 0 & $\operatorname{DIC-01}$ & OXPLATE2 & I.IQOID & $<1.050-1$ & $<1.05 e-1$ \\
\hline
\end{tabular}

\section{Final page for worklist\# 14375}

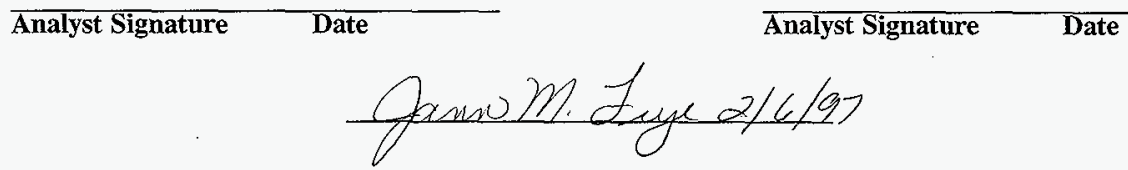

Units shown for QC (BLK/BKG) may not reflect the actual units. 


\section{LABCORE Data Entry Template for Worklist\# 14375}

Analyst: SMF Instrument: ICO 1001 Book\# $36020-C$

Method: LA-533-105 Rev/Mod D-1

Worklist Comment: @IC-01 FOR B-108

RTS!

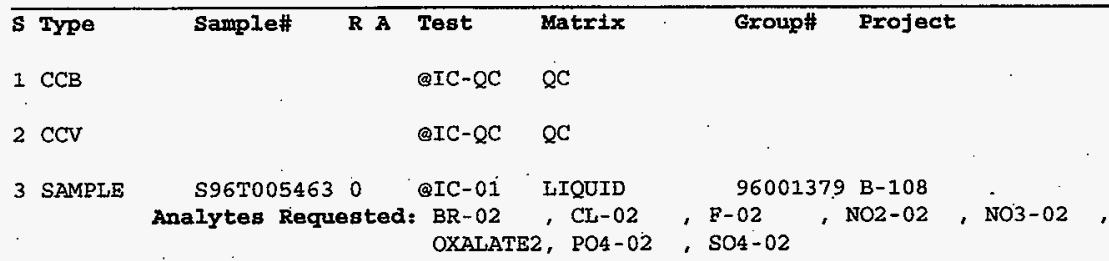

4 DUP S96T005463 $0 . \quad$ @IC-01 IIQUID

\section{Final page for worklist \# 14375}

\section{$\frac{\text { Susee Y7. Pulten 1-7-97 }}{\text { Date }}$

$14375 j \mathrm{j}$. sch

$14375 j y . c 5 V$ revewed 1/28197 कT mfarlond

uploaded of $/$ or $>$ me

Ualudated 2/4/97 gmF

Data Entry Comments:

$S=$ Worklist Slot Number, $R=$ Replicate Number, $A=$ Aliquot Code. 
HNF-SD-WM-DP-219, REV. 0

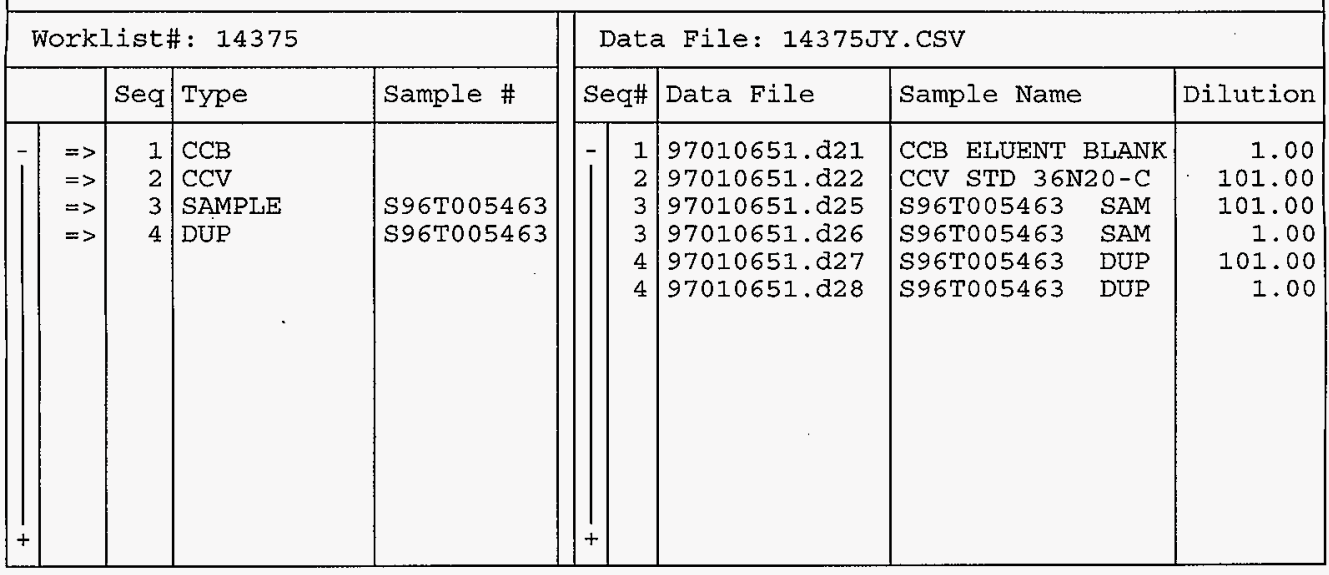

Save(F4) Abort(Shift-F3) ListFiles(Shift-FI) UploadFile(F8) 
Data Reprocessed On $01 / 28 / 199713: 22: 41$

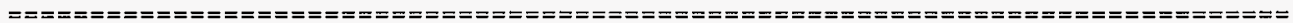

Sample Name: CCB ELUENT BLANK

Date: $01 / 07 / 199704: 19: 18$

Data File : F: \DATA $\backslash 97010651$. d21

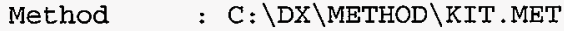

ACI Address: 1 system: 1 Inject\#: 21

Detector: CDM-1

Analyst

Column: AG4A/AS4A anion column

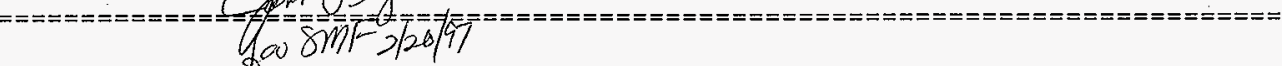

Calibration Volume Dilution Points Rate start stop Area Reject

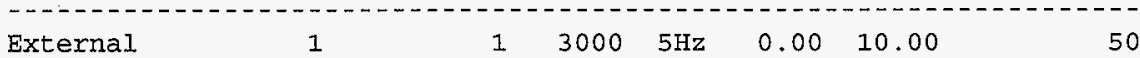

$* * * * * * * * * * * * * * * * * * * * * * * * * * *$ Peak Report: All Peaks $* * \star * * * * * * * * * * * * * * * * * * * * * * * * *$

$\begin{array}{lrrr}\text { Pk. Ret Component } & \text { Concentration } & \text { Height } & \text { Area Bl. } \\ \text { Num } & \text { uglimla } / \mathrm{ml} & \text { Code }\end{array}$

13.48 nitrate

0.033

47

316

$1-2.79$

Totals

0.033

47

316

File: 97010651.d21 Sample: CCB ELUENT BLANK

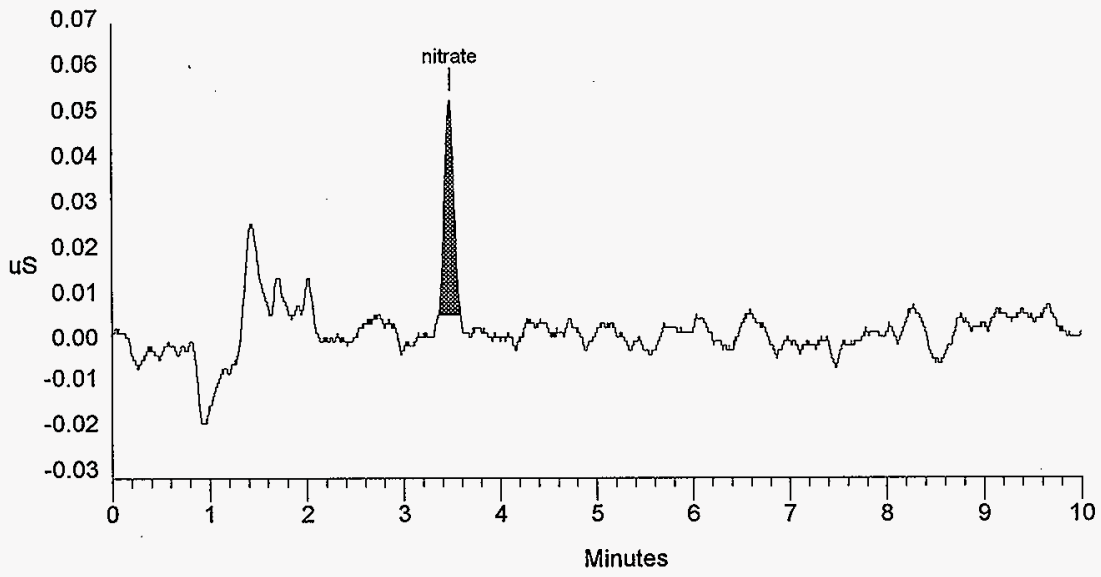

SIGNATURE ABOVE REPRESENTS CHEMICAL TECHNOLOGIST/CHEMIST THAT CONPLETEDNERIFIED THE CALIBRATION/ANALYSIS ON PAGES 272 TO277. 
Data Reprocessed on $01 / 28 / 199713: 22: 44$

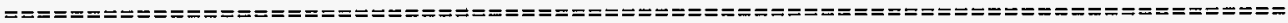

Sample Name: CCV STD 36N20-C

Date: $01 / 07 / 199704: 30: 12$

Data File : F: \DATA \97010651.d22

Method : C: $\backslash \mathrm{DX} \backslash \mathrm{METHOD} \backslash \mathrm{KIT} . \mathrm{MET}$

ACI Address: 1 System: 1 Inject\#: 22 Detector:CDM-1

Analyst : Column: AG4A/AS4A anion column

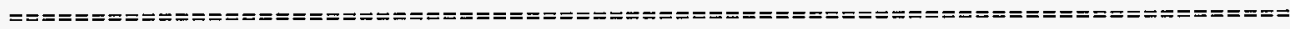

Calibration Volume Dilution Points Rate start Stop Area Reject

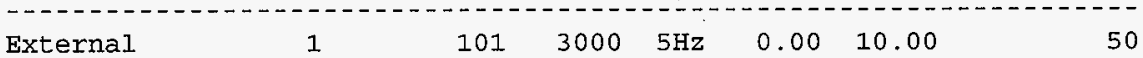

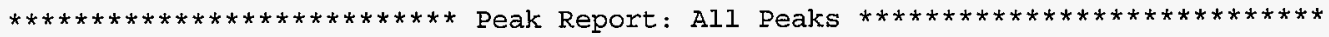

$\begin{array}{lrrr}\text { Pk. Ret Component } & \text { Concentration } & \text { Height } & \text { Area Bl. } \\ \text { Num } & \text { Time Name } / \mathrm{ml} & \text { Code }\end{array}$

21.11 fluoride

31.66 chloride

41.97 nitrite

$5 \quad 2.96$ bromide

$6 \quad 3.37$ nitrate

74.91 phosphate

86.40 sulfate

9

8.43 oxalate

\subsection{4}

74.324

504.809

577.085

565.516

508.855

612.146

538.276
1192

1776

6117

4967

5576

1521

4636

2175
7790

9338

42281

33705

44519

20263

64955

41174

27960

264025

$\begin{array}{rr}1 & 2.47 \\ 1 & -0.40 \\ 1 & -2.95 \\ 1 & 0.00 \\ 1 & -5.77 \\ 1 & -1.08 \\ 1 & -3.23 \\ 1 & -3.07\end{array}$

Totals

3440.043

\section{File: 97010651.d22 Sample: CCV STD 36N20-C}

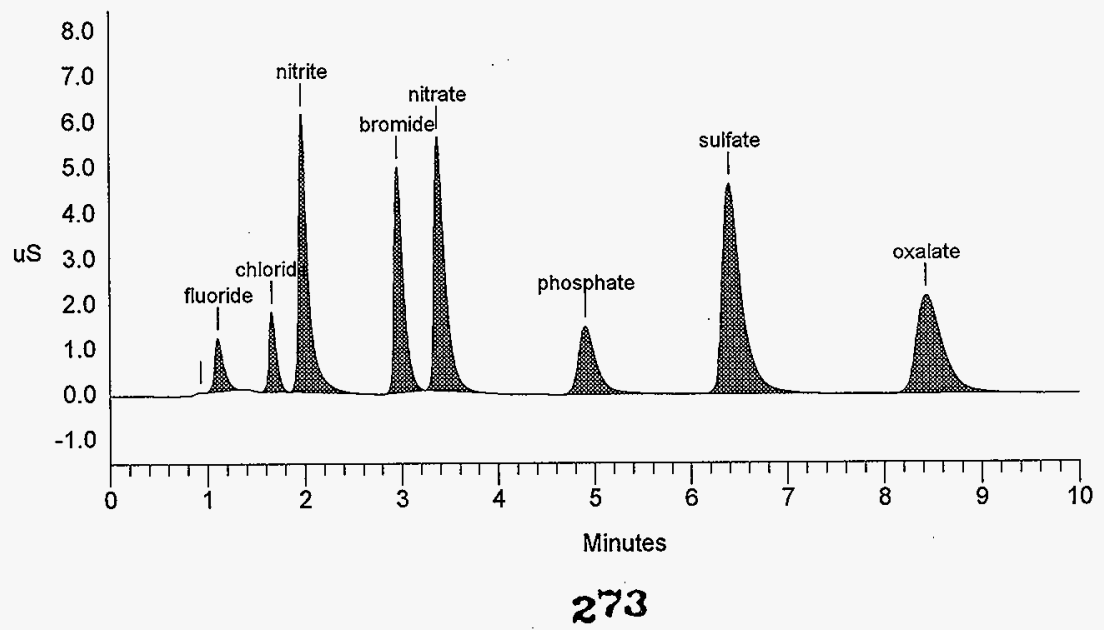


Data Reprocessed on 01/28/1997 13:22:46

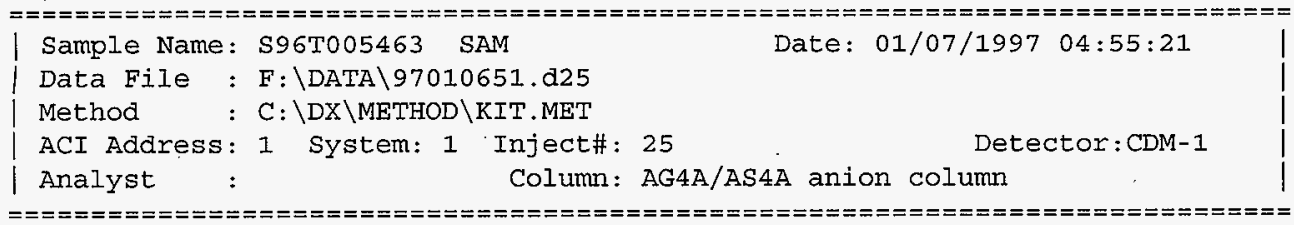

Calibration Volume Dilution Points Rate start Stop Area Reject

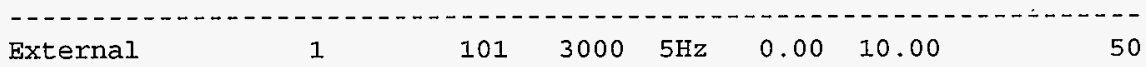

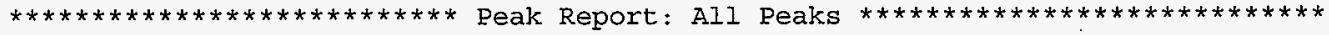

$\begin{array}{lrrr}\text { Pk. Ret component } & \text { Concentration } & \text { Height } & \text { Area BI. } \\ \text { Num Delta } & \text { Code } / \mathrm{ml} & \text { Code Name } & \end{array}$

$1 \quad 1.68$ chloride

22.01 nitrite

33.45 nitrate

46.51 sulfate

$\begin{array}{cccc} & 10.046 & 219 & 1203 \\ 28.847 & 184 & 1263 \\ 43.721 & 463 & 3450 \\ & 6.189 & 106 & 1545 \\ \text { Totals } & 88.803 & 971 & 7462\end{array}$

1203

10.80

$1 \quad-0.98$

$1-3.54$

$1-1.61$

\section{File: 97010651.d25 Sample: S96T005463 SAM}

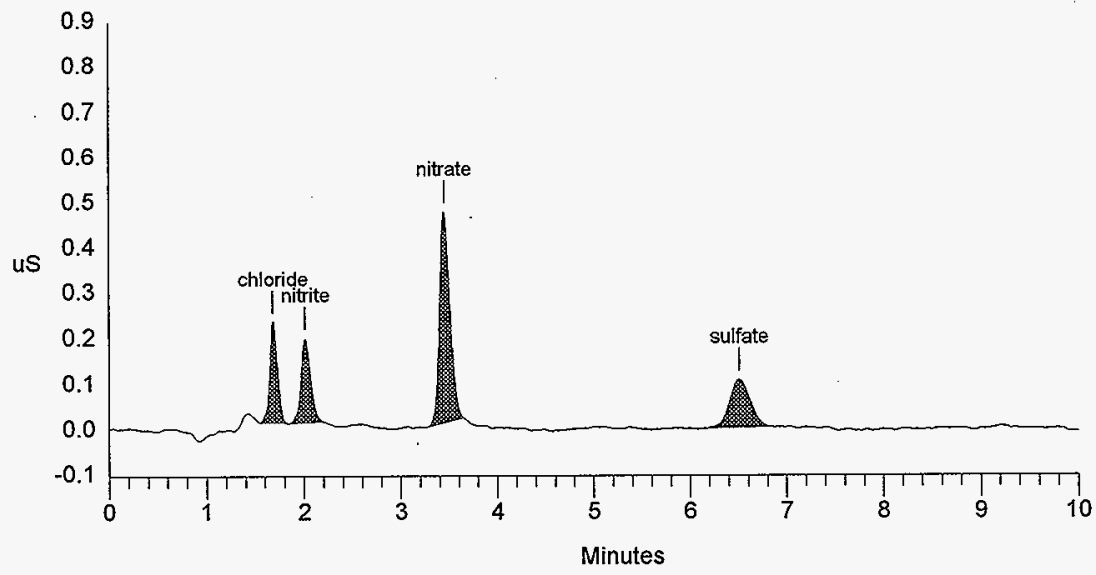


Data Reprocessed on 01/28/1997 13:22:48

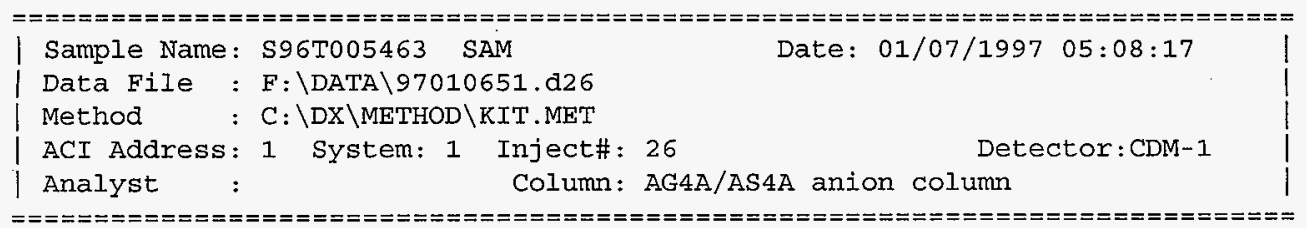

Calibration Volume Dilution Points Rate start stop Area Reject

\begin{tabular}{|c|c|c|c|c|c|c|c|}
\hline External & 1 & 1 & 3000 & $5 \mathrm{~Hz}$ & 0.00 & 10.00 & 50 \\
\hline
\end{tabular}

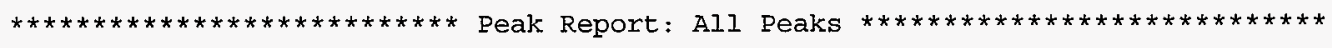

$\begin{array}{lrrr}\text { Pk. Ret Component } & \text { Concentration } & \text { Height } & \text { Area Bl. } \\ \text { Num Delta }\end{array}$
Num Time Name

\begin{tabular}{|c|c|c|c|c|c|}
\hline & 0.254 & 542 & 2958 & 2 & 4.32 \\
\hline & 0.000 & 125 & 877 & 2 & \\
\hline & 9.421 & 32641 & 147428 & 1 & -0.40 \\
\hline & 19.697 & 28201 & 176060 & 1 & -2.30 \\
\hline & 0.433 & 347 & 1934 & 1 & -3.32 \\
\hline & 51.089 & 44163 & 463884 & 1 & 0.10 \\
\hline & 16.090 & 12980 & 176451 & 1 & -4.03 \\
\hline \multicolumn{6}{|c|}{ 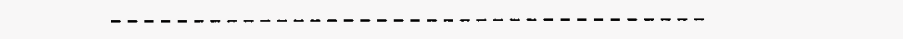 } \\
\hline Totals & 96.984 & 118999 & 969592 & & \\
\hline
\end{tabular}

File: 97010651.d26 Sample: S96T005463 SAM

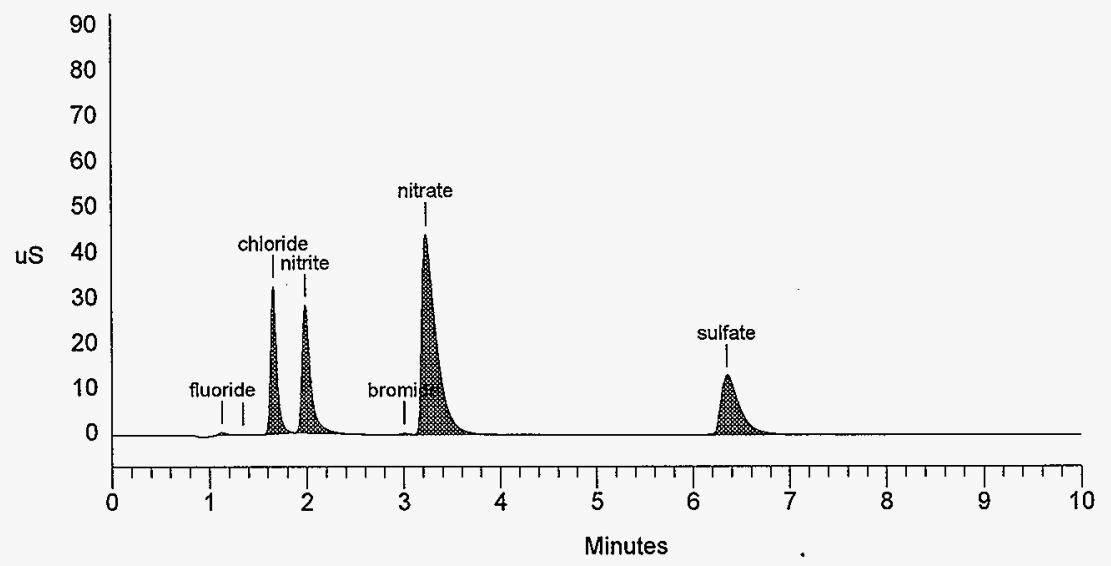


Data Reprocessed On 01/28/1997 13:22:50

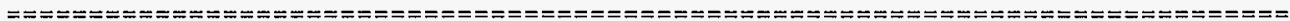

| Sample Name: S96T005463 DUP

Data File : F:\DATA \97010651.d27

Date: $01 / 07 / 199705: 21: 25$

Method : C: $\backslash D X \backslash M E T H O D \backslash K I T . M E T$

ACI Address: 1 System: 1 Inject\#: 27

Detector: $\mathrm{CDM}-1$

Analyst

Column: AG4A/AS4A anion column

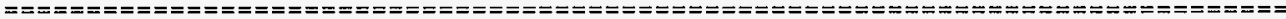

Calibration Volume Dilution Points Rate Start Stop Area Reject Calibration Volume Dilution Points Rate Start

External

$1 \quad 101 \quad 3000 \quad 5 \mathrm{~Hz} \quad 0.00 \quad 10.00$

50

$* * * * * * * * * * * * * * * * * * * * * * * * * * *$ Peak Report: All Peaks $* * * * * * * * * * * * * * * * * * * * * * * * * * * *$

$\begin{array}{lrrr}\text { Pk. Ret Component } & \begin{array}{r}\text { Concentration } \\ \mathrm{ug} / \mathrm{ml}\end{array} & \text { Height } & \text { Area Bl. } \\ \text { Num Dime Name } & & \text { Code }\end{array}$

Num Time Name

22

126

1

$1 \quad 1.43$

0.000

275

1462

$1 \quad 1.20$

1.69 chloride

12.110

227

1523

$1-0.98$

$4 \quad 3.45$ nitrate

61.182

602

4808

$1-3.54$

56.45 sulfate

14.897

134

2440

$1-2.42$

Totals

120.077

1260

10359

File: 97010651.d27 Sample: S96T005463 DUP

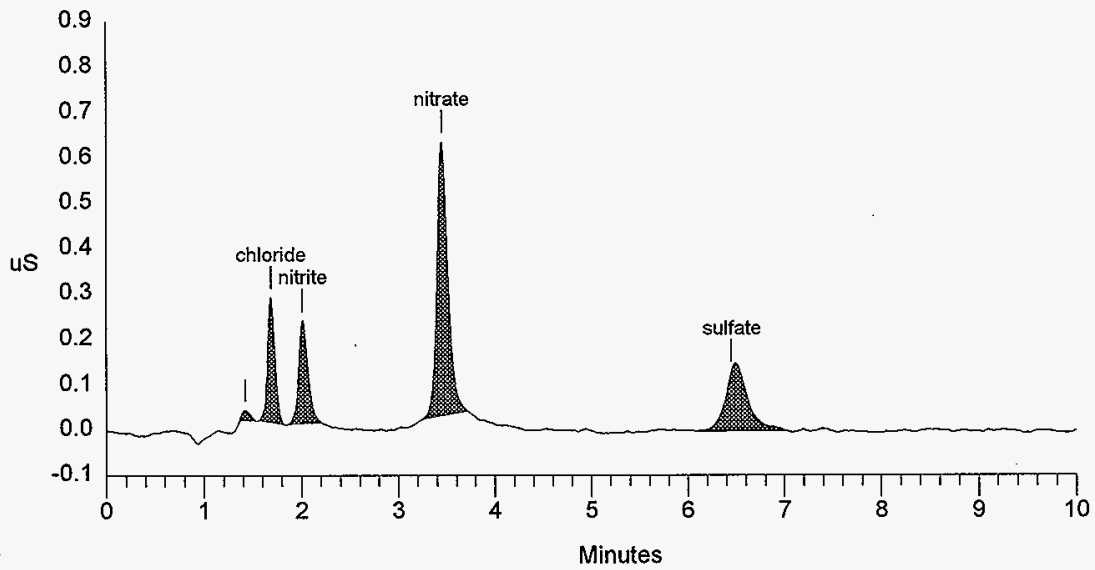


HNF-SD-WM-DP-219, REV. 0

Data Reprocessed On 01/28/1997 13:22:52

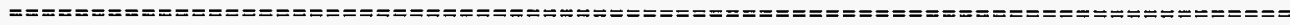

Calibration Volume Dilution Points Rate start Stop Area Reject

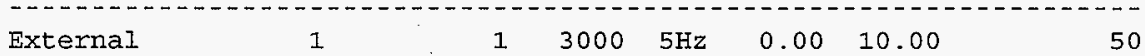

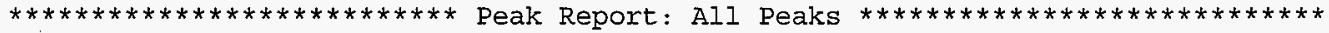

Pk. Ret Component

Num Time Name
Concentration Height

$\mathrm{ug} / \mathrm{ml}$

$\begin{array}{lll}1 & 1.13 & \text { fluoride } \\ 2 & 1.34 & \\ 3 & 1.66 & \text { chloride } \\ 4 & 1.99 & \text { nitrite } \\ 5 & 3.01 \text { bromide } \\ 6 & 3.23 \text { nitrate } \\ 7 & 4.96 \text { phosphate } \\ 8 & 6.35 \text { sulfate }\end{array}$

0.263

0.000

9.509

19.788

0.433

51.374

0.151

16.235
556

121

32737

28239

350

44250

19

12986

\section{Area Bl. \%Delta Code} Code

$\begin{array}{rrr}3095 & 2 & 4.32 \\ 891 & 2 & \\ 149255 & 1 & -0.40 \\ 176915 & 1 & -2.30 \\ 1936 & 1 & -3.32 \\ 466962 & 1 & 0.10 \\ 258 & 1 & 0.00 \\ 178116 & 1 & -4.03\end{array}$

Totals

97.753

119259

977429

File: 97010651.d28 Sample: S96T005463 DUP

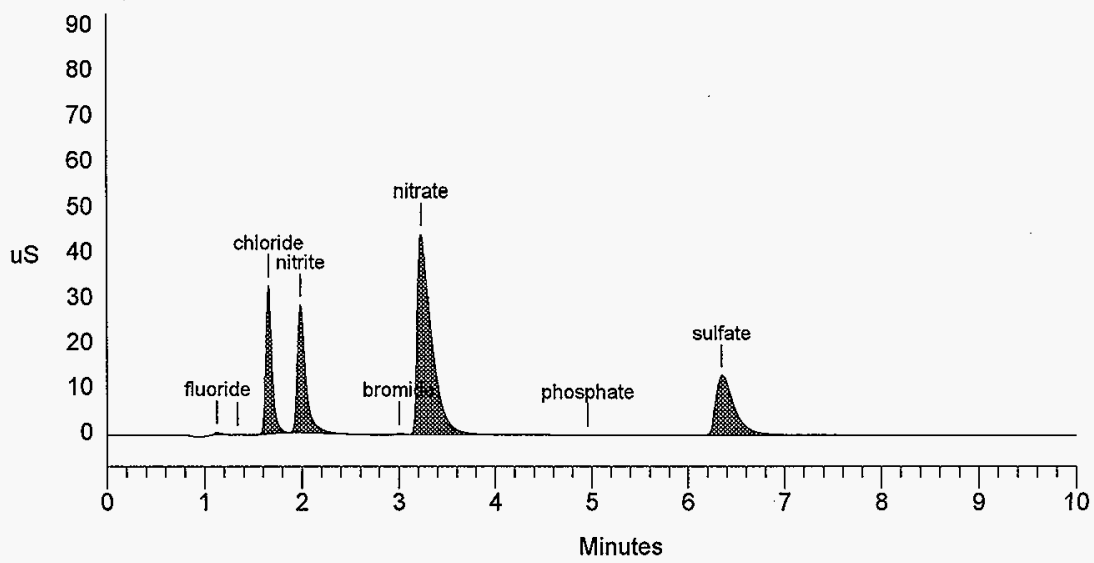




\section{LABCORE Completed Worklist Report for Worklist\# 14415}

Analyst: smf

Instrument: IC02

Book\# $36 \mathrm{N20} \cdot \mathrm{C}$

Method: LA53310S Rev/Mod D-1

Worklist Comment: ic-01 for b-108

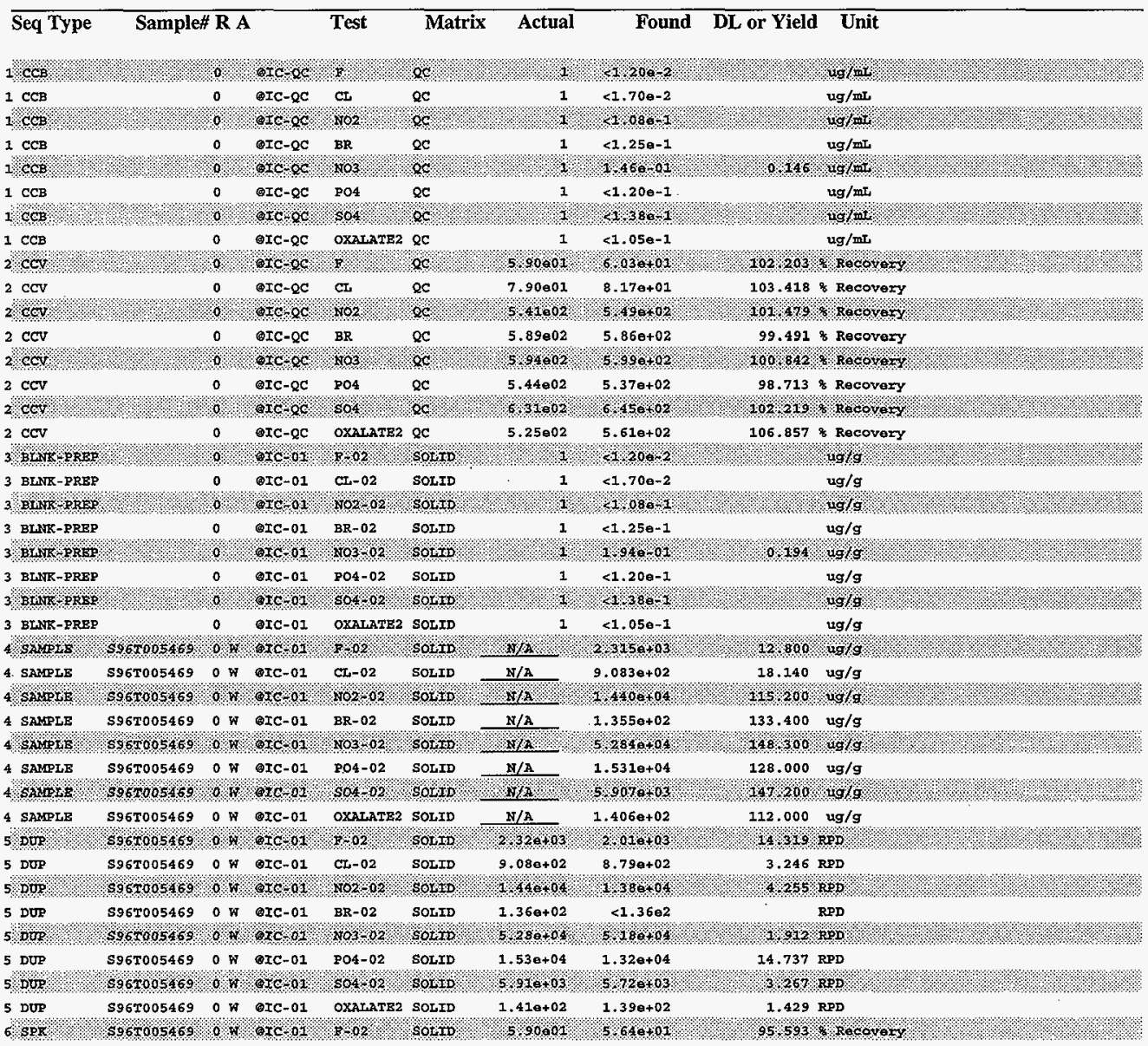




\section{LABCORE Completed Worklist Report for Worklist\# 14415}

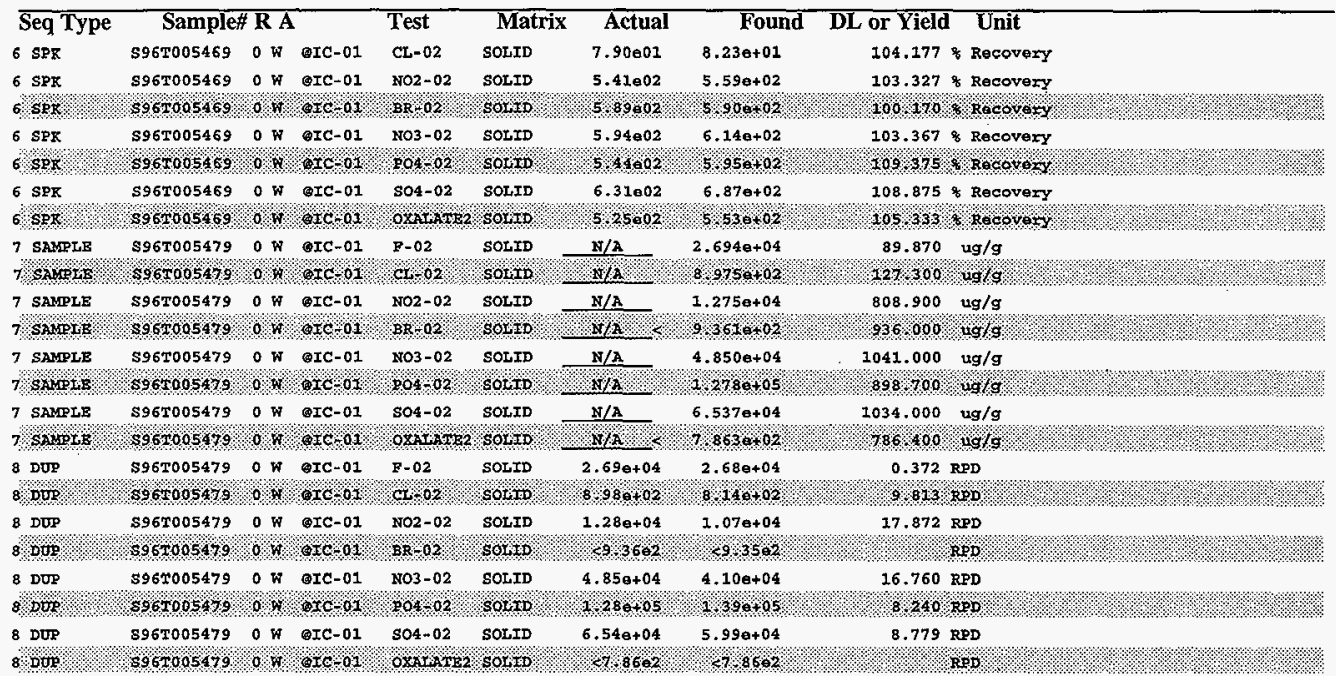

\section{Final page for worklist\# 14415}

$\overline{\text { Analyst Signature }}$ Date $\quad$ Analyst Signature

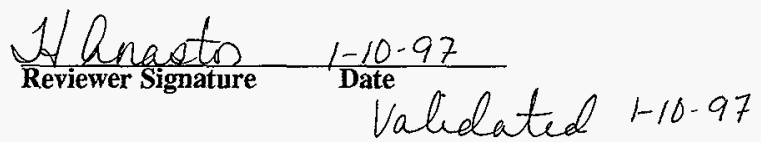




\section{LABCORE Data Entry Template for Worklist\# 14415}

Analyst: $\quad 5 M F$ Instrument: ICO $\mathrm{FCO} 2$ Book\# $36 \mathrm{NO20-C}$

Method: LA-533-105 Rev/Mod

Worklist Comment: ic-01 for b-108

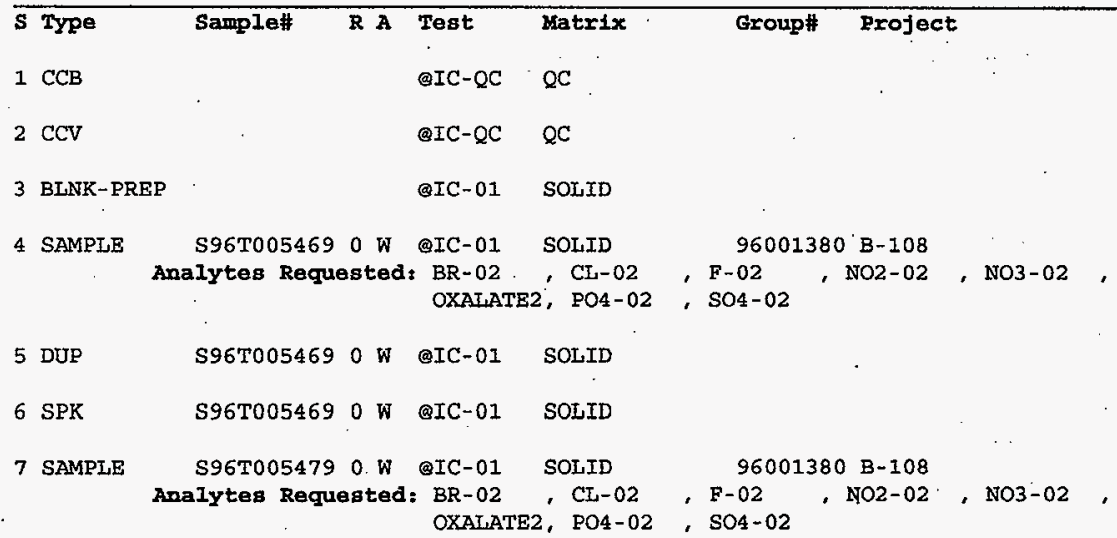

8 DUP S96T005479 O W OIC-01 SOLID

Final page for worklist \# 14415

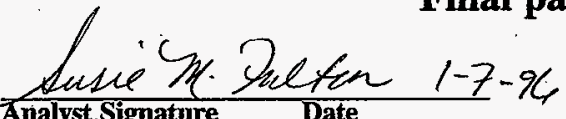

Analyst.Signature Date

Analyst Signature Date

$14415 J A$. CSV uploaded 1-10-97 Hea

Data Entry Comments:

$S=$ Worklist Slot Number, $R=$ Replicate Number, $A=$ Aliquot Code. 
HNF-SD-WN-DP-219, REV.O

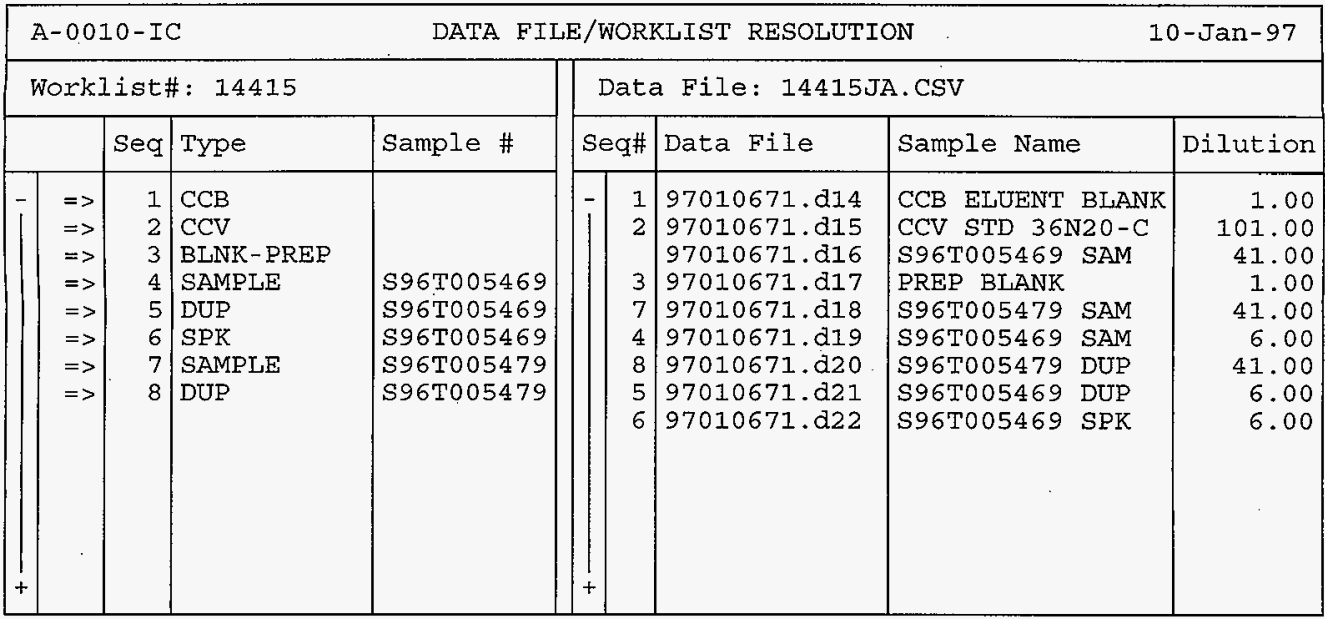

Save (F4) Abort (Shift-F3) ListFiles(Shift-F1) UploadFile(F8) 


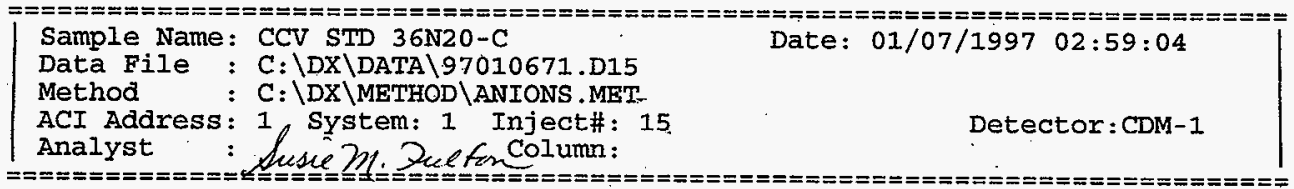

Cailbration Volume Dilution Points Rate start Stop Area Reject

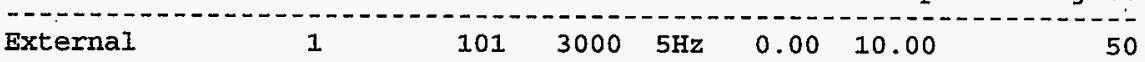

$* * * * * * * * * * * * * * * * * * * * * * * * * * *$ Peak Report: All Peaks $* * * * * * * * * * * * * * * * * * * * * * * * * * * *$

Pk. Ret Component Concentration Height Area Bl. :Delta Num Time Name $\mathrm{ug} / \mathrm{ml}$ Code

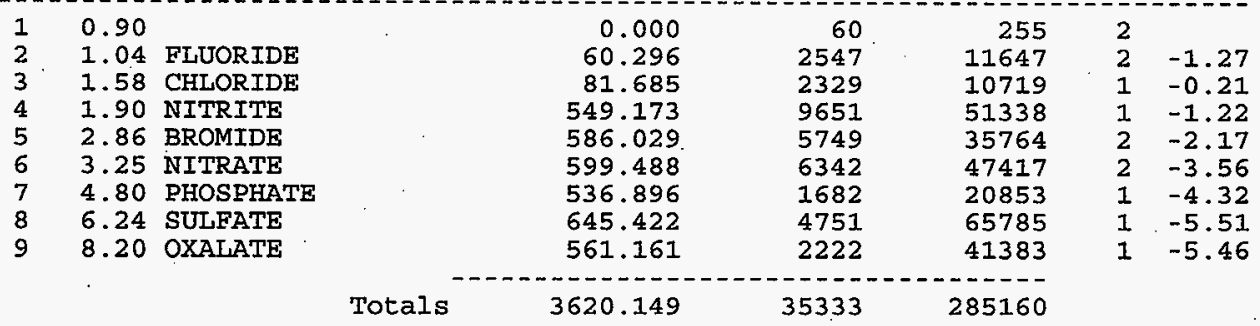

File: 97010671.D15 Sample: CCV STD 36N20-C

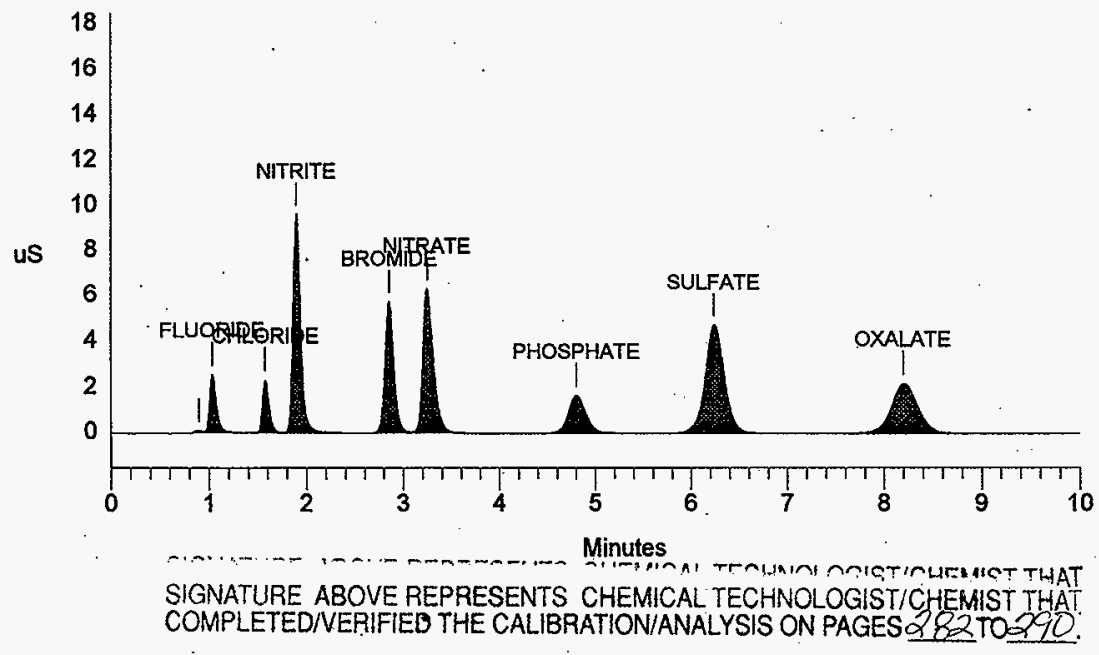


HNF-SD-WMADP-219, REV.O

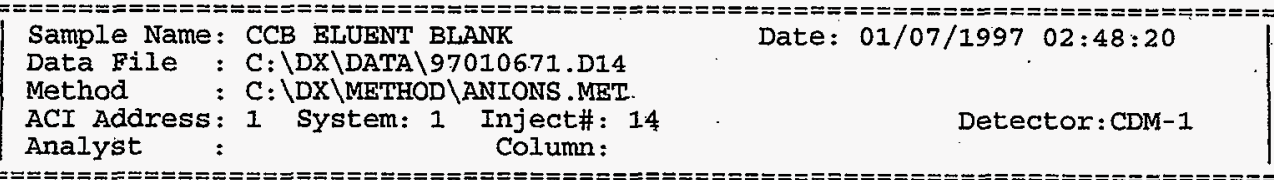

Calibration Volume Dilution Points Rate start Stop Area Reject

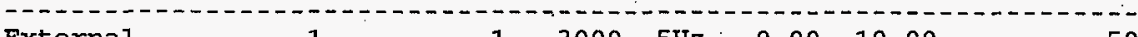

External

1

$13000 \quad 5 \mathrm{~Hz} \cdot 0.00,10.00$

50

$\star * * * * * * * * * * * * * * * * * * * * * * * * * *$ Peak Report: All Peaks $* * * * * * * * * * * * * * * * * * * * * * * * * * * *$

Pk. Ret Component Concentration Height Area Bl. $\%$ Delta

Num Time Name $\mathrm{ug} / \mathrm{ml}$ Code

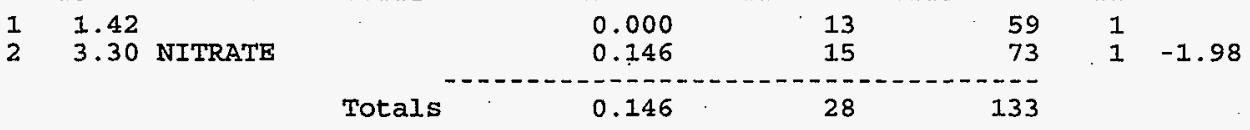

File: 97010671.D14 Sample: CCB.ELUENT BLANK

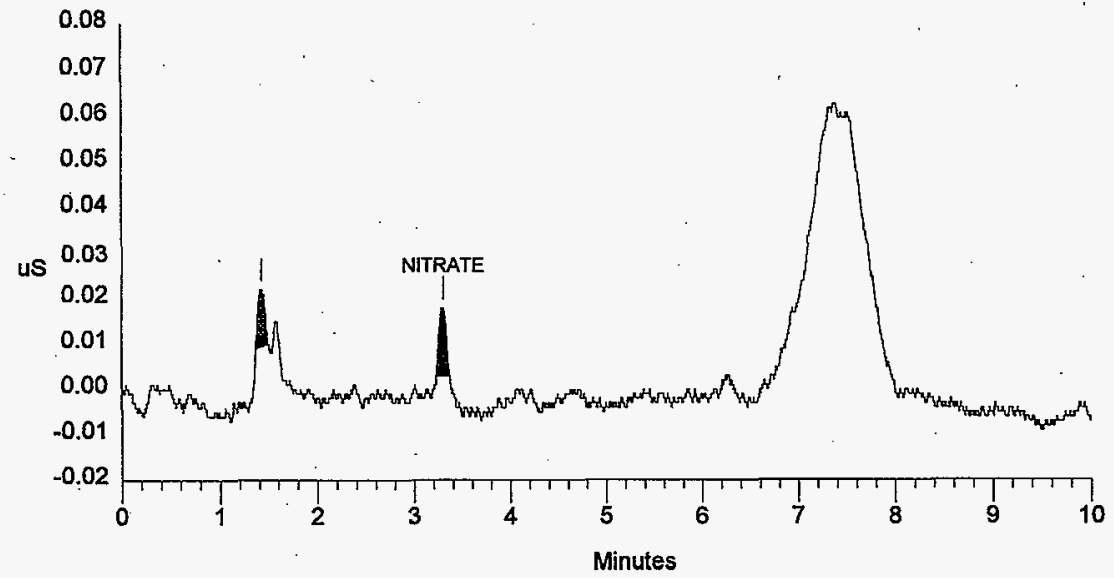




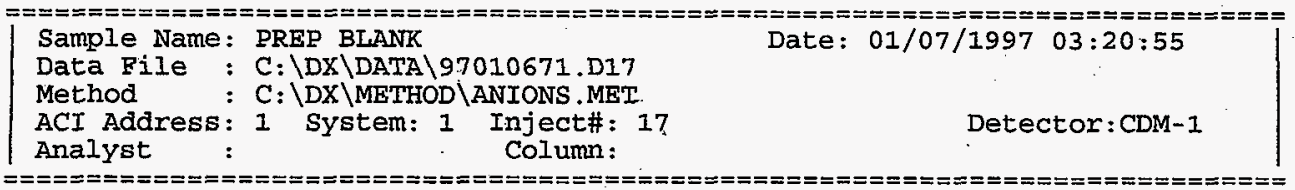

Calibration "Volume Dilution Points Rate start stop Area Reject

External

External

1

$\begin{array}{lllll}1 & 3000 \quad 5 \mathrm{~Hz} & 0.00 & 10.00\end{array}$

50

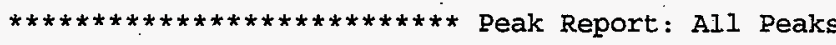

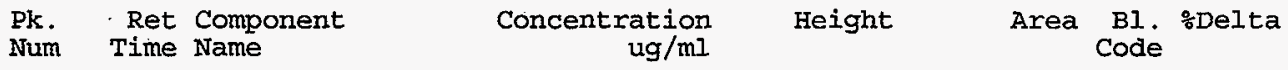

Num Time Name

$1 \quad 1.30$

$3 \quad 3.31$ NITRATE

\begin{tabular}{cccccc} 
& 0.000 & 44 & 429 & 1 & \\
& 0.194 & 73 & 456 & 1 & -1.78 \\
\hline Totals & 0.194 & 117 & 885 & &
\end{tabular}

File: 97010671.D17 Sample: PREP BLANK

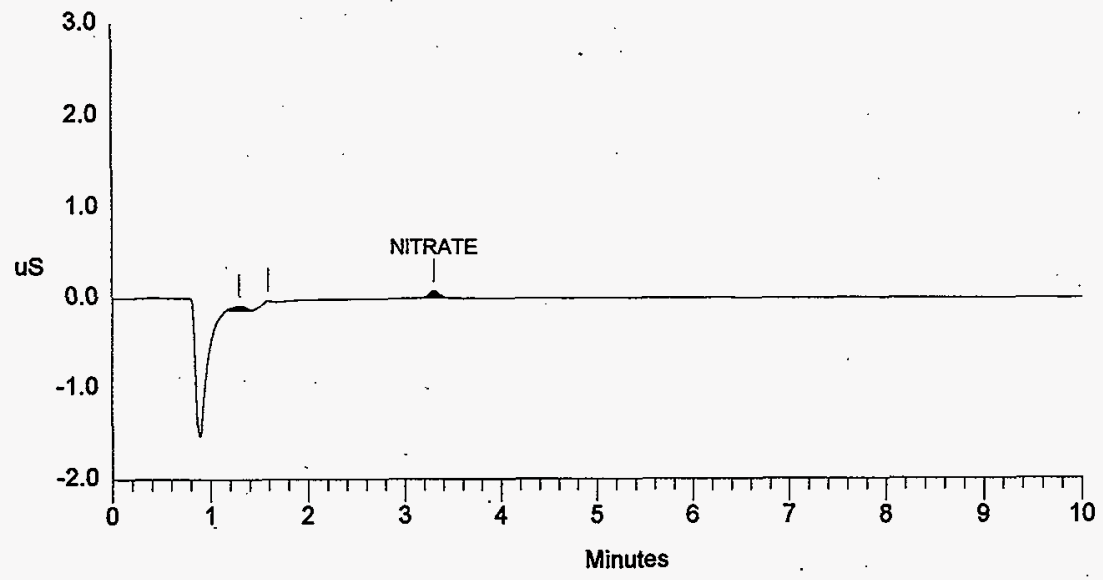




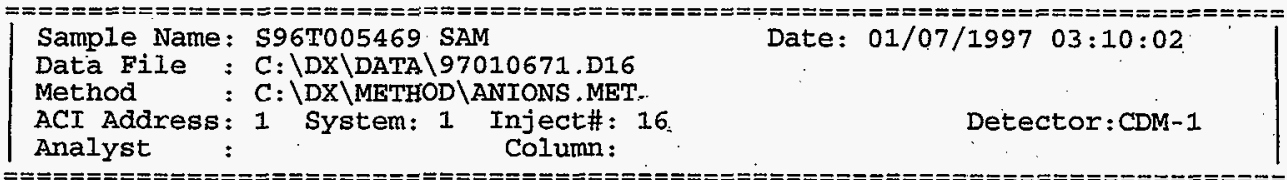

Calibration Volume Dilution Points Rate start stop Area Reject

Cal

External

1

$41 \quad 3000 \quad 5 \mathrm{~Hz} \quad 0.00 \quad 10.00$

50

$\star * * * * * * * * * * * * * * * * * * * * * * * * * *$ Peak Report: AIl Peaks $* * * * * * * * * * * * * * * * * * * * * * * * * * * *$

$\begin{array}{lrrr}\text { Pk. Ret Component } & \text { Concentration } & \text { Height } & \text { Area Bl. } \\ \text { Num } & \text { Time Name } / \mathrm{ml} & & \text { Code }\end{array}$

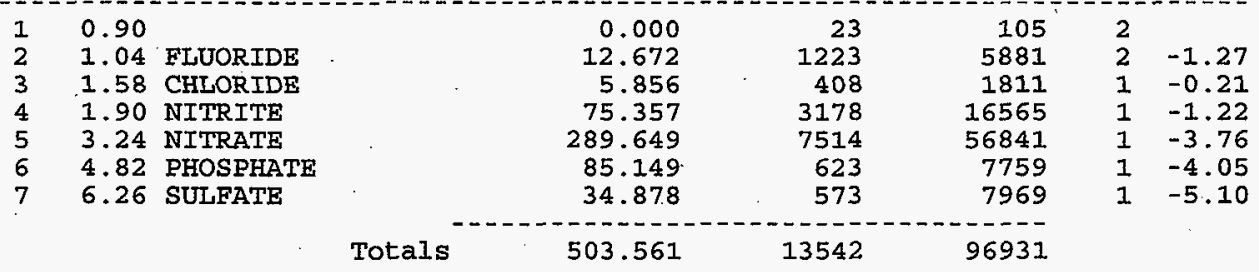

File: 97010671.D16 Sample: S96T005469 SAM

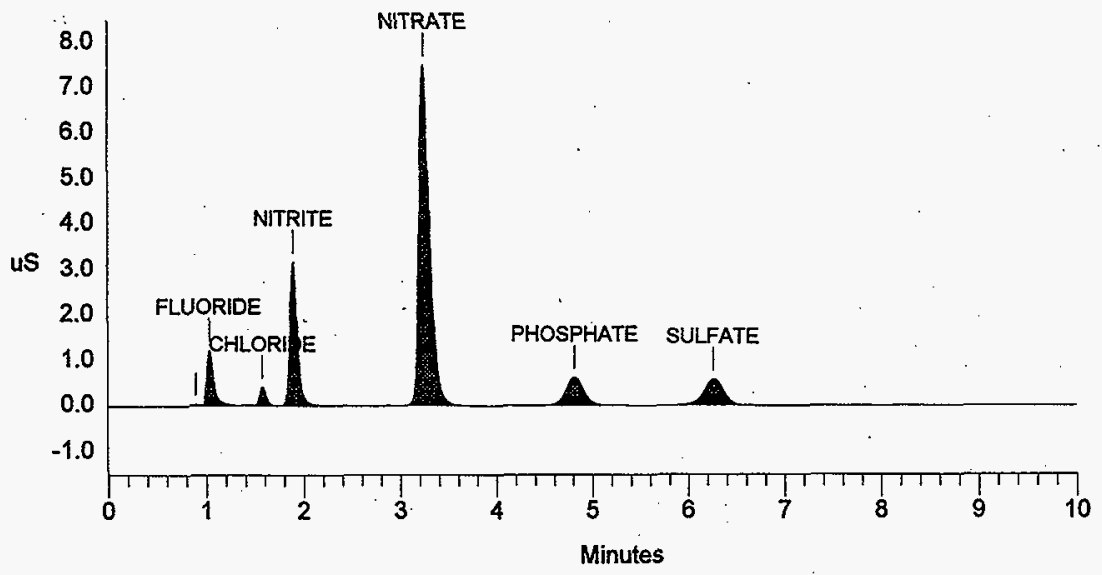

$q^{10} i^{4}$ 
Data Reprocessed on 01/09/1997 14:45:21

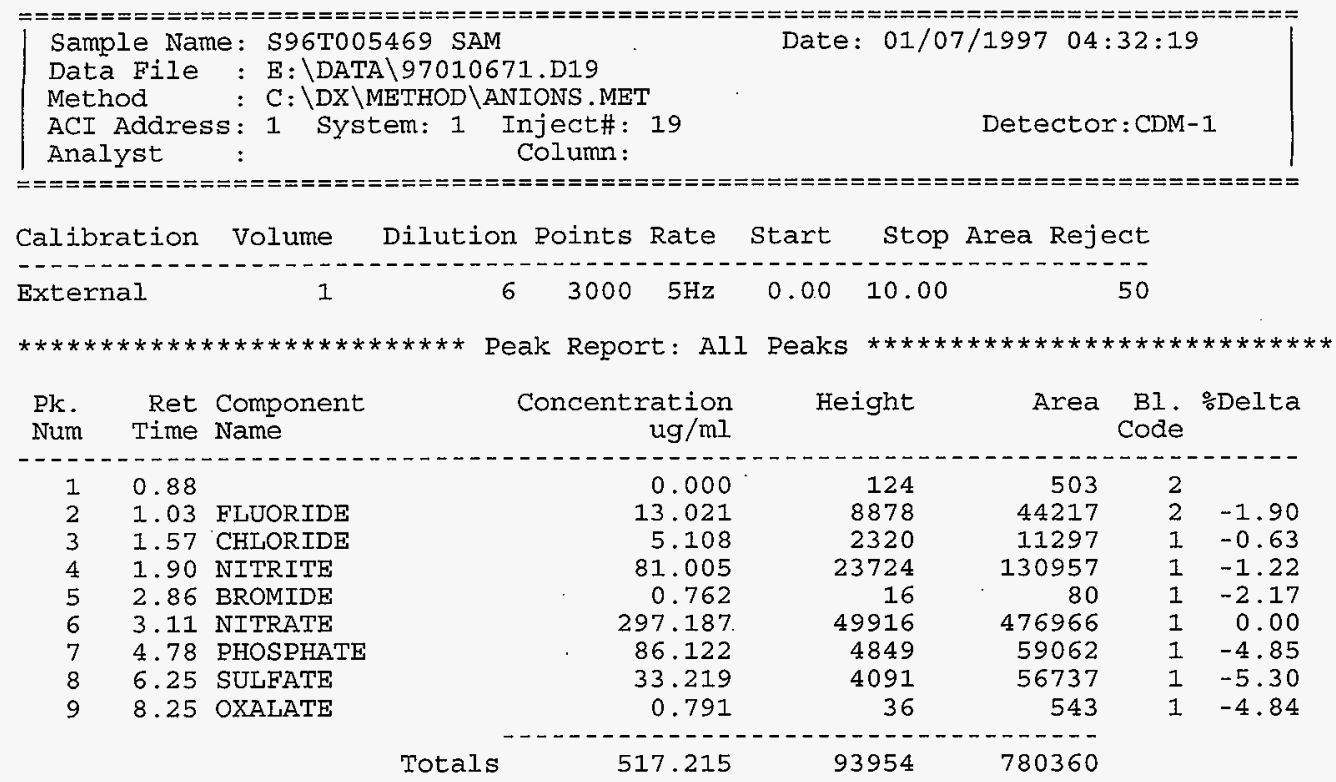

\section{File: 97010671.D19 Sample: S96T005469 SAM}

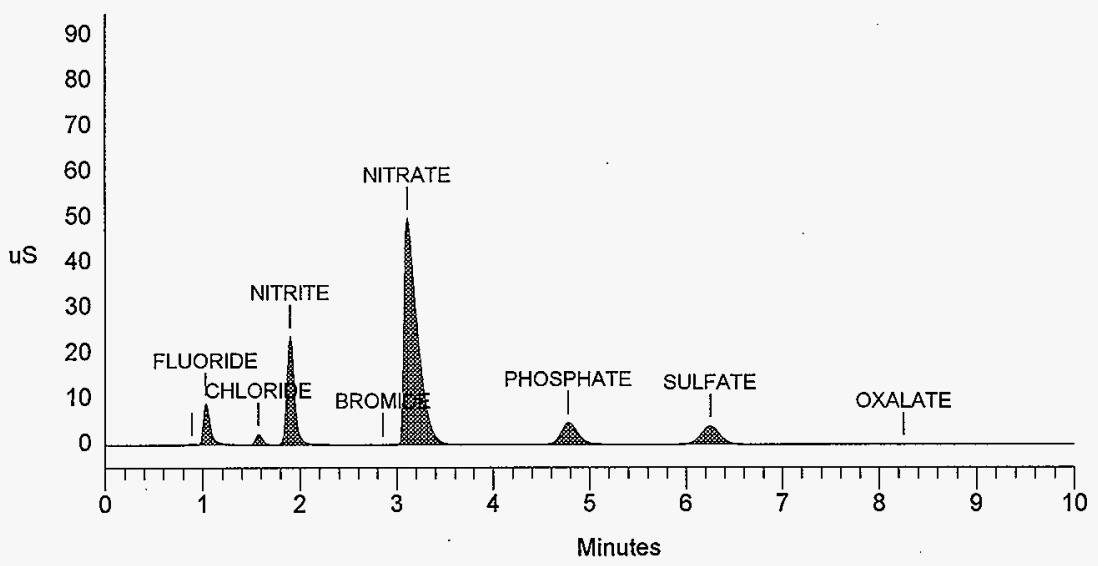


HINF-SD-WN-DP-219, REV.C

Data Reprocessed on 01/09/1997 14:46:26

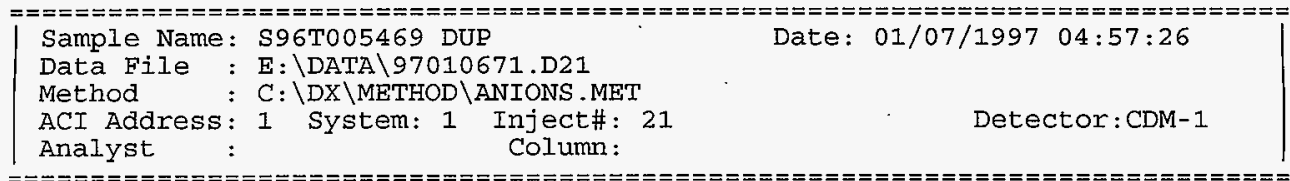

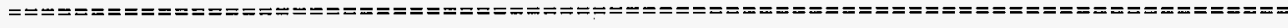

Calibration Volume Dilution Points Rate start stop Area Reject

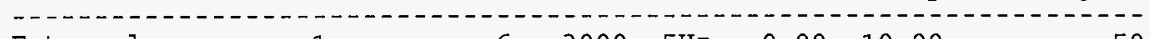

External

1

$\begin{array}{lllll}6 & 3000 & 5 \mathrm{~Hz} & 0.00 & 10.00\end{array}$

50

\begin{tabular}{|c|c|c|c|c|c|c|c|}
\hline $\begin{array}{l}\text { Pk. } \\
\text { Num }\end{array}$ & $\begin{array}{r}\text { Ret } \\
\text { Time }\end{array}$ & $\begin{array}{l}\text { Component } \\
\text { Name }\end{array}$ & $\begin{array}{r}\text { Concentration } \\
\mathrm{ug} / \mathrm{ml}\end{array}$ & Height & \multicolumn{3}{|c|}{ Code } \\
\hline 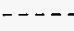 & - & - & & -- & ----- & -- & - \\
\hline 1 & 0.88 & & 0.000 & 131 & 598 & 2 & \\
\hline 2 & 1.04 & FLUORIDE & 11.125 & 7814 & 37523 & 2 & -1.27 \\
\hline 3 & 1.58 & CHLOR IDE & 4.855 & 2362 & 10725 & 1 & -0.21 \\
\hline 4 & 1.90 & NITRITE & 76.260 & 23447 & 123037 & 1 & -0.87 \\
\hline 5 & 2.86 & BROMIDE & 0.746 & 16 & 63 & 1 & -2.17 \\
\hline 6 & 3.12 & NITRATE & 286.369 & 48274 & 455427 & 1 & -0.11 \\
\hline 7 & 4.78 & PHOSPHATE & 73.043 & 4083 & 49604 & 1 & -4.71 \\
\hline 8 & 6.25 & SULFATE & 31.578 & 3903 & 53850 & 1 & -5.30 \\
\hline 9 & 8.26 & OXALATE & 0.770 & 33 & 517 & 1 & -4.69 \\
\hline & & & 484.747 & 90062 & 731344 & & \\
\hline
\end{tabular}

\section{File: 97010671.D21 Sample: S96T005469 DUP}

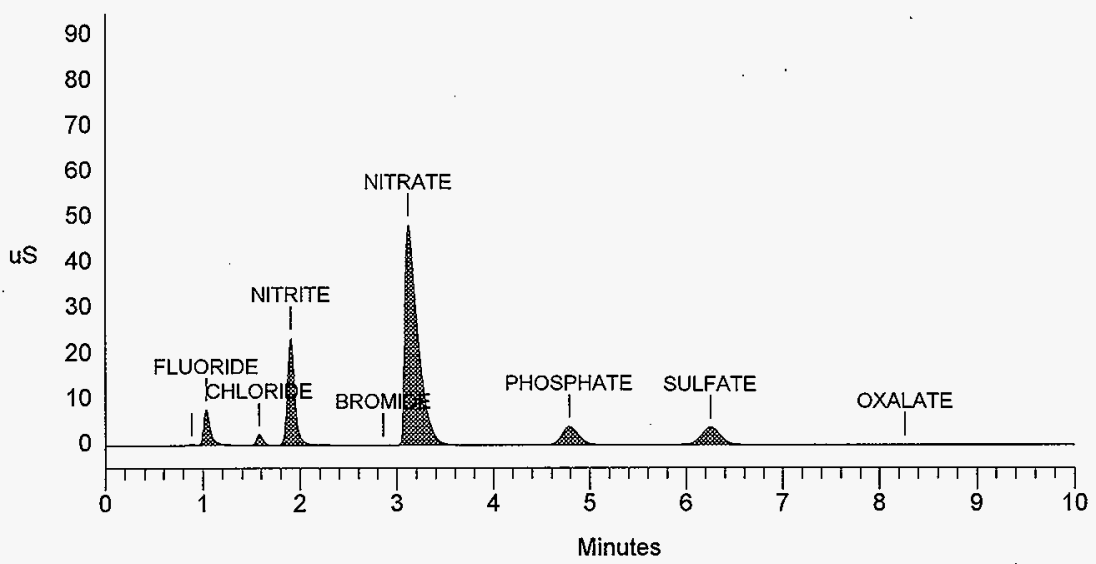


Data Reprocessed On 01/09/1997 14:47:40

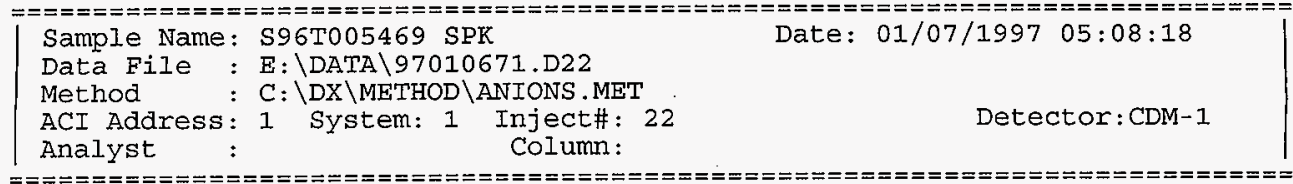

Calibration Volume Dilution Points Rate start stop Area Reject

Calibration Volumen

$\begin{array}{llllllll}\text { External } & 1 & 6 & 3000 & 5 \mathrm{~Hz} & 0.00 & 10.00 & 50\end{array}$

$\star * * * * * * * * * * * * * * * * * * * * * * * * * *$ Peak Report: All Peaks $* * * * * * * * * * * * * * * * * * * * * * * * * * * *$

$\begin{array}{lrrr}\text { Pk. Ret Component } & \text { Concentration } & \text { Height } & \text { Area Bl. } \\ \text { Num } & \text { Time Name } / \mathrm{ml} & & \text { Code }\end{array}$

Num Time Name

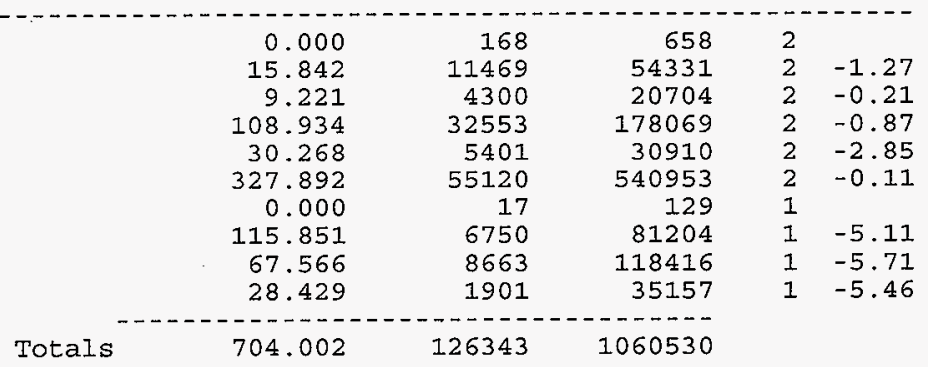

File: 97010671.D22 Sample: S96T005469 SPK

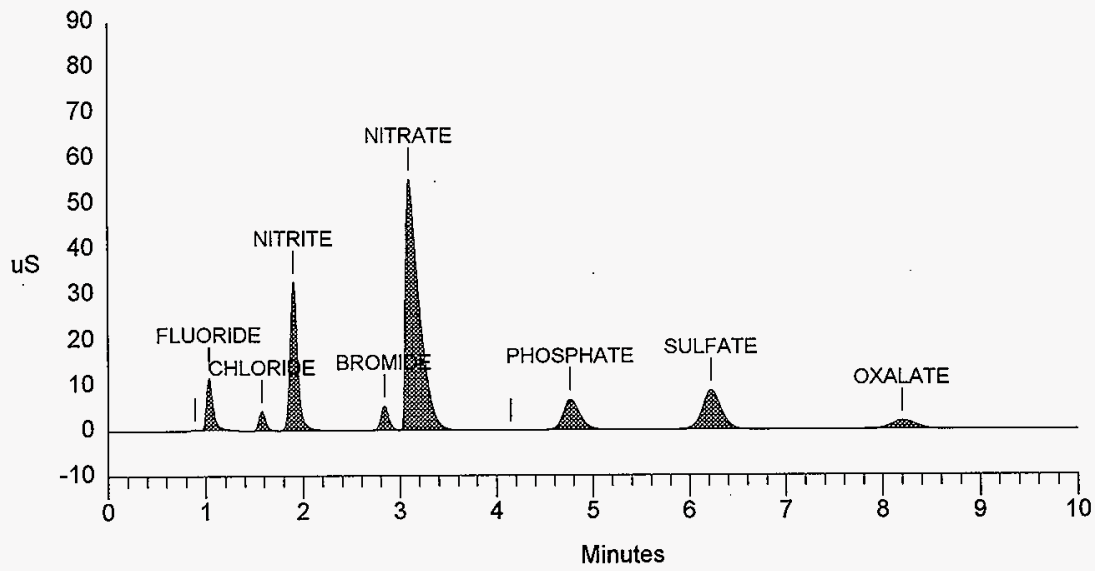




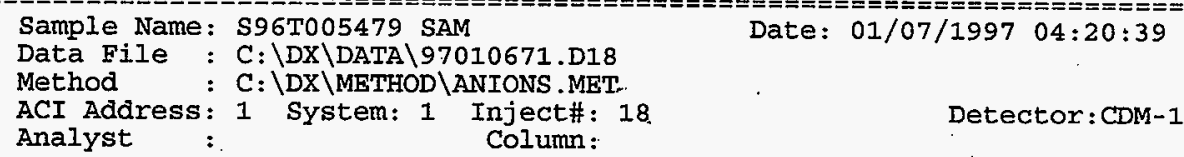

Calibration Volume Dilution Points Rate start stop Area Reject Calibation

$\begin{array}{llllllll}\text { External } & 1 & 41 & 3000 & 5 \mathrm{~Hz} & 0.00 & 10.00 & 50\end{array}$

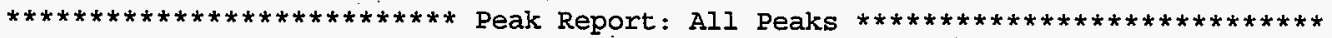
Pk. Ret Component $\begin{array}{r}\text { Concentration Height } \\ \text { ug/mI }\end{array}$ Time Name
Bl.

$\begin{array}{lll}1 & 0.88 & \\ 2 & 1.04 & \text { FLUORIDE } \\ 3 & 1.58 & \text { CHLORIDE } \\ 4 & 1.90 & \text { NITRITE } \\ 5 & 3.24 & \text { NITRATE } \\ 6 & 4.77 & \text { PHOSPHATE } \\ 7 & 6.22 & \text { SULFATE }\end{array}$

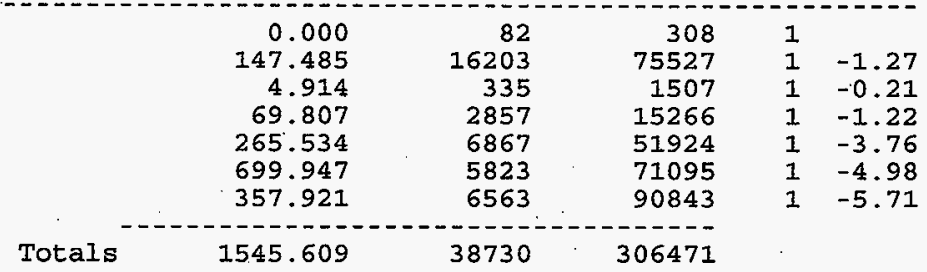

File: 97010671.D18 Sample: S96T005479 SAM

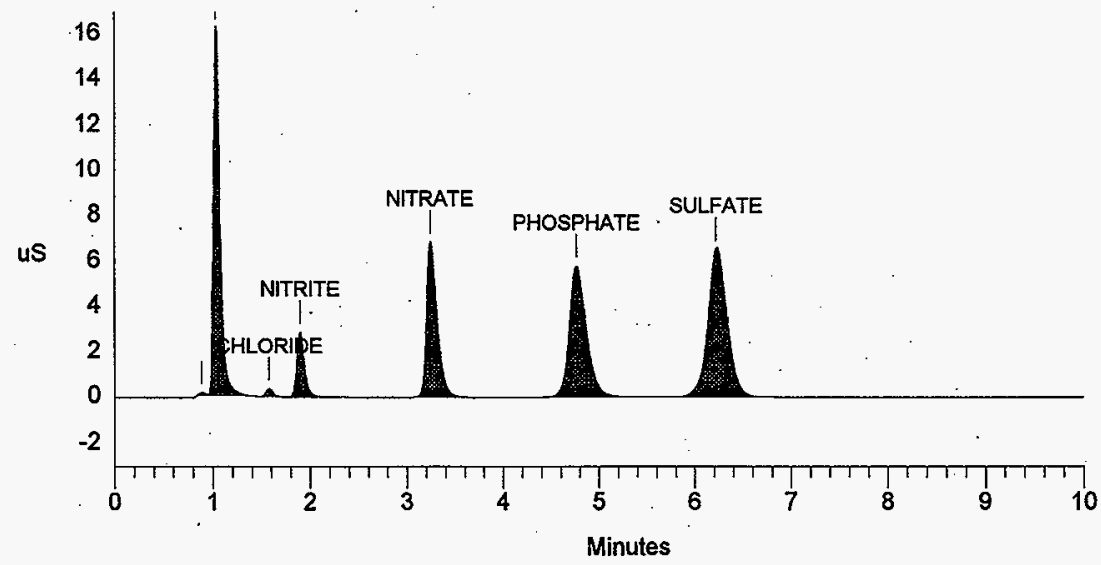


, TNE-SD-WNA-DP-219, REV.O

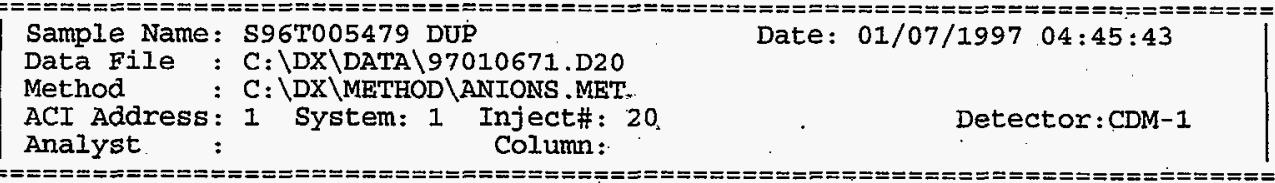

Calibration Volume Dilution Points Rate Start Stop Area Reject

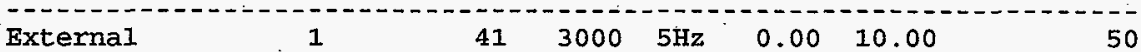

$* * * * * * * * * * * * * * * * * * * * * * * * * * *$ Peak Report All Peaks $* * * * * * * * * * * * * * * * * * * * * * * * * * * *$

Pk. Ret Component Concentration Height Area BI. \%Delta Num Time Name $\mathrm{ug} / \mathrm{ml}$ Code

$\begin{array}{lll}1 & 0.88 & \\ 2 & 1.04 & \text { FLUORIDE } \\ 3 & 1.58 & \text { CHLORIDE } \\ 4 & 1.90 & \text { NITRITE } \\ 5 & 3.25 & \text { NITRATE } \\ 6 & 4.76 & \text { PHOSPHATE } \\ 7 & 6.24 & \text { SULFATE }\end{array}$

$\begin{array}{rrrrr}0.000 & 90 & 363 & 2 & \\ 147.012 & 16349 & 75267 & 2 & -1.27 \\ 4.461 & 311 & 1361 & 1 & -0.21 \\ 58.865 & 2413 & 12707 & 1 & -1.22 \\ 224.847 & 5839 & 43667 & 1 & -3.56 \\ 763.884 & 6326 & 78123 & 2 & -5.11 \\ 328.489 & 6016 & 83112 & 2 & -5.51\end{array}$

Totals

$37343 \quad 294599$

File: 97010671.D20 Sample: S96T005479 DUP

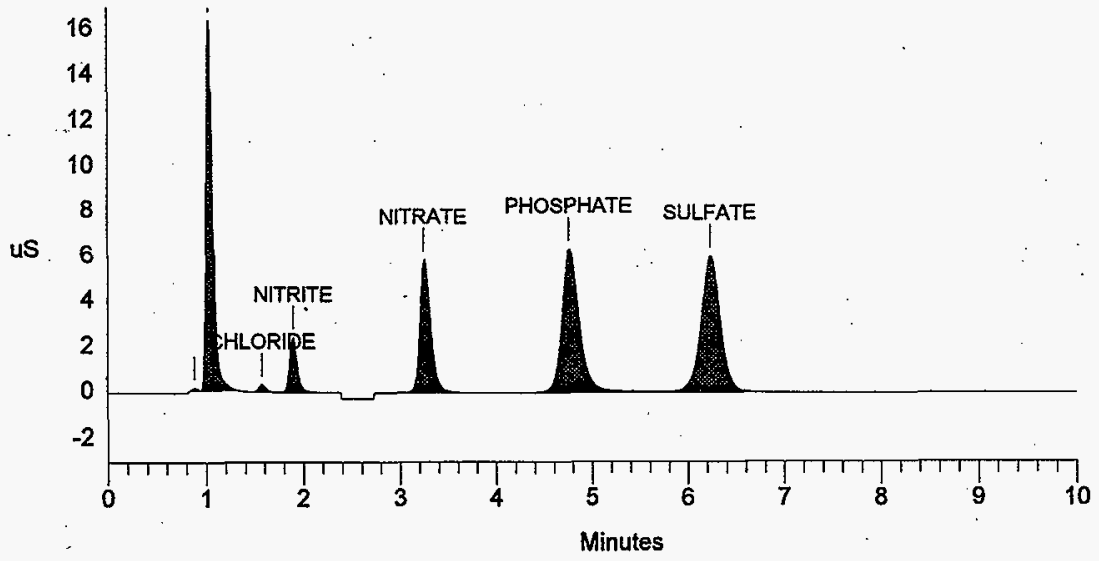




\section{LABCORE Completed Worklist Report for Worklist\# 14416}

Analyst: rag

Method: LA533105 Rev/Mod
Book\# 36N2OC

Worklist Comment: ic-01 for b-108

Instrument; $\mathrm{ICO}, \quad$,

Test Matrix Actual Found DL or Yield Unit

\begin{tabular}{llllll}
\hline Seq Type & Sample\# R A & Test & Matrix Actual Found DL or Yield Unit
\end{tabular}

\begin{tabular}{|c|c|c|c|c|c|c|c|c|c|}
\hline $1 \mathrm{ccB}$ & & 08 & $010<0$ & $\mathrm{~B}$ & $0 \mathrm{c}$ & 1 & $4200 \%$ & & 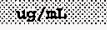 \\
\hline $1 \mathrm{CCB}$ & $\therefore$ & 0 & IC-QC & Cr & $Q C$ & 1 & $<1.70 e-2$ & & $\mathrm{ug} / \mathrm{mi}$ \\
\hline $1 \mathrm{LB}$ & & 0 & $810-0$ & 102 & $0 \%$ & 1 & $41008-1$ & & $0,1,0$ \\
\hline $1 \mathrm{CCB}$ & & $\therefore$ & axc-ec & $\mathrm{BR}$ & $Q C$ & 1 & $<1.25 e-1$ & & $\mathrm{ug} / \mathrm{mr}$ \\
\hline $1 \mathrm{cos}$ & & 0 & $01 C-\alpha c$ & 103 & 80 & 1 & $41390 \%$ & & xplon \\
\hline $1 \mathrm{CCB}$ & & 0 & OIC-QC & PO4 & $Q C$ & 1 & $<1.200-1$ & & $\mathrm{ug} / \mathrm{mL}$ \\
\hline $18 \mathrm{CB}$ & & $\%$ & $\alpha x<\alpha c$ & 804 & $0 \%$ & 1 & 4180001 & & $10 \%$ \\
\hline $1 \mathrm{CCB}$ & & 0 & - IC-QC & OXATATE2 & QC & 1 & $<1.05 e-1$ & & $\mathrm{ug} / \mathrm{mr}$ \\
\hline $2 \mathrm{cct}$ & & 0 & $0 P_{1 C} \alpha c$ & P & 80 & 500000 & 6530001 & 130068 & $8 / 10<0 \times 0 x$ \\
\hline $2 \mathrm{ccV}$ & & 0 & $\operatorname{QIC}-Q \mathrm{CC}$ & CL & $\mathrm{QC}$ & 7.90001 & $7.510+01$ & 95.063 & \& Recovery \\
\hline $20 \%$ & & \% & $400 \%$ & roo & $0 c$ & $5 \times 4100$ & $5<190+0 \%$ & 45933 & $6 \% 200-1 / 4 x$ \\
\hline $2 \operatorname{ccv}$ & & 0 & GIC-OC & $\mathrm{BR}$ & $Q \mathrm{C}$ & 5.89002 & $5.810+02$ & 98.642 & \& Recovery \\
\hline 200 & & 0 & 0 I $\alpha$. & 103 & $8 c$ & 5,94002 & $8000+02$ & 97643 & 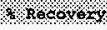 \\
\hline $2 \mathrm{ccV}$ & & 0 & eIC-QC & PO4 & $Q C$ & 5.44002 & $5.13 \theta+02$ & 94.301 & \& Recovery \\
\hline 2601 & & 0 & $\alpha+\alpha \%$ & 504 & $6 c$ & 631002 & $6250+07$ & 990049 & $x<-8<-4 y$ \\
\hline $2 \mathrm{ccV}$ & & 0 & $\operatorname{CrC}-\mathrm{QC}$ & OXALATB2 & $\mathrm{ec}$ & $5.25 e 02$ & $5.47 e+02$ & 104.190 & \& Recovery \\
\hline $3 \mathrm{BH}+\mathrm{H}_{\mathrm{HP}}$ & & \% & $80<01$ & 202 & 501 to & 1 & $1 / 2004$ & & $49 \%$ \\
\hline 3 BLNK-PREP & & 0 & IC-01 & $\mathrm{CL}-02$ & SOLID & 1 & $2.70 e-02$ & 0.027 & $\mathrm{ug} / \mathrm{g}$ \\
\hline $3, \mathrm{H}_{\mathrm{MK}}-\mathrm{pRP}$ & & \% & $61<01$ & 00202 & 80410 & 1 & 6108001 & & 1019 \\
\hline 3 BLTK-PRBP & & 0 & QIC-0I & $\mathrm{BR}-\mathrm{O2}_{2}$ & Sorrt & 1. & $<1.25 e-1$ & & $\mathbf{u g} / \mathrm{g}$ \\
\hline 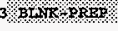 & & 0 & $0 x-01$ & $103-2$ & $\mathrm{sot} 1 \mathrm{~L}$ & 4 & $1 / 60 / 4$ & & 4oto \\
\hline 3 BLNR-PREP & & 0 & arc-01 & PO4-02 & soLID & 1 & $<1.200-1$ & & $\mathrm{ug} / \mathrm{g}$ \\
\hline 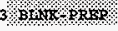 & & \% & 61001 & $804-2$ & $501-10$ & 1. & 310801 & & 19/9 \\
\hline 3 BINRT-PREP & & 0 & $9 x \mathrm{x}-01$ & OXATATE2 & SOLrD & 1 & $<1.05 e-1$ & & $\mathrm{ug} / \mathrm{g}$ \\
\hline 4 SAYPIS & 6596005480 & $0 \%$ & 64601 & 202 & 50110 & Sho & $3 \times 895604$ & $86 \% 40$ & 498 \\
\hline 4 SAMPLE & S96T005480 & $0 \mathrm{w}$ & $\operatorname{arC}-01$ & $\mathrm{CL}-02$ & SOLID & $N / A$ & $4.574 \mathrm{e}+02$ & 122.100 & $\mathrm{ug} / \mathrm{g}$ \\
\hline $4.8 x+2 p$ & $89500500 \%$ & ok & $610-01$ & 40202 & $501+10$ & $2 \%$ & $7 / 270+03$ & 125,300 & 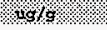 \\
\hline 4 SAMPLE & S96T005480 & $0 \mathrm{w}$ & arC-01 & $\mathrm{BR}-02$ & SOLID & $N / A<$ & $8.974 e+02$ & 897.500 & ug/g \\
\hline $48 x+1=4$ & 8564005480 & $0 \%$ & 01001 & 403402 & 80100 & S1/ & $38350+04$ & 8401000 & $4 \%$ \\
\hline 4 SAMPLE & S96T005480 & $0 \%$ & arc-01 & PO4-02 & soLID & $N / A$ & $2.141 e+05$ & 861.400 & $\mathrm{ug} / \mathrm{g}$ \\
\hline $48 \mathrm{AMP}$ & 8950005980 & $0 \%$ & 201001 & $80 \% 02$ & 5010 & $x_{1} A_{0}$ & $7 / 400004$ & 9900600 & $6 \%$ \\
\hline 4 SAMPLB & S96T005480 & $0 \%$ & QIC-01 & OXALATE2 & SOLID & $N / A<$ & $7.538 e+02$ & 754.000 & $\mathrm{ug} / \mathrm{g}$ \\
\hline $5 \%$ DoP & 8060005480 & 08 & 610001 & 802 & 50110 & 3900004 & $34530+04$ & $6 \% 96$ & $\mathrm{Rpp}$ \\
\hline 5 DUP & S96T005480 & $0 \%$ & IC-01 & $\mathrm{CL}_{1}-02$ & SOLID & $4.57 e+02$ & $5.28 e+02$ & 14.416 & RPD \\
\hline $5 \%$ & 5967005480 & 0 & $8 \mathrm{Hol} 0$ & 40202 & $501+0$ & $7 \times 3000$ & 6221006 & 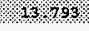 & ppp \\
\hline 5 DUP & S96T005480 & $0 \%$ & exc-01 & $\mathrm{BR}-02$ & SOLID & $<8.97 e_{2}$ & $<9.23 \mathrm{e} 2$ & & RPD \\
\hline $5 \% 0$ & $8-6400580$ & 0.4 & $01-01$ & $400-02$ & solpo & $8080+04$ & 3090004 & $1 / 2,48$ & RDP \\
\hline $5 \mathrm{DUP}$ & $\$ 96 \mathrm{~T} 005480$ & $0 \mathrm{w}$ & erc-01 & PO4-02 & SOLID & $2+14 a+05$ & $1.87 e+05$ & 13.466 & RPD \\
\hline $5 \mathrm{pup}$ & 500000580 & 01 & $010+01$ & $6040 \%$ & 80140 & $6 \times 21604$ & $657000 \%$ & 9889 & pq \\
\hline 5 DUP & $\$ 96 \mathrm{~T} 005480$ & $0 \%$ & erc-01 & OXATATE2 & SOLID & $<7.54 \mathrm{e} 2$ & $<7.75 e 2$ & & RPD \\
\hline & 556500 & 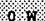 & 801 & 1002 & sotion & 90003 & 42 & & \\
\hline
\end{tabular}

Units shown for $Q C(B L K / B K G)$ may not reflect the actual units. 


\section{LABCORE Completed Worklist Report for Worklist\# 14416}

\begin{tabular}{|c|c|c|c|c|c|c|c|c|c|}
\hline Seq Type & Sample & $\overline{R A}$ & & Test & Matrix & Actual & DL or Yield & Unit & \\
\hline $6 \mathrm{spr}$ & S96T005480 & OW & IC-01 & CL- 02 & SOLID & 7.90001 & $7.810+01$ & Recovery & \\
\hline $6 \operatorname{spx}$ & \$967005480 & 0 พ & eIC-01 & $\mathrm{NO} 2-02$ & SOLID & 5.41002 & $5.14 \theta+02$ & Recovery & \\
\hline $6 \%$ SPR & $896 r 00540 \%$ & $0 \%$ & $8010-1$. & $8 \mathrm{~B}-02$ & $80 .+0$ & $5.8960 \%$ & $5.41-02$. & 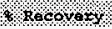 & \\
\hline $6 \mathrm{SPX}$ & 5967005480 & ० $\mathrm{w}$ & बIC-01 & sio3-02 & SOIID & 5.94002 & $5.62 a+02$ & 8 Recovery & \\
\hline $6.6 \mathrm{~s}$ R. & 89600548 & $0 \%$ & 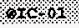 & $101<02$ & SoOn & $541 \% 02$ & 1.57 .02 & 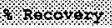 & 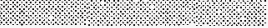 \\
\hline $6 \mathrm{sgK}$ & $S 96 T 005480$ & $0 \mathrm{w}$ & eIC-01 & $304-02$ & SOLID & 6.31002 & $5.53 \theta+02$ & \& Recovary & \\
\hline $6 / 8 p x$ & 5890005480 & $0 \%$ & S 2000 & OXALATE2 & sorro & 5.2500 & $5 / 48+02$ & 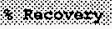 & \\
\hline 7 SAMPIZ & 5967005488 & $0 \%$ & (IC-01 & $8-02$ & SOEID & $N / A$ & 1. $9200+04$ & $u g / \mathrm{s}$ & \\
\hline $78 x p=3$ & 856400588 & $0 . \%$ & $8 x_{1-61}$ & $20.02 \%$ & solin & $\sqrt{2} x_{\lambda x}$ & 8.836 .03 & (2. $2 . \%$ & \\
\hline 7 SAMPLB & $596 \mathrm{~T} 005488$ & $0 \mathrm{w}$ & $\oplus 1 \mathrm{C}-01$ & $\mathrm{NO2-02}$ & SOIID & $\mathbf{N} / \mathbf{A}$ & $5.332 \theta+03$ & $4 g / g$ & \\
\hline $7 / 8 \mathrm{SANP}$ & 596200588 & $0 \%$ & $810-01$ & \&B -02 & Sor. & 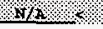 & 255.000 & $4 \%$ & \\
\hline 7 SAMPII & S96T005488 & $0 \mathrm{w}$ & AIC-0I & $\mathrm{NO} 3-02$ & SorID & $N / A$ & 2730.000 & $\mathrm{ug} / \mathrm{g}$ & \\
\hline $7.5 \times 468$ & $896 r 00588$ & $0 \%$ & $8 \mathrm{rov}$ : & $304-02$ & solio & 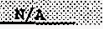 & $233 \% \% 00$ & ilots. & \\
\hline 7 SAMPLE & $596 \mathrm{~T} 005488$ & OW & arc-01 & S04-02 & SOLID & N/A & 2711.000 & ug $/ \mathrm{g}$ & \\
\hline $7 \mathrm{SAMPSB}$ & 596700548 & $0 \%$ & 201000 & $0 \times 4=1,82$ & soirf & 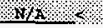 & 2002.000 . & $49 \%$ & \\
\hline 8 DUR & 5967005488 & $0 \mathrm{~W}$ & arc-01 & E. 02 & SOLID & $1.92 a+04$ & $4.568+04$ & RPD & \\
\hline 8 & $896 \times 00548$ & $6 \%$ & $6 \mathrm{rc} 01$. & 20.02 & Solin & $8.44-03$ & $1+14-03$. & ob & \\
\hline 8 DUP & S96T005488 & O W & $\oplus \mathrm{IC}-01$ & $\mathrm{NO2}-02$ & SOLID & $5.33 e+03$ & $1.05 e+04$ & $\mathrm{RPD}$ & \\
\hline 8. Lut & 8967005,88 . & $6 \%$ & 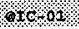 & ${ }_{2}=02$ & soritso & $2 \% 2 \times 6+1$ & $22 * 4 * 3$ & 8.0. & \\
\hline $8 \mathrm{DUP}$ & $s 96 \mathrm{~T} 005488$ & $0 w$ & erc-01 & $\mathrm{x} 03-02$ & SOLID & $1.94 a+04$ & $4.05 e+04$ & RPD & \\
\hline 8 onth & 56400588 & $0 \%$ & $6 x-60 \%$ & $104 \times 62$ & Sol 70 & $4.30=-8$ & $2.640+0 \%$ & 501 \% & \\
\hline 8 DUP & S96T005488 & ० $\mathrm{w}$ & IC-01 & SO4-02 & SOLID & $2.25 e+04$ & $152.000=$ & RPD & \\
\hline 8200 & 596700588 & $\% \%$ & $80<01$ & 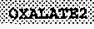 & SOL_L & 4.663 & $\times 2.050 \%$ & BPD. & 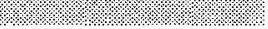 \\
\hline S SPR & 3967005498 & $0 \%$ & IC -01 & $5-02$ & SOLID & 5.90001 & 181.356 & 8 Recovexy & \\
\hline 9. squ & 596700548 & $0 \%$ & 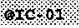 & $4.0 \%$ & sotro & 7.90 .01 & $3.4 \times+2$ & $\gamma \quad \gamma-\sigma \cdot<\gamma \gamma$ & 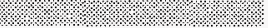 \\
\hline $9 \mathrm{SPR}$ & S96T005488 & $0 \mathrm{~W}$ & IC-01 & $1802-02$ & SOHID & 5.41 .02 & $5.23 e+02$ & \& Recovery & \\
\hline $9.8 \mathrm{sx}$ & 5967005188 & $0 \%$ & 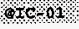 & $8 \mathrm{BR} / 6$ & SOL-D & 589007 & $402 \% 37 \%$ & 1 Roov-4y & 柿 \\
\hline $9 \mathrm{spr}$ & S96T005488 & o $\mathrm{w}$ & aIC-01 & $\mathrm{NO}_{\mathrm{N}}$ & SOLID & $5.94 e^{e 02}$ & 102.862 & Recovery & \\
\hline $9.8 p R$ & $596 r 00580$ & $\% \%$ & $0 \mathrm{CO}-01$. & 804.02 & Soting & $5 . \times 8002$ & $150 / 73$ & 8 secoros & \\
\hline $9 \mathrm{SPR}$ & S96T005488 & ० พ & aIC-01 & S04-02 & SOLID & $6.31 \mathrm{e02}$ & 102.377 & \& Recovery & \\
\hline $98 \mathrm{six}$ & 5264005488 & $0 \%$ & 01001 & $0 \times 1 / 4122$ & Sortid & 5.25002 & $5656 * 02$ & 8. $\mathrm{r} \cdot 0$. & \%: \\
\hline
\end{tabular}

\section{Final page for worklist\# 14416}

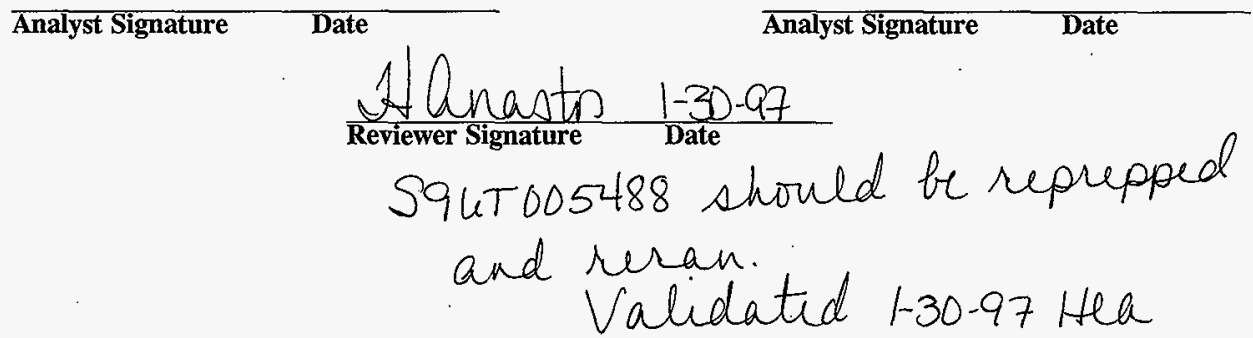

Units shown for $Q C(B L K / B K G)$ may not reflect the actual units. 
10/29/96 10:42.

HNF-SD-WM-DP-219, REV. 0

Page: 1

A-0004-1

LABCORE Data Entry Template for Worklist\# 14416

Analyst: IYS Instrument: ICO IDol Book\# 36N20.C

Method: LA-533-105 Rev/Mod D-1

Worklist Comment: ic-01 for b-108 .

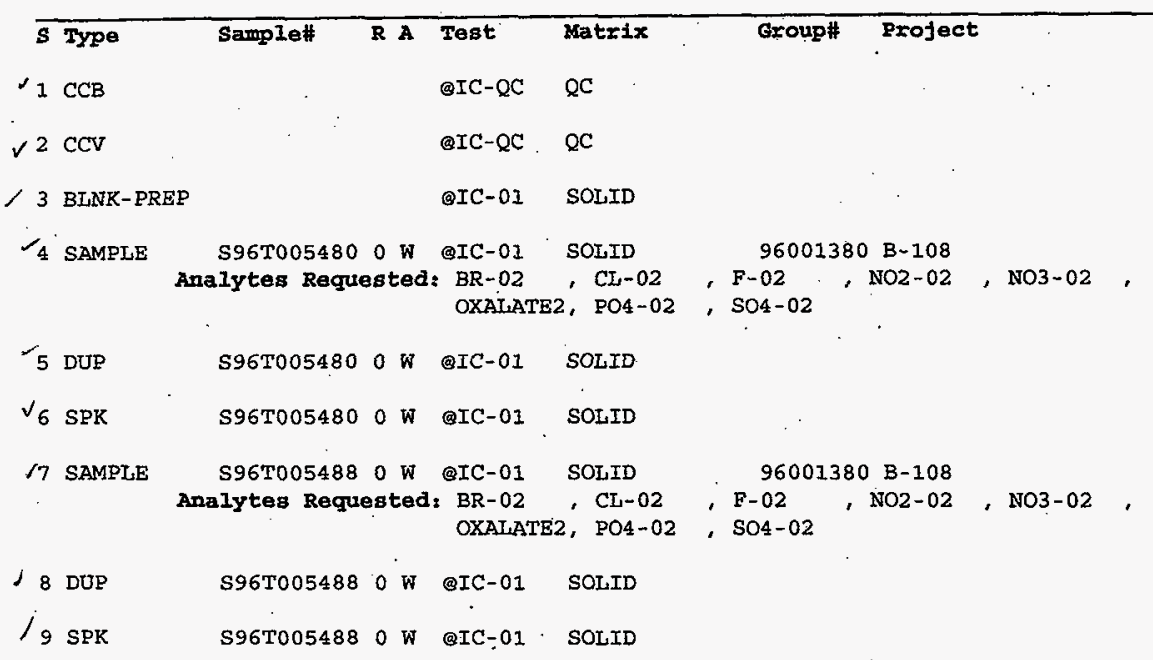

Final page for worklist \# 14416

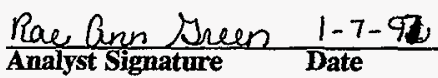

S96T.005488 should be reprepped and reanalyzed. Hel 1-29-97

Data Entry Comments: 5967005480

Ban at dilution factors 41 for Bromide 101 for all others

$S=$ Worklist Slot Number, $R=$ Replicate Number, $A=$ Aliquot Code.

293 
HNF-SD-WM-DP-219, REV. 0

\begin{tabular}{|c|c|c|c|c|c|c|c|c|c|}
\hline \multicolumn{3}{|c|}{$A-0010-I C$} & \multicolumn{5}{|c|}{ DATA FILE/WORKLIST RESOLUTION } & \multicolumn{2}{|c|}{ 29-Jan-97 } \\
\hline \multicolumn{4}{|c|}{ Worklist\# : 14416} & \multicolumn{6}{|c|}{ Data File: 14416JA.CSV } \\
\hline & Seq & Type & Sample \# & Sec & & Data File & Sample Nam & & Dilution \\
\hline $\begin{array}{l}\Rightarrow \\
\Rightarrow \\
\Rightarrow \\
\Rightarrow \\
\Rightarrow \\
\Rightarrow \\
\Rightarrow \\
\Rightarrow \\
=>\end{array}$ & $\begin{array}{l}1 \\
2 \\
3 \\
4 \\
5 \\
6 \\
7 \\
8 \\
9\end{array}$ & $\begin{array}{l}\text { CCB } \\
\text { CCV } \\
\text { BLNK-PREP } \\
\text { SAMPLE } \\
\text { DUP } \\
\text { SPK } \\
\text { SAMPLE } \\
\text { DUP } \\
\text { SPK }\end{array}$ & $\begin{array}{l}\text { S96T005480 } \\
\text { S96T005480 } \\
\text { S96T005480 } \\
\text { S96T005488 } \\
\text { S96T005488 } \\
\text { S96T005488 }\end{array}$ & - & $\begin{array}{l}2 \\
3 \\
4 \\
5 \\
6\end{array}$ & $\begin{array}{l}97010661 \cdot d 01 \\
97010671 \cdot d 02 \\
97010671 \cdot d 03 \\
97010671 \cdot d 04 \\
97010671 \cdot d 16 \\
97010671 . d 10 \\
97010671 . d 07 \\
97010671 . d 17 \\
97010671 . d 09 \\
97010671 . d 11 \\
97010671 . d 12 \\
97010671 . d 13 \\
97010671 . d 14 \\
97010671 \cdot d 15\end{array}$ & 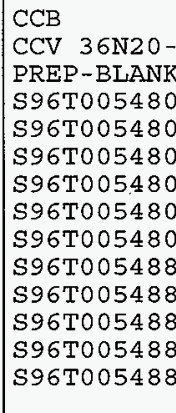 & $\begin{array}{l}\text { DUP } \\
\text { SPIKE } \\
\text { DUP } \\
\text { SPIKE } \\
\text { DUP } \\
\\
\text { DUP } \\
\text { SPIKE }\end{array}$ & $\begin{array}{r}1.00 \\
101.00 \\
1.00 \\
41.00 \\
41.00 \\
41.00 \\
101.00 \\
101.00 \\
101.00 \\
41.00 \\
41.00 \\
101.00 \\
101.00 \\
101.00\end{array}$ \\
\hline
\end{tabular}

Save(F4) Abort(Shift-F3) ListFiles(Shift-F1) UploadFile(F8) 


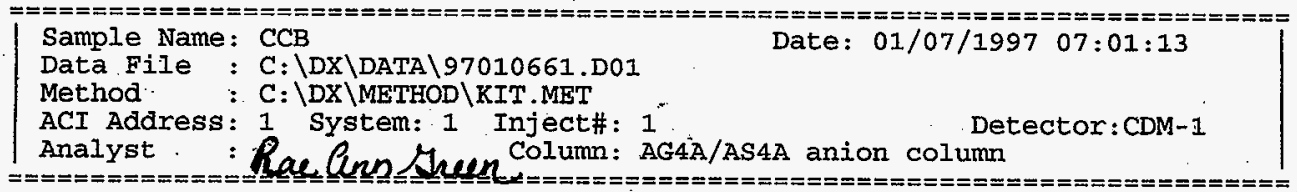

Calibration Volume Dilution Points Rate Start Stop Area Reject

External $1130005 \mathrm{~Hz} 0.0010 .00 \quad 50$

$* * * * * * * * * * * * * * * * * * * * * * * * *$ Peak Report: All Peaks $* * * * * * * * * * * * * * * * * * * * * * * * * * * *$

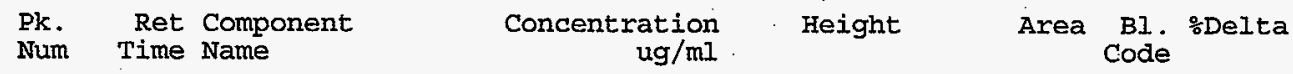

$1 \quad 3.47$ nitrate

0.094

112

790

$1-3.17$

Totals

0.094

112

790

File: 97010661.D01 Sample: CCB

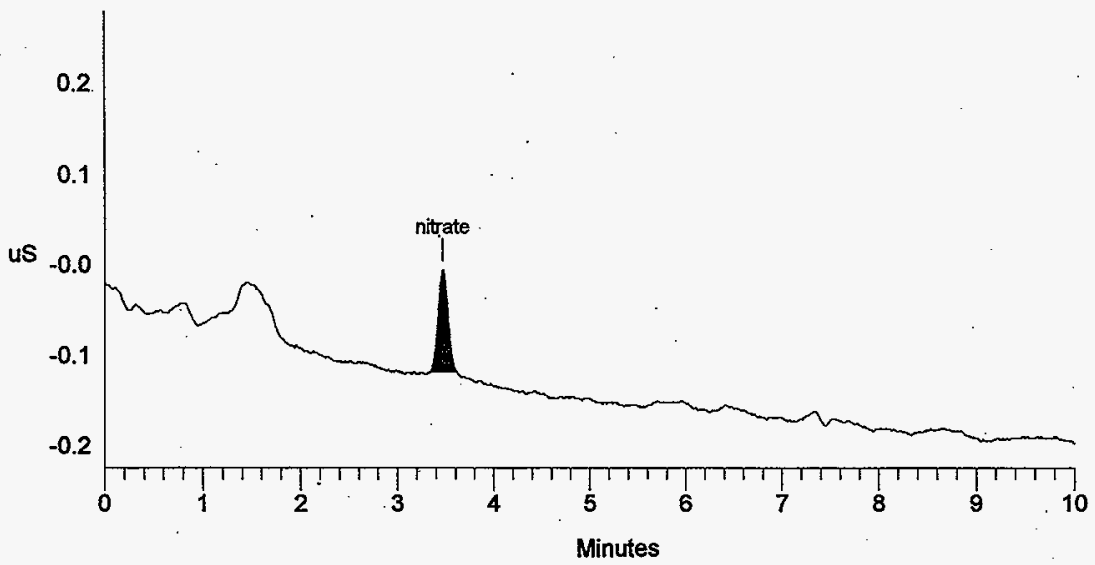

SIGNATURE ABOVE REPRESENTS CHEMICAL TECHNOLOGIST/CHEMIST IHAT COMPLETEDNERIFIED THE CALIBRATION/ANALYSIS ON PAGES 295 TO 303 
Data Reprocessed on 01/17/1997 08:07:39

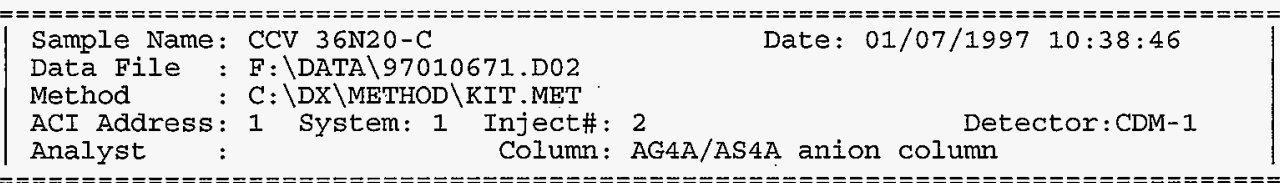

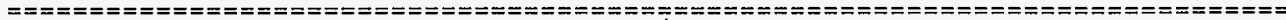

Calibration Volume Dilution Points Rate start stop Area Reject

Calibration

External

1.

$1013000 \quad 5 \mathrm{~Hz}$

$0.00 \quad 10.00$

50

$\star * * * * * * * * * * * * * * * * * * * * * * * * * *$ Peak Report: All Peaks $* * * * * * * * * * * * * * * * * * * * * * * * * * * *$ $\begin{array}{lrrr}\text { Pk. Ret Component } & \text { Concentration } & \text { Height } & \text { Area Bl. } \\ \text { Num } & \text { Time Name } & \text { Code }\end{array}$

\begin{tabular}{|c|c|c|c|c|c|c|c|}
\hline 2 & 1.11 & fluoride & 65.338 & 1120 & 8715 & 2 & 2.47 \\
\hline 3 & 1.66 & chloride & 75.138 & 1749 & 9442 & 1 & -0.40 \\
\hline 4 & 1.97 & nitrite & 519.020 & 6228 & 43519 & 1 & -2.95 \\
\hline 5 & 2.96 & bromide & 580.868 & 5022 & 33934 & 1 & 0.00 \\
\hline 6 & 3.37 & nitrate & 579.942 & 5690 & 45669 & 1 & -5.77 \\
\hline 7 & 4.88 & phosphate & 512.928 & 1502 & 20431 & 1 & -1.61 \\
\hline 8 & 6.35 & sulfate & 625.379 & 4198 & 66365 & 1 & -4.03 \\
\hline 9 & 8.37 & oxalate & 547.322 & 2042 & 41880 & 1 & -3.68 \\
\hline & & & 3505.935 & 27551 & 269956 & & \\
\hline
\end{tabular}

\section{File: 97010671.D02 Sample: CCV 36N20-C}

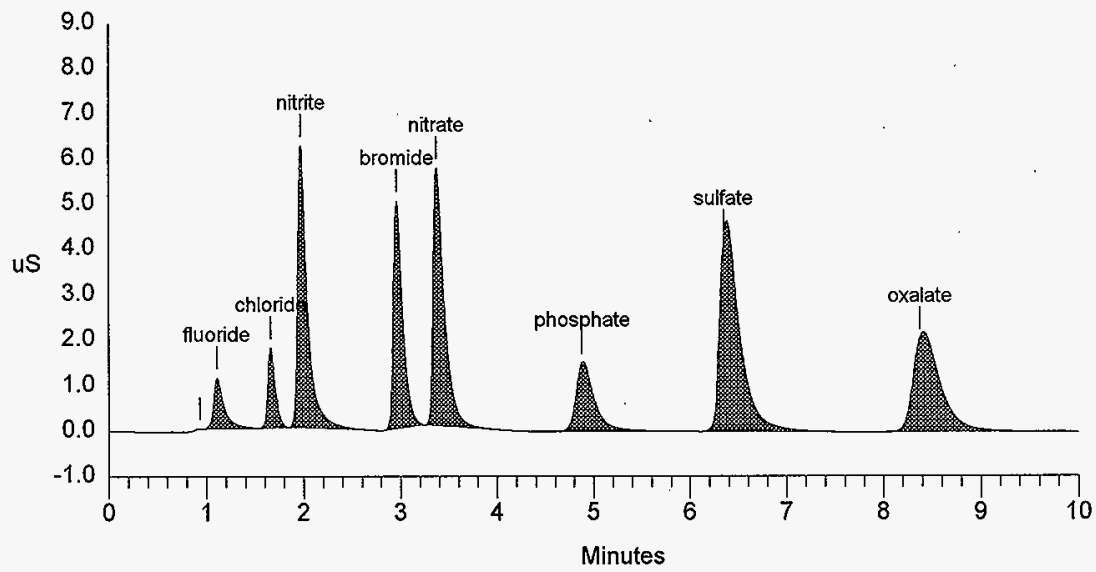




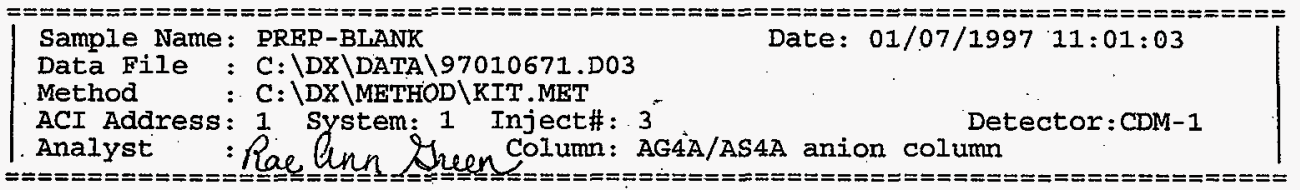

Calibration Volume Dilution Points Rate start Stop Area Reject

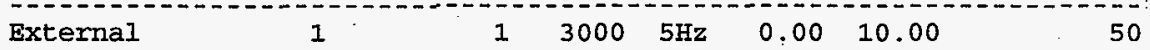

$\star * * * * * * * * * * * * * * * * * * * * * * * * * *$ Peak Report: All Peaks $* * * * * * * * * * * * * * * * * * * * * * * * * * *$

Pk. Ret Component $\quad \begin{gathered}\text { Concentration Height } \\ \text { ug/ml }\end{gathered}$ Area $\begin{gathered}\text { Bl. } \\ \text { Code }\end{gathered}$

Num Time Name

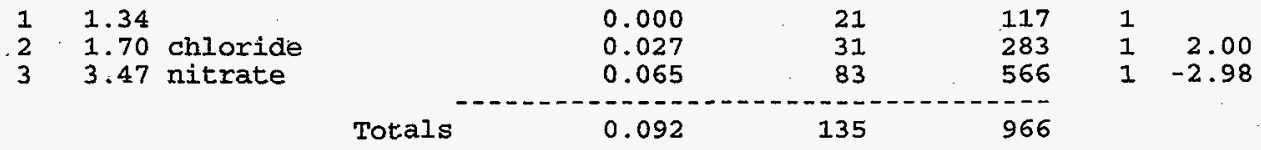

File: 97010671.D03 Sample: PREP-BLANK

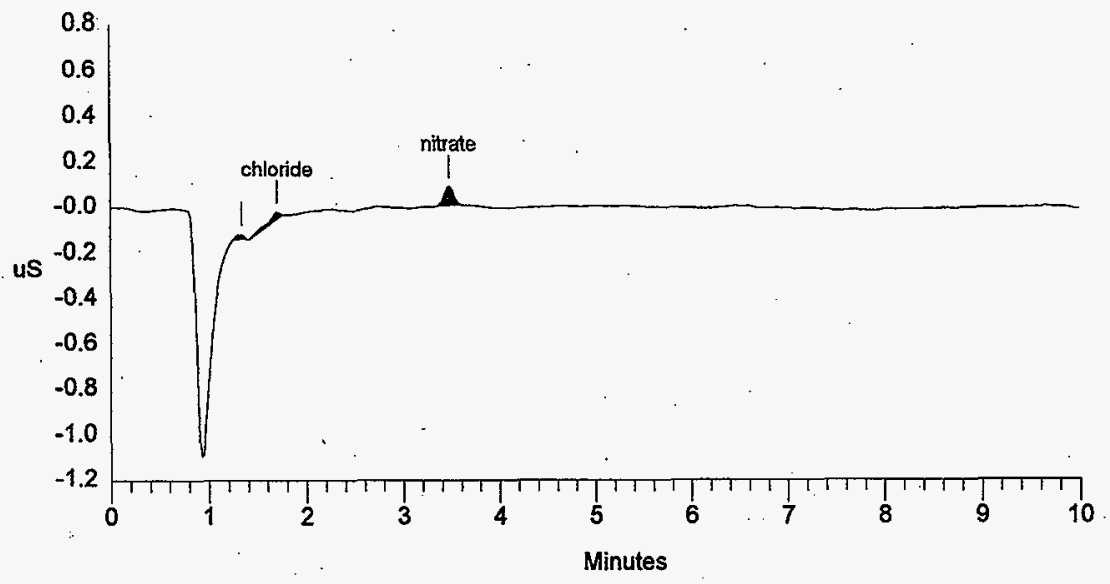




\section{use for Bromide}

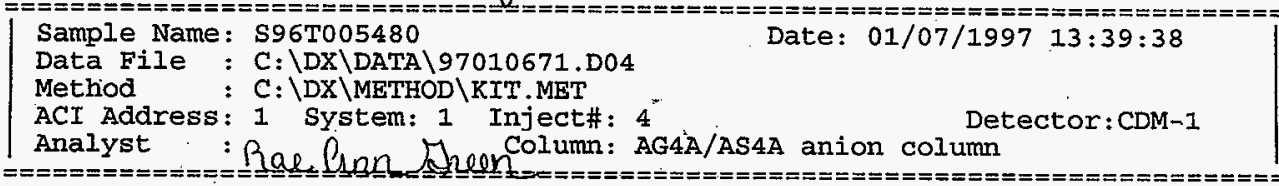

Calibration Volume Dilution Points Rate start stop Area Reject

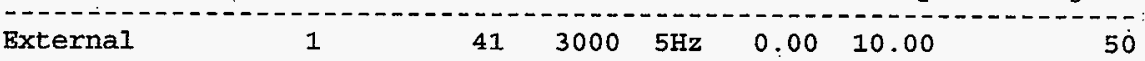

\begin{tabular}{|c|c|c|c|c|c|c|c|}
\hline $\begin{array}{l}\text { Pk. } \\
\text { Num }\end{array}$ & $\begin{array}{l}\text { Ret } \\
\text { Time }\end{array}$ & $\begin{array}{l}\text { Component } \\
\text { Name }\end{array}$ & $\begin{array}{r}\text { Concentration } \\
\mathrm{ug} / \mathrm{ml}\end{array}$ & Height & Area & $\begin{array}{l}\text { BI. } \\
\text { Code }\end{array}$ & 을. \\
\hline $\begin{array}{l}1 \\
2 \\
3 \\
4 \\
5 \\
6 \\
7\end{array}$ & $\begin{array}{l}0.92 \\
1.08 \\
1.67 \\
1.99 \\
3.37 \\
4.80 \\
6.35\end{array}$ & $\begin{array}{l}\text { fluoride } \\
\text { chloride } \\
\text { nitrite } \\
\text { nitrate } \\
\text { phosphate } \\
\text { sulfate }\end{array}$ & $\begin{array}{r}0.000 \\
222.690 \\
2.612 \\
40.700 \\
218.992 \\
1222.493 \\
411.787\end{array}$ & $\begin{array}{r}36 \\
16434 \\
153 \\
1028 \\
5144 \\
10547 \\
7427\end{array}$ & $\begin{array}{r}98 \\
99252 \\
751 \\
7365 \\
42445 \\
133310 \\
108356\end{array}$ & $\begin{array}{r}1 \\
1 \\
1 \\
1 \\
1 \\
1 \\
1\end{array}$ & $\begin{array}{r}-0.00 \\
0.00 \\
-2.30 \\
-5.77 \\
-3.23 \\
-4.03\end{array}$ \\
\hline
\end{tabular}

File: 97010671.D04 Sample: S96T005480

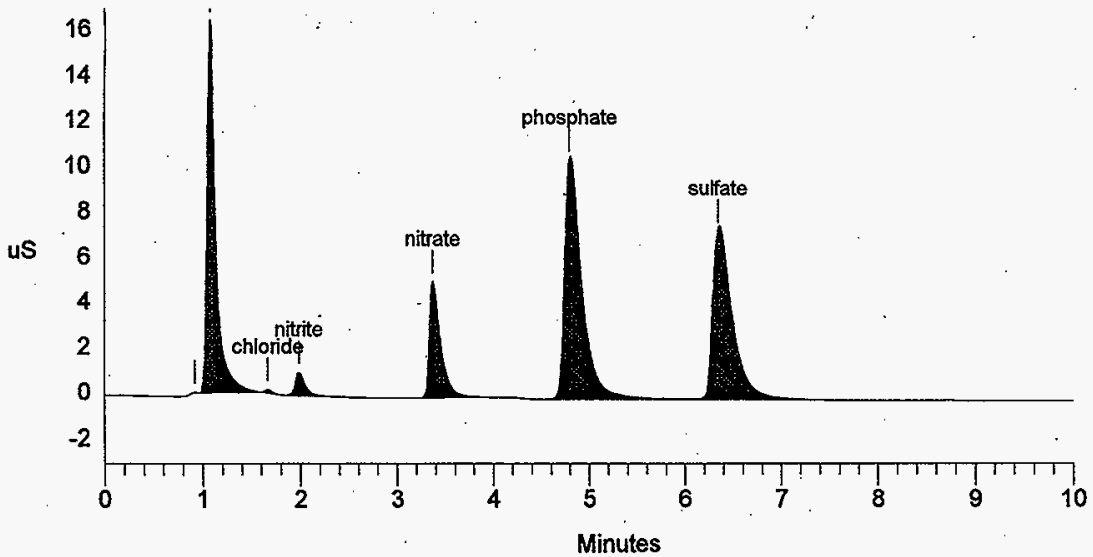




\section{llae For Bromide}

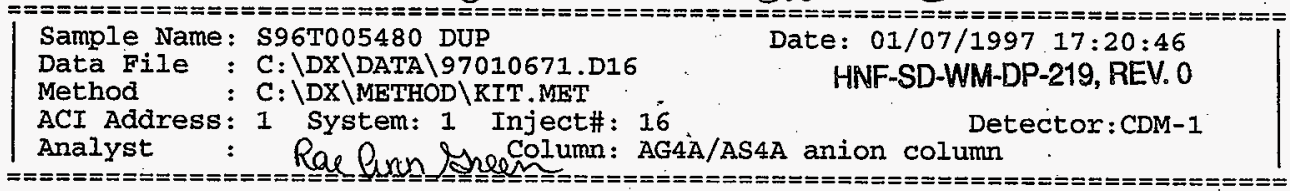

Calibration Volume Dilution Points Rate start stop Area Reject

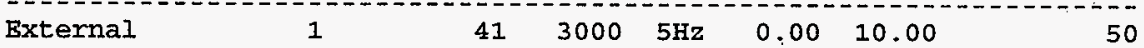

$\star * * * * * * * * * * * * * * * * * * * * * * * * * *$ Peak Report: All Peaks $* * * * * * * * * * * * * * * * * * * * * * * * * * * *$ $\begin{array}{lrrr}\text { Pk. Ret Component } & \text { Concentration } & \text { Height } & \text { Area Bl. } \\ \text { Num } & \text { Time Name } & \text { Code }\end{array}$

$\begin{array}{lll}1 & 0.93 \\ 2 & 1.09 & \text { fluoride } \\ 3 & 1.68 \text { chloride } \\ 4 & 1.99 \text { nitrite } \\ 5 & 3.37 \text { nitrate } \\ 6 & 4.83 \text { phosphate } \\ 7 & 6.35 \text { sulfate }\end{array}$

$\begin{array}{rr}0.000 & 25 \\ 195.945 & 13539 \\ 2.934 & 174 \\ 34.464 & 840 \\ 188.415 & 4454 \\ 1039.330 & 8907 \\ 364.822 & 6528\end{array}$

25

539

174

840

4454

6528

34468

1825.911

Totals
$68 \cdot 1$

$83307 \quad 1 \quad 0.62$

$851 \quad 1 \quad 0.80$

$\begin{array}{lll}6051 & 1 & -1.97\end{array}$

$\begin{array}{lll}36461 & 1 & -5.77\end{array}$

$\begin{array}{lll}111196 & 1 & -2.69\end{array}$

$\begin{array}{lll}95760 & 1 & -4.03\end{array}$

\section{File: 97010671.D16 Sample: S96T005480 DUP}

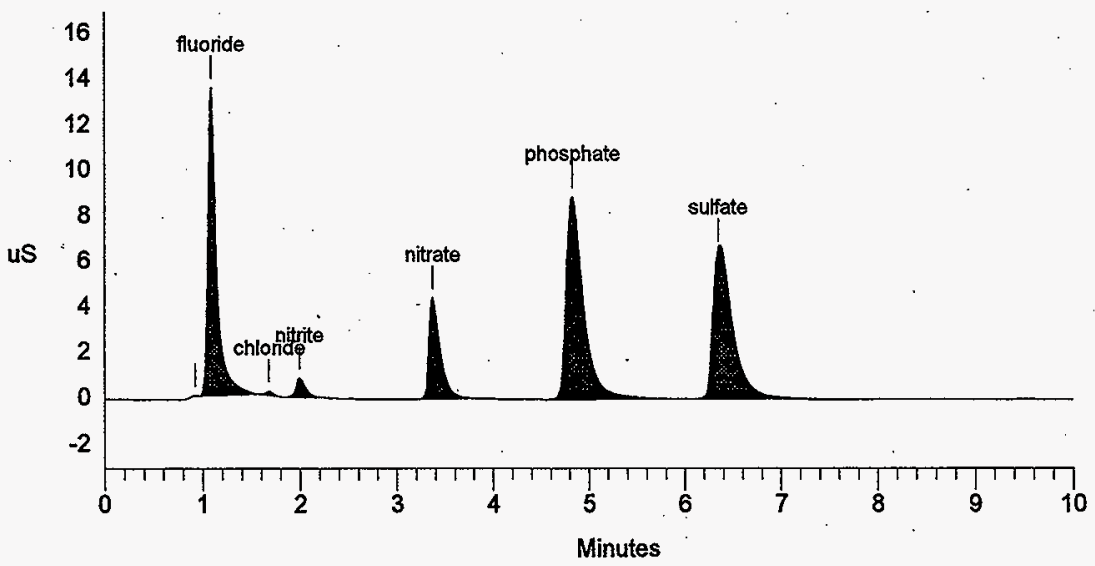


Data Reprocessed on 01/17/1997 08:08:45

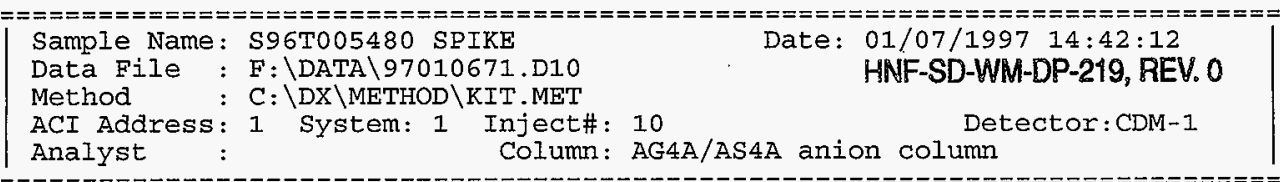

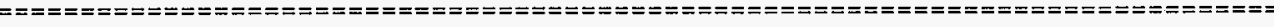

Calibration Volume Dilution Points Rate start stop Area Reject

Cal-

External

1

$413000 \quad 5 \mathrm{~Hz} \quad 0.00 \quad 10.00$

50

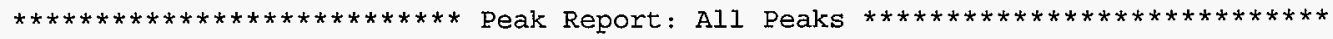

Pk. Ret Component Concentration Height Area Bl. $\frac{\text { Delta }}{}$ Num Time Name ug/ml Code

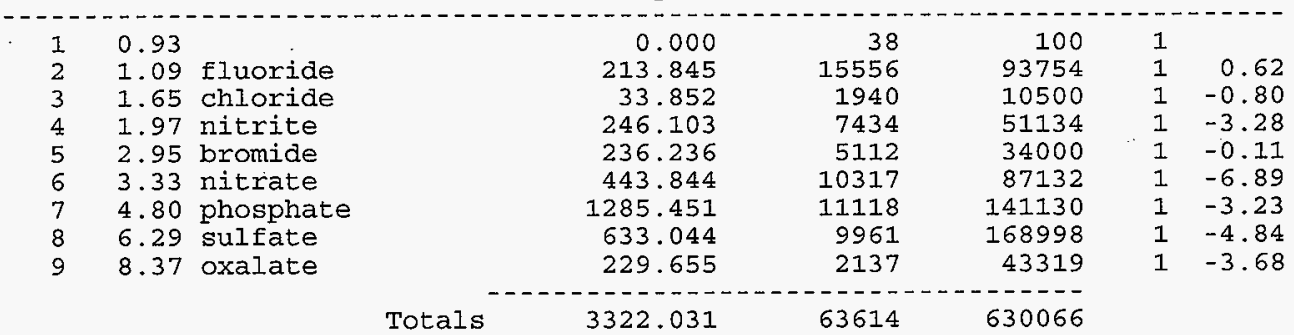

\section{File: 97010671.D10 Sample: S96T005480 SPIKE}

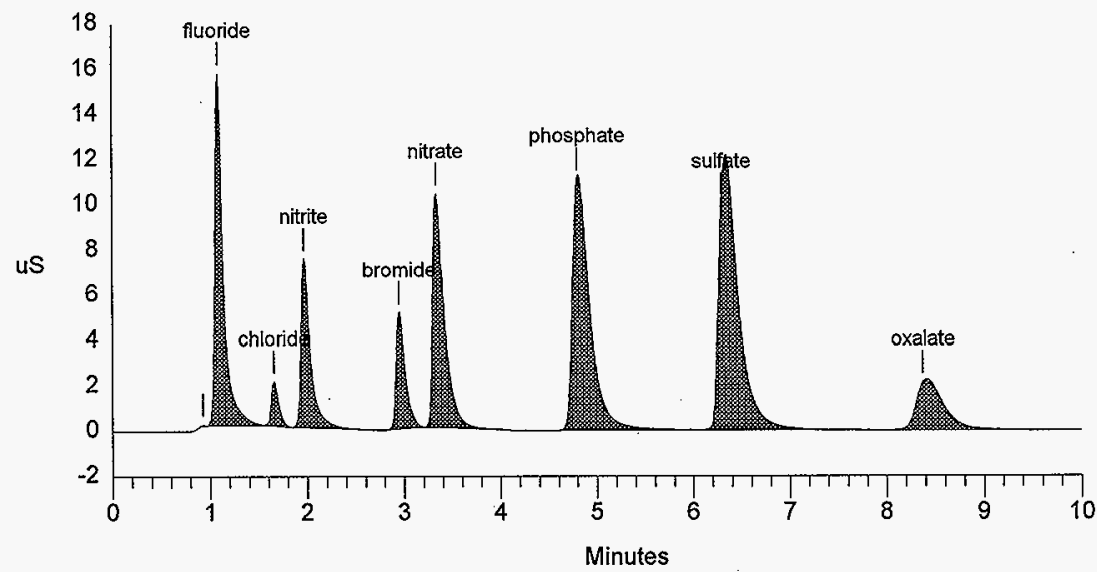




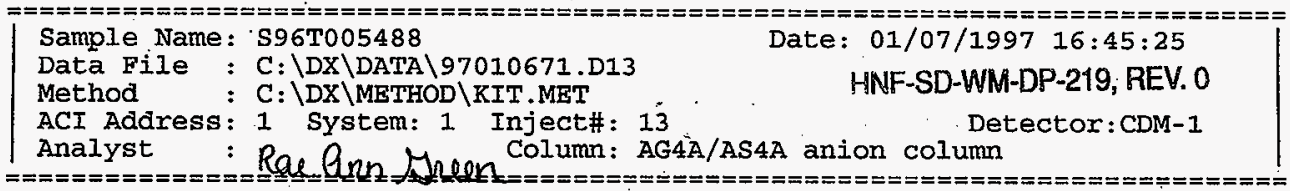

Calibration Volume Dilution Points Rate start stop Area Reject

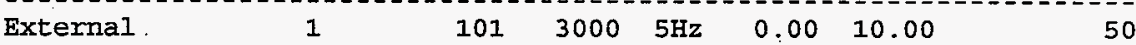

$* * * * * * * * * * * * * * * * * * * * * * * * * * *$ Peak Report: All Peaks $* * * * * * * * * * * * * * * * * * * * * * * * * * * *$

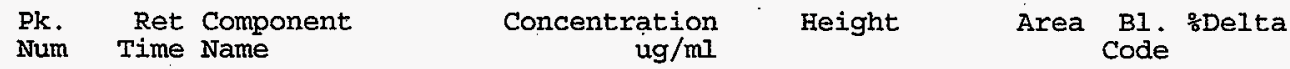

-

$1 \quad 1.10$ fluoride

$2 \quad 1.67$ chloride

98.722

45.433

1836

13683

1.85

2.01 nitrite

27.418

1046

5660

$1 \quad 0.00$

43.43 nitrate

99.480

181

1142

7791

669.580

980
2006

26914

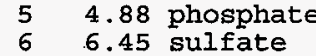

115.466

822

12814

$1-1.31$

$1-4.28$

$\begin{array}{rrrr} & 115.466 & 822 & 12814 \\ \text { Totals } & 1056.099 & 6871 & 68003\end{array}$

File: 97010671.D13 Sample: S96T005488

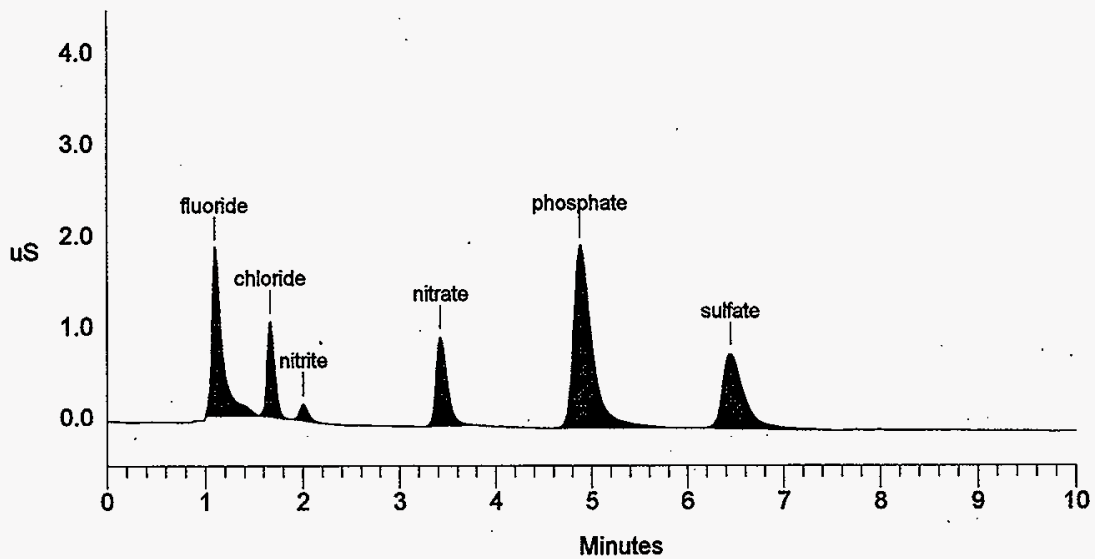




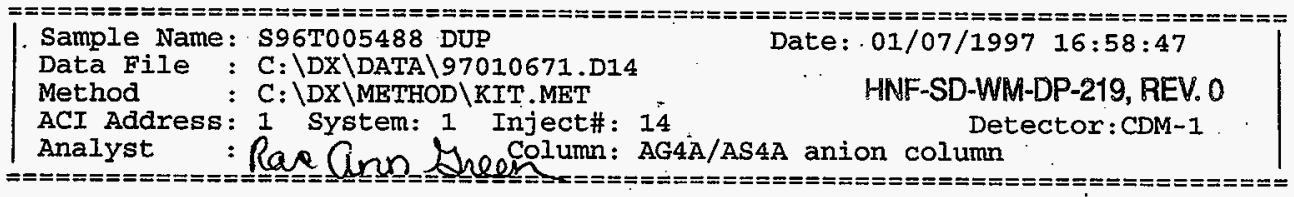

Calibration Volume Dilution Points Rate start stop Area Reject

External

$* * * * * * * * * * * * * * * * * * * * * * * * * * *$ Peak Report: All Peaks $* * * * * * * * * * * * * * * * * * * * * * * * * * * *$

Pk. Ret Component Concentration Height Area BI. $\%$ Delta

Num Time Name $\mathrm{ug} / \mathrm{ml}$ Code

$\begin{array}{lll}1 & 1.09 & \text { fluoride } \\ 2 & 1.68 & \text { chloride } \\ 3 & 2.00 & \text { nitrite } \\ 4 & 3.40 & \text { nitrate } \\ 5 & 4.88 & \text { phosphate } \\ 6 & 6.35 & \text { sulfate }\end{array}$

$\begin{array}{rrrr} & 235.963 & 5344 & 35511 \\ & 5.888 & 137 & 683 \\ 54.485 & 480 & 3452 \\ & 209.734 & 2034 & 16408 \\ & 395.296 & 1077 & 15606 \\ & 856.573 & 6088 & 91196 \\ & & -15160 & 162855\end{array}$

File: 97010671.D14 Sample: S96T005488 DUP

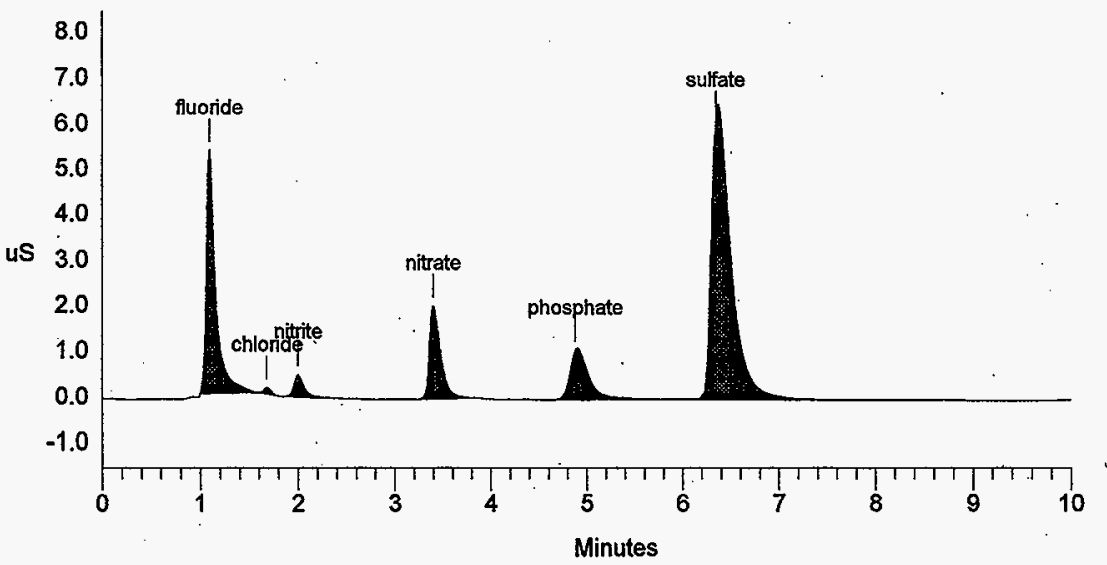


Data Reprocessed On 01/17/1997 08:11:38

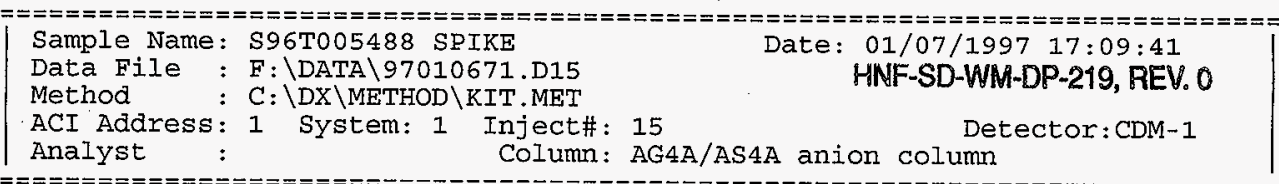

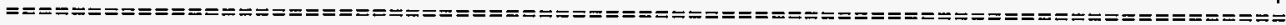

Calibration Volume Dilution Points Rate start stop Area Reject

Cal

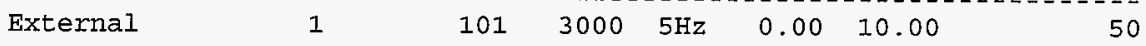

Pk. Ret Component Concentration Height Area Bl. $\frac{\text { Delta }}{}$

Num Time Name ug/ml

Code

$\begin{array}{lll}1 & 0.93 & \\ 2 & 1.09 & \text { fluoride } \\ 3 & 1.65 & \text { chloride } \\ 4 & 1.97 & \text { nitrite } \\ 5 & 2.95 & \text { bromide } \\ 6 & 3.35 & \text { nitrate } \\ 7 & 4.85 & \text { phosphate } \\ 8 & 6.35 & \text { sulfate } \\ 9 & 8.37 \text { oxalate }\end{array}$

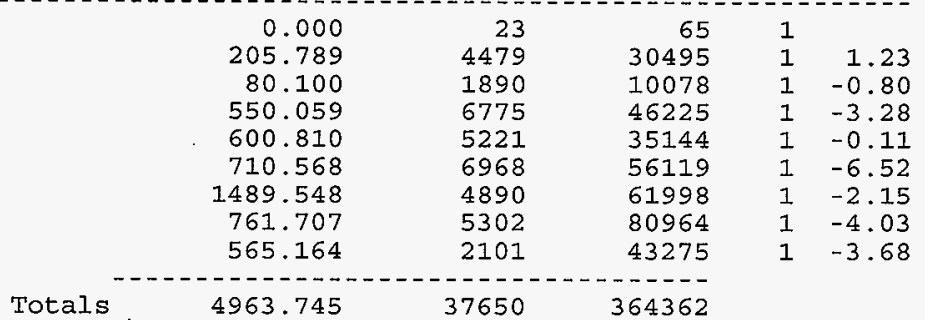

File: 97010671.D15 Sample: S96T005488 SPIKE

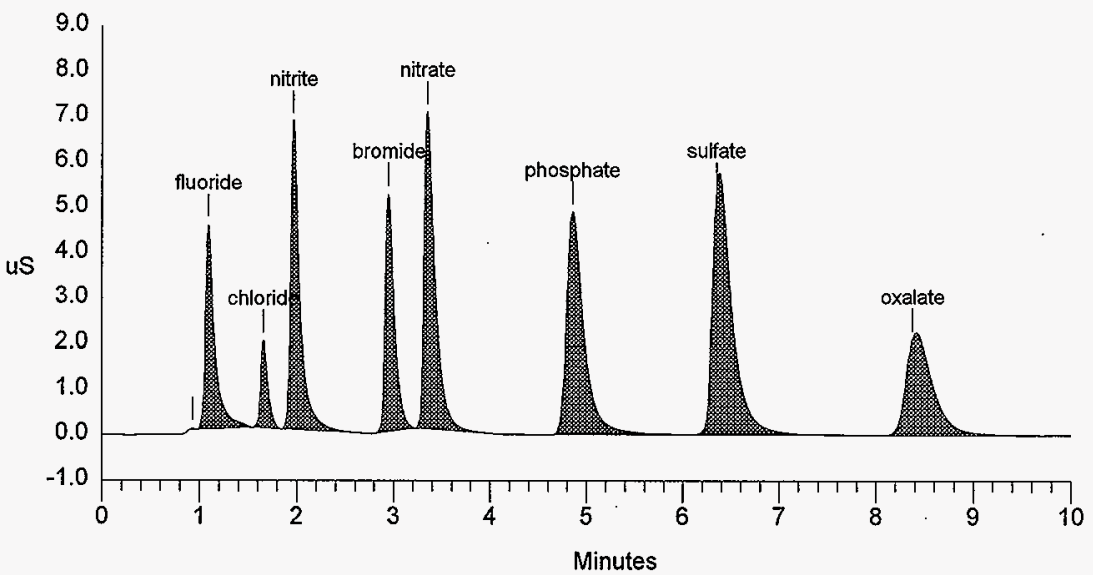




\section{LABCORE Completed Worklist Report for Worklist\# 14654}

Analyst: $\mathrm{kgh}$
Book\# 36N20C

Method: $\quad$ A $-533-105-R e v / M o d ~ D-1$

Worklist Comment: @IC-01 FOR B-108

RTS!

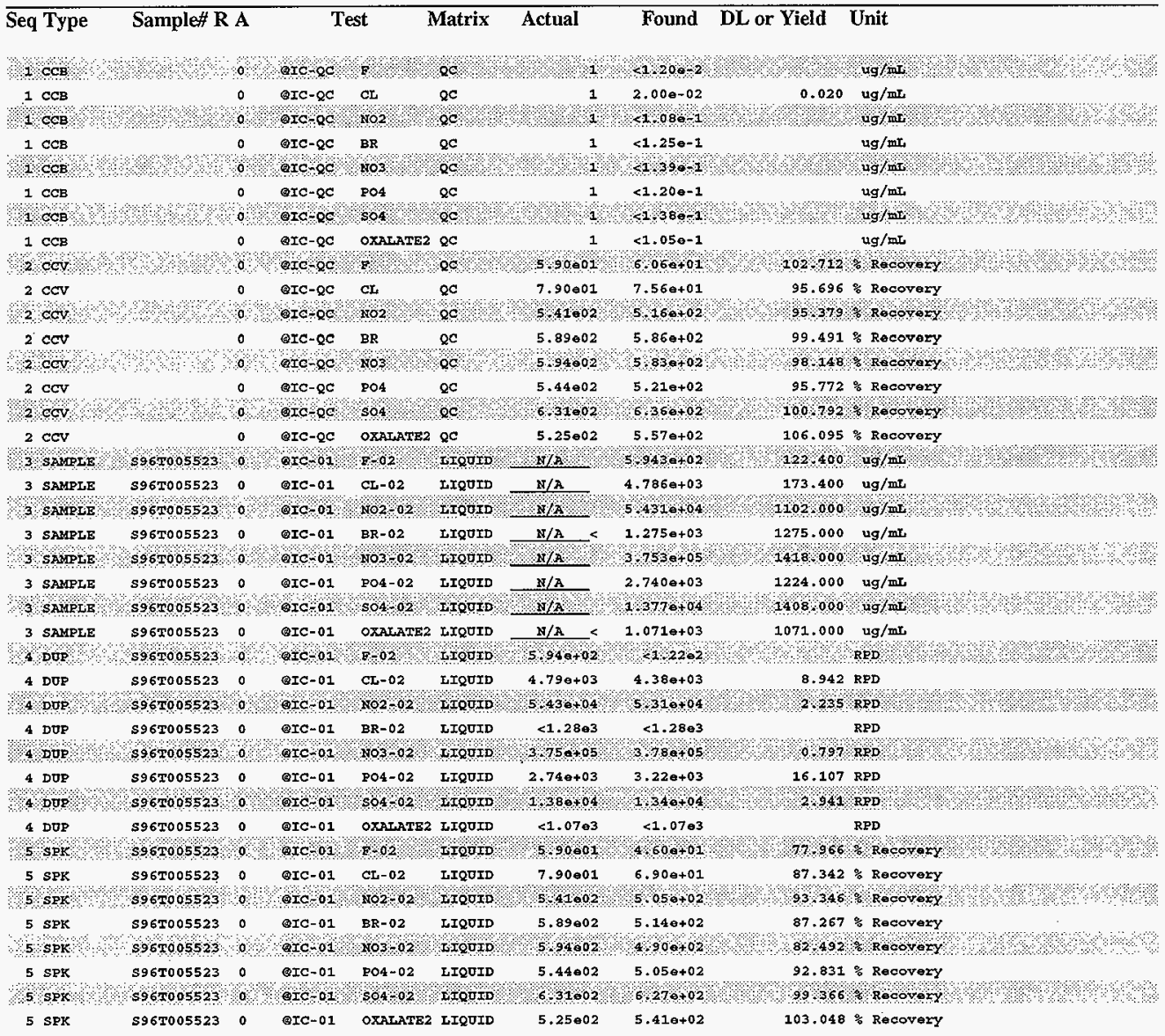




\section{LABCORE Completed Worklist Report for Worklist\# 14654}

Seq Type Sample\# RA

Test Matrix Actual Found DL or Yield Unit

Final page for worklist\# 14654

Analyst Signature Date

Analyst Signature

Date

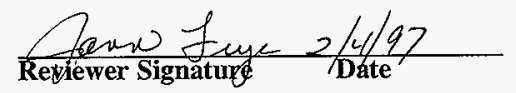




\section{LABCORE Data Entry Template for Worklist\# 14654}

Analyst:

Hetr Instrument: ICO

Method: LA-533-105 Rev/Mod D-1

Worklist Comment: @IC-01 FOR B-108

Book\# $360120-0$

RTS!

\begin{tabular}{|c|c|c|c|c|c|c|c|}
\hline s Type & Sample\# & RA & Test & Matrix & Group\# & Project & \\
\hline $1 \mathrm{CCB}$ & 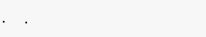 & & $Q I C-Q C$ & $\mathrm{QC}$ & & & \\
\hline $2 \mathrm{ccV}$ & & & QIC-QC & $\mathrm{QC}$ & & - & \\
\hline 3 SAMPLE & S96T005523 & 0 & $@ I C-01$ & LIQUID & 960013 & 379 B-108 & \\
\hline & Analytes Reque & ested: & $\begin{array}{l}=\text { BR-02 } \\
\text { OXAI_ATE2 }\end{array}$ & $\begin{array}{l}\therefore \text { CL-02 } \\
\therefore \text { PO4-02 }\end{array}$ & $\begin{array}{l}\mathrm{F}-02 \\
\mathrm{SO}-02\end{array}$ & , NO2-02 & , $\mathrm{NO} 3-02$ \\
\hline 4 DUP & S96T005523 & 0 & QIC-01 & LIQUID & & & \\
\hline 5 SPK & S96T005523 & 0 & QIC-01 & LIQUID & & & \\
\hline
\end{tabular}

\section{Final page for worklist \# 14654}

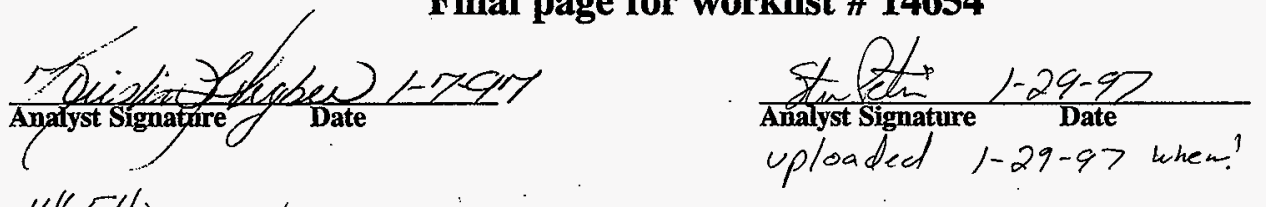

$14654 j a \cdot \operatorname{csv}$

Veledated 2/4a7 gimfue

Data Entry Comments:

$S=$ Worklist Slot Number, $R=$ Replicate Number, $A=$ Aliquot Code. 
HNF-SD-WM-DP-219, REV. 0

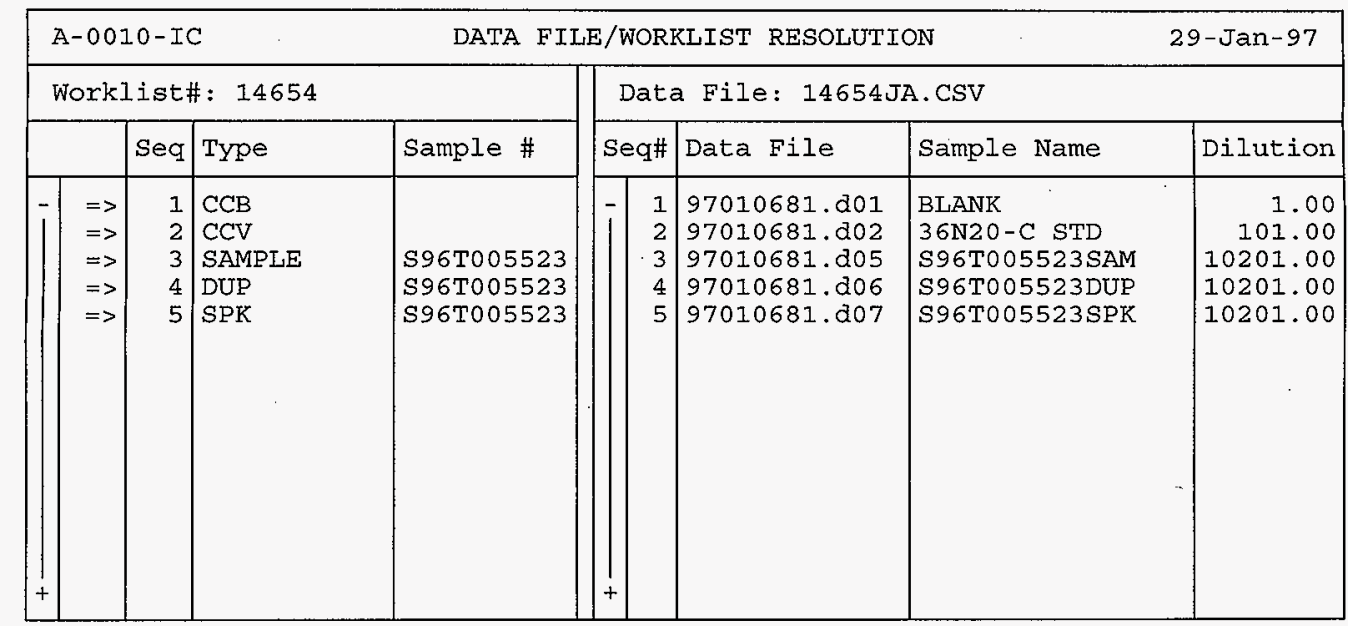

Save(F4) Abort(Shift-F3) ListFiles(Shift-F1) UploadFile(F8) 


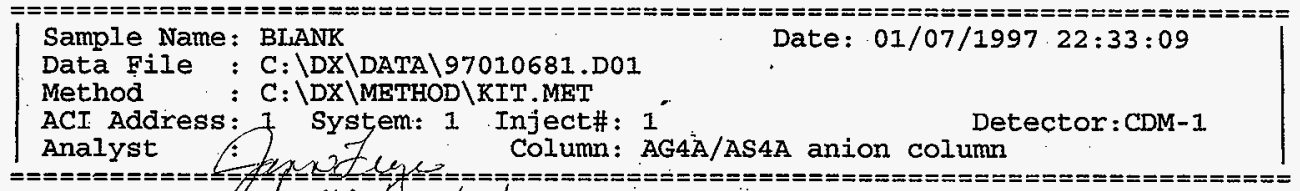

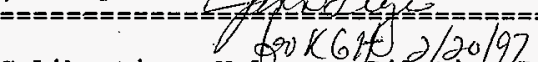

Calibration Volume Difution/points Rate start stop Area Reject

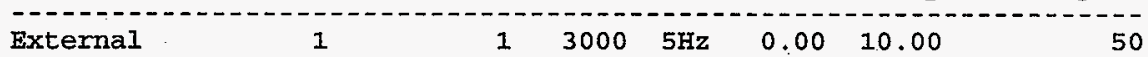

$\star * \star * * * * * * * * * * * * * * * * * * * * * * * *$ Peak Report: All Peaks $* * * * * * * * * * * * * * * * * * * * * * * * * * * *$

$\begin{array}{lrrr}\text { Pk. Ret Component } & \text { Concentration } & \text { Height } & \text { Area Bl. } \\ \text { Num } & \text { Theltal } & \text { Code }\end{array}$

11.43

0.000

75

$537 \quad 1$

2. 1.68 chloride

0.020

44

$194 \quad 1$

3. 3.45 nitrate

0.023

38

231

10.80

$1 .-3.54$

Totals

0.042

156

962

File: 97010681.D01 Sample: BLANK

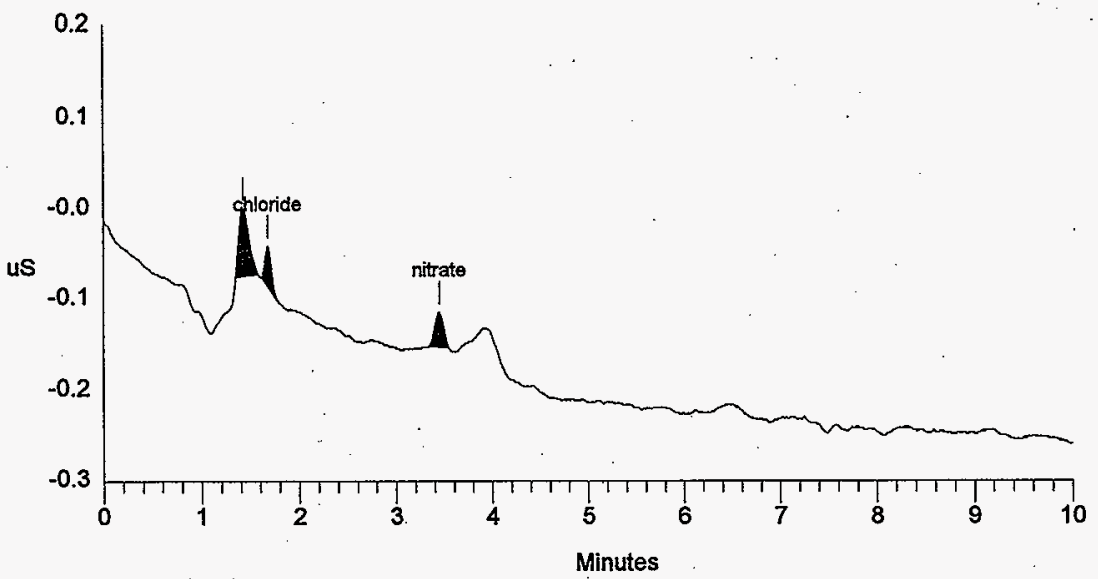

SIGNATURE ABOVE REPRESENTS CHEMICAL TECHNOLOGIST/CHEMIST THAT COMPLETED NERIFIED THE CALKBRATIONIANALYSIS ON PAGES 308 TO $2 / 2$ 
Data Reprocessed on 01/17/1997 08:00:23

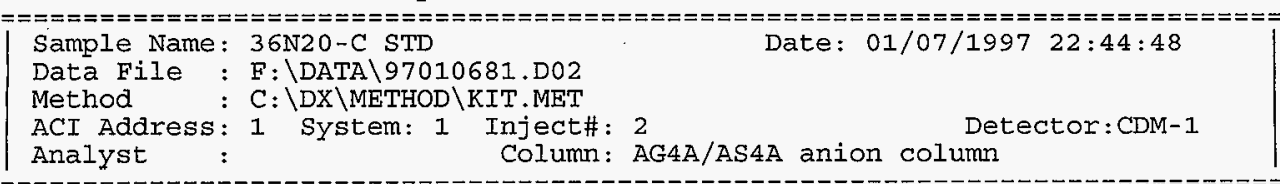

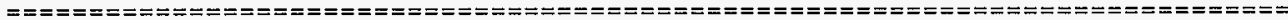

Calibration Volume Dilution Points Rate start Stop Area Reject

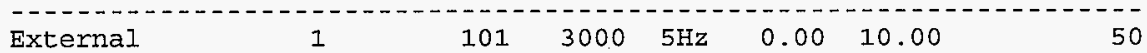

$* * * * * * * * * * * * * * * * * * * * * * * * * * *$ Peak Report: All Peaks $* * * * * * * * * * * * * * * * * * * * * * * * * * * *$

$\begin{array}{lrrr}\text { Pk. Ret Component } & \text { Concentration } & \text { Height } & \text { Area Bl. } \\ \text { Num } & \text { Time Name } / \mathrm{ml} & \text { Code }\end{array}$

\begin{tabular}{|c|c|c|c|c|c|c|c|}
\hline 1 & 0.93 & & 0.000 & 23 & 89 & 1 & \\
\hline 2 & 1.11 & fluoride & 60.583 & 1122 & 8017 & 3 & 3.09 \\
\hline 3 & 1.42 & & 0.000 & 81 & 576 & 4 & \\
\hline 4 & 1.67 & chloride & 75.637 & 1827 & 9506 & $I$ & 0.00 \\
\hline 5 & 1.97 & nitrite & 516.497 & 6366 & 43299 & 1 & -2.95 \\
\hline 6 & 2.95 & bromide & 585.679 & 5098 & 34226 & $I$ & -0.11 \\
\hline 7 & 3.36 & nitrate & 582.950 & 5705 & 45909 & 1 & -6.15 \\
\hline 8 & 4.88 & phosphate & 520.973 & 1522 & 20762 & 1 & -1.61 \\
\hline 9 & 6.35 & sulfate & 636.254 & 4321 & 67525 & 1 & -4.03 \\
\hline 10 & 8.37 & oxalate & 557.451 & 2140 & 42672 & 1 & -3.68 \\
\hline & & & 3536.025 & 28205 & 272582 & & \\
\hline
\end{tabular}

\section{File: 97010681.D02 Sample: 36N20-C STD}

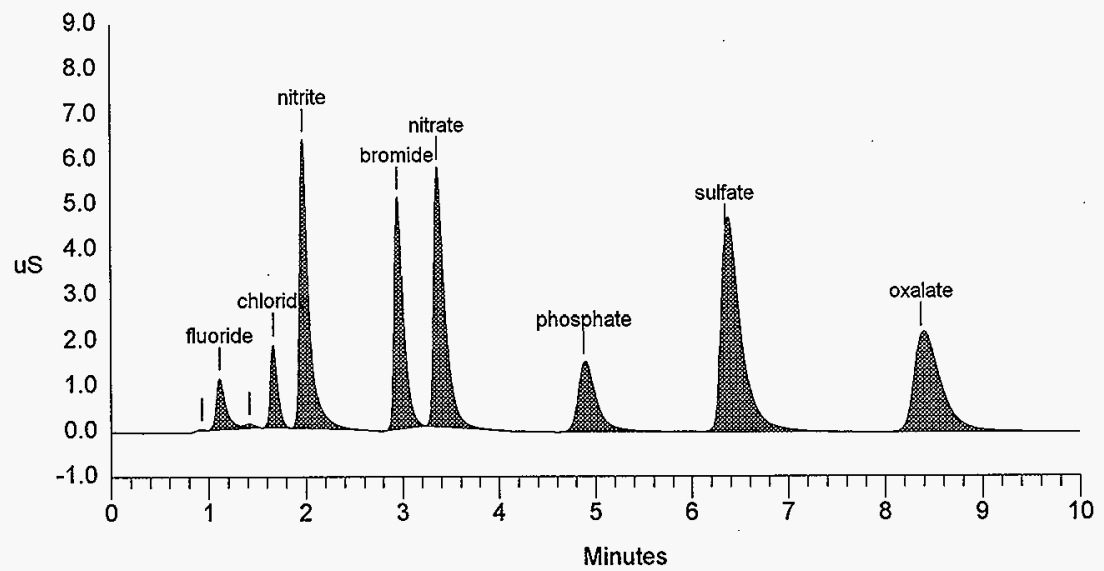


Data Reprocessed on 01/17/1997 08:01:47

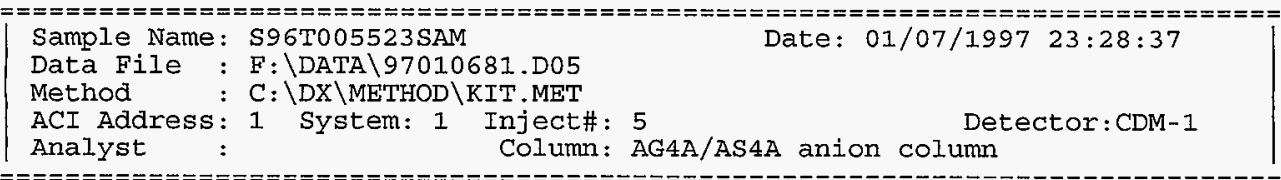

Calibration Volume Dilution Points Rate start stop Area Reject

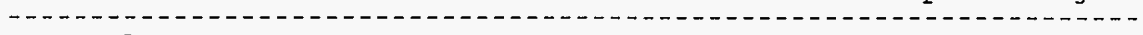

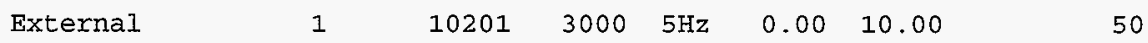

$* * * * * * * * * * * * * * * * * * * * * * * * * * *$ Peak Report: All Peaks $* * * * * * * * * * * * * * * * * * * * * * * * * * * *$

$\begin{array}{lrrr}\text { Pk. Ret Component } & \text { Concentration } & \text { Height } & \text { Area Bl. } \\ \text { Num } & \text { Time Name } & \text { Coded }\end{array}$

Num Time Name

$\mathrm{ug} / \mathrm{ml}$

Code

$\begin{array}{lll}1 & 0.93 & \\ 2 & 1.14 & \text { fluoride } \\ 3 & 1.25 & \\ 4 & 1.42 & \\ 5 & 1.67 & \text { chloride } \\ 6 & 1.97 & \text { nitrite } \\ 7 & 3.25 & \text { nitrate } \\ 8 & 4.91 & \text { phosphate } \\ 9 & 6.40 & \text { sulfate }\end{array}$

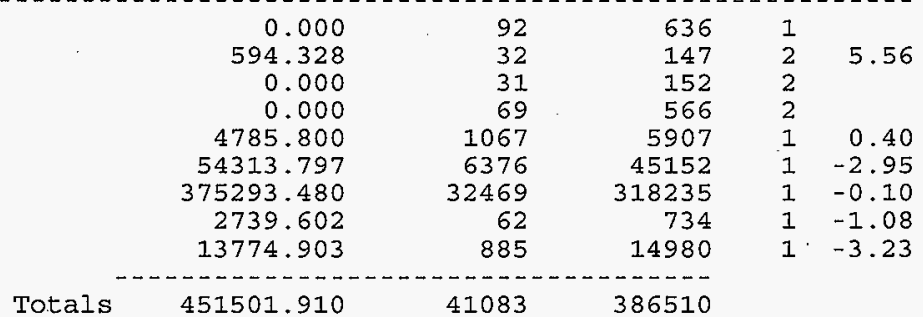

File: 97010681.D05 Sample: S96T005523SAM

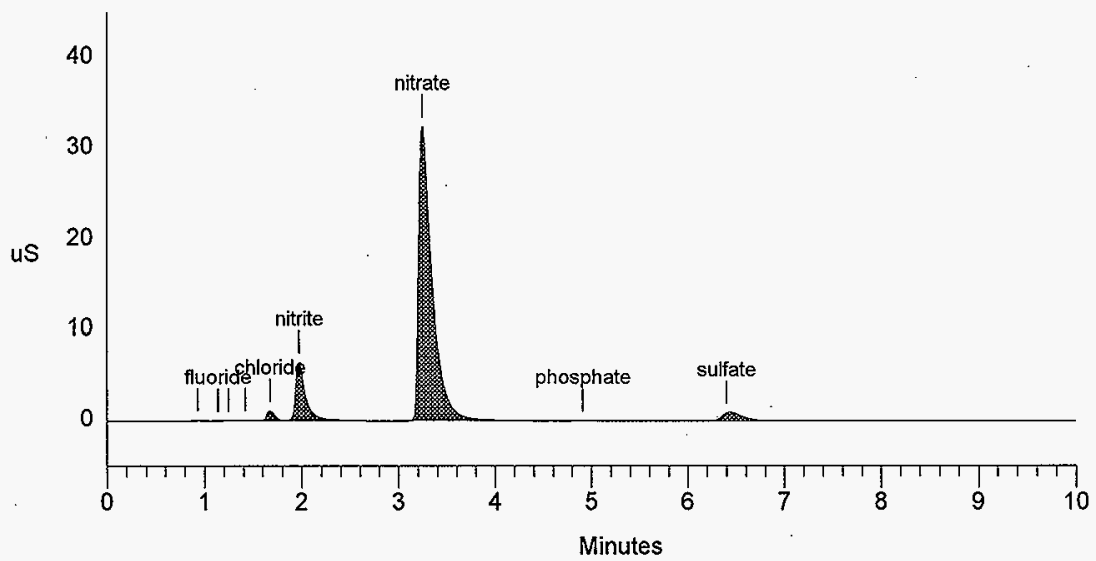


Data Reprocessed On 01/17/1997 08:03:26

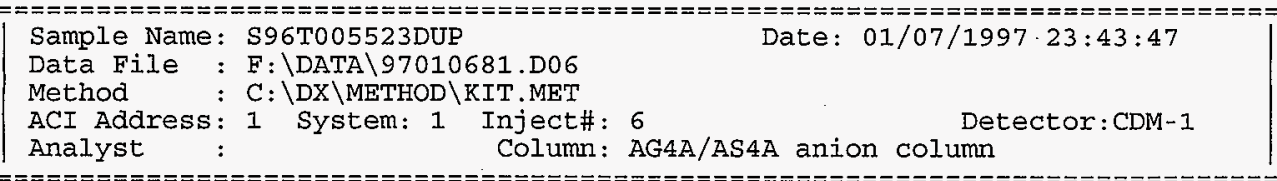

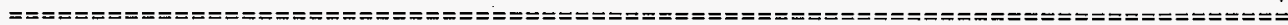

Calibration Volume Dilution Points Rate start stop Area Reject

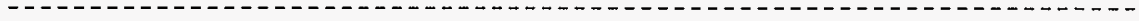

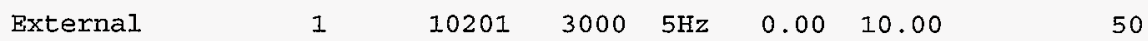

$* * * * * * * * * * * * * * * * * * * * * * * * * * *$ Peak Report: All Peaks $* * * * * * * * * * * * * * * * * * * * * * * * * * * *$

Pk. Ret Component Concentration Height Area Bl. $\%$ Delta

Num Time Name

$\mathrm{ug} / \mathrm{ml}$ Code

$\begin{array}{lll}1 & 0.93 \\ 2 & 1.41 \\ 3 & 1.67 \text { chloride } \\ 4 & 1.97 \text { nitrite } \\ 5 & 3.25 \text { nitrate } \\ 6 & 4.93 \text { phosphate } \\ 7 & 6.40 \text { sulfate }\end{array}$

$\begin{array}{rrrrrr}0.000 & 90 & 634 & 1 & \\ 0.000 & 62 & 648 & 1 & \\ 4380.630 & 1000 & 5399 & 1 & 0.00 \\ 53093.084 & 6284 & 44099 & 1 & -3.28 \\ 378222.060 & 32262 & 321007 & 1 & -0.10 \\ 3215.735 & 73 & 922 & 1 & -0.54 \\ & 13426.554 & 868 & 14623 & 1 & -3.23 \\ \text { Totals } & 452338.064 & 40638 & 387331 & & \end{array}$

File: 97010681.D06 Sample: S96T005523DUP

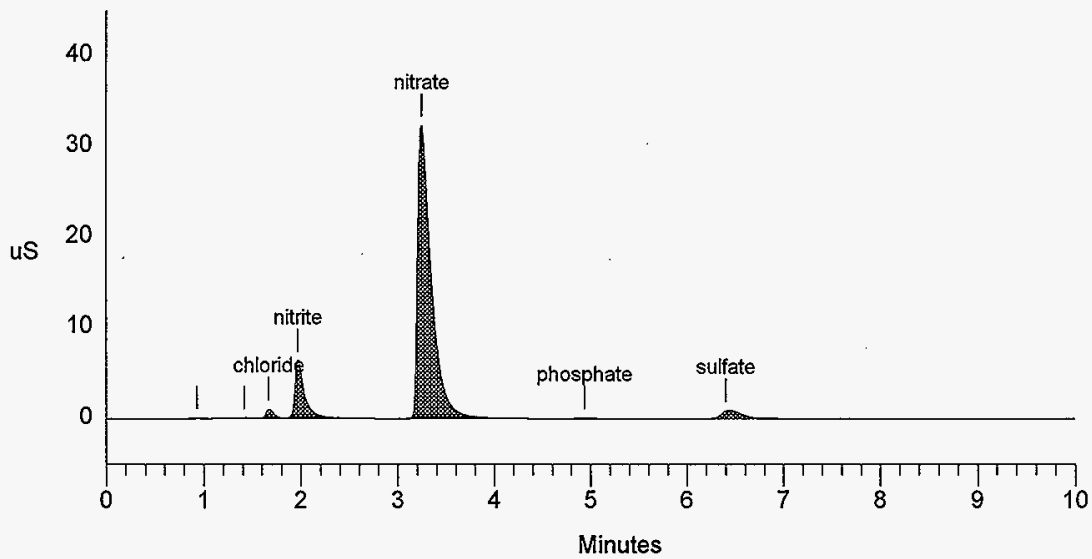


Data Reprocessed on 01/17/1997 08:04:48

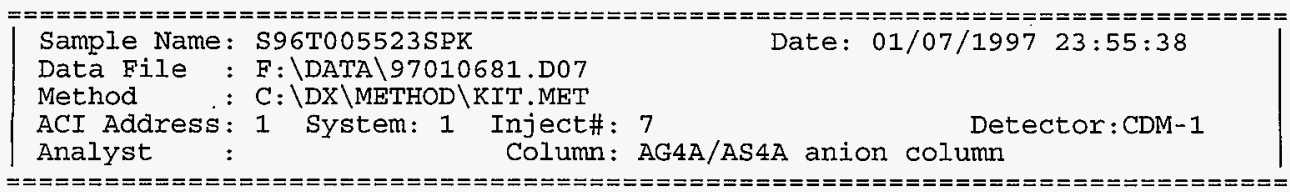

Calibration Volume Dilution Points Rate start stop Area Reject

External 102013000 5Hz $0.0010 .00 \quad 50$

Peak Report: All Peaks

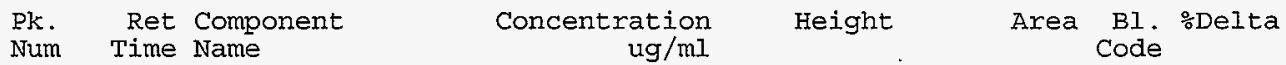

\begin{tabular}{|c|c|c|c|c|}
\hline $\begin{array}{r}0.000 \\
5242.048\end{array}$ & $\begin{array}{r}61 \\
1070\end{array}$ & $\begin{array}{r}232 \\
6749\end{array}$ & $\begin{array}{l}1 \\
1\end{array}$ & 3.09 \\
\hline 11753.259 & 2780 & 14757 & 1 & -0.80 \\
\hline 105318.497 & 13547 & 89687 & 1 & -3.28 \\
\hline 51919.282 & 4988 & 29894 & 1 & 0.00 \\
\hline 424813.114 & 36768 & 365891 & 1 & 0.10 \\
\hline 53709.175 & 1562 & 21207 & 1 & $-I .61$ \\
\hline 77136.724 & 5453 & 81181 & 1 & -4.03 \\
\hline 54677.360 & 2095 & 41415 & 1 & -3.68 \\
\hline 784569.458 & 68323 & 651013 & & \\
\hline
\end{tabular}

File: 97010681.D07 Sample: S96T005523SPK

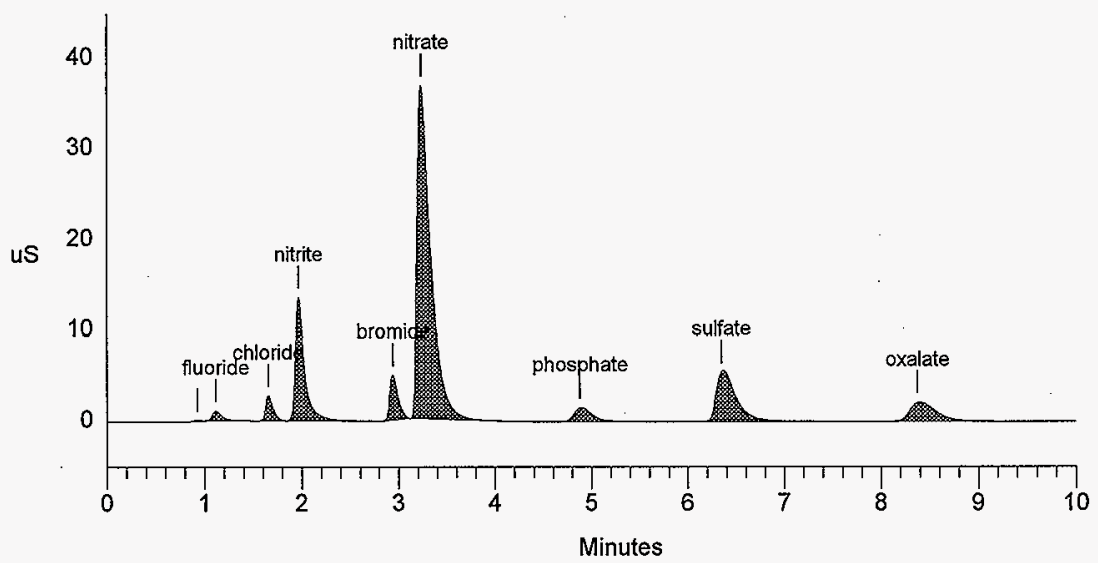




\section{LABCORE Completed Worklist Report for Worklist\# 14853}

Analyst: kgh

Aethod:
Instrument: $\mathrm{ICO}$

Book\# Jonbex

Worklist Comment: B-108 IC. RCJ

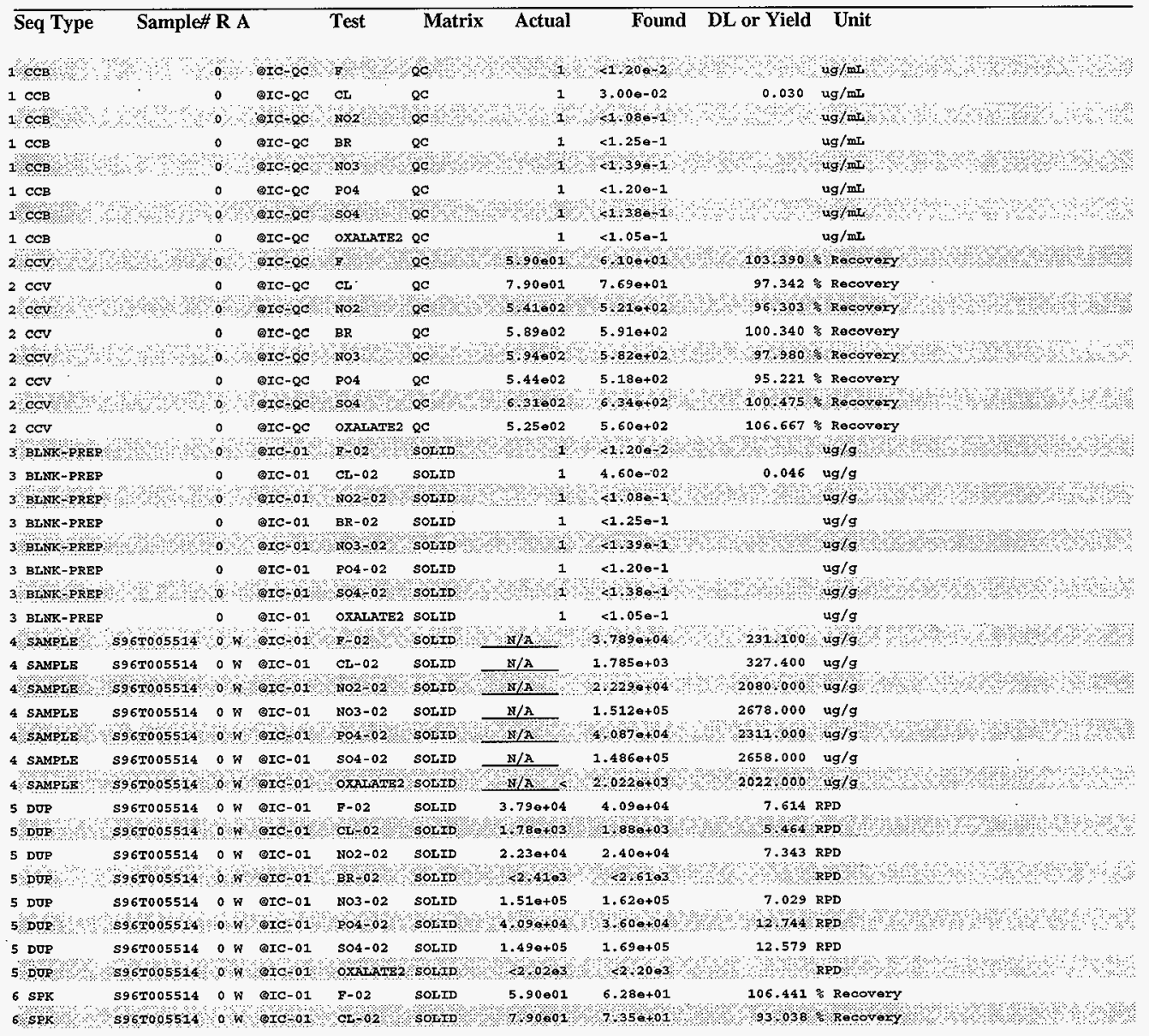




\section{LABCORE Completed Worklist Report for Worklist\# 14853}

\begin{tabular}{|c|c|c|c|c|c|c|c|c|}
\hline Seq Type & Samplet & $\overline{7 \mathbf{R A}}$ & & Test & Matrix & Actual & Found DL or Yield & Unit \\
\hline $6 \mathrm{SPK}$ & S96T005514 & $0 \mathrm{~W}$ & QIC-01 & No2-02 & SOLID & $5.41 \mathrm{e} 02$ & $5.05 e+02$ & \% Recovery \\
\hline $6 \mathrm{sPK}$ & $596 \mathrm{~T} 005514$ & ow & $\otimes I C-01$ & $B R-02$ & SOLID & 5.89 .02 & $5.58 e+02$ & : Recovery \\
\hline $6 \mathrm{spr} / 1$ & 5967005514 & $0 \%$ & $8 \mathrm{IC}-0$ & $103-02$ & SoLXP & 5.9402 & $6350+02$ & 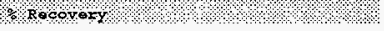 \\
\hline $6 \mathrm{SPK}$ & $596 T 005514$ & $0 \mathrm{w}$ & $\operatorname{arc}-01$ & $804-02$ & SOLID & $5.44 \mathrm{e} 02$ & $5.02 e+02$ & $\because$ Recovery \\
\hline $6 . \mathrm{SPK}$ & $596 r 005514$ & $0 \%$ & Q1C-01 & $\mathrm{so4} 02$ & SOSID & $6 \times 31002$ & $671 \% 02$ & 8 Roovory \\
\hline $6 \mathrm{SPK}$ & $\$ 967005514$ & $0 \%$ & erc-01 & OXALATE2 & SOLID & 5.25002 & $5.43 e+02$ & $\because$ Recovery \\
\hline 7 SAMFE & 8967005513 & ow & $8 \mathrm{CO}-0 \mathrm{O}$ & B-22 & SofID & $\mathrm{N} / \mathrm{A}$ & 1,483004 & $u \$ / g$ \\
\hline 7 SAMPLE & S96T005513 & $0 w$ & arc-01 & $C E-02$ & SOLID & $N / A$ & $1.756 a+03$ & $\mathrm{ug} / \mathrm{g}$ \\
\hline 7 SAMELE & $596 \times 005513$ & $00 \mathrm{~N}$ & QIC- 01 & $102-02$ & 80010 & $\mathrm{r} / \mathrm{A}$ & $17070-04$ & $4.9 /: 9$ \\
\hline 7 SAMPLE & S96T005513 & $0 \mathrm{w}$ & IC-01 & $B R-02$ & SorID & $N / A<$ & $5.3300+02$ & $\mathrm{ug} / \mathrm{g}$ \\
\hline $7 S \mathrm{SMEZ}$ & 896005513 & $0 \mathrm{n}$ & eIC-0x & $803-02$ & SotTo & $\mathrm{N} / \mathrm{N}$ & $77630+04$ & uglo \\
\hline 7 SAMPLE & S96T005513 & $0 \mathrm{w}$ & arc-01 & PO4-02 & SOLID & $\mathbb{N} / \mathrm{A}$ & $1.2720+05$ & $4 g / g$ \\
\hline 7 SAYPLE & $896 \mathrm{T005513}$ & o. W & QIC 01 & 50402 & SOIID & $\pi / A$ & $82130-03$ & $0 / 9$ \\
\hline 7 SAMPLE & S967005513 & $0 \mathrm{w}$ & $\operatorname{arc}-01$ & OXALATE2 & sorrd & $\mathrm{N} / \mathrm{A}<$ & $4.477 e+02$ & $\mathrm{ug} / \mathrm{g}$ \\
\hline 8 DOR & 896005513 & on & $0 x C-01$ & ro2 & SOLID & $1,480+04$ & $124-04$ & RPD \\
\hline $8 \mathrm{DUP}$ & $\$ 96 T 005513$ & $0 \mathrm{w}$ & IC-01 & CL- 02 & SOLID & $1.760+03$ & $8.65 e+02$ & RPD \\
\hline 8 DUR: & s96ro05513 & $0 \%$ & IC- 01 & $\$ 0202$ & SOLTD & $1,110+04$ & $98806+03$ & $\mathrm{RPD}$ \\
\hline 8 DUP & S96T005513. & o w & OIC-0I & $B R-02$ & SOLID & $<5.33 e^{2}$ & $<5.0302$ & RPD \\
\hline 8 Dणp: & s967005513 & $0 \mathrm{w}$ & $0 x<01$ & 103 -2 & SOLID & $7,760+04$ & $6910+04$ & RPD \\
\hline $8 D O P$ & $\$ 967005513$ & $0 \mathrm{~W}$ & OIC-OI & P04-02 & SOLID & $1.27 \bullet+05$ & $1.05 a+05$ & RPD \\
\hline 8 Dणr & $\$ 967005513$ & $0 \%$ & ercors & 50402 & SoLID & $8,210+03$ & $685+03$ & $\mathrm{ReD}$ \\
\hline $8 D O P$ & 5967005513 & $0 \%$ & IC-01 & OXALATE2 & sotro & $<4.4802$ & $<4.23 \cdot 2$ & RPD \\
\hline 10 ovo & 5961005515 & $0 \mathrm{w}$ & $0 \times 0-01$ & $\mathrm{r}-02$ & soL $\mathrm{XD}$ & $572 \theta+04$ & $7600+04$ & $\mathrm{RpD}$ \\
\hline $10 D J P$ & S96T005515 & $0 \mathrm{~K}$ & $01 C-01$ & $\mathrm{CL}-02$ & SOIID & $8.84 a+02$ & $1.25 \bullet+03$ & RPD \\
\hline $10 \mathrm{DPr}$ & 8969005515 & $0 \mathrm{n}$ & $010-01$ & No2 02 & sodid & $9.740+03$ & $10090+04$ & seo \\
\hline 10 DOR & 5967005515 & $0 \mathrm{~W}$ & $\otimes I C-01$ & $B R-02$ & SOLTD & $<2.6003$ & $<2.54 \otimes 3$ & RPD \\
\hline $10 \%$ DOP & 3967005515 & $0 \%$ & $Q x-0.1$ & 10302 & SoL & $6 \not 140+04$ & $60976+04$ & RPD \\
\hline 10 DUP & 5967005515 & $0 \mathrm{n}$ & Orc-01 & $804-02$ & SoLrD & $1.190+05$ & $3.89 e+04$ & RPD \\
\hline 10 Dण & s96To055 & $0 \mathrm{~W}$ & 01001 & 50002 & SOLID & $1.920+05$ & $3.270+05$ & RPD \\
\hline $10 \mathrm{DOP}$ & 5967005515 & 0 พ & QIC-01 & OXALATE2 & SOLID & $<2.19 e 3$ & $<2.14 \mathrm{e3}$ & RPD \\
\hline
\end{tabular}

\section{Analyst Signature Date}




\section{LABCORE Data Entry Template for Worklist\# 14853}

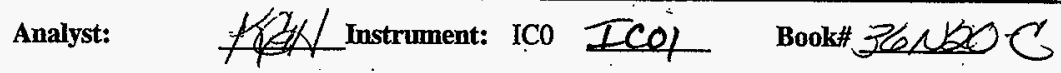

Method: LA-533-105 Rev/Mod $\mathrm{D}-$

Worklist Comment: B-108 IC. RCJ

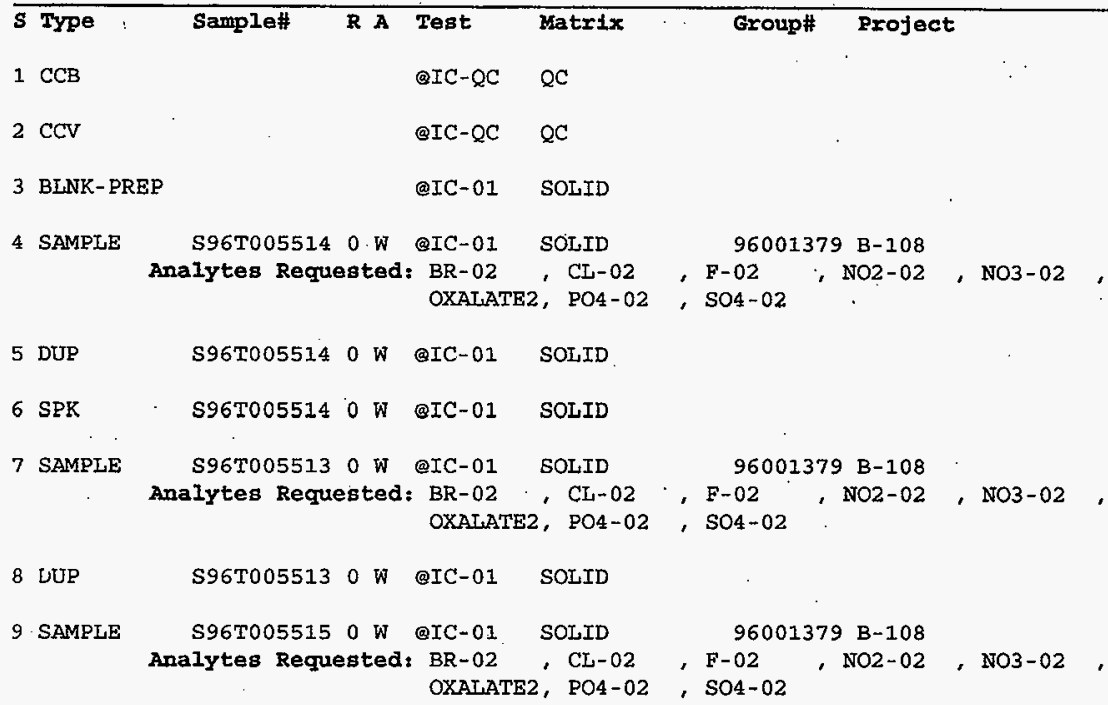

10 DUP

S96T005515 O WIC-01 SOLID

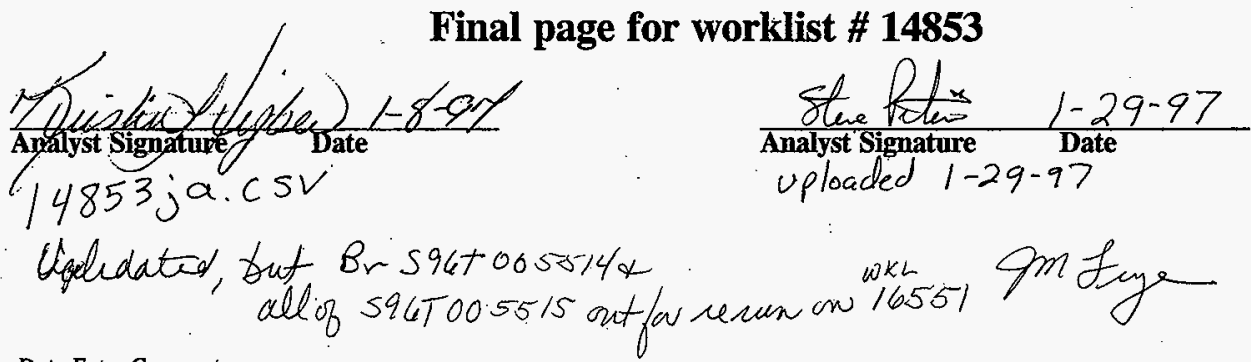

Data Entry Comments: 
HNF-SD-WMM-DP-219, REV. 0

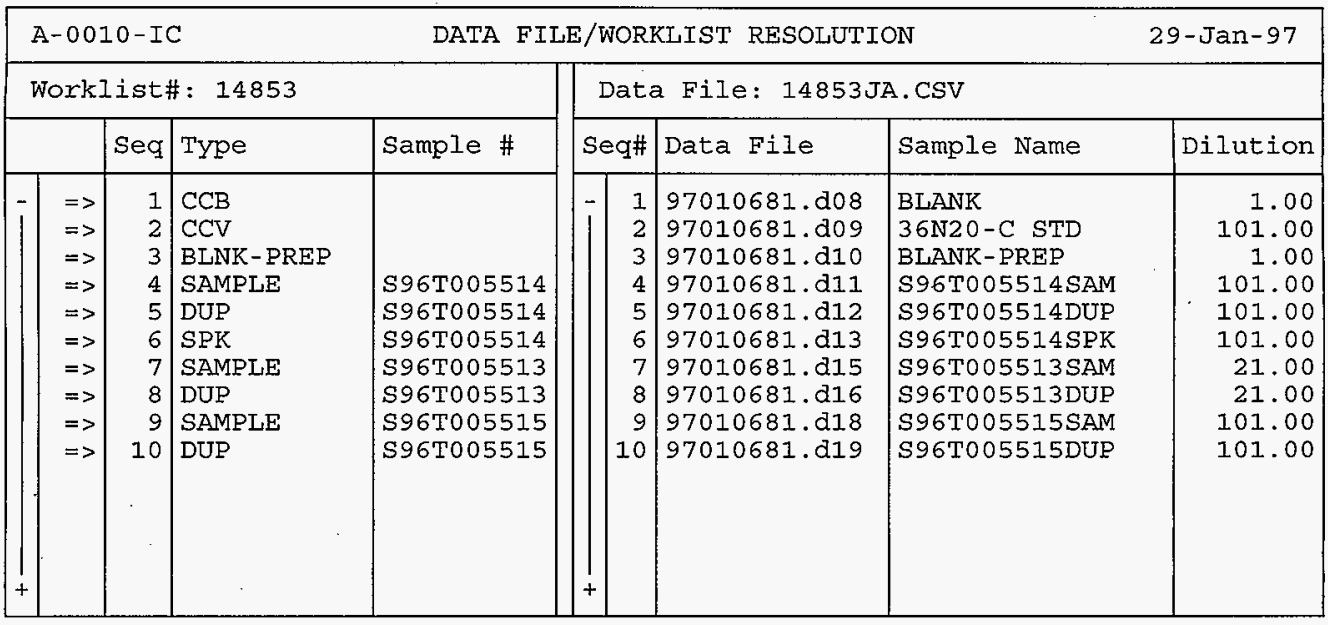

Save (F4) Abort(Shift-F3) ListFiles(Shift-F1) UploadFile(F8) 


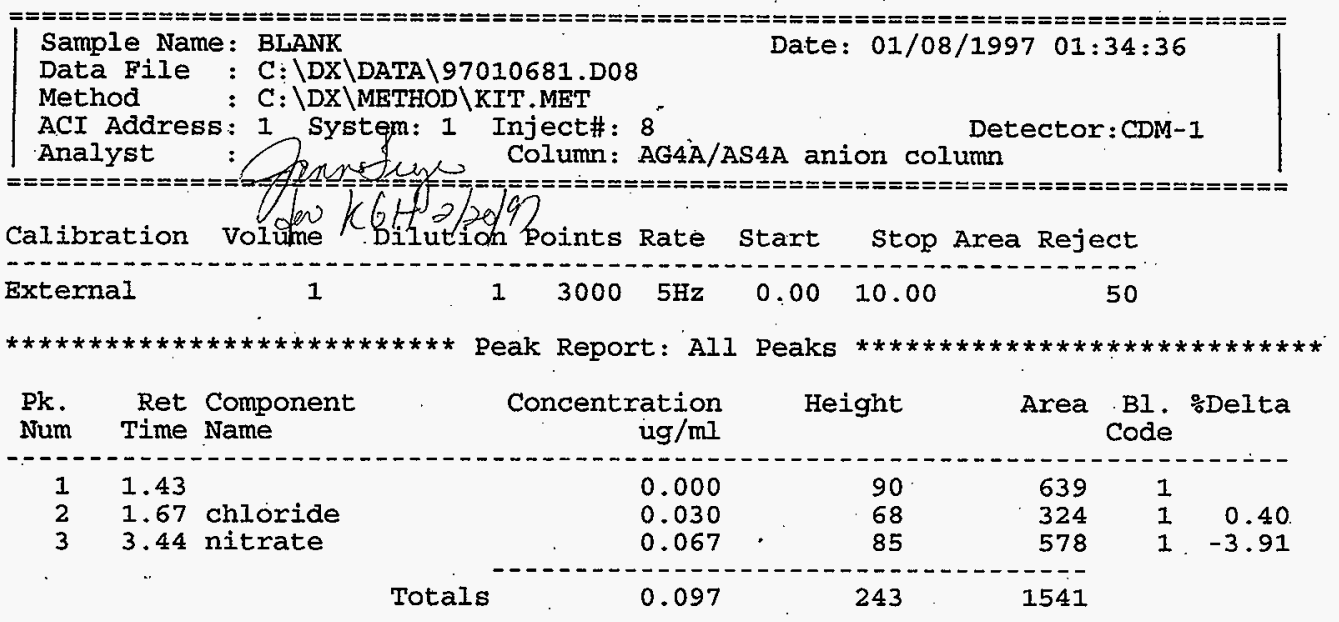

File: 97010681.D08 Sample: BLANK

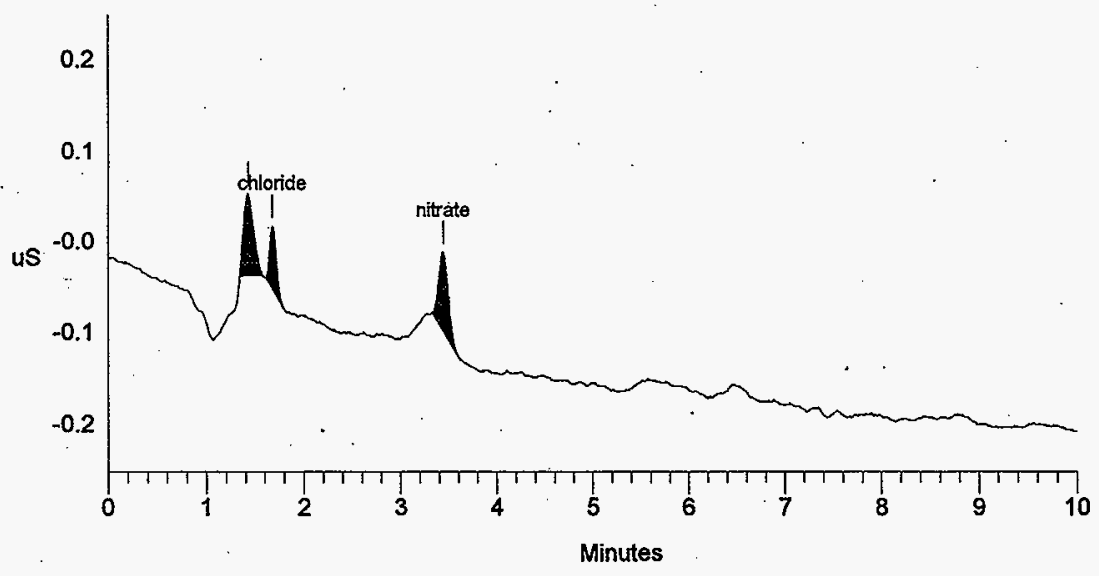

SIGNATURE ABOVE REPRESENTS CHEMICAL TECHNOLOGIST/CHEMIST THAT COMPLFTEDNERIFIED THE CALIERATIONANALYSIS ON PAGES $3 / 7$ TO 326 
Data Reprocessed On 01/17/1997 08:44:53

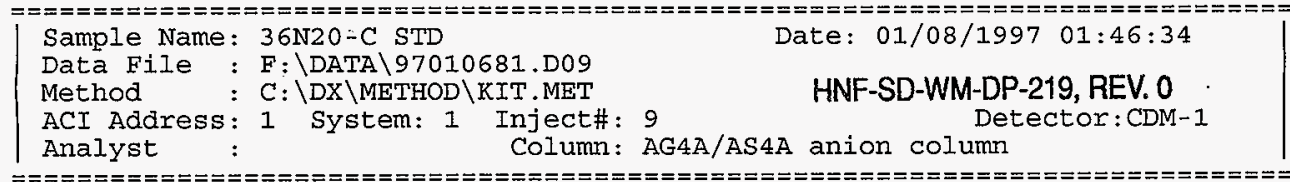

Calibration Volume Dilution Points Rate start stop Area Reject

Cal

External

1

101

$3000 \quad 5 \mathrm{~Hz}$

0.0010 .00

50

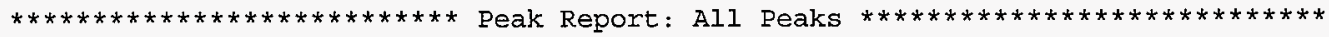

$\begin{array}{lrrr}\text { Pk. Ret Component } & \text { Concentration } & \text { Height } & \text { Area Bl. } \\ \text { Num } & \text { Bime Name } & \text { Code }\end{array}$

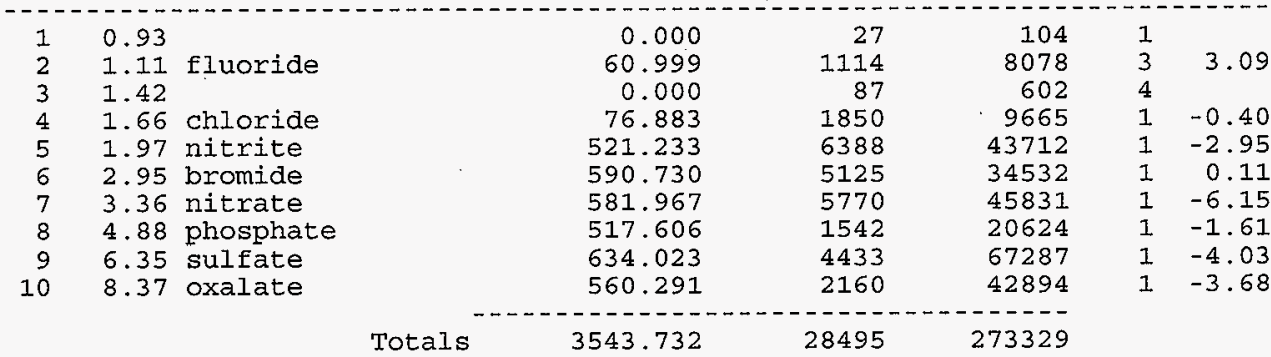

\section{File: 97010681.D09 Sample: 36N20-C STD}

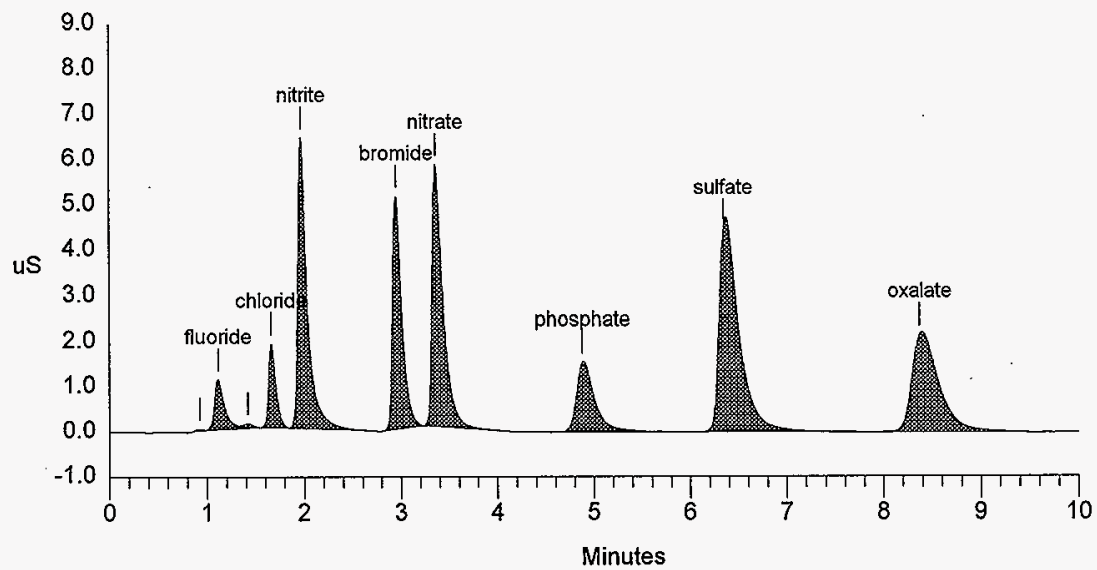


Calibration Volume Dilution Points Rate start stop Area Reject

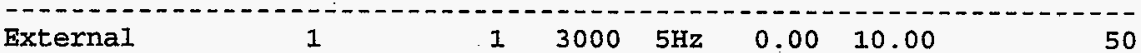

$* * * * * * * * * * * * * * * * * * * * * * * * * * *$ Peak Report: All Peaks $* * * * * * * * * * * * * * * * * * * * * * * * * * * *$

Pk. Ret Component - Concentration Height Area Bl. 5 Delta Num Time Name ug/ml Code

Num Time Name

1. 1.27

21.70 chloride

0.000

44

0.046

57

0.038

54

286

3.45 nitrate

0.083

351

1

12.00

$1-3.54$

Totals

0.083

155

1160

File: 97010681.D10 Sample: BLANK-PREP

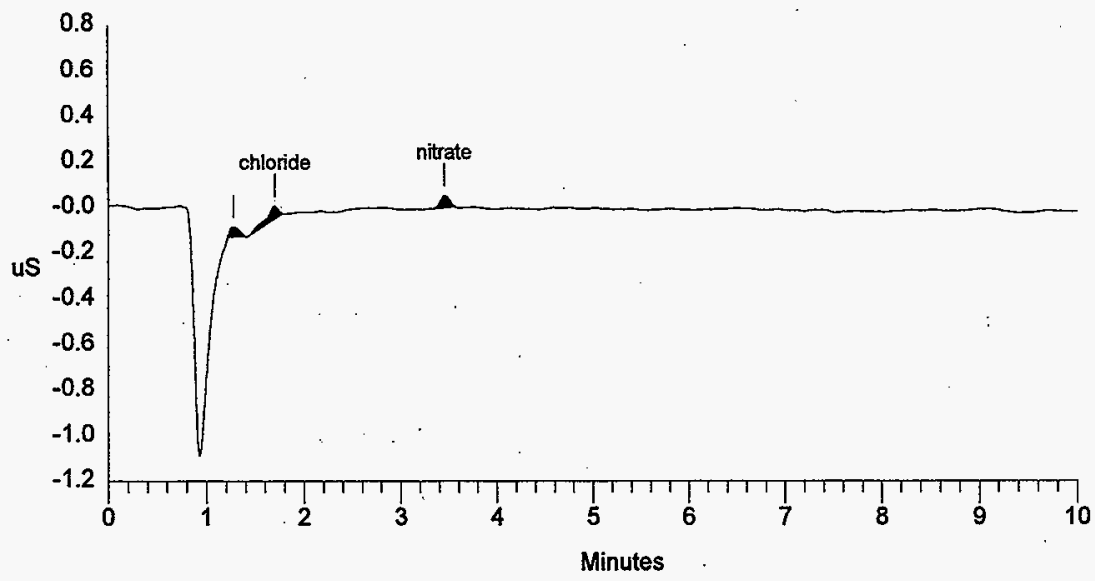




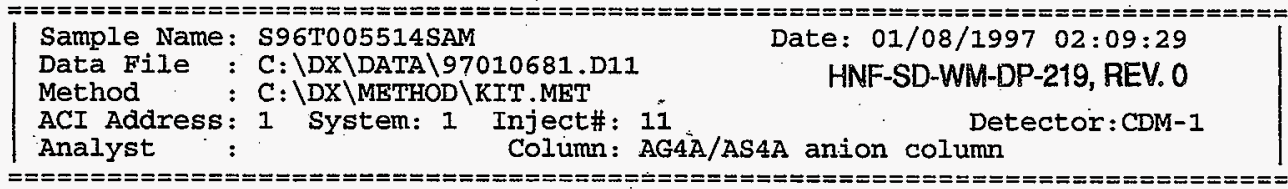

Calibration Volume Dilution Points Rate start stop Area Reject

-

External

$1 \quad 101 \quad 3000 \quad 5 \mathrm{~Hz} \quad 0: 00 \quad 10.00$

50

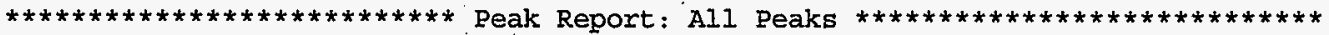

Pk. Ret Component Concentration Height Area Bl. ofDelta

Num Time Name $\mathrm{ug} / \mathrm{ml}$ Code

1.1 .09 fluoride

21.68 chloride

$3 \quad 1.99$ nitrite

43.34 nitrate

54.91 phosphate

$6 \quad 6.35$ sulfate

\begin{tabular}{rrrrrr}
198.677 & 4306 & 29332 & 1 & 1.23 \\
9.360 & 209 & 1117 & 1 & 0.80 \\
116.912 & 1194 & 8790 & 1 & -2.30 \\
793.114 & 7512 & 62757 & 1 & -6.70 \\
& 214.323 & 591 & 8253 & 1 & -1.08 \\
& 779.098 & 5737 & 82835 & 1 & -4.03 \\
\hline & 2111.483 & 19548 & 193084 & &
\end{tabular}

File: 97010681.D11 Sample: S96T005514SAM

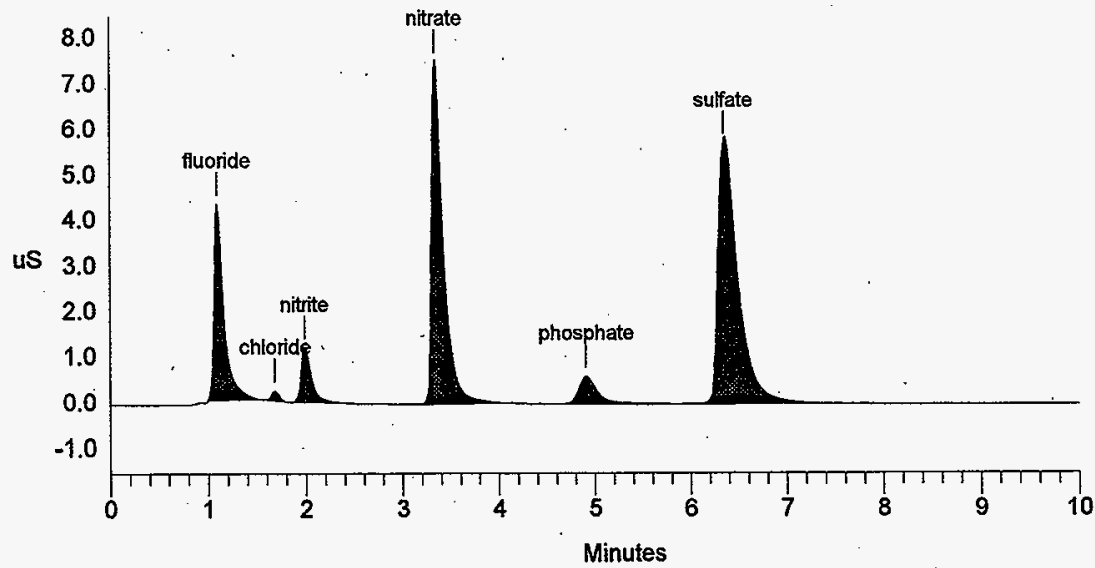




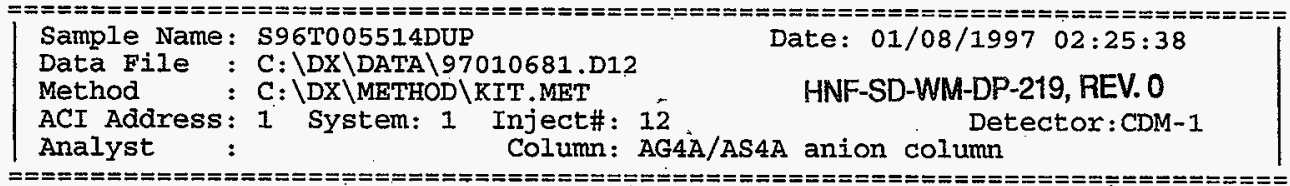

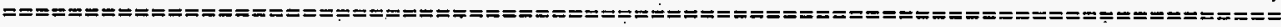
Calibration Volume Dilution Points Rate start stop Area Reject

$\begin{array}{lllll} & & & \\ \text { External } & 1 & & & \end{array}$

$* * * * * * * * * * * * * * * * * * * * * * * * * * *$ Peak Report: All Peaks $* * * * * * * * * * * * * * * * * * * * * * * * * * * *$

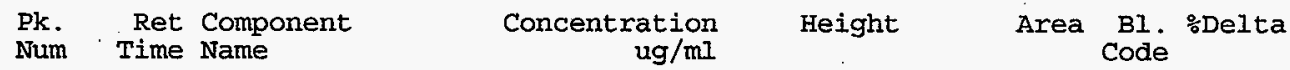

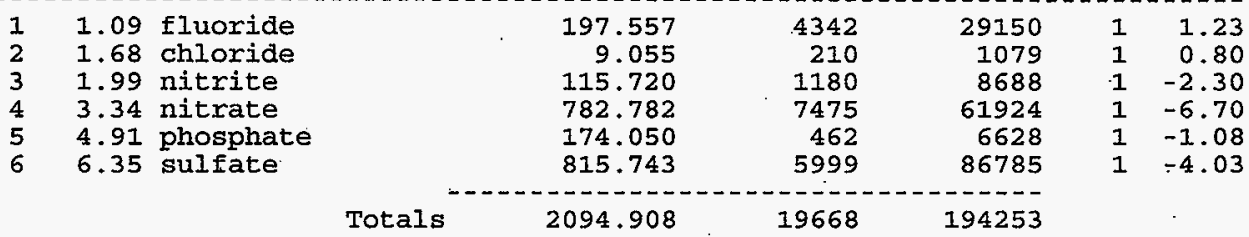

File: 97010681.D12 Sample: S96T005514DUP

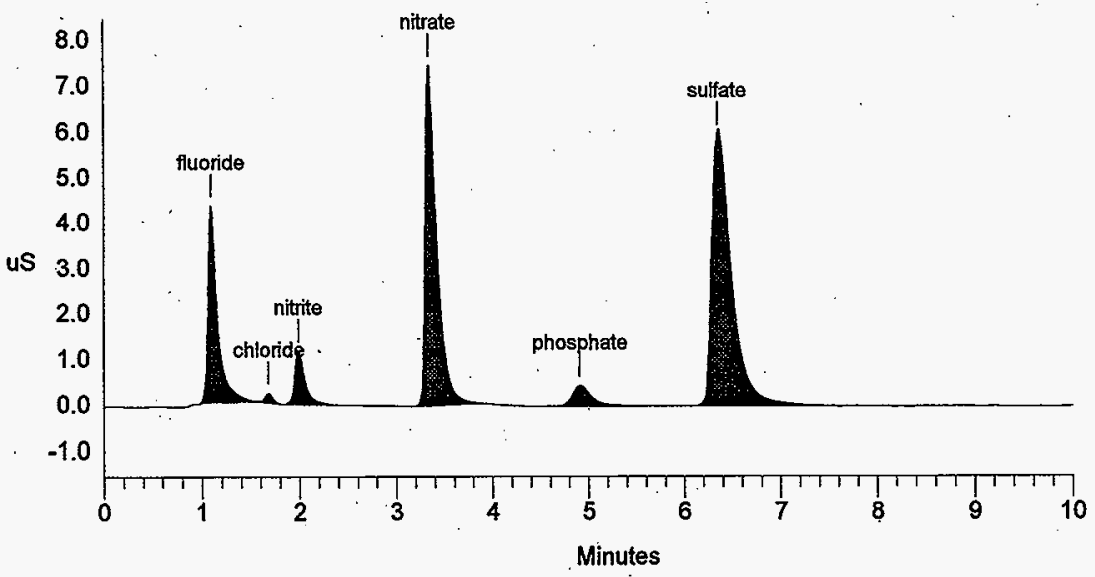


Data Reprocessed On 01/17/1997 08:47:36

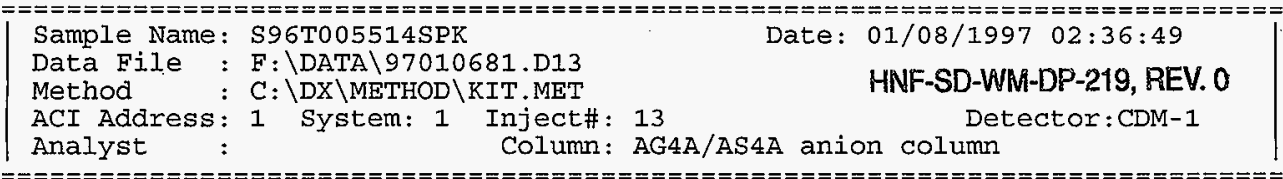

Calibration Volume Dilution Points Rate start stop. Area Reject

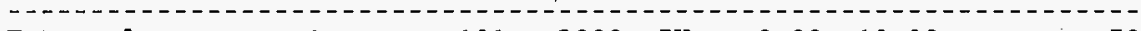

External

1

$101.3000 \quad 5 \mathrm{~Hz} \quad 0.00 \quad 10.00$

50

$* * * * * * * * * * * * * * * * * * * * * * * * * * *$ Peak Report: All Peaks $* * * * * * * * * * * * * * * * * * * * * * * * * * * *$

Pk. Ret Component Concentration Height Area Bl. $\frac{\text { Delta }}{}$ Num Time Name ug/ml Code

\begin{tabular}{|c|c|c|c|c|c|c|c|}
\hline 1 & 0.93 & & 0.000 & 20 & 58 & 1 & \\
\hline 2 & 1.09 & fluoride & 261.527 & 6125 & 39870 & 1 & 0.62 \\
\hline 3 & 1.65 & chlóride & 82.878 & 1929 & 10434 & 1 & -0.80 \\
\hline 4. & 1.97 & nitrite & 622.077 & 7682 & 52518 & 1 & -3.28 \\
\hline 5. & 2.94 & bromide & 558.153 & 4941 & 32559 & 1 & 0.00 \\
\hline 6 & 3.31 & nitrate & 1428.231 & 13338 & 114760 & 1 & 0.10 \\
\hline 7 & 4.88 & phosphate & 716.430 & 2227 & 28866 & 1 & -1.61 \\
\hline 8 & 6.29 & sulfate & 1449.946 & 10103 & 156635 & 1 & -4.84 \\
\hline \multirow[t]{2}{*}{9} & 8.37 & oxalate & 542.707 & 2144 & 41520 & 1 & -3.68 \\
\hline & & & 5661.948 & 48508 & 477219 & & \\
\hline
\end{tabular}

\section{File: 97010681.D13 Sample: S96T005514SPK}

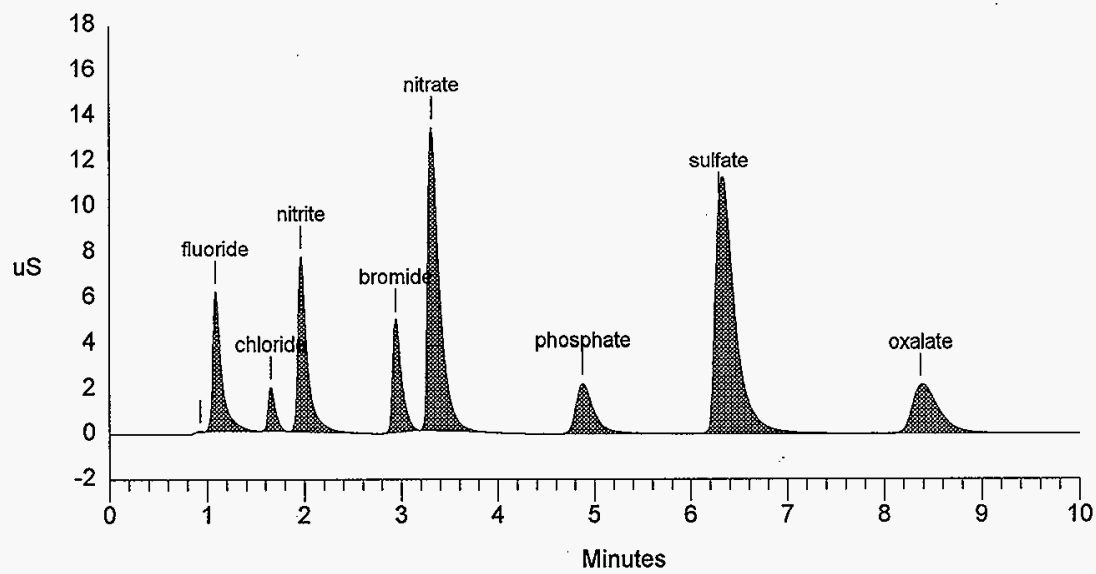


Data Reprocessed On 01/17/1997 08:48:50

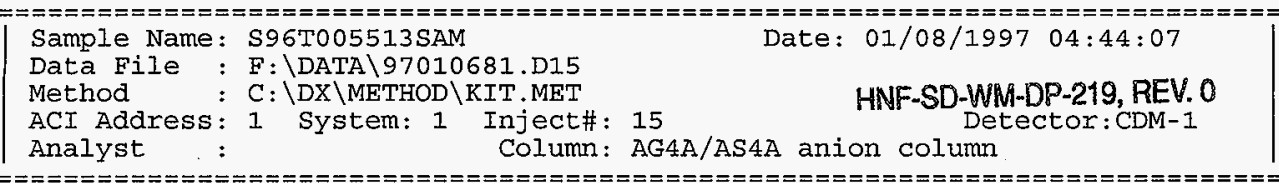

Calibration Volume Dilution Points Rate start stop Area Reject

-

External

1

$\begin{array}{lllll}21 & 3000 & 5 \mathrm{~Hz} & 0.00 & 10.00\end{array}$

50

$\star * * * * * * * * * * * * * * * * * * * * * * * * * *$ Peak Report: All Peaks $* * * * * * * * * * * * * * * * * * * * * * * * * * * *$

Pk. Ret Component Concentration Height Area Bl. $\%$ Delta Num Time Name ug/ml Code

\begin{tabular}{|c|c|c|c|c|c|c|c|}
\hline 1 & 0.93 & & 0.000 & 29 & 80 & 2 & \\
\hline 2 & 1.09 & fluoride & 73.027 & 8999 & 56086 & 2 & 0.62 \\
\hline 3 & 1.66 & chloride & 8.647 & 920 & 5173 & 1 & -0.40 \\
\hline 4 & 1.97 & nitrite & 54.509 & 2911 & 21265 & 1 & -2.95 \\
\hline 5 & 3.29 & nitrate & 382.307 & 16932 & 149300 & 1 & 0.10 \\
\hline 6 & 4.80 & phosphate & 626.554 & 10602 & 133406 & 1 & -3.23 \\
\hline 7 & 6.40 & sulfate & 40.450 & 1404 & 21014 & 1 & -3.23 \\
\hline & & & 1185.493 & 41798 & 386323 & & \\
\hline
\end{tabular}

\section{File: 97010681.D15 Sample: S96T005513SAM}

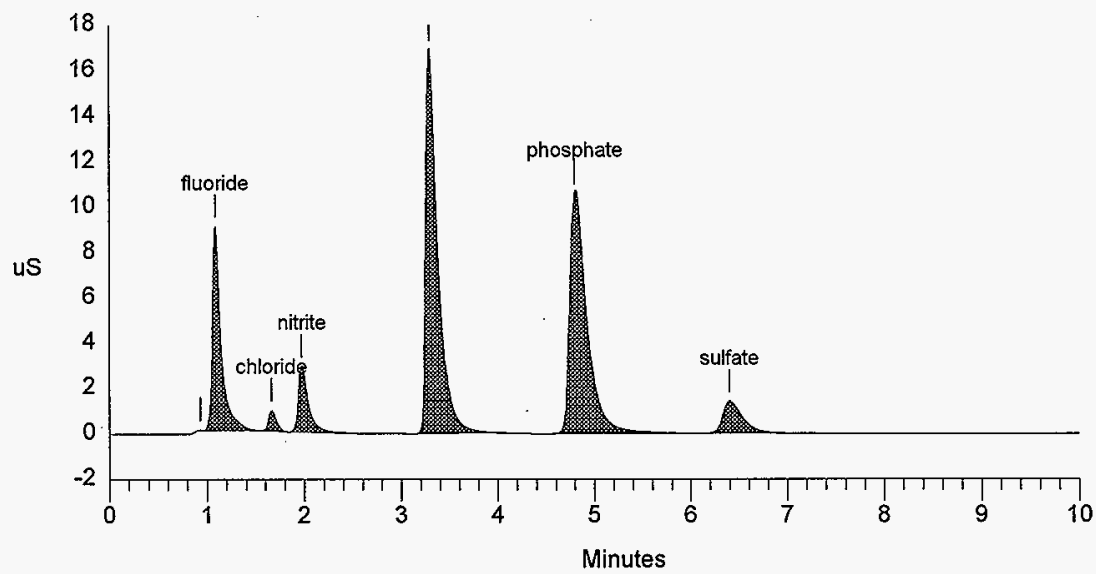


Data Reprocessed On 01/17/1997 08:50:11

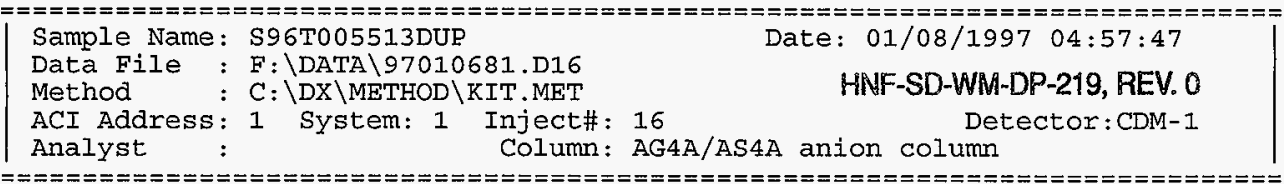

Calibration Volume Dilution Points Rate start stop Area Reject

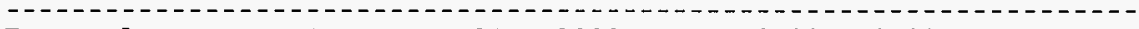

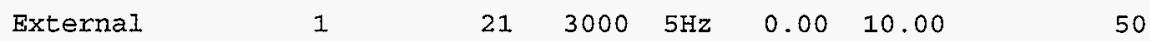

$\star * * * * * * * * * * * * * * * * * * * * * * * * * *$ Peak Report: All Peaks $* * * * * * * * * * * * * * * * * * * * * * * * * * * *$

Pk. Ret Component Concentration Height Area Bl. $\frac{\text { DDelta }}{}$ Num Time Name ug/ml Code

$\begin{array}{rrlrrrr}1 & 0.93 & 0.000 & 26 & 71 & 2 \\ 2 & 1.09 \text { fluoride } & 64.666 & 7617 & 48626 & 2 & 0.62 \\ 3 & 1.67 \text { chloride } & 4.508 & 480 & 2662 & 1 & 0.40 \\ 4 & 1.97 \text { nitrite } & 51.512 & 2743 & 20024 & 1 & -2.95 \\ 5 & 3.30 \text { nitrate } & 360.152 & 16076 & 140260 & 1 & 0.00 \\ 6 & 4.80 \text { phosphate } & 549.669 & 8860 & 115213 & 1 & -3.23 \\ 7 & 6.40 \text { sulfate } & 35.725 & 1247 & 18654 & 1 & -3.23 \\ & & \text { Totals } & 1066.232 & 37050 & 345510 & \end{array}$

\section{File: 97010681.D16 Sample: S96T005513DUP}

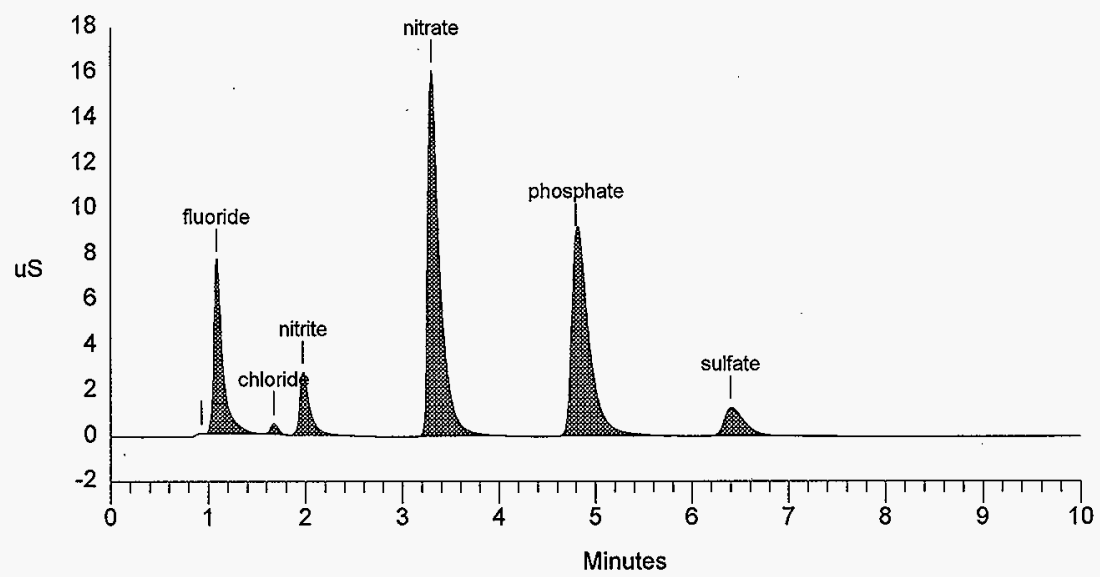




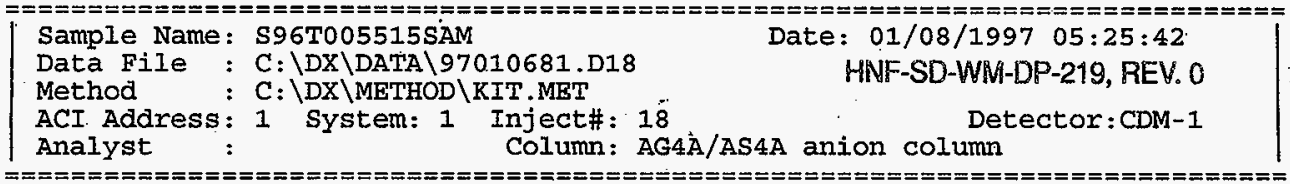

Calibration Volume Dilution Points Rate Start stop Area Reject

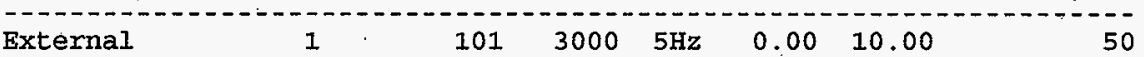

$\star * * * * * * * * * * * * * * * * * * * * * * * * * *$ Peak Report: All Peaks $* * * * * * * * * * * * * * * * * * * * * * * * * * * *$

Pk. Ret component Concentration Height Area BI. $\%$ Delta

Num Time Name $\mathrm{ug} / \mathrm{mI}$ Code

$\begin{array}{ll}1 & 1.09 \\ 2 & 1.68 \text { chloride } \\ 3 & 2.00 \text { nitrite } \\ 4 & 3.39 \text { nitrate } \\ 5 & 4.88 \text { phosphate } \\ 6 & 6.35 \text { sulfate }\end{array}$

$\begin{array}{rrrrrr}277.363 & 6685 & 42625 & 1 & 0.62 \\ 4.288 & 98 & 483 & 1 & 0.80 \\ 47.246 & 297.833 & 2872 & 23325 & 1 & -5.40 \\ & 575.096 & 1711 & 22996 & 1 & -1.61 \\ & 929.045 & 7078 & 99054 & 1 & -4.03 \\ & 2130.871 & 18849 & 191316 & & \end{array}$

File: 97010681.D18 Sample: S96T005515SAM

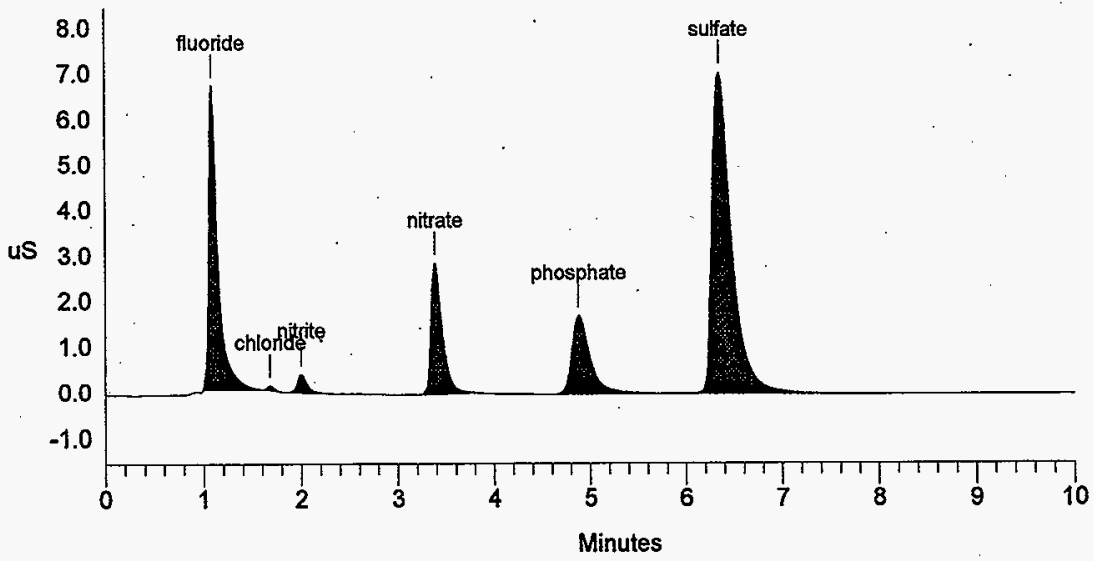




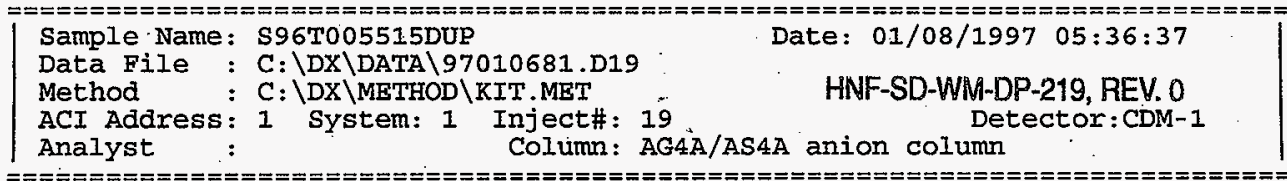

Calibration Volume Dilution Points Rate start stop Area Reject

External

External

1

$\begin{array}{lllll}101 & 3000 & 5 \mathrm{~Hz} & 0.00 & 10.00\end{array}$

50

$* * * * * * * * * * * * * * * * * * * * * * * * * * *$ Peak Report: All Peaks $* * * * * * * * * * * * * * * * * * * * * * * * * * * *$

Pk. Ret Component Concentration Height. Área BI. \%Delta

Num Time Name $\mathrm{ug} / \mathrm{mI}$ Code

$\begin{array}{lll}1 & 1.09 & \text { fluoride } \\ 2 & 1.68 \text { chloride } \\ 3 & 2.00 \text { nitrite } \\ 4 & 3.38 \text { nitrate } \\ 5 & 4.91 \text { phosphate } \\ 6 & 6.29 \text { sulfate }\end{array}$

\begin{tabular}{|c|c|c|c|c|c|}
\hline & $\begin{array}{r}377.001 \\
6.181 \\
54.188 \\
345.852 \\
192.789 \\
1620.580\end{array}$ & $\begin{array}{r}9855 \\
148 \\
480 \\
3418 \\
536 \\
11905\end{array}$ & $\begin{array}{r}61053 \\
720 \\
3426 \\
27107 \\
7384 \\
175940\end{array}$ & $\begin{array}{l}1 \\
1 \\
1 \\
1 \\
1 \\
1\end{array}$ & $\begin{array}{r}0.62 \\
0.80 \\
-1.64 \\
-5.59 \\
-1.08 \\
-4.84\end{array}$ \\
\hline otals & 2596.590 & 26343 & 275629 & & \\
\hline
\end{tabular}

File: 97010681.D19 Sample: S96T005515DUP

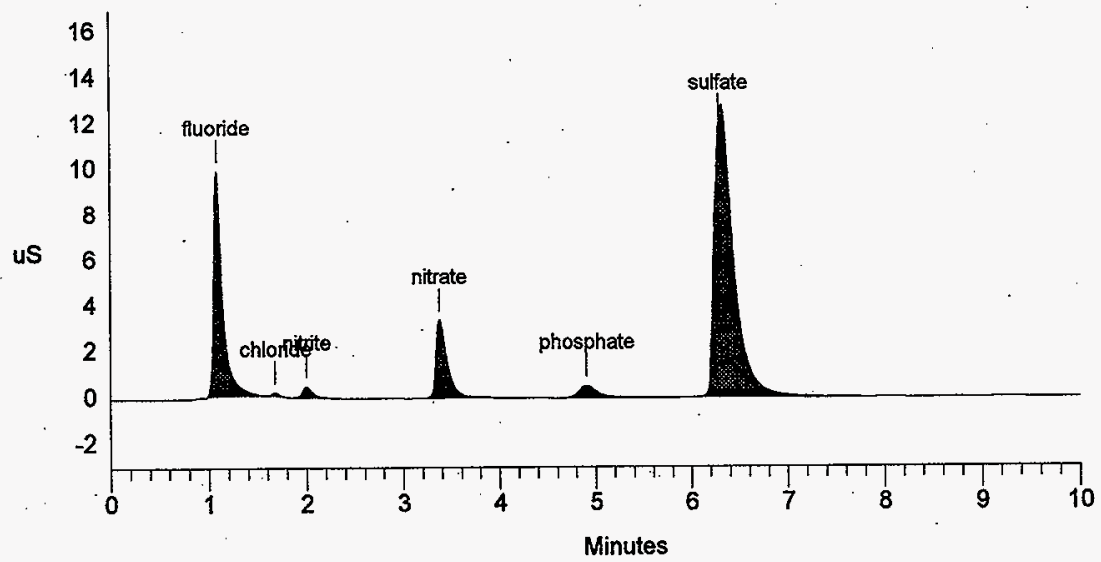




\section{LABCORE Completed Worklist Report for Worklist\# 16305}

Analyst: ded

Instrument: ICO1

Book\# 36NbeD

Method: $533-105-\operatorname{Rev} / \mathrm{Mod} D-1$

Worklist Comment: B-108. Run per procedure. STD: 0.100-10ml.

new

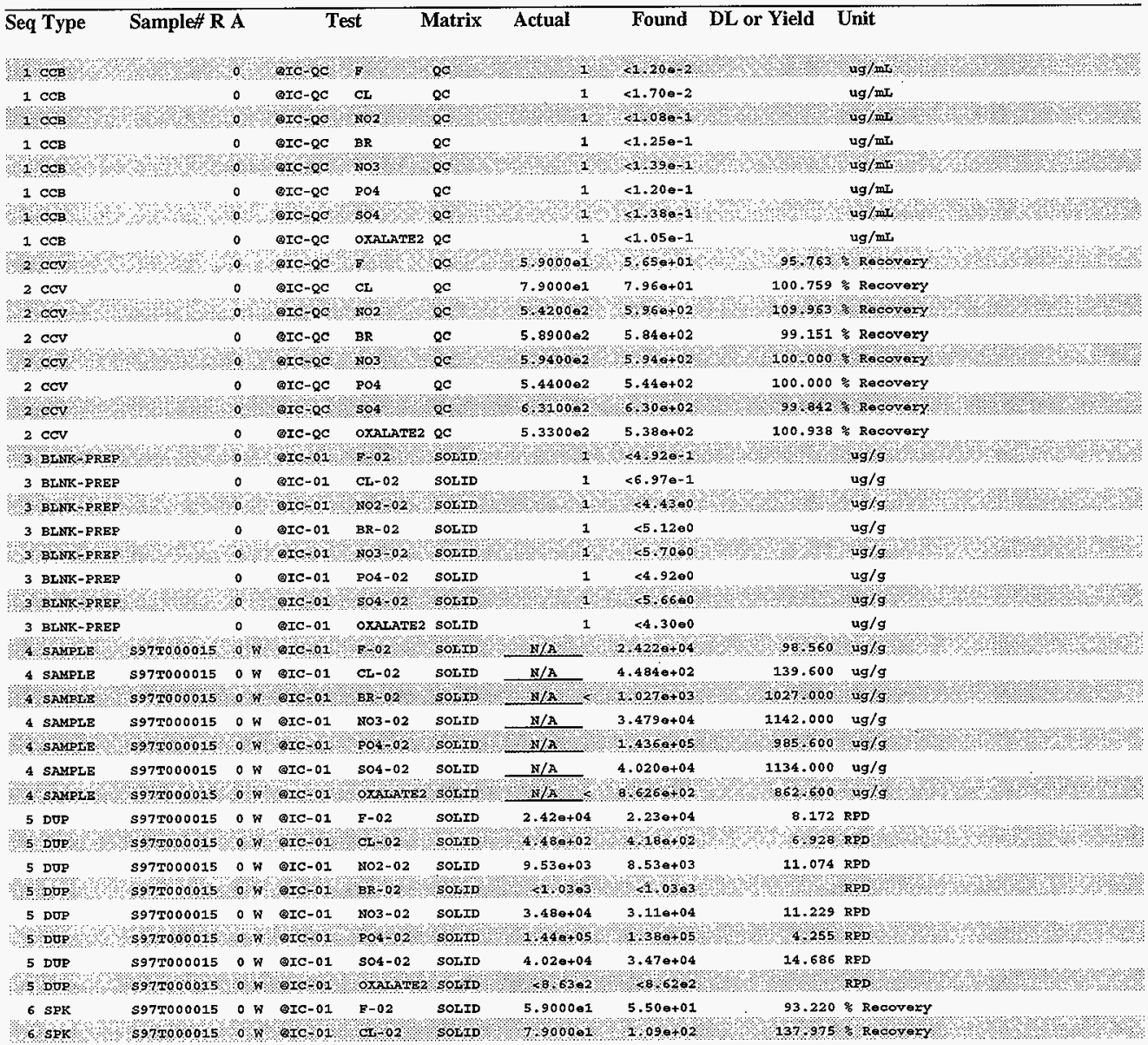

Units shown for $Q C(B L K / B K G)$ may not reflect the actual units. 


\section{LABCORE Completed Worklist Report for Worklist\# 16305}

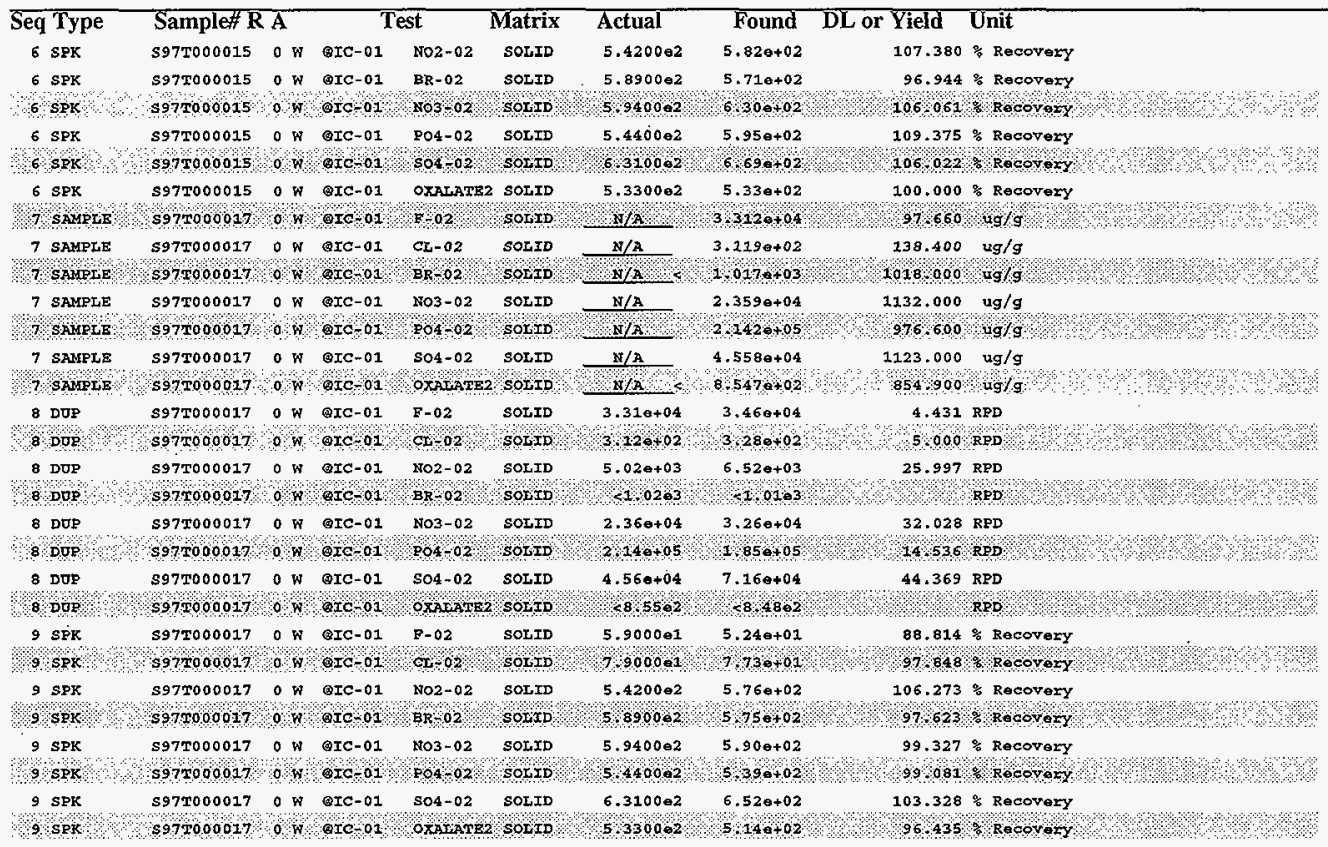

\section{Final page for worklist\# 16305}

Analyst Signature Date


HANF-SD-WM-DP-219, REV. 0

Page: 1

LABCORE Data Entry Template for Worklist\# 16305

Analyst: DCD Instrument: IC0 ICO/ Book\# 36N20-D

Method: LA-533-105 Rev/Mod D-1.

Worklist Comment: B-108. Run per procedure. STD: $0.100-10 \mathrm{ml}$. new

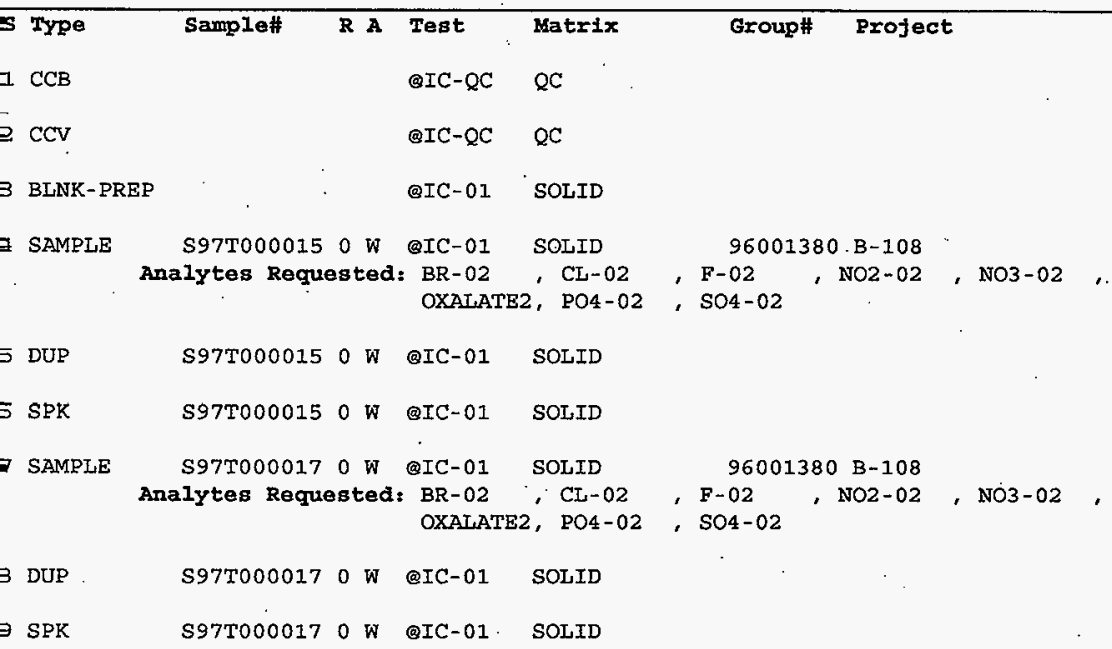

Final page for worklist \# 16305

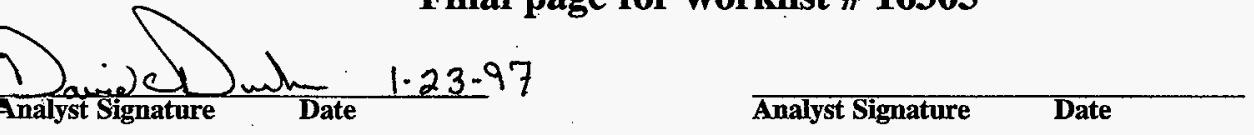

16305 ja.esv

Uploaded ty 6. Ham init 1/26/97

Validated $128 / 97$ gmfuye $-\mathrm{NO}_{2}$ std failed

out foo sewer foo $\mathrm{NO}_{2}$ on WKL

16392

Data Entry Comments:

$=$ Worklist Slot Number, $R=$ Replicate Number, $A=$ Aliquot Code.

329 
Aibration Volume Dilution Points Rate start stop Area Reject

ternal $\quad 1 \quad 41 \quad 3000 \quad 5 \mathrm{~Hz} 0.00 \quad 10.00 \quad 50$

\begin{tabular}{|c|c|c|c|c|c|}
\hline $\begin{array}{l}\text { Ret Component } \\
\text { Time Name }\end{array}$ & $\begin{array}{r}\text { Concentration } \\
\mathrm{ug} / \mathrm{mI}\end{array}$ & Height & Area & $\begin{array}{l}\text { Bl. } \\
\text { Code }\end{array}$ & \&Delta \\
\hline $\begin{array}{l}1.68 \text { chloride } \\
3.39\end{array}$ & $\begin{array}{r}-0.187 \\
0.000\end{array}$ & $\begin{array}{l}20 \\
58\end{array}$ & $\begin{array}{r}88 \\
388\end{array}$ & $\begin{array}{l}1 \\
1\end{array}$ & 3.70 \\
\hline & -0.187 & 78 & 475 & & \\
\hline
\end{tabular}

File: 97012321.D14 Sample: PREP-BLANK

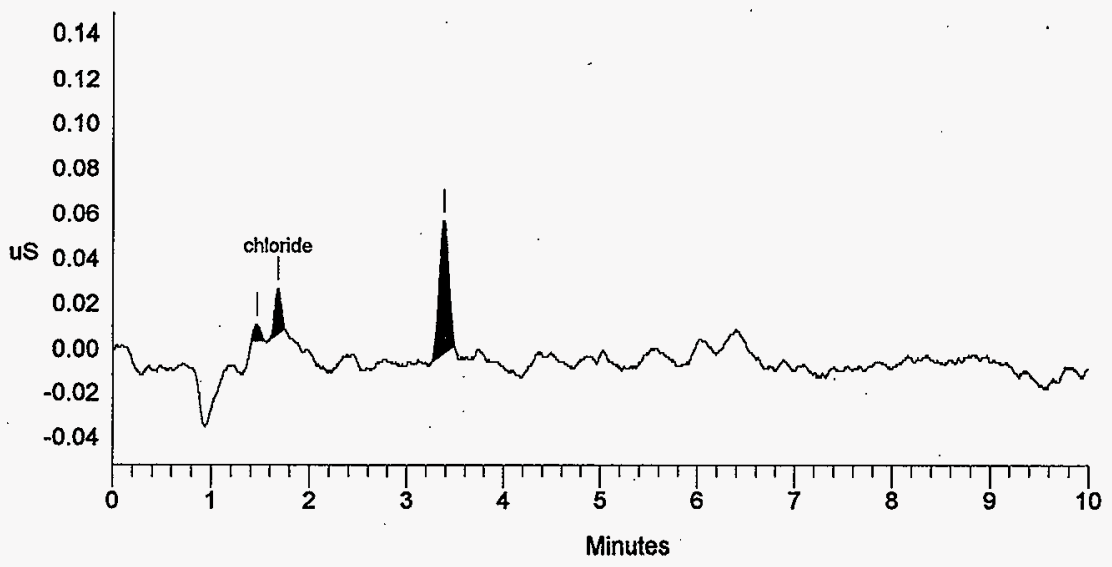


ACI Address: 1 System: 1 Inject\#: 13 Analyst

Column: AG4A/AS4A anion column

Detector: $\mathrm{CDM}-1$

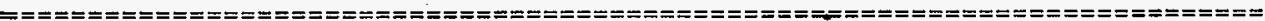

Aibration Volume Dilution Points Rate start stop Area Reject

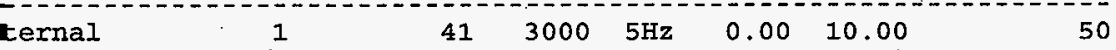

$* * * * * * * * * * * * * * * * * * * * * * * * *$ Peak Report: All Peaks $* * * * * * * * * * * * * * * * * * * * * * * * * * * *$

$\begin{array}{lrrr}\text { ket Component } & \text { Concentration } \\ \text { ug } / \mathrm{ml} & \text { Height } & \text { Area Bl. } & \text { sDelta } \\ \text { Code }\end{array}$

$\begin{array}{lll}1 & 1.09 & \text { fluoride } \\ 2 & 1.67 & \text { chloride } \\ 3 & 1.97 \text { nitrite } \\ 4 & 3.29 \text { nitrate } \\ 5 & 4.77 \text { phosphate } \\ 6 & 6.24 \text { sulfate }\end{array}$

\begin{tabular}{rrrrrr}
120.895 & 8096 & 50441 & 1 & 0.62 \\
2.238 & 157 & 798 & 1 & 2.88 \\
47.546 & 1074 & 7876 & 1 & 0.34 \\
173.637 & 4205 & 33196 & 1 & 4.22 \\
& 716.554 & 5387 & 71121 & 1 & 1.13 \\
& 200.663 & 3765 & 53647 & 1 & 2.63 \\
\hline & 1261.533 & 22684 & 217079 & &
\end{tabular}

File: 97012321.D13 Sample: S97T000015 SAM

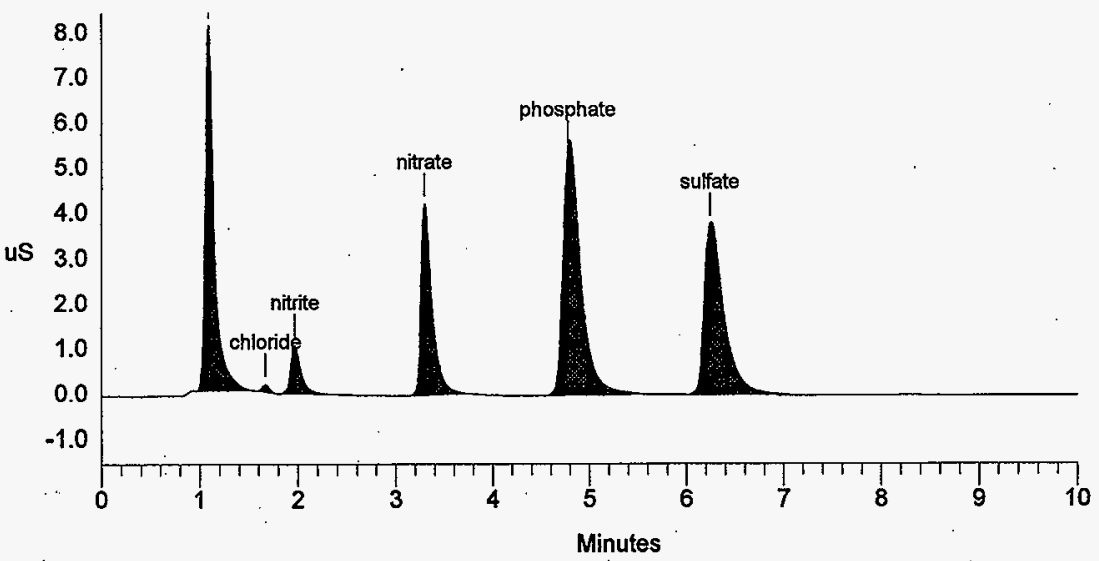




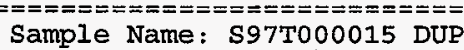 \\ Data File : C: \DX\DATA \97012321.D15 \\ Method : C: \DX\METHOD \KIT.MET \\ ACI Address: 1 System: 1 Inject\#: 15 Analyst \\ Column: AG4A/AS4A anion column \\ Date: $01 / 23 / 199723: 31: 48$}

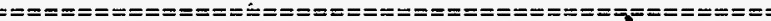

libration Volume Dilution Points Rate start stop Area Reject

ternal $\quad 1 \quad 41 \quad 3000 \quad 5 \mathrm{~Hz} \quad 0.00 \quad 10.00$.

$* * * * * * * * * * * * * * * * * * * * * * * * *$ Peak Report: All Peaks $* * * * * * * * * * * * * * * * * * * * * * * * * * * *$

Ret Component $\begin{array}{r}\text { Concentration } \\ \text { ug/ml }\end{array}$
um Time Name Height

$\begin{array}{lll}1 & 1.09 & \text { fluoride } \\ 2 & 1.67 & \text { Chloride } \\ 3 & 1.97 \text { nitrite } \\ 4 & 3.30 \text { nitrate } \\ 5 & 4.77 \text { phosphate } \\ 6 & 6.24 \text { sulfate }\end{array}$

\begin{tabular}{|c|c|c|c|c|}
\hline $\begin{array}{r}111.204 \\
2.087 \\
42.613 \\
155.504 \\
686.941 \\
173.294\end{array}$ & $\begin{array}{r}7417 \\
149 \\
952 \\
3760 \\
5119 \\
3199\end{array}$ & $\begin{array}{r}45912 \\
754 \\
6983 \\
29718 \\
67974 \\
46483\end{array}$ & $\begin{array}{l}1 \\
1 \\
1 \\
1 \\
1 \\
1\end{array}$ & $\begin{array}{l}0.62 \\
2.88 \\
0.34 \\
4.43 \\
1.13 \\
2.63\end{array}$ \\
\hline 1171.644 & 20597 & 197824 & & \\
\hline
\end{tabular}

File: 97012321.D15 Samp/e: S97T000015 DUP

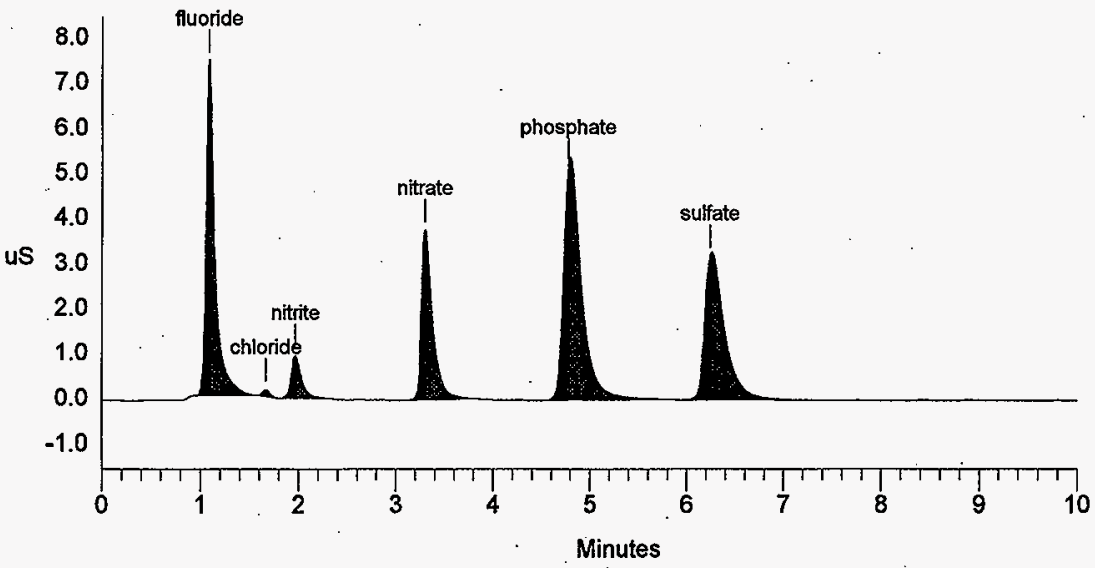


$==========================$
Sample Name: S97T000015 SPIKE

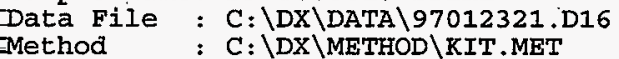

ACI Address: 1 System: 1 Inject\#: 16 Analyst

Column: AG4A/AS4A anion column

Date: $01 / 23 / 1997 \quad 23: 42: 34$

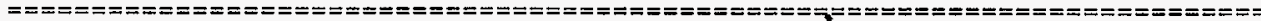

libration Volume Dilution Points Rate Start Stop Area Reject

$\begin{array}{llllllll}\text { ternal } & 1 & 41 & 3000 & 5 \mathrm{~Hz} & 0.00 & 10.00 & 50\end{array}$

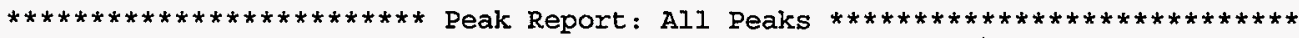

k. Ret Component

um Time Name

Concentration Height

$\mathrm{ug} / \mathrm{ml}$

Area B1. :Delta

Code

$\begin{array}{lll}1 & 0.93 \\ 2 & 1.09 & \text { fluoride } \\ 3 & 1.63 \text { chloride } \\ 4 & 1.95 \text { nitrite } \\ 5 & 2.89 \text { bromide } \\ 6 & 3.27 \text { nitrate } \\ 7 & 4.77 \text { phosphate } \\ 8 & 6.19 \text { sulfate } \\ 9 & 8.21 \text { oxalate }\end{array}$

$\begin{array}{rrrrr}0.000 & 27 & 69 & 2 & \\ 142.883 & 10191 & 61103 & 2 & 0.62 \\ 45.974 & 2627 & 13944 & 1 & 0.82 \\ 280.293 & 7478 & 50670 & 1 & -0.68 \\ 228.268 & 5049 & 32349 & 1 & 1.88 \\ 425.777 & 10185 & 82543 & 1 & 3.38 \\ 954.498 & 7581 & 97197 & 1 & 1.13 \\ 468.410 & 7617 & 125528 & 1 & 1.75 \\ 213.156 & 2234 & 42358 & 1 & 3.36\end{array}$

Totals

2759.259

52988

505761

File: 97012321.D16 Sample: S97T000015 SPIKE

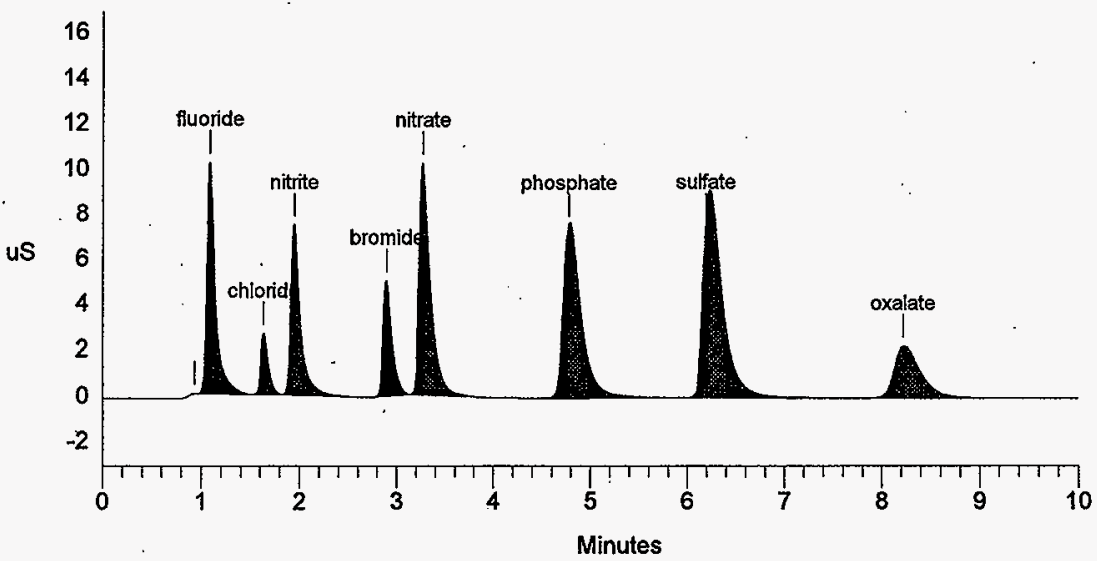


Iibration Volume Dilution Points Rate start Stop Area Reject

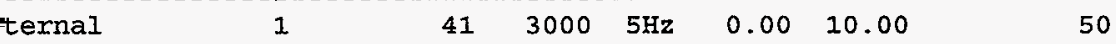

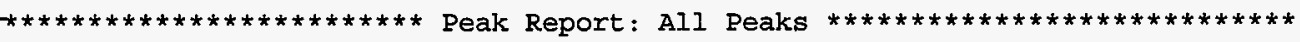

k. Ret Component Concentration Height Area Bl. \%Delta

un

Time Name $\mathrm{ug} / \mathrm{ml}$

1.09 fluoride

1.67 chloride

1.98 nitrite

3.33 nitrate

4.77 phosphate

6.24 sulfate

\begin{tabular}{|c|c|c|c|c|}
\hline $\begin{array}{r}166.816 \\
1.571 \\
25.279 \\
118.832 \\
1078.781 \\
229.577\end{array}$ & $\begin{array}{r}11978 \\
121 \\
534 \\
2873 \\
8512 \\
3947\end{array}$ & $\begin{array}{r}73405 \\
602 \\
3852 \\
22713 \\
111427 \\
61252\end{array}$ & $\begin{array}{l}2 \\
1 \\
1 \\
1 \\
1 \\
1\end{array}$ & $\begin{array}{l}0.62 \\
2.88 \\
1.02 \\
5.27 \\
1.13 \\
2.63\end{array}$ \\
\hline 1620.855 & 27966 & 273251 & & \\
\hline
\end{tabular}

File: 97012321.D17 Sample: S97T000017 SAM

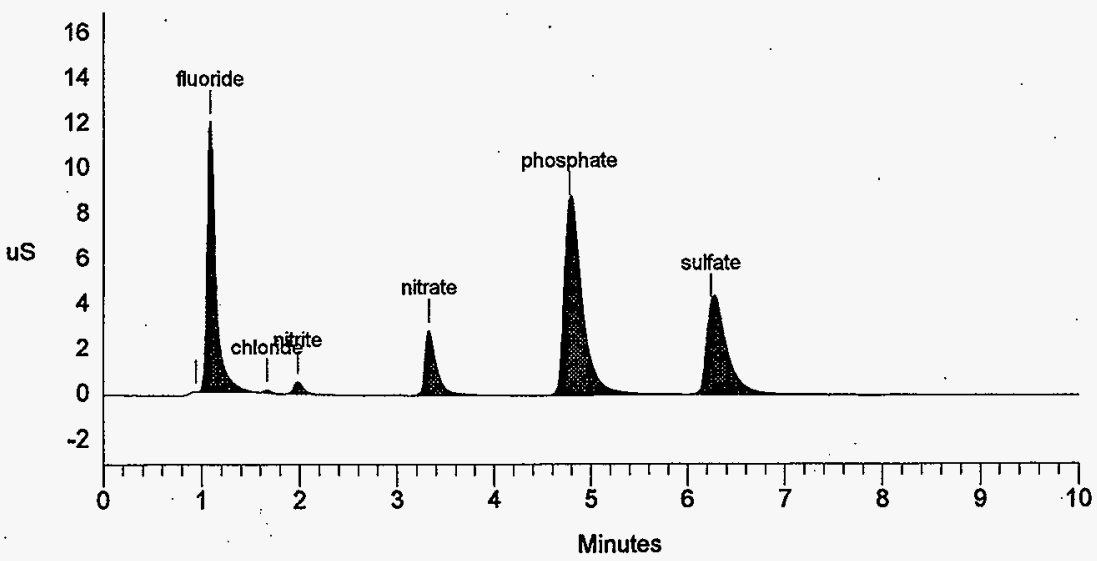


libration Volume Dilution Points Rate Start Stop Area Reject

Cernal $\quad 1 \quad 41 \quad 3000 \quad 5 \mathrm{~Hz} 0.00 \quad 10.00 \quad 50$

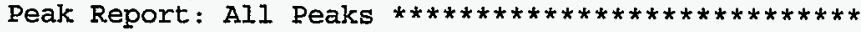

Ret Component Concentration
Time Name Height Area BI.
$\begin{gathered}\text { Code } / \mathrm{ml} \\ \text { Colta }\end{gathered}$

0.93

1.09 fluoxide

1.64 chloride

1:95 nitrite

2.89 bromide

3.27 nitrate

4.75 phosphate

6.19 sulfate

8.21 oxalate

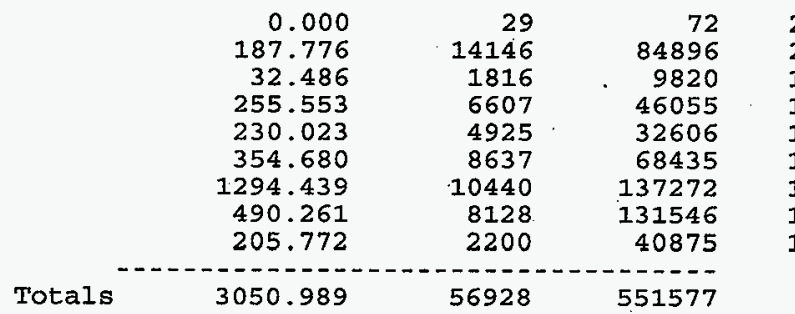

187.776

32.486

255.553

230.023

354.680

294.439

490.261

3050.989

56928

551577

File: 97012321.D19 Sample: S97T000017 SPIKE

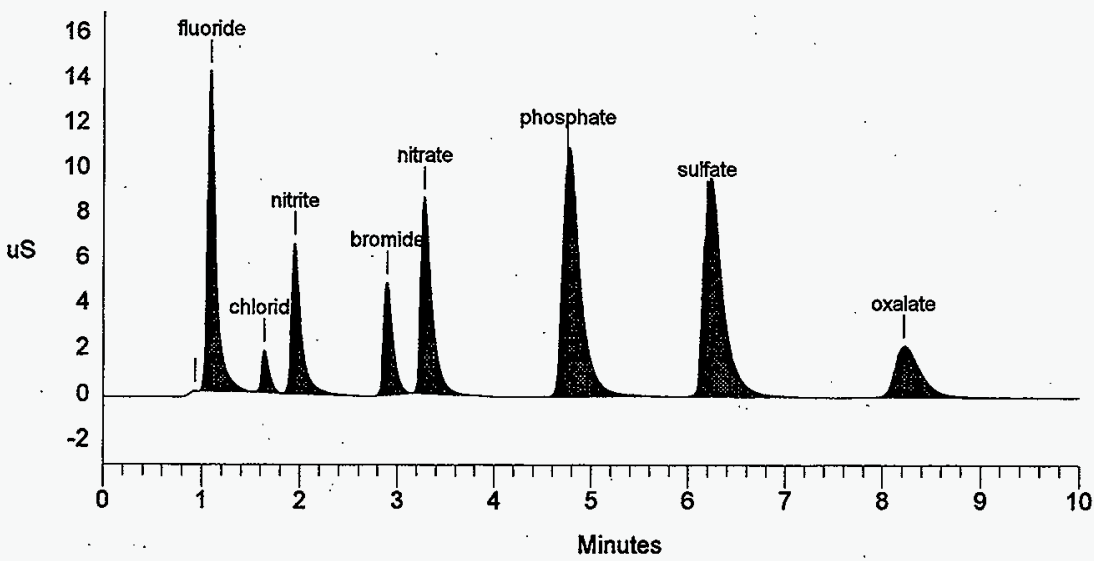


FILE \#O $061120 B . T \times 5$

11/08/96 08:47

$A-0004-1$

HEF-SD-WM-DP-219, REV.O

Page: 1

\section{LABCORE Data Entry Template for Worklist\# 14793}

\begin{tabular}{|c|c|c|}
\hline Analys & 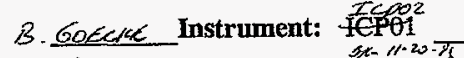 & Bookf $6.2848 B$ \\
\hline
\end{tabular}

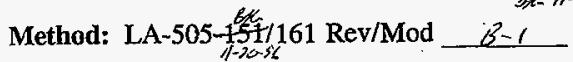

Worklist Comment: ICP B-108 (DIRECT)

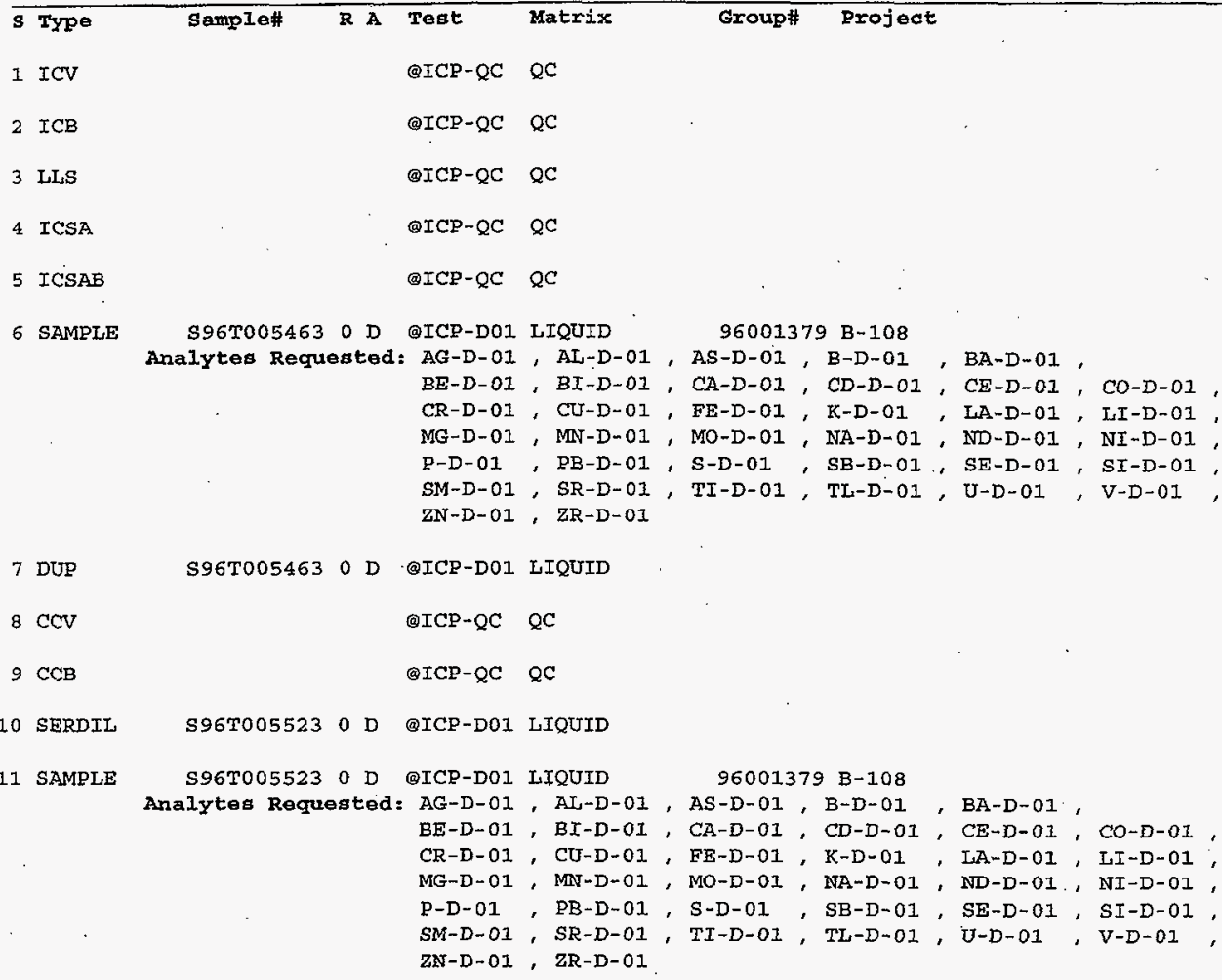

12 DUP S96T005523 O D OICP-DO1 LIQUID

Data Entry Comments:

$\bar{S}=$ Worklist Slot Number, $R=$ Replicate Number, $A=$ Aliquot Code. 
$11 / 20 / 96 \quad 13: 05 \quad$ O509 $3722929 \ldots \quad$ WESTINGHOUSE $\quad \rightarrow \rightarrow \rightarrow$ HO-924 $200 \%$

002

$1 \overline{1} / 08 / 9608: 47$

HNF-SD-WMA-DP-219, REV.O

Page: $\quad 2$

A-0004-1

LABCORE Data Entry Template for Worklist\# 14793

S Type Sample\# R A Test Matxix Group\# Project

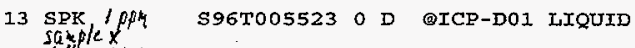

sQ*plex

14 ICSA

$15 \operatorname{ICSAB}$

$16 \mathrm{CCV}$

$17 \mathrm{CCB}$
बICP-QC QC

QICP-QC QC

@ICP-QC QC

बICP-QC $\quad Q C$

Final page for worklist \# 14793

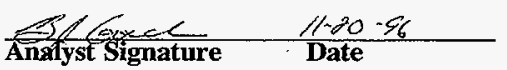

596 T00 5463 1ai-6ul

$5463-1 \quad 191-6-1$

$5967005523-2$, 025nd-10u1-201-801

$5523.055-1.10-1$

$5523-0$.025ar-10u/

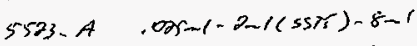

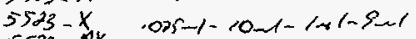

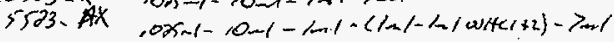

Remwed ty:

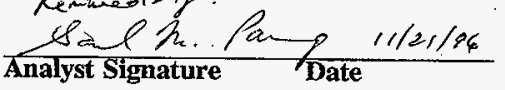

$D, F$

7

2005

401

401

401

4010

Data Entry Comments:

Spite morey colculatin:

$5967005 r^{23}$ al $=\left(\frac{1344}{401}\right)-\left(\frac{88201}{45}\right) \times 100=115.2 \%$

$S=$ Worklist Slot Number, $R=$ Replicate Number, $A=$ Aliquot Code.

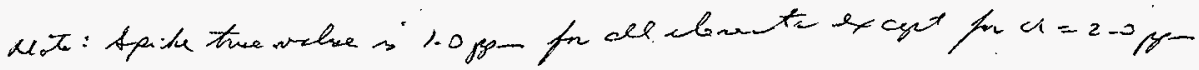

$\mathbf{3 4 0}$ 
HNF-SD-WMA-DP-219, REV. 0

\begin{tabular}{|c|c|c|c|c|c|c|c|}
\hline Analysis Report & ummary & & Wed 11-2 & 51 & $: 0$ & $M$ & \\
\hline \# Sample Nane & File & Method & Date & Time & $O_{p} I D$ & Type & Mode \\
\hline 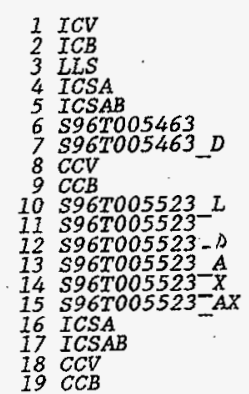 & $\begin{array}{l}961120 B \\
961120 B \\
961120 B \\
961120 B \\
961120 B \\
961120 B \\
961120 B \\
961120 B \\
961120 B \\
961120 B \\
961120 B \\
961120 B \\
961120 B \\
961120 B \\
961120 B \\
961120 B \\
961120 B \\
961120 B \\
961120 B\end{array}$ & $\begin{array}{l}I C P 2 \\
I C P 2 \\
I C P 2 \\
I C P 2 \\
I C P 2 \\
I C P 2 \\
I C P 2 \\
I C P 2 \\
I C P 2 \\
I C P 2 \\
I C P 2 \\
I C P 2 \\
I C P 2 \\
I C P 2 \\
I C P 2 \\
I C P 2 \\
I C P 2 \\
I C P 2 \\
I C P 2 \\
I C P 2\end{array}$ & $\begin{array}{l}11 / 20 / 96 \\
11 / 20 / 96 \\
11 / 20 / 96 \\
11 / 20 / 96 \\
11 / 20 / 96 \\
11 / 20 / 96 \\
11 / 20 / 96 \\
11 / 20 / 96 \\
11 / 20 / 96 \\
11 / 20 / 96 \\
11 / 20 / 96 \\
11 / 20 / 96 \\
11 / 20 / 96 \\
11 / 20 / 96 \\
11 / 20 / 96 \\
11 / 20 / 96 \\
11 / 20 / 96 \\
11 / 20 / 96 \\
11 / 20 / 96\end{array}$ & $\begin{array}{l}10: 37 \\
10: 40 \\
10: 43 \\
10: 46 \\
10: 48 \\
10: 52 \\
10: 55 \\
10: 58 \\
11: 01 \\
11: 06 \\
71: 09 \\
11: 12 \\
11: 15 \\
11: 18 \\
11: 22 \\
11: 25 \\
11: 28 \\
11: 31 \\
11: 34\end{array}$ & $\begin{array}{l}B J G \\
B J G \\
B J G \\
B J G \\
B J G \\
B J G \\
B J G \\
B J G \\
B J G \\
B J G \\
B J G \\
B J G \\
B J G \\
B J G \\
B J G \\
B J G \\
B J G \\
B J G \\
B J G\end{array}$ & $\begin{array}{l}Q \\
\oint \\
\oint \\
\oint \\
\$ \\
S \\
S \\
Q \\
Q \\
S \\
S \\
S \\
S \\
S \\
S \\
S \\
S \\
Q \\
\& \\
Q\end{array}$ & $\begin{array}{l}\text { CONC } \\
\text { CONC } \\
\text { CONC } \\
\text { CONC } \\
\text { CONC } \\
\text { CONC } \\
\text { CONC } \\
\text { CONC } \\
\text { CONC } \\
\text { CONC } \\
\text { CONC } \\
\text { CONC } \\
\operatorname{CONC} \\
\operatorname{CONC} \\
\operatorname{CONC} \\
\operatorname{CONC} \\
\operatorname{CONC} \\
\operatorname{CONC} \\
\operatorname{CONC}\end{array}$ \\
\hline
\end{tabular}

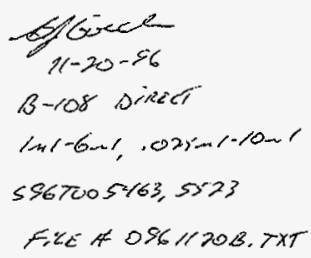


HNF-SD-WM-DP-219, REV.O

Analysis Report Averages Wed 11-20-96 11:37:07 AM page 2

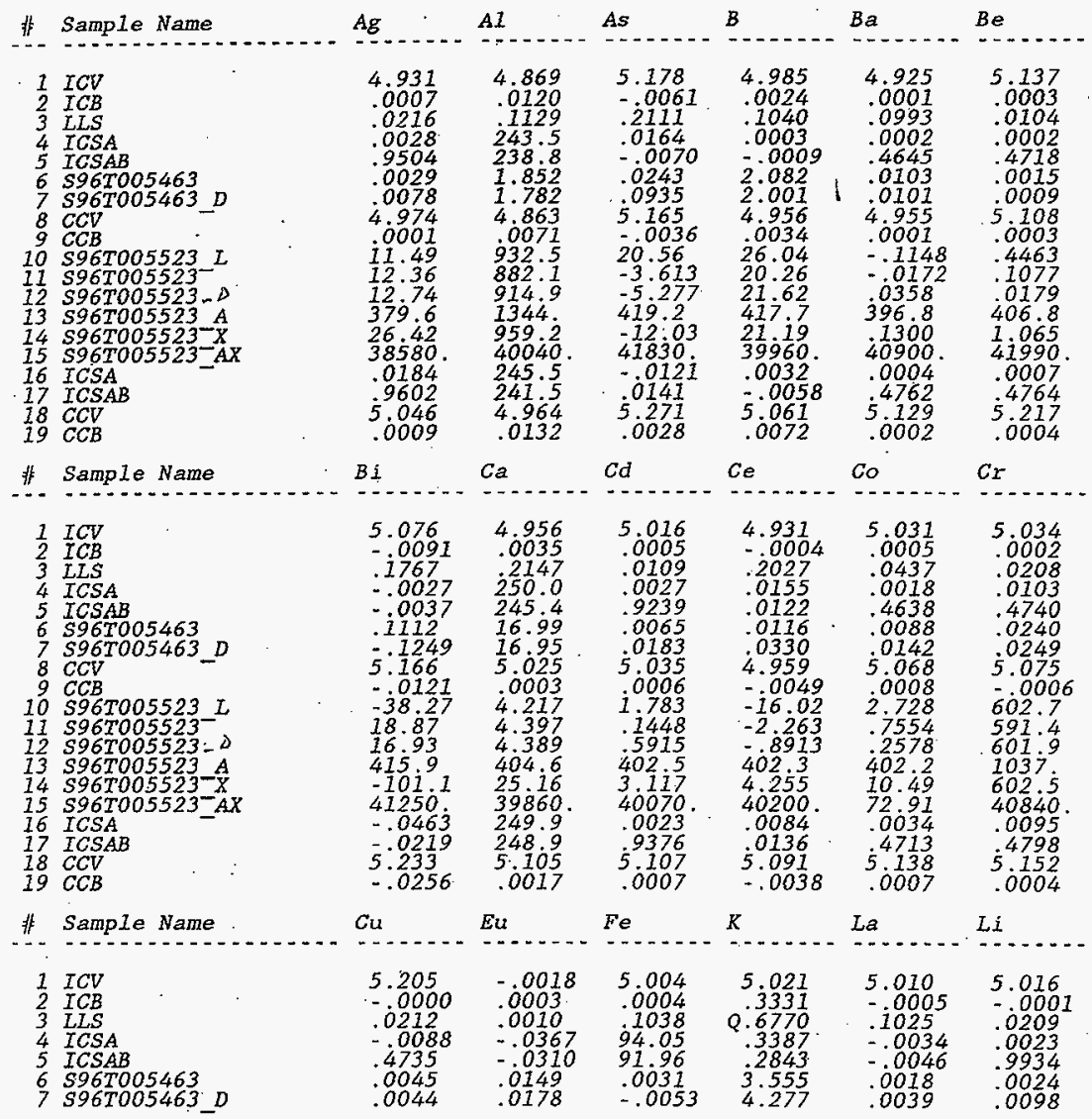


page 3

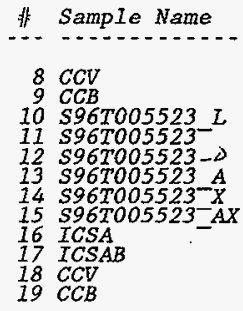

\# Sample Name

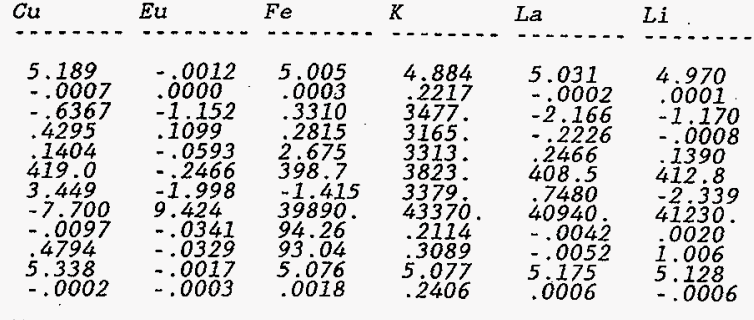

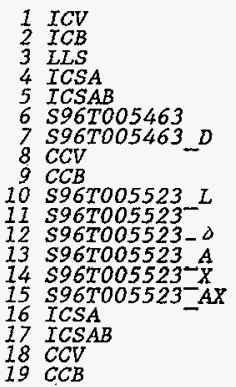

$\mathrm{Mg}$

Mn

Mo

$N a$

$\mathrm{Nd}$

$N i$

$\begin{array}{llllll}4.814 & 4.892 & 5.028 & 4.824 & 5.018 & 5.016 \\ .0063 & .0002 & .0026 & -.0004 & -.0035 & -.0051 \\ .1995 & .0201 & .0998 & .2093 & .1968 & .0379 \\ 254.3 & -.0061 & -.0038 & 194.2 & .0047 & -.0124 \\ 2490 & .4381 & -.0058 & 190.7 & .0030 & .9122 \\ 3.633 & .0163 & .0259 & 56.27 & -.0255 & -.0102 \\ 3.582 & .0175 & .0138 & 55.55 & -.0212 & -.0212 \\ 4.786 & 4.898 & 5.043 & 4.810 & 5.031 & 5.032 \\ .0009 & .0001 & .0002 & -0021 & -10038 & -.0030 \\ 8.928 & -.3959 & 37.91 & 177800 . & -10.10 & 16.20 \\ 4.148 & -.0144 & 35.25 & 171700 . & -17174 & .3271 \\ 3.526 & .2510 & 35.19 & 175700 . & -1.246 & -1.298 \\ 383.5 & 383.13 & 444.3 & 185900 . & 407.2 & 397.0 \\ 34.99 & -1.284 & 45.60 & 178300 . & -15.08 & -1.907 \\ 40710 . & 39740 . & 39580 . & 218300 . & 40920 . & 39840 \\ 256.0 & -.0059 & .0016 & 195.6 & .0047 & -.0135 \\ 251.2 & .4451 & -.0030 & 1925 & .0044 & .9259 \\ 4.874 & 4.993 & 5.148 & 4.919 & 5.171 & 5.098 \\ .0124 & .0001 & .0040 & -.0059 & -.0034 & -.0042\end{array}$

\# Sample Name

P $\quad P b \quad P S$

$1 \mathrm{ICV}$

2 ICB

4.984

.0083

4.989

4 ICSA

5 ICSAB

6 5967005463

7 S96T005463 D

$8 \mathrm{CCV}$

$9 C C B$

10 S96T005523 L

11 S96T005523

$112596 T 005523-\Delta$

13 S96T005523 A

14 S96T005523-X

$\begin{array}{ll}.4124 & .2422 \\ .0151 & .0664\end{array}$

$.0261 \quad .9959$

$.0715 \quad .1145$

.1757

5.051

.1663

5.042

i139.

1164 .

1264 .

1661 .

1063 .

$S b$

Se

Si

\begin{tabular}{llll}
4.937 & 4.787 & 4.862 & 4.993 \\
.0098 & .0083 & .0332 & .0017 \\
.2098 & .1091 & .2201 & .1331 \\
-.0138 & -.0057 & -.0278 & .0059 \\
\hline .0421 & .0013 & .00046 & .0005 \\
8.650 & .0101 & .2885 & 1.908 \\
8.886 & .0746 & .2826 & 1.948 \\
5.003 & 4.816 & 4.867 & 5.078 \\
.0129 & .0077 & .0416 & .0084 \\
4686. & -7.530 & 67.46 & 65.02 \\
4685. & 3.155 & 20.86 & 59.50 \\
4816. & -3.232 & 15.77 & 81.97 \\
$5405:$ & 393.3 & 432.3 & 542.9 \\
4766. & 13.10 & 106.6 & 108.8 \\
44890. & 40230. & 39270. & 42160
\end{tabular}

$51.93 \quad 4686$.

7.123

7.608

409.6

88.61

41980 . 40790 .

$44890^{\circ}$.

40230 .

42160 . 
$11 / 20 / 96 \quad 13: 08$

$8509 \quad 372 \quad 2929$

WESTINGHOUSE

$\rightarrow \rightarrow \rightarrow M 0-924$ 200W

0006

HNF-SD-WVM-DP-219, REV. 0

Analysis Report

Averages

Wed 11-20-96 11:37:07 AM page 4

\# Sample Name

$P$

$\mathrm{Pb} \quad \mathrm{S}$

$S \quad S b$

$\mathrm{Se}$

Si

16 ICSA

17 ICSAB

$18 G C V$

.0291
.0240
5.151
.0070

\section{.0705 \\ 5.110}

-.0313
$=.0253$
5.011
.0116

.0040
.008
4.88
.006

$T h$

$T i$

$\begin{array}{ll}.0121 & .0173 \\ .0029 & .0063 \\ 4.938 & 5.093 \\ .0291 & .0044\end{array}$

IJ Sample Name

$S m$

$S x$

$1 . I C V$

$2 T C B$

4 ICSA

5 ICSAB

65967005463

7 S96T005463 D

$8 \mathrm{CCV}$

$9 C C B$

10. $\$ 96 \mathrm{T005523L} \mathrm{L}$

11 S96T005523-

12 S96T005523-D

13 S96T005523 A

14 S96T005523-X

I5 S96T005523-AX

16 ICSA

I) ICSAB

$18 \mathrm{CCV}$

$19 C C B$

4.857
.0039
.2101
.0008
.0030
-.0151
.0544
4.862
.0018
-18.76
.1969
.6435
392.4
-30.71
39790
-.0039
-.0029
5.009
-.0090

4.875

.0001

.0020

.0019

.0735

.0735
4.899

.0000
-.3099

-3099
.1241

2061

394.6

$-.4090$

39960.

.0022

.0019

-.0000

.0539
.0049
.0008
.0056
-.0041
.0119
.0305
.0474
.0023
3.060
.8336
1.093
7.830
21.52
253.5
-.0083
-.0020
.0521
.0068

4.851

TI

$U$

$\begin{array}{lll}4.8510 & 4.839 & 9.519\end{array}$

$\begin{array}{lll}. .0005 & .0052 & .0040\end{array}$

$.0797 \quad .4308 \quad .5424$

$.0012 \quad .0232 \quad .0245$

$\begin{array}{lll}-.0000 & .0356 & .0363\end{array}$

$\begin{array}{lll}.0035 & .0391 & .2000\end{array}$

- $0035 \quad 1291$

$-.0035$

4.8291

.4297

$-.0002$

. .9791
.1025

.0869

402.0

$-.8894$

36880 .

$-.0003$

5.0000

$-.0177$

$49 \cdot 31$

$-.0046$

4.329

2.372

383.1

$-45.43$

38.80

38750
-.0040

.0145

$\begin{array}{lll}5.020 & 4.953 & 9.817\end{array}$

14.32

783.9

$-116.4$

2992.

\# Sample Name

V

$1 \mathrm{ICV}$

$\frac{2}{3} I C B$

4 ICSA

5 ICSAB

6. $\$ 967005463$

7 S96T005463 D

$8 \mathrm{CCV}$

$9 C C B$

10 S96r005523L

i1 S96T005523-

12 S96T005523_s

13 S96T005523A

14 S96T005523-X

15 S96TO05523-AX

16 ICSA

17 ICSAB

$18 \mathrm{CCV}$

19 CCB

$Y$

$Z n$

$2 x$

$\begin{array}{llll}5.015 & .0061 & 5.076 & 4.908 \\ .0007 & .0001 & .0004 & .0004 \\ .1057 & .0009 & .0238 & .0222 \\ .0008 & .0070 & .0074 & . .0022 \\ .4640 & .0071 & .9510 & -.0022 \\ .0003 & -.0006 & .0133 & -.0015 \\ .0078 & .0015 & .0087 & .0018 \\ 5.038 & .0066 & 5.114 & 4.930 \\ .0002 & -.0001 & -.0000 & .0002 \\ -2.226 & -.7665 & 4.435 & -3.057 \\ .4511 & -.0061 & 2.752 & .0442 \\ .0410 & -.0769 & 2.923 & .0043 \\ 403.6 & .3898 & 414.0 & 396.4 \\ -4.409 & -1.522 & 8.310 & -5.781 \\ 26.13 & 55.62 & -129.5 & 40600 \\ .0000 & .0068 & .0069 & -.0026 \\ .4705 & .0070 & .9577 & -.0017 \\ 5.139 & .0062 & 5.163 & 5.043 \\ -.0009 & -.0005 & .0007 & -.0012\end{array}$

$\begin{array}{llll}5.015 & .0061 & 5.076 & 4.908 \\ .0007 & .0001 & .0004 & .0004 \\ .1057 & .0009 & .0238 & .0222 \\ .0008 & .0070 & .0074 & -.0022 \\ .4640 & .0071 & .9510 & -.0022 \\ .0003 & -.0006 & .0133 & -.0015 \\ .0078 & .0015 & .0087 & .0018 \\ 5.038 & .0066 & 5.114 & 4.930 \\ .0002 & -.0001 & -.0000 & -.0002 \\ -2.226 & -.7665 & 4.435 & -3.057 \\ .4511 & -.0061 & 2.752 & .0442 \\ .0410 & -.0769 & 2.923 & .0043 \\ 403.6 & .3898 & 414.0 & 396.4 \\ -4.409 & -1.522 & 8.310 & -5.781 \\ 26.13 & 55.62 & -129.5 & 40600 \\ .0000 & .0068 & .0069 & -.0026 \\ .4705 & .0070 & .9577 & . .0017 \\ 5.139 & .0062 & 5.163 & 5.043 \\ -.0009 & -.0005 & .0007 & -.0012\end{array}$ 
$12 / 11 / 9608: 23$ A-0004-1

HNF-SD-WM-DP-219, REV. 0

\section{LABCORE Data Entry Template for Worklist\# 14797}

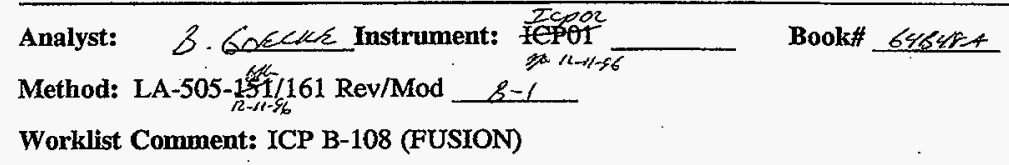

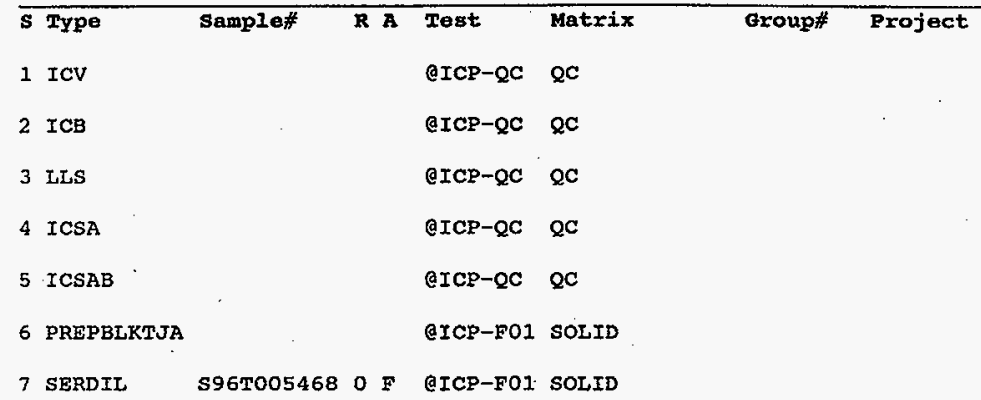

8 SAMPLE S96T005468 O F OICP-FO1 SOLID

96001380 B-108

Analytes Requested: $A G-F-01, A L-F-01, A S-F-01, B-F-01, B A-F-01$,

BE-F-O1， BI-F-01，CA-F-01，CD-F-02，CE-F-O1，CO-F-O2， CR-F-01, CU-F-01, FE-F-01, LA-F-01, LI-F-01, MG-F-01,

MN-F-O1, MO-F-O1, NA-F-O1, ND-F-01, NI-F-O1, P-F-O1, $\mathrm{PB}-\mathrm{F}-01, \mathrm{~S}-\mathrm{F}-\mathrm{O1}, \mathrm{SB}-\mathrm{F}-01, \mathrm{SE}-\mathrm{F}-\mathrm{OI}, \mathrm{SI}-\mathrm{F}-01, \mathrm{SM}-\mathrm{F}-01$, SR-F-01，TI-F-01，TL-F-01，U-F-01，V-F-01，2N-F-01， $\mathbf{Z R}-\mathrm{F}-01$

9 DUP S96TO05468 O F QICP-FOI SOLID

10 SPK S96TO0546B O F EICP-FO1 SOLID

$11 \mathrm{CCV}$ (aICP-QC QC

$12 \mathrm{CCB} \quad$ eICP-QC QC

13 SAMPLE S96T005477 O F OICP-FO1 SOLID $96001380 \mathrm{~B}-108$

Analytes Requested: $A G-F-01, A L-F-01, A S-F-01, B-F-01, B A-F-01$,

$\mathrm{BE}-\mathrm{F}-01, \mathrm{BI}-\mathrm{F}-01, \mathrm{CA}-\mathrm{F}-01, \mathrm{CD}-\mathrm{F}-01 ; \mathrm{CE}-\mathrm{F}-01 ; \mathrm{CO}-\mathrm{F}-01$ ， CR-F-01 , CU-F-01， FE-F-01 , LA-F-01 , II-F-01 , MG-F-01 , $\mathrm{MN}-\mathrm{F}-01, \mathrm{MO}-\mathrm{F}-01, \mathrm{NA}-\mathrm{F}-01, \mathrm{ND}-\mathrm{F}-01, \mathrm{NI}-\mathrm{F}-01, \mathrm{P} \sim \mathrm{F}-01$, PB-F-01, S-F-O1 , SB-F-01, SE-F-01, ST-F-01，SM-F-01，

Data Enitry Comments:

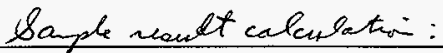

$5967005468 \quad \mathrm{Ol}=438.1 \frac{\mathrm{ug}}{\mathrm{m}} \times \frac{1}{2.082 \frac{\mathrm{g}}{\mathrm{L}} \times \frac{1 \mathrm{l}}{1000}-\mathrm{l}}=2.10 \mathrm{e}+5 \frac{\mathrm{ug}}{\mathrm{g}}$

$S=$ Worklist Slot Number, $R=$ Replicate Number, $A=$ Aliquot Code. 


\section{LABCORE Data Entry Template for Worklist\# 14797}

\begin{tabular}{|c|c|c|c|c|c|c|c|c|c|c|c|c|c|c|}
\hline $\mathbf{s}$ & Type & Sample\# & $\mathbf{R}$ & 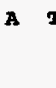 & $\begin{array}{l}\text { Test } \\
\text { SR-F-01 } \\
\text { ZR-F-01 }\end{array}$ & $\begin{array}{l}\text { Matrix } \\
\text { - } T I-F-01\end{array}$ & , & $\begin{array}{l}\text { Group\# } \\
\mathrm{TL},-\mathrm{F}-01\end{array}$ & & $\begin{array}{l}\text { Project } \\
\mathrm{U}-\mathrm{F}-01\end{array}$ & , & $V-F-01$ & , & $\mathrm{ZN}-\mathrm{F}-01$ \\
\hline 14 & DUP & s96T005477 & 0 & $P$ & QICP-FO1 & SOLID & & & & & & & & \\
\hline 15 & SERDIY & s96T005478 & 0 & $F$ & QICP-FO1 & SOLID & & & & & & & & \\
\hline 16 & SAMPLE & $\begin{array}{c}\text { S96T005478 } \\
\text { Analytes Reque }\end{array}$ & $\begin{array}{c}0 \\
\text { est }\end{array}$ & F & $\begin{array}{r}\text { ICP-F01 } \\
\text { AG-F-01 } \\
B E-F-01 \\
C R-F-01 \\
3 N-F-01 \\
P B-F-01 \\
5 R-F-01 \\
Z R-F-01\end{array}$ & $\begin{array}{l}\text { SOLID } \\
\text {, AI-F-0I } \\
\text { BI-F-01 } \\
\text { CU-F-01 } \\
\text {, } M O-F-01 \\
\text { S-F-01 } \\
\text { TI }-F-01\end{array}$ & ; & $\begin{array}{l}960013 \\
A S-F-01 \\
C A-F-01 \\
F E-F-01 \\
N A-F-01 \\
S B-F-01 \\
T L-F-01\end{array}$ & $\begin{array}{l}380 \\
: 1 \\
: 1 \\
: 1\end{array}$ & $\begin{array}{l}B-108 \\
B-F-01 \\
C D-F-01 \\
L A-F-01 \\
N D-F-01 \\
S E-F-01 \\
U-F-01\end{array}$ & ; & $\begin{array}{l}B A-F-01 \\
C E-F-01 \\
I I-F-01 \\
N I-F-01 \\
S I-F-01 \\
V-F-01\end{array}$ & ; & $\begin{array}{l}C O-F-01 \\
M G-F-01 \\
P-F-01 \\
S M \sim F-01 \\
Z N-F-01\end{array}$ \\
\hline 17 & DUP & s96T005478 & 0 & $F$ & QICP-F01 & SOLID & & & & & & & & \\
\hline 18 & $\begin{array}{l}\text { SPK } \\
\text { spk lopd } \\
\text { CCV }\end{array}$ & s96T005478 & 0 & $F$ & $\begin{array}{l}\text { AICP-FOI } \\
\text { AICP-OC }\end{array}$ & $\begin{array}{l}\text { SOLID } \\
\text { QC }\end{array}$ & & & & & & . & & \\
\hline 20 & $\mathrm{CCB}$ & & & & QICP-QC & $\mathrm{OC}$ & & & & & & & & \\
\hline 21 & SERDIL & s96T005487 & 0 & $\mathbf{F}$ & QICP-FOI & SOLID & & & & & & & & \\
\hline 22 & SAMPLE & $\begin{array}{c}\text { s96ro0 } 5487 \\
\text { Analytes Reque }\end{array}$ & & ted: & $\begin{array}{l}\text { ACP-FO1 } \\
A G-F-01 \\
B E-F-01 \\
C R-F-01 \\
M N-F-01 \\
P B-F-01 \\
S R-F-01 \\
Z R-F-01\end{array}$ & $\begin{array}{l}\text { SOLID } \\
\text { AI-F-01 } \\
\text {, BI-F-O1 } \\
\text { CU-F-01 } \\
\text { MO-F-OI } \\
\text { SII-F-OI }\end{array}$ & ! & $\begin{array}{l}960013 \\
A S-F-01 \\
C A-F-01 \\
F E-F-01 \\
N A-F-01 \\
S B-F-01 \\
T L-F-01\end{array}$ & $\begin{array}{l}380 \\
: 1 \\
:\end{array}$ & $\begin{array}{l}B-108 \\
B-F-01 \\
C D-F-01 \\
L A-F-01 \\
N D-F-01 \\
S E-F-01 \\
U-F-01\end{array}$ & : & $\begin{array}{l}B A-F-01 \\
C E-F-01 \\
L I-F-01 \\
N I-F-01 \\
S I-F-01 \\
V-F-01\end{array}$ & $\begin{array}{l}; \\
; \\
;\end{array}$ & $\begin{array}{l}\mathrm{CO}-\mathrm{F}-01 \\
\mathrm{MG}-\mathrm{F}-01 \\
\mathrm{P}-\mathrm{F}-01 \\
\mathrm{SM}-\mathrm{F}-01 \\
\mathrm{ZN}-\mathrm{F}-01\end{array}$ \\
\hline 23 & DƯP & s96T005487. & 0 & F & eICP-FO1. & SOLID & & & & & & & & \\
\hline 25 & $\begin{array}{l}\text { SPK } \\
\text { spk oppr } \\
\text { ICsA }\end{array}$ & S96T005487 & 0 & 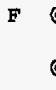 & $\begin{array}{l}\text { बICP-FOI } \\
\text { EICP-QC }\end{array}$ & $\begin{array}{l}\text { SOLID } \\
\text { QC }\end{array}$ & & & & & & & & \\
\hline 26 & ICSAB & & & & CICP $Q \mathrm{CC}$ & $Q C$ & & & & & & & & \\
\hline 27 & $\mathrm{ccV}$ & & & & GICP $-Q C$ & $\mathrm{QC}$ & & & & & & & & \\
\hline
\end{tabular}

Data Entry Comments:

$S=$ Worklist Slot Number, $R=$ Replicate Number, $A=$ Aliquot Code. 
$12 / 11 / 96 \quad 14: 43 \quad 8509 \quad 372 \quad 2929$

WESTINGHOUSE $\rightarrow \rightarrow \rightarrow$ MO-924 200W

四 003

HNF-SD-WM-DP-219, REV. 0

12/11/96 08:23

Page: 3

$A-\infty 004-1$

LABCORE Data Entry Template for Worklist\# 14797

\begin{tabular}{cccc}
\hline Sype Sample\# R A Test Natrix & Group\# Project \\
$28 \mathrm{CCB}$ & GCP-QC $\mathrm{QC}$
\end{tabular}

Final page for worklist \# 14797

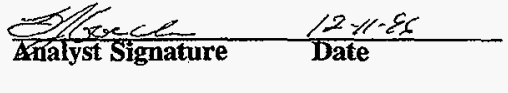

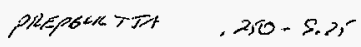

$556 \pi 05468-<.250-8>5-2-8$

$5468 \quad .250-535$

$5 \% 68-0,250-5.35$

$5468-4 \quad .250-2(\sin )-7.35$

$5468-X \quad .250-1-1($ witCIr $)-2.25$

S9670054/17 , 250-9.75

$5472-1230-875$

$58005478-6 \quad .250-577-2-8$

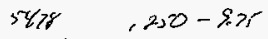

$5478-0 \quad .250-8.75$

$5478-4,200-3(500)-7.75$

$547 x-250-1-1($ wate $1 \times 2)-7.75$

596720

$5487.6 .230-.75-2-8$

5487 .25. 975

5487-1 $-250-5.25$

$5 Y 87-A+250-2(\operatorname{SST})-7.75$

$5487-x \cdot 250-1-1($ arterre $)-7.240$

Kerind by:

D.F

1

292

40

40

40

40

40

40

200

40

40

40

200

40

40

40

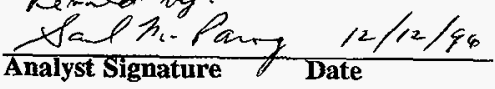

Analyst Signature

Data Entry Comments:

Spite reconeng calculets:

5967005468 al $=\left(\frac{475.6}{40}\right)-\left(\frac{438.1}{40}\right) \times 100=93.8 \%$

$S=$ Worklist Slot Number, $\mathbb{R}=$ Replicate Number, $A=$ Aliquot Code.

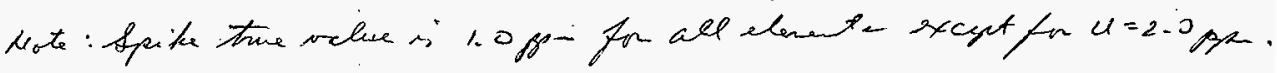

347 
Analys is Report

Summary

Wed 12-11-96 02:34:18 PM.

page 1

\begin{tabular}{|c|c|c|c|c|c|c|c|c|}
\hline \# & Sample Name & Tile & Method & e & & $O p I D$ & & Mode \\
\hline $\begin{array}{l}1 \\
2 \\
3 \\
4 \\
5 \\
6 \\
7 \\
8 \\
9 \\
10 \\
11 \\
12 \\
13 \\
14 \\
15 \\
16 \\
17 \\
18 \\
19 \\
20 \\
21 \\
22 \\
23 \\
24 \\
25 \\
26 \\
27 \\
28 \\
29 \\
30\end{array}$ & 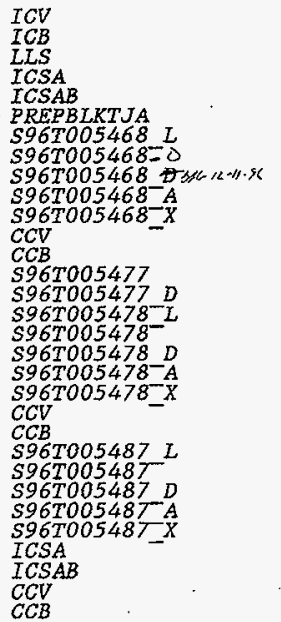 & $\begin{array}{l}961211 D \\
961211 D \\
961211 D \\
961211 D \\
961211 D \\
961211 D \\
961211 D \\
961211 D \\
961211 D \\
961211 D \\
961211 D \\
961211 D \\
961211 D \\
961211 D \\
961211 D \\
961211 D \\
961211 D \\
961211 D \\
961211 D \\
961211 D \\
961211 D \\
961211 D \\
961211 D \\
961211 D \\
961211 D \\
961211 D \\
961211 D \\
961211 D \\
961211 D \\
961211 D \\
961211 D\end{array}$ & $\begin{array}{l}I C P 2 \\
I C P 2 \\
I C P 2 \\
I C P 2 \\
I C P 2 \\
I C C P 2 \\
I C P 2 \\
I C P 2 \\
I C P 2 \\
I C P 2 \\
I C P 2 \\
I C P 2 \\
I C P 2 \\
I C P 2 \\
I C P 2 \\
I C P 2 \\
I C P 2 \\
I C P 2 \\
I C P 2 \\
I C P 2 \\
I C P 2 \\
I C P 2 \\
I C P 2 \\
I C P 2 \\
I C P 2 \\
I C P 2 \\
I C P 2 \\
I C P 2 \\
I C P 2 \\
I C P 2 \\
I C P 2 \\
I C P 2 \\
I C P 2\end{array}$ & $\begin{array}{l}12 / 11 / 96 \\
12 / 11 / 96 \\
12 / 11 / 96 \\
12 / 11 / 96 \\
12 / 11 / 96 \\
12 / 11 / 96 \\
12 / 11 / 96 \\
12 / 11 / 96 \\
12 / 11 / 96 \\
12 / 11 / 96 \\
12 / 11 / 96 \\
12 / 11 / 96 \\
12 / 11 / 96 \\
12 / 11 / 96 \\
12 / 11 / 96 \\
12 / 11 / 96 \\
12 / 11 / 96 \\
12 / 11 / 96 \\
12 / 11 / 96 \\
12 / 11 / 96 \\
12 / 11 / 96 \\
12 / 11 / 96 \\
12 / 11 / 96 \\
12 / 11 / 96 \\
12 / 11 / 196 \\
12 / 11 / 96 \\
12 / 111 / 96 \\
12 / 111 / 96 \\
12 / 11 / 196 \\
12 / 11 / 196 \\
12 / 11 / 96\end{array}$ & $\begin{array}{l}45 \\
48 \\
52 \\
55 \\
58 \\
01 \\
05 \\
08 \\
11 \\
14 \\
19 \\
22 \\
26 \\
29 \\
32 \\
35 \\
38 \\
41 \\
44 \\
47 \\
51 \\
58 \\
01 \\
04 \\
07 \\
10 \\
7\end{array}$ & $\begin{array}{l}B J G \\
B J G \\
B J G \\
B J G \\
B J G \\
B J G \\
B J G \\
B J G \\
B J G \\
B J G \\
B J G \\
B J G \\
B J G \\
B J G \\
B J G \\
B J G \\
B J G \\
B J G \\
B J G \\
B J G \\
B J G \\
B J G \\
B J G \\
B J G \\
B J G \\
B J G \\
B J G \\
B J G \\
B J G \\
B J G \\
B J G\end{array}$ & $\begin{array}{l}Q \\
\oint \\
\oint \\
\oint \\
\& \\
S \\
S \\
S\end{array}$ & $\begin{array}{l}\text { CONC } \\
\text { CONC } \\
\text { CONC } \\
\text { CONC } \\
\text { CONC } \\
\text { CONC } \\
\text { CONC } \\
\text { CONC } \\
\text { CONC } \\
\text { CONC } \\
\text { CONC } \\
\text { CONC } \\
\text { CONC } \\
\text { CONC } \\
\text { CONC } \\
\text { CONC } \\
\text { CONC } \\
\text { CONC } \\
\text { CONC } \\
\text { CONC } \\
\text { CONC } \\
\text { CONC } \\
\text { CONC } \\
\text { CONC } \\
\text { CONC } \\
\text { CONC } \\
\text { CONC } \\
\text { CONC } \\
\text { CONC } \\
\text { CONC } \\
\text { CONC }\end{array}$ \\
\hline
\end{tabular}

$$
\begin{aligned}
& \frac{106-16-56}{12-11-56} \\
& \text { B-k \& Fsin } \\
& 5967005468,547 ?, 547 F, 547) \\
& .250 .1-9.3=1
\end{aligned}
$$

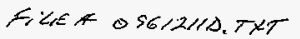

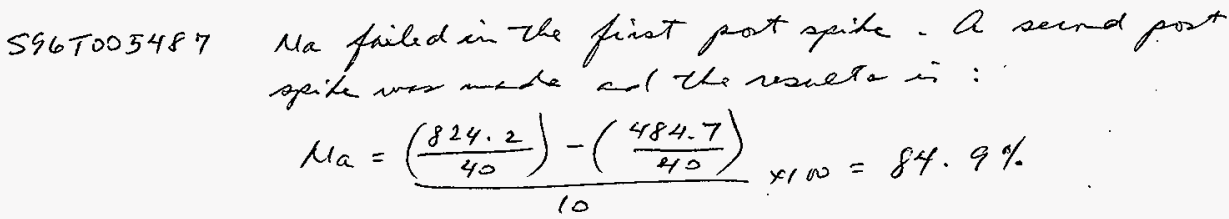
SIGNATURE ABOVE REPRESENTS CHEMICAL TECHNOLOGIST/CHEMIST THATT
COMPLETEDNERIFIED THE CALIBRATIONANALYS ON PAGES 248 TO 323. 
HNF-SD-WM-DP-219, REV. 0

Analysis Report Averages

Wed 12-11-96 02:34:18 PM page 2

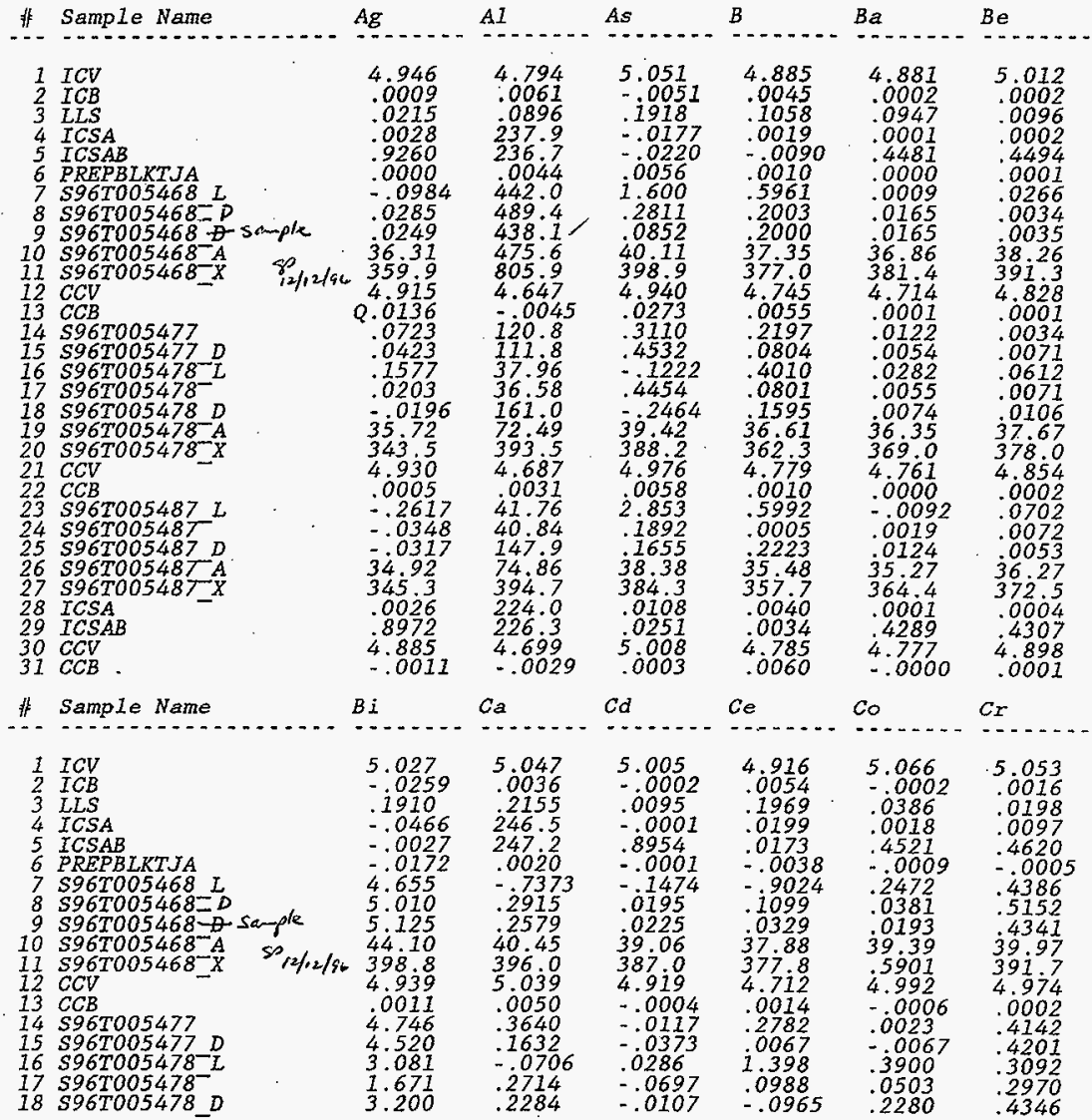


-INF-SD-WRA-DP-219, REV. 0

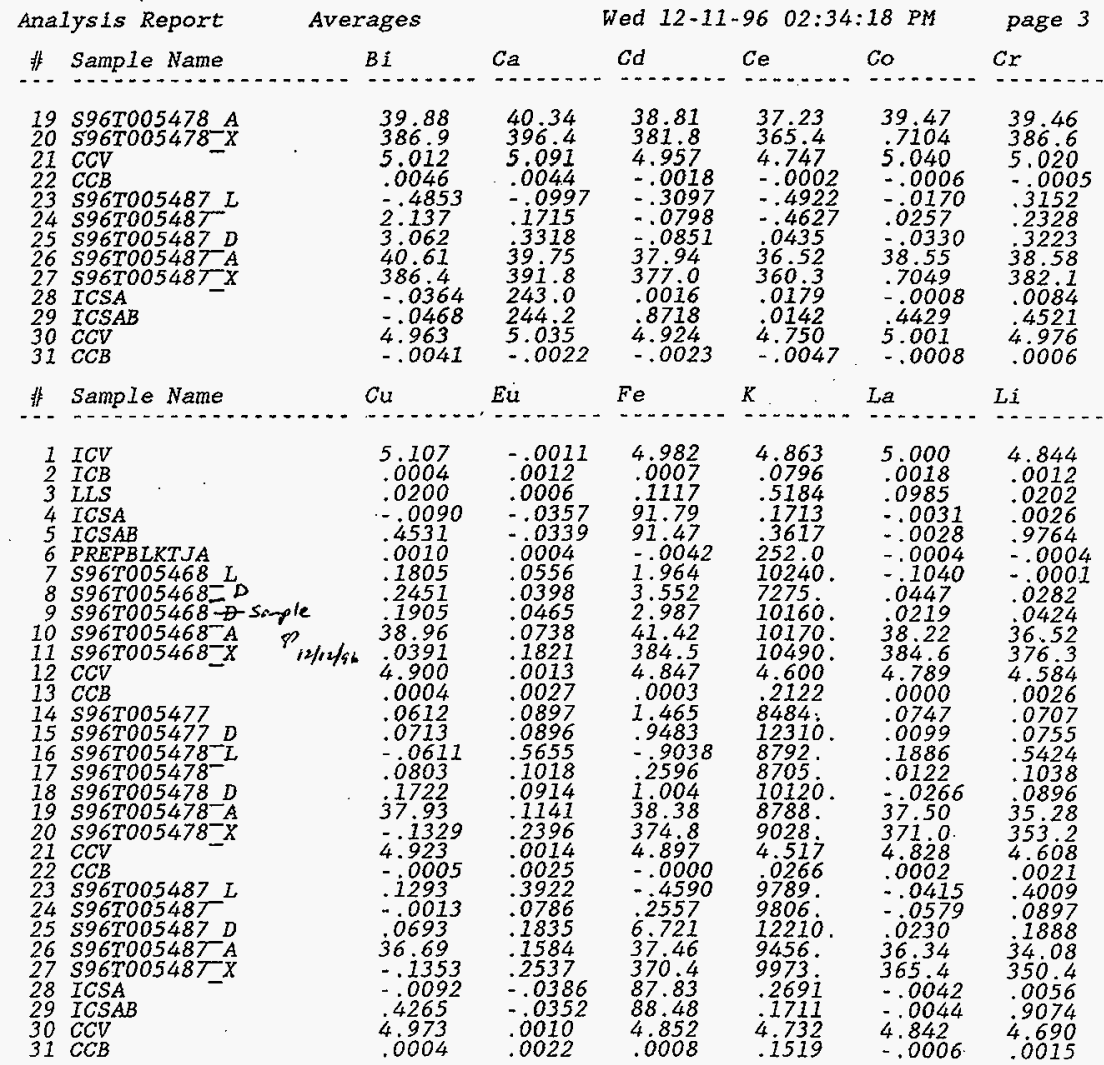


HF-SD-WM-DP-249, REV. O

Analysis Report Averages

Wed 12-11-96 02:34:18 PM page 4

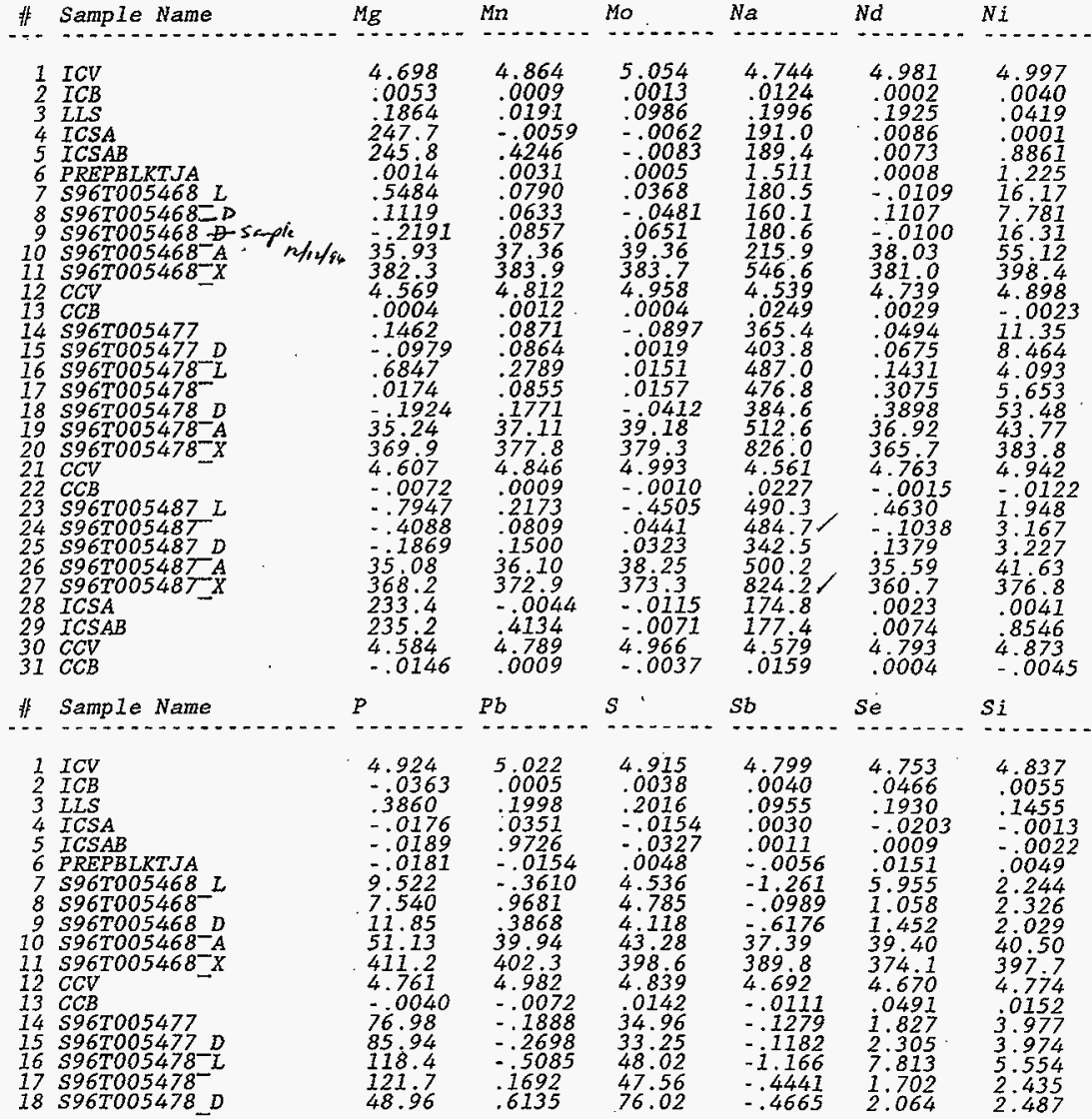


HNF-SD-WM-DP-219, REV. 0

\begin{tabular}{|c|c|c|c|c|c|c|c|}
\hline \multicolumn{2}{|c|}{ Analysis Report } & Averages & \multicolumn{4}{|c|}{ Wed $12-11-5$} & \multirow{2}{*}{$\begin{array}{l}\text { page } 5 . \\
S_{i}\end{array}$} \\
\hline 非 & Sample Name & $p$ & $P b$ & $s$ & $S b$ & $e$ & \\
\hline $\begin{array}{l}19 \\
20 \\
21 \\
22 \\
23 \\
24 \\
25 \\
26 \\
27 \\
28 \\
29 \\
30 \\
31\end{array}$ & $\begin{array}{l}\text { S96T005478 A } \\
\text { S96T005478-X } \\
G C V \\
C C B \\
\text { S96T005487L } \\
\text { S96T005487 } \\
\text { S96T005487 D } \\
\text { S96T005487 A } \\
\text { S96T005487 X } \\
\text { ICSA } \\
I C S A B \\
C C V \\
C C B\end{array}$ & $\begin{array}{l}159.3 \\
506.5 \\
4.935 \\
7.0201 \\
112.1 \\
116.6 \\
72.08 \\
151.2 \\
505.0 \\
-.0214 \\
-.0020 \\
4.862 \\
-.0111\end{array}$ & $\begin{array}{l}39.52 \\
401.3 \\
5.066 \\
-.0123 \\
-.5252 \\
-.2668 \\
.0382 \\
39.08 \\
396.8 \\
.0422 \\
.9479 \\
4.974 \\
. .0125\end{array}$ & $\begin{array}{l}86.98 \\
432.5 \\
4.869 \\
.0111 \\
52.83 \\
50.61 \\
25.35 \\
87.26 \\
435.7 \\
-.0376 \\
-.0203 \\
4.905 \\
.0010\end{array}$ & $\begin{array}{l}37.00 \\
382.7 \\
4.736 \\
-.0084 \\
-1.729 \\
-.5062 \\
-.1373 \\
36.46 \\
379.8 \\
.0007 \\
-.0072 \\
4.729 \\
-.0148\end{array}$ & $\begin{array}{l}38.32 \\
360.6 \\
4.708 \\
.0445 \\
6.600 \\
1.464 \\
2.717 \\
37.31 \\
357.4 \\
.0229 \\
=.0066 \\
4.682 \\
.0580\end{array}$ & $\begin{array}{l}40.43 \\
391.6 \\
4.809 \\
.0106 \\
7.272 \\
5.401 \\
2.972 \\
42.75 \\
391.6 \\
.0134 \\
.0059 \\
4.773 \\
.0081\end{array}$ \\
\hline 非 & Sample Nam & $m$ & $r$ & Th & & 7 & \\
\hline $\begin{array}{l}1 \\
2 \\
3 \\
4 \\
5 \\
6 \\
7 \\
8 \\
9 \\
10 \\
11 \\
12 \\
13 \\
14 \\
15 \\
16 \\
17 \\
18 \\
19 \\
20 \\
21 \\
22 \\
23 \\
24 \\
25 \\
26 \\
27 \\
28 \\
29 \\
30 \\
21\end{array}$ & 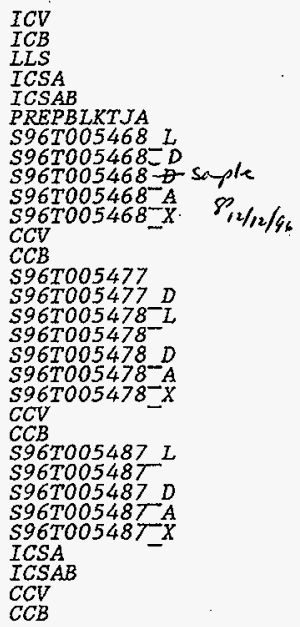 & $\begin{array}{l}4.832 \\
.0164 \\
.1991 \\
.0084 \\
.0090 \\
.0042 \\
.6143 \\
.6008 \\
5703 \\
37.50 \\
371.1 \\
4.624 \\
.0394 \\
1.226 \\
1.301 \\
7.345 \\
1.386 \\
1.457 \\
37.41 \\
357.3 \\
4.667 \\
.0346 \\
5.544 \\
1.258 \\
2.480 \\
36.80 \\
352.4 \\
.0400 \\
.0344 \\
4.683\end{array}$ & $\begin{array}{l}4.853 \\
.0005 \\
.0193 \\
.0020 \\
.0020 \\
.0009 \\
.0833 \\
.0811 \\
.0853 \\
36.96 \\
373.8 \\
4.626 \\
.0006 \\
.0830 \\
.0894 \\
.1644 \\
.0726 \\
.0893 \\
36.13 \\
359.7 \\
4.661 \\
.0005 \\
.1442 \\
.0769 \\
.1140 \\
34.95 \\
354.8 \\
.0019 \\
.0019 \\
4.690 \\
.0005\end{array}$ & $\begin{array}{l}.0388 \\
.0011 \\
.0007 \\
.0069 \\
.0030 \\
.0029 \\
.6991 \\
.0986 \\
.1921 \\
.3810 \\
i .913 \\
.0344 \\
.0086 \\
.0279 \\
=.1003 \\
-.5293 \\
.0272 \\
.0157 \\
.2275 \\
i .556 \\
.0385 \\
-.0066 \\
.2 .130 \\
. .0198 \\
-.2424 \\
.3225 \\
1.574 \\
-.0069 \\
.0041 \\
.0393 \\
=.0092\end{array}$ & $\begin{array}{l}4.869 \\
.0007 \\
.0194 \\
.0010 \\
.0020 \\
.0003 \\
.1016 \\
.1881 \\
.1591 \\
37.78 \\
351.1 \\
4.715 \\
.0002 \\
.0491 \\
.0294 \\
-.0056 \\
.0497 \\
.0893 \\
37.11 \\
341.4 \\
4.784 \\
. .0003 \\
-.0107 \\
.0104 \\
.0087 \\
35.85 \\
337.3 \\
.0010 \\
.0013 \\
4.769 \\
.0002\end{array}$ & $\begin{array}{l}4.730 \\
.0091 \\
.4015 \\
.0079 \\
.0369 \\
.0240 \\
.2296 \\
.1725 \\
.4456 \\
36.29 \\
366.5 \\
4.674 \\
.0146 \\
.9247 \\
.0534 \\
5.055 \\
.2485 \\
2769 \\
35.91 \\
361.0 \\
4.728 \\
.0789 \\
4.756 \\
.8552 \\
1.306 \\
37.30 \\
354.7 \\
.0034 \\
.0332 \\
4.6774 \\
.0174\end{array}$ & $\begin{array}{l}9.492 \\
.0703 \\
.4949 \\
.0328 \\
.0300 \\
-.0657 \\
2.200 \\
3.194 \\
2.236 \\
75.76 \\
33.36 \\
9.111 \\
1534 \\
4.702 \\
3.774 \\
28.05 \\
3.974 \\
3.992 \\
75.14 \\
35.79 \\
9.171 \\
14424 \\
20.91 \\
2.542 \\
7.736 \\
74.90 \\
35.45 \\
.1151 \\
0829 \\
9.201 \\
.1099\end{array}$ \\
\hline
\end{tabular}


HNF-SD-WM-DP-219, REV. 0

Analysis Report

Averages

Wed 12-11-96 02:34:18 PM page 6

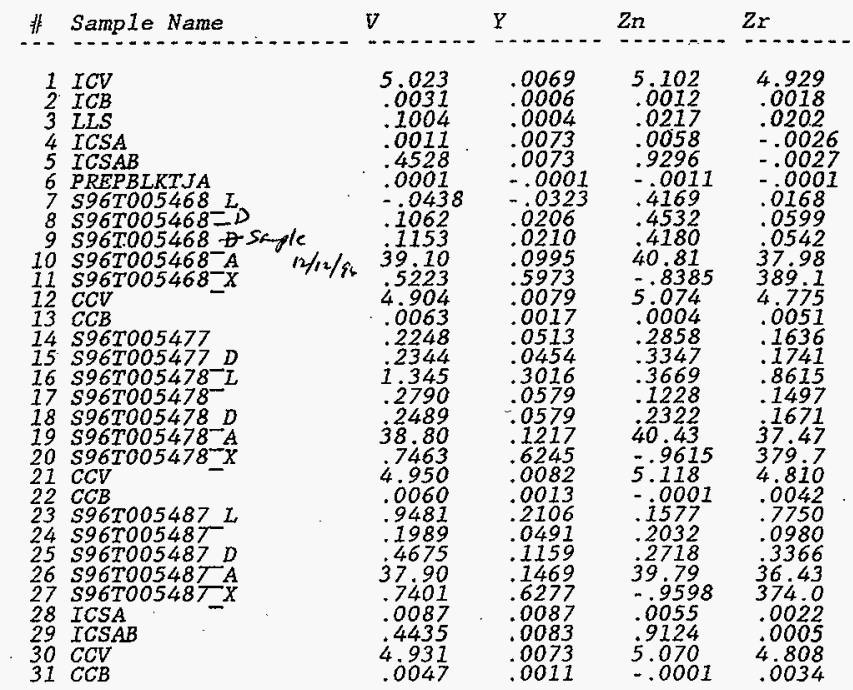




\section{LABCORE Data Entry Template for Worklist\# 14798}

Analyst: B. Gascke Instrument: ICPOO $^{\mathrm{T}}$

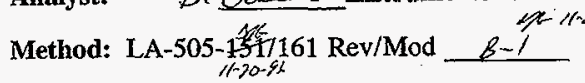

Worklist Comment: ICP B-108 (FUSION)

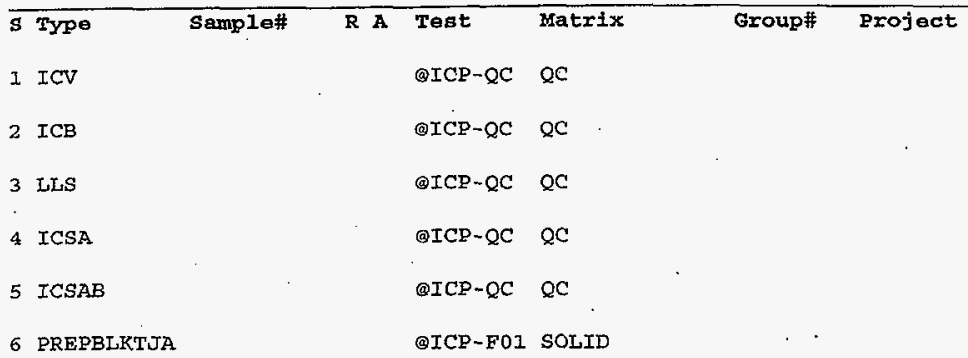

7 SAMPLE S96T005510 OF @ICP-F01 SOLID. 96001379 B-108

Analytes Requested: AG-F-01, AL-F-01, AS-F-01, B-F-01, BA-F-01,

$\mathrm{BE}-\mathrm{F}-01$, $\mathrm{BI}-\mathrm{F}-01, \mathrm{CA}-\mathrm{F}-01, \mathrm{CD}-\mathrm{F}-01, \mathrm{CE}-\mathrm{E}-01, \mathrm{CO}-\mathrm{F}-01$, $\mathrm{CR}-\mathrm{F}-01$, CU-F-01, FE-F-01, IA-F-01, LI-F-01， MG-F-01, MN-F-01, MO-F-01, NA-F-01, ND-F-01, NI-F-01, P-F-01, PB-F-01，S-F-01，SB-F-01，SE-F-01，SI-F-01，SM-F-01， SR-F-01，TI-F-01，TL-F-01, U-F-01，V-F-01， ZN-F-01, $\mathrm{ZR}-\mathrm{F}-01$
8 DUP
S96T005510 O F @ICP-F01 SOLID
$9 \mathrm{CCV}$
@ICP-QC QC
$10 \mathrm{CCB}$
QICP-QC QC
11 SERDIL
S96T005511 0 F @ICP-F01 SOLID
12 SAMPLE
S96T005511 O F QICP-FO1 SOLID

Analytes Requested: $A G-F-01, A L-F-01, A S-F-01, B-F-01$

BE-F-01, BA-F-01

CR-F-01， CU-F-01，FE-F-01， LA-F-01，II-F-01， MG-F-01，

$\mathrm{MN}-\mathrm{F}-01, \mathrm{MO}-\mathrm{F}-01$, NA-F-01, ND-F-01, NI-F-01, P-F-01,

PB-F-OI, S-F-OI , SB-F-OI, SE-F-01, SI-E-0I, SM-F-OI,

SR-F-01, TI-F-01, TL-F-01, U-F-01, V-F-01, ZN-F-01,

$\mathrm{ZR}-\mathrm{F}-01$

Data Entry Comments:

Sappli rauet calculatur: :

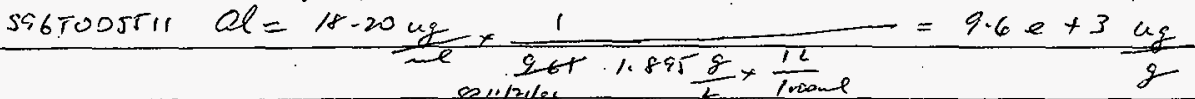

$S=$ Worklist Slot Number, $R=$ Replicate Number, $A=$ Aliquot Code. 
$11 / 20 / 96 \quad 14: 28 \quad \not 509372 \quad 2929$

WESTYNGHOUSE $\quad \rightarrow \rightarrow \rightarrow$ MO-924 $200 \mathrm{~W}$

002

11/08/9609:10

HNF-SD-WHA-DP-219, REV.O

Page:

2

A-0004-1

LABCORE Data Entry Template for Worklist\# 14798

s Type

13 DOP

14

SPK I pph spex $\times$ roppon

15 SAMPLE
Sample\#

S96T005511 0 F
R A Test

Matrix

QICP-F01 SOLID

S96T005511 O F OICE-EOI SOLID

S96T005512 O F OICP-F01 SOLID
16 DUP

17 ICSA

18 ICSAB

$19 \mathrm{CCV}$

$20 \mathrm{CCB}$

$$
\text { Analytes Requested: }
$$
BE-F-01， BI-F-01，CA-F-01，CD-F-01，CE-F-01，CO-F-01， CR-F-01， CU-F-01， FE-F-01， LA-F-01，LI-F-01， MG-F-01 , MN-F-01， MO-F-01，NA-F-01，ND-F-01，NI-F-01， P-F-01 PB-F-01，S-F-01，SB-F-01，SE-F-01，SI-F-01，SM-F-01， SR-F-01 , TI-F-01，TL-F-01，U-F-01，V-F-01，ZN-F-01 , ZR-F-OI

S96T0055I2 O F OICP-F01 SOLID

@ICP-QC QC

@ICP-QC QC

QICP-QC QC

@ICP-QC QC

Final page for worklist \# 14798

kenwery:

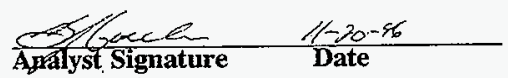

prepserTTAA, 250ur-tons

$5867005510,25001-10.21$

$5510-0$. 250ud c0ut

$5967005511-6 \cdot 03201-1001-2 a 1-8+1$ soll .250 is $1-20 \mathrm{ml}$

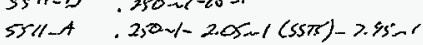

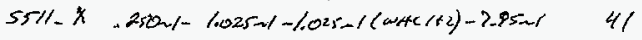

$5967005572.250-1-100-1$

$5512 \rightarrow, 25021-1001$

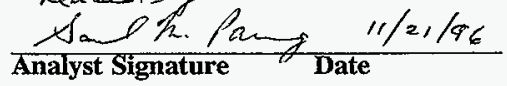

$d F$

41

205

41

41

41

4

41

Data Entry Comments:

Spike neover calculatir:

5967005511 al $=\left(\frac{57.75}{4 i}\right)-\left(\frac{10.20}{41}\right) \times 10=96.5 \%$

1

$S=$ Worklist Slot Number, $R=$ Replicate Number, $A=$ Aliquot Code.

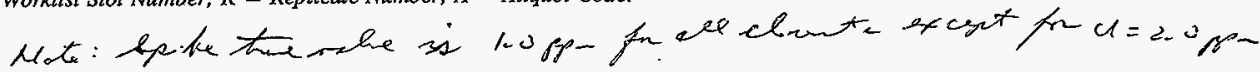

355 
Analysis Report

Summary

HWF-SD-WMM-DP-219, REV.C

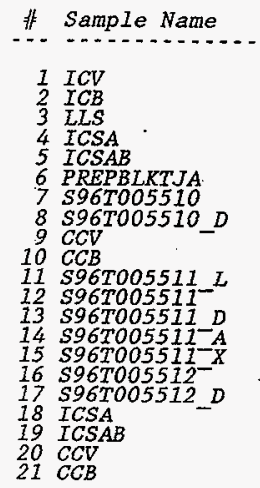

\begin{tabular}{ll} 
File & Method \\
\hline $961120 C$ & ICP2 \\
$961120 C$ & $I C P 2$ \\
$961120 C$ & $I C P 2$ \\
$961120 C$ & $I C P 2$ \\
$961120 C$ & $I C P 2$ \\
$961120 C$ & $I C P 2$ \\
$961120 C$ & $I C P 2$ \\
$961120 C$ & $I C P 2$ \\
$961120 C$ & $I C P 2$ \\
$961120 C$ & $I C P 2$ \\
$961120 C$ & $I C P 2$ \\
$961120 C$ & $I C P 2$ \\
$961120 C$ & $I C P 2$ \\
$961120 C$ & $I C P 2$ \\
$961120 C$ & $I C P 2$ \\
$961120 C$ & $I C P 2$ \\
$961120 C$ & $I C P 2$ \\
$961120 C$ & $I C P 2$ \\
$961120 C$ & $I C P 2$ \\
$961120 C$ & $I C P 2$ \\
$961120 C$ & $I C P 2$
\end{tabular}

Wed 11-20-96 02:20:51 PM

page 1

\begin{tabular}{|c|c|c|c|c|}
\hline ate & Time & pID & e & \\
\hline $\begin{array}{l}11 / 20 / 96 \\
11 / 20 / 96 \\
11 / 20 / 96 \\
11 / 20 / 96 \\
11 / 20 / 96 \\
11 / 20 / 96 \\
11 / 20 / 96 \\
11 / 20 / 96 \\
11 / 20 / 96 \\
11 / 20 / 96 \\
11 / 20 / 96 \\
11 / 20 / 96 \\
11 / 20 / 96 \\
11 / 20 / 96 \\
11 / 20 / 96 \\
11 / 20 / 96 \\
11 / 20 / 96 \\
11 / 20 / 96 \\
11 / 20 / 96 \\
11 / 20 / 96\end{array}$ & $\begin{array}{l}13: 12 \\
13: 15 \\
13: 18 \\
13: 20 \\
13: 23 \\
13: 28 \\
13: 31 \\
13: 35 \\
13: 38 \\
13: 41 \\
13: 44 \\
13: 47 \\
13: 50 \\
13: 53 \\
13: 56 \\
14: 00 \\
14: 03 \\
14: 07 \\
14: 10 \\
14: 14\end{array}$ & $\begin{array}{l}B J G \\
B J G \\
B J G \\
B J G \\
B J G \\
B J G \\
B J G \\
B J G \\
B J G \\
B J G \\
B J G \\
B J G \\
B J G \\
B J G \\
B J G \\
B J G \\
B J G \\
B J G \\
B J G\end{array}$ & $\begin{array}{l}Q \\
\oint \\
\oint \\
\oint \\
\oint \\
S \\
S \\
S \\
Q \\
Q \\
S \\
S \\
S \\
S \\
S \\
S \\
S \\
Q\end{array}$ & $\begin{array}{l}N C \\
N C \\
N C \\
N C \\
N C \\
N C \\
N C \\
N C \\
v C \\
v C \\
N C \\
V C \\
V C \\
v C \\
V C \\
V C \\
V C \\
V C \\
V C\end{array}$ \\
\hline
\end{tabular}

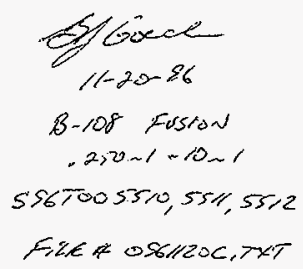
SIGNATURE ABOVE REPRESENTS CHEMICAL TECHNOLOGIST/CHEMIST THAT
COMPLETEDNERIFIED THE CALIBRATION/ANALYSIS ON PAGES 350 TO 260. 
$11 / 20 / 96 \quad 14: 29 \quad 8509372 \quad 2929$

AnaIysis Report

Averages

HNF-SD-WHA-DP-219, REV. 0

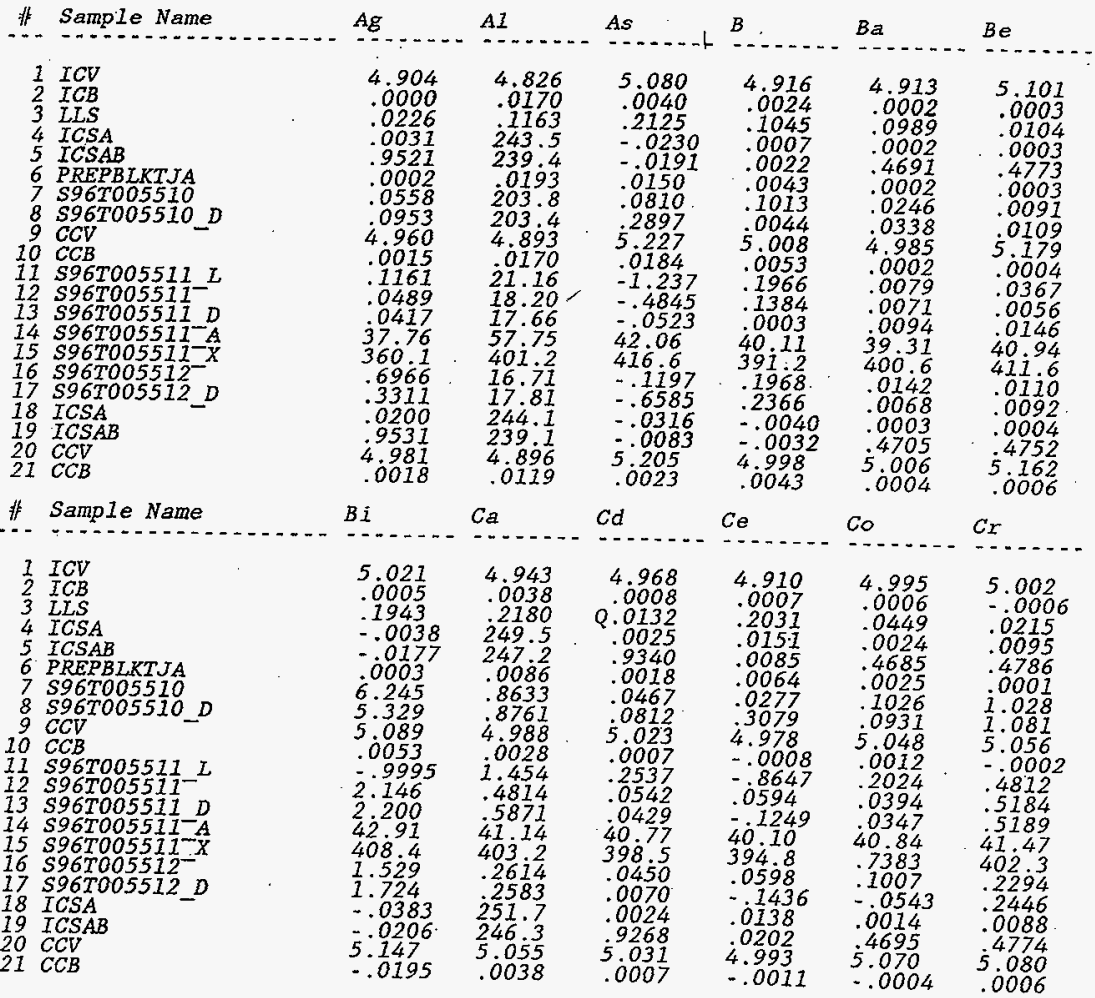

Wed 11-20.96 02:20:51 PM page 2 


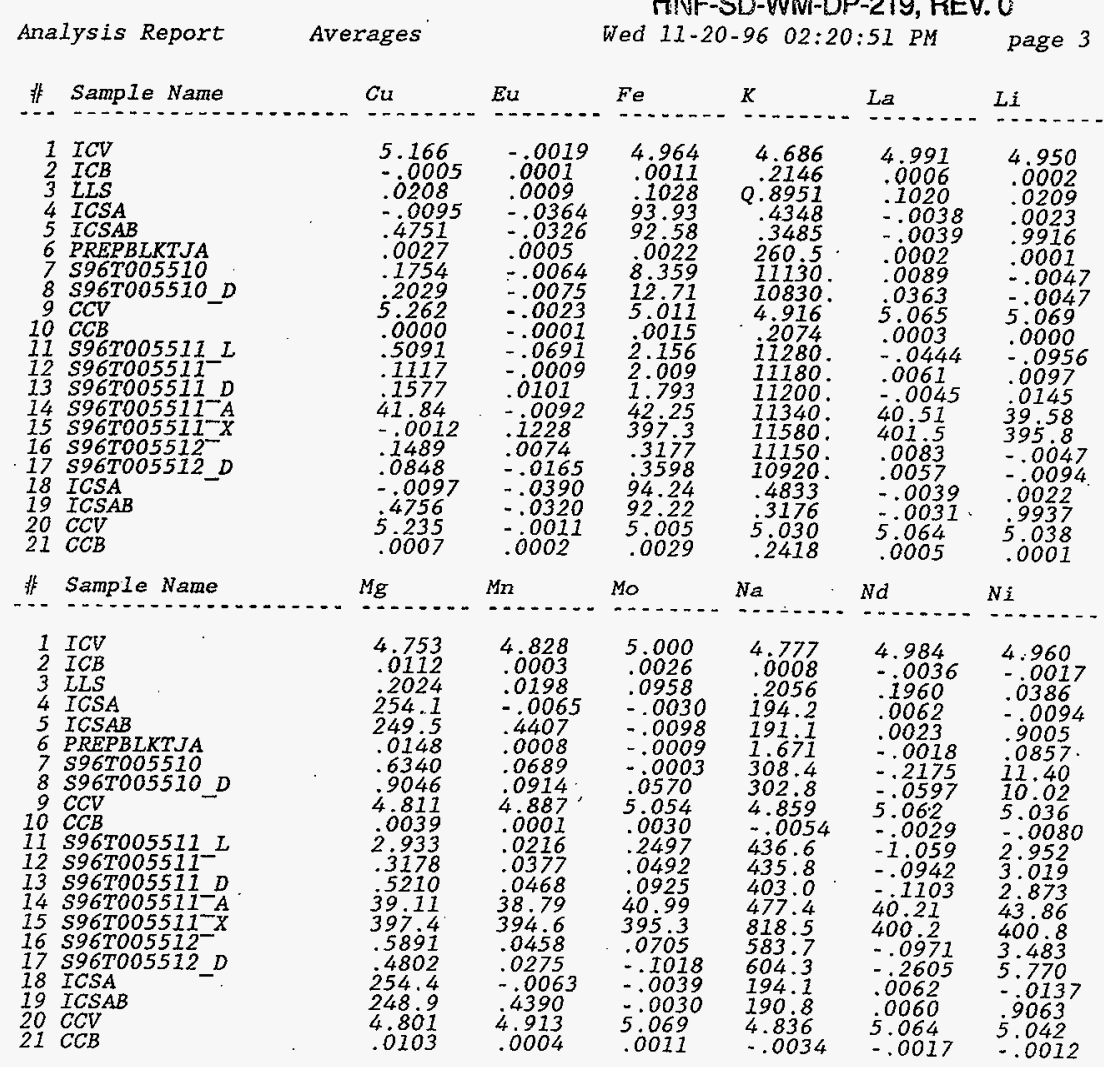


Analysis Report

Averages
$P \quad P b$

4.976

$-.0174$

.0098

.0304

52.11

51.55

4.996

.0083

16.93

22.62

57.89

44.98

24.26

.0223

4.0193

. .0063

HNF-SD-WIV-DP-219, REV. O

Wed 11-20-96 02:20:51 PM page 4

$S m$

$\begin{array}{ll}4.827 & 4.869 \\ .0026 & .0001 \\ .2092 & .0202 \\ -.0012 & .0020 \\ -.0005 & .0020 \\ .0045 & .0012 \\ -.2121 & .1092 \\ -.0729 & .1113 \\ 4.898 & 4.938 \\ -.0039 & .0000 \\ -.6858 & .0540 \\ -.0304 & .0836 \\ 30109 & .0793 \\ 39.08 & 39123 \\ 387.8 & 391.3 \\ -.0109 & .0665 \\ -.2032 & .0559 \\ -.0001 & .0020 \\ -.0043 & .0019 \\ 4.905 & 4.932 \\ .0022 & .0003\end{array}$

$S b$

Se

$S i$

$\begin{array}{ll}4.941 & 4.897 \\ .0223 & .0034 \\ .2411 & .2020 \\ .0474 & -.0193 \\ 1.003 & -.0310 \\ .0258 & -.0012 \\ 1.381 & 7.036 \\ 1.081 & 7.177 \\ 5.000 & 4.963 \\ .0168 & .0117 \\ 4.440 & 87.65 \\ 1.203 & 91.01 \\ .8639 & 76.19 \\ 41.59 & 132.1 \\ 410.7 & 493.3 \\ .8830 & 166.1 \\ .4971 & 192.0 \\ .0620 & -.0235 \\ 1.004 & -.0172 \\ 5.042 & 4.935 \\ .0177 & .0137\end{array}$

Th

4.754
.0062
.1100
$: .0048$
-.0008
.0027
.1271
.3514
4.838
.0065
2.066
-.0893
-.0290
39.12
400.3
.5221
-.0387
-.0037
-.0047
.0033
.0009

4.820

$.0469 \quad 4.943$

.0019

$-.0086 \quad .0092$

$-.0013-.0008$

$.0397 \quad .0054$

$2.192 \quad 4.290$

$1.357 \quad 4.506$

$4.922 \quad 5.005$

.0500

8.314

1.985

1.858

41.30

388.1

1. 673

$-.0019$

$-.0019$

4.884

.0561

$-.0019$

2.322

2.694

2.620

43.65

421.0

1. 326

.0053

.0001

$T i$

$T 2$

.0487
.0065
.0027
.0031
.0049
.0087
.3926
.5461
.0599
.0058
1.607
.3097
.3263
.7563
.647
.4295
.2428
.0015
.0090
.0476
.0057

4.858
.0000
.0195
.0000
.0003
.0004
.0018
.0025
4.931
-.0005
-.1430
-.0180
.0126
39.62
365.4
-.0072
.00482
.0005
.0000
4.943
.0003

4.825

.0044

.4328

.0147

.0208

.0048

.8929

4.885

$-.0048$

4120

1.132

38.89

381.6

.3124

.5451

.0074

.0107

4.893

.0052
$U$

$$
\begin{aligned}
& 9.463 \\
& .0183 \\
& .5310 \\
& .0096 \\
& .0013 \\
& -.0646 \\
& -1.231 \\
& 9.9619 \\
& 9.639 \\
& -.0179 \\
& -6.229 \\
& -2.019 \\
& -1.574 \\
& 74.74 \\
& 29.69 \\
& -2.417 \\
& -3.119 \\
& .0042 \\
& .0031 \\
& 9.631 \\
& .0087
\end{aligned}
$$




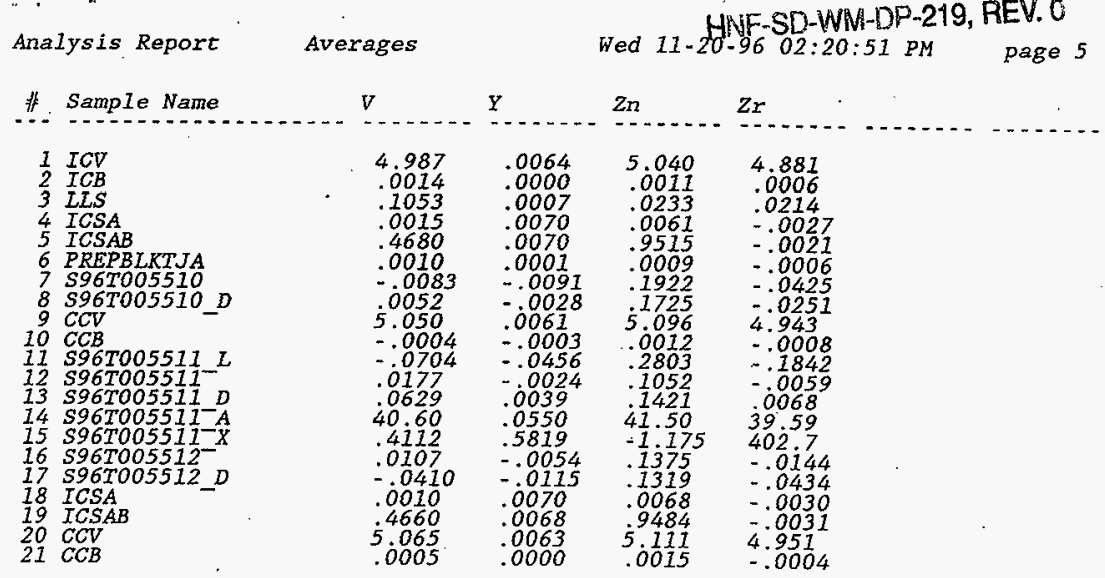


HNF-SD-WM-DP-249, REV.O

$11 / 08 / 9609: 21$

A-0004-1

$F / L E N: 96 / 121 A .7 X T$

LABCORE Data Entry Template for Worklist\# 14799

Page: 1

TWORELL TR $11-21-96$

Analyst: $\pi$, WORREL Instrument: ICPO12

Method: LA-505-15t/161 Rev/Mod B -1

Sov $11-2196$

Worklist Comment: ICP B-108 (SOLID WATER DIGEST)

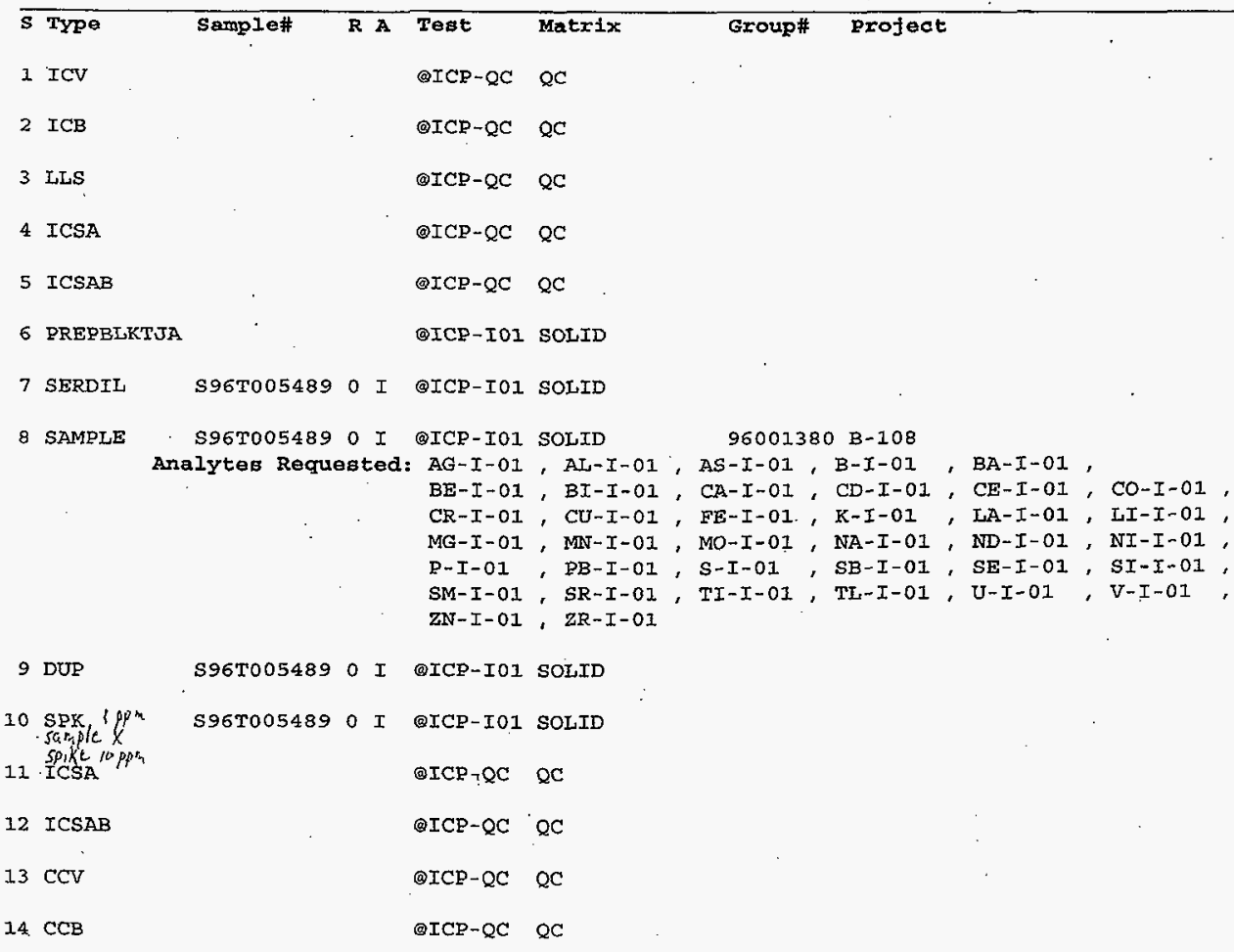

Data Entry Comments: Saych reulet calaulatin:

5967005489 al $=1150 \mathrm{ug} \times \frac{1}{5.009 \frac{\mathrm{g}}{\mathrm{L}} \times \frac{1 \mathrm{~L}}{1000 \mathrm{me}}}=2.30 \mathrm{e}+2 \mathrm{ug}$

$S=$ Worklist Slot Number, $R=$ Replicate Number, $A=$ Aliquot Code. 
HWF-SD-WW-DP-219, REV .O

11/08/9ं 09:21

Page: 2

A-0004-1

LABCORE Data Entry Template for Worklist\# 14799

S Type Sample\# RA Test Matrix Group\# project

Final page for worklist \# 14799
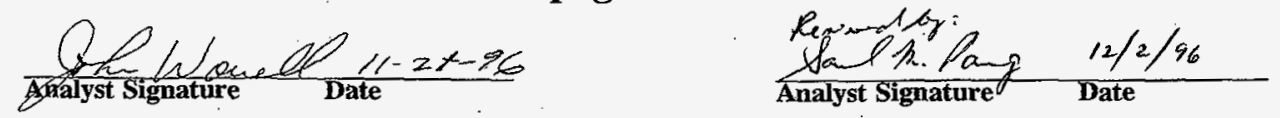

PREP BLK DIRECT

$\frac{D F}{1}$

$\$ 967005489$

DIR $574-4-2-8$

SAM $4-4$

DUP $4-4$

P. SPR 4-2-2

SAM X0.4-9.6

10

10PPM P.SPK 0.4-1-1-7.625

$$
\begin{aligned}
\text { spike true value }= & 2(5)=8(x) \\
x & =1.26 \text { ppm }
\end{aligned}
$$

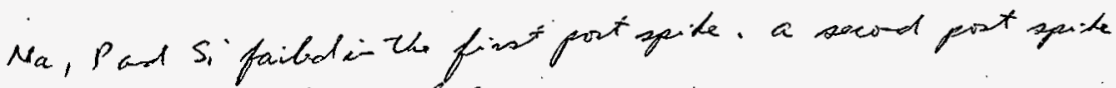
ware wack and the vanes are:

$$
\begin{aligned}
& \mu_{a}=\frac{\left(\frac{1138}{25}\right)-\left(\frac{85.4}{25}\right)}{10} \times 100=10 \% .0 \% \\
& P=\frac{\left(\frac{563.6}{25}\right)-\left(\frac{30 \% .2}{25}\right)}{10} \times 100=10 \% .8 \% \\
& S_{i}=\frac{\left(\frac{245.4}{25}\right)-\left(\frac{8.034}{25}\right)}{10} \times 10=102.9 \%
\end{aligned}
$$

Data Entry Comments:

Ap te reaver calculation:

5167005489 al $=\frac{\left(\frac{3.668}{2}\right)-\left(\frac{1.100}{2}\right)}{1.25} \times 100=101.0 \%$

$S=$ Worklist Slot Number, $R=$ Replicate Number, $A=$ Aliquot Code.

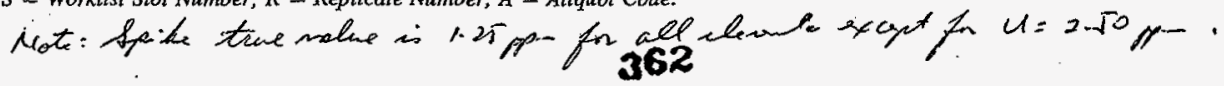

2.00 济

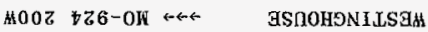

6667 aLE 6098

$D G: 2 T$

$96 /[z / I I$ 
HNF-SD-WN-DP-219, REV.0

\begin{tabular}{|c|c|c|c|c|c|c|c|}
\hline alysis & & & $2 u$ & $96 \quad 05: 4$ & .52 & $M$ & \\
\hline \# Sample Name & File & & & e & $n$ & ype & Mode \\
\hline $\begin{array}{rl}1 & I C V \\
2 & I C B \\
3 & L L S \\
4 & I C S A \\
5 & I C S A B \\
6 & P R E P B L K T J A \\
7 & S 96 T 005489 L \\
8 & S 96 T 005489-1 \\
9 & S 96 T 005489-D \\
10 & S 96 T 005489-A \\
11 & 596 T 005489-X \\
12 & S 96 T 005489-10 P P M \\
13 & I C S A \\
14 & I C S A \\
15 & I C S A B \\
16 & C C V \\
17 & C C B\end{array}$ & $\begin{array}{l}961121 A \\
961121 A \\
961121 A \\
961121 A \\
961121 A \\
961121 A \\
961121 A \\
961121 A \\
961121 A \\
9611121 A \\
961121 A \\
961121 A \\
961121 A \\
961121 A \\
961121 A \\
961121 A \\
961121 A\end{array}$ & $\begin{array}{l}I C P 2 \\
I C P 2 \\
I C P 2 \\
I C P 2 \\
I C P 2 \\
I C P 2 \\
I C P 2 \\
I C P 2 \\
I C P 2 \\
I C P 2 \\
I C P 2 \\
I C P 2 \\
I C P 2 \\
I C P 2 \\
I C P 2 \\
I C P 2 \\
I C P 2 \\
I C P 2\end{array}$ & $\begin{array}{l}11 / 21 / 96 \\
11 / 21 / 96 \\
11 / 21 / 96 \\
11 / 21 / 96 \\
11 / 21 / 96 \\
11 / 21 / 96 \\
11 / 21 / 96 \\
11 / 21 / 96 \\
11 / 21 / 96 \\
11 / 21 / 96 \\
11 / 21 / 96 \\
11 / 21 / 96 \\
11 / 21 / 96 \\
11 / 21 / 96 \\
11 / 21 / 96 \\
11 / 21 / 96 \\
11 / 21 / 96\end{array}$ & $\begin{array}{l}16: 27 \\
16: 31 \\
16: 35 \\
16: 40 \\
76: 43 \\
16: 53 \\
16: 57 \\
17: 01 \\
17: 06 \\
17: 10 \\
17: 15 \\
17: 19 \\
17: 27 \\
17: 31 \\
17: 34 \\
17: 38 \\
17: 42\end{array}$ & $\begin{array}{l}\text { JHW } \\
\text { JHW } \\
\text { JHW } \\
\text { JHW } \\
\text { JHW } \\
\text { JHW } \\
\text { JHW } \\
\text { JHWW } \\
\text { JHW } \\
\text { JHW } \\
\text { JHW } \\
\text { JHW } \\
\text { JHW } \\
\text { JHW } \\
\text { JHW } \\
\text { JHW } \\
\text { JHW }\end{array}$ & 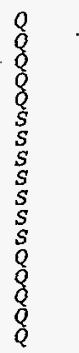 & \\
\hline
\end{tabular}

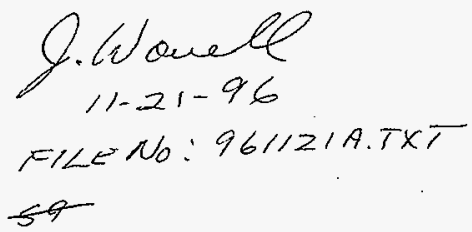

WORKLISTE. 14799

SIGNATUAE ABOVE REPRESENTS CHEMICAL TECHNOLOGIST/CHEMIST THAT
COMPLETEDNERIFIED THE CALIBRATION/ANALYSIS ON PAGES 363 TO 36 L 
WORKBOOK PAGE: SAM4

Sr-89/90 : LA-220-101 (D-1), 102 (E-3), 104 (D-1)

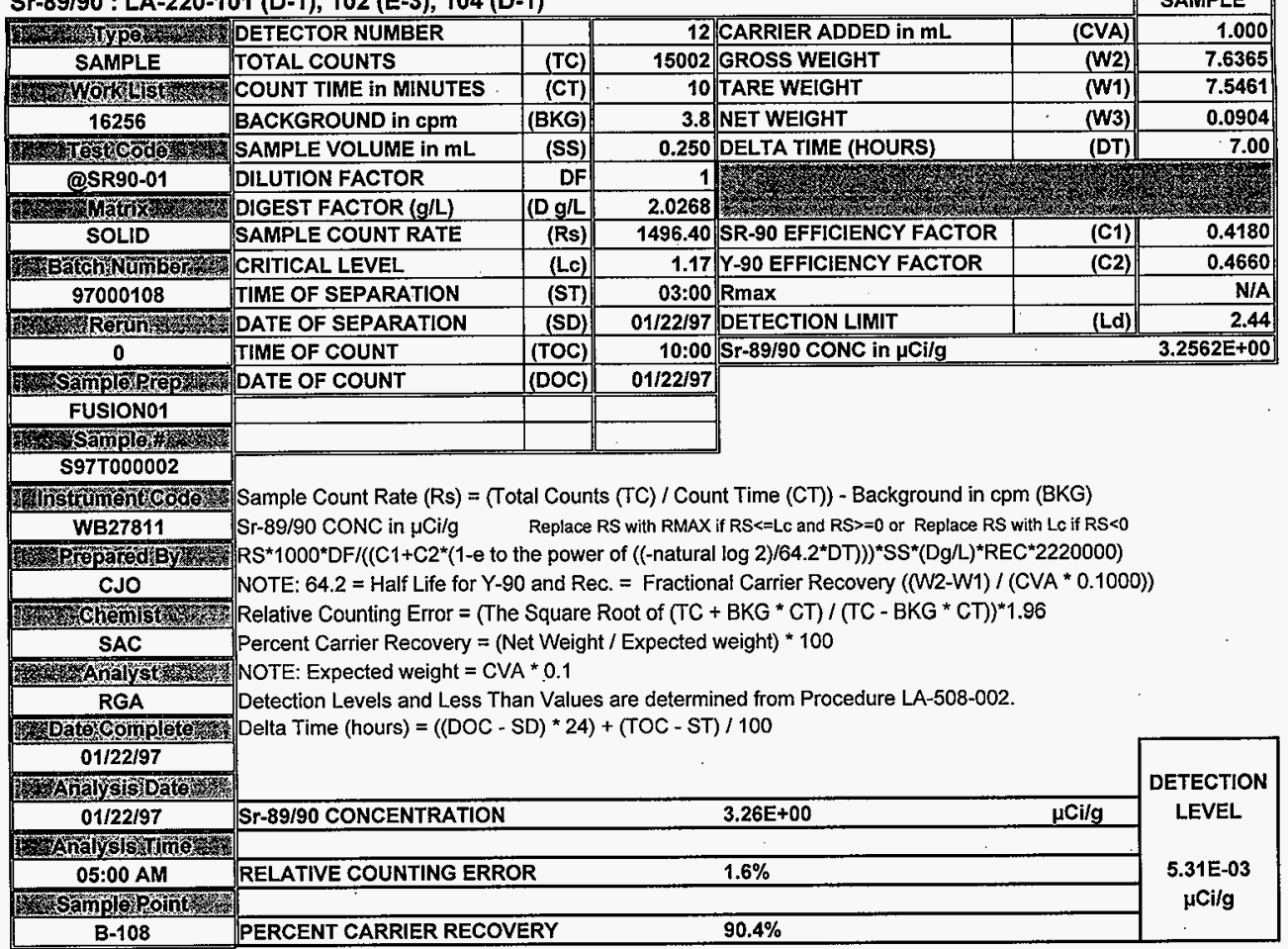

\begin{tabular}{|lllll|}
\hline Analyst: & RGA & Date: & 22-Jan-97 \\
\hline Signature of Chemist: & SN Cattam & SAC & Date: & 2.37\% 97 \\
\hline SAMPLE.WB1 REV 2.0 & 22010NML & &
\end{tabular}


WORKBOOK PAGE: DUP5

Sr-89/90 : LA-220-101 (D-1), 102 (E-3), 104 (D-1)

\begin{tabular}{|c|c|c|c|c|c|c|}
\hline 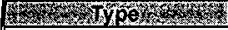 & DETECTOR NUMBER & & 12 & CARRIER ADDED in $\mathrm{mL}$ & (CVA) & 1.000 \\
\hline DUP & TOTAL COUNTS & (TC) & 17959 & GROSS WEIGHT & (W2) & 7.6135 \\
\hline Whormos & COUNT TIME in MINUTES & (CT) & 10 & TARE WEIGHT & (W1) & 7.5215 \\
\hline 16256 & BACKGROUND in cpm & (BKG) & 3.8 & NET WEIGHT & (W3) & 0.0920 \\
\hline Shestoodos & SAMPLE VOLUME in $\mathrm{mL}$ & (SS) & 0.250 & DELTA TIME (HOURS) & (DT) & 7.50 \\
\hline @SR90-01 & DILUTION FACTOR & DF & 1 & \multirow{2}{*}{\multicolumn{3}{|c|}{ 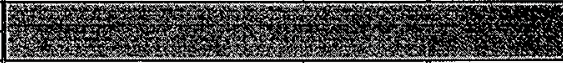 }} \\
\hline 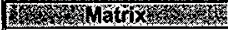 & DIGEST FACTOR (g/L) & $(\mathrm{D} \mathrm{g} / \mathrm{L}$ & 2.0308 & & & \\
\hline SOLID & SAMPLE COUNT RATE & (Rs) & 1792.10 & SR-90 EFFICIENCY FACTOR & (C1) & 0.4180 \\
\hline gegatchonber & CRITICAL LEVEL & (LC) & 1.17 & Y-90 EFFICIENCY FACTOR & (C2) & 0.4660 \\
\hline 97000108 & TIME OF SEPARATION & (ST) & 03:00 & Rmax & & N/A \\
\hline 4 HRoTh & DATE OF SEPARATION & (SD) & $01 / 22 / 97$ & DETECTION LIMIT & (Ld) & 2.44 \\
\hline 0 & TIME OF COUNT & (TOC) & $10: 30$ & \multicolumn{2}{|l|}{ Sr-89/90 CONC in $\mu \mathrm{Ci} / \mathrm{g}$} & $3.8047 E+00$ \\
\hline os samplespops & DATE OF COUNT & (DOC) & $01 / 22 / 97$ & \multicolumn{3}{|l|}{ 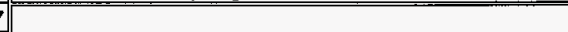 } \\
\hline \multicolumn{7}{|c|}{ FUSION01 } \\
\hline \multicolumn{7}{|l|}{ S } \\
\hline \$97T000002 & & & & & & \\
\hline Whotrumantcoder & \multicolumn{6}{|c|}{ Sample Count Rate (Rs) $=($ Total Counts $(T C) /$ Count Time $(C T))-$ Background in cpm (BKG) } \\
\hline WB27811 & Sr-89/90 CONC in $\mu \mathrm{Ci} / \mathrm{g}$ & \multicolumn{5}{|c|}{ Replace RS with RMAX if RS $<=L c$ and RS $>=0$ or Replace RS with Lc if $R S<0$} \\
\hline 3Prepared By & \multicolumn{6}{|c|}{$R S^{\star} 1000^{\star} \mathrm{DF} /\left(\left(\mathrm{C} 1+\mathrm{C} 2^{\star}\left(1-e \text { to the power of }\left((- \text { natural } \log 2) / 64.2^{\star} \mathrm{DT}\right)\right)\right)^{\star} \mathrm{SS}{ }^{\star}(\mathrm{Dg} / \mathrm{L})^{\star} \mathrm{REC} \mathrm{C}^{\star} 2220000\right)$} \\
\hline CJO & \multicolumn{6}{|c|}{ NOTE: $64.2=$ Half Life for $Y-90$ and Rec. $=$ Fractional Carrier Recovery $($ W2-W1 $) /(C V A * 0.1000))$} \\
\hline 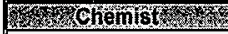 & \multicolumn{6}{|c|}{ Relative Counting Error $=\left(\text { The Square Root of }\left(T C+B K G{ }^{*} \mathrm{CT}\right) /(T C-B K G * C T)\right)^{\star} 1.96$} \\
\hline SAC & \multicolumn{6}{|c|}{ Percent Carrier Recovery $=$ (Net Weight $/$ Expected weight) * 100} \\
\hline 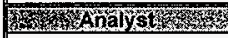 & \multicolumn{6}{|c|}{ NOTE: Expected weight $=\mathrm{CVA} \cdot 0.1$} \\
\hline RGA & \multirow{2}{*}{\multicolumn{6}{|c|}{$\begin{array}{l}\text { Detection Levels and Less Than Values are determined from Procedure LA-508-002. } \\
\text { Delta Time (hours) }=((\mathrm{DOC}-\mathrm{SD}) \cdot 24)+(\mathrm{TOC}-\mathrm{ST}) / 100\end{array}$}} \\
\hline 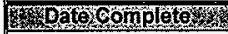 & & & & & & \\
\hline $01 / 22 / 97$ & & & & & & \multirow{4}{*}{$\begin{array}{l}\text { DETECTION } \\
\text { LEVEL }\end{array}$} \\
\hline 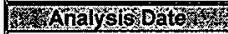 & & & & & & \\
\hline $01 / 22 / 97$ & \multicolumn{3}{|l|}{ Sr-89/90 CONCENTRATION } & $3.80 \mathrm{E}+00$ & $\mu \mathrm{Ci} / \mathrm{g}$ & \\
\hline 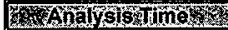 & \multicolumn{5}{|c|}{ (2) } & \\
\hline 05:00 AM & \multicolumn{3}{|c|}{ RELATIVE COUNTING ERROR } & $1.5 \%$ & & \multirow{3}{*}{$\begin{array}{l}5.18 \mathrm{E}-03 \\
\mu \mathrm{Ci} / \mathrm{g}\end{array}$} \\
\hline WSamplepotht & 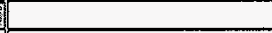 & 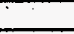 & . & 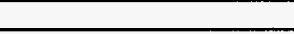 & & \\
\hline B-108 & \multicolumn{3}{|c|}{ PERCENT CARRIER RECOVERY } & \multicolumn{2}{|l|}{$92.0 \%$} & \\
\hline
\end{tabular}

\begin{tabular}{|llll|}
\hline Analyst: & RGA & Date: \\
\hline Signature of Chemist: & St Catfis & SAC & Date: \\
\hline
\end{tabular}




\section{DISTRIBUTION SHEET}

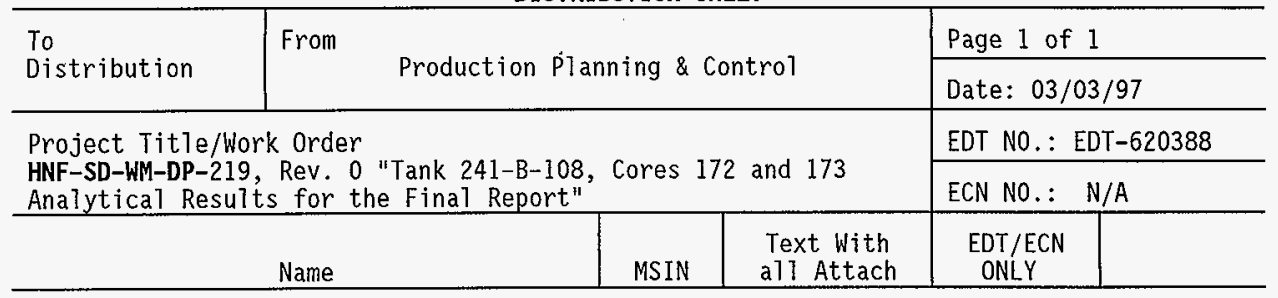

DE\&S Hanford, Inc.

J. E. Meacham

U.S. Department of Energy, RL

C. A. Babel

Lockheed Mart in Hanford Corp.

J. N. Appel

T. J. Kelley

N. W. Kirch

M. J. Kupfer

J. B. Schaffer

R. D. Schreiber

J. A. Voogd

A. E. Young

Lockheed Martin Services. Inc.

Central Files

Los Alamos Technical Associates

M. T. E11 sworth

Pacific Northwest National Laboratory

S. J. Harris

K. L. Silvers

Rust Federal Services of Hanford, Inc.

M. W. Barnes

D. C. Hetzer

J. L. Nuzum

K. L. Powe 11

C. M. Seidel

LTIC

SGN Eurisys Services Corp.

B. A. Higley

Defense Nuclear Federal Safety Board

Rich Tontodonato

625 Indiana Ave. N. W.

S7-14 $\quad X$

$57-54 \quad x$

G3-21

S7-21

R2-11

H5-49

$\mathrm{R} 2-12$

R2-12

H5- 03

R2-12

A3-88

T6-06

$X^{*}$

K7-22

$\mathrm{K} 9-08$

$x$

T6-16

S6-31

T6-50

T6-04

T6-04

T6-03

H5-27

$x$

$x$

$x$

$x$

$x$

Washington D.C. 20004

* Needs only releasing paperwork, not a copy of the released document. 


\begin{tabular}{|c|c|c|c|c|c|c|c|}
\hline 侎 & Sample Name & $g$ & A1 & 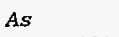 & $B$ & $B a$ & $B e$ \\
\hline & $\begin{array}{l}\text { ICV } \\
\text { ICB } \\
\text { LLS } \\
\text { ICSA } \\
\text { ICSAB } \\
\text { PREPBLKTJA } \\
\text { S96T005489 } L \\
\text { S96T005489- } \\
\text { S96T005489-D. } \\
\text { S96T005489-A } \\
\text { S96T005489-X } \\
\text { S96T005489-10PPM } \\
\text { IESA } 80-1 / 22 / 46 \\
\text { ICSA } \\
\text { ICSAB } \\
C C V \\
C C B\end{array}$ & $\begin{array}{l}5.035 \\
.0003 \\
.0220 \\
.0011 \\
.9667 \\
.0006 \\
.0618 \\
.0625 \\
.0715 \\
2.603 \\
.0847 \\
246.6 \\
0.0022 \\
.0017 \\
.9687 \\
5.108 \\
.0001\end{array}$ & $\begin{array}{l}4.994 \\
.0018 \\
11133 \\
246.0 \\
244.4 \\
3006 \\
1.184 \\
1.150 \\
4.113 \\
3.668 \\
1.268 \\
240.5 \\
246.3 \\
245.4 \\
244.6 \\
4.992 \\
-.0029\end{array}$ & $\begin{array}{l}5.212 \\
.0052 \\
.2220 \\
-.0477 \\
.0451 \\
.0047 \\
-.0362 \\
.0007 \\
.0208 \\
2.716 \\
.0684 \\
254.2 \\
-.0292 \\
-.0030 \\
-.0222 \\
5.263 \\
.0112\end{array}$ & $\begin{array}{l}5.121 \\
.0020 \\
.1013 \\
.0015 \\
-.0041 \\
4.403 \\
2.923 \\
2.794 \\
8.059 \\
5.364 \\
2.889 \\
252.3 \\
.0044 \\
.0024 \\
.00024 \\
5.089 \\
.0035\end{array}$ & $\begin{array}{l}5.115 \\
.0001 \\
.1022 \\
.0002 \\
.4824 \\
.0019 \\
.0036 \\
.0017 \\
.0038 \\
2.530 \\
.0116 \\
252.9 \\
0.0004 \\
.0003 \\
.4865 \\
.0221 \\
.0003\end{array}$ & $\begin{array}{l}5.224 \\
-.0001 \\
.0101 \\
-.0003 \\
.4752 \\
-.0004 \\
-.0022 \\
-.0009 \\
-.0007 \\
2.597 \\
-.0067 \\
254.43 \\
Q-.0001 \\
-.0001 \\
.4789 \\
5.215 \\
-.0002\end{array}$ \\
\hline \# & Sample Name & $B \dot{1}$ & $C a$ & $c d$ & $\mathrm{Ce}$ & Co & $C r$ \\
\hline & 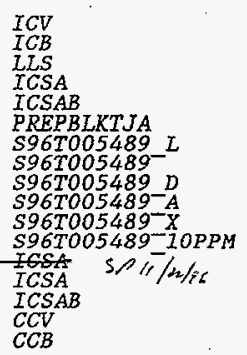 & $\begin{array}{l}5.154 \\
.0288 \\
.1939 \\
-.0056 \\
.0020 \\
.0067 \\
.0886 \\
.1350 \\
.0514 \\
2.693 \\
.0247 \\
253.5 \\
-.0370 \\
.0384 \\
.0204 \\
5.314 \\
.0240\end{array}$ & $\begin{array}{l}4.990 \\
-.0024 \\
2104 \\
250.8 \\
249.6 \\
.0204 \\
.3963 \\
.0891 \\
.0710 \\
2.685 \\
1.989 \\
250.0 \\
250.8 \\
251.9 \\
253.1 \\
5.167 \\
.0020\end{array}$ & $\begin{array}{l}5.079 \\
.0006 \\
.0113 \\
.0011 \\
.9376 \\
.0003 \\
.0047 \\
-.0005 \\
-.0020 \\
2.591 \\
.0253 \\
2471 \\
Q .0034 \\
.0024 \\
.9485 \\
5.161 \\
.0009\end{array}$ & $\begin{array}{l}5.123 \\
-.0019 \\
.2070 \\
.0079 \\
.0171 \\
.0063 \\
-.0152 \\
.0018 \\
-.0233 \\
2.588 \\
.1082 \\
249.4 \\
.0129 \\
.0075 \\
.0103 \\
5.184 \\
.0032\end{array}$ & $\begin{array}{l}5.099 \\
-.0021 \\
.0396 \\
-.0036 \\
.4673 \\
-.0029 \\
-.0141 \\
-.0043 \\
-.0082 \\
2.583 \\
-.0928 \\
.3248 \\
Q-.0032 \\
-.0031 \\
.4726 \\
5.174 \\
-.0038\end{array}$ & $\begin{array}{r}5.100 \\
.0002 \\
.0208 \\
.0069 \\
.4810 \\
.0004 \\
.3755 \\
.3655 \\
.6451 \\
2.963 \\
.3636 \\
249.2 \\
Q .0085 \\
.0068 \\
.4816 \\
5.184 \\
.0001\end{array}$ \\
\hline$\#$ & Sample Name & $C u$ & $E u$ & $\mathrm{Fe}$ & $K$ & La & Li \\
\hline & $\begin{array}{l}I C V \\
I C B \\
L L S \\
I C S A \\
I C S A B \\
\text { PREPBLKTJA } \\
S 96 T 0054899^{-} L \\
S 96 T 005489^{-} \\
S 96 T 0054899^{-} D \\
S 96 T 005489^{-} \\
S 96 T 005489^{-} X\end{array}$ & $\begin{array}{l}5.383 \\
.0009 \\
.0221 \\
.0100 \\
.4865 \\
.0032 \\
.0158 \\
.0044 \\
.0026 \\
2.658 \\
.0336\end{array}$ & $\begin{array}{l}-.0030 \\
.0000 \\
.0010 \\
-.0439 \\
-.0405 \\
.0009 \\
.0056 \\
.0031 \\
.0025 \\
.0014 \\
.0217\end{array}$ & $\begin{array}{l}5.085 \\
-.0004 \\
.1022 \\
94.41 \\
93.60 \\
.0057 \\
-.0070 \\
.0162 \\
.0644 \\
2.562 \\
.0508\end{array}$ & $\begin{array}{l}4.886 \\
.0013 \\
.6132 \\
.0223 \\
.0996 \\
.0832 \\
.8168 \\
1.647 \\
3.080 \\
4.507 \\
4.878\end{array}$ & $\begin{array}{l}5.200 \\
-.0004 \\
.1059 \\
-.0049 \\
-.0037 \\
.0006 \\
.0020 \\
.0001 \\
.0021 \\
2.611 \\
.0403\end{array}$ & $\begin{array}{l}5.242 \\
-.0002 \\
.0215 \\
.0031 \\
i .040 \\
.0036 \\
.0095 \\
.0057 \\
.0060 \\
2.623 \\
.0148\end{array}$ \\
\hline
\end{tabular}




\begin{tabular}{|c|c|c|c|c|c|c|c|}
\hline Ana. & lysis Report & ges & & Thu 11-2 & $9605:$ & $52 P M$ & page 3 \\
\hline \# & Sample Name & $G u$ & $\mathrm{Eu}$ & $F e$ & K & $L a$ & Li \\
\hline $\begin{array}{l}12 \\
19 \\
14 \\
15 \\
16 \\
17\end{array}$ & $\begin{array}{l}S 96 T 005489-10 P P M \\
I C S A \\
I C S A \\
I C S A B \\
C C V \\
C C B\end{array}$ & $\begin{array}{r}-.0414 \\
Q-.0089 \\
-.0089 \\
.4865 \\
5.375 \\
.0009\end{array}$ & $\begin{array}{l}.0538 \\
-.0425 \\
-.0417 \\
=.0431 \\
-.0017 \\
.0009\end{array}$ & $\begin{array}{l}245.7 \\
94.14 \\
94.16 \\
94.15 \\
5.178 \\
.0004\end{array}$ & $\begin{array}{l}24511 \\
-.1092 \\
.1353 \\
.1810 \\
5.059 \\
-.0558\end{array}$ & $\begin{array}{l}252.9 \\
-.0042 \\
-.0049 \\
-.0053 \\
5.276 \\
.0002\end{array}$ & $\begin{array}{r}249.55 \\
0.0030 \\
0.031 \\
1.033 \\
5.165 \\
.0009\end{array}$ \\
\hline$\#$ & Sample Name & $M g$ & $M n$ & Mo & $\mathrm{Na}$ & $\mathrm{Nd}$ & $N i$ \\
\hline $\begin{array}{r}9 \\
10 \\
11 \\
12 \\
119 \\
14 \\
15 \\
16 \\
17\end{array}$ & 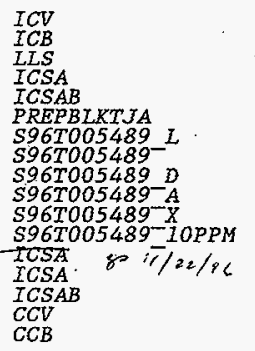 & $\begin{array}{l}4.927 \\
-.0062 \\
.1979 \\
256.0 \\
253.8 \\
.0078 \\
=.0312 \\
.0134 \\
.0001 \\
2.450 \\
.1443 \\
249.1 \\
255.8 \\
254.5 \\
253.2 \\
4.880 \\
-.0024\end{array}$ & $\begin{array}{l}4.941 \\
.0002 \\
.0203 \\
-.0062 \\
.4434 \\
.0006 \\
.0033 \\
.0015 \\
.0020 \\
2.464 \\
.0105 \\
245.6 \\
0-.0061 \\
-.0062 \\
.4476 \\
5.033 \\
.0005\end{array}$ & $\begin{array}{l}5.125 \\
.0022 \\
.0998 \\
-.0107 \\
.0115 \\
.0002 \\
.0431 \\
.0212 \\
.0369 \\
2.641 \\
.0227 \\
250.6 \\
-.0066 \\
=.0115 \\
-.0119 \\
5.205 \\
.0020\end{array}$ & $\begin{array}{l}5.076 \\
-.0017 \\
21663 \\
200.2 \\
198.3 \\
6.170 \\
907.5 \\
890.0 \\
1037.5 \\
889.5 \\
885.4 \\
1138.5 \\
198.5 \\
197.7 \\
196.1 \\
4.965 \\
.0051\end{array}$ & $\begin{array}{l}5.233 \\
.0001 \\
.0050 \\
.0038 \\
.0059 \\
.0017 \\
.0169 \\
.0086 \\
0.0041 \\
2.610 \\
.0601 \\
253.5 \\
.0052 \\
.0061 \\
.0042 \\
5.282 \\
.0023\end{array}$ & $\begin{array}{l}5.058 \\
-.0036 \\
.0434 \\
.0063 \\
.0184 \\
.0019 \\
.01446 \\
.0068 \\
.0122 \\
2.590 \\
.0604 \\
244.9 \\
0-.0036 \\
.0097 \\
.9199 \\
5.127 \\
.0068\end{array}$ \\
\hline$\#$ & Sample Name & $P$ & $P b$ & $s$ & $s b$ & $\mathrm{Se}$ & $S i$ \\
\hline $\begin{array}{r}9 \\
10 \\
11 \\
12 \\
13 \\
14 \\
15 \\
16 \\
17\end{array}$ & 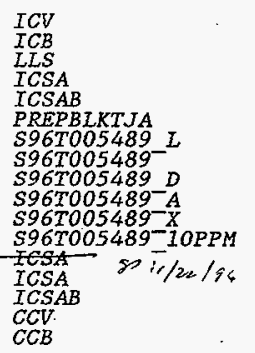 & $\begin{array}{l}5.221 \\
-.0024 \\
.4007 \\
.0134 \\
.0165 \\
.0005 \\
306.5 \\
319.0 \\
138.8 \\
317.6 \\
309.2 \\
563.6 \\
-.0010 \\
.0176 \\
-.0060 \\
5.128 \\
-.0074\end{array}$ & $\begin{array}{l}5.062 \\
.0026 \\
.2042 \\
.0060 \\
.9923 \\
-.0079 \\
-.1949 \\
-.0195 \\
-.0437 \\
2.598 \\
.1606 \\
254.8 \\
0.0206 \\
.0214 \\
1.011 \\
5.144 \\
-.0144\end{array}$ & $\begin{array}{l}5.038 \\
.0055 \\
.2001 \\
-.0355 \\
-.0401 \\
.0420 \\
36.96 \\
37.79 \\
284.9 \\
40.18 \\
37.07 \\
290.3 \\
-.0412 \\
-.0478 \\
.0520 \\
5.025 \\
.0023\end{array}$ & $\begin{array}{l}4.861 \\
.0057 \\
.1127 \\
.0033 \\
.0119 \\
.0118 \\
.1171 \\
.0112 \\
.0228 \\
2.529 \\
5.0400 \\
252.5 \\
.0135 \\
.0106 \\
.0107 \\
4.924 \\
.0106\end{array}$ & $\begin{array}{l}4.927 \\
.0210 \\
.2031 \\
-.0328 \\
-.0311 \\
.0097 \\
.1524 \\
-.0315 \\
-.0044 \\
2.485 \\
.2861 \\
2383 \\
-.0309 \\
-.0259 \\
-.0058 \\
4.967 \\
.0147\end{array}$ & $\begin{array}{l}4.914 \\
.0016 \\
.1413 \\
-.0007 \\
. .0016 \\
1.953 \\
7.136 \\
8.775 \\
62.60 \\
12.16 \\
8.034 \\
265.4 \\
.0138 \\
.0077 \\
.0073 \\
4.972 \\
.0142\end{array}$ \\
\hline
\end{tabular}




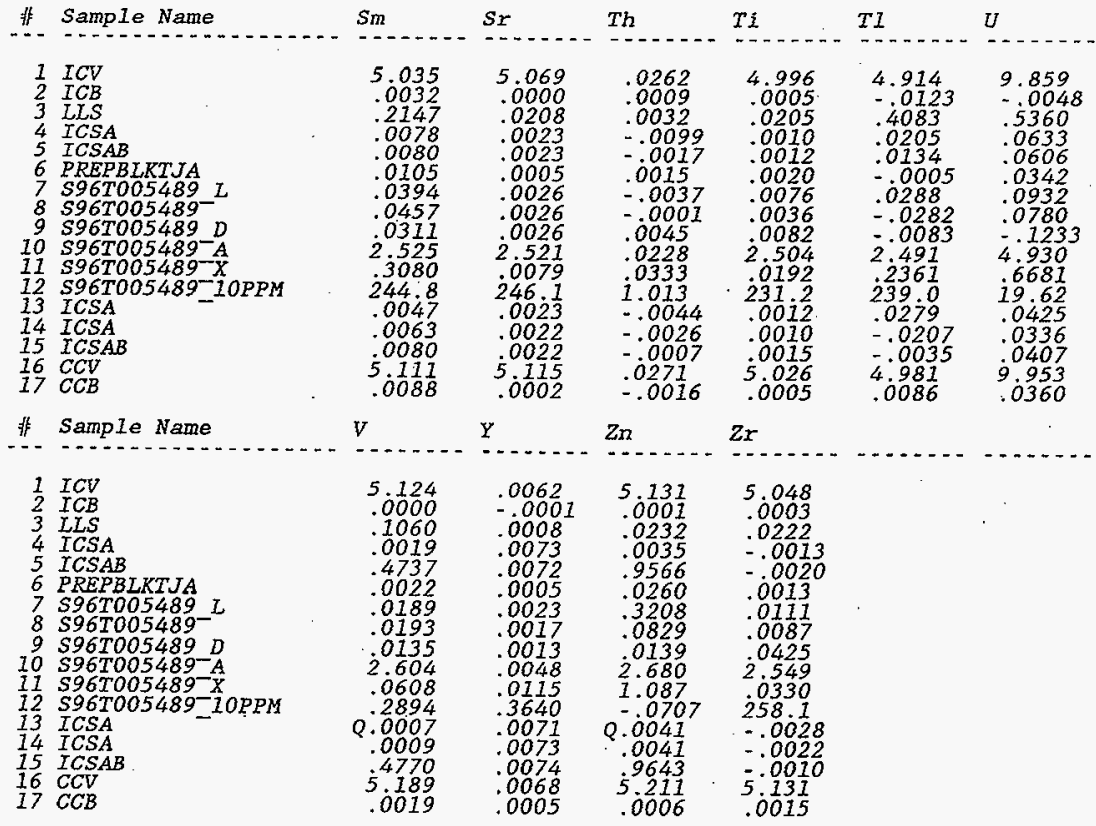




\section{LABCORE Data Entry Template for Worklist\# 15994}

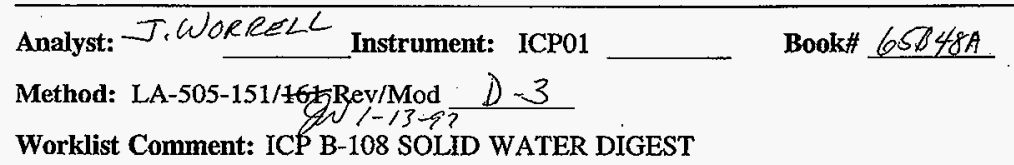

\begin{tabular}{|c|c|c|c|c|c|}
\hline $\bar{s}$ & Type & Sample\# & RA & Test & Matrix \\
\hline 1 & ICV & & & (1CP-QC & $\mathrm{QC}$ \\
\hline 2 & $I C B$ & & & QICP-QC & QC \\
\hline 3 & LLS & & & (1)ICP-QC & $\mathrm{QC}$ \\
\hline 4 & ICSA & & & QICD-QC & $Q C$ \\
\hline 5 & $I C S A B$ & & & QICP-QC & $\mathrm{QC}$ \\
\hline 6 & PREPBLKEF & AR ARL $1 / 1$ & .97 & (QICP-I01 & SOLID \\
\hline 7 & SERDIL & S96T005519 & $0 I$ & @ICP-IOI. & SOLID \\
\hline 8 & SAMPLE & $\begin{array}{l}\text { \$96T005519 } \\
\text { Analytes Reque }\end{array}$ & $\begin{array}{c}0 \mathrm{I} \\
\text { ented }\end{array}$ & $\begin{aligned} \text { QICP-I01 } \\
\text { AG-I-01 } \\
\text { BE-I-01 } \\
\text { CR-I-0I } \\
\text { MG-I-01 } \\
\text { P-I-0I } \\
\text { SM-I-01 } \\
\text { ZN-I-01 }\end{aligned}$ & $\begin{array}{l}\text { SOLID } \\
, \mathrm{AL}-I \\
\mathrm{BI}-\mathrm{CU}-I \\
\mathrm{M} N-I \\
\mathrm{BB}-\mathrm{I} \\
\mathrm{SR}-\mathrm{I} \\
\mathrm{ZR}-\mathrm{I}\end{array}$ \\
\hline 9 & DUP & S96T005519 & $0 I$ & QICP-I01 & SOLID \\
\hline $\begin{array}{l}10 \\
11\end{array}$ & 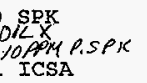 & S96T005519 & $0 I$ & $\begin{array}{l}\text { QICP-I0I } \\
\text { QICP-QC }\end{array}$ & $\begin{array}{l}\text { SOLID } \\
\text { QC }\end{array}$ \\
\hline 22 & ICSAB & & & (-)ICP-QC & $Q \mathrm{C}$ \\
\hline 13 & $\mathrm{CCV}$ & & & . $Q I C P-Q C$ & $Q C$ \\
\hline 14 & $\mathrm{CCB}$ & & & QICP-QC & $\mathrm{QC}$ \\
\hline
\end{tabular}

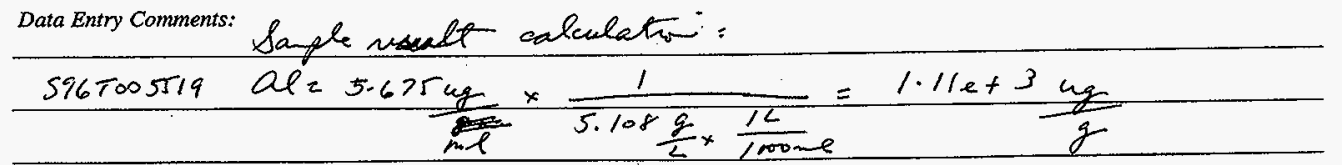

$S=$ Worklist Slot Number, $R=$ Replicate Number, $A=$ Aliquot Code.

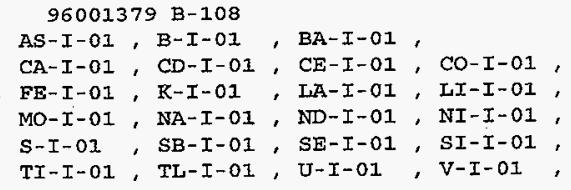

$C-Q C \quad Q C$ 
HNF-SD-WMV-DP-219, REV .O

$01 \% 06 / 97714: 33$

Page: 2

A-0004-1

LABCORE Data Entry Template for Worklist\# 15994

S Type Sample\# RA Test Matrix Group\# Project

Final page for worklist \# 15994

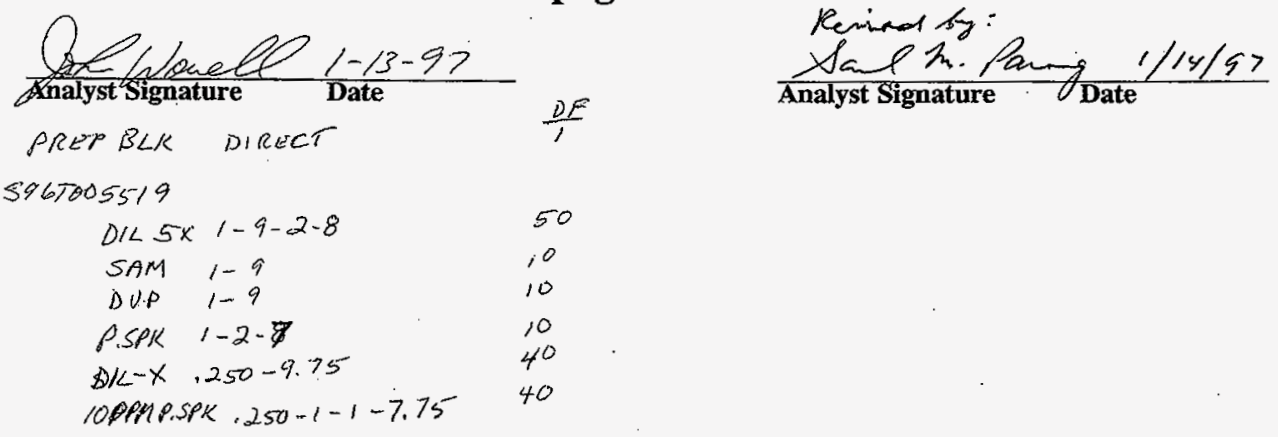

P, S, an Si failed in the first post spike. A second post

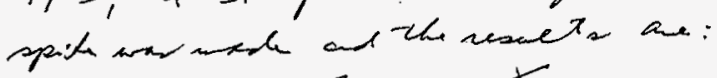

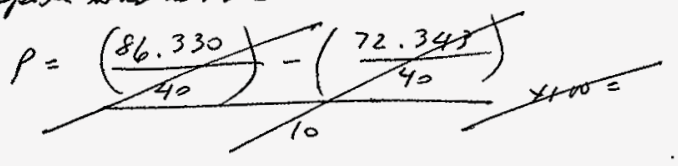

$$
\begin{aligned}
& P=\frac{\left(\frac{510.516}{40}\right)-\left(\frac{93.629}{40}\right)}{10} \times 10=104.2 \% \\
& S=\frac{\left(\frac{708.299}{40}\right)-\left(\frac{316.410}{40}\right)}{10} \times 100=98.0 \% \\
& S_{i}=\frac{\left(\frac{517.340}{40}\right)-\left(\frac{107.250}{40}\right)}{10} \times 100=102.0 \%
\end{aligned}
$$

Data Entry Comments: Spike recovery cal curation:

$5967005719 a l=\left(\frac{15.423}{10}\right)-\left(\frac{5.675}{12}\right) \times 100=97.5 \%$

$S=$ Worktist Slot Number, $R=$ Replicate Number, $A=$ Aliquot Code.

368

7000

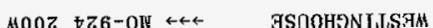

6Z67. ZIS GOG.O to: 


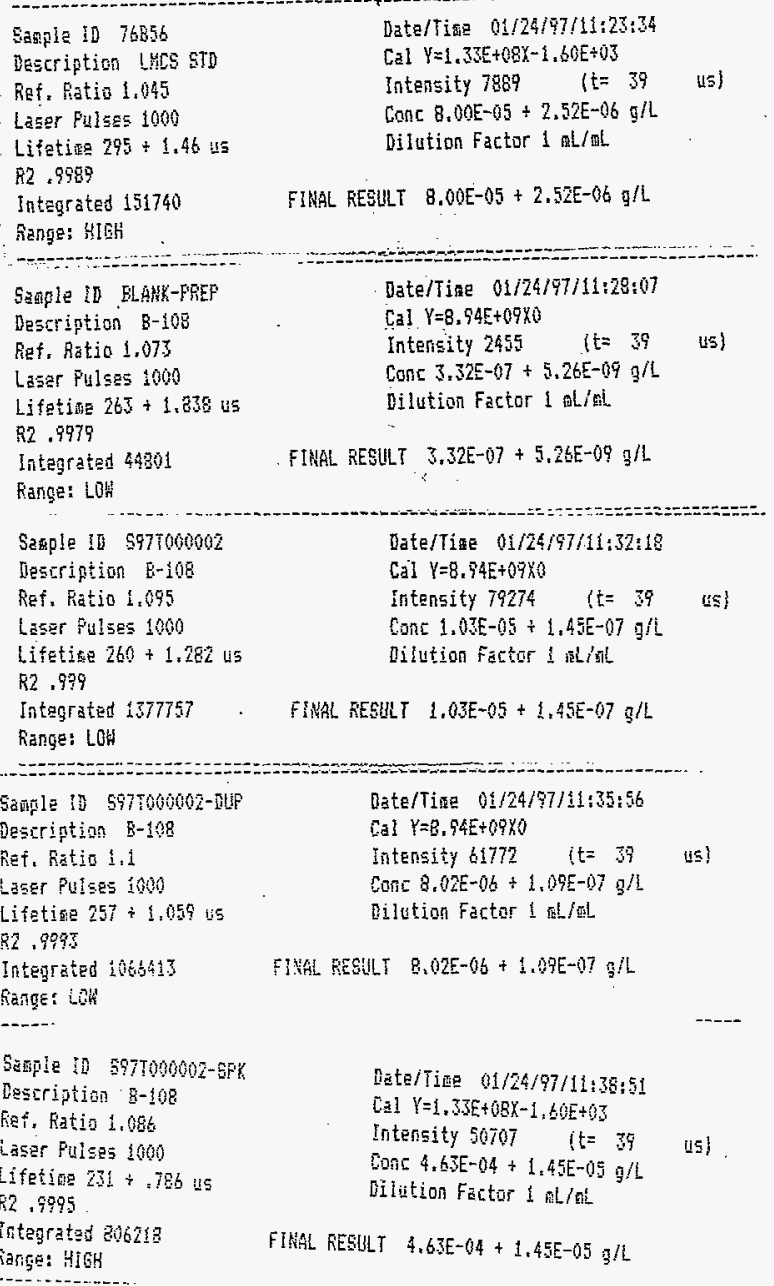

